\title{
Summary of Radioactive Solid Waste Received in the 200 Areas During Calendar Year 1993
}

\author{
J. D. Anderson \\ D. L. Hagel \\ Date Published \\ September 1994
}

Prepared for the U.S. Department of Energy Office of Environmental Restoration and Waste Management

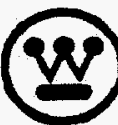

Westinghouse P.O Box 1970 Hanford Company Richland, Washington

Henford Operations and Engineering Contractor for the

U.S. Department of Energy under Contract DE-ACO6-87RL10930 


\section{RELEASE AUTHORIZATION}

Document Number: WHC-EP-0125-6

Document Title: Summary of Radioactive Solid Waste Recevied in the 200 Areas During Calendar Year 1993

Release Date: $\quad 9 / 7 / 94$

This document was reviewed following the procedures described in WHC-CM-3-4 and is:

APPROVED FOR PUBLIC RELEASE

WHC Information Release Administration Specialist:
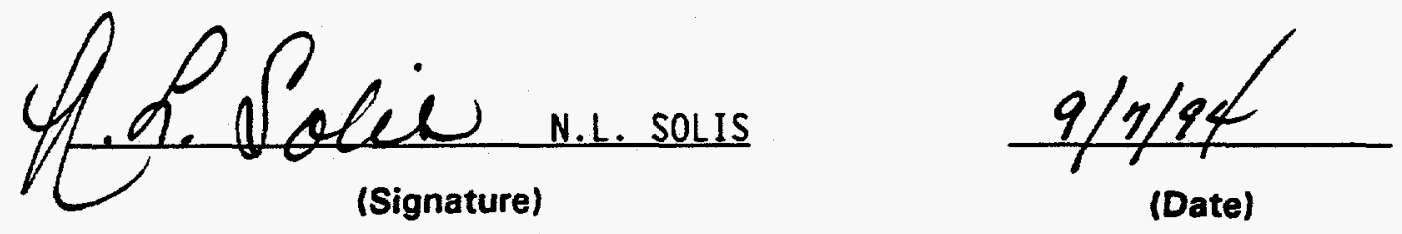


\section{DISCLAIMER}

This report was prepared as an account of work sponsored by an agency of the United States Government. Neither the United States Government nor any agency thereof, nor any of their employees, make any warranty, express or implied, or assumes any legal liability or responsibility for the accuracy, completeness, or usefulness of any information, apparatus, product, or process disclosed, or represents that its use would not infringe privately owned rights. Reference herein to any specific commercial product, process, or service by trade name, trademark, manufacturer, or otherwise does not necessarily constitute or imply its endorsement, recommendation, or favoring by the United States Government or any agency thereof. The views and opinions of authors expressed herein do not necessarily state or reflect those of the United States Government or any agency thereof. 


\section{DISCLAIMER}

Portions of this document may be illegible in electronic image products. Images are produced from the best available original document. 
Document Title: Summary of Radioactive Solid Waste Received in the 200 Areas During Calendar Year 1993

Prepared by:

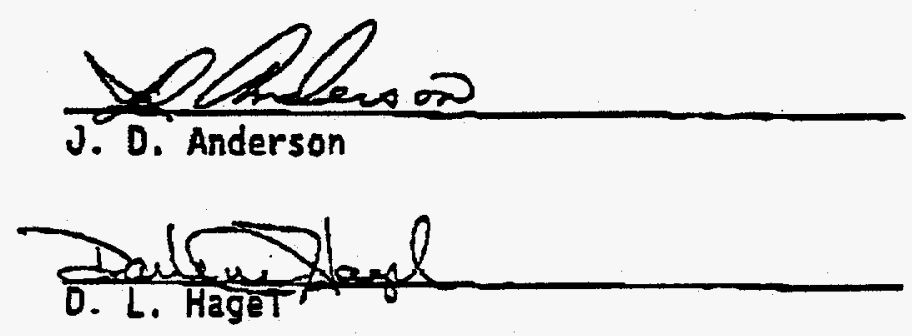

Reviewed by:
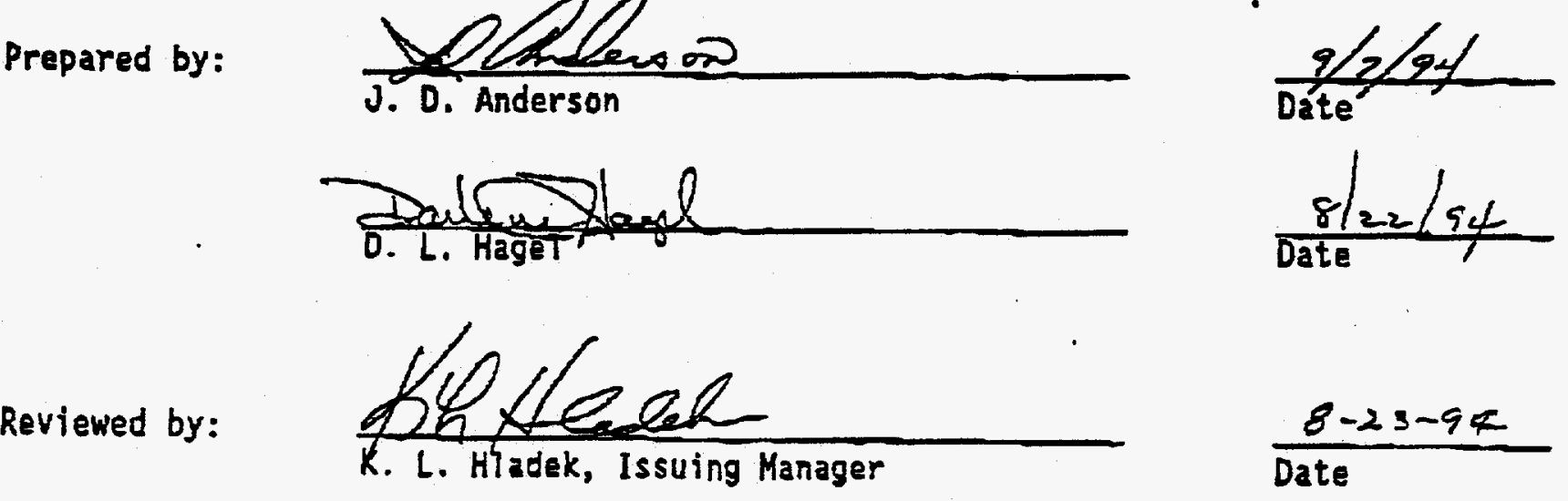

$\frac{8-23-9 k}{\text { Date }}$

Miminccarsen

M. M. HeCarthy, Program dfice

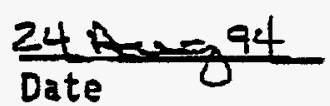


WHC-EP-0125-6

This page intentionally left blank. 


\title{
SULQLAY OF RADIOACTIVE SOLID WASTE \\ RECEIVED IN THE 200 MREAS DURING \\ CALELTAR YEAR 1993
}

\section{J. D. Andergon}

D. L. Hagel

\begin{abstract}
westinghouse Hanford Company manages and operates the Hanford Site 200 Areas radioactive solid waste storage and disposal facilities for the U.S. Department of Energy, Richland Operations office, under contract DE-ACO6-87RL10930. These facilities include radioactive solid waste disposal sites and radioactive solid waste storage areas. This document summarizes the amount of radioactive materials that have been buried and stored in the 200 Areas radioactive solid waste storage and disposal facilities since startup in 1944 through calendar year 1993. This report does not include backlog waste, solid radioactive waste in storage or disposed of in other areas, or facilities such as the underground tank farms. UnIess packaged within the scope of WHC-EP-0063, "Hanford Site Solid Waste-Acceptance Criteria," (WHC 1988), liquid waste data are not included in this document.
\end{abstract}


WHC-EP-0125-6

This page intentionally left blank. 
WAC-EP-0125-6

CONHENTE

Solid Waste Burial Ground Areas Waste Volumes Disposed and Stored Nondecayed Curie Content in the 200 areas from startup to December 31, 1993 . . . . . . . . . . . . . . . . . . . . . . . . . .

Solid Waste Burial Ground Areas Decayed Curie content in the 200 Areas from startup to December 31, $1993 . .$. . . . . . . . . . . . 30

Unsegregated Waste Burial Ground Areas Waste Volumes Buried and Nondecayed Curie Content from Startup through December 31, 1993 . . . .

Unsegregated Haste Burial Ground Areas waste Volumes Buried and Decayed Curie Content from startup through December 31, $1993 . . . . . . . .51$

Post-1970 Nontransuranic Waste Burial Ground Areas Waste Volumes Buried or Stored and Nondecayed Curie Content through

December 31, 1993 . . . . . . . . . . . . . . . . . . . . . . .

Post-1970 Nontransuranic Waste Burial Ground Areas Decayed Curie Content through December 31, 1993 . . . . . . . . . . . . . . . . . . . .

Post-1970 Segregated Transuranic Waste Burial Ground Areas Waste Volumes Stored and Nondecayed Curie Content through December 31, 1993 . . . . . . . . . . . . . . . . . . . . . 119

Poat-1970 Segregated Transuranic Waste Burial Ground Areas Decayed Curie Content through December 31, 1993 . . . . . . . . . . . . . . . 131

Transuranic and Nontransuranic Waste Received for Disposal or Storage for the Calendar Year 1993 . . . . . . . . . . . . . . . . . . 137

Status of Individual Burial Grounds and Facilities Land Disposal or Storage from January 1 to December 31, 1993:

Burial Ground $218 \mathrm{E} 10$

Burial Ground 218E12B 161

Burial Ground 218พ3A 162

Burial Ground 218W3AE 166

Burial Ground $218 \% 4 \mathrm{C}$ 168

Burial Ground 218 w5 174

Transuranic Waste Storage and Assay Facility (TRUSAF) • . . . . . 196

Central Waste Complex 2401W Building . . . . . . . . . . . . . . . 198

Central Waste Complex 2402w Building . . . . . . . . . . . . . 199

Central Waste Complex 2402WB Building . . . . . . . . . . . . . 200

Central Waste Complex 2402wC Building . . . . . . . . . . . . 201

Central waste Complex 2402WG Building . . . . . . . . . . . . . 205

Central Waste Complex 2402WH Building . . . . . . . . . . . . . . 212 


\section{COKHYANYs (cont.)}

Central waste complex 2402\%L Building . . . . . . . . . . . . . . 214

Central Waste complex 2403 wa Building . . . . . . . . . . . . . . 218

Central Waste Complex 2403 WC Building . . . . . . . . . . . . . . 227

Central Waste complex 2403 w Building . . . . . . . . . . . . . . 237

Flammable storage Module 1 . . . . . . . . . . . . . . . . . . 243

Flammable storage Module 11 . . . . . . . . . . . . . . . . . 244

Flammable storage Module 12 . . . . . . . . . . . . . . . . . 246

Flammable storage Module 13 . . . . . . . . . . . . . . . . 248

Flammable storage Module 15 . . . . . . . . . . . . . . . . 250

Flamable storage Module 3 . . . . . . . . . . . . . . . . . . 252

Flammable storage Module 6 . . . . . . . . . . . . . . . . . . 254

Flammable Storage Module 7 . . . . . . . . . . . . . . . . 255

Flammable storage Module 8 . . . . . . . . . . . . . . . . . 256

Grand Totals for Land Disposal or Storage from January 1

to December 31, 1993 . . . . . . . . . . . . . . . . . . . 258

References . . . . . . . . . . . . . . . . . . . . . . . . 259

Appendix A

200 Area Solid Waste storage/Disposal Facility-Coordinates . . . . 261

Appendix B

Burial Ground/Storage Facilities Land Usage . . . . . . . . . . . 267 


\section{IMHRODUCTIOA}

This document summarizes the amount of radioactive materials that have been buried and stored in the 200 Areas burial ground facilities during calendar year 1993 (see Table 1). In addition to the data for calendar year 1993, cumulative data are presented. This document also lists decayed activity for the plant burial sites. It should be noted that storage locations listed in this report are current only on the run date identified at the top of the data sheets, as these locations are subject to frequent change.

Table 1. Summary of Document Data.

\begin{tabular}{|c|c|}
\hline Pages & Data \\
\hline $3-29$ & $\begin{array}{l}\text { Amount of radiouctive materials buried and stored within } \\
\text { the Westinghouse Hanford Company's } 200 \text { Area plateau since } \\
\text { startup }\end{array}$ \\
\hline $30-48$ & $\begin{array}{l}\text { Listing of the decayed activities in the various burial } \\
\text { ground facilities from atartup through calendar year } 1993\end{array}$ \\
\hline $49-50$ & $\begin{array}{l}\text { Estimate of the radioactive materials in the unsegregated } \\
\text { burial sites }\end{array}$ \\
\hline $51-56$ & Decay status of unsegregated waste from startup to present \\
\hline $57-92$ & $\begin{array}{l}\text { Amount of radioactive material in the nontransuranic } \\
\text { disposal or otorage facilities }\end{array}$ \\
\hline $93-118$ & Decay status of nontransuranic waste from 1970 to present \\
\hline $119-130$ & $\begin{array}{l}\text { Amount of radioactive material in the transuranic } \\
\text { segregated storage sites }\end{array}$ \\
\hline $131-136$ & $\begin{array}{l}\text { Decay atatus of transuranic segregated waste from } 1970 \text { to } \\
\text { present }\end{array}$ \\
\hline $137-159$ & $\begin{array}{l}\text { Summary of waste received for disposal or storage for } \\
\text { calendar year } 1993\end{array}$ \\
\hline $160-258$ & $\begin{array}{l}\text { Amount of waste received for each burial ground and } \\
\text { storage facility for calendar } 1993\end{array}$ \\
\hline
\end{tabular}

It should be noted that the summation of ${ }^{90} \mathrm{Sr},{ }^{100} \mathrm{Ru},{ }^{137} \mathrm{Cs}$, and other radionuclides does not equal the total curie amount. The difference is the amount of other mixed fission products present in the burials that are not noted in this report. The reported curie values do not include the activities for the radioactive daughters. The data for plutonium for waste disposed before 1970 could be biased low by about 300 kilograms.

This document was prepared using the solid waste Information and Tracking Sygtem database (SWITS), which results in more accurate and updated data. Radiolsotopic data are based on the book, "Table of Isotopes," seventh Edition, published by wiley-Interecience. As a reault, oome of the data are different from previous editions.

Some numbers for total decayed curies in the 1992 report (WHC 1993) show an increase in value from the 1992 report (Wic 1992). This resulted from previously excluding the presence of daughter producta produced in the decay process. Thus, the values were low by this amount. Facility names were changed to reflect Hanford site etandard naming conventions. In 1993 land 
usage data were removed from the report data and placed in tables in Appendix B for ease of use.

The computer-generated data in the tables are presented using exponential notation. An example of this form is presented below:

$$
\begin{aligned}
& 2.41 \mathrm{E}+06=2.41 \times 10^{+6}=2,410,000 \\
& 2.41 \mathrm{E}-06=2.41 \times 10^{-6}=0.00000241
\end{aligned}
$$

Footnotes for 100 Ares waste are offset 1 isted for convenience in the OTHER RADIOACTIVITY (CURIES) column for information purposes, but are not included in the totals for OTHER RADIOACTIVITY radionuclides. The ${ }^{15} \mathrm{Cs}$ is included in the ${ }^{137} \mathrm{Cs}$ column, however. An example follows:

\section{OTHER RADIOACTIVITY (CURIES)}

$\begin{array}{lll}\text { C-14 } & 5.660 E+02 & \text { (OTHER TOTAL) } \\ \text { CO-60 } & 1.234 \mathrm{E}+01 & \text { (OTHER) } \\ \text { CS137 } & 5.537 \mathrm{E}+02 & \text { (OTHER) } \\ \text { CO58 } & 2.356 \mathrm{E}+03 & (105 \mathrm{~N}) \\ \text { CO60 } & 1.343 \mathrm{E}-07 & (105 \mathrm{~N}) \\ \text { EU155 } & 4.566 \mathrm{E}+01 & (105 \mathrm{~N}) \\ \text { C14 } & 1.846 \mathrm{E}+00 & (105 \mathrm{~N}) \\ \text { EU154 } & 1.249 \mathrm{E}+00 & (105 \mathrm{~N}) \\ \text { FE59 } & 8.697 \mathrm{E}+00 & (105 \mathrm{~N}) \\ \text { MN54 } & 4.619 \mathrm{E}-11 & (105 \mathrm{~N}) \\ \text { EU152 } & 1.964 \mathrm{E}-01 & (105 \mathrm{~N}) \\ & 9.573 \mathrm{E}-01 & (105 \mathrm{~N})\end{array}$


SUIR328A - (Page 1 of 8 )

RUNDATE: 08/20/94

Solid Waste Burial Ground Areas Waste Volumes Disposed and Stored and

Nondecayed Curie Content in the 200 Areas from Startup to December 31, 1993, Including segregated Transuranic Waste.

BURIAL GROUND FACILITIES

2OOE AREA

-........

BURIAL GROUND 218E1

BURIAL GROUND 218E10

BURIAL GROUND 218E12A

BURIAL GROUND 218E12B
VOLUME VOLUME (CUBIC M) (CUBIC M)

-..........

(CUBIC MI

$3.030 E+03$

$2.186 E+04$

$1.525 \mathrm{E}+04$

9.219E+04

$3.030 E+03$

$2.186 E+04$

$1.525 E+04$

$9.219 E+04$
URANIUNA

(GRAMS)

4.000E+05

$8.010 E+05$

$9.900 E+05$

$2.499 E+05$
PLUTONIU

(GRAMS)

(G...........

$9.000 E+02$

$4.941 E+03$

$8.930 E+03$

$1.552 E+03$
PUREX TUNNEL (\#1) 218E14

PUREX TUNNEL (\#2) 218E15

BURIAL GROUNO 218E2

BURIAL GROUND 218E4

BURIAL GROUND 218E5

BURIAL GROUND 218E5A

BURIAL GROUND $218 E 8$

BURIAL GROUND 218EC9

222B VAULTS

Total $200 E$
$5.666 \mathrm{E}+02$

$1.140 E+03$

$9.033 E+03$

$1.586 E+03$

$3.172 E+03$

$6.173 E+03$

$2.265 E+03$

$7.582 E+03$

$1.699 E+02$

$1.640 E+05$
$5.666 E+02$

$1.140 \mathrm{E}+03$

$9.033 E+03$

$1.586 E+03$

$3.172 E+03$

$6.173 E+03$

$2.265 E+03$

$7.582 E+03$

$1.699 E+02$

$1.640 E+05$
$.000 E+00$

$.000 E+00$

3.000E+05

$1.000 E+03$

$1.200 E+05$

$1.200 E+05$

2. $000 E+03$

$.000 E+00$

$1.000 E+03$

$2.985 E+06$
OTHER RADIOACTIVITY (GRAMS)

$\begin{array}{rr}.000 E+00 \\ .000 E+00 \\ \\ .000 E+00 \\ 1.241 E-02 \\ N M-241 & 9.561 E-03 \\ C H-244 & 2.920 E-06 \\ P U-239 & 2.012 E-03 \\ P U-240 & 5.495 E-04 \\ P U-241 & 8.673 E-05 \\ T H-232 & 2.000 E-04 \\ & .000 E+00 \\ & .000 E+00 \\ & .000 E+00 \\ & .000 E+00 \\ & .000 E+00 \\ .000 E+00 \\ .000 E+00 \\ .000 E+00 \\ .000 E+00 \\ .001 \\ 1.241 E-02\end{array}$


SWIR328A - (Page 2 of 8 )

RUNDATE: OB/20/94

Solid Waste Burial Ground Areas Waste Volumes Disposed and stored and Nondecayed Curie Content in the 200 Areas from Startup to December 31, 1993, Including Segregated Transuranic Waste.

BURIAL GROUND FACILITIES
2OOW AREA

212N BUILOING

BURIAL GROND 218W1

BURIAL GROUND 218W11

BURIAL GROUND 218W1A

BURIAL GROUND 218W2

BURIAL GROUND 218H2A

BURIAL GROUND $218 \mathrm{H} 3$

BURIAL GROUND 21813A

VOLUME
(CUBIC M)

PACKED

VOLUME

..........

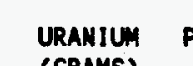

(GRAMS)

PLUTONIUN (GRAMS) (G........

\subsection{E+02}

7.164E+03

$1.160 E+03$

$1.371 E+04$

8.240E+03

$2.514 E+04$

$1.090 E+04$

1.006E+05

2.167E+

7.164E +03

1.160E+03

$1.371 \mathrm{E}+04$

8.240E+03

$2.514 E+04$

$1.090 \mathrm{E}+04$

$1.006 E+05$

$1.000 E+0$

7.000E+05

$.000 E+00$

9.000E+05

$1.400 E+06$

2. $690 E+06$

$7.000 E+07$

$7.240 E+07$

4.280E+0

$9.402 E+04$

$.000 E+00$

2.000E+03

1.260E+05

$6.384 E+03$

$6.800 E+04$

$2.931 E+04$

$A M-241$
$A M-243$

CF-252

$\mathrm{CM}-243$

$\mathrm{CM}-244$

H -2

LI -6

WP -237

PU-238

PU-239

PU-240

PU-241

PU-242

RA-226

TH-228

TH-232

TH-234

U-233

U. 234

U. 235

U-238

BURIAL GROUND 218W3AE

$2.208 E+04$

$2.208 E+04$

$2.656 E+07 \quad 1.222 E+02$

AM-241

CM-242

CM-244

NP-237

PU-238

PU-239

PU -240

PU-241

TH-232

U. 233

BURIAL GROUND 218W4A

BURIAL GROUND 218W4B

BURIAL GROUND 218W4C

$\begin{array}{llll}1.771 E+04 & 1.771 E+04 & 3.941 E+08 & 3.538 E+04 \\ 1.046 E+04 & 1.046 E+04 & 5.893 E+06 & 6.634 E+04\end{array}$

$1.046 E+0$

$5.893 E+06$

$6.634 E+04$

TH-232

NM-241

LI -6

NP- 237

PU-238

TH-232

$\mathrm{U}-233$

$1.642 E+04 \quad 1.642 E+04 \quad 1.619 E+07 \quad 3.833 E+05$
MM-241

$M A-243$

CF-252

CH-242

CM-245
$1.000 E+00$

$1.000 E+00$

$.000 E+00$

$.000 E+00$

$.000 E+00$

$.000 E+00$

$.000 E+00$

$6.000 E+07$

$6.000 E+07$

$1.134 E+06$

$2.280 E+01$

5.007E-03

3.730E-08

1.000E-06

3.050E-05

$2.025 E+03$

$1.636 \mathrm{E}+04$

$1.516 E+01$

$2.113 E+00$

2.064E-04

8.693E-01

4.460E-05

$3.490 E-13$

$2.595 E+00$

1.619E-09

$1.115 E+06$

5.672E-09

$2.022 E+02$

4.330E-04

1.890 E-02

$3.667 \mathrm{E}+02$

$3.063 E+04$

$1.015 E+00$

$9.200 \mathrm{E}-08$

5.862E-08

2.030E-02

$1.060 E-04$

$1.801 E-02$

5.150E-06

8.240E-07

$3.062 E+04$

1.000E+00

$2.500 E+08$

$2.500 E+08$

$6.784 E+05$

1.210E +00

2.007E+03

2.140E+02

$1.432 E+00$

$6.685 \mathrm{E}+05$

$7.607 E+03$

$9.736 E+04$

$1.528 E+02$

5.276E +01

2. $000 E+00$

3.800E-07

$4.822 E+01$ 


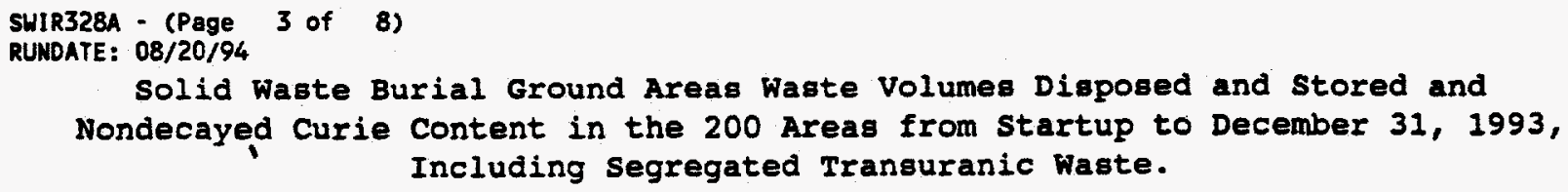

BURIAL GROND FACILITIES

BURIAL GROUND 218W4C

BURIAL GROUND 218W5
$4.856 E+02$

$1.586 \mathrm{E}+02$

$6.796 E+01$

$2.464 E+02$
$4.856 E+02$

$1.586 \mathrm{E}+02$

$6.796 \mathrm{E}+01$

$2.464 E+02$
$.000 E+00$

$7.000 E+02$

$3.000 E+02$

$4.872 E+04$
$.000 E+00$

7.000E-01

3.000E-01

4. $775 E+04$ $\begin{array}{llll}4.455 E+04 & 4.455 E+04 & 2.792 E+07 & 1.681 E+02\end{array}$

PACKED

VOLUME

(CUBIC M)

(n)

URANIUM PLUTONIUN

(GRAMS)

-..........

(GRAMS)

(Ge-......

222S VAULTS

222T VAULTS

TRANSURANIC WASTE STORAGE \& ASSAY

FACILITY(TRUSAF)
$M-241$

AM-243

Cris -243

CH-244

Cin-245

NP-237

PU-238

PU-239

PU-240

PU-241

PU-242

TH-228

TH- 232

U-233
(GRAMS)

$5.900 E+00$

$1.401 E+02$

5.244E+03

5.176E+01

$1.901 E+00$

2.429E-02

7.791E-02

2.480E-02

8.726E+04

8.373E+02

4.040E-04

1.766E-02

$3.566 \mathrm{E}+03$

2. $195 E+06$

3.623E-0

$1.741 \mathrm{E}-02$

$1.838 E-06$

$6.306 E-09$

2.503E-02

$1.659 E-04$

3.148E-05

5.151E-03

1.014E-05

$1.848 E-20$

$1.548 E+01$

$7.358 E+00$

9.151E-01

$9.876 E-02$

1.149E-02

3.190E-02

3.125E-03

$2.915 E+00$

5. $755 E-02$

2.160E+06

5.001E-03

$1.503 E-03$

$1.389 E-01$

$1.209 E+01$

$6.113 E+01$

2. $265 E+00$

$3.454 E+04$

$.000 E+00$

$.000 E+00$

$.000 E+00$

$2.252 E+03$

$3.151 E+01$

5.700E-07

1.287E-03

$1.373 E+00$

$4.998 E+01$

2. $092 E+00$

5.952E-01

$1.922 E+03$

$1.316 E+02$

$1.020 E+01$

2. $041 E+00$

5.375E-04

$1.000 E+02$

2.000E-01 


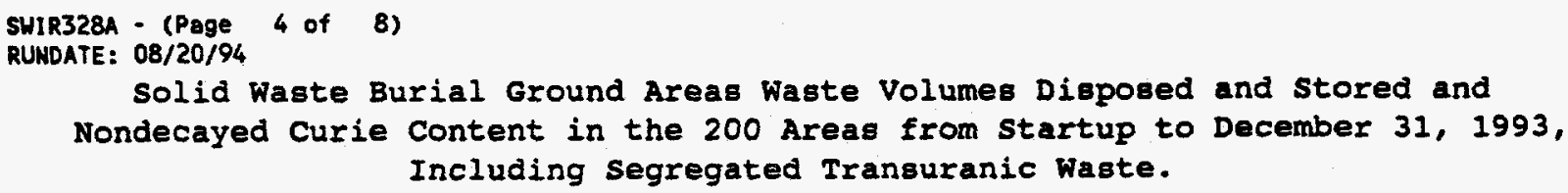

BURIAL GROUHD FACILITIES

CENTRAL WASTE COMPLEX 24014 BUILDING CENTRAL WASTE COMPLEX 2402Y BUILDING

CENTRAL MASTE COMPLEX 2402WB BUILDING

CENTRAL WASTE COMPLEX 2402WC BUILDING

CENTRAL WASTE COMPLEX 240ZWD BUILDING

CENTRAL HASTE COMPLEX 2402WE BUILDING

CENTRAL WASTE COMPLEX 2402WF BUILDING CEMTRAL MASTE COMPLEX 2402WG BUILOING

\begin{tabular}{cccc}
$\begin{array}{c}\text { VOLUME } \\
\text { (CUBIC M) }\end{array}$ & $\begin{array}{c}\text { PACKED } \\
\text { VOLUME }\end{array}$ & $\begin{array}{c}\text { URANILM } \\
\text { (CUBIC M) }\end{array}$ & $\begin{array}{c}\text { PLUTONIUN } \\
\text { (GRAMS) }\end{array}$ \\
\hdashline $1.179 M S$ ) \\
\hdashline $1.179 E+02$ & $1.179 E+02$ & $.000 E+00$ & $9.863 E+02$ \\
$2.554 E+02$ & $2.554 E+02$ & $1.083 E+05$ & $2.418 E+02$
\end{tabular}

$1.326 E+02$

$5.280 E+03$

$2.182 E+02$
$5.009 E+0$

$5.009 E+01$

$5.663 E+02$

$2.781 E+02$

$\begin{array}{llll}1.926 E+02 & 1.926 E+02 & 2.774 E+04 & 1.000 E-06 \\ 1.248 E+00 & 1.248 E+00 & 1.004 E-01 & .000 E+00 \\ & & & \\ 2.207 E+02 & 2.207 E+02 & 5.463 E+04 & .000 E+00 \\ 1.097 E+02 & 1.097 E+02 & 3.751 E+01 & 2.520 E+02\end{array}$

$1.097 \mathrm{E}+02$

$3.751 E+01$

$2.520 \mathrm{E}+02$

OTHER RADIOACTIVITY (GRAMS)

$\begin{array}{ll} & .000 E+00 \\ & 1.000 E+01 \\ \text { NH-241 } & 1.740 E-07 \\ \text { PU-238 } & 4.600 E-08 \\ \text { PU-239 } & 1.470 E-04 \\ \text { PU-240 } & 8.980 E-06 \\ \text { PU-241 } & 7.330 E-07 \\ \text { PU-242 } & 3.080 E-08 \\ \text { TH-232 } & 1.000 E+01 \\ \text { TH-234 } & 2.100 E-12\end{array}$

$2.100 E-12$

AM-241 1.503E-05

MP- 237 7.450E-02

PU-238 1.969E-06

PU-239 3.829E-03

PU-240 2.300E-04

PU-241 1.003E-04

PU-242 6.702E-07

RA-224 3.306E-09

RA-226 5.597E-06

TH-228 5.543E-07

TH-232 6.040E+03

TH-234 6.819E-08

U-234 $2.885 E-05$

U-235 1.261E-03

U-238 2.756E-02

$7.922 E+00$

AM-241 1.289E-04

CM-242 1.179E-15

CM-243 1.200E-10

CM-244 6.010E-10

NP-237 4.920E-01

PU-238 2.200E-03

PU-239 6.937E+00

PU-240 4.475E-01

PU-241 4.030E-02

PU-242 2.700E-03

U-234 4.410E-09

$U-235 \quad 1.040 E-08$

U-238 2.800E-07

$2.800 E-07$
$1.000 E-06$

AM-241 1.000E-06

2.430E-09

CM-242 $1.020 E-16$

PU-241 2.430E-09

$.000 E+00$

4.064E+01

NM-241 4.504E-02

CM-242 5.500E-06

PU-238 1.171E-02

PU-239 3.754E+01

PU-240 2.286E+00

PU-241 2.859E-01

PU-242 7.806E-03

RA-226 2.500E-01

U-234 3.112E-05

U-235 2.373E-03

U-236 $1.420 E-04$

u-238 2.107E-01 
SUIR328A - (Page 5 of 8 )
RUNDATE: 08/20/94

Solid Waste Burial Ground Areas Waste Volumes Disposed and Stored and Nondecayed Curie Content in the 200 Areas from startup to December 31, 1993, Including Segregated Transuranic Waste.

BURIAL GROUND FACILITIES

CENTRAL WASTE COMPLEX $2402 \mathrm{HH}$ BUILOING

CENTRAL MASTE COMPLEX 2402WI BUILDING

CENTRAL WASTE COMPLEX 2402WJ BUILDING CENTRAL WASTE COMPLEX 2402WL BUILDING

CENTRAL WASTE COMPLEX 2403WA BUILDING

CENTRAL HASTE COMPLEX 2403UC BUILDING

\begin{tabular}{cccc}
$\begin{array}{c}\text { VOLLME } \\
\text { (CUBIC M) }\end{array}$ & $\begin{array}{c}\text { PACKED } \\
\text { VOLUME } \\
\text { (CUBIC M) }\end{array}$ & $\begin{array}{c}\text { URANIUM } \\
\text { (GRAMS) }\end{array}$ & $\begin{array}{c}\text { PLUTONIUM } \\
\text { (GRAMS) }\end{array}$ \\
\hline $2.371 E+01$ & $2.371 E+01$ & $1.001 E+03$ & $1.031 E+02$
\end{tabular}

2.371E+01

$2.371 E+01$

$1.001 E+03 \quad 1.031 E+02$

$\begin{array}{llll}2.129 E+02 & 2.129 E+02 & 6.310 E+04 & .000 E+00 \\ & & & \\ 1.891 E+02 & 1.891 E+02 & 2.764 E+04 & .000 E+00 \\ 5.698 E+01 & 5.698 E+01 & 3.299 E-01 & 3.566 E+00\end{array}$

$1.822 E+03 \quad 1.822 E+03 \quad 1.512 E+06 \quad 7.054 E+02$

8.627E+02

$8.627 E+02$

$3.611 E+03$

$1.736 E+03$
OTHER RADIOACTIVITY (GRAMS)

$\begin{array}{ll}M M-241 & 7.528 E+00 \\ \text { CH-242 } & 5.782 E-09 \\ \text { PU-238 } & 1.030 E-16 \\ \text { PU-239 } & 4.038 E-00 \\ \text { PU-240 } & 2.445 E-07 \\ \text { PU-241 } & 8.323 E-07 \\ \text { PU-242 } & 8.370 E-10 \\ \text { RA-226 } & 4.520 E-02 \\ \text { TH-232 } & 1.000 E+00 \\ \text { TH-234 } & 8.620 E-17 \\ U-234 & 5.000 E-04 \\ U-235 & 5.100 E-02 \\ U-236 & 1.170 E-02 \\ U-238 & 5.390 E+00 \\ & 1.264 E-08 \\ \text { CH-242 } & 5.310 E-16 \\ \text { PU-241 } & 1.264 E-08 \\ & .000 E+00 \\ & 7.068 E-03\end{array}$

CH-242 $3.926 \mathrm{E}-12$

CM-243 1.803E-13

CM-244 2.000E-12

NP-237 5.701E-06

PU-238 1.002E-05

PU-239 7.878E-05

PU-240 1.436E-05

PU-241 6.731E-04

PU-242 6.660E-09

$R A-226 \quad 7.690 E-10$

TH-234 2.020E-95

U-234 $6.220 E-06$

U-235 2.720E-04

U-238 $\quad 5.950 E-03$

$1.290 E+03$

AM-241 1.150E+00

AM-243 2.715E-03

CH-242 5.502E-09

CH-243 1.203E-07

CH-244 2.792E-09

NP-237 7.115E+00

PU-238 7.324E-03

PU-239 $\quad 5.200 E+01$

PU-240 $1.681 E+00$

PU-241 8.781E-01

PU-242 9.253E-03

RA-226 1.146E-02

TH-228 1.443E-07

TH-232 4.959E+02

TH-234 1.030E-06

U-233 1.711E-04

U-234 5.020E-02

U-235 $5.280 E+00$

U-236 1.420E-04

U-238 $\quad 7.260 E+02$

$1.716 E+04$

$\begin{array}{ll}\text { AM-243 } & 2.770 E-04\end{array}$ 
SWIR328A - (Page 6 of 8 )

RUHDATE: 08/20/94

Solid Waste Burial Ground Areas Waste Volumes Disposed and Stored and

Nondecayed Curie Content in the 200 Areas from startup to December 31, 1993, Including Segregated Transuranic waste.

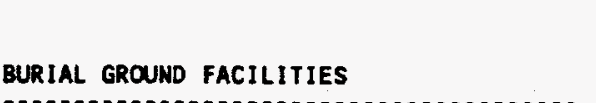

CENTRAL WASTE COMPLEX 2403WC BUILDING

CENTRAL HASTE COMPLEX 2403WD BUILDING

FLAMMABLE STORAGE MOOULE 1

FLAMMABLE STORAGE MODULE 10 FLAMMABLE STORAGE MOOULE 11

fLAMMABLE STORAGE MOOULE 12

\begin{tabular}{|c|c|c|c|}
\hline & PACKED & & \\
\hline $\begin{array}{l}\text { VOLLME } \\
\text { (CUBIC M) }\end{array}$ & $\begin{array}{c}\text { VOLLME } \\
\text { (CUBIC M) }\end{array}$ & $\begin{array}{l}\text { URANIUM } \\
\text { (GRAMS) }\end{array}$ & $\begin{array}{l}\text { PLUTONIUM } \\
\text { (GRAMS) }\end{array}$ \\
\hline
\end{tabular}

OTHER RADIOACTIVITY (GRAMS)

CH-242 1.615E-11

CH-243 7.952E-05

CM-244 5.293E-03

NP-237 2.878E+00

PU-238 2.484E-01

PU-239 2.759E+02

PU-240 2.884E+01

PU-241 3.193E+00

PU-242 8.687E-01

RA-224 9.000E-04

RA-226 4.001E-01

TH-228 1.646E-04

TH-232 $1.571 \mathrm{E}+04$

U-232 4.670E-12

U-233 2.100E-02

U-234 1.073E-01

U-235 $1.023 E+01$

U-236 7.385E-01

U-238 $9.123 E+03$

$4.701 E+02 \quad 4.701 E+02 \quad 8.748 E+04 \quad 7.000 E+01$

MM- 241

$6.605 E+02$

CM-242 2.115E-09

CM-243 3.120E-07

$\mathrm{CM}-244 \quad 3.485 \mathrm{E}-06$

NP $-237 \quad 6.580 E+00$

PU-238 1.220E-01

PU-239 4.030E+02

PU-240 2.456E+01

PU-241 2.434E+00

PU-242 9.937E-02

U-235 1.508E-01

U-236 3.652E-08

U-238 2.230E+02

$\begin{array}{llllll}1.026 E+00 & 1.026 E+00 & 1.035 E-01 \quad .000 E+00 \quad & & & 2.950 E-06 \\ & & & & \end{array}$

CM-242 9.240E-16

PU-238 1.311E-08

PU-239 1.896E-06

PU-241 5.090E-08

$\begin{array}{llllll}4.765 E+00 & 4.765 E+00 & .000 E+00 \quad .000 E+00 & P U-241 & .000 E+00\end{array}$

$\begin{array}{lllll}1.464 E+00 & 1.464 E+00 & 1.521 E-03 & 4.260 E-04 & 5.469 E-06\end{array}$

AM-241 2.852E-08

NM-243 1.930E-06

CM-242 3.650E-15

PU-238 4.110E-11

PU-239 3.273E-06

PU-240 2.040E-07

PU-241 3.399E-08

$\begin{array}{llllll}6.598 E+00 & 6.598 E+00 & 1.565 E-01 & 6.566 E-04 & & 3.942 E-03\end{array}$

NM-241 1.409E-06

NH-243 3.070E-06

CN-242 6.920E-16

CH-243 9.530E-10

CM-244 6.070E-10

MP-237 3.760E-03

PU-238 1.380E-04

PU-239 3.624E-05

PU-240 7.499E-07

PU-241 1.029E-07 


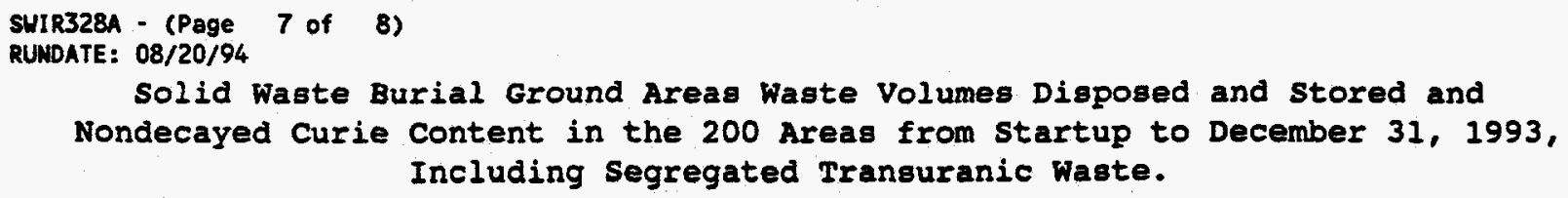

BURIAL GROUND FACILITIES

FLamLable storage moOUle 12

fLAmable STORAGE mODULE 13

FLAMMABLE StORAGE mODULE 15

FLAMMABLE STORAGE MOOULE 2 FLAMMABLE STORAGE MODULE 3

fLAMMABLE STORAGE MOOULE 4

FLAMMABLE STORAGE MOOULE 5

FLAMMABLE STORAGE MODULE 6

FLAMMABLE STORAGE MOOULE 7

flammable storage moOULE 8

VOLUNE
(CUBIC $H$ )

PACKEO (CUBIC Ms (CUBIC H) (C)

$4.977 \mathrm{E}+00$
$4.370 E+00 \quad 4.370 E+00 \quad 1.035 E-01 \quad 4.517 E-05$

$3.150 E+00$ 8. 328E-01

3.150E+00 8. 328E-01

$.000 E+00$
$.000 E+00$

$.000 E+00$ $.000 E+00$

$5.509 E+00$

$5.509 E+00$

$1.200 E-01$

4. 100E-05

$6.660 \mathrm{E}+0$

$3.992 E+00$

$6.660 E+00$

$.000 E+0$

2.820E-03

632E-01 1.014E-02

$891 E+0$

$2.891 E+00$

2.131E-01

$.000 E+00$ 


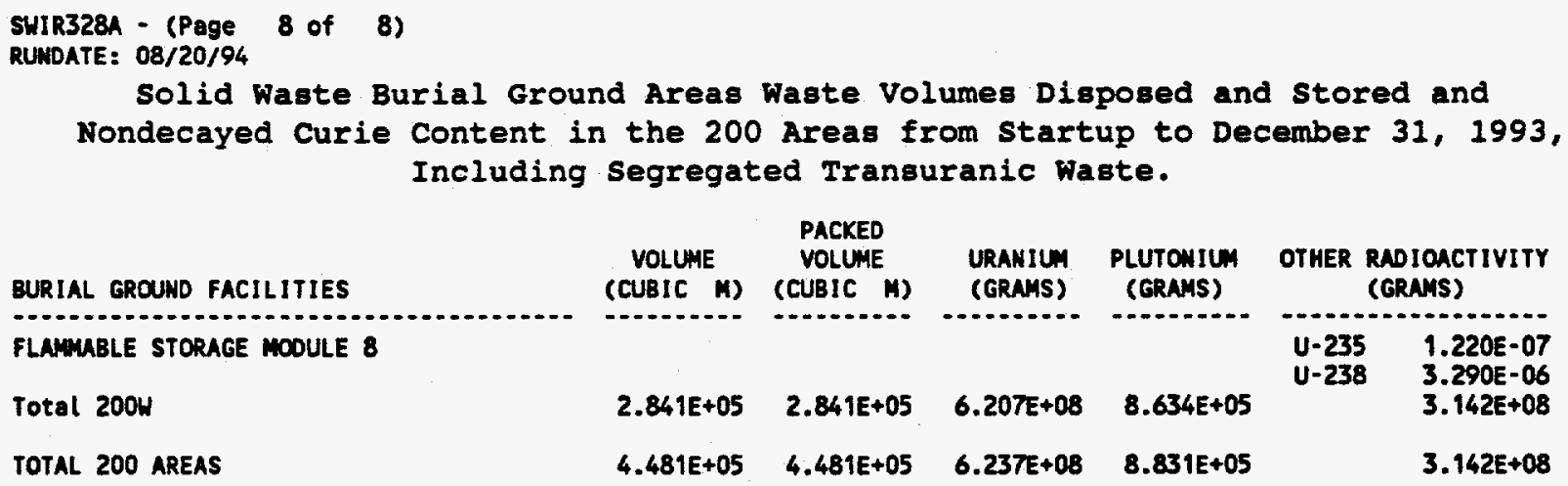

\begin{tabular}{|c|c|c|c|c|c|c|}
\hline BURIAL GROUND FACILITIES & $\begin{array}{l}\text { VOLUHE } \\
\text { (CUBIC } M \text { ) }\end{array}$ & $\begin{array}{l}\text { PACKED } \\
\text { VOLUME } \\
\text { (CUBIC H) }\end{array}$ & $\begin{array}{l}\text { URANIUNA } \\
\text { (GRAMS) }\end{array}$ & $\begin{array}{l}\text { PLUTONIUM } \\
\text { (GRAMS) }\end{array}$ & OTHER & $\begin{array}{l}\text { RADIOACTIVITY } \\
\text { (GRAMS) }\end{array}$ \\
\hline $\begin{array}{l}\text { FLAMABLE STORAGE MOOULE } 8 \\
\text { Total 20OW }\end{array}$ & $2.841 E+05$ & $2.841 E+05$ & $6.207 E+08$ & $8.634 E+05$ & $\begin{array}{l}U-235 \\
U-238\end{array}$ & $\begin{array}{l}1.220 E-07 \\
3.290 E-06 \\
3.142 E+08\end{array}$ \\
\hline TOTAL 200 AREAS & $4.481 E+05$ & $4.481 E+05$ & $6.237 E+08$ & $8.831 E+05$ & & $3.142 E+08$ \\
\hline
\end{tabular}

SPECIAL CAISSON/TRENCH BREAKOUT FOR FACILITY 218W4B

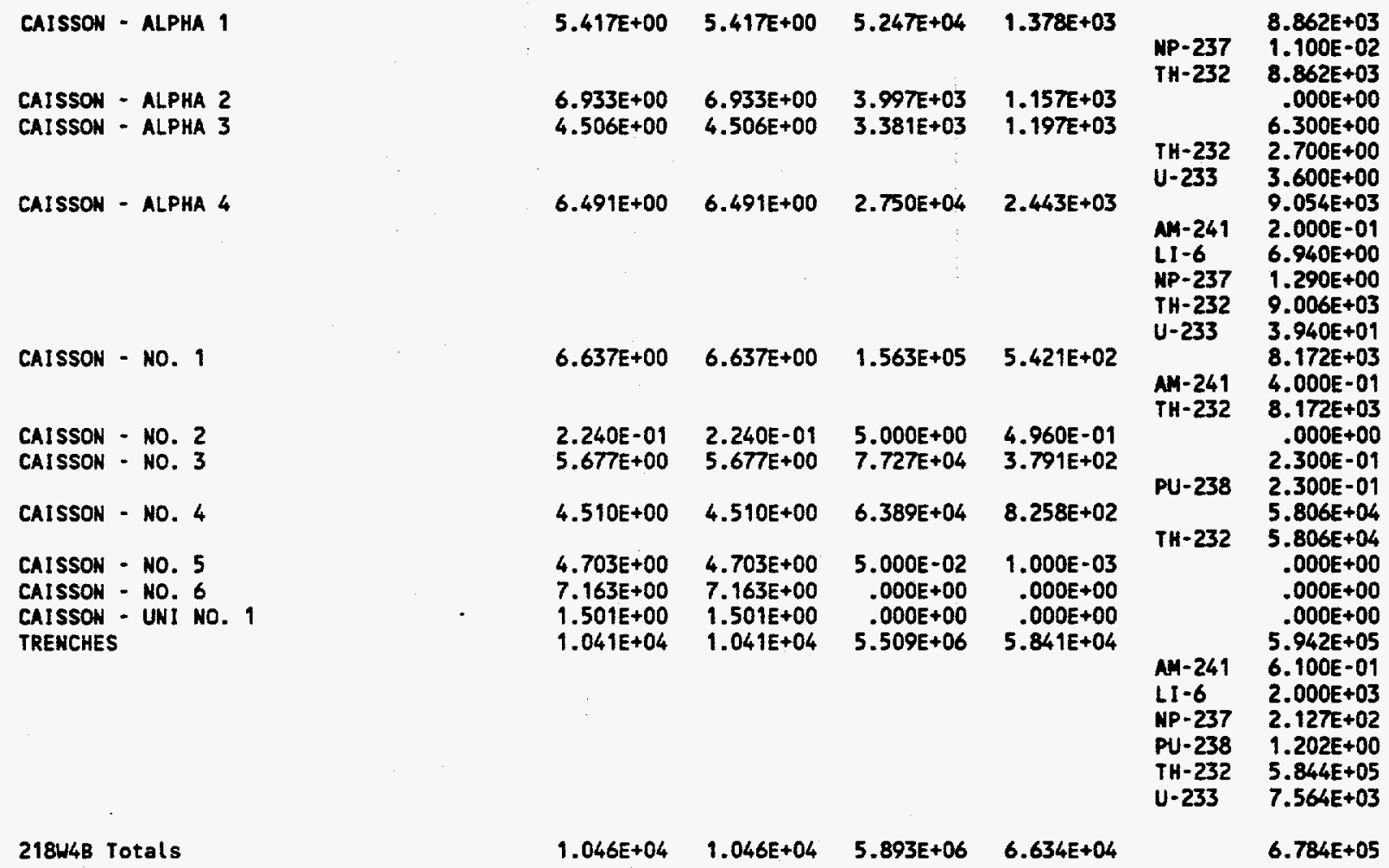


SWIR328B - (Page 1 of 19$)$

RUNDATE: 08/20/94

Solid Waste Burial Ground Areas Waste Volumes Disposed and Stored and Nondecayed Curie Content in the 200 Areas from startup to December 31, 1993, Including segregated Transuranic Waste.

\section{BURIAL GROUND FACILITIES}

2OOE AREA

-..........

BURIAL GROUND 218E1

BURIAL GROUND 218E10

BURIAL GROUND 218E12A BURIAL GROUND 218E12B
PUREX TUNMEL (\#1) 218E14 PUREX TUNMEL (\#2) $218 E 15$ BURIAL GROUND $218 E 2$

TOTAL
(CURIES)

$1.000 E+02$ $4.698 E+06$

$1.989 E+00$ $1.002 E+06$

$4.283 E+00$
$8.900 E+02$ $2.088 E+06$

1. $770 E+01$ $3.072 E+04$
3. $812 E+01$ $7.732 E+02$
$1.890 E+01$ $3.106 E+04$
$2.124 E+00$ $1.180 E+06$
CS-137

(CURIES)

(CURIES)
OTHER RADIOACTIVITY (CURIES)

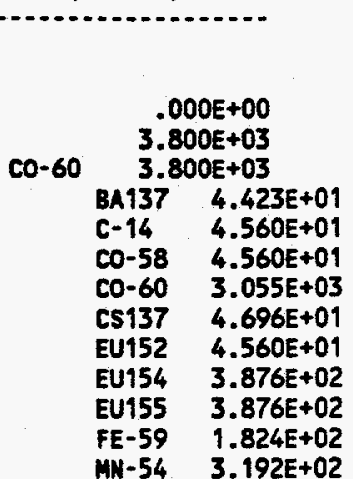

$.000 E+00$

$1.952 E+06$

$B E-10 \quad 6.110 E+01$

BE-7 $\quad 1.300 E-09$

$C-14 \quad 8.120 E+0$

CE-141 4.010E-01

CE-144 3.4T7E+01

CL-36 6.110E+01

CO-58 4.779E+03

C0-60 7.968E+05

CR-51 4.718E+01

CS-134 $1.062 E-01$

FE-55 5.165E+05

FE-59 2.203E+01

$\mathrm{H}-3 \quad 9.122 \mathrm{E}+03$

HF-181 6.010E-01

$I-129 \quad 6.112 E+01$

$K-40 \quad 9.054 E-03$

MN-54 8.014E+03

M0-93 6.900E-02

NB-94 5.674E-01

NB-95 3.903E+00

$N 1-59 \quad 4.211 E+03$

NI-63 6.198E+05

PM-147 3.004E+00

SB-125 1.100E-06

SE-79 2.422E-05

SM-151 4.241E-01

TA-182 1.000E-03

TC-99 1.030E-01

ZN-65 3.316E-01

ZR-93 2.035E-04

2R-95

$1.476 E+00$

BA137 1.166E-02

C- 14 1.202E-02

CO-58 1.202E-02

CO-60 8.054E-01

CS137 1.238E-02

EU152 1.202E-02

EU154 1.022E-01

EU155 1.022E-01

FE-59 4.808E-02

MN-54 8.415E-02
$.000 E+00$ $.000 E+00$ $.000 E+00$

$\begin{array}{lllll}8.780 E+04 & 1.746 E+03 & 3.760 E+03 & 1.865 E+03 & .000 E+00 \\ 5.465 E+04 & 8.358 E+03 & 9.103 E+02 & 9.036 E+03 & .000 E+00 \\ 2.500 E+04 & 4.973 E+02 & 1.071 E+03 & 5.310 E+02 & .000 E+00\end{array}$


WHC-EP-0125-6

SHIR328B - (Page 2 of 19)

RUMDATE: $08 / 20 / 94$

Solid Waste Burial Ground Areas Waste Volumes Disposed and Stored and Nondecayed Curie content in the 200 Areas from startup to December 31, 1993, Including Segregated Transuranic Waste.

BURIAL GROUND FACILITIES

BURIAL GROUND $218 E 4$

BURIAL GROUMD $218 E 5$

BURIAL GROUND 218E5A

BURIAL GROUND $218 E 8$

BURIAL GROUND 218EC9

2228 VAULTS

Total 200E
TOTAL
(CURIES)

$1.000 E+01$

$7.500 E+03$

$1.650 E+04$

$1.000 E+01$

4.348E+01

$6.000 E+02$

$6.979 E+06$
SR-90

(CURIES)

1.989E-01

$1.492 E+02$

$3.282 E+02$

$1.989 E-01$

$1.318 E+01$

$1.193 E+01$

$1.044 E+06$
RU-106 CS-137
(CURIES) (CURIES)

RU-106

4.283E-01

$3.212 E+02$

$7.067 \mathrm{E}+02$

4.283E-01

1.326E-01

2.570E+01

$2.533 E+04$
CS-137

(CURIES)

2.124E-01

$1.593 E+02$

$3.505 E+02$

2.124E-01

$7.974 E+00$

$1.274 E+01$

$1.223 E+06$
OTHER RADIOACTIVITY (CURIES)

$.000 E+00$ $.000 E+00$

$.000 E+D 0$

$.000 E+00$

$1.100 E-06$

EU-154 1.100E-06

$.000 E+00$

$1.955 E+06$ 
SHIR328B - (Page 3 of 19)

RUNDATE: 08/20/94

Solid Waste Burial Ground Areas Waste Volumes Disposed and Stored and

Nondecayed Curie content in the 200 Areas from Startup to December 31, 1993, Including segregated Transuranic Waste.

\section{BURIAL GROUND FACILITIES}

20OW AREA

212N BUILLING

BURIAL GROUND 218 1

BURIAL GROUND 218W19

BURIAL GROUND 218WIA

BURIAL GROUND 218W2

BURIAL GROUND 218W2A

BURIAL GROUND 21813

BURIAL GROUND 218W3A

TOTAL
(CURIES)

SR-90

(CURIES)

RU-106

(CURIES)

Cs-137

(CURIES)

(CURIES)

OTHER RADIOACTIVITY (CURIES)

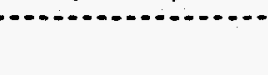

$.000 E+00$

$2.000 E+02$

$1.000 E-01$

$4.800 E+04$

$5.000 E+02$

$2.474 E+05$

$.000 E+00$
$.078 E+00$

$3.978 E+00$

$1.989 E-03$

$9.547 \mathrm{E}+02$

$9.945 E+00$

5.179E+03

$9.000 E+02$

$1.790 E+01$

$1.953 E+06$ $\begin{array}{lr}.000 E+00 & .000 E+00 \\ 8.566 E+00 & 4.248 E+00 \\ 4.283 E-03 & 2.124 E-03 \\ 2.056 E+03 & 1.020 E+03 \\ 2.142 E+01 & 1.062 E+01 \\ 1.065 E+04 & 5.537 E+03\end{array}$

$3.855 E+01$

$2.032 E+04$
$1.912 E+01$

$3.579 E+05$
$6.083 E+05$

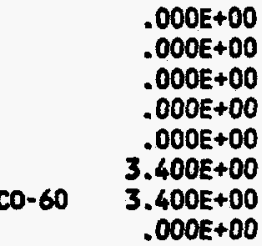

AG-108 9.100 E-06

AG-110 3.069E+00

AG-110 3.400E-06

BA-133 1.575E-04

BE-10 5.600E-10

BE-7 1.745E-01

BI-207 2.696E-03

C- $14 \quad 1.740 E+00$

CA-45 $1.000 E-04$

CD-109 5.080E-04

CE-141 1.746E+02

CE-144 5.747E+04

CL-36 2.002E-04

CO-57 1.302E-03

CO-58 $2.532 \mathrm{E}+01$

CO-60 4.980E+04

CR-51 9.964E-03

CS-134 1.727E+04

EU-152 1.377E+00

EU-154 4.426E-01

EU-155 3.810E+01

FE-55 1.702E+00

FE-59 3.329E-01

CD-152 3.390E-03

CD-154 2.310E-03

GE-68 2.399E-02

$H-3 \quad 4.376 E+05$

I-125 1.510E-02

I-129 $1.439 \mathrm{E}-02$

$I-131 \quad 1.371 E-03$

K-40 2.124E-03

KR-85 3.730E+03

$M N-54 \quad 4.354 E+00$

MA-22 6.578E+00

MB-94 1.000E-04

MI-59 3.000E-04

MI-60 2.979E-01

$M 1-63 \quad 3.840 E+00$

P-32 4.710E-02

$P M-147 \quad 4.024 E+04$

PR-144 1.240E-05

RB-86 5.017E-03

RE-187 2.000E-04

S-35 4.235E-02

S8-122 3.000E-07

SB-125 1.493E+03

SB-126 3.993E+0D

SC-46 1.200E-04

SE-75 8.600E-05

SI-31 1.500E-05 
SHIR328B - (Page 4 of 19)

RUNDATE: $08 / 20 / 94$

Solid Waste Burial Ground Areas Waste Volumes Disposed and Stored and

Nondecayed Curie Content in the 200 Areas from startup to December 31, 1993, Including Segregated Transuranic Waste.

BURIAL GROUND FACILITIES

BURIAL GROUND 218W3A

BURIAL GROUND 218W3AE
$2.757 E+05 \quad 1.343 E+04 \quad 1.261 E+03 \quad 2.985 E+04$ \begin{tabular}{cccc}
$\begin{array}{c}\text { TOTAL } \\
\text { (CURIES) }\end{array}$ & $\begin{array}{c}\text { SR-90 } \\
\text { (CURIES) }\end{array}$ & $\begin{array}{c}\text { RU-106 } \\
\text { (CURIES) }\end{array}$ & $\begin{array}{c}\text { CS-137 } \\
\text { (CURIES) }\end{array}$ \\
\hline
\end{tabular}

OTHER RADIOACTIVITY (CURIES)

SH- $147 \quad 2.000 E-04$

SW-151 2.000E-13

SR-85 2.882E-03

TA-182 1.251E-03

TC-99 1.999E-01

TE-125 3.394E+02

TL-204 5.000E-10

TM-170 2.000E-04

$v-49 \quad 4.000 E-08$

$Y-88 \quad 1.000 E-06$

$Y-90 \quad 2.000 E-04$

2N-65 3.982E+01

BA137 2.807E+02

C-14 $2.894 E+02$

CO-5B 2.894E+02

CO-60 $1.939 E+04$

CS137 2.981E+02

EU152 $2.894 E+02$

EU154 $2.460 E+03$

EU155 2.460E+03

FE-59 1.158E+03

MN-54 2.026E+03

BE-10 1.731E+05

BE-10 4.600E-11

C-14 2.017E+01

CD-113 1.260E-05

CE-144 9.565E-02

CL-36 1.840E-08

CO-58 2.616E+02

CO-60 4.837E+04

CR-51 8.591E-03

CS-134 1.840E+02

CS-135 3.800E-07

EU-152 1.316E+03

EU-154 2.032E+03

EU-155 4.860E+02

FE-55 1.617E+04

FE-59 1.351E+02

$\mathrm{H}-3 \quad 2.716 \mathrm{E}+04$

HF-181 1.051E-02

$1-129 \quad 1.805 E-07$

KR-85 1.302E+01

MN-54 3.797E+02

NA-22 7.599E-01

NB-93M 2.600E-05

NB- $94 \quad 5.609 E-01$

MB-95 1.420E+03

NI-59 $4.000 E+02$

N1-60 1.798E+03

NI $-63 \quad 4.833 E+04$

PM-147 9.500E-02

SB-125 9.000E+03

SB-126 7.200E-08

SE-75 7.400E-07

SE-79 1.784E-07

SM-151 3.484E-04

SN-119 $1.280 E+04$

SN-121 1.000E-07

SN- $123 \quad 1.080 E+02$

SN-126 5.100E-07

TA-182 1.240E+02 
SWIR328B - (Page 5 of 19)

RUNDATE: 08/20/94

Solid Waste Burial Ground Areas Waste Volumes Disposed and stored and

Nondecayed Curie Content in the 200 Areas from startup to December 31, 1993, Including Segregated Transuranic Waste.

BURIAL GROUND FACILITIES

BURIAL GROUND 2184BAE

BURIAL GROUND 218W4A

BURIAL GROUND 218W4B

BURIAL GROND 218448
BURIAL GROUND 218W4C

TOTAL
(CURIES)

SR-90 (CURIES)

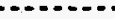

C........ (CURIES)

-

(CURIES)
OTHER RADIOACTIVITY (CURIES)

$\begin{array}{lll}\text { TC-99 } & 5.590 E-05 \\ \text { TE-125 } & 1.920 E+03 \\ 2 R-93 & 8.184 E-06 \\ 2 R-95 & 6.600 E+02 \\ \text { BA137 } & 1.092 E+01 \\ C-14 & 1.126 E+01 \\ \text { CO-58 } & 1.126 E+01 \\ \text { CO-60 } & 7.544 E+02 \\ \text { CS137 } & 1.160 E+01 \\ \text { EU152 } & 1.126 E+01 \\ \text { EU154 } & 9.570 E+01 \\ \text { EU155 } & 9.570 E+01 \\ \text { FE-59 } & 4.504 E+01 \\ \text { HN }-54 & 7.882 E+01\end{array}$

$5.000 E+02$

PH-147 5.000E+02

2. $617 \mathrm{E}+05$

CE- $144 \quad 8.420 E+0$ ?

CO-60 2.094E+04

CR-51 1.000E-01

CS-134 3.000E-01

EU-154 2.200E-01

FE-59 2.046E+00

$\mathrm{H}-3 \quad 2.311 \mathrm{E}+05$

1-129 5.000E-01

$M H-54 \quad 3.623 E+00$

PM-147 9.586E+03

PO-210 7.000E+00

SB-125 2.000E-02

ZR-95 3.000E+01

BA137 1.109E+01

C-14 $1.144 E+01$

Co-58 $\quad 1.144 E+01$

Co-60 7.663E+02

CS137 $1.178 E+01$

EU152 1.144E+01

EU154 9.721E+01

EU155 9.721E+01

FE-59 4.575E+0?

MN-54 8.006E+01

$1.086 \mathrm{E}+06$

$1.890 E-10$

$\begin{array}{ll}C-14 & 8.326 E+00 \\ C D-113 & 5.181 E-05\end{array}$

CE-141 1.370E-02

CE-144 1.282E+03

CL-36 7.580E-08

Co-58 7.766E+03

Co-60 1.022E+06

CR-51 $9.380 E+03$

CS-134 8.593E+02

CS-135 1.582E-06

EU-152 2.097E+0

EU- $154 \quad 3.026 E+02$

EU-155 6.600E-03

FE-55 $1.096 E+04$

FE-59 1.697E+04

$\mathrm{H}-3$ 4.556E+01

I-125 7.000E-06

$1-129 \quad 1.008 E-03$

KR-85 6.300E-02 
SUIR3288 - (Page 6 of 19)

RUHOATE: 08/20/94

Solid Waste Burial Ground Areas Waste Volumes Disposed and stored and Nondecayed Curie Content in the 200 Areas from Startup to December 31, 1993, Including Segregated Transuranic waste.

\begin{tabular}{|c|c|c|c|c|}
\hline BURIAL GROUND FACILITIES & $\begin{array}{l}\text { TOTAL } \\
\text { (CURIES) }\end{array}$ & $\begin{array}{l}5 R-90 \\
\text { (CURIES) }\end{array}$ & $\begin{array}{c}\text { RU-106 } \\
\text { (CURIES) }\end{array}$ & $\begin{array}{c}\text { CS-137 } \\
\text { (CURIES) }\end{array}$ \\
\hline
\end{tabular}
BURIAL GROUND 218W4C

OTHER RADIOACTIVITY (CURIES)

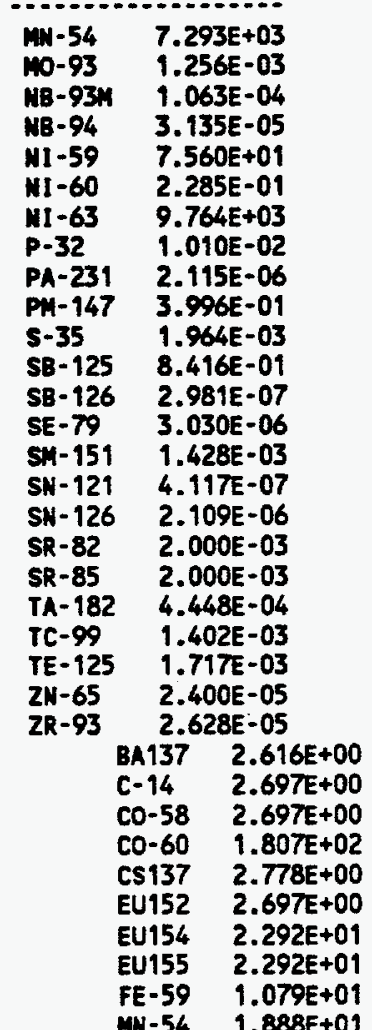

AC-227 3.686E-05

AC-228 1.200E-04

AG-108 5.215E-06

AG-110 1.620E-05

AG- $110 \quad 5.449 E-04$

AL-28 3.400E-05

AU-195 4.780E-05

BA-133 6.320E-02

BA-134 1.330E-07

BA-137 2.090E-07

BA-140 1.000E-06

$B E-7 \quad 1.042 E+00$

BI-207 1.669E-03

BI-212 7.006E-03

$81-214 \quad 6.356 E-04$

C-14 $4.342 E+00$

CA-45 1.691E-02

CD-109 1.492E-02

CE-141 1.206E-03

CE-144 6.737E+00

CL-36 1.368E-05

CO-56 9.407E-OG

CO-57 4.612E-02

CO-58 2.468E-01

C0-59 4.000E-06

CO-60 4.589E+03 
WHC-EP-0125-6

SUIR328B - (Page

RUNDATE: 08/20/94

Solid Waste Burial Ground Areas Waste Volumes Disposed and Stored and Nondecayed Curie Content in the 200 Areas from startup to December 31, 1993, Including Segregated Transuranic Waste.

BURIAL GROUND FACILITIES

TOTAL

BURIAL GROUND 218 W

(CURIES)

SR-90

(CURIES)

RU-106

(CURIES)

CS-137

(CURIES)

OTHER RNIOACTIVITY

(CURIES)

CR-51 2.145E-01

CS-133 2.800E-03

CS-134 3.428E-01

CU-65H 7.020E-03

ES-254 1.152E-04

EU-152 $1.708 E+00$

EU-154 1.272E+02

EU-155 1.263E+01

FE-55 2.090E+01

FE-59 $3.710 E+00$

CA-68 5.973E-02

CD-152 8.468E-02

CD-153 5.877E-04

CD-154 4.420E-02

GE-68 2.015E-03

$\mathrm{H}-3 \quad 1.574 \mathrm{E}+04$

HF- $175 \quad 1.002 E-06$

HF- $181 \quad 3.360 \mathrm{E}-06$

HG-203 1.200E-05

$I-123 \quad 5.610 E-04$

$1-125 \quad 1.543 \mathrm{E}-01$

$1-129 \quad 3.266 \mathrm{E}-02$

$I-131 \quad 1.284 \mathrm{E}-02$

$K-40 \quad 6.081 E-03$

$K R-85 \quad 4.181 E-02$

$M N-54 \quad 4.464 E+00$

$\% 0-93 \quad 1.292 E+00$

MA-22 1.315E+01

NB-91 2.902E-02

NB-93M 9.001E-05

NB-94 7.295E-04

MB-95 $1.318 E-02$

NB-95M 2.000E-07

MD- $147 \quad 6.000 E-09$

NI-59 8.359E-03

$M I-60 \quad 3.164 E+00$

$N I-63 \quad 3.345 E+00$

P-32 3.686E-01

PA-231 7.937E-01

PB-208 9.203E-08

PB-212 3.320E-03

PB-214 2.310E-04

PH- 147 3.586E+00

PO-210 3.066E-06

PT-195 9.000E-10

RA-228 1.200E-03

RB-85M 3.000E-06

RB-86 $6.197 E-03$

RE-187 3.000E-06

RU-103 8.371E-04

S-35 $1.430 E-01$

SB-124 1.818E-04

SB-125 1.786E-02

SB-126 2.500E-04

SC-46 4.491E-03

SE-7S $1.190 E-02$

SE-79 3.038E-03

\$I-31 1.000E-04

SN-151 8.013E-01

SN- $113 \quad 1.834 E-03$

SN-121 3.300E-07 
SWIR328B - (Page 8 of 19)
RUNOATE: 08/20/94.

Solid Waste Burial Ground Areas Waste Volumes Disposed and Stored and

Nondecayed Curie content in the 200 Areas from Startup to December 31, 1993, Including segregated Transuranic Waste.

BURIAL GROUND FACILITIES

BURIAL GROUND 218 US
TOTAL

(CURIES)
SR-90
(CURJES)

(CURJES)
RU-106

(CURJES)
CS-137 (CURIES)

\subsection{E-01 \\ 4.030E+03 \\ $7.700 E+02$ \\ $1.879 E+02$ \\ $1.989 E-03$ \\ $8.016 E+01$ \\ $1.532 E+01$ \\ 4.283E-03 \\ $1.726 E+02$ \\ $3.298 E+01$ \\ 4.980E-01 \\ 2.124E-03 \\ $8.560 E+01$ \\ $1.635 E+01$ \\ $1.506 E+01$}

TRANSURANIC WAST
FACILITY(TRUSAF)

CENTRAL MASTE COMPLEX 2401W BUILDING

OTHER RADIOACTIVITY (CURIES) (n)

SN-123 2.000E-05

SR-82 4.325E-04

SR-85 7.650E-02

SR-89 5.000E-04

TA-182 2.510E-01

TC- 9 1.212E-01

TE-129 1.000E-04

TE-125 4.047E-04

TE-127 2.500E-04

TE- $129 \quad 1.000 E-07$

TL-204 8.500E-04

$\mathrm{TL}-208 \quad 2.109 \mathrm{E}-03$

TK-170 3.300E-07

$V-49 \quad 6.753 E-06$

W-183M 5.770E-06

$U-185 \quad 2.120 E-05$

$Y-87 \quad 2.530 E-03$

$Y-88 \quad 8.744 E-05$

$Y-90 \quad 1.706 E-01$

$2 N-65 \quad 3.780 E-01$

2R-95 1.043E-02

BA137 $1.065 E+00$

C- $14 \quad 1.098 E+00$

Co-58 $1.098 E+00$

CO-60 7.355E+01

CS137 $1.131 E+00$

EU152 $1.098 E+00$

EU154 9.331E+00

EU155 $9.331 E+00$

FE-59 4.391E+00

MN-54 7.685E+00 $.000 E+00$ $.000 E+00$ $.000 E+00$

$1.907 \mathrm{E}-03$

Co-60 7.267E-04

CS-134 4.589E-04

EU-152 8.290E-05

EU-154 3.261E-04

MN-54 2.900E-07

SB-125 1.550E-04

TE- $125 \quad 1.550 E-04$

ZR-95 2.100E-06

BA137 5.345E-05

C- $14 \quad 5.510 E-05$

CO-58 5.510E-05

CO-60 3.692E-03

CS137 5.675E-05

EU152 5.510E-05

EU154 4.684E-04

EU155 4.684E-04

FE-59 2.204E-04

MII-54 3.857E-04

2. 100E+02

CO-60 3.00OE-04

EU-154 4.000E-05

$\mathrm{H}-3 \quad 2.100 \mathrm{E}+\mathrm{O2}$

BA137 $3.143 E-08$

C-14 3.240E-08

CO-58 3.240E-08

CO-60 2.171E-06 
SWIR328B - (Page 9 of 19)

RUNDATE: 08/20/94

Solid Waste Burial Ground Areas Waste Volumes Disposed and stored and Nondecayed Curie Content in the 200 Areas from Startup to December 31, 1993, Including segregated Transuranic waste.

$\begin{array}{llccc}\begin{array}{l}\text { BURIAL GROUD FACILITIES } \\ \text { CENTRAL WASTE COMPLEX } 2401 \mathrm{H} \text { BUILOING }\end{array} & \begin{array}{c}\text { TOTAL } \\ \text { (CURIES) }\end{array} & \begin{array}{c}\text { SR-90 } \\ \text { (CURIES) }\end{array} & \begin{array}{c}\text { RU-106 } \\ \text { (CURIES) }\end{array} \\ \text { (CURIES) }\end{array}$

CENTRAL WASTE COMPLEX 2401W BUILDING

CENTRAL MASTE COMPLEX 2402W BUILDING

$9.621 E+00$

7.367E-0

4.631E-01

$1.557 E+00$

AC- 228

BI -212

BI -214

C -14

CE-144

CO-60

CS-134

EU-152

EU- 154

EU-155

FE-59

$H-3$

$M N-54$

MA-22

MB-95M

NI -60

PA-231

PB-212

PB-214

PM-147

PO-210

RA-228
CTIVITY CUR IES)

CS137 3.337E-08

EU152 3.240E-08

EU154 2.754E-07

EU155 2.754E-07

FE-59 1.296E-07

MU-54 2.268E-07

8.070E-01

3.760E-03

7.261E-04

3.855E-01

1.505E-01

8.968E-03

1.597E-03

2.400E-05

1.379E-03

6.806E-04

1.600E-02

5.200E-09

3.114E-03

1.200E-07

7.130E-05

$1.764 \mathrm{E}-01$

$1.162 E-04$

$1.410 E-03$

5.060E-02

4.570E- 03

$1.586 \mathrm{E}-03$

2.642E-05

BA137 1.627E-06

C-14 1.677E-06

CO-58 1.677E-06

CO-60 1.124E-04

CS137 1.727E-06

EU152 1.677E-06

EU154 1.425E-05

EU155 1.425E-05

FE-59 6.708E-06

MN-54 1.174E-05

3.490E-02

1.755E-08

$3.435 E-11$

$1.494 \mathrm{E}-13$

$1.110 \mathrm{E}-08$

$1.820 E-02$

$4.845 E-03$

4.455E-08

2.940E-05

5.023E-05

2.010E-05

2.200E-05

1.741E-05

3. $500 E-05$

1.780E-05

$8.120 E-10$

2.770E-05

4.010E- 16

$1.374 E-05$

8.110E-06

8.712E-03

$1.855 E-23$

7.497E-04 
SUIR328B - (Page 10 of 19)

RUNDATE: OB/20/94

Solid Waste Burial Ground Areas Waste Volumes Disposed and Stored and Nondecayed Curie Content in the 200 Areas from Startup to December 31, 1993, Including Segregated Transuranic Waste.

\begin{tabular}{lllll} 
BURIAL GROUND FACILITIES & $\begin{array}{c}\text { TOTAL } \\
\text { (CURIES) }\end{array}$ & $\begin{array}{c}\text { SR-90 } \\
\text { (CURIES) }\end{array}$ & $\begin{array}{c}\text { RU-106 } \\
\text { (CURIES) }\end{array}$ & $\begin{array}{c}\text { CS-137 } \\
\text { (CURIES) }\end{array}$ \\
\hline
\end{tabular}

CENTRAL MASTE COMPLEX 2402UB BUILDING

CENTRAL WASTE COMPLEX 2402WC BUILDING

$4.147 E+00 \quad 4.518 E-01 \quad 1.021 E-01 \quad 4.589 E-01$

OTHER RADIOACTIVITY (CURIES)

SW-151 2.100E-03

TC-99 1.131E-06

TL-208 4.623E-05

$2 R-95 \quad 5.030 E-06$

BA137 3.159E-07

C. $14 \quad 3.256 E-07$

CO-58 3.256E-07

CO-60 2.182E-05

CS137 3.354E-07

EU152 3.256E-07

EU154 2.768E-06

EU155 $2.768 E-06$

FE-59 $1.302 E-06$

MN-54 2.279E-06

$1.350 E-06$

C-14 $1.026 E-05$

CE- $141 \quad 1.450 E-08$

CE-144 1.851E-02

CO-60 6.164E-02

CS-134 4.043E-05

EU-152 4.318E-04

EU-154 7.469E-04

EU-155 3.000E-04

FE-55 4.330E-01

FE-59 5.340E-04

$\mathrm{H}-3$ 4.316E-04

I-129 $1.426 E-09$

MN-54 4.180E-02

HA-22 2.620E-04

NB-94 9.467E-15

NB-95 1.000E-12

NB-95M 1.150E-08

NI-60 1.001E-10

NI $-63 \quad 2.340 E-04$

PM-147 8.716E-03

SB- $125 \quad 3.000 E-10$

SE-75 3.000E-10

SE-79 6.256E-11

SM- $151 \quad 2.400 E-03$

TC-99 5.011E-06

2R-95 3.908E-04

BA137 4.819E-08

C- $14 \quad 4.968 E-08$

CD-58 4.968E-08

CO-60 $3.329 E-06$

CS137 5.117E-08

EU152 4.968E-08

EU154 4.223E-07

EU155 4.223E-07

FE-59 1.987E-07

CENTRAL WASte COMPLEX 240210 BUilding $\quad 2.024 E+00 \quad 7.165 E-03 \quad 8.789 E-03 \quad 7.826 E-03$

MN-54 3.478E-07

C-14 7.530E-12

CE-144 2.760E-02

EU-155 4.000E-04

$\mathrm{H}-3 \quad 1.700 \mathrm{E}+0 \mathrm{O}$

PM-147 1.530E-02

SH- 151 4.400E-03

SN-123 1.000E-04

TC-99 2.013E-01 
SUIR328B - (Page 11 of 19)

RUNDATE: $08 / 20 / 94$

Solid haste Burial Ground Areas Waste Volumes Disposed and stored and Nondecayed Curie Content in the 200 Areas from Startup to December 31, 1993, Including Segregated Transuranic Waste.

$\begin{array}{lllll} & \text { TOTAL } & \text { SR-90 RU-106 CS-137 OTHER RADIOACTIVI }\end{array}$

BURIAL GROUND FACILITIES (CURIES (CURIES) (CURIES) CENTRAL UASTE COMPLEX 2402WE BUILDING 2.019E-03

$2.774 \mathrm{E}-04$ (CURIES) 2.908E-04

CENTRAL WASTE COMPLEX 2402WF BUILDING
4.432E-01
$9.623 E-03 \quad 8.166 E-03$ (CURIES)

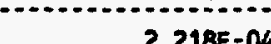

2.218E-04

C-14 1.460E-15

CE-144 4.300E-07

CO-60 2.200E-04

H-3 4.950E-09

$1-129 \quad 3.580 E-13$

$\mathrm{MB}-94 \quad 8.190 \mathrm{E}-16$

PH-147 1.320E-06

SE-79 5.400E-12

TC- 99 2.000E-10

$3.529 E-01$
C-14

$680 E-12$

CE-144 4.080E-02

H-3 3.888E-05

PM-147 1.920E-02

SM-151 6.400E-03

TC-99 2.864E-01

1.662E-01

C-14 1.001E-04

CE-144 2.280E-05

CO-60 1.257E-01

CS-134 1.110E-06

EU-152 6.160E-05

EU-154 1.692E-04

EU-155 4.670E-05

FE-59 1.441E-02

H-3 $1.163 E-03$

I-129 1.129E-06

$1-131 \quad 2.400 E-03$

MN-54 2.166E-02

NB-94 5.280E-14

NI-59 6.540E-11

$N I-60 \quad 1.438 E-04$

MI-63 7.547E-06

P-32 7.500E-06

PM-147 2.601E-04

S-35 4.750E-05

SE-79 3.485E-10

TA-182 2.220E-07

TC- 99 1.593E-06

2N-65 7.400E-06

BA137 2.506E-07

C-14 2.584E-07

CO-58 2.584E-07

CO-60 1.731E-05

CS137 2.661E-07

EU152 2.584E-07

EU154 2.196E-06

EU155 2.196E-06

FE-59 1.034E-06

MN-54 1.809E-06

$1.026 E+00$

$\begin{array}{ll}C-14 & 5.018 E-10 \\ C E-144 & 1.160 E-02\end{array}$

CO-60 9.066E-0G

CS-135 $1.000 E-04$

EU-152 5.001E-05

EU- $154 \quad 9.004 E-05$

EU-155 4.700E-04

FE-59 6.000E-05

$H-3 \quad 1.000 E+00$ 
SWIR3288 - (Page 12 of 19 )

RUNDATE: 08/20/94

Solid Waste Burial Ground Areas Waste Volumes Disposed and Stored and

Nondecayed Curie Content in the 200 Areas from startup to December 31, 1993, Including segregated Transuranic Waste.

BURIAL GROUND FACILITIES

TOTAL

(CURIES)

SR-90

(CURIES)

CENTRAL HASTE COMPLEX 2402UH BUILDING

CENTRAL haste COMPLEX 2402WI BUILDING

CENTRAL WASTE COMPLEX 2402WJ BUILDING

CENTRAL WASTE COMPLEX 2402WL BUILDING
$1.137 E+00 \quad 7.828 E-03 \quad 1.054 E-02 \quad 8.636 E-03$

2.683E-01

$5.385 E-03$

3.721E-03

4.168E-01 5.600E-02 1.674E-02 3.091E-02
OTHER RADIOACTIVITY (CURIES)

$1-129 \quad 1.000 E-04$ MN-54 9.500E-05

NB-94 3.044E-14

WI $-59 \quad 2.500 E-10$

MI $-60 \quad 9.146 E-07$

WI-63 2.500E-08

PD-107 1.000E-04

PU-147 5.837E-03

SE-79 3.730E-11

SN-151 1.000E-03

SN-123 6.000E-05

TC- $99 \quad 5.625 \mathrm{E}-03$

ZR-90 1.000E-04

ZR-93 1.696E-13

$1.096 E-13$
$1.041 E+00$

$\begin{array}{ll}C-14 & 1.012 E-11 \\ \text { CE- } 144 & 4.270 E-02\end{array}$

EU-155 1.700E-04

H-3 7.001E-01

I-129 1.864E-12

NB-94 4.270E-15

PM-147 2.027E-02

SE-79 2.820E-11

SM-151 6.300E-03

SN-123 4.000E-05

TC- 99 2.713E-01

2.165E-0

C-14 1.000E-06

CE-144 9.300E-03

$\mathrm{H}-3 \quad 8.880 \mathrm{E}-06$

PH-147 4.500E-03

SH-151 1.200E-03

TC-99 2.014E-01

6.220E-02

$\begin{array}{ll}C-14 & 5.637 E-02 \\ C A-45 & 3.439 E-07\end{array}$

CE-144 1.704E-03

CL-36 3.560E-05

CO-57 2.610E-06

CO-60 1.158E-03

CS-134 6.823E-05

CS-135 5.650E-13

EU-154 7.002E-07

EU-155 7.440E-08

FE-59 4.311E-12

H-3 1.302E-03

I-129 7.429E-07

MN-54 8.400E-11

MA-22 5.500E-07

MB-93M 1.285E-10

UB- $04 \quad 1.520 E-12$

N1-60 2.216E-08

NI-63 6.565E-06

NI $-63 \quad 8.400 E-11$

PD-107 1.140E-13

PM-147 9.187E-04

RA-228 2.530E-10

RU-103 1.930E-07

S-35 1.000E-05

SE-75 2.540E-07

SE-79 1.490E-06 
SWIR328B - (Page 13 of 19)

RUNDATE: 08/20/94

Solid Waste Burial Ground Areas Waste Volumes Disposed and Stored and

Nondecayed Curie Content in the 200 Areas from Startup to December 31, 1993, Including Segregated Transuranic Waste.

BURIAL GROUND FACILITIES

CENTRAL UASTE COMPLEX 2402WL BUILOING
TOTAL (CURIES)

SR-90 (CURIES)

RU-106 (CURIES) CS-137
(CURIES)

CENTRAL HASTE COMPLEX 2403WA BUILDING

$3.144 E+01 \quad 5.464 E+00$

$6.360 E-01 \quad 5.854 E+00$
OTHER RADIOACTIVITY (CURIES)

SH-151 4.000E-04

TC-99 2.155E-04

ZN-65 1.110E-08

ZR-93 3.960E-12

BA137 $9.933 E-15$

C-14 $\quad 1.024 E-14$

Co-58 1.024E-14

CO-60 6.861E-13

CS137 $1.055 \mathrm{E}-14$

EU152 $1.024 E-14$

EU154 8.704E-14

EU155 8.704E-14

FE-59 4.096E-14

MN-54 7.168E-14

$4.754 E+00$

AC-228 8.200E-07

AG-110 2.235E-07

AG-110 8.526E-08

BE-7 5.224E-03

B]-214 1.288E-05

C-14 2.321E-04

CA-45 1.300E-06

CE-144 9.733E-01

CO-56 2.642E-06

CO-57 7.318E-05

CO-58 1.174E-05

CO-60 3.606E-01

CS-134 5.593E-03

CS-135 6.620E-13

EU-152 1.737E-03

EU-154 3.306E-03

EU-155 6.865E-04

FE-55 3.516E-01

FE-59 3.810E-05

CD-153 5.000E-OL

CD-154 1.919E-0L

$\mathrm{H}-3 \quad 1.156 \mathrm{E}-02$

HF- $175 \quad 3.200 E-04$

HG-203 2.133E-04

$1-125 \quad 1.050 E-05$

$1-129 \quad 2.475 E-04$

$K-40 \quad 1.393 E-02$

KR-85 2.486E-03

MN-54 1.152E-01

MO-93 2.400E-07

NA-22 6.049E-04

NB-93M 2.292E-07

NB-94 2.003E-08

MB-95M 2.387E-08

MI-59 7.650E-06

$M I-60 \quad 7.494 E-02$

MI-63 9.564E-D

P-32 $5.430 E-04$

$P B-212 \quad 5.140 E-07$

PB-214 1.288E-05

PD-107 1.336E-13

$P M-147$ 4.695E-01

PT-195 2.800E-05

S-35 4.619E-03

SB-125 1.940E-02

SC-46 2.600E-08 
SWIR328B - (Page 14 of 19)

RUNDATE: 08/20/94

Solid Waste Burial Ground Areas Waste Volumes Disposed and stored and

Nondecayed Curie Content in the 200 Areas from startup to December 31, 1993, Including Segregated Transuranic Waste.

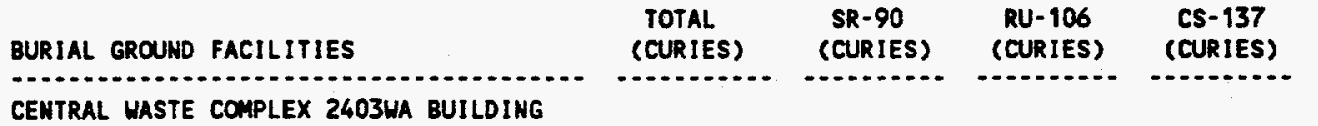

CENTRAL WASTE COMPLEX 2403WA BUILDING

CENTRAL WASTE COMPLEX 2403WC BUILDING

$7.927 E+01$

$2.710 E+01$

$2.429 E-01$

$1.022 E+01$

OTHER RADIOACTIVITY (CURIES)

SE-TO $5.146 E-08$

SE-79 5.146E-08

SH-151 1.115E-01

TA-182 1.000E-05

TC- $99 \quad 2.207 E+00$

TE-125 1.589E-02

TE-125 1.792E-03

TL-208 3.960E-07

$V-49$ 1.301E-04

XE-131 2.600E-05

2N-65 5.566E-06

$2 R-93 \quad 4.630 E-12$

2R-95 2.098E-04

BA137 $1.063 E-08$

C-14 1.096E-08

CO-58 1.096E-08

CO-60 7.344E-07

CS137 1.129E-08

EU152 1.096E-08

EU154 $9.317 E-08$

EU155 9.317E-08

FE-59 4.384E-08

MN-54 7.672E-08

$1.200 E-02$

AC-228 1.200E-03

$A G-110 \quad 1.840 E-06$

AG-110 1.311E-04

BI-212 1.500E-03

BI-214 4.000E-01

C-14 6.463E-04

CE-141 1.810E-06

CE-144 1.841E-01

CO-57 2.541E-09

CO-58 2.344E-03

CO-60 8.765E-02

CR-51 7.900E-03

CS-134 2.875E-02

EU-152 1.002E-01

EU-154 2.080E-01

EU-155 5.189E-02

FE-55 2.931E-02

FE-59 7.320E-04

60-154 6.042E-04

H-3 1.280E-01

$1-129 \quad 1.506 E-04$

$K-40 \quad 2.769 E-04$

MN-54 6.123E-03

MO-93 3.990E-08

MA-22 $9.498 E-06$

MB-93M 1.556E-06

NB-94 1.946E-08

MB-95 2.095E-09

MB-95N 1.603E-08

M1 $-59 \quad 2.627 E-06$

M1-60 1.467E-04

N1-63 1.652E-03

MI-63 2.100E-10

P-32 1.670E-04

PA-231 2.400E-07

PB-212 9.000E-04

PB-214 4.000E-01 
SUIR328B - (Page 15 of 19)

RUNDATE: 08/20/94

Solid Waste Burial Ground Areas Waste Volumes Disposed and stored and Nondecayed Curie Content in the 200 Areas from Startup to December 31, 1993, Including Segregated Transuranic Waste.

BURIAL GROUND FACILITIES

CENTRAL HASTE COAPLEX 2403UC BUILOING
(CURIES)

SR- 90

(CURIES)
RU- 106 (CURIES)
CS-137 (CURIES)
CENTRAL HASTE COMPLEX 2403WD BUILDING

FLAMAGLE STORAGE MOOULE 1
$3.712 E+00$

$3.141 E-02$

$2.357 E+01$

$1.964 E-04$
$1.362 E-05$

$1.945 E-06$
2.401E-05
OTHER RADIOACTIVITY (CURIES)

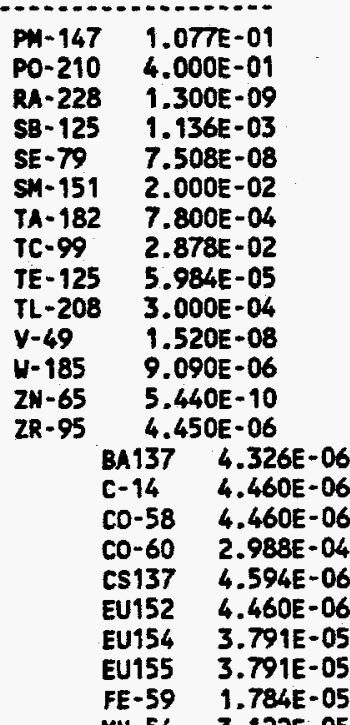

MN-54 3.122E-05

C. $14 \quad 380 E-05$

CE-144 6.483E-03

CO- 60 3.000E+03

EU-152 8.120E-03

EU-154 2.278E-01

EU-155 1.777E-03

$\mathrm{H}-3 \quad 4.279 \mathrm{E}-05$

$1-129 \quad 1.641 \mathrm{E}-03$

NB-93M 2.230E-04

NB-94 4.100E-06

WI-59 1.092E+00

$N I-63 \quad 1.200 E+02$

PN-147 4.302E-03

SE-79 1.865E-05

SM-151 7.000E-04

TC-99 3.642E-01

$8 A 137 \quad 1.455 E-05$

C-14 1.500E-05

CO-58 1.500E-05

C0-60 1.005E-03

CS137 1.545E-05

EU152 1.500E-05

EU154 1.275E-04

EU155 1.275E-04

FE-59 6.000E-05

MN-54 $\quad 1.050 E-04$

$\begin{array}{ll}C-14 & 1.136 E-04 \\ C E-144 & 5.010 E-06 \\ C S-134 & 5.560 E-07 \\ \text { EU-152 } & 3.080 E-05 \\ E U-154 & 5.500 E-05 \\ E U-155 & 8.560 E-06 \\ H-3 & 4.108 E-07 \\ 1-129 & 4.683 E-09 \\ N B-94 & 7.420 E-15\end{array}$




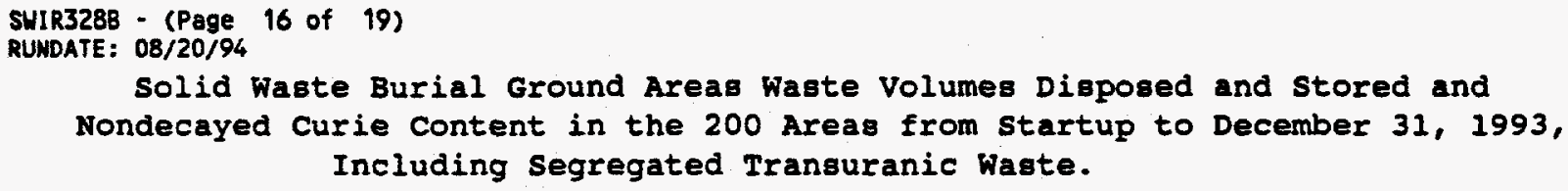

flamaAabe storage mOOULE 10

$3.621 E+00 \quad 9.897 E-01 \quad 1.612 E-02 \quad 7.518 E-01$

$$
\begin{aligned}
& \text { PH-147 1.267E-05 } \\
& \text { SE-79 4.900E-11 } \\
& \text { TA-182 1.110E-07 } \\
& \text { TC-99 4.854E-07 } \\
& \begin{array}{ll} 
& 8.232 E-03 \\
C-14 & 6.010 E-03
\end{array} \\
& \text { CO-60 1.222E-03 } \\
& \mathrm{H}-3 \quad 1.000 \mathrm{E}-03 \\
& \text { BA137 4.831E-06 }
\end{aligned}
$$

fLAMMABLE STORAGE MODULE 11

FLAMMABLE STORAGE MOOULE 12

FLAMAMABLE STORAGE MOOULE 13
$6.200 E-04 \quad 4.742 E-06 \quad 3.439 E-07 \quad 1.971 E-04$

2.541E-02 $1.588 E-03 \quad 1.246 E-03 \quad 3.236 E-03$

$2.602 E+00 \quad 1.124 E-04 \quad 1.548 E-05 \quad 1.210 E-04$
$M N-54 \quad 3.486 E-05$ $2.254 \mathrm{E}-04$

AU-195 $1.166 E-05$

C- $14 \quad 5.500 E-05$

CE-144 7.700E-07

CO-58 4.400E-07

CO-60 8.800E-09

$H-3 \quad 1.390 E-04$

$1-129 \quad 1.280 E-12$

MN-54 1.969E-08

NA-22 8.006E-07

NB-94 2.930E-15

NI-60 8.800E-09

PM-147 4.730E-06

SE-79 1.900E-11

TC-99 1.300E-05

7.936E-03

C- $14 \quad 1.637 \mathrm{E}-04$

CE-144 4.703E-03

CO-60 6.592E-06

CS-134 1.690E-06

CS-135 5.610E-13

EU-152 $1.320 E-07$

EU-154 3.430E-06

EU- $155 \quad 2.446 E-06$

H-3 $7.665 \mathrm{E}-05$

$1-129 \quad 9.293 E-07$

MB-94 5.560E-15

NI-60 6.592E-06

PD-107 1.130E-13

PH- $147 \quad 2.450 E-03$

SB-125 1.840E-08

SE-79 3.670E-11

SH- 151 5.000E-04

TC-99 2.125E-05

TE-125 1.840E-OB

2R-93 3.930E-12

$2.601 E+00$

C-14 4.828E-02

Co-58 6.100E-07

CO-60 8.600E-08

EU-154 1.600E-07

EU-155 3.530E-08

FE-55 $1.200 E-06$ 
SUIR328B - (Page 17 of 19$)$
RUNDATE: $08 / 20 / 94$

Solid Waste Burial Ground Areas Waste Volumes Disposed and Stored and

Nondecayed Curie Content in the 200 Areas from startup to December 31, 1993, Including segregated Transuranic Waste.

\section{BURIAL GROUND FACILITIES}

flaMAable storage mODULE 13

flammable stoRAge mOOULE 15

FLAMMABLE STORAGE MOOULE 2

FLAMMABLE STORAGE MOOULE 3

FLAMMABLE STORAGE MOOULE 4

FLAMABLE STORAGE MOOULE 5
TOTAL
(CURIES)

SR-90

(CURIES)

(CURIEs)

RU-106

(CURIES)

(C)......

CS-137
(CURIES)

3.

$3.492 E-04$

2.993E-05

2.154E-04

$1.538 E-01$

$1.200 \mathrm{E}-03$

$1.223 E-03$

$1.322 E-03$

$1.025 E-10$

2.076E-12

$6.922 E-02$

2.195E-03

2.564E-03

$1.501 E-02$

AG- 110

C- 14

CE- 144

CO-60

CS- 134

EU- 152

EU-154

EU- 155

H-3

$1-129$

MN -54

PN-147

SB- 125

SM- 151

TC- 99

2R-95

4.732E-02

2.879E-OS

2.575E-03

3. 172E-03
C- 14

CE-144

H-3

PM-147

SM-151
RADIOACTIVITY (CURIES)

2.553E+00

$1.719 E-10$

6.300E-07

8.600E-08

$1.400 E-07$

$6.552 E-08$

$1.331 \mathrm{E}-10$

$1.401 E+00$

8.216E-03

$1.580 \mathrm{E}-06$

$1.392 E+00$

$1.815 E-08$

$1.580 E-06$

1.680E- 10

3.710E-08

4.802E-07

1.407E-01

6.280E-04

5.347E-03

6.980E-03

7.671E-04

3.042E-03

$1.000 E-04$

4.810E-08

2.974E-03

$1.385 E-03$

$1.054 E-04$

2. 400 E- 03

4.900E-05

6.000E-04

$1.156 \mathrm{E}-01$

4.390E-04

2.860E-04

3.540E-03

5.760E-08

3.537E-03

2.000E-06

6.800E-07

1.966E-02

2.700E-06

$7.210 E-04$

$1.032 E-02$

$6.722 E-06$

$6.513 E-05$

2.481E-06

2.951E-05

1.261E-05

2.590E-03

$3.866 E-10$

2.957E-07

4. $800 \mathrm{E}-03$

$7.569 E-06$

$1.100 E-03$

$3.002 E-10$

5.360E-07

$1.841 E-02$

$1.186 \mathrm{E}-03$

$1.030 E-02$

$6.355 E-04$

4.800E-03

$1.400 \mathrm{E}-03$ 
SHIR328B - (Page 18 of 19)

RUNOATE: 08/20/94

Solid Waste Burial Ground Areas Waste Volumes Disposed and stored and Nondecayed Curie Content in the 200 Areas from startup to December 31, 1993, Including segregated Transuranic Waste.

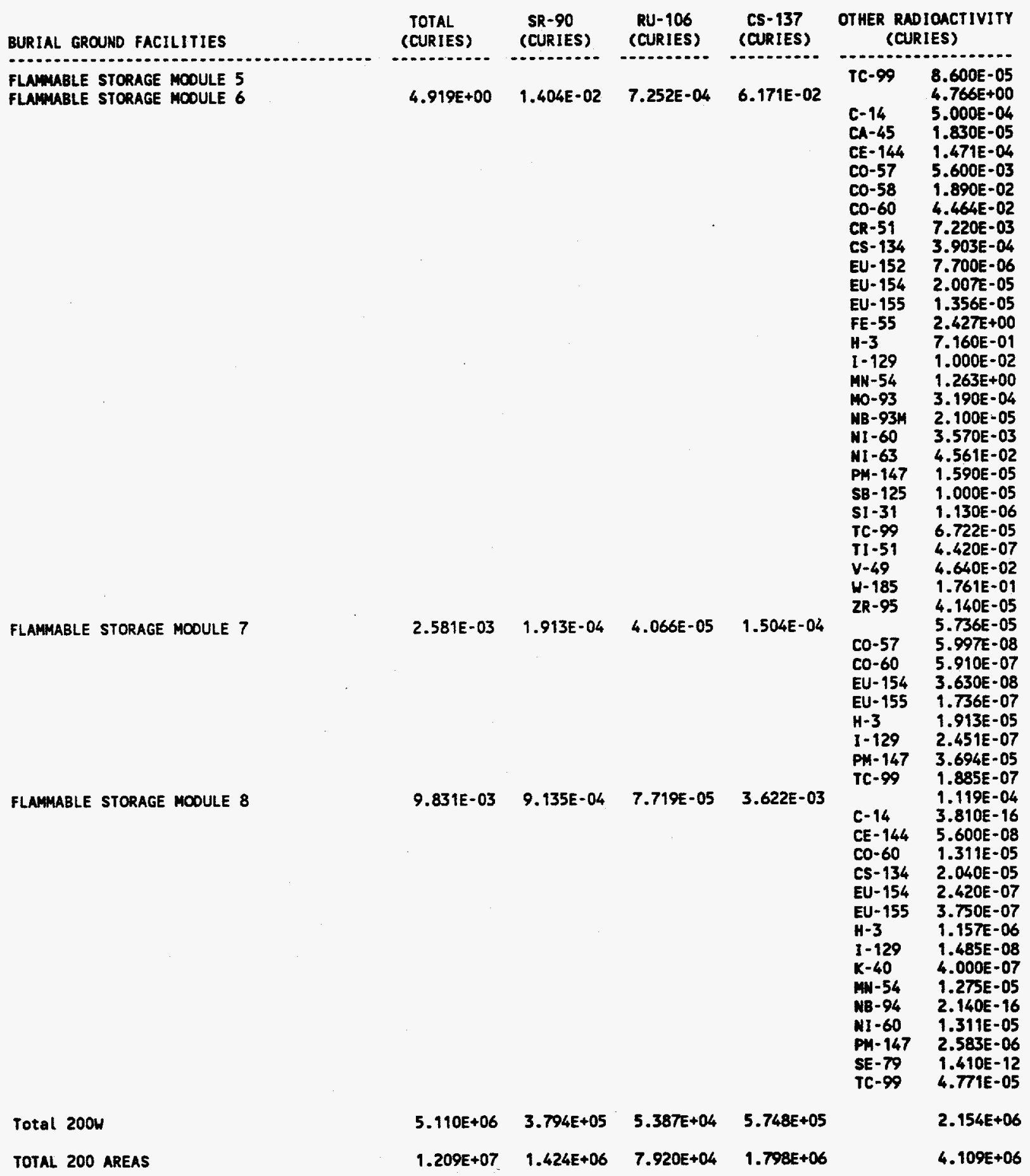


SWIR3288 - (Page 19 of 19)

RUMDATE: $08 / 20 / 94$

Solid Waste Burial Ground Areas Waste Volumes Disposed and stored and Nondecayed Curie Content in the 200 Areas from startup to December 31, 1993, Including segregated Transuranic waste.

\begin{tabular}{|c|c|c|c|c|c|}
\hline URIAL GROUND FACILITIES & $\begin{array}{l}\text { TOTAL } \\
\text { (CURIES) }\end{array}$ & $\begin{array}{l}\text { SR-90 } \\
\text { (CURIES) }\end{array}$ & $\begin{array}{l}\text { RU-106 } \\
\text { (CURIES) }\end{array}$ & $\begin{array}{l}\text { CS-137 } \\
\text { (CURIES) }\end{array}$ & $\begin{array}{l}\text { OTHER RADIOACTIVITY } \\
\text { (CURIES) }\end{array}$ \\
\hline
\end{tabular}

SPECIAL CAISSON/TRENCH BREAROUT FOR FACILITY 218W4B

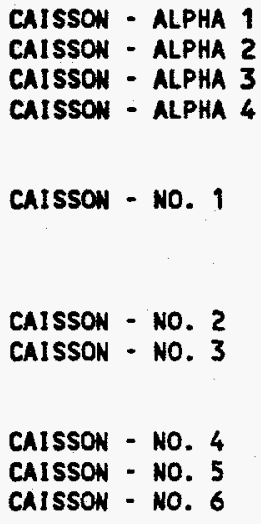

$\begin{array}{llll}3.627 E+04 & 7.501 E+02 & 1.550 E+03 & 7.990 E+02 \\ 2.055 E+04 & 2.472 E+03 & 8.968 E+02 & 2.648 E+03 \\ 2.274 E+04 & 4.336 E+03 & 2.066 E+02 & 4.660 E+03 \\ 1.985 E+04 & 3.878 E+03 & 4.506 E+01 & 4.178 E+03 \\ & & & \\ 1.490 E+04 & 4.748 E+02 & 4.840 E+02 & 2.400 E+02 \\ & & & \\ & & & \\ 2.000 E+01 & 3.978 E-01 & 8.566 E-01 & 4.248 E-01 \\ 6.345 E+03 & 1.169 E+02 & 2.517 E+02 & 1.338 E+02 \\ & & & \\ 5.811 E+04 & 1.191 E+03 & 2.492 E+03 & 1.272 E+03 \\ 1.564 E+03 & 2.015 E+02 & 3.031 E+01 & 3.662 E+02 \\ 2.903 E+04 & 1.492 E+03 & 2.469 E+02 & 1.760 E+03\end{array}$

$1.250 E+02$

$.000 E+00$

$.000 E+00$

$1.288 E+\infty$

$2.975 E+05$

$7.469 E+03$

$9.246 E+03$

$8.063 E+03$

\section{CE}

$\mathrm{H}-3$

$1-129$

PM-147

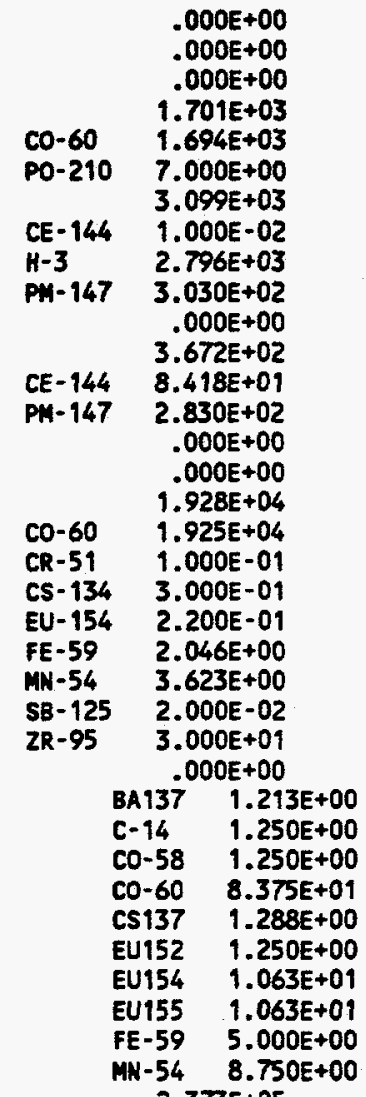

$2.373 E+05$

6. $000 E-03$

2.283E+05

5.000E-01

$9.000 E+03$

BA $1379.881 E+00$

C-14 1.019E+01

Co-58 1.019E+01

CO-60 $\quad 6.825 E+02$

CS137 1.049E+01

EU152 1.019E+01

EU154 $8.659 E+01$

EU155 8.659E+01

FE-59 4.075E+01

NN-54 , 7.131E+01 2.617E+05 
SUIR328C - (Page 1 of 19)

RUNDATE: 08/20/94

Solid Waste Burial Ground Areas Decayed Curie Content in the 200 Areas from Startup to December 31, 1993, Including Segretated Transuranic Haste.

BURIAL GROUND FACILITIES

2OOE AREA

C.........

BURIAL GROUND $218 E 1$

BURIAL GROUND $218 E 10$

BURIAL GROUND 218E12A

BURIAL GROUND 218E12B

TOTAL
(CURIES)

$2.845 E+00$ $3.118 E+06$
SR-90

(CURIES)

(C)........

6.817E-01 $7.157 E+05$

3.540E+01 $1.588 E+06$
8.550E +00 2.330E+04
4.476E- 13 $9.036 E-01$

7.635E-01
$8.656 E+05$

RU-106

(CURIES)

CS-137

(CURIES)

(CURIEs)

$9.431 E+00$
$2.385 E+04$
$1.504 E-07$ $3.440 E+00$
PUREX TUNNEL (\#1) 218E14

PUREX TUNNEL (\$2) $218 E 15$

BURIAL GROUND 218E2

BURIAL GROUND $218 E 4$
$3.311 E+03$

$2.958 E+04$

$7.431 E+02$

3.283E-01
$7.989 E+02$ $7.103 E+03$ $1.783 E+02$ $7.898 E-02$
OTHER RADIOACTIVITY (CURIES)

$\begin{array}{cc} & .000 E+00 \\ C 0-60 & 6.393 E+02 \\ & 6.393 E+02 \\ \text { CS137 } & 3.467 E+01 \\ \text { CO-58 } & 1.857 E-13 \\ \text { CO-60 } & 5.712 E+02 \\ \text { EU155 } & 6.551 E+01 \\ \text { C- } 14 & 4.552 E+01 \\ \text { BA137 } & .000 E+00 \\ \text { EU154 } & 1.347 E+02 \\ \text { FE-59 } & 1.700 E-20 \\ \text { MN-54 } & 4.466 E-02 \\ \text { EU152 } & 2.293 E+01\end{array}$

EU152 2.293E+01

$$
.000 E+00
$$

$1.495 E+06$

6.110E+09

$\begin{array}{ll}B E-7 & 2.315 E-15 \\ C-14 & 8.117 E+01\end{array}$

CE-141 2.162E-28

CE-144 2.096E-01

$C L-36 \quad 6.110 E+01$

Co-58 $4.826 E+02$

CO-60 5.673E+05

CR-51 2.127E+00

CS-134 2.632E-02

FE-55 3.102E+05

FE-59 $1.517 E+00$

$\mathrm{H}-3 \quad 9.477 \mathrm{E}+02$

HF-181 4.465E-02

$1-129 \quad 6.112 E+01$

K-40 9.054E-03

MN-54 $2.613 E+03$

40-93 6.895E-02

NB-94 5.673E-01

MB-95 2.306E-01

NI-59 4.211E+03

NI-63 6.090E+05

PM- $147 \quad 9.199 E-01$

SB- 125 3.161E-07

SE-79 2.422E-05

SM-151 4.099E-01

TA-182 7.229E-25

TC-99 1.030E-01

2N-65 8.718E-02

2R-93 2.035E-04

2R-95 2.252E-01

CS137 1.031E-02

CO-58 5.304E-15

CO-60 2.831E-01

EU155 3.362E-02

$c-14 \quad 1.201 E-02$

BA137 .000E+00

EU154 5.342E-02

FE-59 $1.14 \pi E-21$

MN-54 1.329E-04

EU152 7.916E-03 $.000 E+00$

$.000 E+00$

$.000 E+00$

$.000 E+00$ 
SHIR328C - (Page 2 of 19)

RUNDATE: 08/20/94

Solid Waste Burial Ground Areas Decayed Curie Content in the 200 Areas from Startup to December 31, 1993, Including Segretated Transuranic waste.

BURIAL GROUND FACILITIES

BURIAL GROUND $218 E 5$

BURIAL GROUND 218E5A

BURIAL GROUND $218 E 8$

BURIAL GROUND 218EC9

2228 VAULTS

Total 200E
TOTAL (CURIES)

$2.470 E+02$

5.77BE+02

3.558E-01

$3.784 E+01$

$1.730 E+01$

$4.741 E+06$
SR- 90

(CURIES)

$5.941 E+01$

$1.392 E+02$

8.574E-02

$1.186 E+01$

4. $147 E+00$

7.473E+05
RU-106

(CURIES)

$1.209 E-09$

$1.715 E-08$

$1.438 E-11$

$1.556 E-03$

3.008E-12

$4.824 E+00$
CS-137

(CURIES)

$6.610 E+01$

$1.544 E+02$

$9.507 E-02$

$7.190 E+00$

4.642E+00

$8.985 E+05$
OTHER RADIOACTIVITY (CURIES)

$.000 E+00$ $.000 E+00$

$.000 E+00$

$7.417 \mathrm{E}-07$

EU-154 7.417E-07 $.000 E+00$

$1.496 E+06$ 
SUIR328C - (Page 3 of 19)

RUNDATE: 08/20/94

Solid Waste Burial Ground Areas Decayed Curie Content in the 200 Areas from Startup to December 31, 1993, Including Segretated Transuranic Waste.

$\begin{array}{llcccc}\text { BURIAL GROUND FACILITIES } & \begin{array}{c}\text { TOTAL } \\ \text { (CURIES) }\end{array} & \begin{array}{c}\text { SR-90 } \\ \text { (CURIES) }\end{array} & \begin{array}{c}\text { RU-106 } \\ \text { (CURIES) }\end{array} \text { (CURIES) } & \begin{array}{c}\text { OTHER RADIOACTIVITY } \\ \text { (CURIES) }\end{array}\end{array}$

2OOU AREA

212N BUILDING

BURIAL GROUND 21841

BURIAL GROUND 218W11

BURIAL GROUND 218WIA

BURIAL GROUND 218H2

BURIAL GROUND 218H2A

BURIAL GROUND 21843

BURIAL GROUND 218W3A

$\begin{array}{rrrr}.000 E+00 & .000 E+00 & .000 E+00 & .000 E+00 \\ 5.697 E+00 & 1.365 E+00 & 1.071 E-12 & 1.529 E+00 \\ 3.685 E-03 & 8.888 E-04 & 3.827 E-13 & 9.839 E-04 \\ 1.422 E+03 & 3.411 E+02 & 6.358 E-10 & 3.813 E+02 \\ 1.634 E+01 & 3.929 E+00 & 6.967 E-11 & 4.373 E+00 \\ 9.675 E+03 & 2.331 E+03 & 3.495 E-04 & 2.582 E+03\end{array}$

$3.202 E+01$

$1.030 E+06$

$7.718 E+00$

$1.032 E+05$

1.607E-09

$9.476 E+03$

\subsection{E+00}

$2.827 E+05$

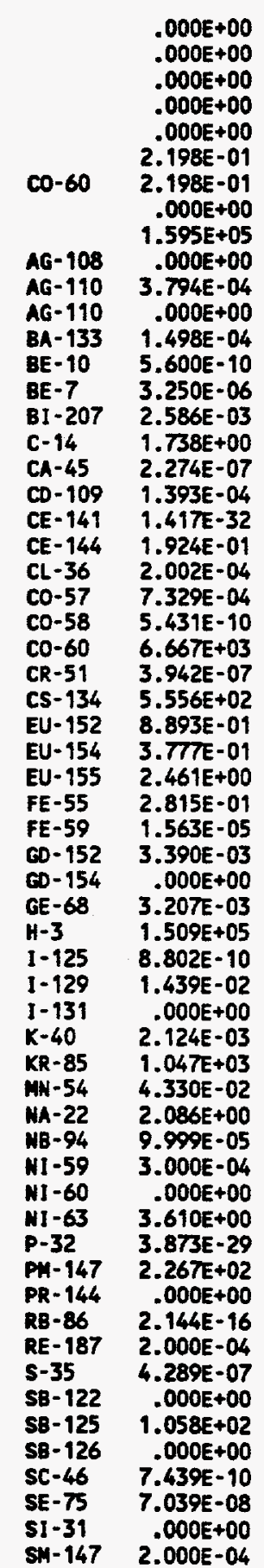


SWIR328C - SPage

RUNDATE: 08/20/94

Solid Waste Burial Ground Areas Decayed Curie Content in the 200 Areas from Startup to December 31, 1993, Including Segretated Transuranic waste.

BURIAL GROND FACILITIES

TOTAL (CURIES)

BURIAL GROUND 218W3A

BURIAL GROUND 218U3AE
$2.043 E+05 \quad 1.292 E+04 \quad 4.463 E+02 \quad 2.736 E+04$
OTHER RADIOACTIVITY (CURIES)

(CURIES) (CURIES) (CURIES)

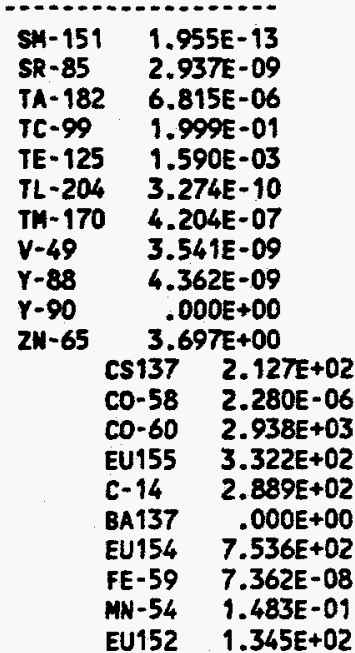

BE-10 $\quad 4.600 E-11$

$C-14 \quad 2.016 E+01$

CD-113 1.155E-05

CE-144 6.908E-03

CL-36 1.840E-08

CO-58 3.400E-01

CO-60 3.658E+04

CR-51 5.206E-05

CS- $134 \quad 1.710 E+02$

CS- $135 \quad 3.800 E-07$

EU-152 1.202E+03

EU-154 $1.778 E+03$

EU-155 3.820E+02

FE-55 9.293E+03

FE-59 2.882E+01

$\mathrm{H}-3 \quad 1.946 \mathrm{E}+04$

HF- 181 4.151E-04

$1-129 \quad 1.805 E-07$

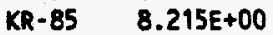

MH-54 1.867E+02

MA-22 7.653E-02

MB-93M 2.368E-05

MB-94 5.608E-01

NB-95 1.192E-03

NI-59 4.000E+02

$M 1-60 \quad .000 E+00$

NI-63 4.761E+04

PN- $147 \quad 5.856 E-02$

SB-125 5.166E+03

SB-126 4.166E-24

SE-TS $\quad 1.479 E-08$

SE-79 1.784E-07

SW-151 3.434E-04

SN-119 1.421E+03

SN-121 9.772E-08

$S N-123 \quad .000 E+00$

SN-126 5.100E-07

TA-182 $1.050 E+00$

TC-99 5.590E-05

TE-125 1.475E-01 
SUIR328C - (Page 5 of 19)

RUNDATE: $08 / 20 / 94$

Solid Waste Burial Ground Areas Decayed Curie Content in the 200 Areas from Startup to December 31, 1993, Including Segretated Transuranic Waste.

BURIAL GROUND FACILITIES BURIAL GROUND 218U3AE

BURIAL GROUND 218W4A

BURIAL GROUND 218H4B

BURIAL GROUND 218W4C $\begin{array}{lllll}\text { TOTAL } & \text { SR-90 } & \text { RU-106 } & \text { CS-137 OTHER RADIOACTIVITY }\end{array}$

$\begin{array}{llll}\text { (CURIES) } & \text { (CURIES) } & \text { (CURIES) } & \text { (CURIES) } \\ \end{array}$

$\begin{array}{llll}1.387 E+02 & 3.339 E+01 & 1.037 E-06 & 3.675 E+01 \\ 1.342 E+05 & 1.548 E+04 & 3.264 E+00 & 1.702 E+04\end{array}$
(CURIES)

$20-03 \quad 8.184 E-06$

2R-95 $1.266 E-01$ CS137 $9.530 E+00$

CO-58 2.113E-11

Co-60 $2.465 E+02$

EU155 2.916E+01

C-14 1.125E+01

BA137 .000E+00

EU154 4.776E+01

FE-59 9.366E-16

HN-54 8.978E-02

EU152 7.193E+00

OM- $147 \quad 5.390 E-01$

PM-147 5.390E-01

CE-144 9.459E-08

CO-60 $9.107 E+04$

CR-51 1.388E-31

CS-134 8.250E-02

EU-154 1.594E-01

FE-59 3.389E-10

$1=129$

$5.856 E+04$

$9.813 E-02$

PH-147 1.639E+01

PO-210 4.064E-11

SB-125 7.100E-03

ZR-95 6.939E-10

CS137 7.129E+00

Co-58 1.298E-21

CO-60 4.883E+01

EU155 5.267E+00

C-14 1.141E+01

BA137 .000E+00

EU154 1.698E+01

FE-59 2.372E-33

HN-54 1.619E-04

EU152 3.671E+00

$1.625 E+05$

BE-10 1.890E-10

$C-14 \quad 8.322 E+00$

CD-113 4.762E-05

CE-141 1.091E-04

CE-144 3.860E+01

CL-36 7.580E-08

CD-58 $9.909 E-02$

CO-60 1.496E+05

CR-51 6.866E-17

CS-134 2.198E+02

CS-135 1.582E-06

EU-152 1.698E+01

EU-154 2.157E+02

EU-155 5.143E-03

FE-55 2.913E+03

FE-59 2.594E-09

$\mathrm{H}-3 \quad 2.468 \mathrm{E}+01$

$1-125 \quad 5.202 E-07$

1.129 1.008E-03

XR-85 5.615E-02

MW-54 8.837E+01

MO-93 1.254E-03

NB-93M 9.710E-05 
SHIR328C - (Page 6 of 19)

RUNDATE: 08/20/94

Solid Waste Burial Ground Areas Decayed Curie Content in the 200 Areas from Startup to December 31, 1993, Including Segretated Transuranic Waste.

BURIAL GROUND FACILITIES

BURIAL GROUND 218W4C
TOTAL (CURIES)

SR-90 (CURIES)

RU-106 CS-137
(CURIES) (CURIES)

OTHER RADIOACTIVITY (CURIES)

\begin{tabular}{ll} 
NB-94 & $3.135 E-05$ \\
MI-59 & $7.559 E+01$ \\
NI -60 & $.000 E+00$ \\
NI-63 & $9.329 E+03$ \\
P-32 & $1.742 E-07$ \\
PA-231 & $2.115 E-06$ \\
PH-147 & $2.517 E-01$ \\
S-35 & $3.271 E-04$ \\
SB-125 & $2.619 E-01$ \\
SB-126 & $7.435 E-22$ \\
SE-79 & $3.030 E-06$ \\
SH-151 & $1.408 E-03$ \\
SN-121 & $4.026 E-07$ \\
SN-126 & $2.109 E-06$ \\
SR-82 & $3.804 E-06$ \\
SR-85 & $1.787 E-04$ \\
TA-182 & $3.668 E-04$ \\
TC-99 & $1.402 E-03$ \\
TE-125 & $1.057 E-06$ \\
ZN-65 & $2.287 E-05$ \\
ZR-93 & $2.628 E-05$ \\
\multicolumn{3}{c}{ CS137 } & $2.186 E+00$ \\
CO-58 & $1.587 E-07$ \\
CO-60 & $4.727 E+01$ \\
EU155 & $5.526 E+00$ \\
C-14 & $2.693 E+00$ \\
BA137 & $.000 E+00$ \\
EU154 & $9.886 E+00$ \\
FE-59 & $1.736 E-07$ \\
MN-54 & $1.902 E-02$ \\
EU152 & $1.564 E+00$
\end{tabular}

BURIAL GROUND 218W5
$1.533 E+04$

AC-227 3.656E-05

AC-228 .000E+00

AG-108 .000E+00

AG-110 1.127E-05

AG- $110 \quad .000 E+00$

AL-28 .000E+00

AU-195 3.686E-07

$B A-133$ 5.805E-02

BA-134 .000E+00

$B A-137 \quad .000 E+00$

BA-140 5.936E-08

BE-7 2.802E-03

BI-207 1.646E-03

BI-212 .000E+00

BI-214 .000E+00

C. $14 \quad 4.339 E+00$

Ch-45 6.105E-03

CD-109 3.854E-03

CE-141 4.947E-06

CE-144 4.763E-01

CL-36 1.368E-05

CO-56 $\quad 9.410 E-05$

CO-57 1.662E-02

CO-58 1.059E-03

CO-59 .000E+00

CO-60 2.313E+03

CR-51 7.621E-04

CS-133 .000E+00

CS-134 1.301E-01

CU-65M . .000E+00 
SWIR328C - (Page 7 of 19)

RUNDATE: 08/20/94

Solid Waste Burial Ground Areas Decayed Curie Content in the 200 Areas from startup to December 31, 1993, Including Segretated Transuranic Waste.

\begin{tabular}{|c|c|c|c|}
\hline IPI & $\begin{array}{l}\text { TOTAL } \\
\text { (CURIES) }\end{array}$ & $\begin{array}{c}\text { SR-90 } \\
\text { (CURIES) }\end{array}$ & $\begin{array}{l}\text { RU-106 } \\
\text { (CURIES) }\end{array}$ \\
\hline
\end{tabular}

OTHER RADIOACTIVITY BURIAL GROUND 218W5

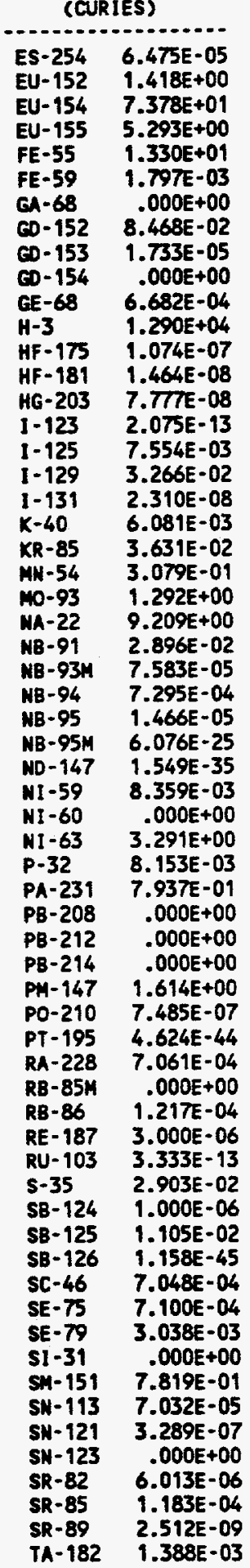


SWIR328C - (Page 8 of 19)

RUNDATE: 08/20/94

Solid Waste Burial Ground Areas Decayed Curie Content in the 200 Areas from Startup to December 31, 1993, Including segretated Transuranic Waste.

BURIAL GROUND FACILITIES

BURIAL GROUND 218 H5
TOTAL (CURIES)
\$R-90 (CURIES)
RU-106

(CURIES)
CS-137 (CURIES)

OTHER RADIOACTIVITY (CURIES)

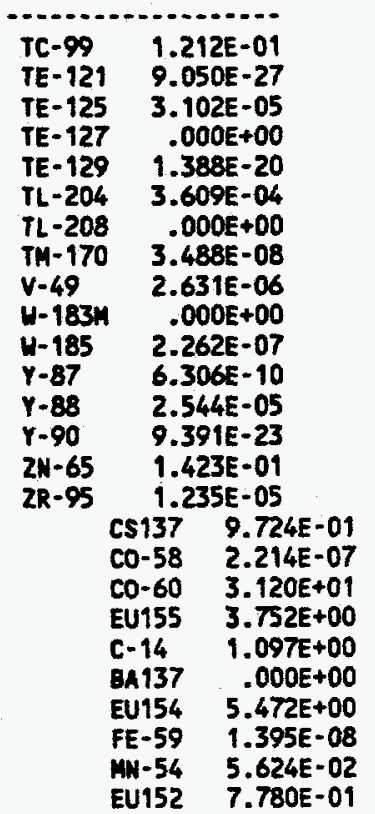

3.217E-03 $1.371 E+02$

$2.231 E+01$

$1.708 E+02$ $3.301 E+01$ $5.349 E+00$

$7.134 E+01$

$7.015 E-15$

2.803E-09

$4.376 E-12$

3.146E-02

8.614E-04

$3.668 E+01$

$5.986 E+00$

$1.351 E+01$
CENTRAL WASTE COMPLEX $2401 \mathrm{H}$ BUILOING
$1.569 E+05$
$7.834 E+04$
$1.265 E-05 \quad 1.253 E-02$
$.000 E+00$
$.000 E+000$ $.000 E+00$ $.000 E+00$ $1.199 \mathrm{E}-03$

$\begin{array}{lr}\text { CO-60 } & 4.241 E-04 \\ \text { CS- } 134 & 2.940 E-04\end{array}$ EU-152 7.757E-05

EU-154 2.922E-04

MN-54 1.933E-08

SB-125 1.105E-04

TE-125 4.774E-07

2R-95

4. $110 \mathrm{E}-08$
1.

CS137 5.519E-05

CO-58 3.328E-06

CO-60 3.149E-03

EU155 3.955E-04

C-14 5.509E-05

BA137 .000E+00

EU154 4.242E-04

FE-59 8.480E-06

MW-54 1.507E-04

EU152 5.169E-05

$2.091 E+02$

Co-60 $\quad 1.748 E-04$

EU-154 2.889E-05

H-3

$2.091 E+02$

CS137 3.045E-08

CO-58 2.046E-14

CO-60 1.285E-06

EU155 1.5T7E-07

C- $14 \quad 3.238 E-08$

BA137 .000E+00

EU154 1.989E-07

FE-59 1.840E-17

MN-54 8.916E-09

EU152 2.627E-08 
SWIR328C - (Page 9 of 19)

RUNDATE: 08/20/94

Solid Waste Burial Ground Areas Decayed Curie Content in the 200 Areas from Startup to December 31, 1993, Including segretated Transuranic Waste.

\begin{tabular}{llccc} 
BURIAL GROUND FACILITIES & $\begin{array}{c}\text { TOTAL } \\
\text { (CURIES) }\end{array}$ & $\begin{array}{c}\text { SR-90 } \\
\text { (CURIES) }\end{array}$ & $\begin{array}{c}\text { RU-106 } \\
\text { (CURIES) }\end{array}$ & $\begin{array}{c}\text { CS-137 } \\
\text { (CURIES) }\end{array}$ \\
\hline CENTRAL HASTE COMPLEX 2402W BUILDING & $4.730 E+00$ & $6.556 E-01$ & $1.936 E-02$ & $1.412 E+00$
\end{tabular}

OTHER RADIOACTIVITY

CENTRAL MASTE COMPLEX 2402W BUILDING

$4.730 E+00 \quad 6.556 E-01 \quad 1.936 E-02 \quad 1.412 E+00$ (CURIES)

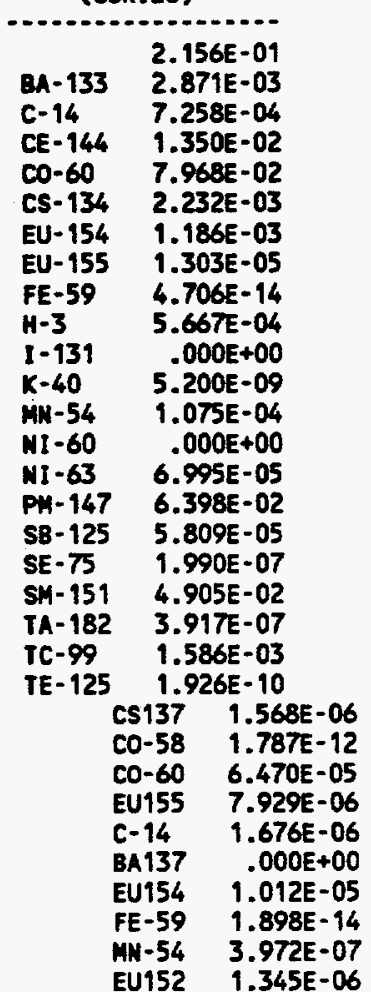

AC -228

BI-212

$B I-214$

C-14

CE-144

CO-60

CS-134

EU-152

EU-154

EU-155

FE-59

$\mathrm{H}-3$

MN -54

NA-22

MB-95M

NI- 60

PA-231

PB-212

PB-214

PN-147

PO-210

RA-228

SM-151

TC- 99

TL-208

ZR-95
1.006E-02 $.000 E+00$ $.000 E+00$ $.000 E+00$

$1.110 E-08$

$9.293 E-04$

$2.885 E-03$

$1.559 E-08$

$2.889 E-05$

$4.889 \mathrm{E}-05$

$1.919 E-05$

2.491E-15

$1.420 E-05$

$1.275 E-06$

1.630E-05

$6.549 E-20$

$.000 E+00$

4.010E-16

$.000 E+00$ $.000 E+00$

$3.425 E-03$

$1.872 E-24$

6.449E-04

2.041E-03

1.131E-06

$.000 E+00$

$1.357 \mathrm{E}-06$

CS137 3.044E-07

Co-58 9.157E-14

CO-60 1.253E-05 
SWIR328C - (Page 10 of 19)

RUNDATE : 08/20/94

Solid Waste Burial Ground Areas Decayed Curie Content in the 200 Areas from Startup to December 31, 1993, Including Segretated Transuranic Waste.

BURIAL GROUND FACILITIES

CENTRAL WASTE COMPLEX 240ZWB BUILDING

CENTRAL WASTE COMPLEX 2402WC BUILDING

CENTRAL WASTE COMPLEX 2402WD BUILDING

CENTRAL WASTE COMPLEX 240ZWE BUILDING
TOTAL
(CURIES)

SR-90

(CURIES)

RU-106

(CURIES)

CS- 137

(CURIES)

\section{.}

西

$1.948 E+00$

3.90
$3.904 E-01 \quad 1.799 E-03 \quad 3.954 E-01$

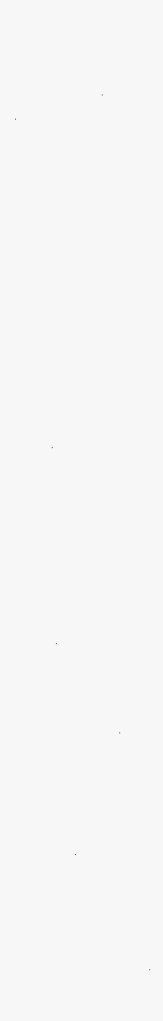

$1.617 E+00$

6.507E-0

5.520E-04

$7.138 E-03$

\begin{abstract}
$1.430 E-03 \quad 2.597 E-04 \quad 2.930 E-05 \quad 2.728 E-04$
\end{abstract}
OTHER RADIOACTIVITY (CURIES)

$\begin{array}{ll}\text { EU155 } & 1.535 E-06 \\ \text { C-14 } & 3.255 E-07 \\ \text { BA137 } & .000 E+00 \\ \text { EU154 } & 1.962 E-06 \\ \text { FE-59 } & 5.124 E-17 \\ \text { MN-54 } & 7.456 E-08 \\ \text { EU152 } & 2.609 E-07\end{array}$

2.936E-01

AG-110 .000E+00

C-14 1.026E-05

CE-141 3.219E-11

CE-144 5.859E-04

CO-60 3.803E-02

CS-134 1.519E-05

EU-152 4.187E-04

EU-154 7.100E-04

EU- 155 2.751E-04

FE-55 2.450E-01

FE-59 2.635E-14

$\mathrm{H}-3 \quad 3.800 \mathrm{E}-04$

I-129 1.426E-09

MN-54 2.253E-03

MA-22 2.240E-04

NB-94 $9.467 E-15$

MB-95 2.148E-15

MB-95M $1.097 E-25$

MI $-60 \quad .000 E+00$

NI-63 2.304E-04

PN-147 3.129E-03

SB-125 1.343E-10

SE-T3 3.642E-13

SE-79 6.256E-11

SM-151 2.327E-03

TC-99 5.011E-06

ZR-95 3.743E-05

CS137 $4.769 E-08$

CO-58 $8.500 E-13$

CO-60 2.224E-06

EU155 2.750E-07

C-14 4.966E-08

$8 A 137.000 E+00$

EU154 3.288E-07

FE-59 5.332E-15

HN-54 $2.888 E-08$

EU152 4.229E-08

C- $14 \quad 1.587 \mathrm{E}+00$

$\begin{array}{ll}C-14 & 7.526 E-12 \\ \text { CE-144 } & 7.619 E-04\end{array}$

EU-155 2.360E-04

$H-3 \quad 9.375 E+00$

PM-147 5.272E-03

SH-151 4.261E-03

SN-123 .000E+0O

TC-99 2.013E-01

C-14 1.681E-04

C- $14 \quad 1.460 E-15$

CE-144 1.250E-07

CO-60 1.671E-04

H-3 4.579E-09

I-129 3.580E-13

MB-94 8.190E-16

PI-147 9.148E-07 
SHIR328C - (Page 11 of 19)

RUNDATE: $08 / 20 / 94$

Solid Waste Burial Ground Areas Decayed Curie Content in the 200 Areas from Startup to December 31, 1993, Including Segretated Transuranic Waste.

BURIAL GROUND FACILITIES

CENTRAL WASTE COMPLEX 2402UE BUILDING

CENTRAL WASTE COMPLEX 2402WF BUILDING

CEMTRAL HASTE COMPLEX 2402WG BUILOING
TOTAL (CURIES) (CURIES) (CURIES)

3.309E-01 6.685E-03

$0.179 E-04$

5.648E-01 1.057E-01 5.223E-04 1.320E-01

(CURIES)

(CURIES)

-.........

(CURIES)
OTHER RADIOACTIVITY

(CURIES)

SE-79 5.400E-12

TC- $99 \quad 2.000 E-10$

C-14 3.006E-01

CE-144 1.187E-03

H-3 3.110E-05

PM- $147 \quad 6.718 E-03$

SH- $151 \quad 6.200 E-03$

TC-99 2.864E-01

c- $14 \quad 1.000 E-04$

CE-144 1.071E-05

CO-60 7.104E-02

CS-134 6.606E-07

EU-152 5.680E-05

EU-154 1.536E-04

EU-155 4.117E-05

FE-59 2.019E-13

$\mathrm{H}-3 \quad 1.114 \mathrm{E}-03$

$1-129 \quad 1.129 E-06$

$1-131 \quad 6.041 E-25$

MN-54 6.255E-04

NB-94 $5.280 E-14$

MI-59 6.540E-11

NI $-60 \quad .000 E+00$

NI-63 7.519E-06

P-32 1.043E-09

PN-147 1.826E-04

S-35 1.113E-05

SE-79 $3.485 E-10$

TA-182 7.419E-09

TC-99 $\quad 1.593 E-06$

ZN-65 4.158E-06

CS137 2.446E-07

C0-58 $1.369 E-09$

CO-60 1.073E-05

EU155 1.321E-06

C-14 2.583E-07

BA137 .000E+00

EU154 1.631E-06

FE-59 1.700E-09

MN-54 1.248E-07

EU152 $2.132 E-07$

8.201E-01

C-14 $\quad 5.018 E-10$

CE-144 8.709E-04

CO-60 5.252E-04

CS- 935 1.000E-04

EU-952 4.670E-05

EU- 954 8.096E-05

EU-155 3.309E-04

FE-59 1.011E-14

$\mathrm{H}-3$ 8.089E-01

I-129 1.000E-04

MN-54 3.939E-06

MB-94 3.044E-14

NI-59 2.500E- 90

NI $-60 \quad .000 E+00$

NI $-63 \quad 2.492 E-08$

PD-107 1.000E-04

PW-147 2.488E-03

SE-79 3.730E-11 
SHIR328C - (Page 12 of 19 )

RUNDATE: 08/20/94

Solid Waste Burial Ground Areas Decayed Curie Content in the 200 Areas from Startup to December 31, 1993, Including segretated Transuranic Waste.

BURIAL GROUND FACILITIES

CENTRAL WASTE COMPLEX 2402WH BUILDING

CENTRAL MASTE COMPLEX 240ZWI BUILDIHG

CENTRAL WASTE COMPLEX 2402WJ BUILDING

CENTRAL HASTE COMPLEX 2402WL BUILDING
TOTAL (CURIES)

(...........

SR-90 (CURIES)

RU-106 (CURIES)

CS-137 (CURIES)

(c........

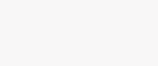

8.842E-01 7.109E-03 6.637E-04 $\quad 7.876 E-03$

2.269E-01

4.815E-03

$1.574 E-04$

5.358E-03

2.347E-0
5.197E-02 4.971E-04 2.751E-02
OTHER RADIOACTIVITY (CURIES)

ST-151 $9.694 \mathrm{E}-04$ SH-123 $\quad .000 \mathrm{E}+00$

TC- $99 \quad 5.625 E-03$

$2 R-90 \quad .000 E+00$

ZR-93 $1.696 E-13$

8.520E-0

C-14 1.011E-11

CE-144 1.202E-03

EU-155 1.003E-04

$\mathrm{H}-3 \quad 5.663 \mathrm{E}-01$

$1-129 \quad 1.864 E-12$

NB-94 4.270E-15

PM-147 7.025E-03

SE-79 2.820E-11

SH-151 6.102E-03

SN-123 .000E+00

TC-99 2.713E-0

2.042E-01

c-14 9.995E-07

CE-144 1.738E-04

$H-3 \quad 6.905 E-06$

PM-147 1.378E-03

SM-151 $\quad 1.158 E-03$

TC-99 2.014E-01

5.938E-02

C-14 5.634E-02

CA-45 2.806E-08

CE-144 6.373E-05

CL-36 3.560E-05

CO-57 5.392E-07

Co-60 6.764E-04

CS-134 5.398E-05

CS-135 5.650E-13

EU- $154 \quad 6.426 E-07$

EU-155 6.439E-08

FE-59 7.031E-14

$\mathrm{H}-3 \quad 1.199 \mathrm{E}-03$

I-129 7.429E-07

MN-54 1.558E-11

HA-22 3.505E-07

MB-93M $1.238 E-10$

MB-94 1.520E-12

MI $-60 \quad .000 E+00$

$M I-63 \quad 6.553 E-06$

NI-63 8.280E-11

PD-107 1.140E-13

PM-147 3.841E-04

RA-228 2.455E-10

RU-103 4.487E-09

S-35 7.111E-06

SE-75 . 2.664E-10

SE-79 1.490E-06

SM-151 3.883E-04

TC-99 2.155E-04

2N-65 8.548E-09

$2 R-93 \quad 3.960 E-12$

CS137 1.006E-14

Co-58 6.066E-18

C0.60 $5.221 E-13$

EU155 6.511E-14

C-14 1.024E-14

BA137 .000E+00 
SWIR328C - (Page 13 of 19)

RUNDATE: 08/20/94

Solid Waste Burial Ground Areas Decayed Curie Content in the 200 Areas from Startup to December 31, 1993, Including Segretated Transuranic Waste.

BURIAL GROUND FACILITIES

CENTRAL WASTE COMPLEX 2402HL BUILOING

CENTRAL WASTE COMPLEX 2403WA BUILDING
TOTAL
(CURIES)

(C...........

(CURIES) (CURIES)

(CURIES)

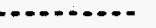

(c.........

(C)........

$2.373 E+01 \quad 4.800 E+00$

$6.455 E-02$

$5.161 E+00$

OTHER RADIOACTIVITY (CURIES)

EU154 7.348E-14

FE-59 3.061E-19

MN-54 1.330E-14

EU152 $9.181 E-15$

AC-228 $3.374 E+00$

AG-110 1.244E-07

$A G-110 \quad .000 E+00$

BE-7 3.274E-04

BI-214 .000E+00

C-14 2.320E-04

CA-45 7.596E-07

CE-144 8.730E-02

CO-56 4.055E-07

CO-57 4.249E-05

CO-58 1.459E-06

CO-60 2.739E-01

CS-134 2.684E-03

CS-135 6.620E-13

EU-152 1.686E-03

EU-154 3.082E-03

EU-155 6.113E- 04

FE-55 3.212E-01

FE-59 9.966E-06

CD-153 2.630E-04

GD-154 .000E+00

$\mathrm{H}-3 \quad 1.104 \mathrm{E}-\mathrm{O} 2$

HF-175 2.429E-05

HG-203 5.675E-06

$1-125 \quad 1.280 E-06$

$1-129 \quad 2.475 E-06$

$K-40 \quad 9.393 E-02$

$K R-85 \quad 2.412 E-03$

MN-54 8.533E-02

MO-93 2.400E-07

MA-22 5.136E-04

NB-93M 2.250E-07

NB-94 2.003E-08

NB-95M 1.291E-17

NI-59 7.650E-06

NI $-60 \quad .000 E+00$

MI-63 9.532E-04

P-32 7.547E-08

PB-212 .000E+00

PB-214 .000E+00

$P D-107 \quad 1.336 E-13$

PM-147 2.276E-01

PT-195 1.273E-23

S-35 1.080E-03

SB-125 1.803E-02

SC-46 9.022E-09

SE-79 5.146E-08

SM-151 1.090E-01

TA-182 4.232E-09

TC-99 2.207E+00

TE-125 4.877E-03

TE-125 5.491E-04

TL-208 .000E+00

$v-49$

$9.922 E-05$

$X E-131 \quad 2.644 E-17$

2N-65 3.040E-06

2R-93 4.630E-12 
SWIR328C - (Page 14 of 19)

RUNDATE: 08/20/94

Solid Waste Burial Ground Areas Decayed Curie Content in the 200 Areas from Startup to December 31, 1993, Including Segretated Transuranic Waste.

BURIAL GROUND FACILITIES

CENTRAL WASTE CONPLEX 2403WA BUILDING

CENTRAL WASTE COMPLEX 2403WC BUILOING

\begin{tabular}{ccc}
$\begin{array}{c}\text { TOTAL } \\
\text { (CURIES) }\end{array}$ & $\begin{array}{c}\text { SR-90 } \\
\text { (CURIES) }\end{array}$ & $\begin{array}{c}\text { RU-106 } \\
\text { (CURIES) }\end{array}$ \\
\hline
\end{tabular}
(..........
Cs-137 (CURIES)

OTHER RADIOACTIVITY (CURIES)

2R-95

1.911E-05

CS137 $1.044 E-08$

CO-58 $1.110 \mathrm{E}-13$

CO-60 4.689E-07

EU155 5.783E-08

C-14 1.096E-08

BA137 .000E+00

EU154 7.054E-08

FE-59 1.366E-14

MN $-54 \quad 4.829 E-09$

EU152 $9.161 \mathrm{E}-09$

$7.040 E+01 \quad 2.554 E+01 \quad 1.996 E-02 \quad 9.226 E+00$

AC -227
AC- 228

$9.058 E-01$

$A G-110$

AG- 110

BI-212

BI-214

C- 14

$1.183 E-02$

$.000 E+00$

3.143E-07

$.000 E+00$

$.000 E+00$

$.000 E+00$

$6.463 \mathrm{E}-04$

CE-141

CE-144

CO-57

$1.202 E-11$

2.351E-02

Co-58

$6.444 \mathrm{E}-10$

$6.545 E-04$

CO-60 6.978E-02

CR-51 1.273E-04

CS-134 1.669E-02

EU- 152 9.756E-02

EU-154 1.990E-01

EU-155 4.562E-02

FE-55 2.701E-02

FE-59 1.097E-09

GD-154 .000E+00

$\mathrm{H}-3$ 1.217E-01

$I-129 \quad 1.506 E-04$

K-40 2.769E-04

MN-54 3.987E-03

M0-93 3.989E-08

MA-22 9.347E-06

MB-93M 1.507E-06

MB -94

NB- 95

$1.946 E-08$

$2.865 E-13$

MB-95M $1.121 E-11$

NI-59 2.627E-06

NI -60

NI -63

NI -63

P-32

$P A-231$

$P B-212$

$P B-214$

$P M-147$

PO-210

RA-228

SB-125

SE- 79

SN-151

TA-182

TC- 99

TE- 125

$T L-208$

$v-49$

$.000 E+00$

1.648E-03

$2.070 E-10$

7.682E-05

2.400E-07

$.000 E+00$

$.000 E+00$

$6.088 E-02$

1.751E-01

1.223E-09

$1.013 E-03$

7.508E-08

1.961E-02

1.419E-04

2.878E-02

5.625E-07

$.000 E+00$

4.847E-09

U-185

8.415E-08 
SWIR328C - (Page 15 of 19)

RUNDATE: $08 / 20 / 94$

Solid Waste Burial Ground Areas Decayed Curie Content in the 200 Areas from Startup to December 31, 1993, Including Segretated Transuranic Waste.

BURIAL GROUND FACILITIES

CENTRAL WASTE COMPLEX 2403WC BUILDING

CENTRAL WASTE COMPLEX 2403WD BUILDING

\section{$3.098 E+03 \quad 3.634 E+00 \quad 1.650 E-02 \quad 2.307 E+01$}

$1.774 E-04 \quad 1.336 E-05 \quad 1.380 E-06 \quad 2.343 E-05$

$3.124 E+00 \quad 8.886 E-01 \quad 4.979 E-04 \quad 6.781 E-01$
OTHER RADIOACTIVITY (CURIES)

$2 N-65 \quad 5.082 E-10$ ZR-95 3.235E-06

CS137 4.345E-06 CO-58 1.337E-09 CO-60 2.175E-04 EU155 2.705E-05 C- $14 \quad 4.458 E-06$ BA137 .000E+00 EU154 3.113E-05 FE-59 1.672E-10

MW-54 4.438E-06 EU152 $3.928 E-06$ $3.045 E+03$ C- $14 \quad 5.379 E-05$ CE-144 6.178E-04 C0-60 2.924E+03 EU-152 8.034E-03 EU-154 2.123E-01 EU-155 1.699E-03 $\mathrm{H}-3 \quad 4.024 \mathrm{E}-05$ I-129 $\quad 1.641 \mathrm{E}-03$ NB-93M 2.126E-04 MB-94 4.100E-06 NI-59 1.092E+00 NI $-63 \quad 1.198 E+02$ $P M-147 \quad 2.438 E-03$ SE-79 1.865E-05 SK-151 6.832E-04 TC-99 3.642E-01 CS137 1.541E-05 C0-58 $\quad 9.981 E-06$ C0-60 9.901E-04 EU155 1.255E-04 C. $14 \quad 1.500 E-05$ BA137 .000E+00 EU154 1.263E-04 FE-59 3.143E-05 MN-54 9.573E-05 EU152 1.491E-05

9.940E-05

1.322E-14

C-14 $14.322 E-14$

$\begin{array}{ll}C E-144 & 3.007 E-06 \\ \text { CS-134 } & 3.339 E-07\end{array}$

EU-152 2.844E-05

EU-154 4.860E-05

EU-155 6.926E-06

H-3

$1-129$

3.785E-07

$4.683 E-09$

NB-94 7.420E-15

PH-147 1.121E-05

SE-79 4.900E-11

TA-182 3.939E-09

TC-99 4.854E-07

7.493E-03

C-14 6.007E-03

Co-60 6.978E-04

$\mathrm{H}-3 \quad 7.881 \mathrm{E}-\mathrm{OH}$

CS137 4.572E-06

CO-58 8.237E-14

CO-60 1.72TE-04

EU155 2.102E-05

C-14 4.977E-06 
WHC-EP-0125-6

SWIR328C - (Page 16 of 19$)$

RUNDATE: 08/20/94

Solid Wagte Burial Ground Areas Decayed Curie Content in the 200 Areas from Startup to December 31, 1993, Including Segretated Transuranic Waste.

\section{BURIAL GROUND FACILITIES}

FLAMABLE STORAGE MOOULE 10

flamiale storage mOOULE 11

flammable storage module 12

FLAMMABLE STORAGE MODULE 13

FLAMHABLE STORAGE MODULE 15

\begin{tabular}{lccc}
$\begin{array}{l}\text { TOTAL } \\
\text { (CURIES) }\end{array}$ & $\begin{array}{c}\text { SR-90 } \\
\text { (CURIES) }\end{array}$ & $\begin{array}{c}\text { RU-106 } \\
\text { (CURIES) }\end{array}$ & $\begin{array}{c}\text { CS-137 } \\
\text { (CURIES) }\end{array}$ \\
\hline
\end{tabular}
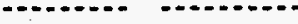

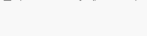

$6.014 E-04 \quad 4.654 E-06 \quad 1.137 E-07 \quad 1.958 E-04$
$2.356 E-04$

3.101E-03
OTHER RADIOACTIVITY (CURIES)

(c).......

BA137 .000E+00

EU154 2.814E-05

FE-59 8.693E-18

MN-54 5.999E-07

EU152 3.828E-06 2. $106 E-04$

Uu-195 1.005E-06

C- $14 \quad 5.498 E-05$

CE-144 1.807E-07

CO-58 7.806E-10

CO-60 6.972E-09

$H-3 \quad 1.378 E-04$

$1-129 \quad 1.280 \mathrm{E}-12$

MN-54 4.682E-09

MA-22 4.995E-07

NB-94 2.930E-15

NI $-60 \quad .000 E+00$

PM-147 3.076E-06

SE-79 1.900E-11

TC-99 1.300E-05

$2.519 E-03$

C-14 1.637E-04

CE-144 4.872E-04

CO-60 5.410E-06

CS-134 1.020E-06

CS-135 5.610E-13

EU-152 1.220E-07

EU-154 3.035E-06

EU-155 1.983E-06

I- $129 \quad 9.293 E-07$

$\mathrm{MB}-94 \quad 5.560 \mathrm{E}-15$

$N I-60 \quad .000 E+00$

PD-107 1.130E-13

PN- 147 1.274E-03

SB-125 1.542E-08

SE-79 3.670E-11

SM-151 4.900E-04

TC-99 2.125E-05

TE-125 9.060E-10

ZR-93 3.930E-12

$2.481 E+00 \quad 1.111 E-04 \quad 8.923 E-06 \quad 1.197 E-04$

C- 14

$2.480 E+00$

4.828E-02

CO-58 4.090E-08

CO-60 7.787E-08

EU-154 1.517E-07

EU-155 3.223E-08

FE-55 9.874E-07

H-3 2.432E+0O

$1-129 \quad 1.719 E-10$

HN-54 3.414E-07

$M 1-60 \quad 000 E+00$

N1-63 1.393E-07

PN- $147 \quad 5.294 E-08$

TC-99 1.331E-10

$1.336 E+00 \quad 3.365 E-04 \quad 6.846 E-06 \quad 2.055 E-04$

\section{C- 14}

Co-60 1.403E-06

$\mathrm{H}-3 \quad 1.326 \mathrm{E}+0 \mathrm{O}$

$1-129$ 1.815E-08

$N t-60 \quad .000 E+00$

NI $-63 \quad 1.670 E-10$ 
SWIR328C - (Page 17 of 19)

RUNDATE: $08 / 20 / 94$

Solid Waste Burial Ground Areas Decayed Curie Content in the 200 Areas from Startup to December 31, 1993, Including Segretated Transuranic Waste.

BURIAL GROUND FACILITIES

FLAMABLE STORAGE MOOULE 15

FLAMMABLE STORAGE MOOULE 2

flammable storage mOOULE 3

FLAMMABLE STORAGE MOOULE 4

fLAMMABLE STORAGE MOOULE 5

FLAMMABLE STORAGE MOOULE 6

TOTAL
(CURIES)

SR-90 (CURIES)

RU-106
(CURIES) (CURIES)

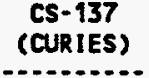

$1.160 E-02$

2.893E- 04

-..........

1.139E-03

\begin{abstract}
$3.530 E-03 \quad 1.025 E-10$
$2.072 E-12$

1.103E-10
\end{abstract}

$2.010 E-03 \quad 1.969 E-04 \quad 1.400 E-02$

$1.907 E-02 \quad 2.653 E-03 \quad 3.254 E-04 \quad 2.943 E-03$

$3.020 E+00 \quad 1.292 E-02 \quad 6.025 E-05 \quad 5.677 E-02$
OTHER RADIOACTIVITY (CURIES)

....

PM-147 2.564E-08

TC-99 4.802E-07

C-14 5.298E-03

CE-144 8.279E-04

CO-58 5.361E-07

CO-60 4.963E-04

CR-51 2.559E-13

FE-59 1.218E-16

$H-3 \quad 4.280 E-08$

MN-54 1.932E-04

WA-22 6.675E-04

NB-95 1.714E-12

PH- 147 1.385E-03

SC-47 .000E+00

SM-151 5.902E-04

TA-182 4.873E-04

TA-183 .000E+00

2N-65 2.188E-05

3.530E-03

$\mathrm{H}-3 \quad 3.528 \mathrm{E}-03$

P-32 $9.200 E-07$

IC- $99 \quad 6.800 E-07$

6.164E-03

AG-110 .000E+00

C-14 7.207E-04

CE-144 3.745E-04

CO-60 4.570E-06

CS-134 2.432E-05

EU-152 2.124E-06

EU-154 2.321E-05

EU-155 8.364E-06

H-3 2.153E-03

I-129 3.866E-10

MN-54 2.678E-08

PM-147 1.782E-03

SB-125 3.579E-06

SM-151 1.068E-03

TC-99 3.002E-10

ZR-95 4.359E-12

$6.144 E-03$

C-14 1.186E-03

CE-144 7.573E-04

$\mathrm{H}-3 \quad 5.548 \mathrm{E}-04$

PM-147 2.194E-03

SM-151 1.366E-03

TC- 99 8.600E-05

$2.883 E+00$

C-14 4.998E-04

CA-45 4.767E-07

CE-144 9.055E-06

CO-57 6.098E-04

CO-58 3.814E-06

CO-60 3.303E-02

CR-51 1.614E-07

CS-134 1.145E-04

EU-152 6.648E-06

EU-154 1.634E-05

EU-155 9.030E-06

FE-55 1.691E+00

$H-3 \quad 6.274 E-01$ 
SHIR328C - (Page 18 of 19 )

RUNDATE: $08 / 20 / 94$

Solid Waste Burial Ground Areas Decayed Curie Content in the 200 Areas from Startup to December 31, 1993, Including Segretated Transuranic Waste.

BURTAL GROUND FACILITIES

FLAMABLE STORAGE MOOULE 6

FLAMABLLE STORAGE MODULE 7

fLAMMABLE STORAGE MOOULE 8

Total 200W

TOTAL 200 AREAS
8.198E-04 1.840E-04 $1.236 E-05 \quad 1.448 E-04$

$9.038 E-03$

$8.788 E-0$

2.151E-05

$3.512 E \cdot 03$
OTHER RADIOACTIVITY (CURIES)

(CURIES)

\begin{tabular}{|c|c|}
\hline 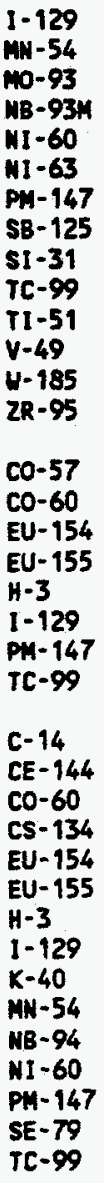 & $\begin{array}{l}1.000 E-02 \\
4.547 E-01 \\
3.188 E-04 \\
1.860 E-05 \\
.000 E+00 \\
4.514 E-02 \\
1.093 E-05 \\
4.467 E-06 \\
.000 E+00 \\
6.722 E-05 \\
.000 E+00 \\
1.626 E-02 \\
3.392 E-03 \\
4.015 E-07 \\
4.257 E-05 \\
1.314 E-08 \\
4.771 E-07 \\
3.178 E-08 \\
1.383 E-07 \\
1.745 E-05 \\
2.451 E-07 \\
2.402 E-05 \\
1.885 E-07 \\
8.199 E-05 \\
3.810 E-16 \\
1.908 E-08 \\
1.140 E-05 \\
1.356 E-05 \\
2.192 E-07 \\
3.164 E-07 \\
1.080 E-06 \\
1.485 E-08 \\
4.000 E-07 \\
5.401 E-06 \\
2.140 E-16 \\
.000 E+00 \\
1.872 E-06 \\
1.410 E-12 \\
4.771 E-05\end{array}$ \\
\hline & $5.340 E+05$ \\
\hline
\end{tabular}

$2.030 E+06$

SPECIAL CAISSON/TRENCH BREAKOUT FOR FACILITY 218W4B

\begin{tabular}{|c|c|c|c|c|c|}
\hline $\begin{array}{l}\text { CAISSON } \\
\text { CAISSON } \\
\text { CAISSON } \\
\text { CAISSON }\end{array}$ & $\begin{array}{l}\text { - ALPHA } 1 \\
\text { - ALPHA } 2 \\
\text { - ALPHA } 3 \\
\text { - ALPHA } 4\end{array}$ & $\begin{array}{l}1.779 E+03 \\
6.490 E+03 \\
1.217 E+04 \\
1.197 E+04\end{array}$ & $\begin{array}{l}4.306 E+02 \\
1.569 E+03 \\
2.941 E+03 \\
2.793 E+03\end{array}$ & $\begin{array}{l}2.047 E-04 \\
1.446 E-03 \\
2.710 E-03 \\
2.192 E-02\end{array}$ & $\begin{array}{l}4.702 E+02 \\
1.715 E+03 \\
3.216 E+03 \\
3.054 E+03\end{array}$ \\
\hline CAIsson & - No. 1 & $1.444 E+03$ & $2.598 E+02$ & $1.193 E-05$ & $1.338 E+02$ \\
\hline $\begin{array}{l}\text { CAIsson } \\
\text { caIsson }\end{array}$ & $\begin{array}{l}-10.2 \\
-10.3\end{array}$ & $\begin{array}{l}9.083 E-01 \\
2.798 E+02\end{array}$ & $\begin{array}{l}2.198 E-01 \\
6.511 E+01\end{array}$ & $\begin{array}{l}3.363 E-08 \\
1.332 E-05\end{array}$ & $\begin{array}{l}2.410 E-01 \\
7.665 E+01\end{array}$ \\
\hline
\end{tabular}

$\begin{array}{llll}2.110 E+06 & 2.876 E+05 & 1.007 E+04 & 4.533 E+05 \\ 6.851 E+06 & 1.035 E+06 & 1.007 E+04 & 1.352 E+06\end{array}$

$2.798 E+02 \quad 6.511 E+01 \quad 1.332 E-05 \quad 7.665 E+01$

$\begin{array}{lr} & .000 E+00 \\ & .000 E+00 \\ & .000 E+00 \\ & 4.054 E+02 \\ C 0-60 & 4.054 E+02 \\ P 0-210 & 4.064 E-11 \\ & 6.645 E+02 \\ C E-144 & 1.078 E-12 \\ H-3 & 6.641 E+02 \\ P W-147 & 4.044 E-01 \\ & .000 E+00 \\ & 4.344 E-01 \\ C E-144 & 9.459 E-08 \\ P W-147 & 4.344 E-01\end{array}$

OOOE+00 .054E+02

$054 E+02$ $6.645 E+02$

OTBE-12

.044E-01

$344 E-01$

PN-147 4.344E-01 
SUIR328C - (Page 19 of 19)

RUNDATE: 08/20/94

Solid Waste Burial Ground Areas Decayed Curie Content in the 200 Areas from Startup to December 31, 1993, Including Segretated Transuranic Waste.

\begin{tabular}{|c|c|c|c|c|c|}
\hline BURIAL GROUND FACILITIES & $\begin{array}{l}\text { TOTAL } \\
\text { (CURIES) }\end{array}$ & $\begin{array}{l}\text { SR-90 } \\
\text { (CURIES) }\end{array}$ & $\begin{array}{l}\text { RU-106 } \\
\text { (CURIES) }\end{array}$ & $\begin{array}{l}\text { CS-137 } \\
\text { (CURIES) }\end{array}$ & $\begin{array}{l}\text { OTHER RADIOACTIVITY } \\
\text { (CURIES) }\end{array}$ \\
\hline
\end{tabular}

SPECIAL CAISSON/TRENCH BREAKOUT FOR FACILITY 218W4B

CAISSON - NO. 4

CAISSON - NO. 5

CAISSON - NO. 6

$\begin{array}{llll}2.786 E+03 & 6.740 E+02 & 1.980 E-04 & 7.383 E+02 \\ 7.358 E+02 & 1.311 E+02 & 1.209 E-04 & 2.427 E+02 \\ 1.604 E+04 & 1.178 E+03 & 3.233 E+00 & 1.422 E+03\end{array}$

CAISSON - UNI NO. 1

2.281E+01

$.000 E+00$

9.417E-01

TRENCHES

$8.049 E+04$

$5.440 E+03$

4.796E-03

$5.954 E+03$

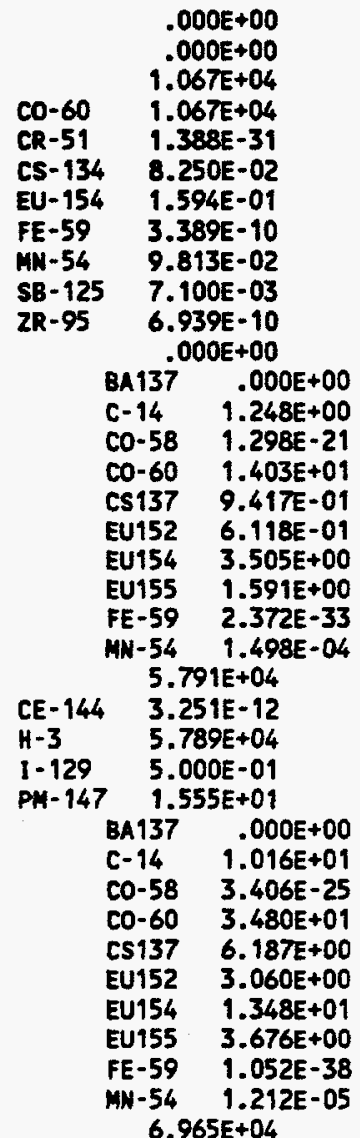

$6.965 E+04$ 


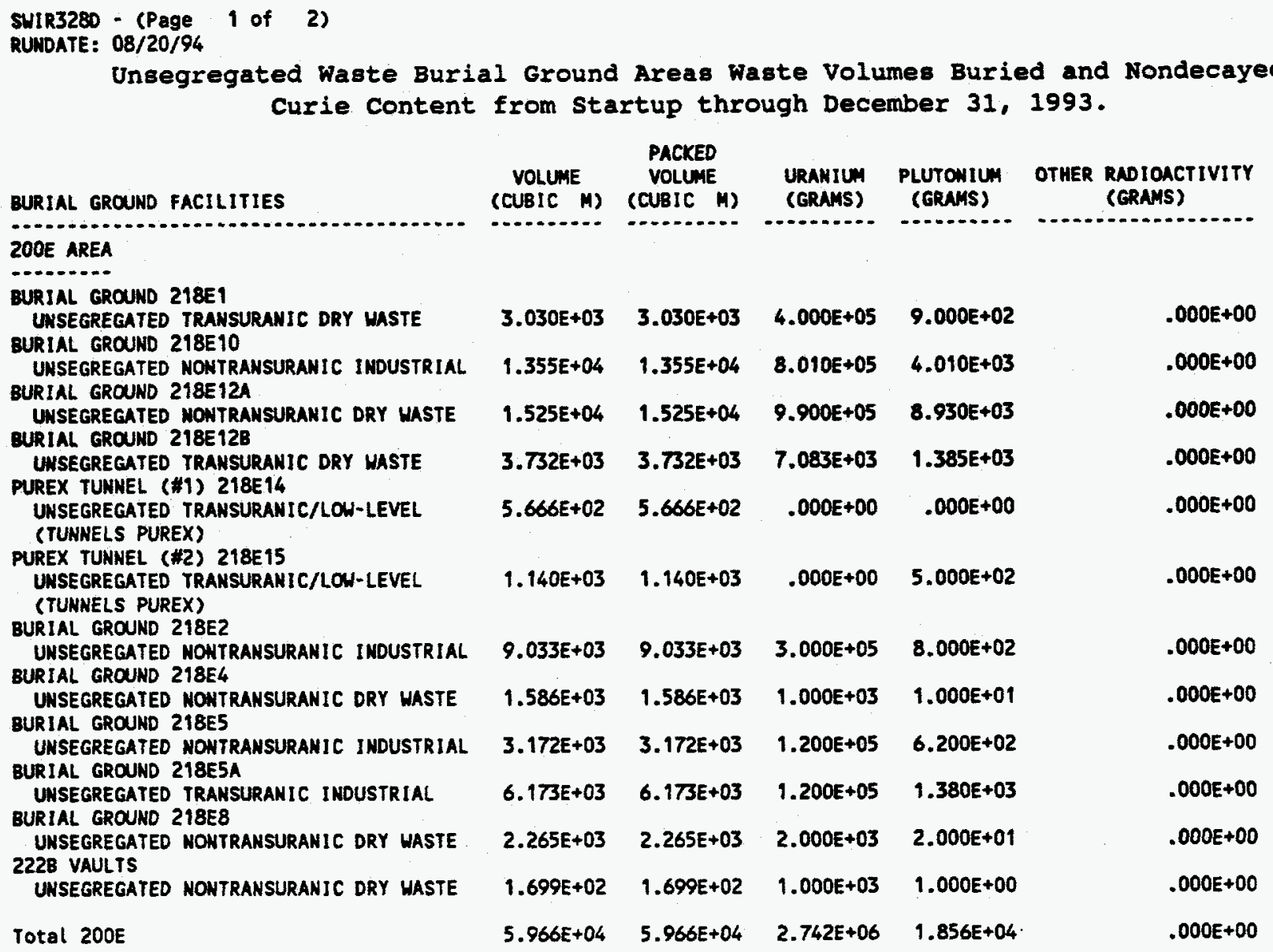




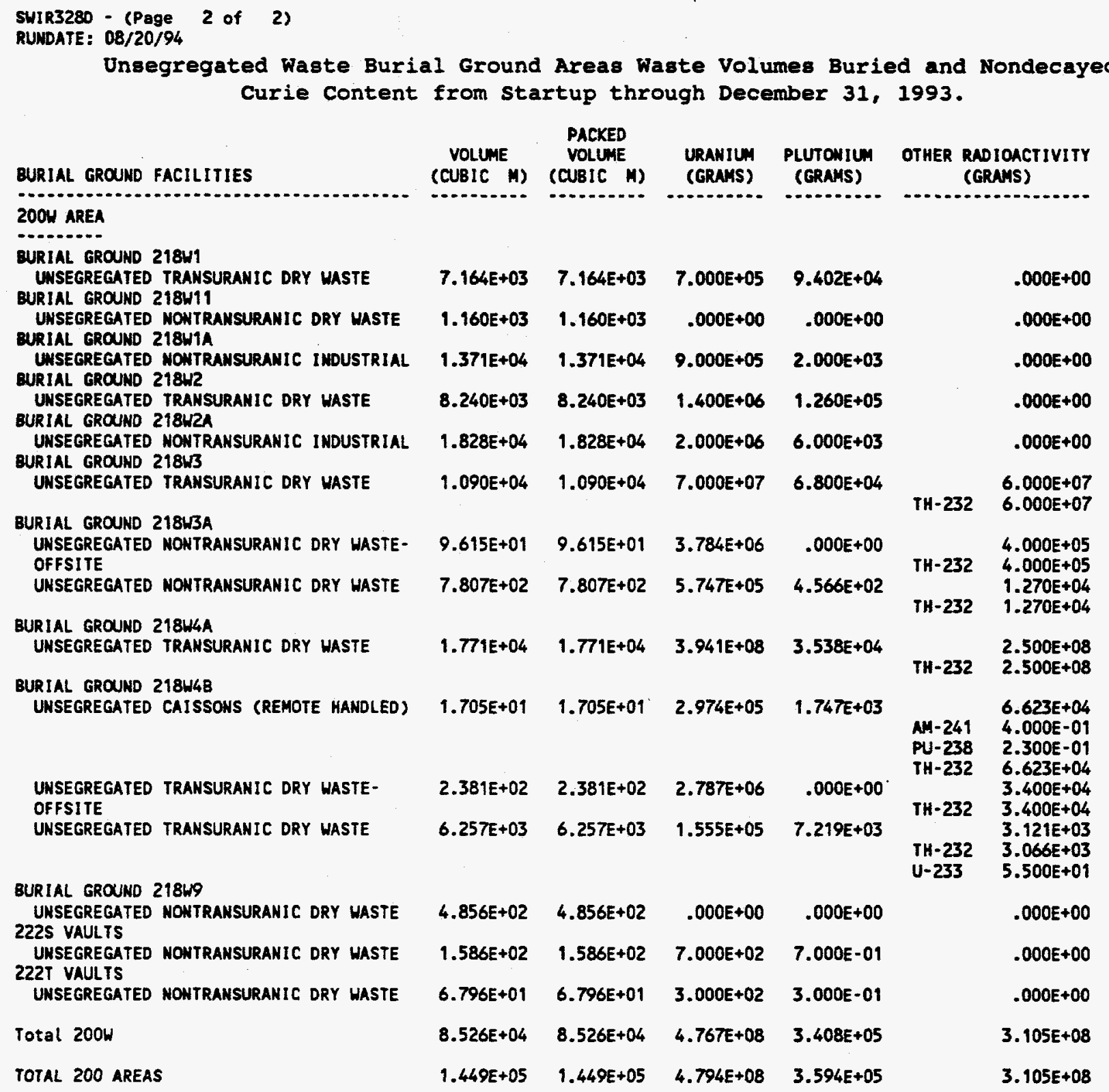


SHIR328E - (Page 1 of 3)

RUNDATE: 08/20/94

Unsegregated Waste Burial Ground Areas Waste Volumes Buried and Nondecayed Curie Content from startup through December 31, 1993.

\begin{tabular}{|c|c|c|c|c|c|}
\hline BURIAL GROUND FACILITIES & $\begin{array}{l}\text { TOTAL } \\
\text { (CURIES) }\end{array}$ & $\begin{array}{l}\text { SR-90 } \\
\text { (CURIES) }\end{array}$ & $\begin{array}{l}\text { RU-106 } \\
\text { (CURIES) }\end{array}$ & $\begin{array}{l}\text { CS-137 } \\
\text { (CURIES) }\end{array}$ & $\begin{array}{l}\text { OTHER RADIOACTIVITY } \\
\text { (CURIES) }\end{array}$ \\
\hline \multicolumn{6}{|l|}{ 2OOE AREA } \\
\hline $\begin{array}{l}\text { BURIAL GROUND } 218 E 1 \\
\text { UNSEGREGATED TRANSURANIC DRY WASTE }\end{array}$ & $1.000 E+02$ & $1.989 E+00$ & $4.283 E+00$ & $2.124 E+00$ & $.000 E+00$ \\
\hline $\begin{array}{l}\text { UNSEGREGATED NONTRANSURANIC INDUSTRIAL } \\
\text { BURIAL GROUND } 218 E 12 A\end{array}$ & $1.815 E+05$ & $3.610 E+03$ & $7.773 E+03$ & $3.855 E+03$ & $.000 E+00$ \\
\hline $\begin{array}{l}\text { UHSEGREGATED NOWTRANSURANIC DRY WASTE } \\
\text { BURIAL GROUND } 218 E 128\end{array}$ & $8.900 E+02$ & $1.770 E+01$ & $3.812 E+01$ & $1.890 E+01$ & $.000 E+00$ \\
\hline $\begin{array}{l}\text { UNSEGREGATED TRANSURANIC DRY MASTE } \\
\text { PUREX TUNMEL (\#1) } 218 E 14\end{array}$ & $6.352 E+03$ & $1.263 E+02$ & $2.720 E+02$ & $1.349 E+02$ & $.000 E+00$ \\
\hline $\begin{array}{l}\text { UNSEGREGATED TRANSURANIC/LON-LEVEL } \\
\text { (TUNHELS PUREX) }\end{array}$ & $8.780 E+04$ & $1.746 E+03$ & $3.760 E+03$ & $1.865 E+03$ & $.000 E+00$ \\
\hline $\begin{array}{l}\text { PUREX TUNNEL (" } 218 \text { ) } 215 \\
\text { UNSEGREGATED TRANSURANIC/LOW-LEVEL } \\
\text { (TUNNELS PUREX) }\end{array}$ & $.465 E+04$ & 8.358E+03 & $9.103 E+02$ & $9.036 E+03$ & $.000 E+00$ \\
\hline $\begin{array}{l}\text { BURIAL GROUND } 218 E 2 \\
\text { UNSEGREGATED NONTRANSURANIC INDUSTRIAL } \\
\text { BURIAL GROUND } 218 E 4\end{array}$ & $.500 E+04$ & $4.973 E+02$ & $1.071 E+03$ & $5.310 E+02$ & $.000 E+00$ \\
\hline $\begin{array}{l}\text { UNSEGREGATED NONTRANSURANIC DRY WASTE } \\
\text { BURIAL GROUND } 218 E 5\end{array}$ & $1.000 E+01$ & $1.989 E-01$ & 4.283E-01 & $2.124 E-01$ & $.000 E+00$ \\
\hline $\begin{array}{l}\text { UNSEGREGATED NONTRANSURANIC INDUSTRIAL } \\
\text { BURIAL GROUND } 218 E 5 A\end{array}$ & $7.500 E+03$ & $1.492 E+02$ & $3.212 E+02$ & $1.593 E+02$ & $.000 E+00$ \\
\hline $\begin{array}{l}\text { UNSEGREGATED TRANSURANIC INDUSTRIAL } \\
\text { BURIAL GROUND } 218 E 8\end{array}$ & $1.650 E+04$ & $3.282 E+02$ & $7.067 E+02$ & $3.505 E+02$ & $.000 E+00$ \\
\hline $\begin{array}{l}\text { UNSEGREGATED NONTRANSURANIC DRY WASTE } \\
222 B \text { VAULTS }\end{array}$ & $1.000 E+01$ & $1.989 E-01$ & $4.283 \mathrm{E}-01$ & $2.124 E-01$ & $.000 E+00$ \\
\hline UNSEGREGATED NONTRANSURANIC DRY HASTE & $6.000 E+02$ & $1.193 E+01$ & $2.570 E+01$ & $1.274 E+01$ & $.000 E+00$ \\
\hline Total 200E & $3.809 E+05$ & $1.485 E+04$ & $1.488 E+04$ & $1.597 E+04$ & $.000 E+00$ \\
\hline
\end{tabular}


SUIR328E - (Page 2 of 3 )

RUNDATE: $08 / 20 / 94$

Unsegregated Waste Burial Ground Areas Waste Volumes Buried and Nondecayed Curie Content from startup through December 31, 1993.

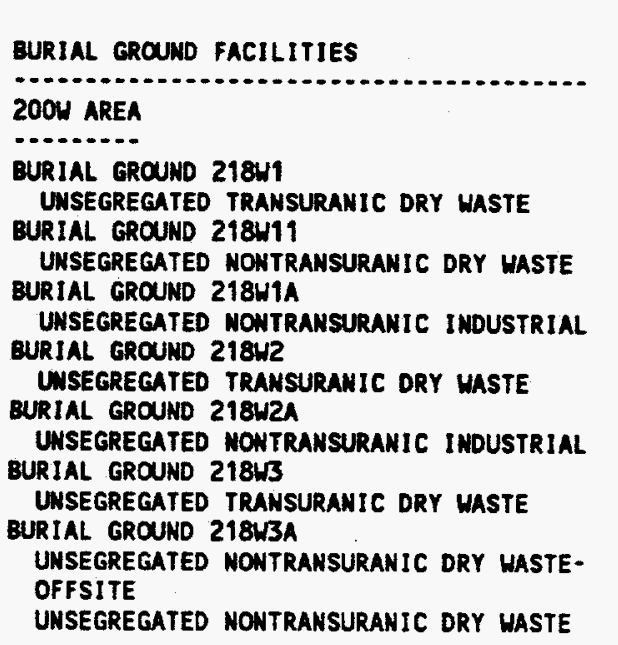

BURIAL GROUND 218W4A

UNSEGREGATED TRANSURANIC DRY WASTE

BURIAL GROUND 218U4B

UNSEGREGATED CAISSONS (REMOTE HANDLED)

UNSEGREGATED TRANSURAHIC DRY WASTE-

OFFSITE

UNSEGREGATED TRAKSURAHIC DRY WASTE

\begin{tabular}{cccc}
$\begin{array}{c}\text { TOTAL } \\
\text { (CURIES) }\end{array}$ & $\begin{array}{c}\text { SR-90 } \\
\text { (CURIES) }\end{array}$ & $\begin{array}{c}\text { RU-106 } \\
\text { (CURIES) }\end{array}$ & $\begin{array}{c}\text { CS-137 } \\
\text { (CURIES) }\end{array}$ \\
\hline $2.000 E+02$ & $3.978 E+00$ & $8.566 E+00$ & $4.248 E+00$ \\
$1.000 E-01$ & $1.989 E-03$ & $4.283 E-03$ & $2.124 E-03$ \\
$4.800 E+04$ & $9.547 E+02$ & $2.056 E+03$ & $1.020 E+03$ \\
$5.000 E+02$ & $9.945 E+00$ & $2.142 E+01$ & $1.062 E+01$ \\
$2.351 E+05$ & $4.677 E+03$ & $1.007 E+04$ & $4.994 E+03$ \\
$9.000 E+02$ & $1.790 E+01$ & $3.855 E+01$ & $1.912 E+01$ \\
$9.700 E-01$ & $1.929 E-02$ & $4.154 E-02$ & $2.060 E-02$ \\
$1.885 E+03$ & $1.362 E+01$ & $2.932 E+01$ & $1.677 E+02$
\end{tabular}

3.815E+03

$6.594 E+01$

$1.420 E+02$

7.042E+01

$7.937 E+04$

$1.783 E+0$

$3.229 E+03$

$1.647 E+03$

$3.393 E+04$

$7.120 E-03$

$1.533 E \cdot 02$

2.297E+05

$5.358 E+02$

$1.154 E+03$

$5.764 E+02$

(2)

GURIAL GROUND 218 W

UNSEGREGATED MOWTRANSURANIC DRY WASTE 2225 VAULTS

UNSEGREGATED NOWTRAMSURANIC DRY WASTE 2221 VAULTS

UNSEGREGATED NONTRANSURANIC DRY WASTE

\author{
$1.000 E-01$ \\ 1.989E-03 \\ 4.030E+03 \\ 8.016E+01 \\ $7.700 E+02$
}

4.283E-03

2.124E-03

$1.726 E+02$
OTHER RADIOACTIVITY (CURIES)

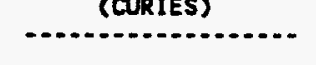

\begin{tabular}{|c|c|c|}
\hline \multicolumn{3}{|c|}{$.000 E+00$} \\
\hline \multicolumn{3}{|c|}{$.000 E+00$} \\
\hline \multicolumn{3}{|c|}{$.000 E+00$} \\
\hline \multicolumn{3}{|c|}{$.000 E+00$} \\
\hline \multicolumn{3}{|c|}{$.000 E+00$} \\
\hline \multicolumn{3}{|c|}{$.000 E+00$} \\
\hline \multicolumn{3}{|c|}{$.000 E+00$} \\
\hline co- 60 & $\begin{array}{l}6.00 \\
6.00\end{array}$ & $\begin{array}{l}1 E+02 \\
1 E+02\end{array}$ \\
\hline & $\begin{array}{l}\text { BA137 } \\
\text { C-14 } \\
\text { CO-58 } \\
\text { CO-60 } \\
\text { CS137 } \\
\text { EU152 } \\
\text { EU154 } \\
\text { EU155 } \\
\text { FE-59 } \\
\text { MN }-54\end{array}$ & $\begin{array}{l}2.989 E+00 \\
3.081 E+00 \\
3.081 E+00 \\
2.064 E+02 \\
3.173 E+00 \\
3.081 E+00 \\
2.619 E+01 \\
2.619 E+01 \\
1.232 E+01 \\
2.157 E+01\end{array}$ \\
\hline
\end{tabular}

PH-147 $\begin{aligned} & 5.000 E+02 \\ & 5.000 E+02\end{aligned}$

$3.466 E+03$

CE-144 8.419E+01

$H-3 \quad 2.796 E+03$

PN-147 5.860E+02

$3.393 \mathrm{E}+04$

$H-3 \quad 3.393 E+04$

CE-144 2.024E+05

6.000E-03

$H-3 \quad 1.934 E+05$

$1-129 \quad 5.000 E-01$

PM-147 9.000E+03

$\begin{array}{ll}\text { BA137 } & 3.977 E+00 \\ \text { C- } 94 & 4.100 E+00 \\ \text { CO-58 } & 4.100 E+00 \\ \text { CO-60 } & 2.747 E+02 \\ \text { CS137 } & 4.223 E+00 \\ \text { EU152 } & 4.100 E+00 \\ \text { EU154 } & 3.485 E+01 \\ \text { EU155 } & 3.485 E+01 \\ \text { FE-59 } & 1.640 E+01 \\ \text { MW-54 } & 2.870 E+01\end{array}$

$.000 E+00$

$.000 E+00$

$.000 E+00$ 
SWIR328E - (Page 3 of 3 )

RUNDATE: 08/20/94

Unsegregated Waste Burial Ground Areas Waste Volumes Buried and Nondecayed Curie Content from Startup through December 31, 1993.

BURIAL GROUND FACILITIES

Total 200w

TOTAL 200 AREAS
SR-90 (CURIES) (CURIES

C.383E+05

$6.383 E+05$

$1.019 E+06$
$R U-106$

(CURIES)

CS-137

(CURIES)

OTHER RADIOACTIVITY (CURIES)

$8.158 E+03 \quad 1.695 E+04 \quad 8.611 E+03$

$2.409 E+05$

$2.300 E+04$

3.184E+04

$2.458 E+04$

2.409E+05 


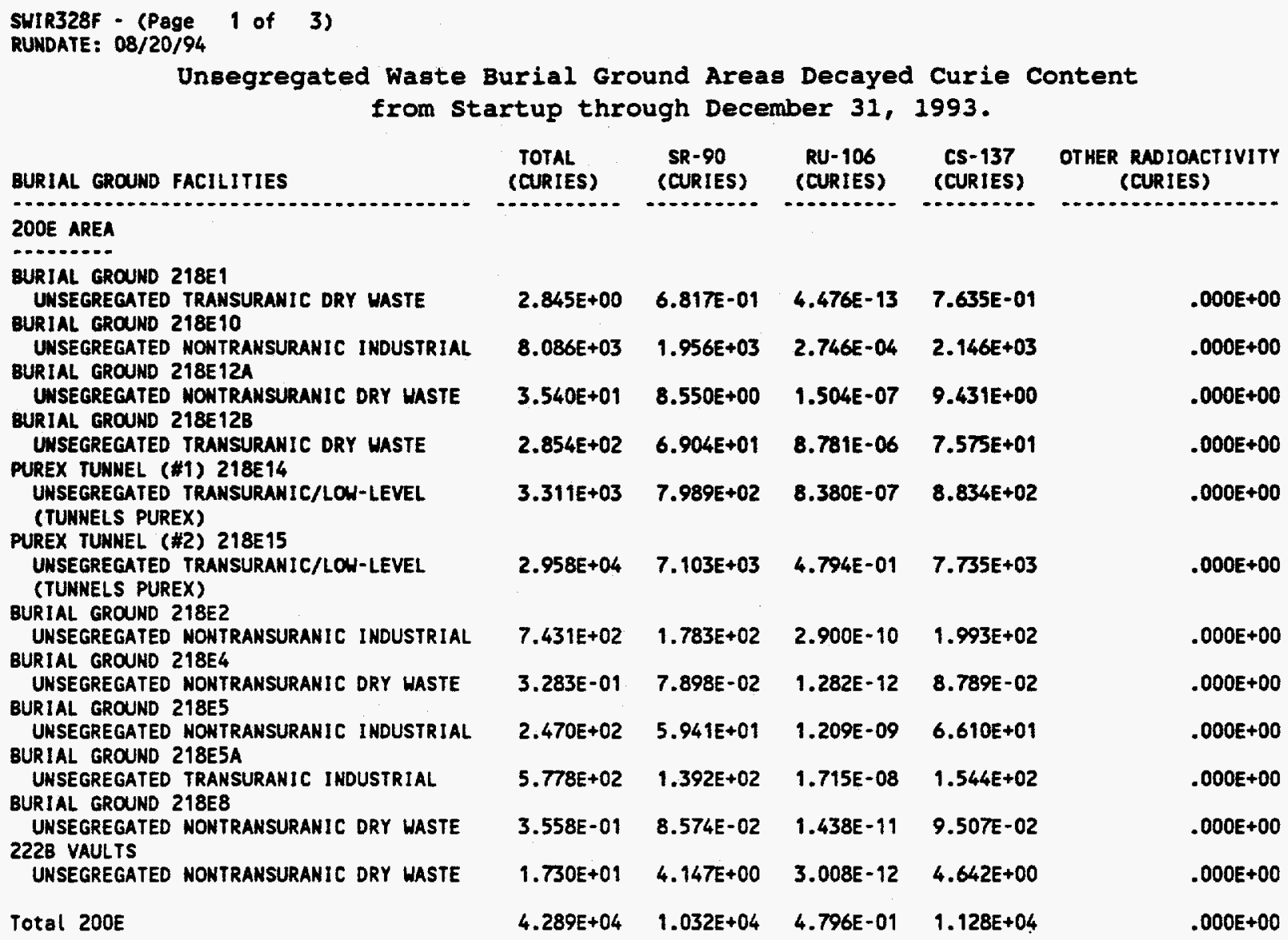


SUIR328f - (Page 2 of 3 )

RUNDATE: $08 / 20 / 94$

Unsegregated Waste Burial Ground Areas Decayed Curie Content from Startup through December 31, 1993.

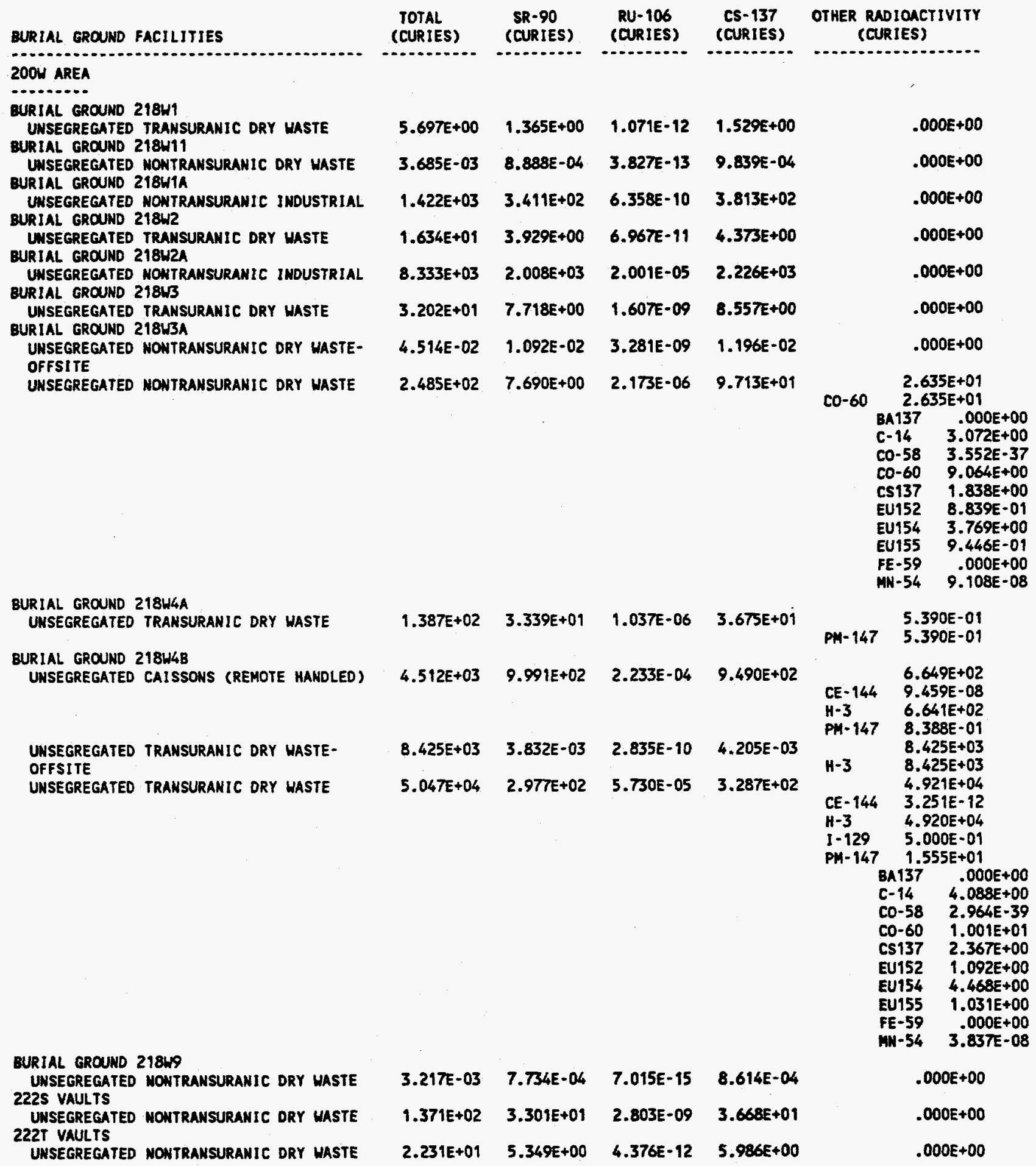


WHC-EP-0125-6

SWIR328F - (Page 3 of 3)

RUNDATE: $08 / 20 / 94$

Unsegregated Waste Burial Ground Areas Decayed Curie Content from startup through December 31, 1993.

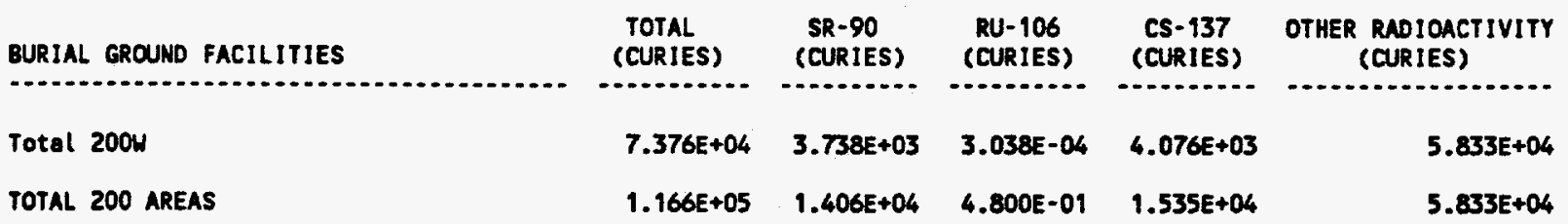




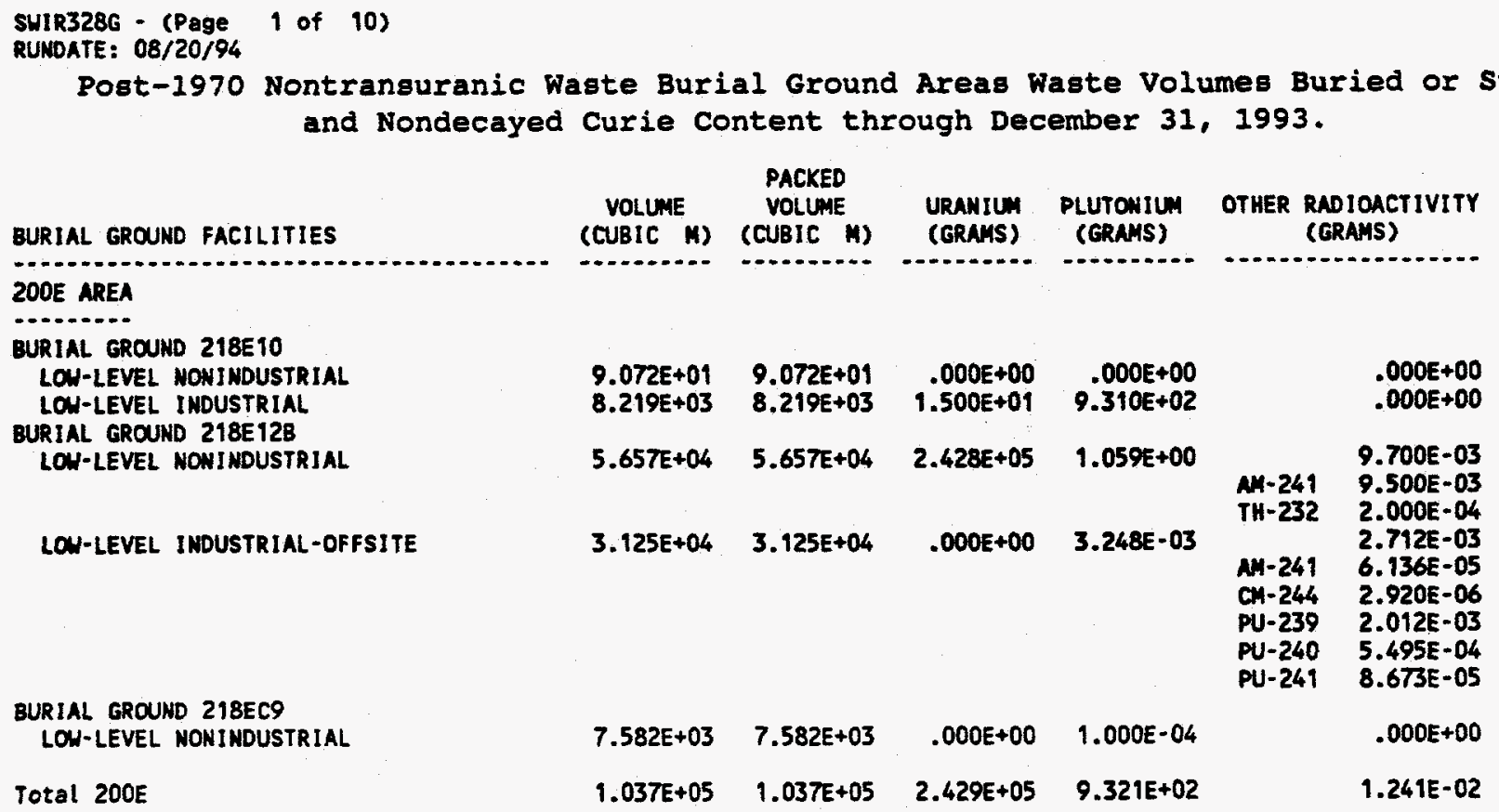




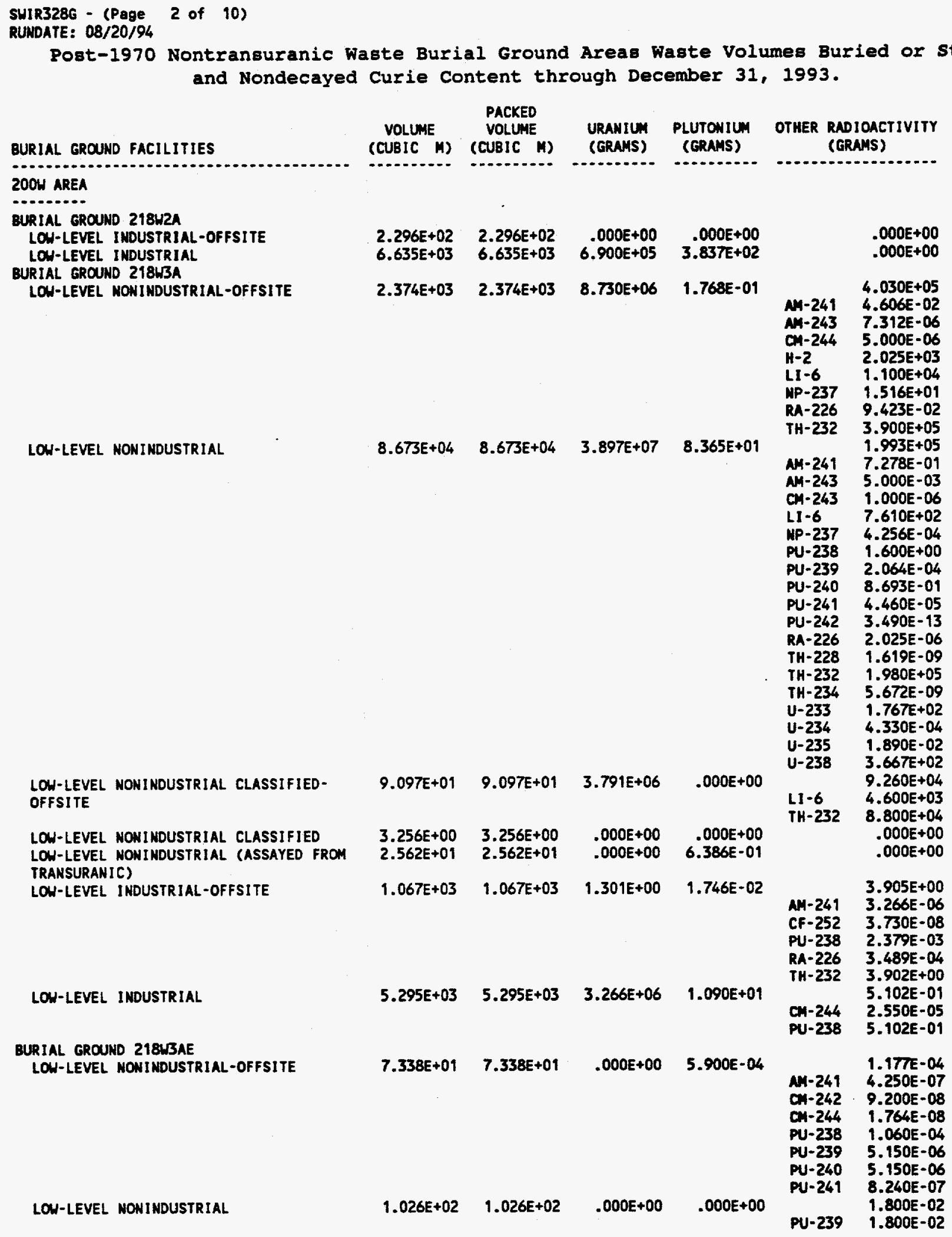




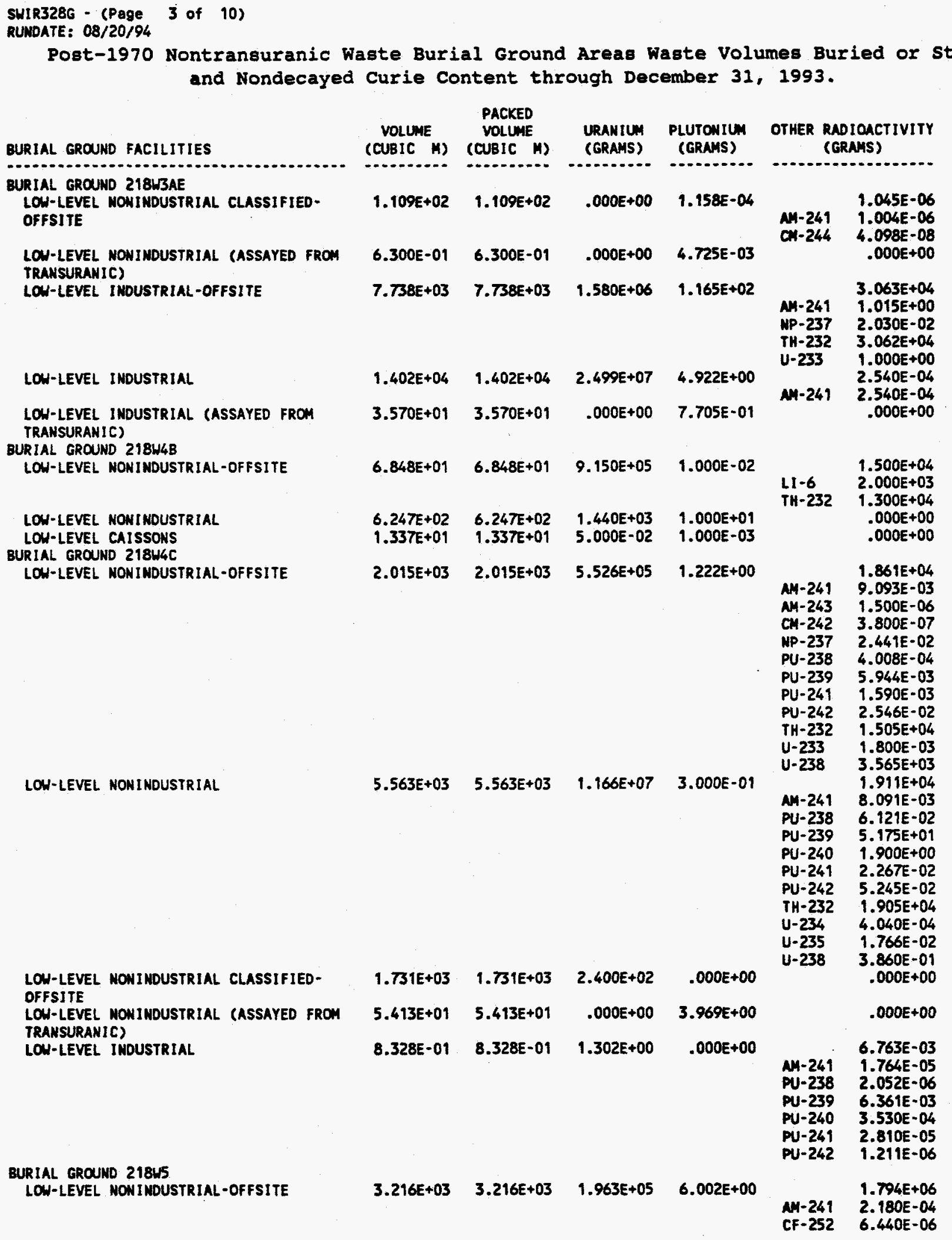




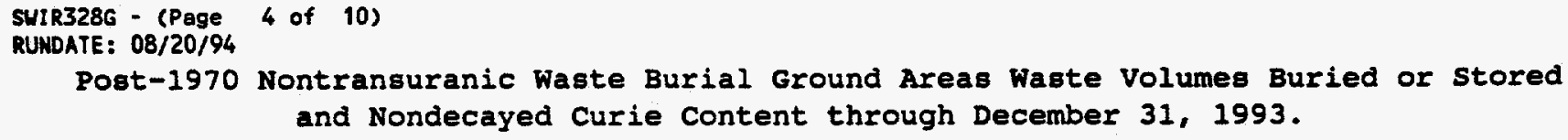

\section{USTRIAL-OFFSITE}

LOW-LEVEL NONINDUSTRIAL

LOW-LEVEL NONINDUSTRIAL (ASSAYED FROM TRANSURANIC)

LOW-LEVEL INDUSTRIAL-OFFSITE

LON-LEVEL INDUSTRIAL

$1.298 E+04 \quad 1.298 E+04 \quad 1.029 E+06 \quad 1.224 E+00$

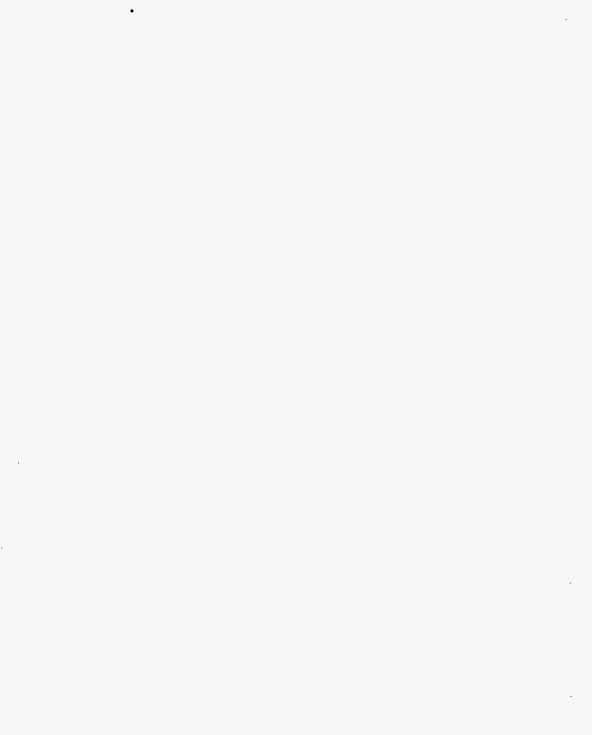

$3.780 E+00 \quad 3.780 E+00 \quad .000 E+00 \quad 5.094 E-02$

$9.323 E+03 \quad 9.323 E+03 \quad 1.144 E+07 \quad 1.360 E+02$

$\begin{array}{lr}\text { PU-238 } & 2.319 E-04 \\ \text { PU-239 } & 5.775 E-05 \\ \text { PU-241 } & 2.160 E-10 \\ \text { PA-226 } & 2.047 E-04 \\ \text { TH-228 } & 9.667 E-08 \\ \text { TH-232 } & 1.794 E+06 \\ & 1.493 E+03 \\ \text { NH-241 } & 8.590 E-04 \\ \text { CH-244 } & 4.960 E-10 \\ \text { NP-237 } & 2.257 E-01 \\ \text { PU-238 } & 2.276 E-06 \\ \text { PU-239 } & 8.759 E-04 \\ \text { PU-240 } & 5.400 E-06 \\ \text { PU-241 } & 4.520 E-08 \\ \text { PU-242 } & 1.830 E-06 \\ \text { RA-226 } & 2.000 E-06 \\ \text { TH-232 } & 1.492 E+03 \\ \text { TH-234 } & 1.110 E-09 \\ & .000 E+00\end{array}$

\begin{tabular}{|c|c|}
\hline $\begin{array}{l}A M-241 \\
A M-243 \\
C F-250 \\
C F-251 \\
C F-252 \\
C H-242 \\
C M-243 \\
C M-244 \\
C H-245 \\
C M-250 \\
M P-237 \\
P U-238 \\
P U-239 \\
P U-240 \\
P U-241 \\
P U-242 \\
R A-224 \\
R A-226 \\
T H-228 \\
T H-232 \\
T H-234 \\
U-232 \\
U-233 \\
U-234 \\
U-235 \\
U-238 \\
M H-241 \\
M H-243 \\
C F-252 \\
C M-242 \\
C M-243 \\
C M-244 \\
C H-245 \\
M P-237 \\
P U-238 \\
P U-239 \\
P U-240\end{array}$ & $\begin{array}{l}1.925 E-02 \\
1.674 E-02 \\
1.838 E-06 \\
6.306 E-09 \\
2.502 E-02 \\
3.300 E-07 \\
5.183 E-08 \\
2.041 E-03 \\
1.401 E-07 \\
1.848 E-20 \\
1.264 E+00 \\
7.310 E+00 \\
3.604 E-01 \\
7.187 E-03 \\
2.905 E-04 \\
3.127 E-02 \\
3.125 E-03 \\
2.914 E+00 \\
4.129 E-02 \\
3.600 E+05 \\
5.000 E-03 \\
1.515 E-06 \\
4.388 E-02 \\
1.180 E+01 \\
3.118 E+01 \\
3.118 E+04 \\
7.724 E+03 \\
3.420 E-01 \\
6.704 E-04 \\
5.800 E-06 \\
1.655 E-04 \\
3.143 E-05 \\
3.110 E-03 \\
1.000 E-05 \\
1.399 E+01 \\
4.787 E-02 \\
5.538 E-01 \\
9.157 E-02\end{array}$ \\
\hline
\end{tabular}




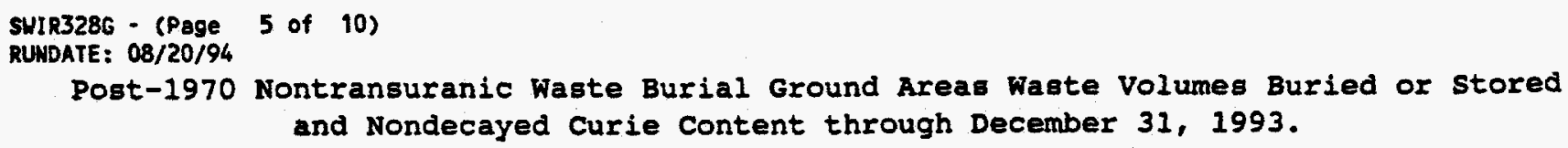

LOU-LEVEL INDUSTRIAL (ASSAYED FRON TRANSURANIC)

CENTRAL WASTE COMPLEX $2401 \mathrm{~W}$ BUILDING LOH-LEVEL NONINDUSTRIAL-OFFSITE LOU-LEVEL NONINDUSTRIAL

CENTRAL UASTE COMPLEX $2402 \mathrm{~N}$ BUILDING LOW-LEVEL NONIMDUSTRIAL-OFFSITE

LOH-LEVEL NONINDUSTRIAL

$\begin{array}{llll}8.324 E+02 & 8.324 E+02 & .000 E+00 & 2.405 E+01 \\ 1.522 E+00 & 1.522 E+00 & .000 E+00 & .000 E+00 \\ 3.859 E+01 & 3.859 E+01 & .000 E+00 & .000 E+00 \\ 6.465 E+01 & 6.465 E+01 & 9.432 E+04 & .000 E+00 \\ 1.717 E+02 & 1.717 E+02 & 1.401 E+04 & 3.461 E-03\end{array}$

PU-242 (GRAMS)

(n)

RA-226

TH-228

TH-232

$\mathrm{TH}-234$

U-232

U. 233

U-234

$\mathrm{U}-235$

vi-236

U-238

$1.717+02$

(2)

(1)

(GRAMS)

$1.120 E-02$

$6.289 E-04$

6.255E-04

1.626E-02

$4.315 E+03$

$1.352 E-06$

$1.501 E-03$

$9.504 E-02$

2.842E-01

$2.995 E+01$

$2.265 E+00$

$3.362 E+03$

$.000 E+00$

$.000 E+00$ $.000 E+00$

1. $000 E+01$

TH-232 1.000E+01

1.570E-04

M-241 1.740E-07

PU-238 4.600E-08

PU-239 1.470E-04

PU-240 8.980E-06

PU-241 7.330E-07

PU-242 3.080E-08

TH-234 2.100E-12

CENTRAL WASTE COMPLEX 2402WB BUILDING LOW-LEVEL NONINDUSTRIAL-OFFSITE

$8.647 E+01 \quad 8.647 E+01 \quad 5.280 E+03 \quad .000 E+00$

$\mathrm{RA}-224$

$6.040 E+03$

RA-226 5.597E-06

TH-228 5.543E-07

TH-232 6.040E+03

TH-234 6.819E-08

$3.168 E+01 \quad 3.168 E+01 \quad 3.047 E-02 \quad 5.065 E-04$

AN -241

$1.075 E-01$

LON-LEVEL NONINDUSTRIAL

$3.168 \mathrm{E}+01$

NP- 237

$1.503 E-05$

7.450E-02

PU-238 1.969E-06

PU-239 $\quad 3.829 E-03$

PU-240 2.300E-04

PU-241 1.003E-04

PU-242, 6.702E-07

U-234 2.885E-05

U-235 $1.261 E-03$

$U-238 \quad 2.756 E-02$

CENTRAL WASTE COMPLEX 2402WC BUILOING LOH-LEVEL NONINDUSTRIAL-OFFSITE LOW-LEVEL NONINDUSTRIAL

$\begin{array}{rrrr}2.082 E-01 & 2.082 E-01 & .000 E+00 & .000 E+00 \\ 2.152 E+01 & 2.152 E+01 & 5.663 E+02 & 5.026 E-04\end{array}$ 


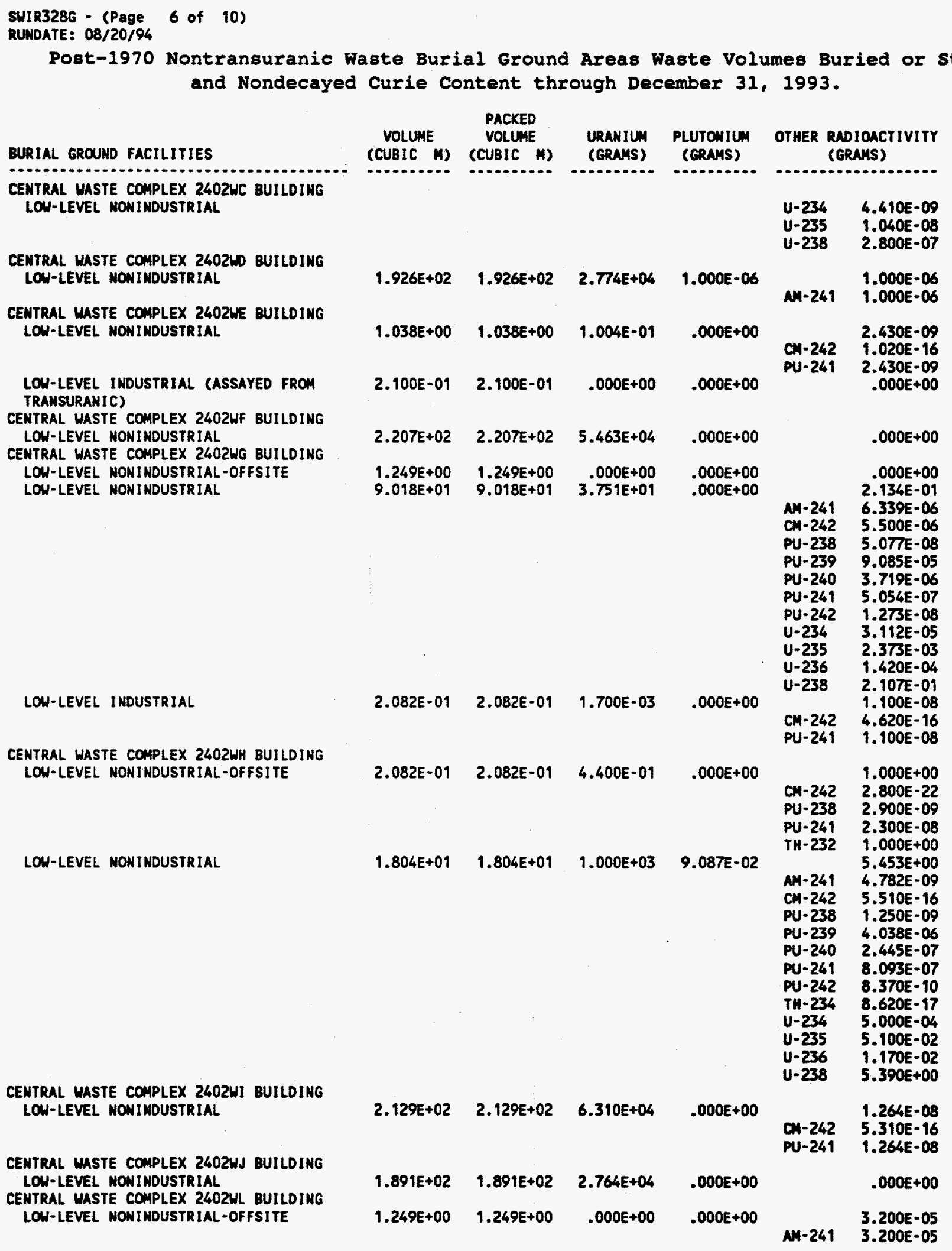




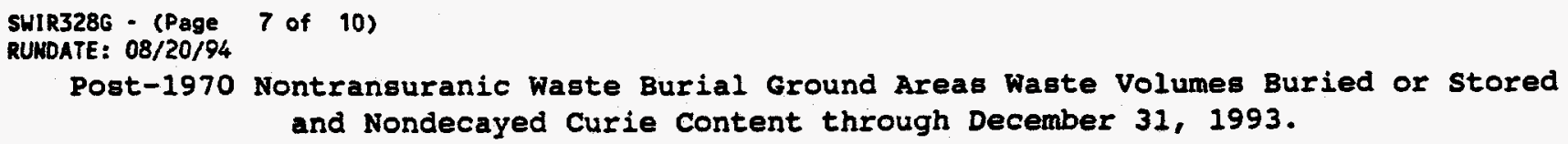

CENTRAL WASTE COMPLEX 2403HA BUILDING LOW-LEVEL NONINDUSTRIAL-OFFSITE

LOW-LEVEL NOWINDUSTRIAL

LOW-LEVEL INDUSTRIAL

$2.082 E-01$

2.082E-0

$.000 E+00$

$.000 E+00$
6.326E-03

5.614E-06

$3.926 E-12$

$1.803 E-13$

2.000E-12

5.701E-06

$1.807 \mathrm{E}-08$

$5.878 E-05$

1.436E-05

1.315E-05

6.660E-09

$7.690 E-10$

2.020E-15

$6.220 \mathrm{E}-06$

2.720E-04

5.950E-03

$1.229 E+03$

AM-241 1.870E-02

AM-243 2.697E-03

CM-242 5.500E-09

NP-237 2.430E+00

PU-238, $1.659 E-05$

PU-239 5.600E-05

PU-240 2.533E-05

PU-241 5.957E-05

RA-226 1.146E-02

TH-228 $1.400 \mathrm{E}-0 \mathrm{~T}$

TH-232 4.959E+02

TH-234 2.900E-10

U-233 1.711E-04

U-234 5.000E-02

U-235 5.270E+00

$\mathrm{U}-238 \quad 7.256 \mathrm{E}+02$

$1.641 E+01$

AM-241 5.910E-01

MM-243 1.800E-05

CM-242 $1.741 \mathrm{E}-12$

CM-243 2.506E-10

CH-244 2.792E-09

MP- $237 \quad 2.685 E+00$

PU-238 1.830E-03

PU-239 1.121E+01

PU-240 6.978E-01

PU-241 8.086E-01

PU-242 1.795E-03

TH-228 4.300E-09

TH-232 9.200E-06

TH-234 1.030 E-06

$U-234 \quad 1.996 E-04$

$U-235 \quad 9.743 E-03$

U-236 1.420E-06

U-238 3.942E-01

$8.138 E-04$
$7.900 E-06$

$\begin{array}{ll}\text { AM-241 } & 7.900 E-06 \\ \text { PU-238 } & 2.850 E-05\end{array}$

PU-239 $\quad 7.700 E-04$

PU-240 7.400E-06

CENTRAL WASTE COMPLEX 2403WC BUILOING 


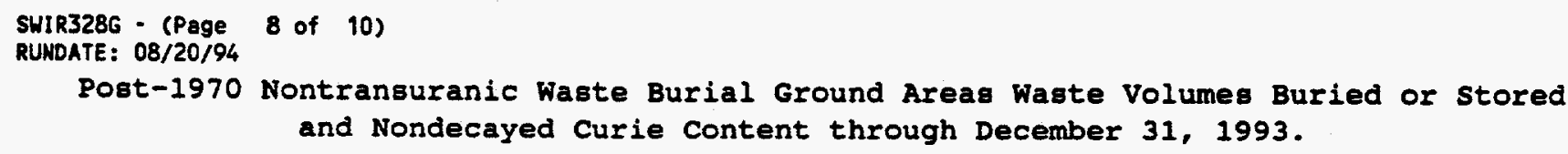

BURIAL GROUND FACILITIES

CEMTRAL WASTE COMPLEX 2403 HC BUILDING LOW-LEVEL NONINDUSTRIAL-OFFSITE

LON-LEVEL NONINDUSTRIAL

LOW-LEVEL NONINDUSTRIAL (ASSAYED FROM TRANSURANIC)

CENTRAL WASTE COMPLEX 2403WD BUILDING

LOU-LEVEL NONINOUSTRIAL-OFFSITE

LON-LEVEL NONIMDUSTRIAL

VOLUME
(CUBIC H)

$1.428 E+02$

1
PACKED

VOLLUE (CUBIC M)

$428 E+02$

20
URANIUN PLUTONIUM

(GRAMS)

$.000 E+00$

(GRAMS)

$.000 E+00$

$5.067 E+02$

$5.067 E+02$

2.682E+03

$1.769 E-02$

2.100E-01

2. 100E-01

$.000 E+00$

$.000 E+00$

4. 182E-01 $4.466 \mathrm{E}+02$

4. 182E-01 $4.466 E+02$

4.454E+01 $8.744 E+04$

$.000 E+00$ $.000 E+00$

$1.026 E+00$

1.

$1.035 E-01$

$.000 E+00$

NM-241

CM-242

PU-238

PU-239.

PU-241

$\begin{array}{rrrr}4.765 E+00 & 4.765 E+00 & .000 E+00 & .000 E+00 \\ 4.182 E-01 & 4.182 E-01 & .000 E+00 & .000 E+00 \\ 1.045 E+00 & 1.045 E+00 & 1.521 E-03 & 4.260 E-04\end{array}$

AM-241

CM-242

$\mathrm{CH}-244$

MP -237

PU-238

PU-239

PU-240

PU-241

PU-242

U-235

$u-236$
$9.260 E-0.4$

2.037E-05

PU-238 1.000E-10

PU-239 8.231E-04

PU-241 5.000E-08

RA-226 8.045E-05

TH-228 3.975E-07

$U-233 \quad 1.616 E-06$

$1.684 \mathrm{E}+04$

$1.666 E-02$
$1.6615 E-11$

CM-242 1.615E-11

CH-243 3.614E-09

CH-244 2.451E-08

MP-237 2.296E-02

PU-238 2.823E-05

PU-239 3.637E-01

PU-240 1.516E-02

PU-241 3.038E-03

PU-242 3.659E-06

RA-224 9.000E-04

RA-226 1.586E-05

TH-228 1.642E-O4

TH-232 1.571E+04

U-232 4.670E-12

U-233 2.100E-02

U-234 1.073E-01

$U-235 \quad 1.023 E+01$

U-236 7.385E-01

U-238 1.123E+03

$.000 E+00$

$.000 E+00$

$2.309 E+02$

2.917E-03

2.115E-09

3.120E-07

3.485E-06

$6.580 E+00$

2.821E-06

7.116E-01

1.311E-02

4.739E-01

2.369E-03

1.508E-01

3.652E-08

2.230E+02

2.950E-06

$9.902 E-07$

$9.240 E-16$

$1.311 E-08$

$1.896 E-06$

5.090E-08

$.000 E+00$

LOW-LEVEL NONINDUSTRIAL

FLAMMALLE STORAGE MOOULE 11

LOW-LEVEL NONINDUSTRIAL-OFFSITE

LOW-LEVEL NONINDUSTRIAL
$.000 E+00$ $5.469 E-06$ 


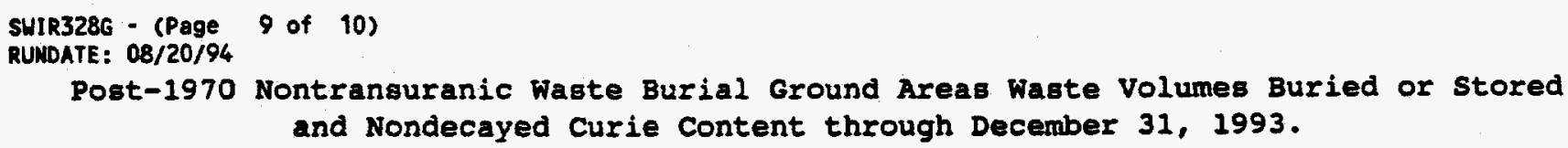

BURIAL GROUND FACILITIES

FLAMABLE STORAGE MODULE 11

LOH-LEVEL NONINDUSTRIAL

FLAMMABLE STORAGE MOOULE 12 LON-LEVEL NONINDUSTRIAL-OFFSITE LOW-LEVEL NONINDUSTRIAL

VOLLME
(CUBIC M)

PACKED

VOLLME

(CUBIC M)

URANIU⿴囗十

(GRAMS)

(...........

$1.874 E+00$

$4.724 E+00$

$1.874 E+00$

$4.724 E+00$

7.140E-02

$8.508 E-02$

$.000 E+00$

$6.566 E-04$

$3.952 E+00$ $1.025 E+00$

$3.952 E+00$

LOU-LEVEL MONINDUSTRIAL-OFFSITE LON-LEVEL NONINDUSTRIAL
FLAMMABLE STORAGE MODULE 15 LOW-LEVEL NOWINOUSTRIAL-OFFSITE LON-LEVEL NONINDUSTRIAL
$1.664 E+00$ $2.706 E+00$

$1.664 E+00$ $2.706 E+00$

$.000 E+00$
$1.035 E-01$

$.000 E+00$ 4.517E-05

$\begin{array}{ll}3.150 E+00 & 3.150 E+00 \\ 2.082 E-09 & 2.082 E-01 \\ 6.246 E-01 & 6.246 E-01\end{array}$

$.000 E+00$

$.000 E+00$ LOW-LEVEL NONINDUSTRIAL flaMmaBle storage mOOULE 3 LOW-LEVEL MON INDUSTRIAL-OFFSITE LOW-LEVEL MONINDUSTRIAL

FLAMUBLE STORAGE MOOULE \& LON-LEVEL NONINDUSTRIAL

FLAMUBLE STORAGE MOOULE 5 LOW-LEVEL NOWINDUSTRIAL

FLAMARLLE STORAGE MOOULE 6

$\begin{array}{llll}6.246 E-01 & 6.246 E-01 & .000 E+00 & .000 E+00 \\ & .000 E+00\end{array}$

$U-23$

U. 235

U-236

U-238

$5.509 E+00 \quad 5.509 E+00 \quad 1.200 E-01 \quad 4.100 E-05$

NH-241

PU-238

PU-239

$6.660 E+00 \quad 6.660 E+00 \quad .000 E+00 \quad 2.820 E-03$
OTHER RNDIOACTIVITY (GRAMS)

AH-241

AN-243

PU-239

PU-240

PU-241

$2.852 E-08$

$1.930 E-06$

$3.650 E-15$

4. $110 E-11$

3.273E-06

2.040E-07

$3.399 E-08$

NM-241

NH-243

CN-242

$\mathrm{CN}-243$

NP-
PU-238

PU-239

PU -240

PU-241

$R A-226$

TH-234

$.000 E+00$

$3.942 E-03$

$1.409 E-06$

3.070E-06

$6.920 E-16$

$9.530 E-10$

$6.070 E-10$

$3.760 E-03$

$1.380 E-04$

3.624E-05

7.419E-07

$1.029 E-07$

8.610E-07

2.020E-06

$.000 E+00$

2.079E-05

AM-241 5.721E-07

PU-238 4.734E-09

PU-239 1.965E-05

PU-240 5.120E-07

PU-241 4.160E-08

PU-242 1.760E-09

AM-241

$.000 E+00$

5.331E-03

5.417E-10

5. $121 \mathrm{E}-09$

PU-240

PU-241

$6.571 \mathrm{E}-07$
$1.000 \mathrm{E}-11$

$.000 E+00$

$.000 E+00$

$4.005 E+00$

3.000E-04

3.800E-02

2.000E-03

$3.965 E+00$

2.52TE- 04

2.527E-04

8.823E-13

$7.882 E-09$

1.312E-05 AM-241 $5.116 E-08$ AM-243 1.307E-05 


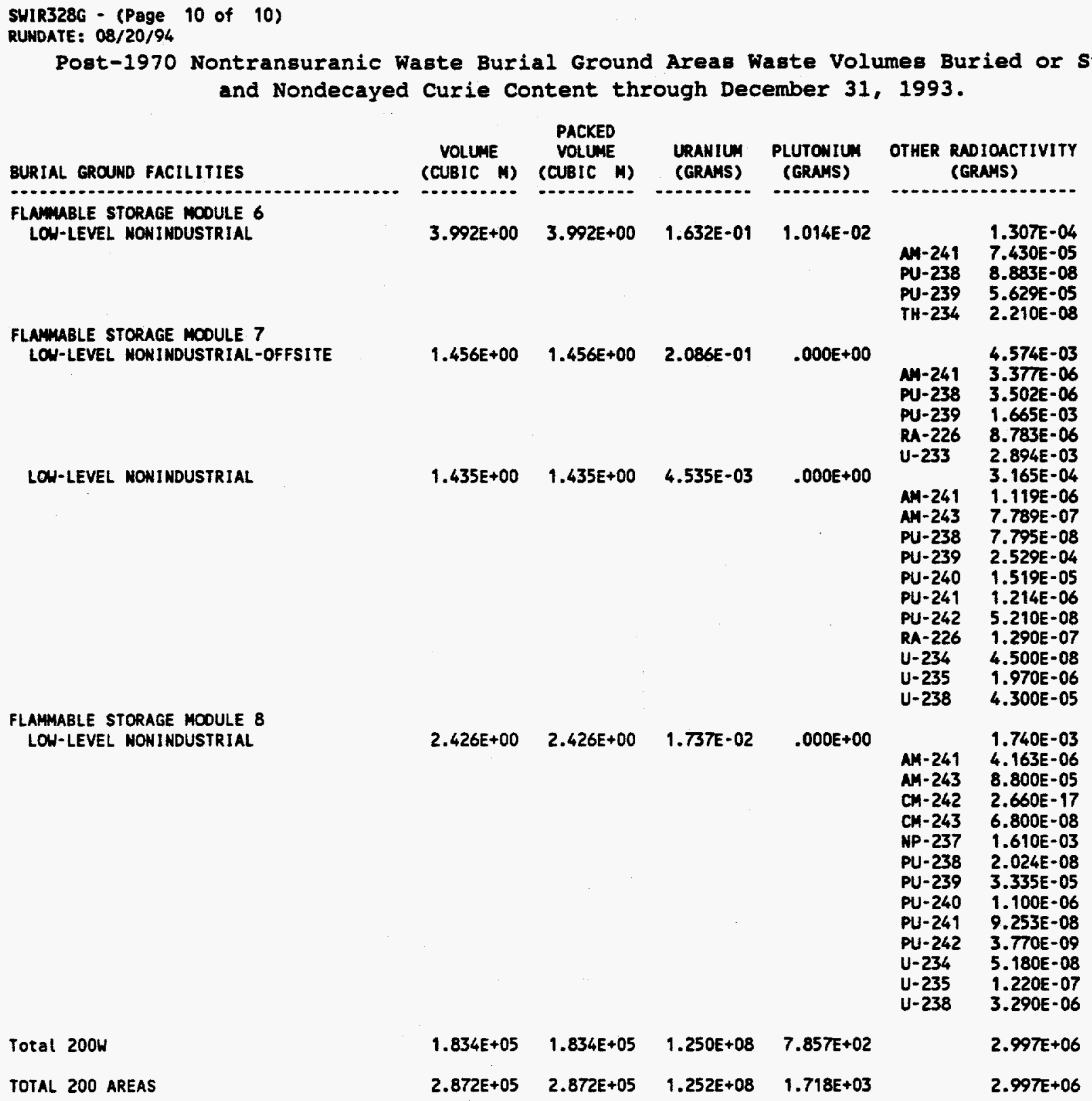




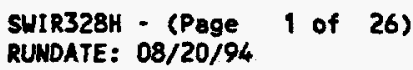

BURIAL GROUND FACILITIES
$200 E$ AREA

\section{O0E AREA}

BURIAL GROUND 218E10

LOH-LEVEL NONINDUSTRIAL

LOU-LEVEL INDUSTRIAL
TOTAL

(CURIES)

(2...........

SR-90

(CURIES)

RU-106

(CURIES)

CS-137

(CURIES)

(CURIES)

$1.912 E+03$

$4.515 E+06$

$5.870 E+02$

$9.982 E+05$

5. $223 E-03$

3.793E+02

$1.176 E+06$

BURIAL GROUND 218E12B LOW-LEVEL NONINDUSTRIAL

LOW-LEVEL INDUSTRIAL-OFFSITE

OTHER RADIOACTIVITY (CURIES)
$1.904 E+06 \quad 3.124 E+00 \quad 6.273 E+00 \quad 6.442 E+01$

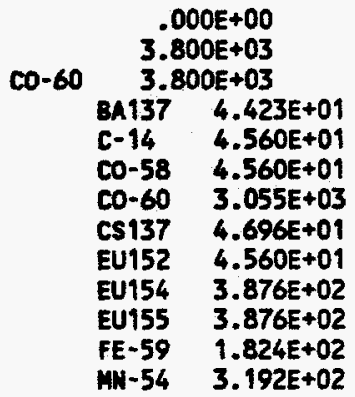

$1.771 E+05 \quad 3.059 E+04 \quad 4.817 E+02 \quad 3.086 E+04$

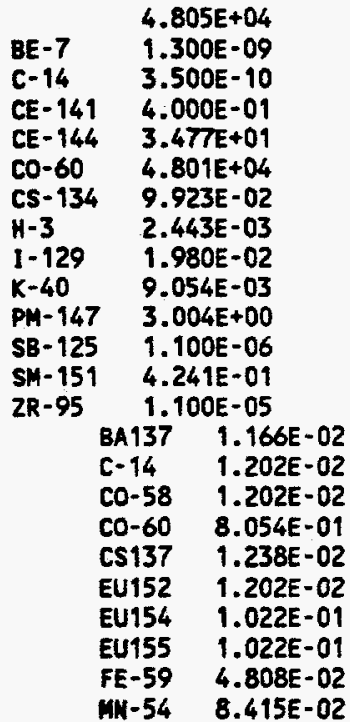

$1.904 E+06$

BE- $10 \quad 6.110 E+01$

C-14 8.120E+01

CL-36 6.110E+01

CO-58 $4.779 E+03$

CO-60 7.488E+05

CR-51 4.718E+01

CS- 134 7.003E-03

FE-55 5.165E+05

FE-59 2.203E+0

$\mathrm{H}-3 \quad 1.122 \mathrm{E}+03$

HF-181 6.010E-01

$1-129 \quad 6.110 E+0$

MN-54 8.014E+03

M-93 6.900E-02

$\mathrm{MB}-94 \quad 5.674 \mathrm{E}-01$

MB-95 3.903E+00

$N I-59 \quad 4.211 E+03$

$\mathrm{HI}-63 \quad 6.198 \mathrm{E}+05$

SE-79 2.422E-05 
WHC-EP-0125-6

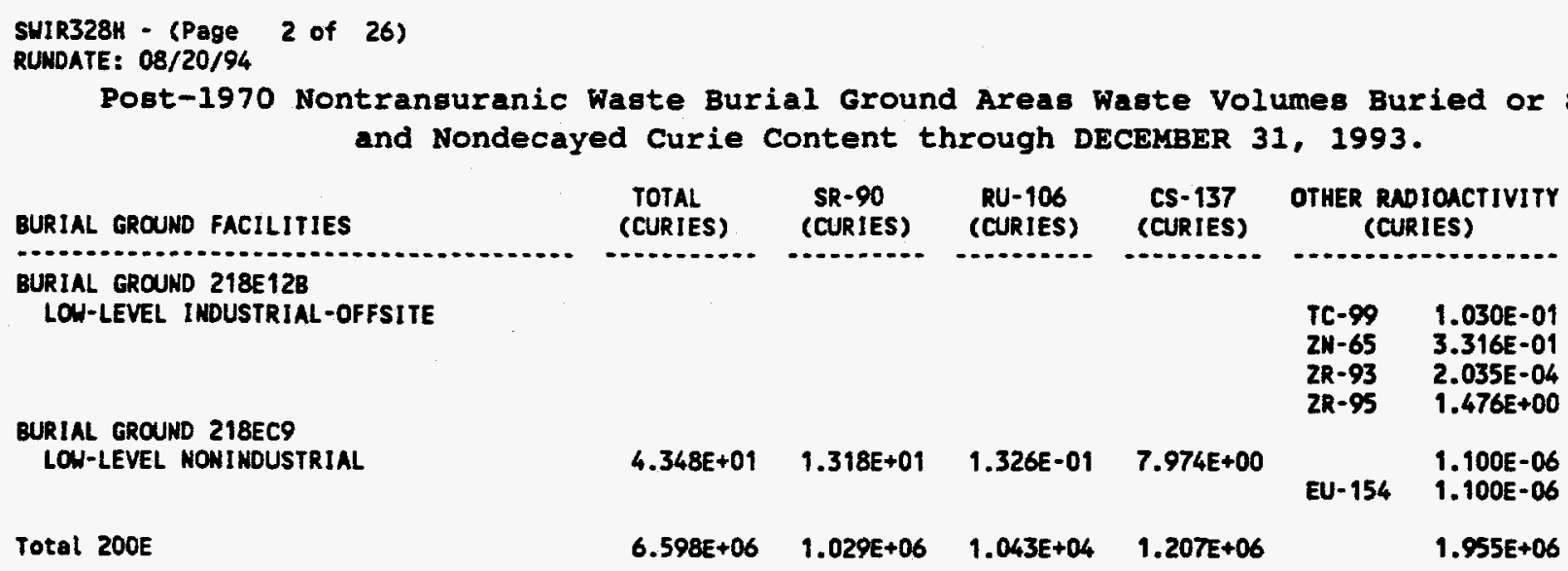


SUIR328H - (Page 3 of 26)

RUNDATE: 08/20/94

Post-1970 Nontransuranic Waste Burial Ground Areas Waste Volumes Buried or stored and Nondecayed Curie Content through DECEMBER 31, 1993.

BURIAL GROUND FACILITIES

\section{W AREA}

BURIAL GROUND 218URA

LOU-LEVEL IMDUSTRIAL-OFFSITE LOW-LEVEL IHOUSTRIAL

BURIAL GROUWD 218WBA

LOW-LEVEL NONINDUSTRIAL-OFFSITE
TOTA

(CURIES)

(CURIES)

$S R-90$

(CURIES)

RU- 106

(CURIES)

CS- 137

(CURIES)

OTHER RNOIOACTIVITY (CURIES)

$\begin{array}{llll}4.184 E+01 & 8.321 E-01 & 1.792 E+00 & 8.88 \pi E-01 \\ 1.223 E+04 & 5.010 E+02 & 5.776 E+02 & 5.418 E+02\end{array}$

$8.011 E+05 \quad 4.107 E+04 \quad 1.319 E+04 \quad 2.126 E+05$

$\cos 60$

LOW-LEVEL NONINDUSTRIAL

$7.871 E+05 \quad 4.589 E+04 \quad 1.383 E+03 \quad 9.698 E+04$

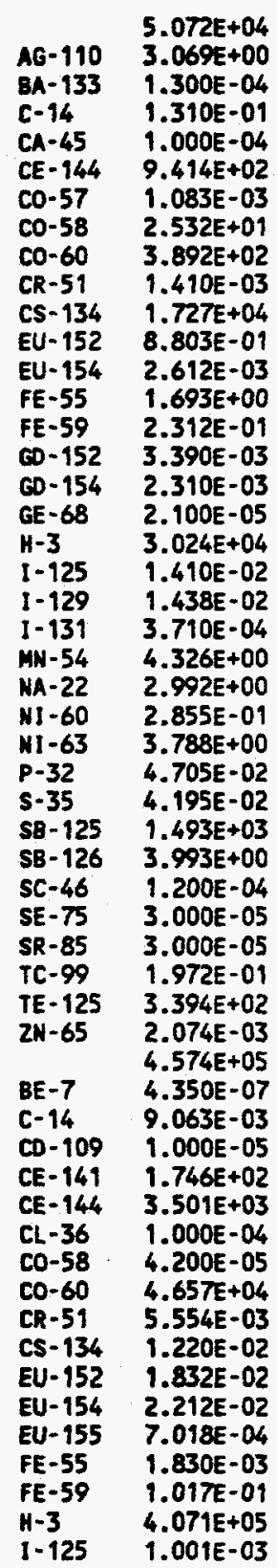




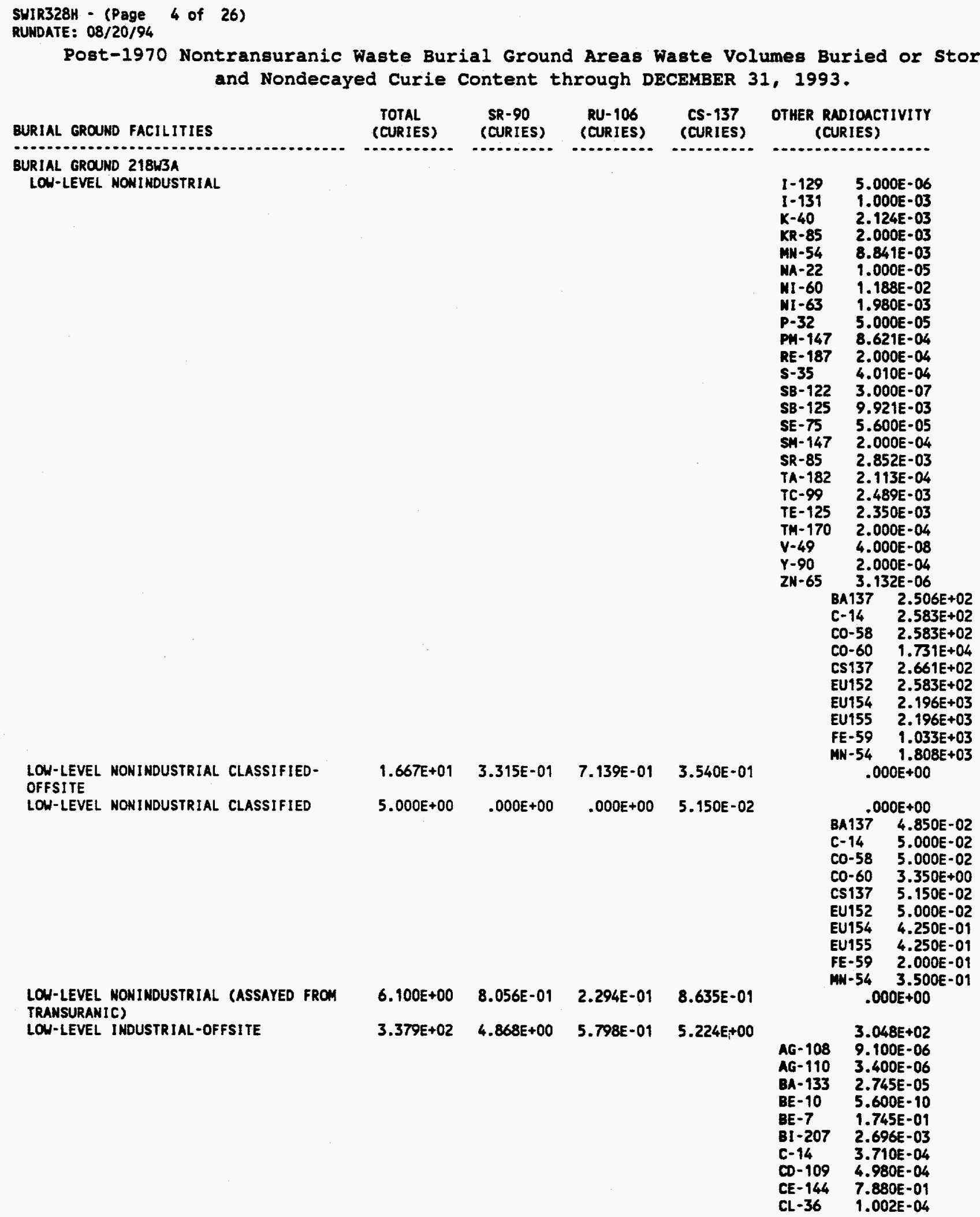


SHIR328H - (Page 5 of 26)

RUNDATE: 08/20/94

Post-1970 Nontransuranic Waste Burial Ground Areas waste Volumes Buried or stored and Nondecayed Curie Content through DECEMBER 31, 1993.

BURIAL GROUND FACILITIES

BURIAL GROUND 218W3A

LOW-LEVEL INOUSTRIAL-OFFSITE

LON-LEVEL INDUSTRIAL

BURIAL GROUND 21843AE

LOW-LEVEL NONINDUSTRIAL-OFFSITE
TOTAL

(CURIES)

$5 R-90$

(CURIES)

$R U-106$

(CURIES)

cs-137

(CURIES)

OTHER RADIOACTIVITY

(CURIES)

-

co-60

2.190E-04

CR-51

3.017E+00

Cs-134

EU-152

EU-156

EU-155

FE-55

FE-59

GE- 68

$H-3$

$1-125$

$1-129$

$k-40$

KR-85

MN -54

MA-22

NB- 94

NI -59

N1 -63

PN- 147

PR- 144

RB- 86

$5-35$

3.000E-03

$1.461 E+00$

3.964E-01

2.809E-01

$2.090 E-04$

7.568E-03

8.801E-06

2.397E-02

$2.526 \mathrm{E}+02$

4.000E-07

5.200E-07

$7.490 E-08$

3.580E-01

1.944E-02

$3.586 E+00$

$9.000 E-04$

3.000E- 04

5.000E-02

$2.134 E+00$

$1.240 E-05$

5.017E-03

SI-31 $1.000 E-08$

TA-182 1.040E-03

TC-99 2.400E-04

TL-204 $\quad 5,000 E-10$

$Y-88$

$1.000 E-06$

$2 N-65$

$3.982 E+01$

$6.523 E+00$

C- 14

3.000E-22

CE-144 $1.000 E-12$

CO-60 4.332E+00

CS-134 4.000E-02

EU-152 8.230E-02

EU-154 1.370E-01

FE-55 1.300E-09

H-3 1.890E+00

MN-54 1.300E-09

NA-22 5.000E-07

NI-60 4.570E-04

PM-147 6.000E-13

SB-125 3.350E-02

SM-151 2.000E-13

TE-125 7.310E-03

BA137 2.405E+01

C-14 2.480E+01

CO-58 2.480E+01

Co-60 1.661E+03

CS137 2.554E+01

EU152 2.480E+01

EU154 2.108E+02

EU155 2.108E+02

FE-59 $9.919 E+01$

MN-54 $1.736 E+02$

$1.746 E+01 \quad 1.622 E-01 \quad 6.104 E-03 \quad 1.612 E-01$

$\begin{array}{ll}B E-10 & 1.674 E+01 \\ C-14 & 4.600 E-11 \\ 2.922 E-02\end{array}$




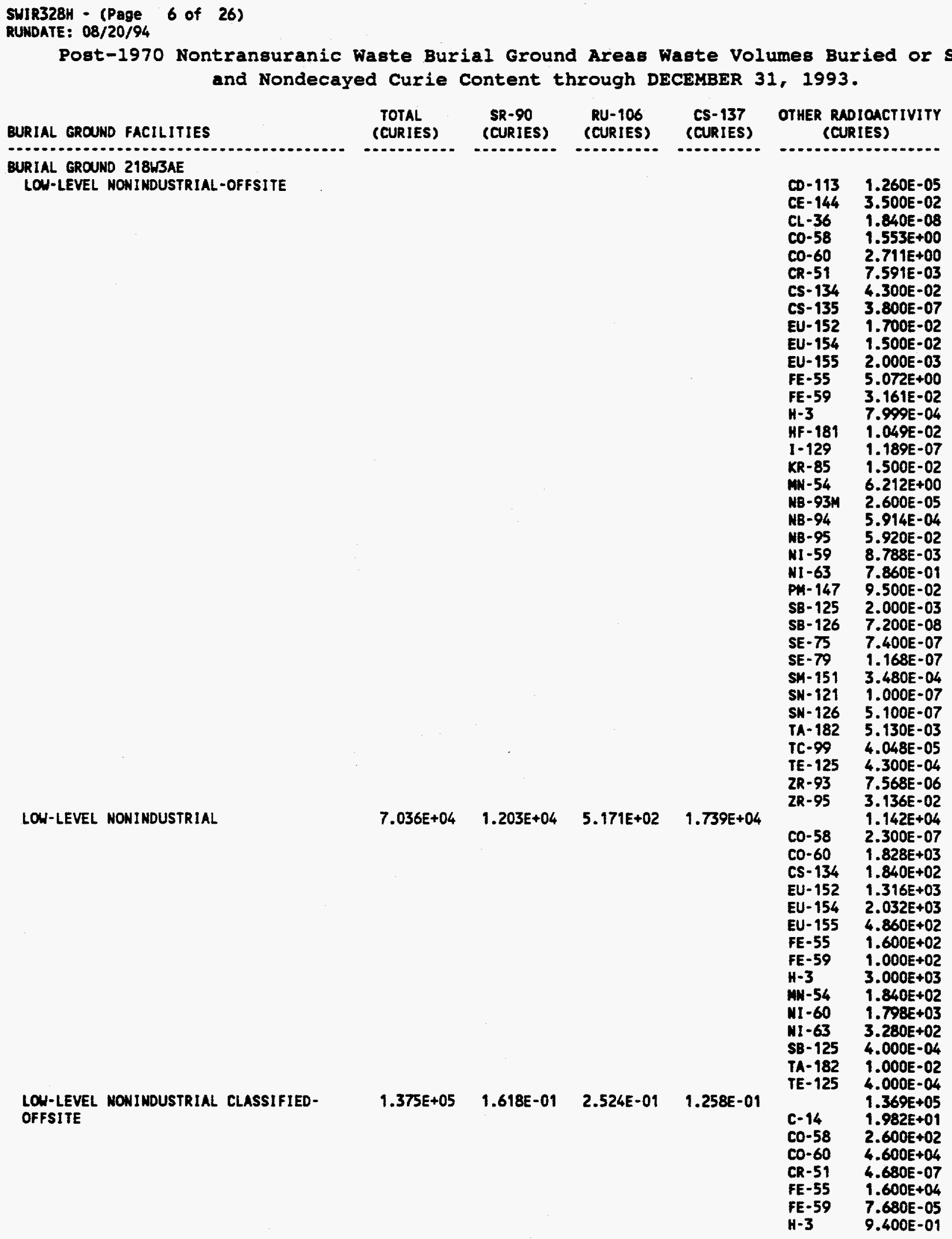


SHIR328H - (Page 7 of 26)
RUNDATE: 08/20/94

Post-1970 Nontransuranic Waste Burial Ground Areas Waste Volumes Buried or stored and Nondecayed Curie Content through DECEMBER 31, 1993.

GURIAL GRONW FACILITIES
BURIAL GROUND 218U3AE
LOH-LEVEL NONINDUSTRIAL CLASSIFIED-
OFFSITE

OFFSITE
LON-LEVEL NONINDUSTRIAL (ASSAYED FROM TRANSURANIC)

LON-LEVEL INDUSTRIAL-OFFSITE

LON-LEVEL INDUSTRIAL

LOU-LEVEL INOUSTRIAL (ASSAYED FROM

TRANSURANIC

QURIAL GROUND 218W4B

LOW-LEVEL MONINDUSTRIAL-OFFSITE

LOW-LEVEL MONINDUSTRIAL

LOW-LEVEL CAISSONS

TOTAL
(CURIES)

$5 R-90$

(CURIES)

(CURIES)

CS- 137 (CURIES)

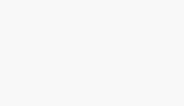

\section{$1.500 E-03$}

2.376E-04

$3.557 E-05$

2.549E-04

$6.044 E+04$

$3.673 E+02$

$6.098 E+02$

$1.136 E+04$

$7.284 E+03$

$1.026 E+03$

$1.336 E+02$

$.087 E+03$

\begin{tabular}{|c|c|c|c|c|c|}
\hline $8.549 E+00$ & $1.188 E+00$ & 2.798E-01 & $1.273 E+00$ & CE-144 & $\begin{array}{r}5.000 E-0 \\
5.000 E-0\end{array}$ \\
\hline $.750 E+$ & $2.188 E-01$ & 4.711E-01 & 2.337E-01 & $n-3$ & $\begin{array}{l}9.640 E+0 \\
9.640 E+0\end{array}$ \\
\hline $\begin{array}{l}.077 E+04 \\
.071 E+04\end{array}$ & $\begin{array}{l}6.720 E+03 \\
1.694 E+03\end{array}$ & $\begin{array}{l}5.493 E+01 \\
2.773 E+02\end{array}$ & $\begin{array}{l}7.252 E+03 \\
2.127 E+03\end{array}$ & & $\begin{array}{r}.000 E+0 \\
1.928 E+0\end{array}$ \\
\hline
\end{tabular}

OTHER RADIOACTIVITY (CURIES)

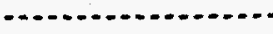

$6.160 E-08$

MB-94 5.603E-01

MB-95 $1.420 E+03$

$N 1.59 \quad 4.000 E+02$

$M I-63 \quad 4.800 E+04$

SB-125 $9.000 E+03$

SE-79 6.160E-08

SN-119 1.280E+04

SN-123 $1.080 E+02$

$T A-182 \quad 1.240 E+02$

TC- 99 1.542E-05

TE-125 1.920E+03

2R-93 6.160E-07

$2 R-95 \quad 6.600 E+02$

$.000 E+00$

$2.450 E+04$

C- $14 \quad 3.208 E-01$

CE-144 1.065E-02

CO-58 4.600E-02

CO-60 3.127E+02

EU-152 2.738E-02

EU-154 1.300E-05

FE-55 2.700E+00

FE-59 5.000E-03

$H-3 \quad 2.416 E+04$

$K R-85 \quad 1.300 E+01$

MN-54 1.106E+01

MA-22 7.599E-01

2.750E+02

$\begin{array}{ll}c-14 & 3.000 E-04 \\ C E-144 & 1.125 E-06\end{array}$

CO-60 2.274E+02

CR-51 1.000E-03

EU-154 1.590E-01

EU-155 6.500E-10

FE-59 3.507E+01

$\mathrm{H}-3 \quad 2.525 \mathrm{E}-09$

MN-54 1.242E+01

PM-147 5.323E-07

SM-151 4.228E-07

BA137 $1.092 E+01$

C- $14 \quad 1.126 E+01$

Co-58 1.126E+01

CO-60 7.544E+02

CS137 1.160E+01

EU152 $1.126 E+01$

EU154 9.570E+01

EU155 $9.570 E+01$

FE-59 4.504E+01

NW-54 7.882E+01

$$
\text { 5.000E-02 }
$$


SUIR328H - (Page 8 of 26)

RUNDATE: $08 / 20 / 94$

Post-1970 Nontransuranic Waste Burial Ground Areas Waste Volumes Buried or stored and Nondecayed Curie Content through DECEMBER 31, 1993.

BURIAL GROUND FACILITIES

BURIAL GROUND $218 \mathrm{BH}$

LOW-LEVEL CAISSONS
BURIAL GROUND 218W4C

LOW-LEVEL NONINDUSTRIAL-OFFSITE
TOTAL

(CURIES)

SR-90
(CURIES)

(CURIES)

RU-106

(CURIES)

CS- 137

(CURIES)

OTHER RADIOACTIVITY (CURIES)

-......................

$3.879 E+04 \quad 2.557 E+00 \quad 4.099 E+00 \quad 2.674 E+00$

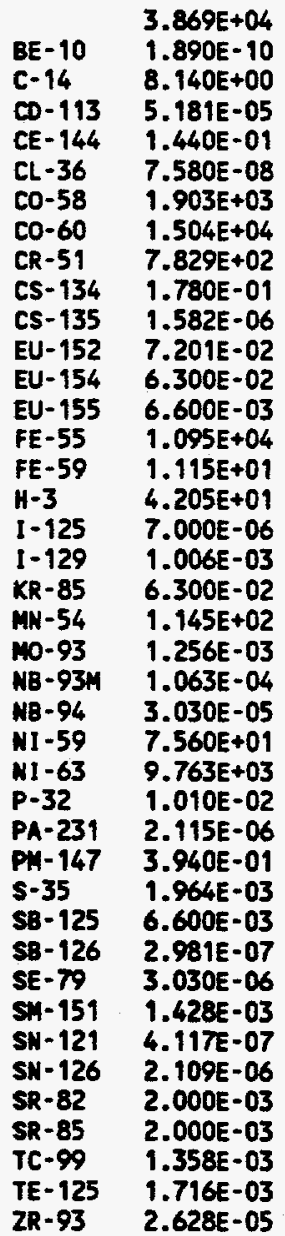




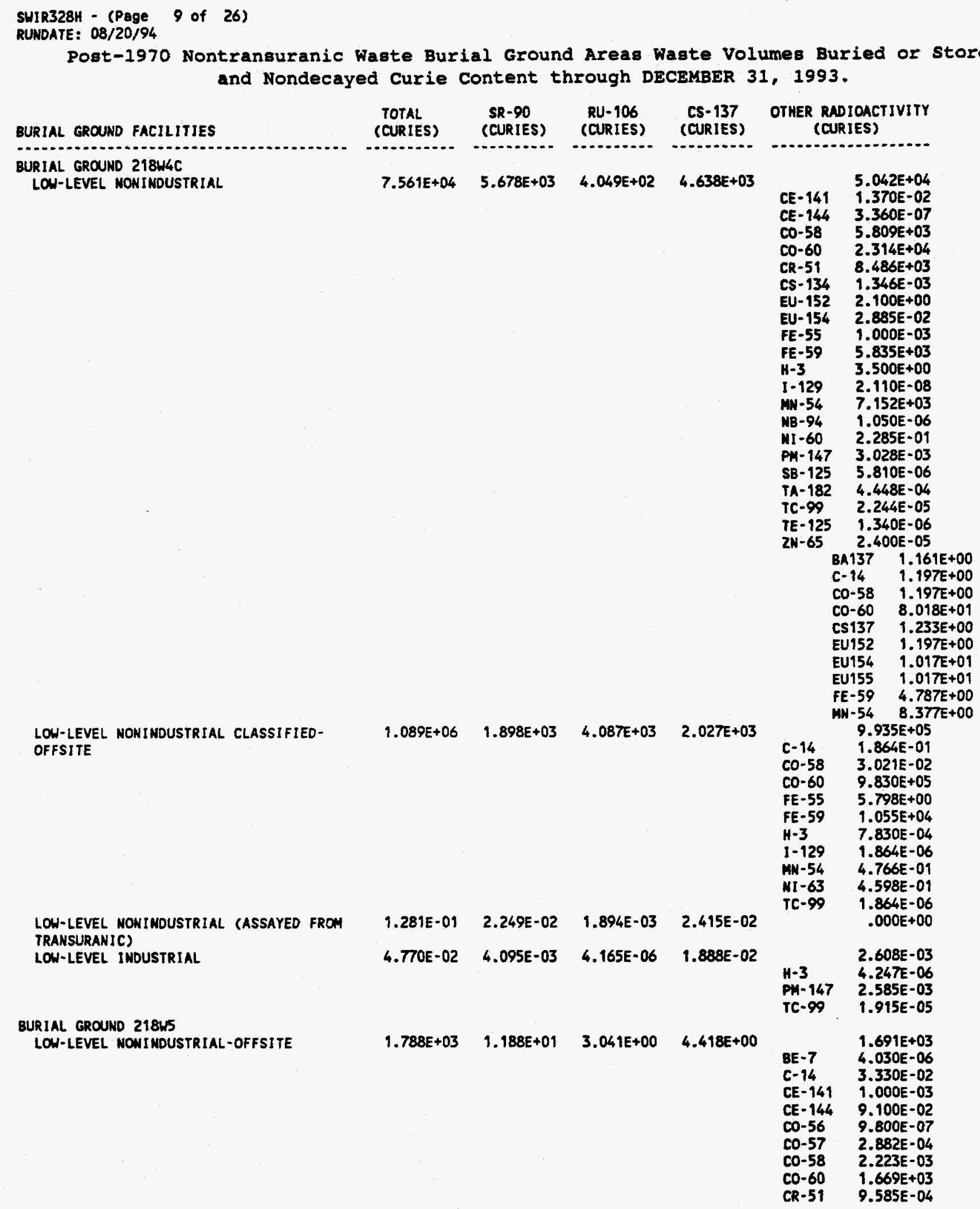


SWIR328H - (Page 10 of 26 )

RUNDATE: 08/20/94

Post-1970 Nontransuranic Waste Burial Ground Areas Waste Volumes Buried or Stored and Nondecayed Curie Content through DECEMBER 31, 1993.

BURIAL GROUND FACILITIES

BURIAL GROUND 218W5

LON-LEVEL NONINDUSTRIAL-OFFSITE
LOW-LEVEL NONINOUSTRIAL
TOTAL (CURIES)

SR -90
(CURIES) RU-106 (CURIES)

CS-137 (CURIES) (CURIES) -.......... -..........

OTHER RADIOACTIVITY (CURIES)

(CURIES)

CS-134 1.414E-01 EU-152 8.097E-02 EU-154 5.747E-02 EU-155 9.660E-O4 FE-55 $\quad 6.638 E+00$ FE-59 1.774E-06 GA-68 1.202E-05 CD-153 4.066E-04 GE-68 2.680E-05 $\mathrm{H}-3$ 1.080E+01 I-125 4.303E-03 I-129 1.000E-05 $1-1318.200 E-04$ KR-85 4.100E-02 MN-54 2.523E-09 NA-22 4.710E-02 MB-95 6.236E-04 MI-60 3.721E-02 $\mathrm{NI}-63 \quad 2.964 E+00$ PN-147 2.480E-01 RB-86 3.620E-03 RU-103 6.661E-04 5-35 1.784E-02 SC-46 1.203E-05 SE-75 2.102E-04 SH-113 5.102E-04 SR-82 1.000E-04 SR-85 6.800E-07 TC- $99 \quad 3.300 E-04$ $r-88 \quad 1.400 E-07$ ZN-65 1.878E-03 2R-95 3.000E-05 $9.515 E+03 \quad 1.808 E+03 \quad 1.507 E+01 \quad 2.783 E+03$

BA- 134

BE -7 $2.520 E+02$ 6.000E-09 $1.027 \mathrm{E}-07$

C-14 4.125E+00

CD-109 3.500E-06

CE-144 1.012E-01

CO-57 6.000E-04

C0-58 $\quad 1.046 E-01$

Co-60 $\quad 9.535 E+01$

CS-134 1.116E-01

EU-152 1.415E-01

EU-154 1.230E+02

EU-155 1.262E+01

FE-55 1.860E-01

FE-59 4.107E-01

H-3 $1.504 E+01$

I-125 5.651E-03

$1-129$ 4.101E-03

I-131 1.095E-02

K-40 $1.766 \mathrm{E}-04$

MW-54 5.513E-01

MA-22 1.051E-03

MB-95 8.100E-07

MI -59 1.000E-03

MI -60 1.270E-08

MI-63 2.987E-02

P-32 1.000E-04

PN-147 4.368E-02 
SWIR328H - (Page 11 of 26)

RUNDATE: 08/20/94

Post-1970 Nontransuranic Waste Burial Ground Areas Waste Volumes Buried or Stored and Nondecayed Curie Content through DECEABER 31, 1993.

\section{BURIAL GROUND FACILITIES}

BURLAL GROUNO $218 \mathrm{H} 5$

LOW-LEVEL MONINDUSTRIAL
TOTAL (CURIES)
RU- 106
(CURIES) (CURIES)
CS- 137 (CURIES)
OTHER RADIOACTIVITY (CURIES)
LOW-LEVEL NONINDUSTRIAL (ASSAYED FROM TRANSURANIC)

LOW-LEVEL INDUSTRIAL-OFFSITE
$1.800 E-02 \quad 1.797 E-03 \quad 9.832 E-04 \quad 1.924 E-03$

$1.935 E+04 \quad 1.998 E+02 \quad 1.599 E+01 \quad 7.508 E+01$

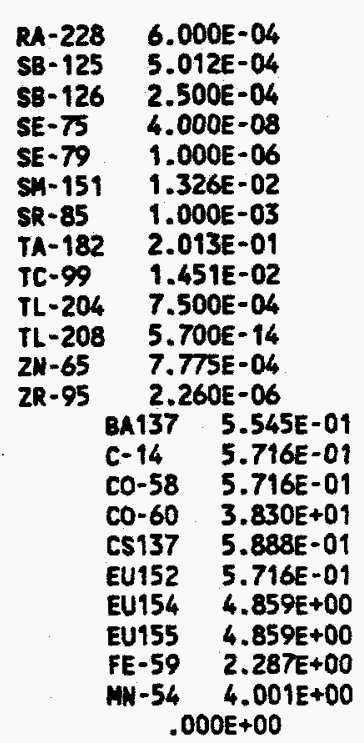

$\begin{array}{ll}A C-227 & 1.846 E+04 \\ A C-228 & 2.015 E-05 \\ A C-228 & 1.193 E-04 \\ A G-108 & 5.215 E-06 \\ A G-110 & 1.620 E-05 \\ A B-110 & 2.402 E-04 \\ A L-28 & 3.400 E-05 \\ A U-195 & 4.780 E-05 \\ B A-133 & 6.320 E-02 \\ B A-134 & 1.270 E-07 \\ B A-137 & 2.000 E-07 \\ B A-140 & 1.000 E-06 \\ B E-7 & 1.042 E+00 \\ B 1-207 & 1.669 E-03 \\ B I-212 & 7.005 E-03 \\ B I-214 & 3.048 E-04 \\ C-14 & 1.070 E-01 \\ C A-45 & 1.690 E-02 \\ C D-109 & 1.799 E-03 \\ C E-141 & 1.701 E-04 \\ C E-144 & 1.363 E-04 \\ C L-36 & 1.142 E-05 \\ C O-56 & 9.397 E-04 \\ C O-57 & 4.492 E-02 \\ C O-58 & 2.804 E-02 \\ C O-59 & 4.000 E-06 \\ C O-60 & 2.741 E+03 \\ C R-51 & 2.112 E-01 \\ C S-134 & 1.997 E-02 \\ C D-654 & 1.983 E-05 \\ E S-254 & 1.152 E-04 \\ E U-152 & 1.083 E+00 \\ E U-154 & 1.263 E-01 \\ E U-155 & 7.256 E-04 \\ \text { FE-55 } & 1.374 E+01 \\ & \end{array}$


SUIR328H - (Page 12 of 26)

RUNDATE: 08/20/94

Post-1970 Nontransuranic Waste Burial Ground Areas Waste Volumes Buried or stored and Nondecayed Curie Content through DECEMBER 31, 1993.

BURIAL GROUND FACILITIES

BURIAL GROUND 218 WS

LOW-LEVEL INDUSTRIAL-OFFSITE
TOTAL SR-90 RU-106 CS-137 OTHER RADIOACTIVITY (CURIES) (CURIES) (CURIES) (CURIES) (CURIES)

\begin{tabular}{|c|c|}
\hline 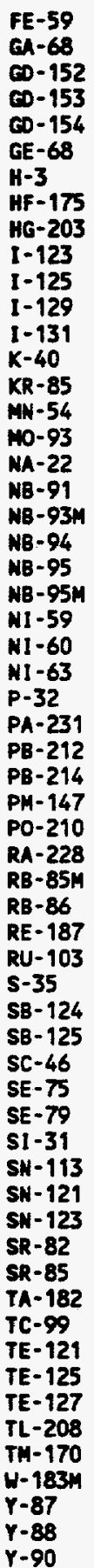 & $\begin{array}{l}3.874 E-02 \\
5.972 E-02 \\
8.468 E-02 \\
1.811 E-04 \\
4.420 E-02 \\
1.989 E-03 \\
1.568 E+04 \\
1.002 E-06 \\
1.200 E-05 \\
5.610 E-04 \\
6.664 E-02 \\
6.062 E-05 \\
2.021 E-05 \\
8.861 E-04 \\
8.074 E-04 \\
1.205 E+00 \\
1.292 E+00 \\
1.310 E+01 \\
1.020 E-03 \\
9.000 E-05 \\
7.513 E-05 \\
1.111 E-02 \\
2.000 E-07 \\
2.588 E-04 \\
2.895 E+00 \\
3.233 E-01 \\
3.593 E-01 \\
7.937 E-01 \\
3.315 E-03 \\
2.087 E-04 \\
1.221 E-05 \\
3.016 E-06 \\
6.000 E-04 \\
3.000 E-06 \\
2.577 E-03 \\
3.000 E-06 \\
1.710 E-04 \\
1.243 E-01 \\
1.818 E-04 \\
1.120 E-02 \\
4.320 E-03 \\
1.066 E-02 \\
2.933 E-03 \\
1.000 E-04 \\
1.324 E-03 \\
3.300 E-03 \\
2.000 E-07 \\
3.325 E-05 \\
1.764 E-02 \\
3.121 E-05 \\
3.146 E-02 \\
1.000 E-04 \\
1.777 E-05 \\
2.500 E-04 \\
2.109 E-03 \\
3.300 E-03 \\
5.770 E-06 \\
1.030 E-03 \\
8.730 E-05 \\
2.000 E-05\end{array}$ \\
\hline
\end{tabular}


SUIR328H - (Page 13 of 26)

RUNDATE: $08 / 20 / 94$

Post-1970 Nontransuranic Waste Burial Ground Areas Haste Volumes Buried or Stored and Nondecayed Curie Content through DECEMBER 31, 1993.

BURIAL GROUND FACILITIES

BURIAL GROUND 218 HS

LOU-LEVEL INDUSTRIAL-OFFSITE

LOW-LEVEL INDUSTRIAL
TOTAL

(CURIES)

(CURIES)

(CURIES)

(............

(CURIES)

(C)........

$7.883 E+02 \quad 7.002 E+01 \quad 2.192 E+01 \quad 1.199 E+02$
OTHER RRDIOACTIVITY (CURIES)

2N-65

$1.006 E-02$

$1.363 E+02$

AC-227 1.672E-05

AC-228 7.275E-07

AG-110 3.04TE-04

$B A-133 \quad 2.719 E-06$

BA-137 9.000E-09

BI-212 4.855E-07

BI-214 3.307E-04

c-14 7.692E-02

CA-45 $\quad 1.035 E-05$

CD-109 1.312E-02

CE-141 3.609E-05

CE-144 $6.545 E+00$

CL-36 2.262E-06

CO-57 3.137E-04

C0-58 $\quad 9.119 E-01$

C0-60 8.393E+01

CR-51 2.307E-03

CS-133 2.800E-03

CS-134 6.976E-02

CU-65M 7.000E-03

EU-152 4.028E-01

EU-154 4.099E+00

EU-155 8.682E-03

FE-55 3.375E-01

FE-59 3.260E+00

$\mathrm{K}-3 \quad 3.009 \mathrm{E}+09$

HF- $1813.360 E-06$

$1-125 \quad 7.771 E-02$

1-129 2.849E-02

I-131 1.052E-03

$K-40 \quad 5.018 E-03$

KR-85 1.458E-08

MN-54 2.455E+00

MO-93 1.000E-05

MA-22 2.137E-04

NB-91 2.800E-02

MB-93M 1.005E-08

MB-94 6.544E-04

NB-95 1.454E-03

MD-147 6.000E-09

NI-59 7.100E-03

NI -60 2.288E-01

$M I-63 \quad 2.765 E-02$

P-32 9.212E-03

PA-231 4.800E-11

PB-208 9.203E-08

PB-212 4.902E-06

PB-214 2.238E-05

PH-147 3.295E+00

PO-210 5.000E-08

PT-195 9.000E-10

S-35 8.002E-04

SB-125 6.161E-03

SC-46 1.587E-04

SE-75 1.035E-03

SE-79 1.038E-04

SH-151 7.881E-01 


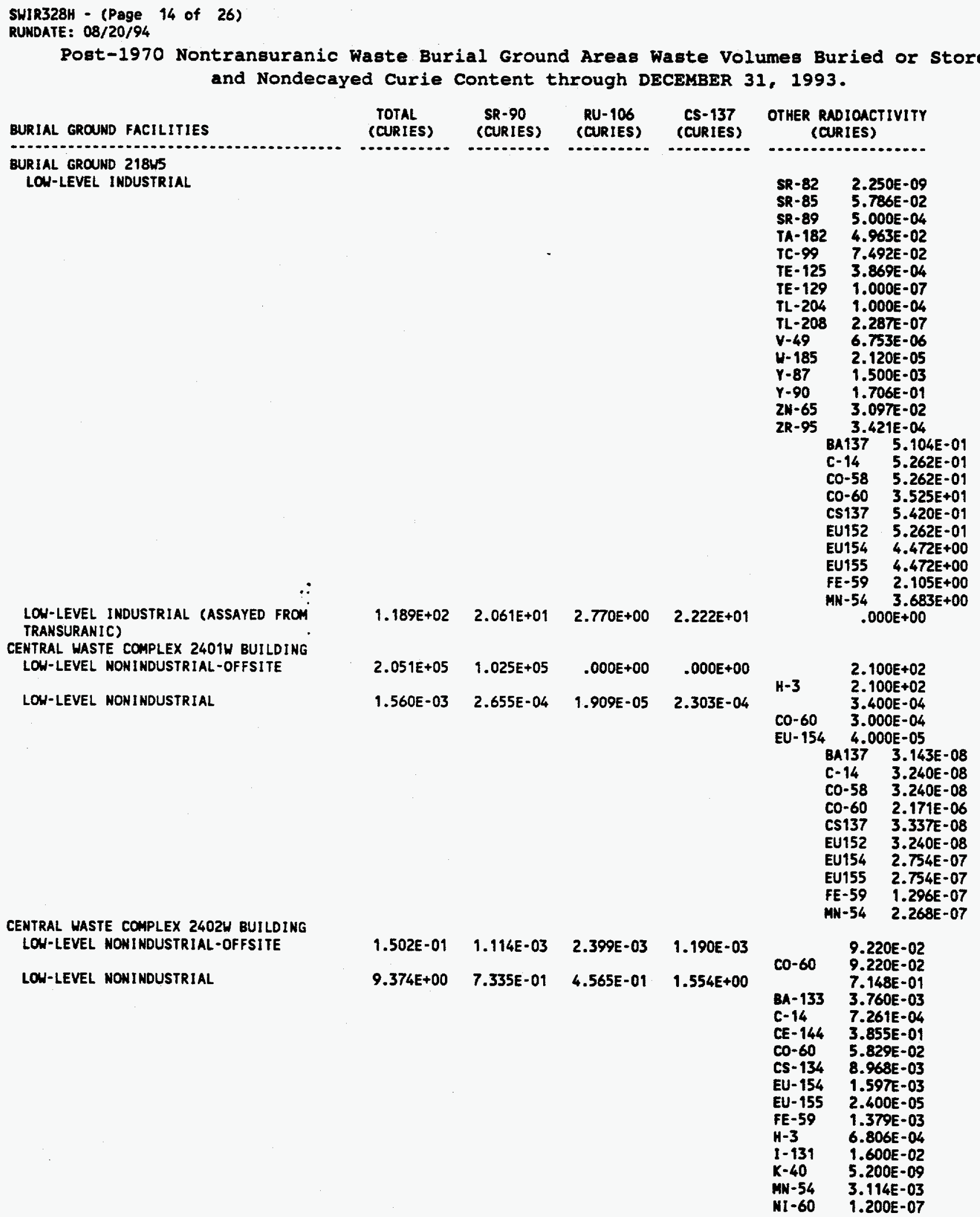


SHIR328H - (Page 15 of 26)

RUNDATE: 08/20/94

Post-1970 Nontransuranic Waste Burial Ground Areas Waste Volumes Buried or Stored and Nondecayed Curie Content through DECEMBER $31,1993$.

\section{BURIAL GROUND FACILITIES}

CENTRAL UASTE COMPLEX 2402H BUILDING LOW-LEVEL NONINDUSTRIAL

CENTRAL WASTE COMPLEX 2402WB BUILDING LON-LEVEL NONINDUSTRIAL-OFFSITE

LON-LEVEL NONINDUSTRIAL

CENTRAL WASTE COMPLEX 2402WC BUILDING LOW-LEVEL NOWINOUSTRIAL-OFFSITE

\begin{tabular}{cccc}
$\begin{array}{c}\text { TOTAL } \\
\text { (CURIES) }\end{array}$ & $\begin{array}{c}\text { SR-90 } \\
\text { (CURIES) }\end{array}$ & $\begin{array}{c}\text { RU-106 } \\
\text { (CURIES) }\end{array}$ & $\begin{array}{c}\text { CS-137 } \\
\text { (CURIES) }\end{array}$ \\
\hdashline
\end{tabular}

OTHER RADIOACTIVITY (CURIES)

\begin{tabular}{ll} 
WI-63 & $7.130 E-05$ \\
PH-147 & $1.7664 E-01$ \\
SB-125 & $1.162 E-04$ \\
SE-75 & $1.410 E-03$ \\
SH-151 & $5.060 E-02$ \\
TA-182 & $4.570 E-03$ \\
TC- 99 & $1.586 E-03$ \\
TE-125 & $2.642 E-05$ \\
\multicolumn{3}{c}{ BA137 } & $1.627 E-06$ \\
C-14 & $1.677 E-06$ \\
CO-58 & $1.677 E-06$ \\
CO-60 & $1.124 E-04$ \\
CS137 & $1.727 E-06$ \\
EU152 & $1.677 E-06$ \\
EU154 & $1.425 E-05$ \\
EU155 & $1.425 E-05$ \\
FE- 59 & $6.708 E-06$ \\
MN-54 & $1.174 E-05$
\end{tabular}

\subsection{E-03 1.275E-05 2.745E-05 2.812E-05}

$1.312 E+01$

6.695E-09

1.960E-01

7.134E-01
$1.000 E-07 \quad .000 E+00$
$.000 E+00$

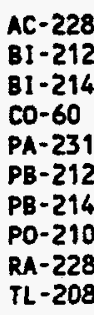

TL-208

C-14 CE-144

CO-60

CS- 134

EU-152

EU-154

EU-155

FE-59

$\mathrm{H}-3$

MN-54

$M A-22$

NB-95M

$11-60$

PM- 147

sit-151

TC- 99

$2 R-95$
8.399E-04

1. $755 E-08$

$3.435 E-11$

1.494E-13

2.216E-05

4.010E-16

1.374E-05

8.110E-06

$1.855 E-23$

7.497E-04

4.623E-05

3.406E-02

1.110E-08

$1.820 \mathrm{E}-02$

4.823E-03

$4.455 \mathrm{E}-08$

$2.940 E-05$

5.023E-05

2.010E-05

2.200E-05

1.741E-05

3.500E-05

$1.780 E-05$

8.120E-10

2.770E-05

8.712E-03

2.100E-03

$1.131 E-06$

5.030E-06

BA137 3.159E-07

C-14 3.256E-07

CO-58 3.256E-07

C0-60 2.182E-05

CS137 3.354E-07

EU152 3.256E-07

EU154 2.768E-06

EU155 2.768E-06

FE-59 1.302E-06

MN-54 2.279E-06

1.000E-07 
SUIR328H - (Page 16 of 26)

RUNDATE: 08/20/94

Post-1970 Nontransuranic Waste Burial Ground Areas Waste Volumes Buried or stored and Nondecayed Curie Content through DECEMBER 31, 1993.

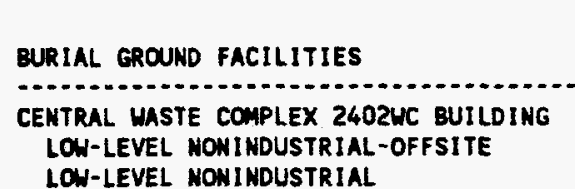

LOW-LEVEL MONIMDUSTRIAL
TOTAL

(CURIES)

SR-90

(CURIES)

RU-106
(CURIES)

CS- 137

(CURIES)

7.143E-01

4.066E-02
$1.669 E-02$
OTHER RADIOACTIVITY (CURIES)

-...........................

CENTRAL HASTE COMPLEX 2402WD BUILOING LOW-LEVEL NONINDUSTRIAL

2.024

7

$165 E-03$

8

8.78

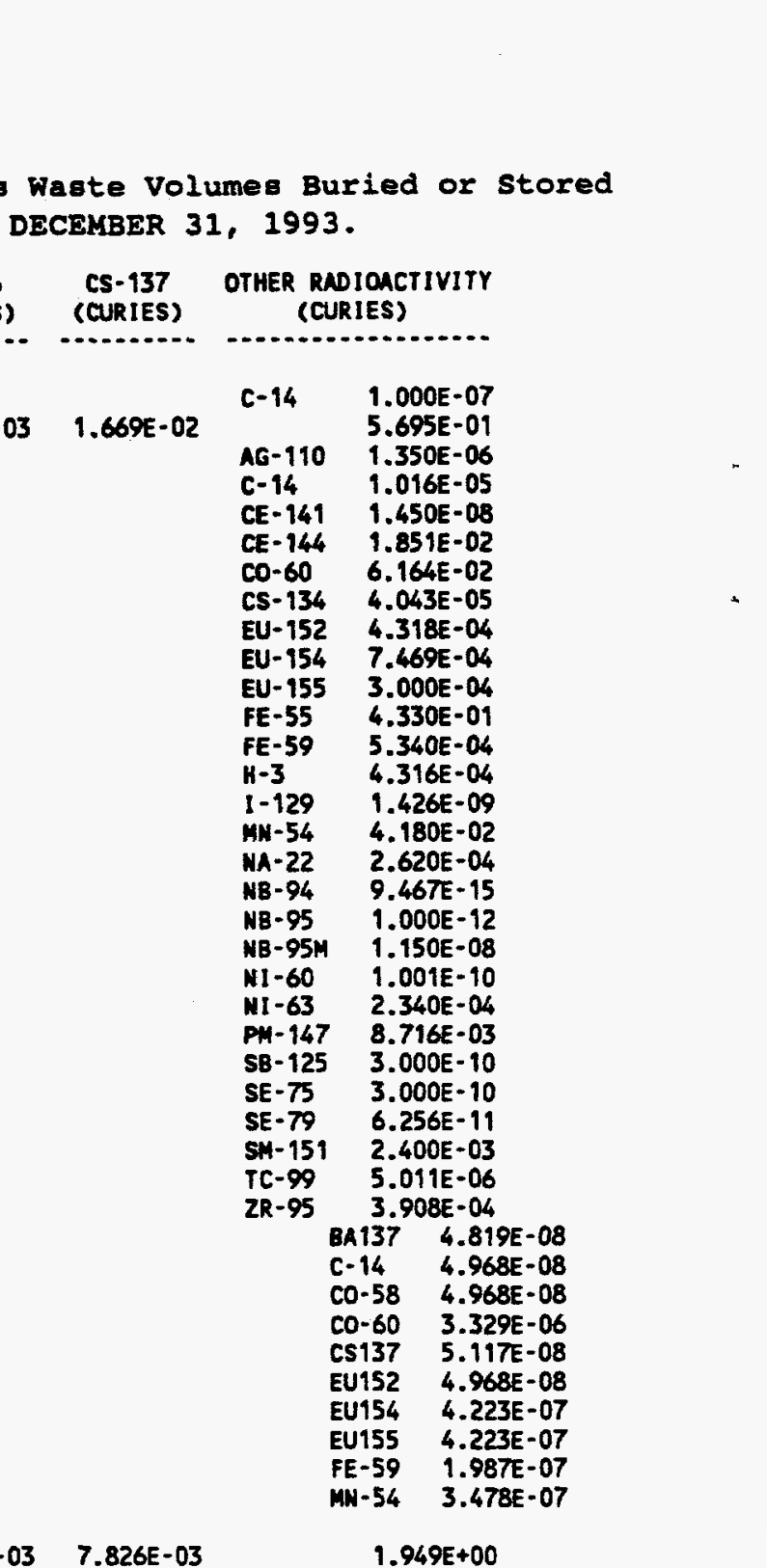


WHC-EP-0125-6

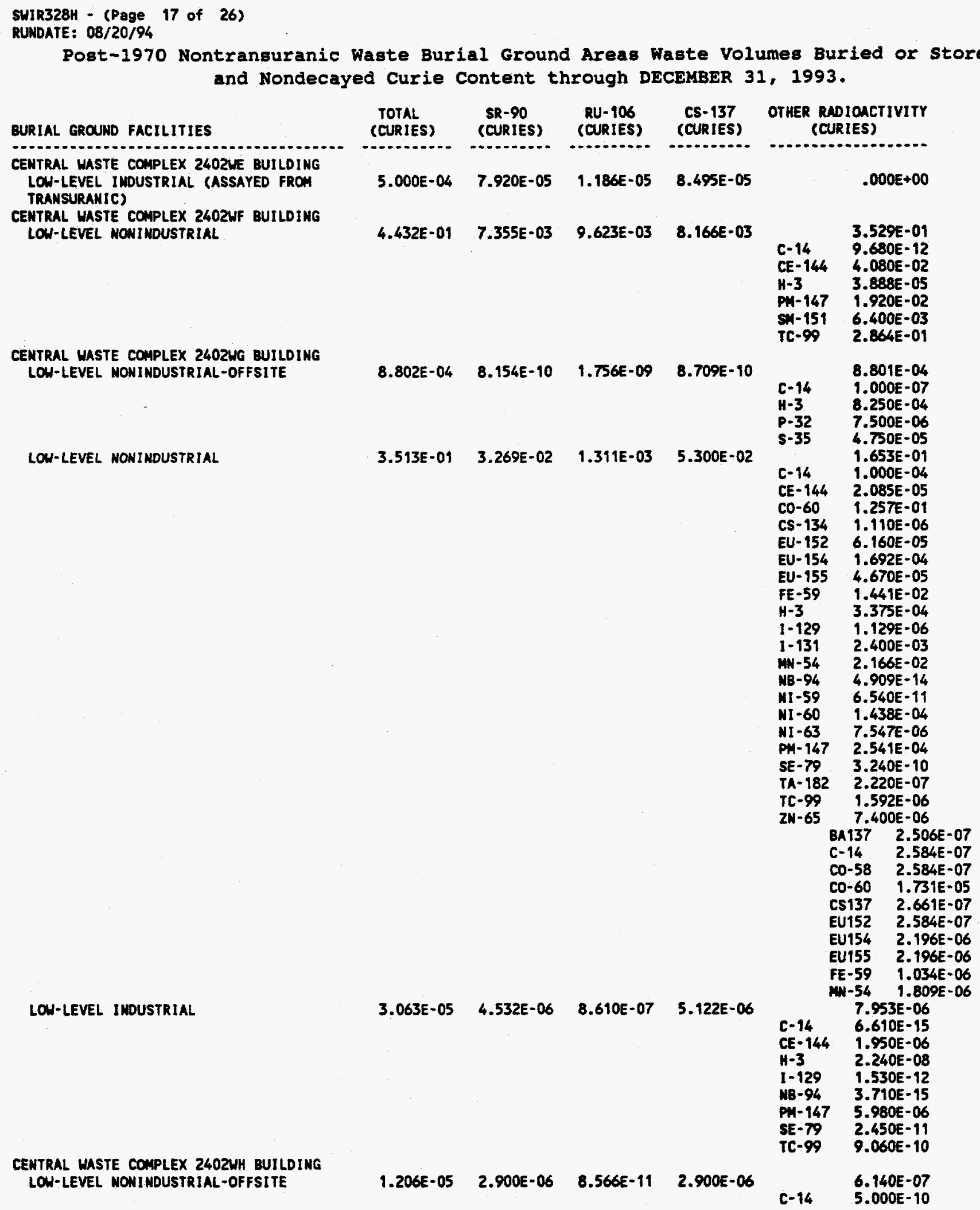




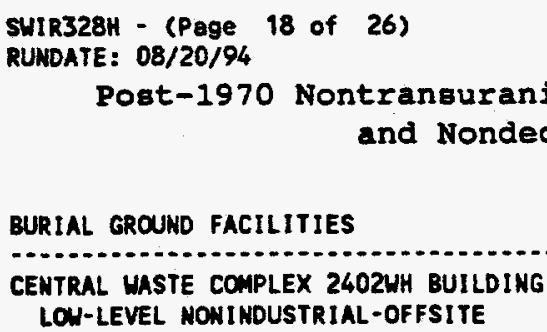

LOW-LEVEL NONINDUSTRIAL

CENTRAL WASTE COMPLEX 2402WI BUILDING LOW-LEVEL NONINOUSTRIAL

CENTRAL WASTE COMPLEX 2402WJ BUILDING LOW-LEVEL. NONINDUSTRIAL

CENTRAL MASTE COMPLEX 2402WL BUILDING LOW-LEVEL MONINDUSTRIAL-OFFSITE

LOW-LEVEL MONINDUSTRIAL
TOTAL $\quad$ SR-90 RU-106 CS-137 OTHER RADIOACTIVITY (CURIES) (CURIES) (CURIES) (CURIES) (CURIES)

(C).........

(C)

(CURIES)

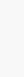

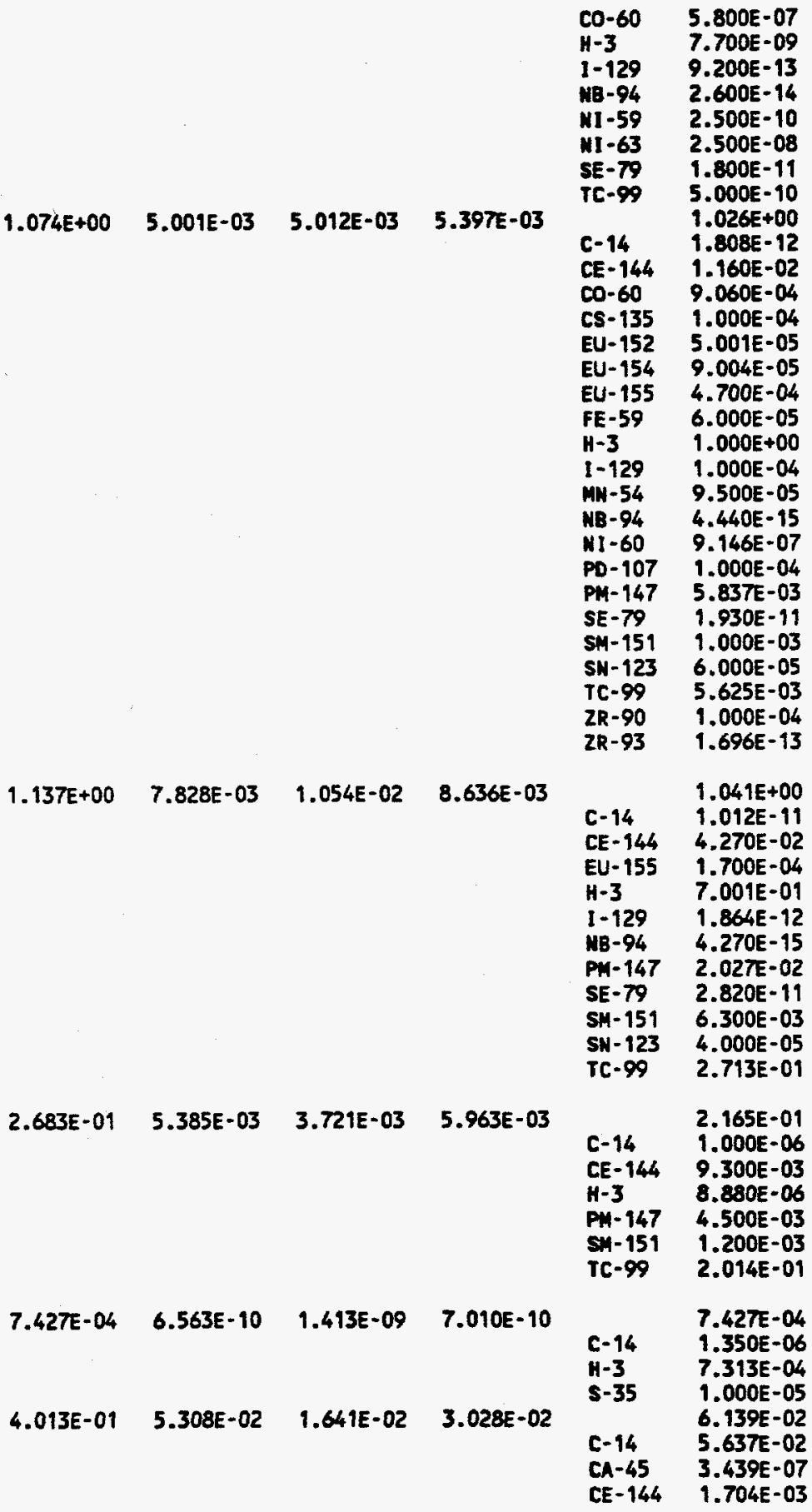


SUIR328H - (Page 19 of 26)

RUNDATE: 08/20/94

Post-1970 Nontransuranic Waste Burial Ground Areas Waste Volumes Buried or Stored and Nondecayed Curie Content through DECEMBER 31, 1993.

BURIAL GROUND FACILITIES

TOTAL \$R-90 RU-106

CENTRAL WASTE COMPLEX 2402WL BUILOING

LOW-LEVEL NONINDUSTRIAL

(CURIES)

(CURIES)

(CURIES)

CS- 137

(CURIES)

OTHER RADIOACTIVITY (CURIES)

CENTRAL WASTE COMPLEX 2403WA BUILDING LOW-LEVEL NONINDUSTRIAL-OFFSITE

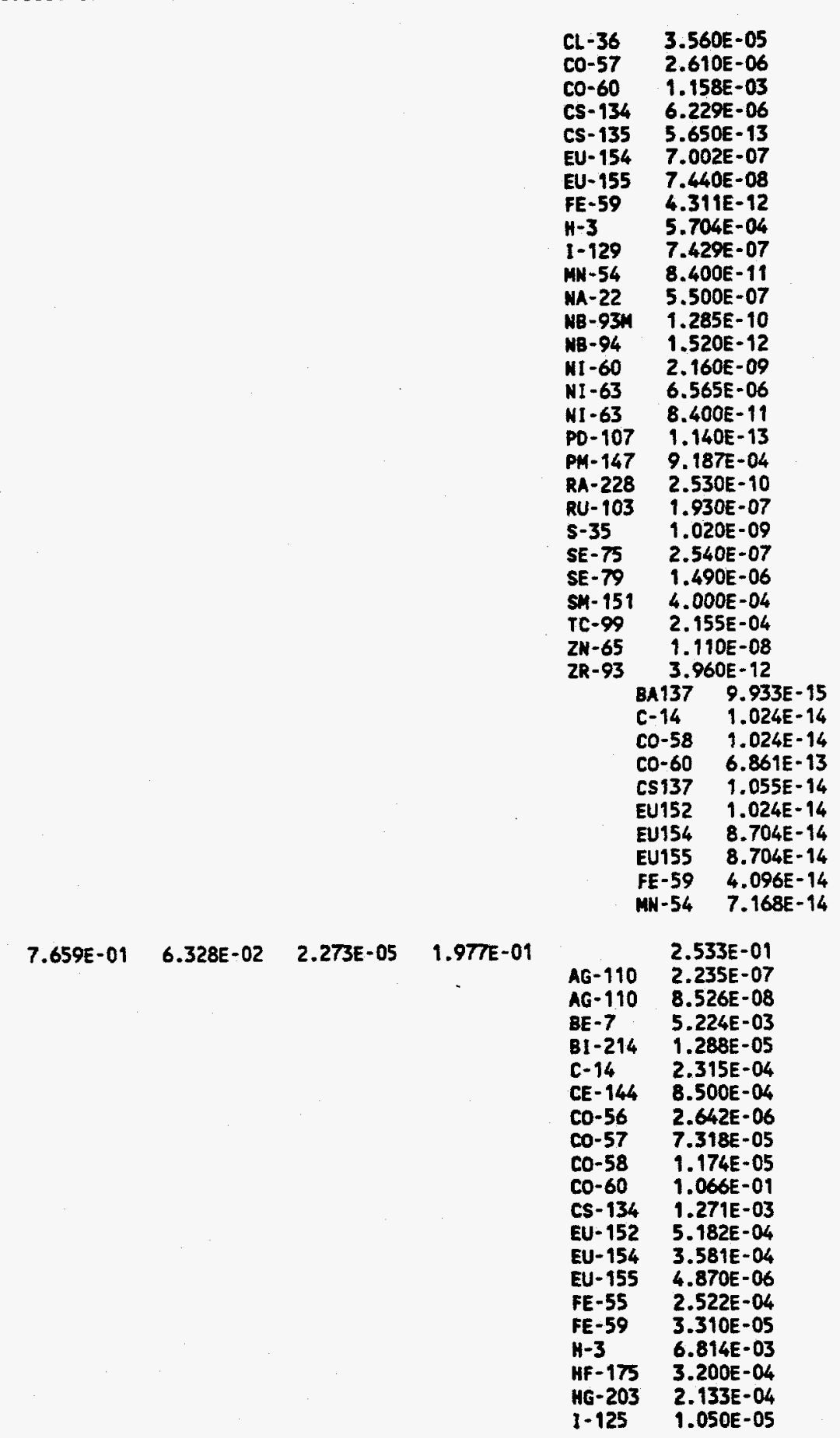


SWIR328H - (Page 20 of 26)

RUWDATE: $08 / 20 / 94$

Post-1970 Nontransuranic waste Burial Ground Areas Waste Volumes Buried or stored and Nondecayed Curie Content through DECEMBER 31, 1993.

$\begin{array}{lllll}\text { BURIAL GROUMD FACILITIES } & \begin{array}{c}\text { TOTAL } \\ \text { (CURIES) }\end{array} & \begin{array}{c}\text { SR-90 } \\ \text { (CURIES) }\end{array} & \begin{array}{c}\text { RU-106 } \\ \text { (CURIES) }\end{array} & \begin{array}{c}\text { CS-137 } \\ \text { (CURIES) }\end{array}\end{array}$

CENTRAL WASTE COMPLEX 2403WA BUILDING LOH-LEVEL NONIMDUSTRIAL-OFFSITE

LOW-LEVEL NONINDUSTRIAL

OTHER RADIOACTIVITY (CURIES)

\begin{tabular}{|c|c|}
\hline 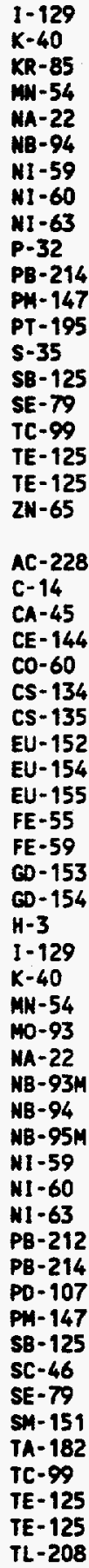 & $\begin{array}{l}.544 E-06 \\
.386 E-02 \\
.486 E-03 \\
.211 E-04 \\
.338 E-04 \\
.793 E-08 \\
.644 E-06 \\
.824 E-02 \\
.517 E-04 \\
.430 E-04 \\
.288 E-05 \\
.403 E-03 \\
.800 E-05 \\
.611 E-03 \\
.940 E-02 \\
.592 E-08 \\
.156 E-06 \\
.589 E-02 \\
.792 E-03 \\
.566 E-06 \\
.500 E+00 \\
.200 E-07 \\
.672 E-07 \\
.300 E-06 \\
.724 E-01 \\
.539 E-01 \\
.322 E-03 \\
.620 E-13 \\
.219 E-03 \\
.948 E-03 \\
.816 E-04 \\
.513 E-01 \\
.000 E-06 \\
.000 E-04 \\
1.919 E-04 \\
.741 E-03 \\
.439 E-04 \\
.704 E-05 \\
.150 E-01 \\
.400 E-07 \\
.711 E-04 \\
.292 E-07 \\
.096 E-09 \\
.387 E-08 \\
.984 E-09 \\
.701 E-03 \\
.661 E-06 \\
.140 E-07 \\
.090 E-09 \\
.336 E-13 \\
.671 E-01 \\
.960 E-06 \\
.600 E-08 \\
.554 E-08 \\
.115 E-01 \\
.000 E-05 \\
.207 E+00 \\
.840 E-07 \\
.290 E-07 \\
.960 E-07\end{array}$ \\
\hline
\end{tabular}


SUIR328H - (Page 21 of 26 )

RUNDATE: 08/20/94

Post-1970 Nontransuranic Waste Burial Ground Areas waste Volumes Buried or stored and Nondecayed Curie Content through DECEMBER 31, 1993.

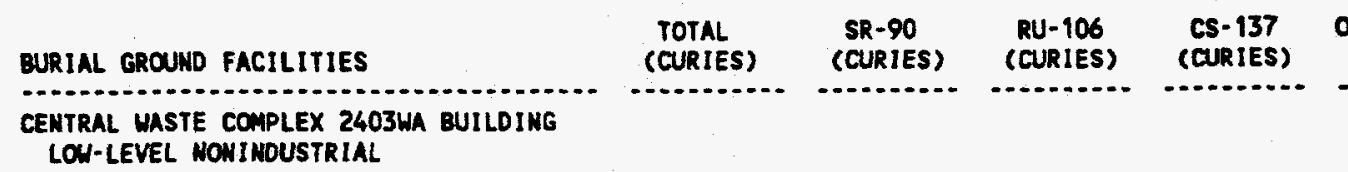

LOW-LEVEL IMDUSTRIAL

CENTRAL WASTE COMPLEX 2403WC BUILDING LOW-LEVEL NOWINDUSTRIAL-OFFSITE $\begin{array}{cccc}7.162 E-07 & 1.435 E-11 & 2.906 E-13 & 3.680 E-07 \\ 1.516 E-01 & 9.804 E-05 & 2.025 E-04 & 3.906 E-04\end{array}$

$5.297 E+01 \quad 2.172 E+01 \quad 9.068 E-02 \quad 4.369 E+00$
OTHER RNDIOACTIVITY (CURIES)

$\begin{array}{ll}V-49 & 1.301 E-04 \\ \text { XE-131 } & 2.600 E-05 \\ 2 N-65 & 1.360 E-10 \\ 2 R-93 & 4.630 E-12 \\ 2 R-95 & 2.098 E-04 \\ \text { BA137 } & 1.063 E-08 \\ \text { C-14 } & 1.095 E-08 \\ \text { CO-58 } & 1.095 E-08 \\ \text { CO-60 } & 7.339 E-07 \\ \text { CS137 } & 1.128 E-08 \\ \text { EU152 } & 1.095 E-08 \\ \text { EU154 } & 9.311 E-08 \\ \text { EU155 } & 9.311 E-08 \\ \text { FE-59 } & 4.382 E-08 \\ M N-54 & 7.668 E-08\end{array}$
$.000 \mathrm{E}+00$

1.463E-01

$81-214 \quad 3.657 E-05$

C- $14 \quad 6.459 E-04$

Co-58 1.354E-03

CO-60 2.703E-02

FE-55 2.698E-02

$\mathrm{H}-3 \quad 8.408 \mathrm{E}-02$

$1-129 \quad 5.399 E-09$

MN-54 4.587E-03

NI $-60 \quad 5.247 \mathrm{E}-05$

NI $-63 \quad 1.354 \mathrm{E}-03$

P-32 1.670E-04

PB-214 3.657E-05

TC- 99 5.399E-09

$5.159 E-01$

AC-228 1.200E-03

$A G-110 \quad 1.840 E-06$

AG-110 1.311E-04

$B 1-212 \quad 1.500 E-03$

C- $14 \quad 3.852 E-07$

CE-149 1.810E-06

CE-144 1.803E-01

CO-57 2.541E-09

CO-60 5.901E-02

CR-51 $1.824 \mathrm{E}-07$

CS-134 2.683E-02

EU-152 1.088E-04

EU-154 1.775E-02

EU-155 2.289E-02

FE-55 3.099E-05

FE-59 $7.320 E-04$

CD-154 6.042E-04

H-3 4.392E-02

$1-129 \quad 1.505 E-04$

$K-40 \quad 2.318 E-04$

MN-54 1.036E-03

MO-93 1.890E-08

MA-22 9.498E-06

MB-93M $1.556 E-06$

MB-94 9.558E-09

MB-95 2.095E-09

NB-95M 1.603E-DB

NI-59 2.733E-08 
SUIR328H - (Page 22 of 26)

RUNDATE: 08/20/94

Post-1970 Nontransuranic Waste Burial Ground Areas Waste Volumes Buried or Stored and Nondecayed Curie Content through DECEMBER 31, 1993.

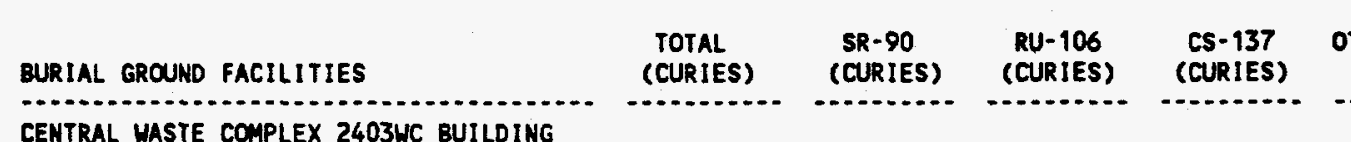

LOW-LEVEL NONINDUSTRIAL (ASSAYED FROM TRANSURANIC)

CENTRAL WASTE COMPLEX 2403WD BUILDING

LOW-LEVEL NONINOUSTRIAL-OFFSITE

LOH-LEVEL NONINDUSTRIAL

$\begin{array}{llll}1.000 E-03 & 1.310 E-04 & 3.818 E-05 & 1.404 E-04 \\ 3.080 E-05 & 1.989 E-07 & 4.283 E-07 & 2.124 E-07 \\ 3.173 E+03 & 3.110 E+00 & 7.933 E-03 & 2.292 E+01\end{array}$

$7.933 E-03$
FLAMMABLE STORAGE MODULE 1

LOW-LEVEL NONINDUSTRIAL
$1.964 E-04 \quad 1.362 E-05 \quad 1.945 E-06 \quad 2.401 E-05$
OTHER RADIOACTIVITY (CURIES)

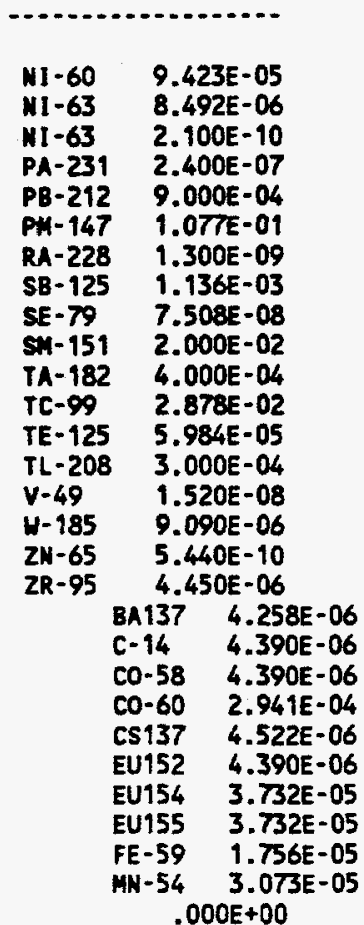

\begin{tabular}{|c|c|}
\hline $\mathrm{H}-3$ & $\begin{array}{l}2.080 E-05 \\
2.080 E-05 \\
3.122 E+03\end{array}$ \\
\hline $\begin{array}{l}C-14 \\
\text { CE- } 144 \\
\text { CO-60 } \\
\text { EU-152 } \\
\text { EU-154 } \\
\text { EU-155 } \\
H-3 \\
I-129 \\
\text { NB-93M } \\
\text { NB-94 } \\
\text { NI -59 } \\
\text { HI }-63 \\
\text { PH-147 } \\
\text { SE-79 } \\
\text { SH-151 } \\
\text { TC-99 }\end{array}$ & $\begin{array}{l}5.380 E-05 \\
6.483 E-03 \\
3.000 E+03 \\
8.120 E-03 \\
2.278 E-01 \\
1.777 E-03 \\
2.199 E-05 \\
1.641 E-03 \\
2.230 E-04 \\
4.100 E-06 \\
1.092 E+00 \\
1.200 E+02 \\
4.302 E-03 \\
1.865 E-05 \\
7.000 E-04 \\
3.642 E-01\end{array}$ \\
\hline $\begin{array}{l}C-14 \\
C E-144 \\
C S-134 \\
\text { EU-152 } \\
\text { EU- } 154 \\
\text { EU-155 } \\
H-3 \\
I-129\end{array}$ & $\begin{array}{l}36 E-04 \\
322 E-14 \\
110 E-06 \\
60 E-07 \\
80 E-05 \\
00 E-05 \\
60 E-06 \\
08 E-07 \\
83 E-09\end{array}$ \\
\hline
\end{tabular}


SHIR328H - (Page 23 of 26)

RUNDATE: 08/20/94

Post-1970 Nontransuranic Waste Burial Ground Areas Waste Volumes Buried or stored and Nondecayed Curie Content through DECEMBER 31, 1993.

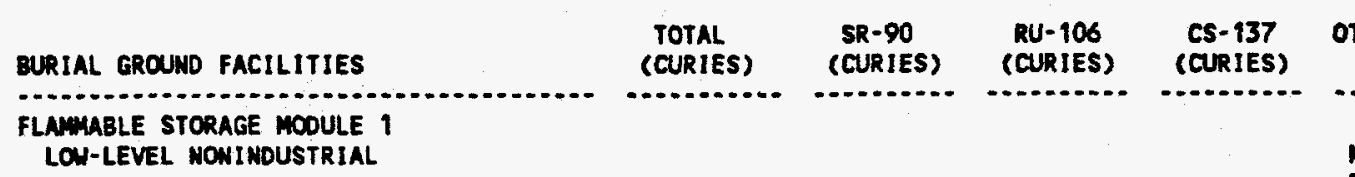

FLAMABLE STORAGE MOOULE 10 LOW-LEVEL NONINOUSTRIAL

$3.621 E+$

$9.897 E-01$

$1.612 E-02$

$7.518 E-0$

FLAMMABLE STORAGE MODULE 11

LOU-LEVEL NOWINDUSTRIAL-OFFSITE

$1.537 E-04$

$3.560 E-09$

$1.765 E-09$

LOW-LEVEL NONINDUSTRIAL

4.663E- 04

4.740E-06

3.403E-07

1.971E-04

FLAMMABLE STORAGE MOOULE 12

LOW-LEVEL MONINDUSTRIAL-OFFSITE LOW-LEVEL NOWINOUSTRIAL

OTHER RADIOACTIVITY (CURIES)

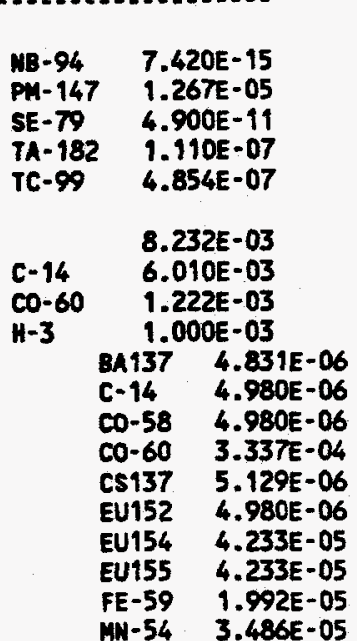

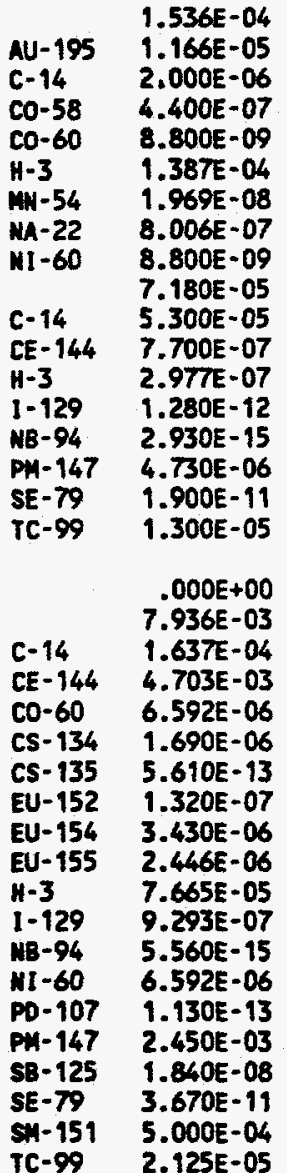




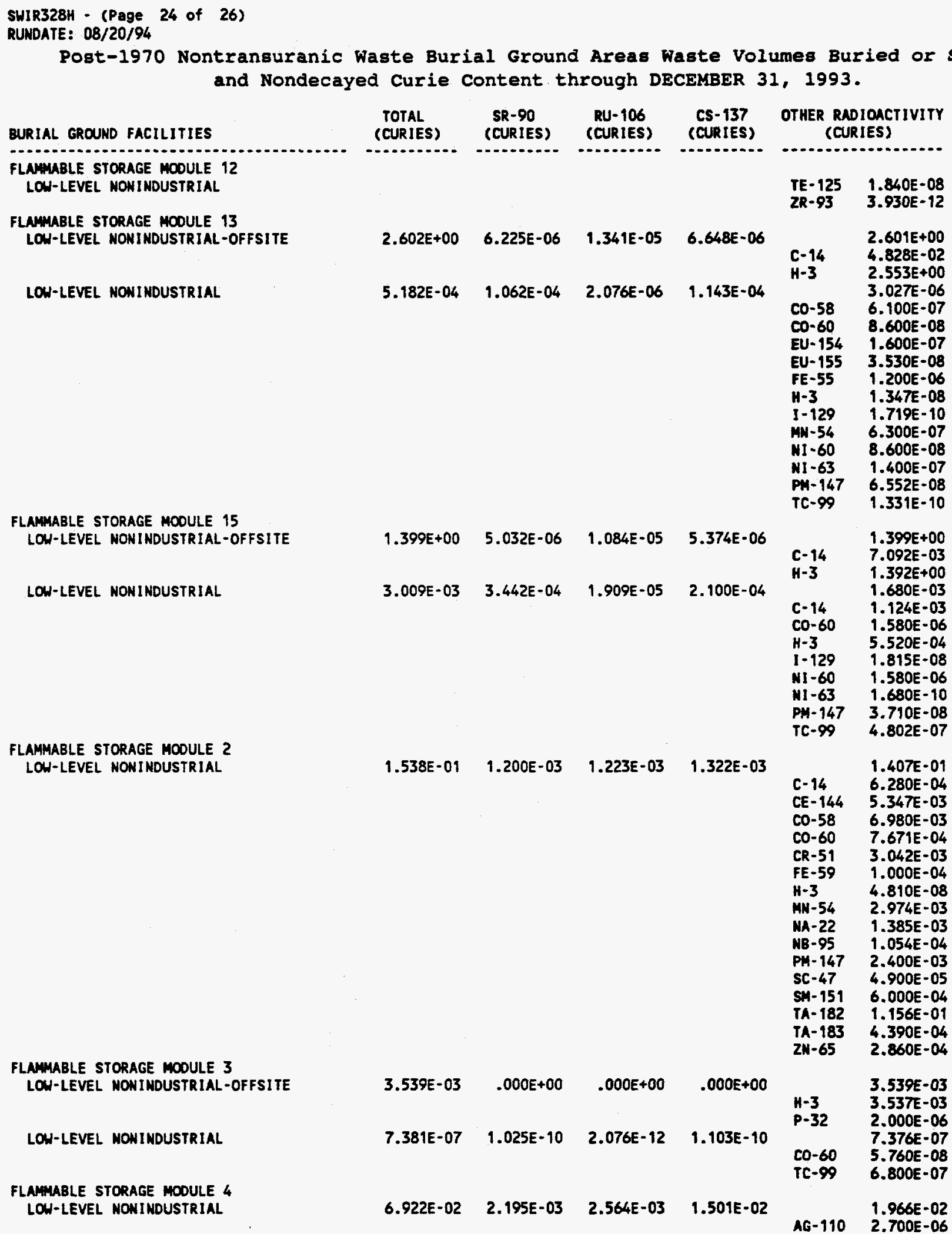


SUIR328H - (Page 25 of 26)

RUNDATE: 08/20/94

Post-1970 Nontransuranic Waste Burial Ground Areas Waste Volumes Buried or stored and Nondecayed Curie Content through DECEMBER 31, 1993.

\section{BURIAL GROUND FACILITIES}

FLAMMABLE STORAGE MOOULE 4 LOH-LEVEL NONINDUSTRIAL

FLAMMABLE STORAGE MOOULE 5 LOW-LEVEL NONINDUSTRIAL

FLAMMABLE STORAGE MOOULE 6 LON-LEVEL NOWINDUSTRIAL

FLAMUABLE STORAGE MODULE 7

LON-LEVEL MONINDUSTRIAL-OFFSITE LON-LEVEL NONI NDUSTRIAL
TOTAL
(CURIES)

SR- 90

(CURIES)

(CURIES)

CS-137

(CURIES)

OTHER RADIOACTIVITY (CURIES)

\begin{tabular}{|c|c|c|c|c|c|}
\hline & & . & & $\begin{array}{l}C-14 \\
C E-144 \\
C O-60 \\
C S-134 \\
E U-152 \\
E U-154 \\
E U-155 \\
H-3 \\
I-129 \\
M U-54 \\
P H-147 \\
S B-125 \\
S N-151 \\
T C-99 \\
2 R-95\end{array}$ & $\begin{array}{l}7.210 E-04 \\
1.032 E-02 \\
6.722 E-06 \\
6.513 E-05 \\
2.481 E-06 \\
2.951 E-05 \\
1.261 E-05 \\
2.590 E-03 \\
3.866 E-10 \\
2.957 E-07 \\
4.800 E-03 \\
7.569 E-06 \\
1.100 E-03 \\
3.002 E-10 \\
5.360 E-07\end{array}$ \\
\hline $4.752 E-02$ & $2.879 E-03$ & $2.575 E-03$ & 3.172E-03 & $\begin{array}{l}C-14 \\
C E-144 \\
H-3 \\
P M-147 \\
5 M-151 \\
T C-99\end{array}$ & $\begin{array}{l}1.841 E-02 \\
1.186 E-03 \\
1.030 E-02 \\
6.355 E-04 \\
4.800 E-03 \\
1.400 E-03 \\
8.600 E-05\end{array}$ \\
\hline $4.919 E+00$ & $1.404 E-02$ & $7.252 E-04$ & 6. $171 E-02$ & $\begin{array}{l}C-14 \\
C A-45 \\
C E-144 \\
C O-57 \\
C O-58 \\
C O-60 \\
C R-51 \\
C S-134 \\
E U-152 \\
E U-154 \\
E U-155 \\
F E-55 \\
H-3 \\
I-129 \\
M N-54 \\
M O-93 \\
N B-93 H \\
N I-60 \\
M I-63 \\
P H-147 \\
S B-125 \\
S I-31 \\
T C-99 \\
T I-51 \\
V-69 \\
W-185 \\
2 R-95\end{array}$ & $\begin{array}{l}4.766 E+00 \\
5.000 E-04 \\
1.830 E-05 \\
1.471 E-04 \\
5.600 E-03 \\
1.890 E-02 \\
4.464 E-02 \\
7.220 E-03 \\
3.903 E-04 \\
7.700 E-06 \\
2.007 E-05 \\
1.356 E-05 \\
2.427 E+00 \\
7.160 E-01 \\
1.000 E-02 \\
1.263 E+00 \\
3.190 E-04 \\
2.100 E-05 \\
3.570 E-03 \\
4.561 E-02 \\
1.590 E-05 \\
1.000 E-05 \\
1.130 E-06 \\
6.722 E-05 \\
4.420 E-07 \\
4.640 E-02 \\
1.761 E-01 \\
4.140 E-05\end{array}$ \\
\hline $\begin{array}{l}9.200 E-04 \\
1.661 E-03\end{array}$ & $\begin{array}{l}1.830 E-05 \\
1.731 E-04\end{array}$ & $\begin{array}{l}3.940 E-05 \\
1.259 E-06\end{array}$ & $\begin{array}{l}1.954 E-05 \\
1.309 E-04\end{array}$ & $\begin{array}{l}C O-57 \\
C O-60 \\
E U-154 \\
E U-155 \\
H-3\end{array}$ & $\begin{array}{l}.000 E+00 \\
5.736 E-05 \\
5.997 E-08 \\
5.910 E-07 \\
3.630 E-08 \\
1.736 E-07 \\
1.913 E-05\end{array}$ \\
\hline
\end{tabular}




\begin{tabular}{|c|c|c|c|c|c|c|}
\hline \multirow{3}{*}{$\begin{array}{l}\text { BURIAL GROUND FACILITIES } \\
\text { FLAMABLE STORAGE MOOULE } 7 \\
\text { LOW-LEVEL NOWINDUSTRIAL }\end{array}$} & \multicolumn{3}{|c|}{$\begin{array}{l}\text { Waste Burial Ground Areas } \\
\text { ed Curie Content through DE }\end{array}$} & \multicolumn{3}{|c|}{$\begin{array}{l}\text { laste Volumes Buried or } \\
\text { CEMBER } 31,1993 .\end{array}$} \\
\hline & $\begin{array}{l}\text { TOTAL } \\
\text { (CURIES) }\end{array}$ & $\begin{array}{l}\text { SR-90 } \\
\text { (CURIES) }\end{array}$ & $\begin{array}{l}\text { RU-106 } \\
\text { (CURIES) }\end{array}$ & $\begin{array}{l}\text { CS-137 } \\
\text { (CURIES) }\end{array}$ & $\begin{array}{l}\text { OTHER RAD } \\
\text { (CUR }\end{array}$ & $\begin{array}{l}\text { lOACTIVITY } \\
\text { (ES) }\end{array}$ \\
\hline & 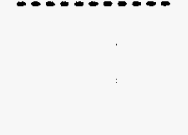 & (2) & & & $\begin{array}{l}1-129 \\
\text { PN- } 147 \\
\text { TC- } 99\end{array}$ & $\begin{array}{l}2.451 E-07 \\
3.694 E-05 \\
1.885 E-07\end{array}$ \\
\hline $\begin{array}{l}\text { FLAMAUABLE STORAGE MOOULE } 8 \\
\text { LOW-LEVEL NOWINDUSTRIAL }\end{array}$ & 7.331E-03 & 4.724E-04 & $3.651 E-05$ & $3.148 E-03$ & $\begin{array}{l}C-14 \\
C E-144 \\
C O-60 \\
C S-134 \\
E U-154 \\
E U-155 \\
H-3 \\
I-129 \\
K-40 \\
M N-54 \\
N B-94 \\
N I-60 \\
P N-147 \\
\text { SE-79 } \\
T C-99\end{array}$ & $\begin{array}{l}1.119 E-04 \\
3.810 E-16 \\
5.600 E-08 \\
1.311 E-05 \\
2.040 E-05 \\
2.420 E-07 \\
3.750 E-07 \\
1.157 E-06 \\
1.485 E-08 \\
4.000 E-07 \\
1.275 E-05 \\
2.140 E-16 \\
1.311 E-05 \\
2.583 E-06 \\
1.410 E-12 \\
4.771 E-05\end{array}$ \\
\hline Total $200 \mathrm{H}$ & $3.389 E+06$ & $2.222 E+05$ & $2.130 E+04$ & $3.605 E+05$ & & $1.808 E+06$ \\
\hline TOTAL 200 AREAS & & & & $1.568 E+06$ & & \\
\hline
\end{tabular}


WHC-EP-0125-6

SHIR328I - SPage

RUNDATE: $08 / 20 / 94$

1 of 26)

Post-1970 Nontransuranic Waste Burial Ground Areas

Decayed Curie Content through DECEMBER 31, 1993.

BURIAL GROUND FACILITIES

\section{E AREA}

BURIAL GROUND 218E10

LON-LEVEL NOWINDUSTRIAL

LON-LEVEL INOUSTRIAL
(CURIES)

(CURIES)

BURIAL GROUND 218E12B

LOW-LEVEL NONINDUSTRIAL

LOW-LEVEL INDUSTRIAL-OFFSITE

$8.826 E+03$
$S R-90$

(CURIES)

RU- 106 (CURIES)

cs- 137 (CURIES)

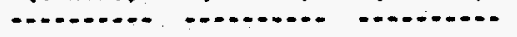
$3.108 E+06$

$5.828 E+02$ $7.131 E+05$

5.074E-03 8.983E-01

$3.768 E+02$ $8.630 E+05$

\begin{tabular}{|c|c|c|}
\hline$\infty 0-60$ & \multicolumn{2}{|c|}{$\begin{array}{r}.000 E+00 \\
6.393 E+02 \\
6.393 E+02\end{array}$} \\
\hline & $\begin{array}{l}\text { BA137 } \\
\text { C- } 14 \\
\text { CO-58 } \\
\text { CO-60 } \\
\text { CS137 } \\
\text { EU152 } \\
\text { EU154 } \\
\text { EU155 } \\
\text { FE }-59 \\
\text { MN-54 }\end{array}$ & $\begin{array}{r}.000 E+00 \\
4.552 E+01 \\
1.857 E-13 \\
5.712 E+02 \\
3.467 E+01 \\
2.293 E+01\end{array}$ \\
\hline
\end{tabular}

$1.486 E+06 \quad 2.974 E+00 \quad 2.365 E+00 \quad 5.923 E+01$
OTHER RADIOACTIVITY (CURIES)

(n)

\section{$1.016 E+05 \quad 2.323 E+04 \quad 1.073 E+00 \quad 2.371 E+04$}

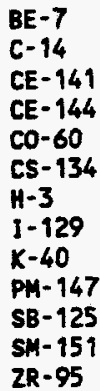

$8.827 E+03$

$2.315 E-15$

$3.498 \mathrm{E}-10$

$2.162 E-28$

2.096E-01

2.394E-02

$1.930 E-03$

$1.980 E-02$

9.054E-03

$9.199 \mathrm{E}-01$

3.161E-07

4.099E-01

$4.608 E-14$

BA137 .000E+00

C- $14 \quad 1.201 E-02$

CO-58 5.304E-15

CO-60 2.831E-01

CS137 1.031E-02

EU152 7.916E-03

EU154 5.342E-02

EU155 3.362E-02

FE-59 1.147E-21

MN-54 1.329E-04

$1.486 E+06$

$B E-10 \quad 6.110 E+01$

C-14 B.117E+01

$C L-36 \quad 6.110 E+01$

CO-58 4.826E+02

CO-60 $\quad 5.585 E+05$

CR-51 2.127E+00

CS-134 2.384E-03

FE-55 3.102E+05

FE-59 1.517E+0D

$\mathrm{H}-3 \quad 9.47 \mathrm{TE}+02$

HF-181 4.465E-02

I-129 $6.110 E+01$

MA-54 2.613E+03

$10-93 \quad 6.895 E-02$

NB-94 5.673E-01

MB-95 2.306E-01

$M I-59 \quad 4.211 E+03$

NII-63 6.090E+05

SE-79 2.422E-05 
WHC-EP-0125-6

\begin{tabular}{|c|c|c|c|c|c|c|}
\hline \multirow[b]{2}{*}{ BURIAL GROUND FACILITIES } & \multicolumn{6}{|c|}{$\begin{array}{l}\text { Nontransuranic Waste Burial Ground Areas } \\
\text { Curie Content through DECEMBER } 31,1993 .\end{array}$} \\
\hline & $\begin{array}{l}\text { TOTAL } \\
\text { (CURIES) }\end{array}$ & $\begin{array}{l}\text { SR- } 90 \\
\text { (CURIES) }\end{array}$ & $\begin{array}{l}\text { RU-106 } \\
\text { (CURIES) }\end{array}$ & $\begin{array}{l}\text { CS-137 } \\
\text { (CURIES) }\end{array}$ & $\begin{array}{r}\text { OTHER } R / \\
\text { (CL }\end{array}$ & $\begin{array}{l}\text { IOACTIVITY } \\
\text { IES) }\end{array}$ \\
\hline $\begin{array}{l}\text { BURIAL GROUND 218E12B } \\
\text { LOW-LEVEL INDUSTRIAL-OFFSITE }\end{array}$ & & & & & $\begin{array}{l}T C-99 \\
2 N-65 \\
2 R-93 \\
2 R-95\end{array}$ & $\begin{array}{l}1.030 E-01 \\
8.718 E-02 \\
2.035 E-04 \\
2.252 E-01\end{array}$ \\
\hline $\begin{array}{l}\text { BURIAL GROUND } 218 E C 9 \\
\text { LOW-LEVEL NONINDUSTRIAL }\end{array}$ & $3.784 E+01$ & $1.186 E+01$ & $1.556 E-03$ & $7.190 E+00$ & $E U-154$ & $\begin{array}{l}\text { 7.417E-07 } \\
7.417 E-07\end{array}$ \\
\hline Total 200E & $4.698 E+06$ & $7.369 E+05$ & $4.345 E+00$ & $8.872 E+05$ & & $1.496 E+06$ \\
\hline
\end{tabular}


SUIR3281 - (Page 3 of 26) RUNDATE: 08/20/94 Post-1970 Nontransuranic Waste Burial Ground Areas
Decayed Curie Content through DECEMBER 31, 1993.

BURIAL GROUND FACILITIES

2004 AREA

BURIAL GROUND 218W2A LOW-LEVEL INOUSTRIAL-OFFSITE LOU-LEVEL INOUSTRIAL.

BURIAL GROUND 218WBA

LOU-LEVEL MONINDUSTRIAL-OFFSITE
TOTAL
(CURIES)

SR-90

(CURIES)

RU-106

(CURIES)

C5- 137

(CURIES)

OTHER RADIOACTIVITY (CURIES)

\section{$2.408 E+00 \quad 5.736 E-01 \quad 4.108 E-05 \cdot 6.230 E-01$ $1.340 E+03 \quad 3.226 E+02 \quad 2.884 E-04 \quad 3.557 E+02$}

\section{$5.272 E+05 \quad 3.329 E+04 \quad 9.457 E+03 \quad 1.688 E+05$}

LOW-LEVEL NONINDUSTRIAL

$3.580 E+05 \quad 3.560 E+04 \quad 1.854 E+01 \quad 7.630 E+04$

$.688 E+05$




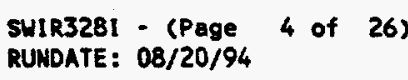

\section{BURIAL GROUND FACILITIES}

BURIAL GROUND 218U3A

LOH-LEVEL NOWINDUSTRIAL.
LOW-LEVEL NONINDUSTRIAL CLASSIFIEDOFFSITE

LON-LEVEL NONINDUSTRIAL CLASSIFIED
LOW-LEVEL NOWIHDUSTRIAL (ASSAYED FRON TRANSURANIC)

LOW-LEVEL INDUSTRIAL-OFFSITE

$\begin{array}{cccc}\begin{array}{c}\text { TOTAL } \\ \text { (CURIES) }\end{array} & \begin{array}{c}\text { SR-90 } \\ \text { (CURIES) }\end{array} & \begin{array}{c}\text { RU-106 } \\ \text { (CURIES) }\end{array} & \begin{array}{c}\text { CS-137 } \\ \text { (CURIES) }\end{array}\end{array}$

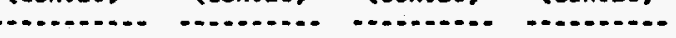

OTHER RADIOACTIVITY (CURIES)

a.....................

\begin{abstract}
8.266E-01 1.996E-01 1.903E-06 2.181E-01
$1.423 E+00 \quad .000 E+00 \quad .000 E+00 \quad 4.136 E-02$
\end{abstract}

$1-131$

$K-40$

$\mathrm{KR}-85$

MH -54

MA-22

NI-60

$N I-63$

P-32

PW-147

RE-187

S-35

$S B-122$

$S B-125$

SE -75

SH- 147

SR-85

TA-182

TC- 99

TE- 125

TM- 170

$V-49$

$Y-90$

ZN-65$$
\text { co-1 }
$$$$
\text { Esi }
$$$$
\text { cou }
$$

$$
\text { E }
$$$$
\text { FU }
$$$$
\text { FE-5 }
$$$$
\begin{gathered}
.000 E+00 \\
.000 E+00
\end{gathered}
$$$$
\text { BA } 137^{.000 E+00} .000 E+00
$$$$
\text { C-14 4.994E-02 }
$$$$
\text { CO-58 7.300E-17 }
$$$$
\text { CO-60 9.548E-01 }
$$$$
\text { CS137 4.135E-02 }
$$$$
\text { EU152 3.028E-02 }
$$$$
\text { EU154 1.951E-01 }
$$$$
\text { EU155 1.119E-01 }
$$$$
\text { FE-59 5.442E-25 }
$$$$
\text { AN-54 1.516E-04 }
$$$$
.000 E+00
$$

$\begin{array}{llll}2.928 E+00 & 6.679 E-01 & 1.047 E-03 & 7.217 E-01 \\ 2.513 E+02 & 4.509 E+00 & 7.559 E-03 & 4.859 E+00\end{array}$




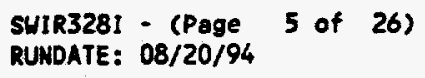

\section{BURIAL GROUND FACILITIES}

BURIAL GROUND 218IB3A

LON-LEVEL INDUSTRIAL-OFFSITE

LOW-LEVEL INDUSTRIAL

BURIAL GROUND 218U3AE

LON-LEVEL NONINDUSTRIAL-OFFSITE

TOTAL
(CURIES)

SR -90

(CURIES)

RU-106

(CURIES)

CS-137

(CURIES)

OTHER RADIOACTIVITY (CURIES)

$5.216 E+02$

8.268E-04

$1.251 E+03$

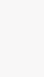

(2)


WHC-EP-0125-6

SUIR3281 - (Page 6 of 26)
RUNDATE: 08/20/94

Post-1970 Nontransuranic Waste Burial Ground Areas Decayed Curie Content through DECEMBER 31, 1993.

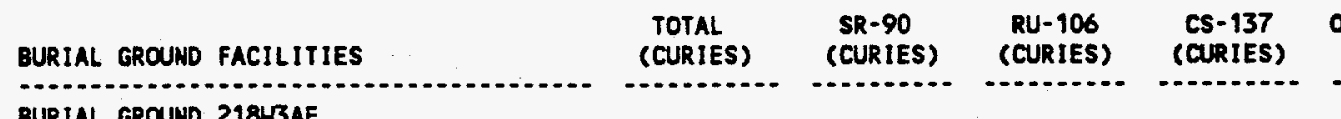

OTHER RADIOACTIVITY

BURIAL GROUHD 218U3AE

LOW-LEVEL NONINDUSTRIAL-OFFSITE :

LON-LEVEL NONINDUSTRIAL

LON-LEVEL NOWIMDUSTRIAL CLASSIFIEDOFFSITE
$6.690 E+04 \quad 1.182 E+04 \quad 4.445 E+02 \quad 1.718 E+04$

$9.805 E+04 \quad 1.536 E-01 \quad 5.634 E-02 \quad 1.196 E-01$

\begin{tabular}{|c|c|}
\hline 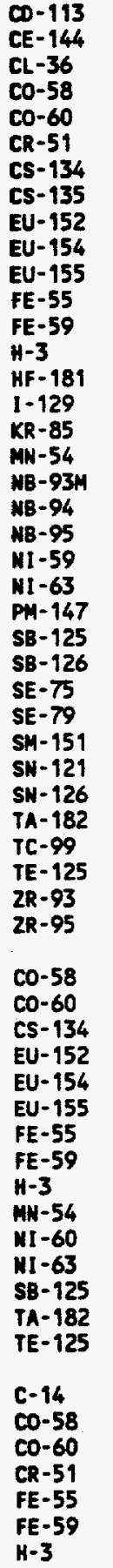 & $\begin{array}{l}1.155 E-05 \\
6.860 E-03 \\
1.840 E-08 \\
2.296 E-01 \\
2.530 E+00 \\
5.206 E-05 \\
2.323 E-02 \\
3.800 E-07 \\
1.544 E-02 \\
1.292 E-02 \\
1.549 E-03 \\
4.431 E+00 \\
1.466 E-03 \\
7.259 E-04 \\
4.151 E-04 \\
1.189 E-07 \\
1.332 E-02 \\
3.927 E+00 \\
2.368 E-05 \\
5.914 E-04 \\
9.792 E-04 \\
8.788 E-03 \\
7.832 E-01 \\
5.856 E-02 \\
1.252 E-03 \\
4.166 E-24 \\
1.479 E-08 \\
1.168 E E-07 \\
3.430 E-04 \\
9.772 E-08 \\
5.100 E-07 \\
1.646 E-03 \\
4.048 E-05 \\
1.452 E-07 \\
7.568 E-06 \\
3.760 E-03 \\
8.942 E+03 \\
1.051 E-07 \\
1.788 E+03 \\
1.709 E+02 \\
1.202 E+03 \\
1.778 E+03 \\
3.820 E+02 \\
1.512 E+02 \\
2.882 E+01 \\
2.960 E+03 \\
1.541 E+02 \\
.000 E+00 \\
3.275 E+02 \\
3.782 E-04 \\
6.175 E-03 \\
1.538 E-04 \\
9.804 E+04 \\
1.981 E+01 \\
1.104 E-01 \\
3.458 E+04 \\
1.133 E-15 \\
9.137 E+03 \\
3.383 E-10 \\
8.322 E-01\end{array}$ \\
\hline
\end{tabular}



SHIR3281 - (Page 7 of 26 )
RUNDATE: $08 / 20 / 94$

Post-1970 Nontransuranic Waste Burial Ground Areaa Decayed Curie Content through DECEMBER 31, 1993.

\section{BURIAL GROUND FACILITIES \\ BURIAL GROUND $218 \mathrm{~W} 3 \mathrm{AE}$ \\ LON-LEVEL NONINDUSTRIAL CLASSIFIED- OFFSITE}

\begin{abstract}
LON-LEVEL NONINDUSTRIAL (ASSAYED FROM TRANSURANIC)
\end{abstract}

LOW-LEVEL INDUSTRIAL-OFFSITE

LOW-LEVEL INDUSTRIAL

LOW-LEVEL INDUSTRIAL (ASSAYED FRON TRANSURANIC)

BURIAL GROUND 218W4B

LOU-LEVEL NONINDUSTRIAL-OFFSITE

LOW-LEVEL NONINOUSTRIAL

LOW-LEVEL CAISSONS

\begin{tabular}{llll}
$\begin{array}{l}\text { TOTAL } \\
\text { (CURIES) }\end{array}$ & $\begin{array}{c}\text { SR-90 } \\
\text { (CURIES) }\end{array}$ & $\begin{array}{c}\text { RU-106 } \\
\text { (CURIES) }\end{array}$ & $\begin{array}{c}\text { CS-137 } \\
\text { (CURIES) }\end{array}$ \\
\hline
\end{tabular}

OTHER RADIOACTIVITY (CURIES)

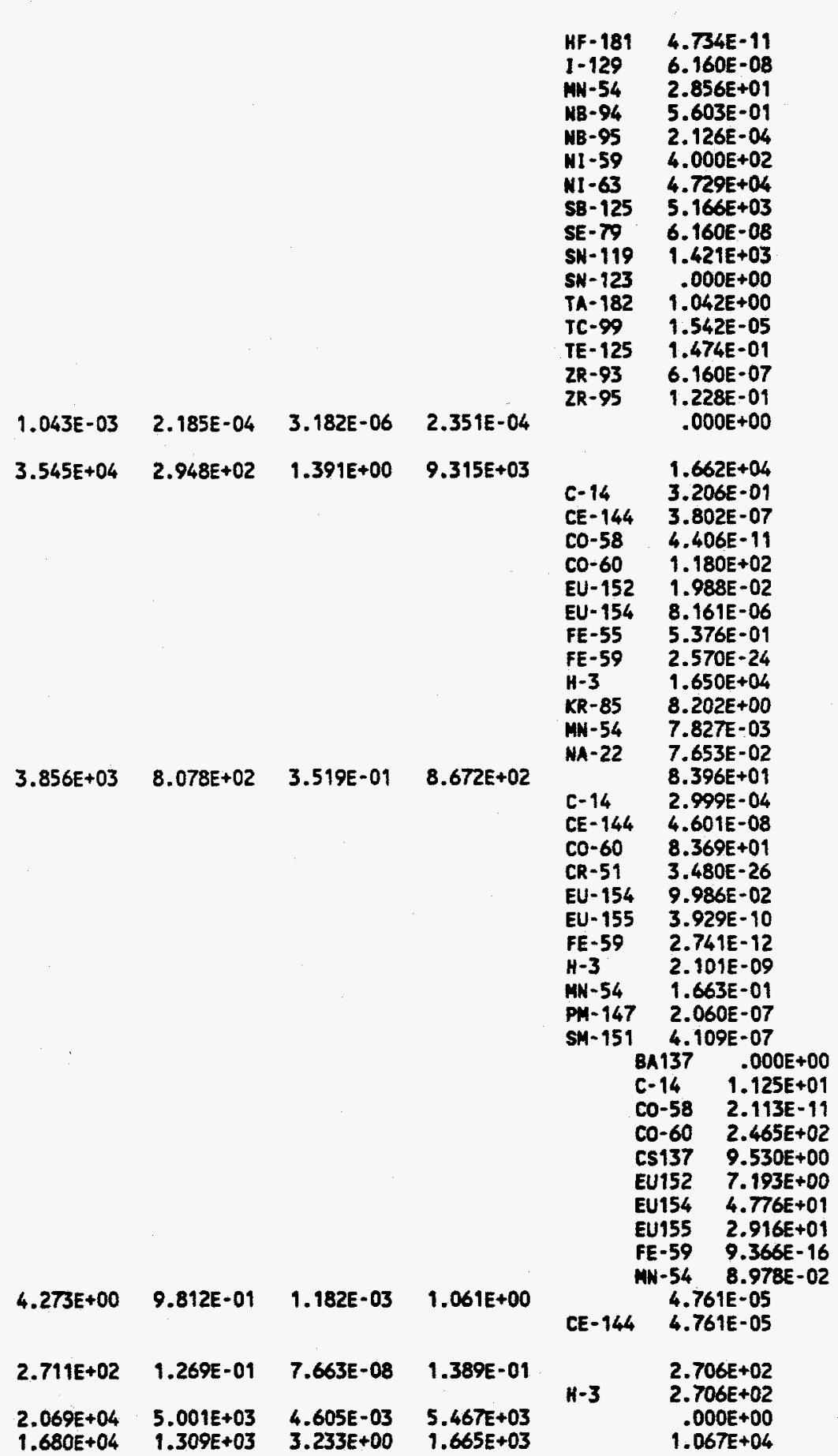


WHC-EP-0125-6

SUIR328I - (Page 8 of 26)
RUNDATE: 08/20/94

Post-1970 Nontransuranic Waste Burial Ground Areas

Decayed Curie Content through DECEMBER 31, 1993.

\begin{tabular}{|c|c|c|c|c|}
\hline BURIAL GROUND FACILI & $\begin{array}{l}\text { TOTAL } \\
\text { (CURIES) }\end{array}$ & $\begin{array}{c}\text { SR-90 } \\
\text { (CURIES) }\end{array}$ & $\begin{array}{l}\text { RU-106 } \\
\text { (CURIES) }\end{array}$ & $\begin{array}{c}\text { CS-137 } \\
\text { (CURIES) }\end{array}$ \\
\hline
\end{tabular}

OTHER RADIOACTIVITY

BURIAL GROUND 218W4B

LOU-LEVEL CAISSONS

(CURies)

(CURIEs)

(CURIES)

(CURIES)

\begin{tabular}{|c|c|c|}
\hline \multirow[t]{2}{*}{$\begin{array}{l}\text { CO-60 } \\
\text { CR-51 } \\
\text { CS- } 134 \\
\text { EU-154 } \\
\text { FE-59 } \\
\text { MN-54 } \\
S B-125 \\
2 R-95\end{array}$} & \multicolumn{2}{|c|}{$\begin{array}{l}1.067 E+04 \\
1.388 E-31 \\
8.250 E-02 \\
1.594 E-01 \\
3.389 E-10 \\
9.813 E-02 \\
7.100 E-03 \\
6.939 E-10\end{array}$} \\
\hline & $\begin{array}{l}8 A 137 \\
C-14 \\
C 0-58 \\
\text { CO-60 } \\
\text { CS137 } \\
\text { EU152 } \\
\text { EU154 } \\
\text { EU155 } \\
\text { FE-59 } \\
\text { MN-54 }\end{array}$ & $\begin{array}{l}.000 E+00 \\
1.248 E+00 \\
1.298 E-21 \\
1.403 E+01 \\
9.417 E-01 \\
6.118 E-01 \\
3.505 E+00 \\
1.591 E+00 \\
2.372 E-33 \\
1.498 E-04\end{array}$ \\
\hline
\end{tabular}

BURIAL GROUND 218W4C

LON-LEVEL NONINDUSTRIAL-OFFSITE

\begin{abstract}
$1.957 E+04$
2.164E+00

3.467E-02 2.273E+00
\end{abstract}

BE-10

C- 14

CD. 113

CE- 144

CL-36

Co-58

Co-60

CR-51

CS- 134

Cs-135

EU-152

EU-154

EU-155

FE- 55

FE-59

$\mathrm{H}-3$

1- 125

I. 129

KR-85

MN-54

MO-93

NB-93M

NB-94

N1 -59

NI -63

P-32

PA-231

PN- 147

s-35

s8-125

SB-126

SE-79

SN- 151

SN- 121

SN-126

SR-82

SR-85

TC- 99

TE- 125

2R-93
$1.956 E+04$

$1.890 \mathrm{E}-10$

$8.135 E+00$

4.762E-05

2.994E-02

$7.580 E-08$

$9.444 E-02$

$7.218 E+03$

9.097E-25

$9.810 E-02$

1.582E-06

6.561E-02

5.450E-02

$5.143 E-03$

2.909E+03

2.013E-16

$2.119 E+01$

5.202E-07

1.006E-03

5.615E-02

$2.807 E+00$

1.254E-03

9.710E-05

3.030E-05

7.559E+01

$9.329 E+03$

1.742E-07

2.115E-06

2.466E-01

3.271E-04

4.182E-03

$7.435 E-22$

3.030E-06

$1.408 E-03$

4.026E-07

2.109E-06

3.804E-06

$1.787 \mathrm{E}-04$

$1.358 E-03$

9.069E-07

2.628E-05 

SUIR328I - (Page 9 of 26)
RUNDATE: $08 / 20 / 94$

Post-1970 Nontransuranic Waste Burial Ground Areas Decayed Curie Content through DECEMBER 31, 1993.

BURIAL GROUNO FACILITIES

LON-LEVEL NONINDUSTRIAL

\begin{tabular}{cccc}
$\begin{array}{c}\text { TOTAL } \\
\text { (CURIES) }\end{array}$ & $\begin{array}{c}\text { SR-90 } \\
\text { (CURIES) }\end{array}$ & $\begin{array}{c}\text { RU-106 } \\
\text { (CURIES) }\end{array}$ & $\begin{array}{c}\text { CS-137 } \\
\text { (CURIES) }\end{array}$ \\
\hline
\end{tabular}

$2.785 E+04 \quad 4.725 E+03 \quad 5.847 E+00 \quad 3.982 E+03$

$1.378 E+05 \quad 1.351 E+03 \quad 3.330 E-01 \quad 1.464 E+03$ OFFSITE

LOW-LEVEL NONINOUSTRIAL (ASSAYEO FROM TRAMSURANIC)

LOW-LEVEL INDUSTRIAL

1.017E-01 2.127E-02 3.151E-04 2.290E-02

$\begin{array}{lll}4.055 E-03 & 4.053 E-06 & 1.870 E-02\end{array}$

BURIAL GROUND 218VS

LOW-LEVEL MONINDUSTRIAL-OFFSITE
4.699E-02

\begin{tabular}{|c|c|c|c|c|c|}
\hline $4.699 \varepsilon-02$ & $4.055 E-03$ & $4.053 E-06$ & $1.870 E-02$ & $\begin{array}{l}H-3 \\
P H-147 \\
T C-99\end{array}$ & $\begin{array}{l}2.331 E-03 \\
4.146 E-06 \\
2.308 E-03 \\
1.915 E-05\end{array}$ \\
\hline $1.124 E+03$ & $1.072 E+01$ & $7.305 E-02$ & $3.985 E+00$ & $\begin{array}{l}B E-7 \\
C-14 \\
C E-141 \\
C E-144 \\
C O-56 \\
C 0-57 \\
C O-58 \\
C O-60 \\
C R-51\end{array}$ & $\begin{array}{l}1.093 E+03 \\
5.731 E-09 \\
3.328 E-02 \\
4.973 E-25 \\
1.293 E-02 \\
3.177 E-07 \\
8.808 E-05 \\
4.508 E-06 \\
1.078 E+03 \\
1.330 E-18\end{array}$ \\
\hline
\end{tabular}

OTHER RADIOACTIVITY (CURIES)

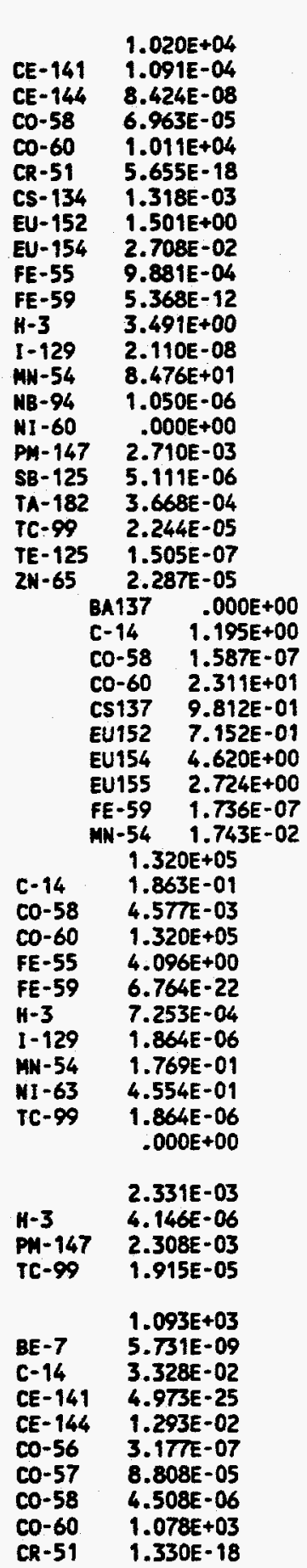


SUIR328I - (Page 10 of 26) RUNDATE: 08/20/94

$$
\begin{aligned}
& \text { Post-1970 Nontransuranic waste Burial Ground Areas } \\
& \text { Decayed Curie Content through DECEMBER 31, } 1993 .
\end{aligned}
$$

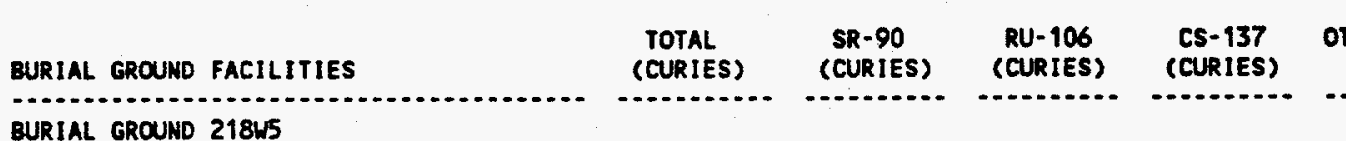

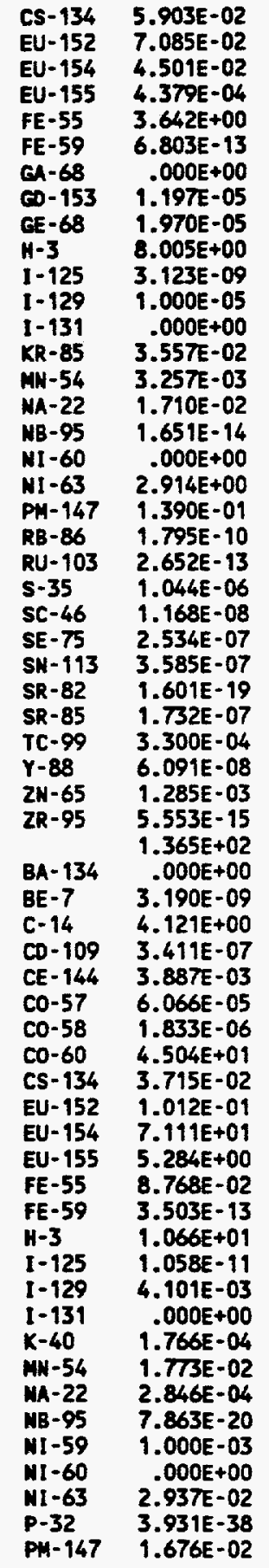


SUIR3281 - (Page 11 of 26)

RUNDATE: $08 / 20 / 94$

Post-1970 Nontransuranic Waste Burial Ground Areas Decayed Curie Content through DECEMBER 31, 1993.

BURIAL GROUND FACILITIES

BURIAL GROUND 218W5

LOW-LEVEL NONINDUSTRIAL
LOW-LEVEL NOWINDUSTRIAL (ASSAYED FROM TRANSURANIC)

LOW-LEVEL INDUSTRIAL-OFFSITE

\section{$7.457 \mathrm{~T}-03$ \\ $1.453 E+04$}

SR -90
(CURIES)

(CURIES)
RU- 106

(CURIES)
CS-137
(CURIES) c..........

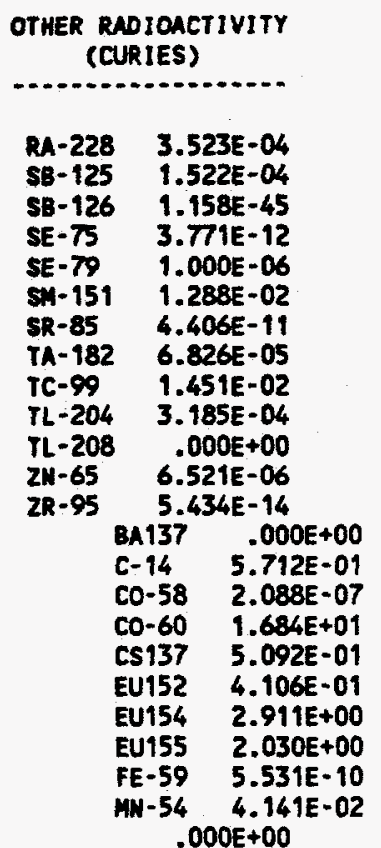

AC- 227

AC- 228

AG-108

$A G-110$

AG-110

AL- 28

$A U-195$

BA-133

$B A-134$

$B A-137$

BA- 140

BE-7

BI-207

BI-212

B1-214

C -14

CA-45

CD- 109

CE-141

CE-144

$\mathrm{CL}-36$

CO-56

CO-57

CO-58

Co.59

co-60

CR-51

CS-134

CU-65M

ES-254

EU-152

EU-156

EU-155

FE -55
$1.403 E+04$

2.001E-05 $.000 E+00$ $.000 E+00$

$1.127 \mathrm{E}-05$ $.000 E+00$ $.000 E+00$

3.686E-07

5.805E-02

$.000 E+00$ $.000 E+00$ 5.936E-08

2.802E-03

$1.646 E-03$

$.000 E+00$

$.000 E+00$

1.069E-01

$6.105 E-03$

1.291E-03

4.011E-06

$1.406 E-05$

1.142E-05

9.378E-05

1.644E-02

6.017E-04 $.000 E+00$

$1.146 E+03$

7.576E-04

4.515E-03

$.000 E+00$

6.475E-05

9.534E-01

1.047E-01

$6.996 E-04$

$9.378 E+00$ 
SUIR3281 - (Page 12 of 26)

RUNDATE: $08 / 20 / 94$

Post-1970 Nontransuranic Waste Burial Ground Areas

Decayed Curie Content through DECEMBER 31, 1993.

\begin{tabular}{llccc} 
BURIAL GROUND FACILITIES & $\begin{array}{c}\text { TOTAL } \\
\text { (CURIES) }\end{array}$ & $\begin{array}{c}\text { SR-90 } \\
\text { (CURIES) }\end{array}$ & $\begin{array}{c}\text { RU-106 } \\
\text { (CURIES) }\end{array}$ (CURIES) \\
\hline
\end{tabular}

OTHER RADIOACTIVITY

BURIAL GROUND 29815

LOW-LEVEL INDUSTRIAL-OFFSITE

(CURIES)

\begin{tabular}{|c|c|}
\hline 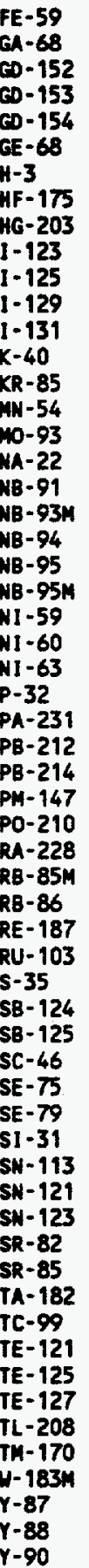 & $\begin{array}{r}1.779 E-03 \\
.000 E+00 \\
8.468 E-02 \\
5.355 E-06 \\
.000 E+00 \\
6.485 E-04 \\
1.286 E+04 \\
1.074 E-07 \\
7.777 E-08 \\
2.075 E-13 \\
7.052 E-03 \\
6.062 E-05 \\
2.310 E-08 \\
8.861 E-04 \\
7.430 E-04 \\
2.060 E-01 \\
1.292 E+00 \\
9.191 E+00 \\
1.020 E-03 \\
7.582 E-05 \\
7.513 E-05 \\
1.225 E-05 \\
6.076 E-25 \\
2.588 E-04 \\
.000 E+00 \\
3.202 E-01 \\
8.153 E-03 \\
7.937 E-01 \\
.000 E+00 \\
.000 E+00 \\
1.043 E-05 \\
7.485 E-07 \\
3.538 E-04 \\
.000 E+00 \\
1.217 E-04 \\
3.000 E-06 \\
6.809 E-14 \\
2.903 E-02 \\
1.000 E-06 \\
8.081 E-03 \\
7.044 E-04 \\
7.032 E-04 \\
2.933 E-03 \\
.000 E+00 \\
6.997 E-05 \\
3.289 E-07 \\
.000 E+00 \\
6.013 E-06 \\
1.150 E-04 \\
1.114 E-05 \\
3.146 E-02 \\
9.050 E-27 \\
5.855 E-06 \\
.000 E+00 \\
.000 E+00 \\
3.488 E-08 \\
.000 E+00 \\
6.306 E-10 \\
2.538 E-05 \\
.90\end{array}$ \\
\hline
\end{tabular}


SUIR328I - (Page 13 of 26)

RUNDATE: $08 / 20 / 94$

Post-1970 Nontransuranic Waste Burial Ground Areas

Decayed Curie Content through DECEMBER 31, 1993.

\begin{tabular}{|c|c|c|c|c|}
\hline BURIAL GROUND FACILITIES & $\begin{array}{l}\text { TOTAL } \\
\text { (CURIES) }\end{array}$ & $\begin{array}{l}\text { SR-90 } \\
\text { (CURIES) }\end{array}$ & $\begin{array}{c}\text { RU-106 } \\
\text { (CURIES) }\end{array}$ & $\begin{array}{c}\text { CS-137 } \\
\text { (CURIES) }\end{array}$ \\
\hline
\end{tabular}

BURIAL GROUND 218W5

LOU-LEVEL INOUSTRIAL-OFFSITE

OTHER RADIOACTIVITY (CURIES)

LOU-LEVEL INOUSTRIAL

$4.785 E+02 \quad 6.487 E+01 \quad 1.024 E+00 \quad 1.123 E+02$

$211-65 \quad 1.116 E-01$

2R-95 2.636E-06

AC-227 $7.589 E+01$

$\begin{array}{rr}A C-227 & 1.655 E-05 \\ A C-228 & .000 E+00\end{array}$

AG-110 .000E+00

BA-133 2.471E-06

BA- $-137 \quad .000 E+00$

81-212 .000E+00

$81-214 \quad .000 E+00$

$c-14 \quad 7.688 E-02$

CA-45 5.411E-07

CD-109 2.562E-03

CE-149 9.357E-07

CE-144 4.594E-01

CL-36 2.262E-06

CO-57 2.905E-05

CO-58 4.508E-04

CO-60 4.404E+01

CR-51 4.512E-06

CS-133 .000E+00

CS-134 2.937E-02

CU-65M .000E+00

EU-152 2.926E-01

EU-154 2.519E+00

EU-155 7.349E-03

FE-55 1.916E-01

FE-59 1.801E-05

H-3 2.576E+OI

$H F-181 \quad 1.464 E-08$

I-125 5.019E-04

1-129 2.849E-02

$\mathrm{I}-131 \quad 1.252 \mathrm{E}-18$

$K-40 \quad 5.018 E-03$

KR-85 1.327E-08

MN-54 8.085E-02

MO-93 9.997E-06

NA-22 1.024E-04

NB-91 2.794E-02

NB-93M 9.987E-09

NB-94 6.544E-04

NB-95 2.409E-06

ND- $147 \quad 1.549 E-35$

MI-59 7.100E-03

$M 1-60 \quad .000 E+00$

MI-63 2.715E-02

P-32 4.880E-14

$P A-231$ 4.800E-11

PB-208. .000E +00

$P B-212 \quad .000 E+00$

PB-214 .000E+00

PM-147 $1.459 E+00$

PO-210 $9.655 E-12$

PT-195 4.624E-44

s-35 6.213E-07

SB-125 2.814E-03

SC-46 3.407E-07

$S E-75 \quad 6.568 E-06$

SE-79 1.038E-04

SH-151 7.690E-01 


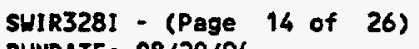

\begin{tabular}{|c|c|c|c|c|}
\hline BURIAL GROUND FACILITIES & $\begin{array}{l}\text { TOTAL } \\
\text { (CURIES) }\end{array}$ & $\begin{array}{l}\text { SR-90 } \\
\text { (CURIES) }\end{array}$ & $\begin{array}{c}\text { RU-106 } \\
\text { (CURIES) }\end{array}$ & $\begin{array}{c}\text { CS-137 } \\
\text { (CURIES) }\end{array}$ \\
\hline
\end{tabular}

LOW-LEVEL INDUSTRIAL (ASSAYED FROM TRANSURANIC)

CENTRAL WASTE COMPLEX 2401W BUILDING LOW-LEVEL NONINDUSTRIAL-OFFSITE

LOW-LEVEL NONINDUSTRIAL
OTHER RADIOACTIVITY (CURIES)
CENTRAL MASTE COMPLEX 2402W BUILDING LOW-LEVEL NONINDUSTRIAL-OFFSITE

LOH-LEVEL NONINDUSTRIAL $\begin{array}{llll}7.565 E+01 \quad 1.762 E+01 & 4.191 E-02 & 1.913 E+01\end{array}$

$1.569 E+05 \quad 7.834 E+04 \quad .000 E+00 \quad .000 E+00$

1.133E-03

\begin{tabular}{|c|c|c|c|c|c|c|}
\hline & & & & $\begin{array}{l}S R-82 \\
S R-85 \\
S R-89 \\
T A-182 \\
T C-99 \\
T E-125 \\
T E-129 \\
T L-204 \\
T L-208 \\
V-49 \\
U-185 \\
Y-87 \\
Y-90 \\
Z N-65 \\
Z R-95\end{array}$ & \multicolumn{2}{|c|}{$\begin{array}{l}2.687 E-15 \\
3.081 E-06 \\
2.512 E-09 \\
1.308 E-03 \\
7.492 E-02 \\
2.517 E-05 \\
1.388 E-20 \\
4.236 E-05 \\
.000 E+00 \\
2.631 E-06 \\
2.262 E-07 \\
.000 E+00 \\
.000 E+00 \\
2.935 E-02 \\
9.719 E-06\end{array}$} \\
\hline $7.565 E+01$ & $1.762 \mathrm{E}+01$ & $4.191 E-02$ & $1.913 E+01$ & & $\begin{array}{l}1137 \\
-14 \\
0-58 \\
0-60 \\
b 137 \\
j 152 \\
J 154 \\
J 155 \\
-59 \\
-54 \\
-54 \\
.0\end{array}$ & $\begin{array}{l}.000 E+00 \\
5.257 E-01 \\
1.257 E-08 \\
1.436 E+01 \\
4.632 E-01 \\
3.674 E-01 \\
2.561 E+00 \\
1.721 E+00 \\
1.340 E-08 \\
1.483 E-02 \\
0 E+00\end{array}$ \\
\hline $1.569 E+05$ & $7.834 E+04$ & $.000 E+00$ & $.000 E+00$ & \multirow{2}{*}{$\begin{array}{l}H-3 \\
C O-60 \\
E U-154\end{array}$} & \multicolumn{2}{|c|}{$\begin{array}{l}2.091 E+02 \\
2.091 E+02\end{array}$} \\
\hline $1.133 \mathrm{E}-03$ & $2.399 E-04$ & $9.060 \mathrm{E}-07$ & $2.088 E-04$ & & $\begin{array}{r}2.0 \\
1.7 \\
2.8 \\
1137 \\
-14 \\
j-58 \\
0-60 \\
b 137 \\
j 152 \\
J 154 \\
J 155 \\
-59 \\
-54\end{array}$ & $\begin{array}{l}7 E-04 \\
8 E-04 \\
9 E-05 \\
.000 E+00 \\
3.238 E-08 \\
2.046 E-14 \\
1.285 E-06 \\
3.045 E-08 \\
2.627 E-08 \\
1.989 E-07 \\
1.577 E-07 \\
1.840 E-17 \\
8.916 E-09\end{array}$ \\
\hline $5.159 E-02$ & $9.813 E-04$ & $6.321 E-05$ & $1.054 E-03$ & & \multirow{2}{*}{\multicolumn{2}{|c|}{$\begin{array}{l}4.624 E-02 \\
4.624 E-02 \\
1.694 E-01 \\
2.871 E-03 \\
7.258 E-04 \\
1.350 E-02 \\
3.344 E-02 \\
2.232 E-03 \\
1.186 E-03 \\
1.303 E-05 \\
4.706 E-14 \\
5.667 E-04 \\
.000 E+00 \\
5.200 E-09 \\
1.075 E-04 \\
.000 E+00\end{array}$}} \\
\hline $4.669 E+00$ & $6.529 E-01$ & $1.925 E-02$ & $1.409 E+00$ & $\begin{array}{l}\text { BA- } 133 \\
C-14 \\
\text { CE- } 144 \\
\text { CO-60 } \\
\text { CS- } 134 \\
\text { EU-154 } \\
\text { EU-155 } \\
\text { FE-59 } \\
H-3 \\
I-131 \\
K-40 \\
M H-54 \\
N I-60\end{array}$ & & \\
\hline
\end{tabular}


SHIR328I - (Page 15 of 26) RUNDATE: 08/20/94

Post-1970 Nontranguranic Waste Burial Ground Areas Decayed Curie Content through DECEMBER 31, 1993.

BURIAL GROUND FACILITIES

CENTRAL WASTE COMPLEX $2402 W$ BUILDING LOW-LEVEL NOWINDUSTRIAL

$\begin{array}{cccc}\text { TOTAL } & \text { SR-90 } & \text { RU-106 } & \text { CS-137 } \\ \text { (CURIES) } & \text { (CURIES) } & \text { (CURIES) } & \text { (CURIES) }\end{array}$
(CURIES) (CURIES) (CURIES) (CURIES)
CENTRAL WASTE COMPLEX 240ZUB BUILOING LOW-LEVEL NONIHOUSTRIAL-OFFSITE

LOW-LEVEL NOWI NDUSTRIAL

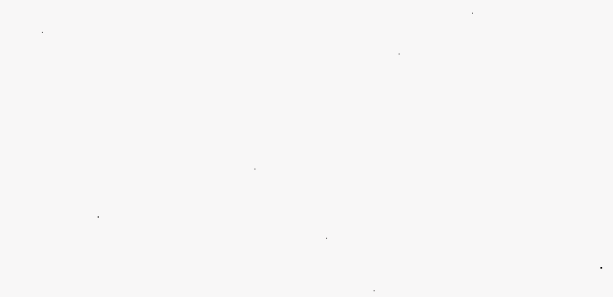

LON-LEVEL NONINDSTRIAL
$7.812 E-04$

(c.........

OTHER RADIOACTIVITY (CURIES)
CENTRAL HASTE COMPLEX 240ZUC BUILDING LOW-LEVEL NOW INDUSTRIAL-OFFSITE
$1.000 E-07 \quad .000 E+00 \quad .000 E+00 \quad .000 E+00$

\begin{tabular}{ll} 
(CURIES) \\
$\ldots 1-63$ & $6.995 E-05$ \\
MI-147 & $6.398 E-02$ \\
PN-147 & $\mathbf{5 . 8 0 9 E - 0 5}$ \\
SB-125 & 5.809 \\
SE-75 & $1.990 E-07$ \\
SN-151 & $4.905 E-02$ \\
TA-182 & $3.917 E-07$ \\
TC-99 & $1.586 E-03$ \\
TE-125 & $1.926 E-10$ \\
\multicolumn{3}{c}{ BA137 } & $.000 E+00$ \\
C-14 & $1.676 E-06$ \\
CO-58 & $1.787 E-12$ \\
CO-60 & $6.470 E-05$ \\
CS137 & $1.568 E-06$ \\
EU152 & $1.345 E-06$ \\
EU154 & $1.012 E-05$ \\
EU155 & $7.929 E-06$ \\
FE-59 & $1.898 E-14$ \\
MH-54 & $3.972 E-07$
\end{tabular}

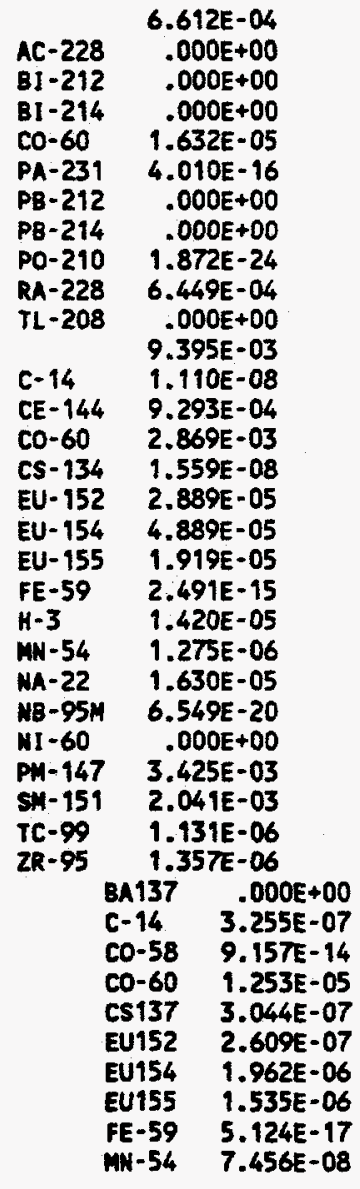

1.000E-07 

SUIR3281 - (Page 16 of 26 )
RUNDATE: $08 / 20 / 94$

Post-1970 Nontransuranic Waste Burial Ground Areas Decayed Curie Content through DECEMBER 31, 1993.

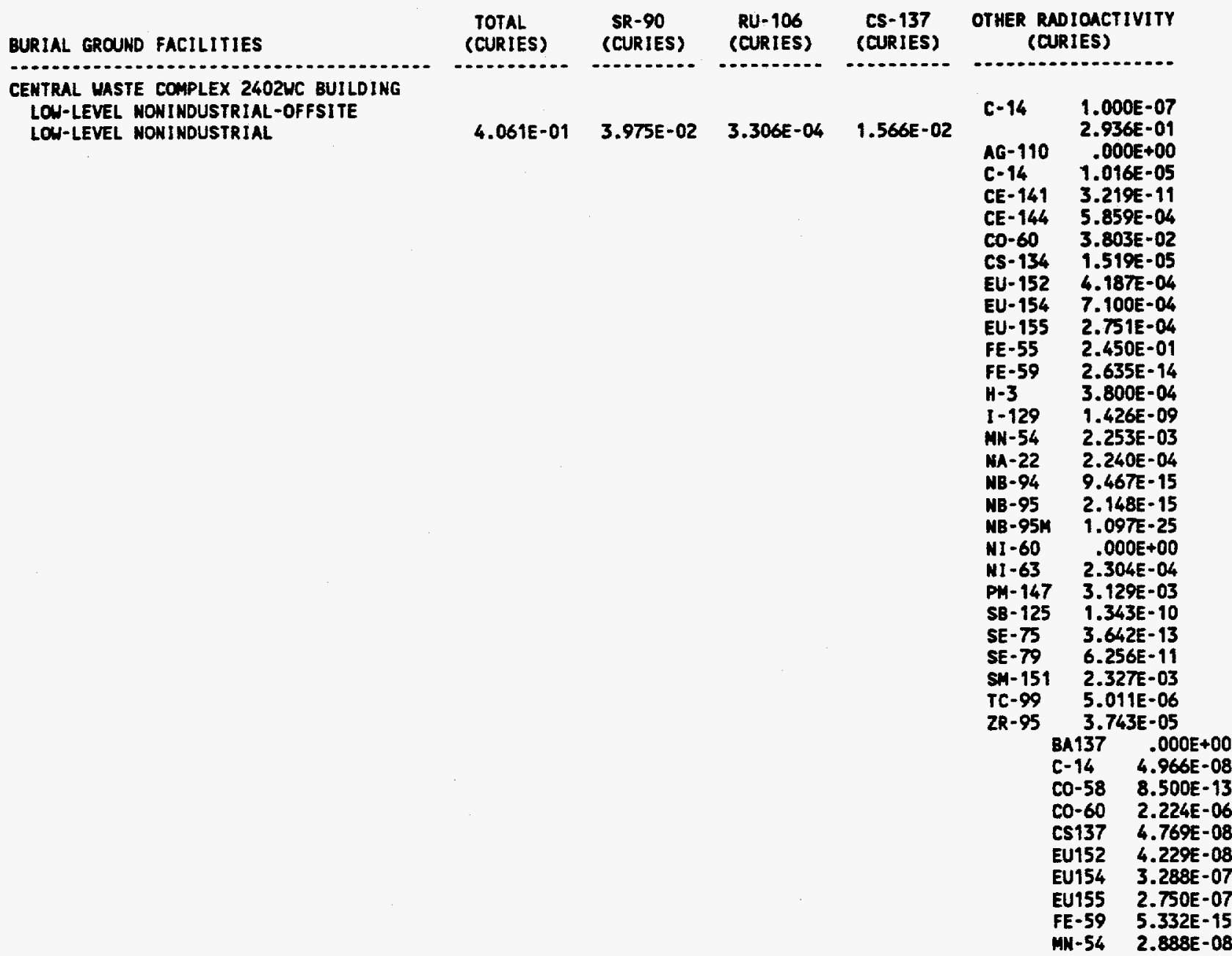

CENTRAL HASTE COMPLEX 2402WD BUILDING LOW-LEVEL NONINDUSTRIAL
$1.617 E+00 \quad 6.507 E-03 \quad 5.520 E-04 \quad 7.138 E-03$

C-14

$7.526 \mathrm{E}-12$

CE-144 7.619E-04

EU- $\{55 \quad 2.360 E-04$

$\mathrm{H}-3 \quad 1.373 \mathrm{E}+0 \mathrm{O}$

PM-147 5.272E-03

SM-151 4.261E-03

SN-123 $\quad .000 E+00$

TC- $99 \quad 2.013 E-01$

CENTRAL WASTE COMPLEX 240ZWE BUILDING LON-LEVEL NONINDUSTRIAL
$1.083 E-03 \quad 1.869 E-04 \quad 2.827 E-05 \quad 1.945 E-04$

$\begin{array}{ll} & 1.681 E-04 \\ C-14 & 1.460 E-15 \\ C E-144 & 1.250 E-07 \\ C O-60 & 1.671 E-04 \\ H-3 & 4.579 E-09 \\ I-129 & 3.580 E-13 \\ M B-94 & 8.190 E-16 \\ \text { PN-147 } & 9.148 E-07 \\ \text { SE-79 } & 5.400 E-12 \\ \text { TC-99 } & 2.000 E-10\end{array}$


SWIR328I - (Page 17 of 26)

RUNDATE: $08 / 20 / 94$

Post-1970 Nontransuranic Waste Burial Ground Areas Decayed Curie Content through DECEMBER 31, 1993.

BURJAL GROUND FACILITIES
CENTRAL WASTE COMPLEX 2402IVE BUILDING
LOU-LEVEL INDUSTRIAL (ASSAYED FROM
TRANSURANIC)
CENTRAL MASTE CONPLEX 2402WF BUILDING
LOW-LEVEL NOWINDUSTRIAL

CENTRAL MASTE COMPLEX 2402WG BUILDING LOW-LEVEL NOWINDUSTRIAL-OFFSITE

LOW-LEVEL NONINDUSTRIAL

LOU-LEVEL INDUSTRIAL

CENTRAL MASTE COMPLEX 2402WH BUTLDING LOW-LEVEL NONINDUSTRIAL-OFFSITE

\begin{tabular}{|c|c|c|c|c|c|}
\hline $\begin{array}{l}\text { TOTAL } \\
\text { (CURIES) }\end{array}$ & $\begin{array}{l}S R-90 \\
\text { (CURIES) }\end{array}$ & $\begin{array}{c}\text { RU-106 } \\
\text { (CURIES) }\end{array}$ & $\begin{array}{c}\text { CS-137 } \\
\text { (CURIES) }\end{array}$ & $\begin{array}{r}\text { OTHER RA } \\
\text { CCU }\end{array}$ & $\begin{array}{l}\text { OACrIVITY } \\
\text { (ES) }\end{array}$ \\
\hline $3.469 E-04$ & $7.275 E-05$ & $1.034 E-06$ & $7.830 E-05$ & & $.000 E+00$ \\
\hline $3.309 E-01$ & $6.685 E-03$ & $6.179 E-04$ & $7.453 E-03$ & $\begin{array}{l}C-14 \\
C E-144 \\
H-3 \\
P W-147 \\
\text { SW-151 } \\
\text { TC-99 }\end{array}$ & $\begin{array}{l}3.006 E-01 \\
9.675 E-12 \\
1.187 E-03 \\
3.110 E-05 \\
6.718 E-03 \\
6.200 E-03 \\
2.864 E-01\end{array}$ \\
\hline
\end{tabular}

$8.134 E-04 \quad 8.057 E-10 \quad 1.242 E-09 \quad 8.610 E-10$

C-14 $\quad 8.134 E-04$

H-3 8.022E-04

P-32 1.043E-09

s-35 1.113E-05

2.384E-01 3.120E-02 9.684E-05 5.106E-02

$\begin{array}{ll}\text { C-14 } & 7.253 E-02 \\ 9.995 E-05\end{array}$

CE-144 9.525E-06

CO-60 7.104E-02

CS-134 6.606E-07

EU- $152 \quad 5.680 E-05$

EU-154 1.536E-04

EU-155 4.117E-05

FE-59 2.019E-13

$H-3 \quad 3.119 E-04$

$1-129 \quad 1.129 E-06$

$1-131 \quad 6.041 E-25$

MN-54 6.255E-04

NB-94 4.909E-14

NI-59 $\quad 6.540 E-11$

NI-60 . .000E+00

NI-63 7.519E-06

PH-147 1.775E-04

SE-79 3.240E-10

TA-182 7.419E-09

TC- 99 1.592E-06

2N-65 4.158E-06

BA137 .000E+00

$\begin{array}{ll}\text { C-14 } & 2.583 \mathrm{E}-07\end{array}$

CO-58 1.369E-09

CO-60 1.073E-05

CS137 2.446E-07

EU152 2.132E-07

EU154 1.631E-06

EU155 1.321E-06

FE-59 1.700E-09

MN-54 $1.248 E-07$

\subsection{E-05 4.471E-06 5.844E-07 5.056E-06}

\section{C-14}

$6.362 E-06$

CE-144 1.183E-06

H-3 2.171E-08

$1-129 \quad 1.530 \mathrm{E}-12$

MB-94 3.710E-15

PN-147 5.156E-06

SE-79 $2.450 E-11$

TC- $99 \quad 9.060 \mathrm{E}-10$

$1.190 E-05 \quad 2.868 E-06 \quad 6.224 E-11 \quad 2.869 E-06$
$5.795 E-07$

C-14 $\quad 5.000 E-10$ 


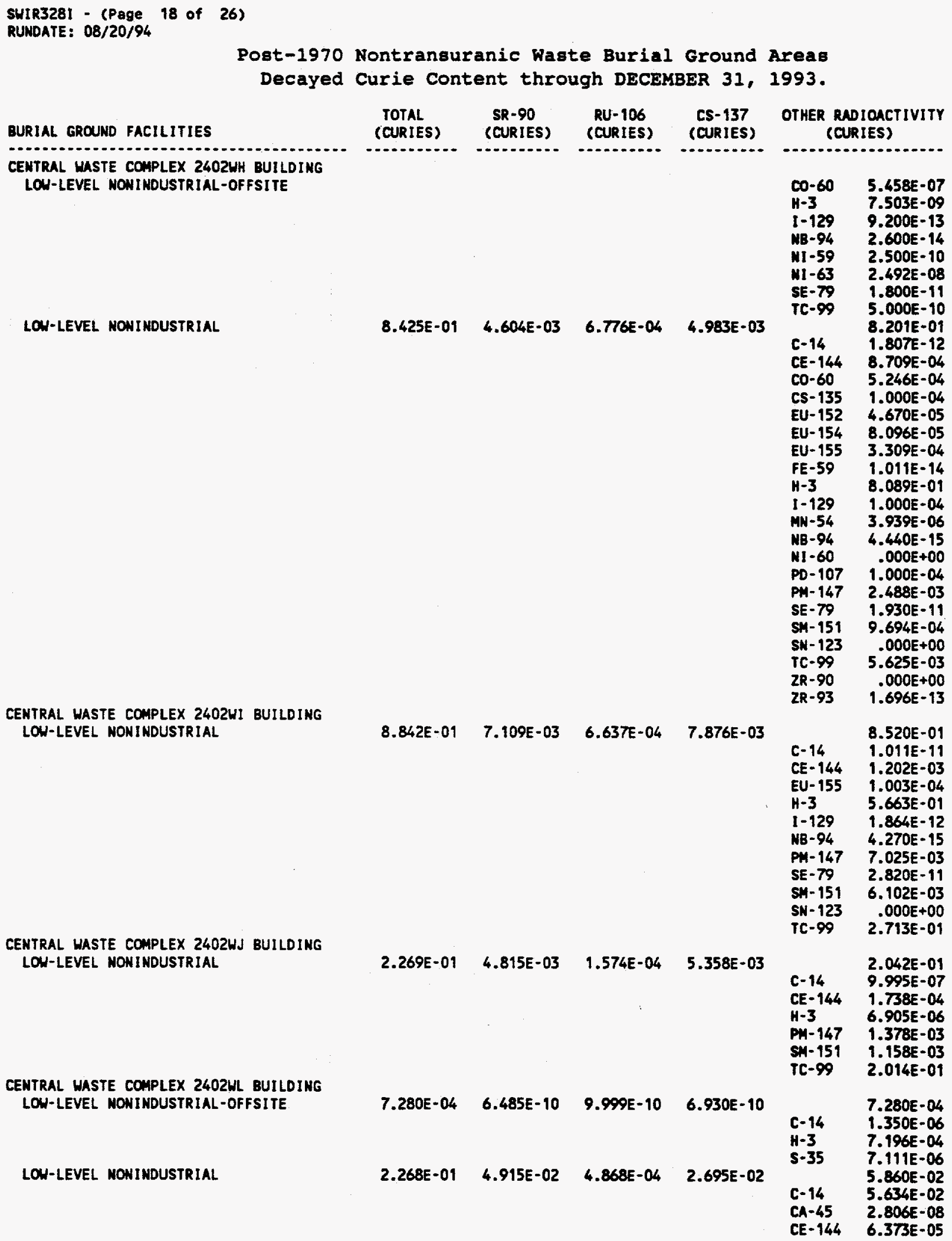


SUIR3281 - (Page 19 of 26) RUNDATE: $08 / 20 / 94$

Post-1970 Nontransuranic Waste Burial Ground Areas Decayed Curie Content through DECEMBER 31, 1993.

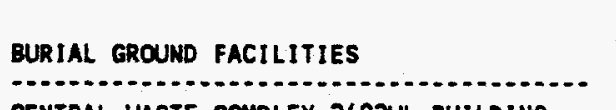

CENTRAL MASTE COMPLEX 2402WL BUILOING LON-LEVEL NONINDUSTRIAL
CENTRAL WASTE CONPLEX 2403WA BUILDING LOU-LEVEL NONINDUSTRIAL-OFFSITE

$\begin{array}{lccc}\text { TOTAL } & \text { SR-90 } & \text { RU-106 } & \text { CS-137 } \\ \text { (CURIES) } & \text { (CURIES) } & \text { (CURIES) } & \text { (CURIES) }\end{array}$

OTHER RADIOACTIVITY (CURIES) $\begin{array}{llll}\text { (CURIES) (CURIES) (CURIES) (CURIES) } & \end{array}$

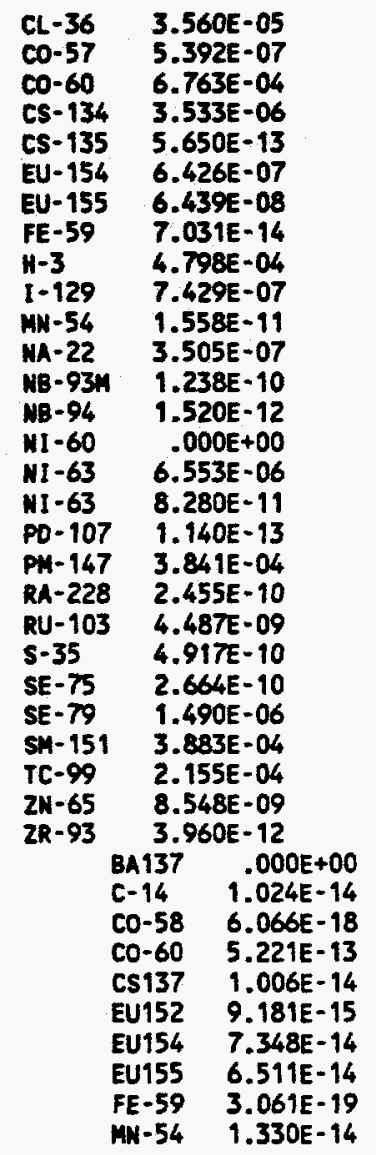

\subsection{E-01 6.276E-02 1.151E-05 1.960E-01}


SWIR3281 - (Page 20 of 26)

RUNDATE: $08 / 20 / 94$

Post-1970 Nontransuranic Waste Burial Ground Areas Decayed Curie Content through DECEMBER 31, 1993.

BURIAL GROUND FACILITIES

CENTRAL WASTE COMPLEX 2403WA BUILOING

LOW-LEVEL NONINDUSTRIAL-OFFSITE
TOTAL

(CURIES)

(CURIES) (CURIES)

RU-106

(CURIES)

CS-137

(CURIES)

OTHER RADIOACTIVITY

LON-LEVEL NONINDUSTRIAL

$7.945 E+00$

$1.123 E+00$

6.320E-02 $1.031 E+00$

$K-40 \quad 1.386 E-02$
(CURIES)

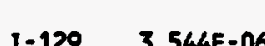

3.544E-06

KR-85 2.412E-03

MN-54 7.547E-05

MA-22 1.140E-04

NB-94 $1.793 E-08$

$\mathrm{NI}-59 \quad 7.644 \mathrm{E}-06$

NI $-60 \quad .000 E+00$

MI-63 9.486E-04

P-32 7.54TE-08

PB-214 .000E+00

PM-147 2.126E-03

PT-195 1.273E-23

S-35 1.080E-03

SB-125 1.803E-02

SE-79 3.592E-08

TC- 99 1.156E-06

TE-125 4.877E-03

TE-125 5.491E-04

ZN-65 3.040E-06

$3.234 E+00$

AC-228 .000E+00

C-14 5.672E-07

CA-45 7.596E-07

CE-144 8.674E-02

CO-60 1.883E-01

CS-134 1.596E-03

CS-135 6.620E-13

EU-152 1.181E-03

EU-154 2.738E-03

EU-155 6.067E-04

FE-55 3.209E-01

FE-59 2.002E-15

CD-153 2.630E-04

CD-154 .000E+00

$\mathrm{H}-3 \quad 4.418 \mathrm{E}-03$

$I-129 \quad 2.439 E-04$

$\mathrm{K}-40 \quad 6.704 \mathrm{E}-05$

MN-54 8.525E-02

MO-93 2.400E-07

NA-22 3.996E-04

NB-93M 2.250E-07

NB-94 2.096E-09

MB-95M 1.291E-17

NI-59 5.984E-09

NI $-60 \quad .000 E+00$

MI-63 4.649E-06

PB-212 .000E+00

PB-214 .000E+00

PD-107 1.336E-13

PM-147 2.255E-01

SB-125 1.917E-06

SC-46 9.022E-09

SE-79 1.554E-08

SH-151 1.090E-01

TA-182 4.232E-09

TC- 9 2.207E+00

TE- 125 9.544E-09

TE-125 1.576E-08

TL-208 .000E+00 
SUIR328I - (Page 21 of 26)
RUNDATE: 08/20/94

Post-1970 Nontransuranic Waste Burial Ground Areas

Decayed Curie Content through DECEMBER 31, 1993.

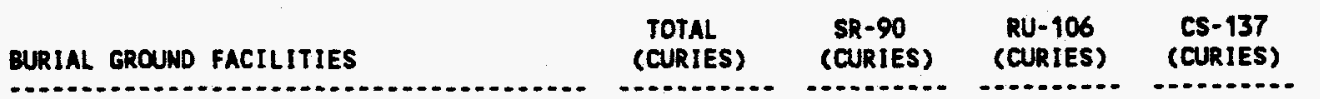

CENTRAL WASTE COMPLEX 2403UA BUILDING

LOW-LEVEL NONINDUSTRIAL

(CURIES) (CURIES) (CURIES) (CURIES)

OTHER RADIOACTIVITY

LOW-LEVEL INDUSTRIAL

CENTRAL WASTE COMPLEX 2403HC BUILDING LOW-LEVEL NONINDUSTRIAL-OFFSITE

\subsection{E-07 1.423E-19 2.2TTE-13 3.650E-07 \\ 1.402E-01 9.423E-05 6.204E-05 3.862E-04}

LON-LEVEL MONINDUSTRIAL

$5.022 E+01 \quad 2.090 E+01 \quad 1.613 E-02 \quad 4.139 E+00$
(CURIES)

\begin{tabular}{|c|c|c|}
\hline $\begin{array}{l}V-49 \\
X E-131 \\
Z N-65 \\
Z R-93 \\
Z R-95\end{array}$ & \multicolumn{2}{|c|}{$\begin{array}{l}9.922 E-05 \\
2.644 E-17 \\
7.449 E-11 \\
4.630 E-12 \\
1.911 E-05\end{array}$} \\
\hline & $\begin{array}{l}\text { BA137 } \\
C-14 \\
\text { CO-58 } \\
\text { CO-60 } \\
\text { CS137 } \\
\text { EU152 } \\
\text { EU154 } \\
\text { EU155 } \\
\text { FE-59 } \\
\text { NN-54 }\end{array}$ & $\begin{array}{r}.000 E+00 \\
1.095 E-08 \\
5.626 E-14 \\
4.685 E-07 \\
1.043 E-08 \\
9.156 E-09 \\
7.049 E-08 \\
5.778 E-08 \\
1.877 E-16 \\
4.814 E-09\end{array}$ \\
\hline
\end{tabular}

$1.386 E-01$

$\begin{array}{lr}B 1-214 & .000 E+00 \\ C-14 & 6.459 E-04\end{array}$

$\begin{array}{lr}B 1-214 & .000 E+00 \\ C-14 & 6.459 E-04\end{array}$

CO-58 4.577E-04

CO-60 2.597E-02

FE-55 2.495E-02

$\mathrm{H}-3 \quad 8.156 \mathrm{E}-02$

I-129 5.399E-09

MN-54 3.587E-03

NI-60 $\quad .000 E+00$

NI-63 1.351E-03

P.32 7.682E-05

PB-214 .000E+00

TC-99 5.399E-09

2.646E-01

$\begin{array}{lr}A C-228 & .000 E+00 \\ A G-110 & 3.143 E-07\end{array}$

AG-110 .000E+00

$B I-212 \quad .000 E+00$

C. $14 \quad 3.851 E-07$

CE-141 1.202E-11

CE-144 2.108E-02

CO-57 6.444E-10

CO-60 4.228E-02

CR-51 1.081E-12

CS-134 1.508E-02

EU-152 1.028E-04

EU-154 1.650E-02

EU-155 1.861E-02

FE-55 2.129E-05

FE-59 1.097E-09

CD-154 .000E+00

$\mathrm{H}-3$ 4.018E-02

$1-129 \quad 1.505 E-04$

$K=40 \quad 2.318 E-04$

MN-54 5.376E-05

MO-93 1.890E-08

MA-22 9.347E-06

NB-93M 1.507E-06

MB-94 9.558E-09

NB-95 2.865E-13

MB-95M 1.121E-11

NI-59 2.733E-08 
SWIR3281 - (Page 22 of 26)

RUNDATE: $08 / 20 / 94$

Post-1970 Nontransuranic Waste Burial Ground Areas

Decayed Curie Content through DECEMBER 31, 1993.

$\begin{array}{lcccc}\text { BURIAL GROUND FACILITIES } & \begin{array}{c}\text { TOTAL } \\ \text { (CURIES) }\end{array} & \begin{array}{c}\text { SR-90 } \\ \text { (CURIES) }\end{array} & \begin{array}{c}\text { RU-106 } \\ \text { (CURIES) }\end{array} & \begin{array}{c}\text { CS-137 } \\ \text { (CURIES) }\end{array}\end{array}$

OTHER RADIOACTIVITY CENTRAL WASTE COMPLEX 2403WC BUILDING LOU-LEVEL NONINDUSTRIAL

LOW-LEVEL NONINDUSTRIAL (ASSAYED FROM TRANSURANIC)

CENTRAL WASTE COMPLEX 2403WO BUILDING LOW-LEVEL NONINDUSTRIAL-OFFSITE

LOW-LEVEL NONINDUSTRIAL
5.458E-04 1.163E-04 1.237E-06 1.253E-04

2.233E-05 1.905E-07 1.251E-07 2.039E-07

$3.095 \mathrm{E}+03$

$3.048 E+00$

$2.244 E+01$

FLAMmable storage mOOULE 1 LON-LEVEL NONINDUSTRIAL

1.774E-04 1.336E-05 1.380E-06 2.343E-05
$H \cdot 3$

C- 14

CE-144

CO-60

EU-152

EU-154

EU-155

$\mathrm{H}-3$

I -129

MB-93M

MB -94

N1-59

MI-63

PN-147

SE-79

SN-151

TC -99

(CURIES)

(CURIES)

8.455E-06

2.070E-10

2.400E-07

$.000 E+00$

$6.088 E-02$

1.223E- 09

$1.013 E-03$

$7.508 E-08$

$1.961 E-02$

$1.802 E-05$

2.878E-02

5.625E-07

$.000 E+00$

$4.847 \mathrm{~F}-09$

$8.415 E-08$

5.082E- 10

3.235E-06

BA137 .000E+00

C-14 4.389E-06

Co-58 7.283E-10

C0-60 2.136E-04

CS137 4.276E-06

EU152 3.863E-06

EU154 3.060E-05

EU155 2.656E-05

FE-59 1.738E-11

MN-54 4.272E-06 $.000 E+00$

2.022E-05

2.022E-05

$3.045 E+03$

5.379E-05

$6.178 E-04$

$2.924 E+03$

8.034E-03

2.123E-01

$1.699 \mathrm{E}-03$

2.002E-05

$1.641 \mathrm{E}-03$

2.126E- 04

$4.100 E-06$

$1.092 E+00$

$1.198 E+02$

$2.438 E-03$

$1.865 \mathrm{E}-05$

$6.832 E-04$

3.642E-01

C- 14

9.940E-05

$1.322 E-14$

CE-144 3.007E-06

CS-134 3.339E-07

EU-152 2.844E-05

EU-154 4.860E-05

EU-155 6.926E-06

$\mathrm{H}-3 \quad 3.785 \mathrm{E}-07$

$1-129 \quad 4.683 E-09$ 
WHC-EP-0125-6 SHIR328I - (Page 23 of 26 )
RUNDATE: $08 / 20 / 94$

Post-1970 Nontransuranic Waste Burial Ground Areas Decayed Curie Content through DECEMBER 31, 1993.

\begin{tabular}{|c|c|c|c|c|c|c|c|}
\hline BURIAL GROUND FACILITIES & $\begin{array}{l}\text { TOTAL } \\
\text { (CURIES) }\end{array}$ & $\begin{array}{l}\text { SR-90 } \\
\text { (CURIES) }\end{array}$ & $\begin{array}{l}\text { RU-106 } \\
\text { (CURIES) }\end{array}$ & $\begin{array}{l}\text { CS-137 } \\
\text { (CURIES) }\end{array}$ & \multicolumn{3}{|c|}{$\begin{array}{l}\text { OTHER RADIOACTIVITY } \\
\text { (CURIES) }\end{array}$} \\
\hline $\begin{array}{l}\text { FLAMUABLE STORAGE MOOULE } 1 \\
\text { LOW-LEVEL NONINDUSTRIAL }\end{array}$ & & & & & $\begin{array}{l}\text { NB-94 } \\
\text { PH-147 } \\
\text { SE-79 } \\
\text { TA-182 } \\
\text { TC-99 }\end{array}$ & \multicolumn{2}{|c|}{$\begin{array}{l}7.420 E-15 \\
1.121 E-05 \\
4.900 E-11 \\
3.939 E-09 \\
4.854 E-07\end{array}$} \\
\hline $\begin{array}{l}\text { FLAMMABLE STORAGE MOOULE } 10 \\
\text { LOW-LEVEL. NOWINDUSTRIAL }\end{array}$ & $3.124 E+00$ & 8.866E-01 & 4.979E-04 & 6.781E-01 & $\begin{array}{l}C-14 \\
c 0-60 \\
H-3\end{array}$ & \multicolumn{2}{|c|}{$\begin{array}{l}7.493 E-03 \\
6.007 E-03 \\
6.978 E-04 \\
7.881 E-04\end{array}$} \\
\hline & & & & & & $\begin{array}{l}137 \\
14 \\
-58 \\
-60 \\
137 \\
1152 \\
154 \\
155 \\
-59 \\
-54\end{array}$ & $\begin{array}{r}.000 E+00 \\
4.977 E-06 \\
8.237 E-14 \\
1.727 E-04 \\
4.572 E-06 \\
3.828 E-06 \\
2.814 E-05 \\
2.102 E-05 \\
8.693 E-18 \\
5.999 E-07\end{array}$ \\
\hline
\end{tabular}

Flammable storage moOULE 11 LOW-LEVEL NONINDUSTRIAL-OFFSITE

LOW-LEVEL NONINDUSTRIAL
$1.411 E-04$

$1.642 E-09$

$3.069 E-09$

$1.754 E-09$

4.603E-04

4
1.106E-07

$1.958 E-04$ fla MMABLE STORAGE mOOULE 12

LON-LEVEL NONINOUSTRIAL-OFFSITE LOW-LEVEL NOWINDUSTRIAL

$\begin{array}{llll}3.760 E-04 & 1.770 E-05 & 2.801 E-05 & 1.892 E-05 \\ 1.246 E-02 & 1.493 E-03 & 2.076 E-04 & 3.082 E-03\end{array}$

\section{C-14}

CE- 144

CO-60

CS- 134

CS-135

EU- 152

EU-154

EU-155

$\mathrm{H}-3$

$1-129$

MB-94

NI -60

PD- 107

PM-147

SB-125

SE-79

SH-151

TC- 99
$1.491 E-04$

$1.005 E-06$

2.000E-06

$7.806 E-10$

6.972E-09

$1.375 E-04$

4.682E-09

4.995E-07

$.000 E+00$

$6.949 E-05$

$5.298 E-05$

$1.807 E-07$

2.484E-07

1.280E-12

$2.930 E-15$

3.076E-06

1.900E-11

1.300E-05

$.000 E+00$

2.519E-03

1.637E-04

4.872E-04

5.410E-06

$1.020 E-06$

$5.610 E-13$

1.220E-07

3.035E-06

1.983E-06

$7.023 E-05$

$9.293 E-07$

5.560E-15

$.000 E+00$

$1.130 \mathrm{E}-93$

1.274E-03

1.542E-08

$3.670 E-11$

4.900E-04

2.125E-05 
SHIR328I - (Page 24 of 26) RUNDATE: 08/20/94

Post-1970 Nontransuranic Waste Burial Ground Areas Decayed Curie Content through DECEMBER 31, 1993.

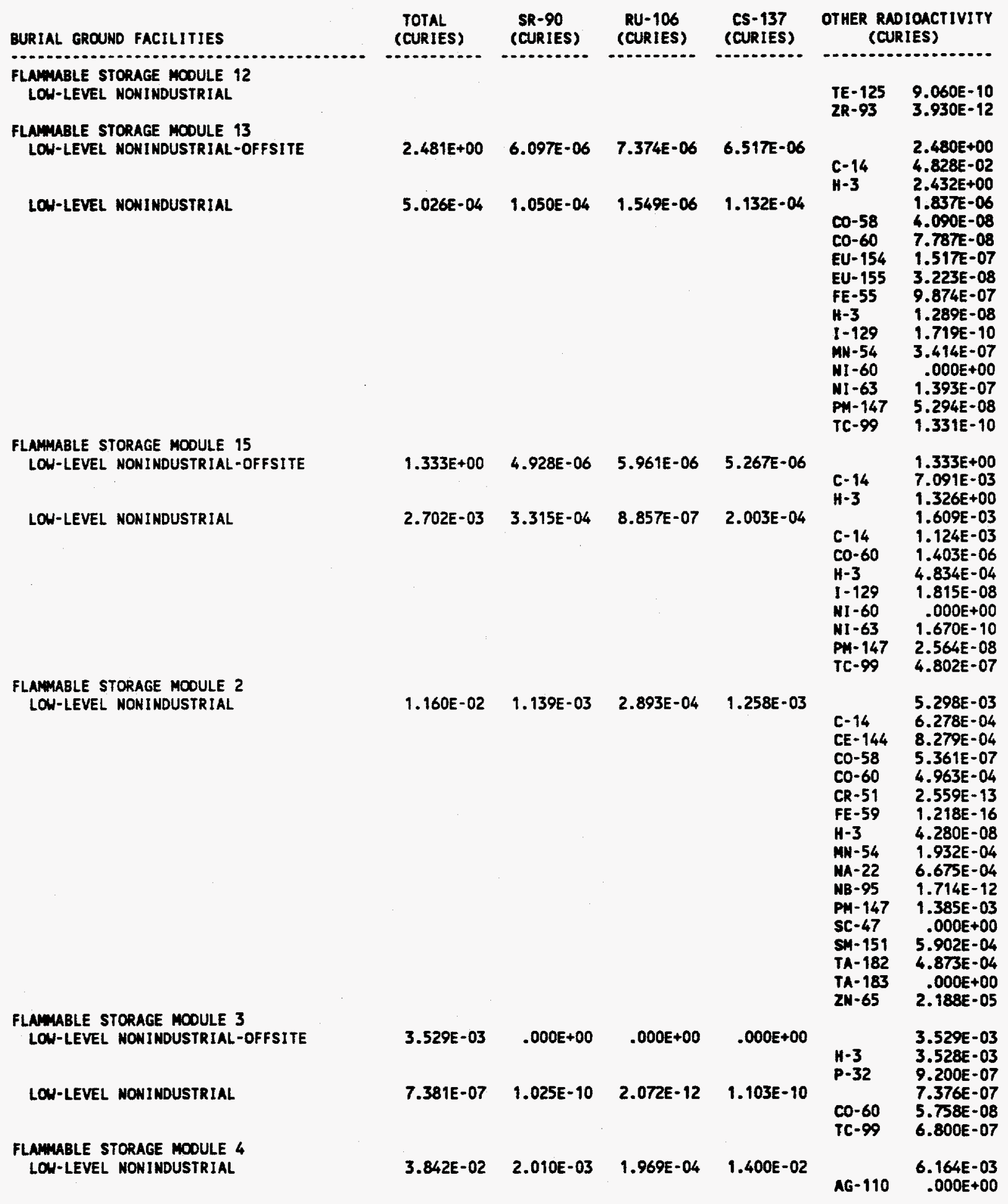


SUIR328I - (Pege 25 of 26)

RUNOATE: $08 / 20 / 94$

Post-1970 Nontransuranic Waste Burial Ground Areas

Decayed Curie content through DECEMBER 31, 1993.

\section{BURIAL GROUND FACILITIES}

FLAMMBLE STORAGE MOOULE 4

LON-LEVEL NOWINDUSTRIAL

\section{FLAMMABLE STORAGE MOOULE 5}

LOW-LEVEL NONINDUSTRIAL

FLAMMABLE STORAGE MOOULE 6 LON-LEVEL NONINDUSTRIAL

\begin{tabular}{cccc}
$\begin{array}{c}\text { TOTAL } \\
\text { (CURIES) }\end{array}$ & $\begin{array}{c}\text { SR-90 } \\
\text { (CURIES) }\end{array}$ & $\begin{array}{c}\text { RU-106 } \\
\text { (CURIES) }\end{array}$ & $\begin{array}{c}\text { CS-137 } \\
\text { (CURIES) }\end{array}$ \\
\hline
\end{tabular}

(CUR1ES)

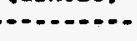

OTHER RADIOACTIVITY (CURIES)

\begin{tabular}{|c|c|c|c|c|c|}
\hline & & & & $\begin{array}{l}C-14 \\
C E-144 \\
C O-60 \\
C S-134 \\
\text { EU-152 } \\
\text { EU-154 } \\
\text { EU-155 } \\
H-3 \\
I-129 \\
\text { HN-54 } \\
\text { PN-147 } \\
\text { SB-125 } \\
\text { SH-151 } \\
\text { TC-99 } \\
\text { ZR-95 }\end{array}$ & $\begin{array}{l}7.207 E-04 \\
3.745 E-04 \\
4.570 E-06 \\
2.432 E-05 \\
2.124 E-06 \\
2.321 E-05 \\
8.364 E-06 \\
2.153 E-03 \\
3.866 E-10 \\
2.678 E-08 \\
1.782 E-03 \\
3.579 E-06 \\
1.068 E-03 \\
3.002 E-10 \\
4.359 E-12\end{array}$ \\
\hline $1.907 \mathrm{E}-02$ & $2.653 E-03$ & $3.254 E-04$ & 2.943E-03 & $\begin{array}{l}C-14 \\
C E-144 \\
H-3 \\
\text { PM- } 147 \\
\text { SH-159 } \\
\text { TC-99 }\end{array}$ & $\begin{array}{l}6.144 E-03 \\
1.186 E-03 \\
7.573 E-04 \\
5.548 E-04 \\
2.194 E-03 \\
1.366 E-03 \\
8.600 E-05\end{array}$ \\
\hline $3.020 E+00$ & $1.292 E-02$ & $6.025 E-05$ & $5.6777-02$ & $\begin{array}{l}C-14 \\
C A-45 \\
C E-144 \\
C O-57 \\
C O-58 \\
C O-60 \\
C R-51 \\
C S-134 \\
E U-152 \\
E U-154 \\
E U-155 \\
F E-55 \\
H-3 \\
1-129 \\
M N-54 \\
M O-93 \\
N B-93 M \\
N I-60 \\
N I-63 \\
P N-147 \\
S B-125 \\
S I-31 \\
T C-99 \\
T I-51 \\
V-49 \\
N-185 \\
\text { ZR- } 95\end{array}$ & $\begin{array}{l}2.883 E+00 \\
4.998 E-04 \\
4.767 E-07 \\
9.055 E-06 \\
6.098 E-04 \\
3.814 E-06 \\
3.303 E-02 \\
1.614 E-07 \\
1.145 E-04 \\
6.648 E-06 \\
1.634 E-05 \\
9.030 E-06 \\
1.691 E+00 \\
6.274 E-01 \\
1.000 E-02 \\
4.547 E-01 \\
3.188 E-04 \\
1.860 E-05 \\
.000 E+00 \\
4.514 E-02 \\
1.093 E-05 \\
4.467 E-06 \\
.000 E+00 \\
6.722 E-05 \\
.000 E+00 \\
1.626 E-02 \\
3.392 E-03 \\
4.015 E-07\end{array}$ \\
\hline $\begin{array}{l}1.983 E-04 \\
6.215 E-04\end{array}$ & $\begin{array}{l}1.755 E-05 \\
1.664 E-04\end{array}$ & $\begin{array}{l}1.195 E-05 \\
4.088 E-07\end{array}$ & $\begin{array}{l}1.878 E-05 \\
1.261 E-04\end{array}$ & $\begin{array}{l}\text { CO-57 } \\
C O-60 \\
\text { EU-154 } \\
\text { EU- } 155 \\
H-3\end{array}$ & $\begin{array}{r}.000 E+00 \\
4.257 \mathrm{E}-05 \\
1.314 E-08 \\
4.771 E-07 \\
3.178 E-08 \\
1.383 E-07 \\
1.745 E-05\end{array}$ \\
\hline
\end{tabular}

FLAMABLE STORAGE MOOULE 7

LON-LEVEL NOHINDUSTRIAL-OFFSITE LON-LEVEL NONIMDUSTRIAL

.


SUIR328I - (Page 26 of 26) RUNDATE: $08 / 20 / 94$

Post-1970 Nontransuranic Waste Burial Ground Areas Decayed Curie Content through DECEMBER 31, 1993.

BURIAL GROUND FACILITIES

FLAMMBLLE STORAGE MODULE 7 LOW-LEVEL NONINDUSTRIAL

FLAMABLE STORAGE MOOULE 8 LOW-LEVEL NONINDUSTRIAL

Total 200W

TOTAL 200 AREAS

\begin{tabular}{cccc}
$\begin{array}{c}\text { TOTAL } \\
\text { (CURIES) }\end{array}$ & $\begin{array}{c}\text { SR-90 } \\
\text { (CURIES) }\end{array}$ & $\begin{array}{c}\text { RU-106 } \\
\text { (CURIES) }\end{array}$ & $\begin{array}{c}\text { CS-137 } \\
\text { (CURIES) }\end{array}$ \\
\hline
\end{tabular}

OTHER RADIOACTIVITY (CURIES)

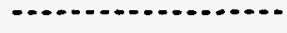

$1-129 \quad 2.451 E-07$

PH-147 2.402E-05

TC-99 $1.885 E-07$

7.051E-03 4.604E-04 1.594E-05 3.062E-03

8.199E-05

C-14 3.810E-16

CE-144 $1.908 E-08$

CO-60 1.140E-05

CS-134 1.356E-05

EU-154 2.192E-07

EU-155 3.164E-07

$\mathrm{H}-3$ 1.080E-06

$1-129 \quad 1.485 E-08$

K-40 4.000E-07

MN-54 5.401E-06

MB-94 2.140E-16

NI $-60 \quad .000 E+00$

PH-147 $1.872 E-06$

SE-79 1.410E-12

TC-99 4.771E-05

$\begin{array}{lllll}1.503 E+06 & 1.733 E+05 & 9.934 E+03 & 2.895 E+05 & 4.731 E+05 \\ 6.201 E+06 & 9.123 E+05 & 9.939 E+03 & 1.177 E+06 & 1.969 E+06\end{array}$


SWIR328J - (Page 1 of 6)

RUNDATE: $08 / 20 / 94$

Post-1970 Segregated Transuranic Waste Burial Ground Areas Waste Volumes Stored and Nondecayed Curie Content through December 31, 1993.

BURIAL GROUND FACILITIES

PACKED

VACLE

(CUBIC H) (CUBIC

URANIU:

PLUTONILA

(GRAMS)

(GRAMS)

OTHER RADIOACTIVITY

2OOE AREA

BURIAL GROUND 218E12B

TRANSURANIC (NOT PROCESSED THROUGH

TRUSAF)

Total for Facility $218 E 12 B$

$\begin{array}{llll}6.379 E+02 & 6.379 E+02 & .000 E+00 & 1.657 E+02\end{array}$

$.000 E+00$

$6.379 E+02$

$6.379 E+02$

$.000 E+00 \quad 1.657 E+02$

$.000 E+00$

Total 200E

$6.379 E+02 \quad 6.379 E+02$

$.000 E+00 \quad 1.657 E+02$

$.000 E+00$ 


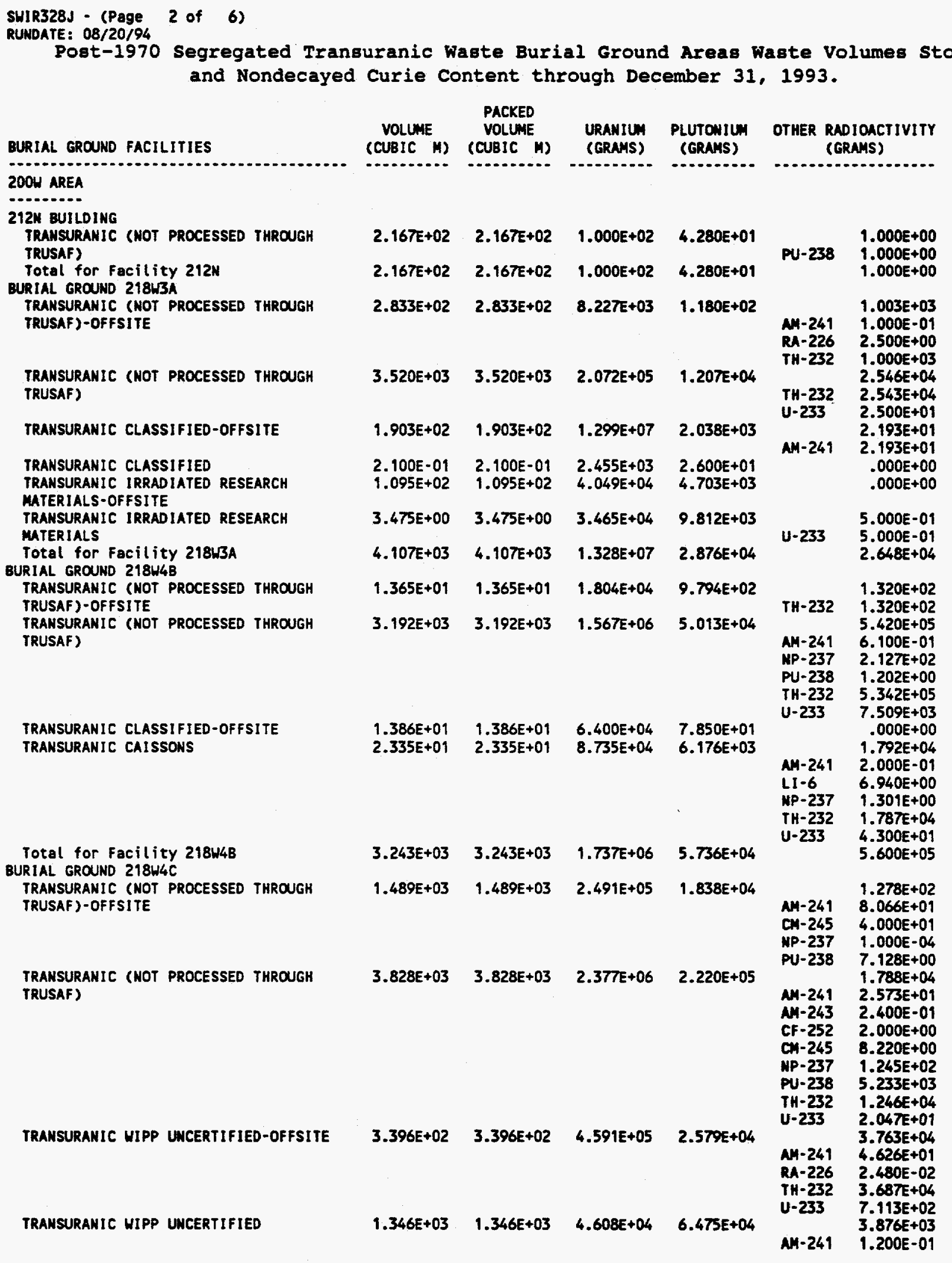




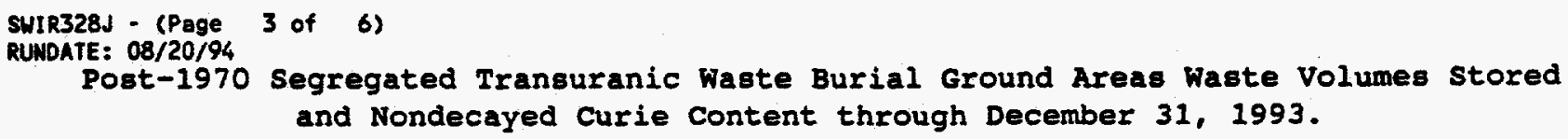

TRANSURANIC SPECIAL CASE-OFFSITE TRANSURANIC IRRADIATED RESEARCH

$2.226 E+01$

4.170E+00

$2.226 E+01$

4.170E+00

$6.380 E+05$

$6.327 E+04$

$1.965 E+04$

$2.297 E+01$

2.297E+01

$1.421 E+05$

$5.925 E+02$

$7.052 E+03$

Total for Facility 218W4C

$7.052 E+03$

TRANSURANIC UASTE STORAGE \& ASSAY FACILITY(TRUSAF)

TRANSURANIC (MOT PROCESSED THROUGH

TRUSAF?

4. $234 E+01$

$4.234 E+01$

$3.974 E+06$

$3.213 E+04$

$3.833 E+05$

$3.293 E+04$

$1.904 E+03$

TRANSURANIC HIPP CERTIFIED

$1.059 E+02$

$1.059 E+02$

$7.883 E+03$

$2.422 E+04$
TRANSURANIC WIPP UNCERTIFIED

$9.817 E+01 \quad 9.817 E+01 \quad 7.912 E+03 \quad 2.163 E+04$

$9.817 E+01 \quad 9.817 E+01 \quad 7.912 E+03 \quad 2.163 E+04$

$9.817 E+01 \quad 9.817 E+01 \quad 7.912 E+03 \quad 2.163 E+04$

Total for Facility 224T

CENTRAL MASTE COMPLEX $2401 \mathrm{~W}$ BUILDING TRANSURANIC (NOT PROCESSED THROUGH TRUSAF)

TRANSURANIC WIPP UNCERTIFIED

Total for Facility $2401 \mathrm{~W}$

CENTRAL WASTE COMPLEX $2402 \mathrm{~N}$ BUILDING

TRANSURANIC WIPP UNCERTIFIED-OFFSITE

TRANSURANIC MIPP UNCERTIFIED

Fotal for Facility 24024

CENTRAL WASTE COMPLEX 2402UB BUILDING

TRANSURANIC (NOT PROCESSED THROUGH

TRUSAF)

TRANSURANIC HIPP CERTIFIED
$2.464 E+02$

8.400E-01

7.691E+01

7.775E+01

$7.560 E+00$

$1.155 E+01$

1.911E+01

4. $200 E+00$

$8.820 E+00$
2.464E+02

8.400E-01

7.691E+01

7.775E+0

$7.560 E+00$

$1.155 E+01$

1.911E+01

$4.200 E+00$

$8.820 E+00$
$4.872 E+04$

$.000 E+00$

$.000 E+00$

$.000 E+00$

$1.428 E+01$

$.000 E+00$

$1.428 E+01$

$.000 E+00$

$.000 E+00$
4. $775 E+04$

$.000 E+00$

$9.863 E+02$

$9.863 E+02$

7.4TTE+01

$1.670 E+02$

$2.418 E+02$

$9.554 E+00$

2.080E+02
(GRAMS)

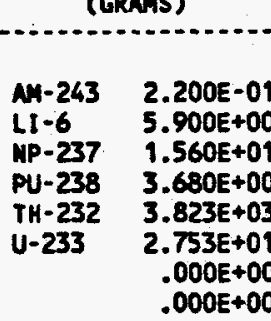

$1.303 E+02$

NM-243 5.230E+01

$U-233 \quad 7.800 E+01$

5.964E+04

$1.773 E+03$

AM-241 1.366E+01

PU-238 3.970E-01

PU-239 $1.656 E+03$

PU-240 9.585E+01

$\mathrm{PU}-241 \quad 6.933 \mathrm{E}+00$

PU-242 2.740E-01

$3.692 E+02$

MH-241 7.835E+00

CM-243 1.287E-03

$\mathrm{CM}-244 \quad 1.364 \mathrm{E}+00$

MP $-237 \quad 1.078 \mathrm{E}+00$

PU-238 1.459E-01

PU-239 2.250E+02

PU-240 2.996E+01

PU-241 2.056E+00

PU-242 $9.768 E+00$

$T H-228,9.750 E-05$

$T H-232 \quad 1.000 E+02$

$1.092 E+02$

AM-241 1.001E+01

AM-243 5.700E-07

CM-243 4.699E-07

CH-244 9.493E-03

CW-245 4.998E+01

$M P-237 \quad 1.014 E+00$

PU-238 5.230E-02

PU-239 4.095E+01

PU-240 5.791E+00

PU-241 1.211E+00

TH-228 5.200E-04

U-233 2.000E-01

2.252E+03

$.000 E+00$

$.000 E+00$

$.000 E+00$

$.000 E+00$

$.000 E+00$

$.000 E+00$

$.000 E+00$

$.000 E+00$ 
WHC-EP-0125-6

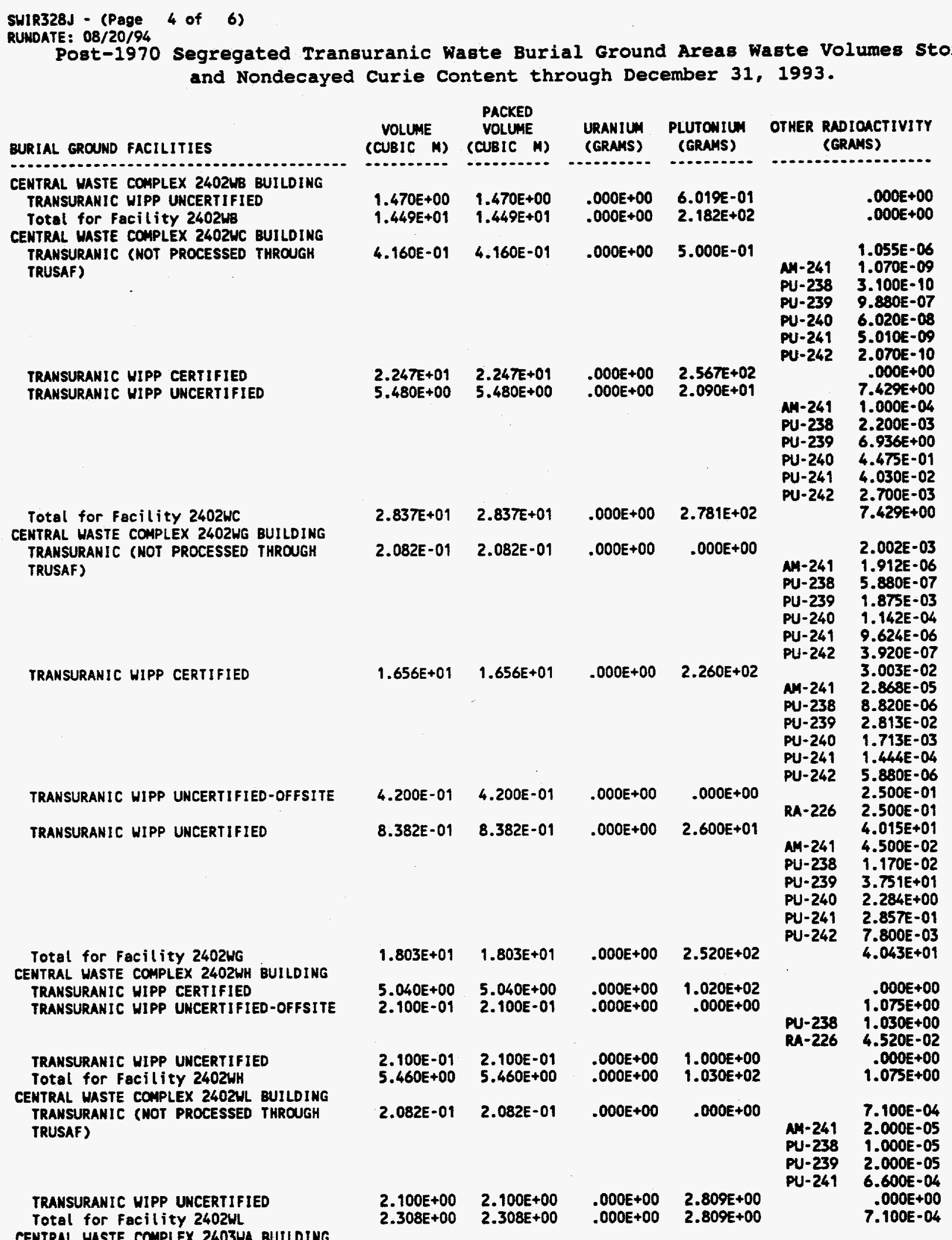




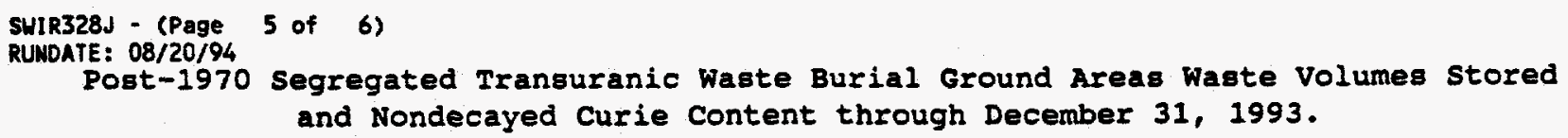

BURIAL GROUND FACILITIES

CENTRAL WASTE COMPLEX 2403WA BUILDING TRANSURANIC (NOT PROCESSED THROUEH TRUSAF)

TRAHSURANIC WIPP CERTIFIED

TRANSURANIC UIPP UNCERTIFIED

Total for Facility 2403WA

CENTRAL WASTE CONPLEX 2403WC BUILDING TRANSURANIC (NOT PROCESSED THROUGH TRUSAF)-OFFSITE

TRANSURANIC (NOT PROCESSED THROUGH TRUSAF )

TRANSURANIC HIPP CERTIFIED

TRANSURAHIC MIPP UNCERTIFIED

Total for Facility $24034 \mathrm{C}$

CENTRAL MASTE COMPLEX 2403LD BUILDING TRANSURANIC (NOT PROCESSED THROUGH TRUSAF )

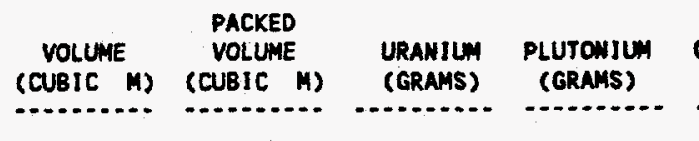

1.186E+01

$1.186 E+01$

$.000 E+00$

$1.989 E+02$

$5.976 E+01$

$5.976 E+01$

$.000 E+00$

$3.775 E+02$

$7.770 E+00$

$7.770 E+D$

$.000 E+0$

$1.290 E+02$

$\begin{array}{cccc}7.939 E+01 & 7.939 E+01 & .000 E+00 & 7.054 E+02 \\ 2.082 E-01 & 2.082 E-01 & .000 E+00 & .000 E+00 \\ 5.135 E+01 & 5.135 E+01 & .000 E+00 & 5.147 E+02\end{array}$

THER RADIOACTIVITY (GRAMS)

$\begin{array}{ll} & 1.415 E-01 \\ N H-241 & 1.415 E-01 \\ & 1.430 E+01 \\ \text { NH-241 } & 3.383 E-01 \\ C H-243 & 1.200 E-07 \\ \text { PU-238 } & 5.433 E-03 \\ \text { PU-239 } & 1.291 E+01 \\ \text { PU-240 } & 9.783 E-01 \\ \text { PU-241 } & 6.705 E-02 \\ \text { PU-242 } & 7.448 E-03 \\ & 2.995 E+01 \\ \text { AH-241 } & 6.082 E-02 \\ \text { NP-237 } & 2.000 E+00 \\ \text { PU-238 } & 1.590 E-05 \\ \text { PU-239 } & 2.788 E+01 \\ \text { PU-240 } & 4.910 E-03 \\ \text { PU-241 } & 2.416 E-03 \\ \text { PU-242 } & 1.080 E-05\end{array}$

PU-242 1.080E-05

4.440E+01

RA-226

4.000E-01

4.000E-01

$1.392 E+02$

AM-241 4.180E+00

AM-243 2.770E-04

CM-243 5.95OE-07

CM-244 1.930E-04

NP-237 3.265E-02

PU-238 1.140E-01

PU-239 1.154E+02

PU-240 $1.702 E+01$

PU-241 1.810E+00

PU-242 6.380E-01

TH-228 3.012E-09

$5.350 E+01 \quad 5.350 E+01 \quad 9.250 E+02 \quad 5.530 E+02$

Ait -241

$9.045 E+01$

$-249 \quad 1.374 E+00$

$\mathrm{CM}-243 \quad 7.893 \mathrm{E}-05$

CM-244 5. 100E-03

NP- $237 \quad 8.223 E-01$

PU-238 3.327E-02

PU-239 8.033E+01

PU-240 6.696E+00

PU-241 $1.102 E+00$

PU-242. 8.267E-02

TH-228 3.960E-08

$8.826 E+01$

$1.079 E+02 \quad 1.079 E+02 \quad 4.000 E+00 \quad 6.681 E+02$

$2.129 E+02 \quad 2.129 E+02 \quad 9.290 E+02 \quad 1.736 E+03$

$3.900 E+01$
AM-241

NP-237

PU-238

PU-239

PU-240

PU-241

PU-242

8.700E-01

2.000E +00

1.010E-01

$7.975 \mathrm{E}+01$

5.113E+00

2.779E-01

$1.480 E-01$

$3.183 E+02$

4. 164E+02

AM-241

4.530E-01

PU-238 1.181E-01

PU-239 3.901E+02

PU-240 2.381E+01 


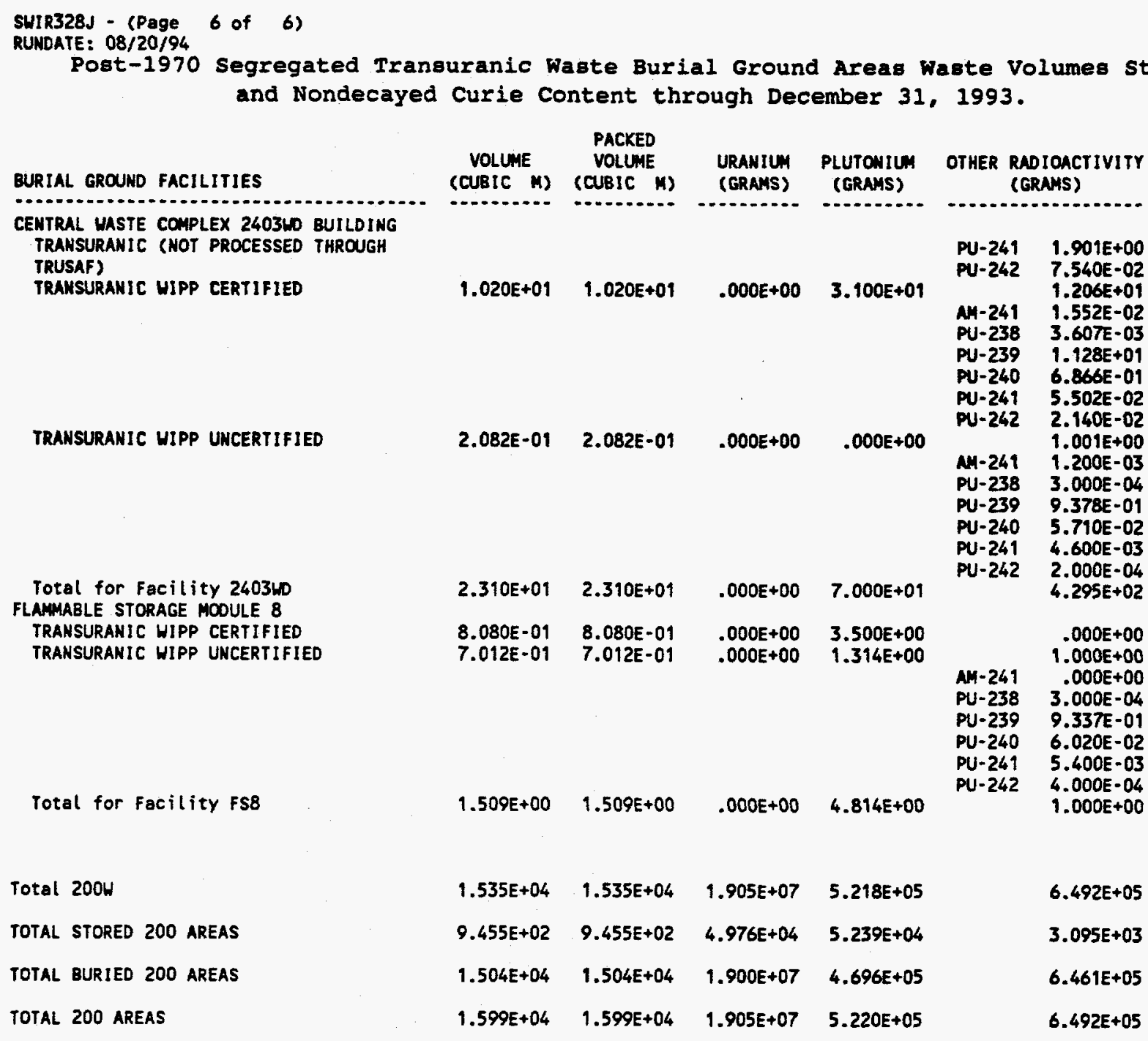


SUIR328K - (Page RUNDATE: 08/20/94

Post-1970 Segregated Transuranic Waste Burial Ground Areas Waste Volumes stored and Nondecayed Curie Content through December 31, 1993.

\begin{tabular}{|c|c|c|c|c|c|c|}
\hline BURIAL GROUND FACILITIES & $\begin{array}{l}\text { TOTAL } \\
\text { (CURIES) }\end{array}$ & $\begin{array}{l}\text { SR-90 } \\
\text { (CURIES) }\end{array}$ & $\begin{array}{l}\text { RU-106 } \\
\text { (CURIES) }\end{array}$ & $\begin{array}{c}\text { CS-137 } \\
\text { (CURIES) }\end{array}$ & \multicolumn{2}{|c|}{$\begin{array}{l}\text { DTHER RADIOACTIVITY } \\
\text { (CURIES) }\end{array}$} \\
\hline \multicolumn{7}{|l|}{$\begin{array}{l}\text { 200E AREA } \\
-\ldots . . .\end{array}$} \\
\hline $\begin{array}{l}\text { BURIAL GROUND } 218 E 128 \\
\text { TRANSURANIC (NOT PROCESSED THROUGH } \\
\text { TRUSAF) }\end{array}$ & $3.070 E+02$ & $6.107 E+00$ & $1.315 E+01$ & $6.521 E+00$ & $\begin{array}{l}\text { CE-141 } \\
T A-182\end{array}$ & $\begin{array}{l}2.000 E-03 \\
1.000 E-03 \\
1.000 E-03\end{array}$ \\
\hline Total for Facility 218E12B & $3.070 E+02$ & $6.107 E+00$ & $1.315 E+01$ & $6.521 E+00$ & & 2.000E-03 \\
\hline Total & $3.070 E+02$ & $6.107 E+00$ & $1.315 E+01$ & $6.521 E+00$ & & $2.000 E-03$ \\
\hline
\end{tabular}




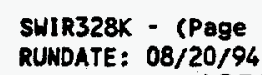

BURIAL GROUND FACILITIES

2OOH AREA

-..........

212N BUILDING

TRANSURANIC (NOT PROCESSED THROUGH TRUSAF)

Total for Facility 212N

BURIAL GROUND 218IJA

TRAHSURAHIC CNOT PROCESSEO THROUGH TRUSAF)-OFFSITE

TRANSURAMIC (NOT PROCESSED THROUGH TRUSAF)
(CURIES)

$\mathbf{S R}-90$

(CURIES)

RU-106

(CURIES)

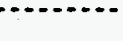

(CRIES)

$.000 E+00 \quad .000 E+00 \quad .000 E+00 \quad .000 E+00$

$.000 E+00$

$7.343 E+01$

$.000 E+00$

$.000 E+00$

$.000 E+00$

$2.785 E+00 \quad 1.381 E+00$

$5.531 E+02$

$1.293 E+00$

$.053 E+0$

1.231E+01

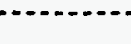

8.151E+01

5.000E-02

2. $371 E+05$

$1.621 E+00$

$5.755 E-03$

TRANSURANIC CLASSIFIED

TRANSURANIC IRRADIATED RESEARCH

MATERIALS-OFFSITE

TRANSURANIC IRRADIATEO RESEARCH MATERIALS

Total for Facility 21843A

BURIAL GROUND 21844B

TRANSURANIC (NOT PROCESSED THROUGH

TRUSAF)-OFFSITE

TRANSURANIC (NOT PROCESSED THROUGH TRUSAF)
TRANSURANIC CLASSIFIED-OFFSITE TRANSURANIC CAISSONS

Total for Facility 29844B

BURIAL GROUND $218 M 4 \mathrm{C}$

TRANSURANIC (NOT PROCESSED THROUGH TRUSAF)-OFFSITE

TRANSURANIC (NOT PROCESSEO THROUGH TRUSAF)

\section{$1.184 E+05$ \\ $3.562 \mathrm{E}+05$ \\ $2.743 \mathrm{E}+04$ \\ $1.984 E+01$ \\ $2.968 E+04$ \\ $4.417 \mathrm{E}+04$ \\ 5. $720 E+03$ \\ $4.669 \mathrm{E}+04$ \\ $7.306 E+00$ \\ $1.453 E-01$ \\ $3.129 E-01$ \\ $1.552 E-01$ \\ $2.087 E+03$ \\ 2. 122E+02 \\ $2.342 E+02$}

1.731E+00

6. 160E-03

$1.6995+04$
OTHER RADIOACTIVITY (CURIES)

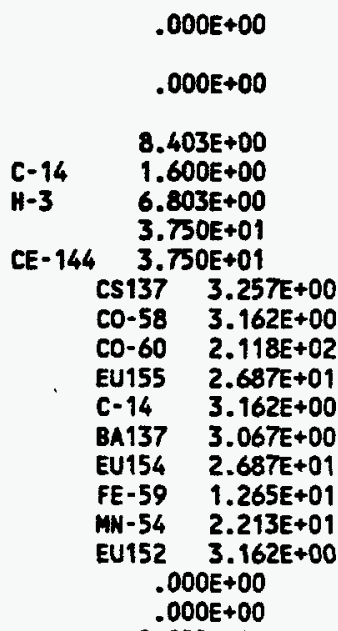

$9.922 E+04$

CE-144 5.299E+04

CO-60 2.232E+03

EU-155 3.810E+O1

KR-85 3.730E+03

PM- $147 \quad 4.023 E+04$

$.000 E+00$

$9.927 E+04$

$.000 E+00$

.000E+00

$\begin{array}{ll}\operatorname{cs} 137 & 6.269 E+00\end{array}$

CO-58 6.087E+0O

CO-60 4.078E+02

EU155 $5.174 E+01$

C- $14 \quad 6.087 E+00$

BA137 $5.904 E+00$

EU154 5.174E+01

FE-59 2.435E+01

MN-54 4.261E+01

EU152 6.087E+00

$.000 E+00$

$3.788 E+00 \quad 7.534 E-02 \quad 1.623 E-01 \quad 8.047 E-02$

$9.941 E+04 \quad 1.144 E+04 \quad 2.698 E+03 \quad 1.229 E+04$

$1.015 E+05 \quad 1.165 E+04 \quad 2.735 E+03 \quad 1.252 E+04$

co-60 $1.694 E+03$

PO-210 7.000E+00

1.701E+03

$1.279 E+02 \quad 2.543 E+00 \quad 5.477 E+00 \quad 2.733 E+00$

$.000 E+00$

$3.805 E+05$

$8.025 E+04$

$1.062 E+03$

$8.642 E+04$
$.000 E+00$

CS137 3.090E-05

CO-58 3.000E-05

CO-60 2.010E-03

EU155 $2.550 E-04$ 
SWIR328K - (Page 3 of 6$)$
RUNDATE: $08 / 20 / 94$

Post-1970 Segregated Transuranic Waste Burial Ground Areas Waste Volumes stored and Nondecayed Curie Content through December 31, 1993.

\author{
BURIAL GROUND FACILITIES \\ BURIAL GROUHO 218 W4 \\ TRAMSURANIC (NOT PROCESSED THROUGH \\ TRUSAF)
}

TRANSURANIC WIPP UNCERTIFIEO-OFFSITE TRANSURANIC MIPP UNCERTIFIED

TOTAL
(CURIES)

SR-90 (CURIES)

(c)

RU-106

(CURIES)

CS-137

(CURIES)

(C...........

$3.398 E+04$ $3.315 E+03$

6. $758 E+02$

$1.455 E+03$

$7.218 E+02$

$5.028 E+02$

$5.393 E+02$
TRANSURANIC SPECIAL CASE-OFFSITE

TRANSURANIC IRRADIATED RESEARCH

MATERIALS-OFFSITE

TRANSURANIC IRRADIATED RESEARCH

MATERIALS

\begin{abstract}
$5.300 E+00$
$5.997 \mathrm{E}+04$

1.054E-01

$1.193 E+03$

2.270E-01

$2.569 E+03$

$1.126 E-01$

$1.274 E+03$
\end{abstract}

$1.464 E+05$

$1.059 E+04$

$1.970 E+03$

$5.744 E+04$

$6.243 E+05$

Total for Facility 218U4C

TRANSURANIC WASTE STORAGE \& ASSAY FACILITY(TRUSAF)

TRANSURANIC (NOT PROCESSED THROUGH

TRUSAF)

$1.168 E+02$

$9.322 E+04$

7.153E+03

1.464E+05

$5.475 E+01$

2.028E-02

$3.368 E+00$

TRANSURANIC MIPP CERTIFIED

$1.306 E+0$

1.581E-01

$3.732 E+00$

\section{OTHER RADIOACTIVITY (CURIES)}

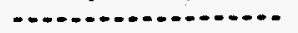

$$
\begin{array}{cc}
C-14 & 3.000 E-05 \\
\text { BA137 } & 2.910 E-05 \\
\text { EU154 } & 2.550 E-04 \\
\text { FE-59 } & 1.200 E-04 \\
\text { MN-54 } & 2.100 E-04 \\
\text { EU152 } & 3.000 E-05 \\
\multicolumn{1}{|c|}{\text {.000E }+00} \\
.000 E+00
\end{array}
$$

CS137 $5.150 E-06$

CO-58 5.000E-06

CO-60 3.35OE-04

EU155 4.250E-05

C-14 5.000E-06

BA137 4.850E-06

EU154 $4.250 E-05$

FE-59 2.000E-05

MN-54 3.500E-05

EU152 5.000E-06 $.000 E+00$ $.000 E+00$

\section{$3.679 E+03$}

CE-144 $1.282 E+03$

CO-58 $\quad 5.452 E+01$

CO-60 4.519E+02

CR-51 $1.116 E+02$

CS-134 8.591E+02

EU-152 1.880E+01

EU-154 3.026E+02

FE-59 5.713E+02

MN-54 2.603E+01

SB-125 8.350E-01

Cs137 1.545E+00

CO-58 $1.500 E+00$

CO-60 $\quad 1.005 E+02$

EU155 $1.275 E+01$

$C-14 \quad 1.500 E+00$

BA137 $1.455 E+00$

EU154 1.275E +01

FE-59 6.000E+00

MN-54 $1.050 E+01$

EU152 $1.500 E+00$

$3.679 E+03$

\begin{tabular}{ll}
\multicolumn{2}{c}{$.000 E+00$} \\
CS137 & $5.150 E-06$ \\
CO-58 & $5.000 E-06$ \\
CO-60 & $3.350 E=04$ \\
EU155 & $4.250 E-05$ \\
C-14 & $5.000 E-06$ \\
BA137 & $4.850 E-06$ \\
EU154 & $4.250 E-05$ \\
FE-59 & $2.000 E-05$ \\
WN-54 & $3.500 E-05$ \\
EU152 & $5.000 E-06$
\end{tabular}

5.000E-06

1.191E-03

C0-60 3.153E-05

CS-134 4.589E-04

EU-152 8.290E-05

EU-154 3.052E-04 


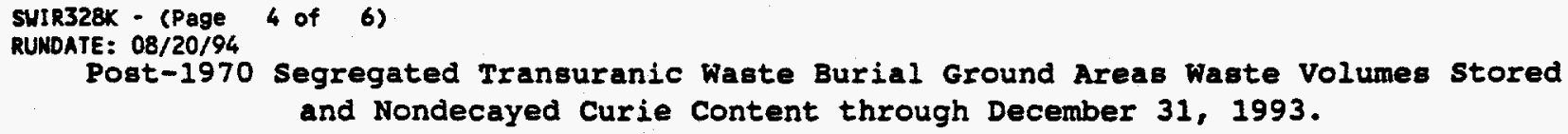

TRANSURANIC NIPP UNCERTIFIED

Total for Facility 224T

CENTRAL HASTE COMPLEX 2401W BUILDING TRANSURANIC (HOT PROCESSED THROUGH TRUSAF)

TRANSURANIC WIPP UNCERTIFIED

Total for Facility $2401 \mathrm{~W}$ CENTRAL WASTE COMPLEX $2402 \mathrm{~W}$ BUILDING TRANSURANIC MIPP UNCERTIFIED-OFFSITE TRANSURANIC WIPP UNCERTIFIED Total for Facility $2402 \mathrm{~W}$

CENTRAL WASTE COMPLEX 2402UB BUILDING TRANSURANIC (NOT PROCESSED THROUGH TRUSAF)

TRANSURANIC WIPP CERTIFIED

TRANSURANIC WIPP UNCERTIFIED

Total for Facility $2402 W B$

CENTRAL WASTE COMPLEX 2402WC BUILDING TRANSURANIC (HOT PROCESSED THROUGH TRUSAF)

TRANSURANIC HIPP CERTIFIED

TRANSURANIC WIPP UNCERTIFIED

Total for Facility 2402WC

CENTRAL HASTE COMPLEX 2402WG BUILDING TRANSURANIC (NOT PROCESSED THROUGH TRUSAF)

TRANSURANIC HIPP CERTIFIED

TRANSURANIC WIPP UNCERTIFIED-OFFSITE TRANSURANIC WIPP UNCERTIFIED Total for Facility 2402WG CENTRAL WASTE COMPLEX 2402WH BUILDING TRANSURANIC WIPP CERTIFIED TRANSURANIC WIPP UNCERTIFIED-OFFSITE TRANSURANIC WIPP UNCERTIFIED

Total for Facility 2402WH

CENTRAL HASTE COMPLEX 2402HL BUILDING TRAHSURANIC (NOT PROCESSED THROUGH TRUSAF)

TRANSURANIC UIPP UNCERTIFIED Total for Facility 2402WL CENTRAL UASTE COMPLEX 2403HA BUILDING

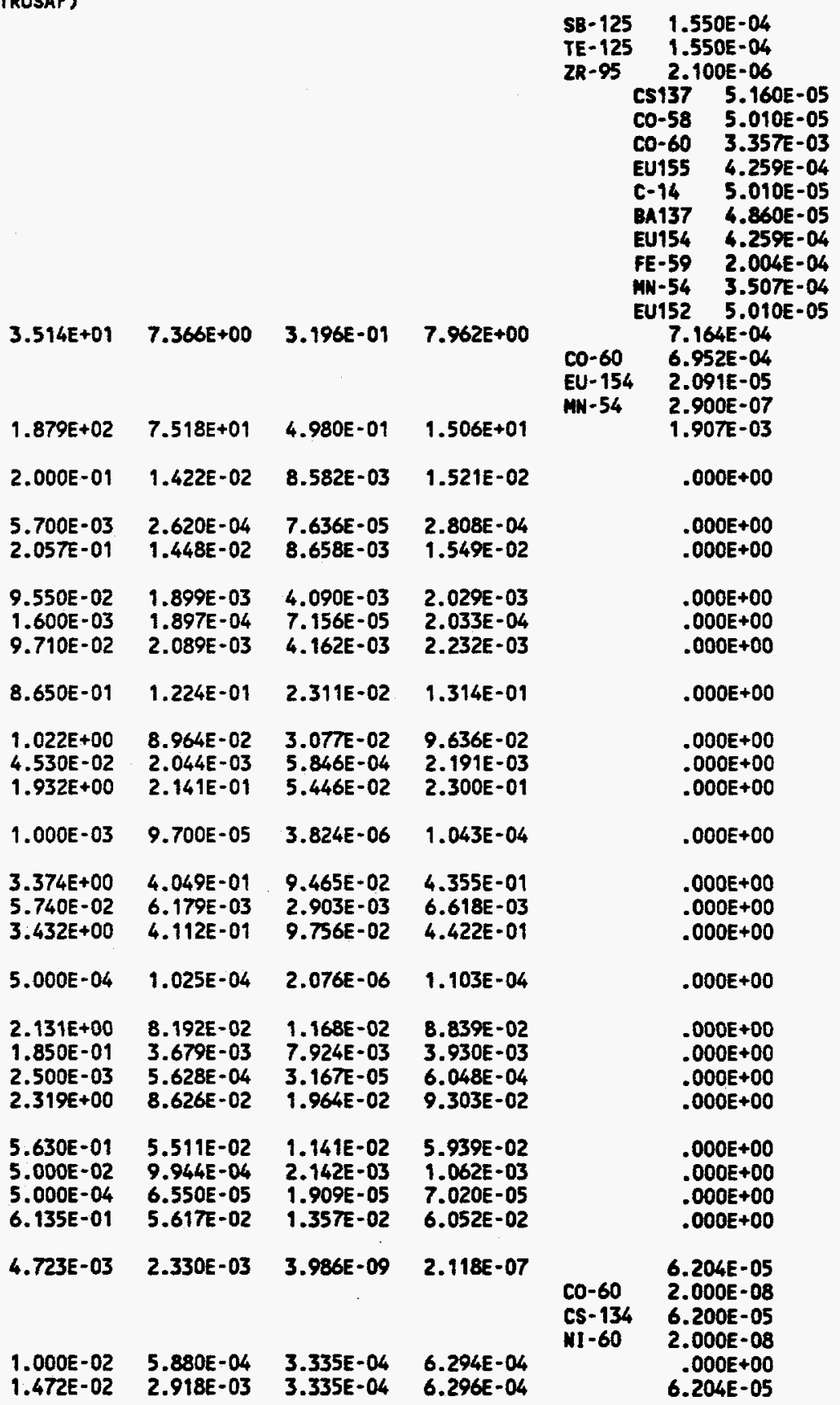

OTHER RADIOACTIVITY (CURIES) 
WHC-EP-0125-6

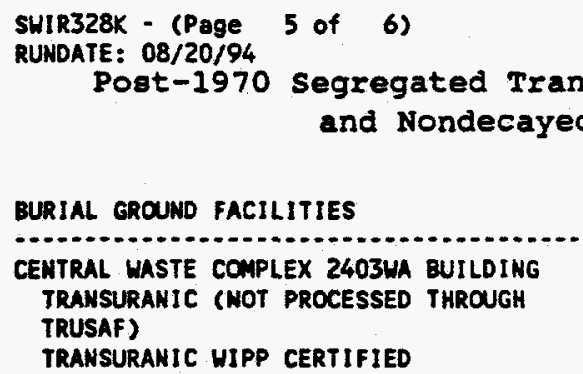

TRANSURANIC (NOT PROCESSED THROUGH TRUSAF)

TRANSURANIC WIPP CERTIFIED

TRANSURANIC MIPP UNCERTIFIED Total for Facility 2403WC

\begin{tabular}{cccc}
$\begin{array}{c}\text { TOTAL } \\
\text { (CURIES) }\end{array}$ & $\begin{array}{c}\text { SR-90 } \\
\text { (CURIES) }\end{array}$ & $\begin{array}{c}\text { RU-106 } \\
\text { (CURIES) }\end{array}$ & $\begin{array}{c}\text { CS-137 } \\
\text { (CURIES) }\end{array}$ \\
\hline $2.900 E-02$ & $5.514 E-03$ & $2.241 E-04$ & $5.926 E-03$ \\
\hline $1.666 E-01$ & $3.059 E-01$ & $8.403 E-02$ & $3.280 E-01$
\end{tabular}

$\begin{array}{llll}1.737 E+01 & 3.903 E+00 & 4.789 E-02 & 4.224 E+00 \\ 1.756 E+01 & 4.215 E+00 & 1.321 E-01 & 4.558 E+00 \\ 1.226 E+00 & 1.426 E-05 & 3.071 E-05 & 1.523 E-05\end{array}$

4.890E-01

2.504E-02

$1.207 E-03$

$4.542 E-02$

$7.212 E+00 \quad 1.494 E+00 \quad 1.035 E-01 \quad 1.630 E+00$

$\begin{array}{llll}1.722 E+01 & 3.861 E+00 & 4.716 E-02 & 4.176 E+00\end{array}$

$2.615 E+01 \quad 5.380 E+00 \quad 1.519 E-01 \quad 5.851 E+00$
OTHER RADIOACTIVITY (CURIES)

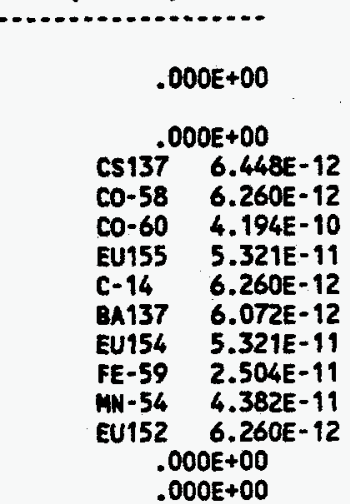

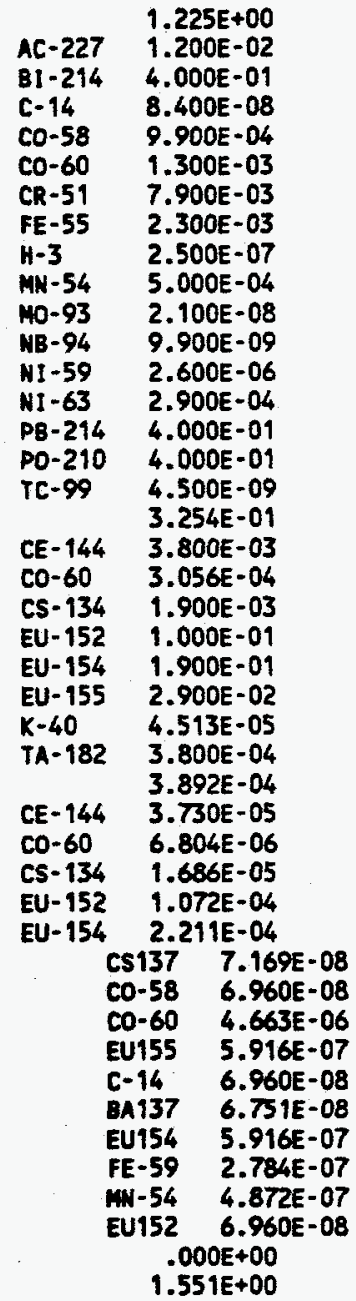


WHC-EP-0125-6

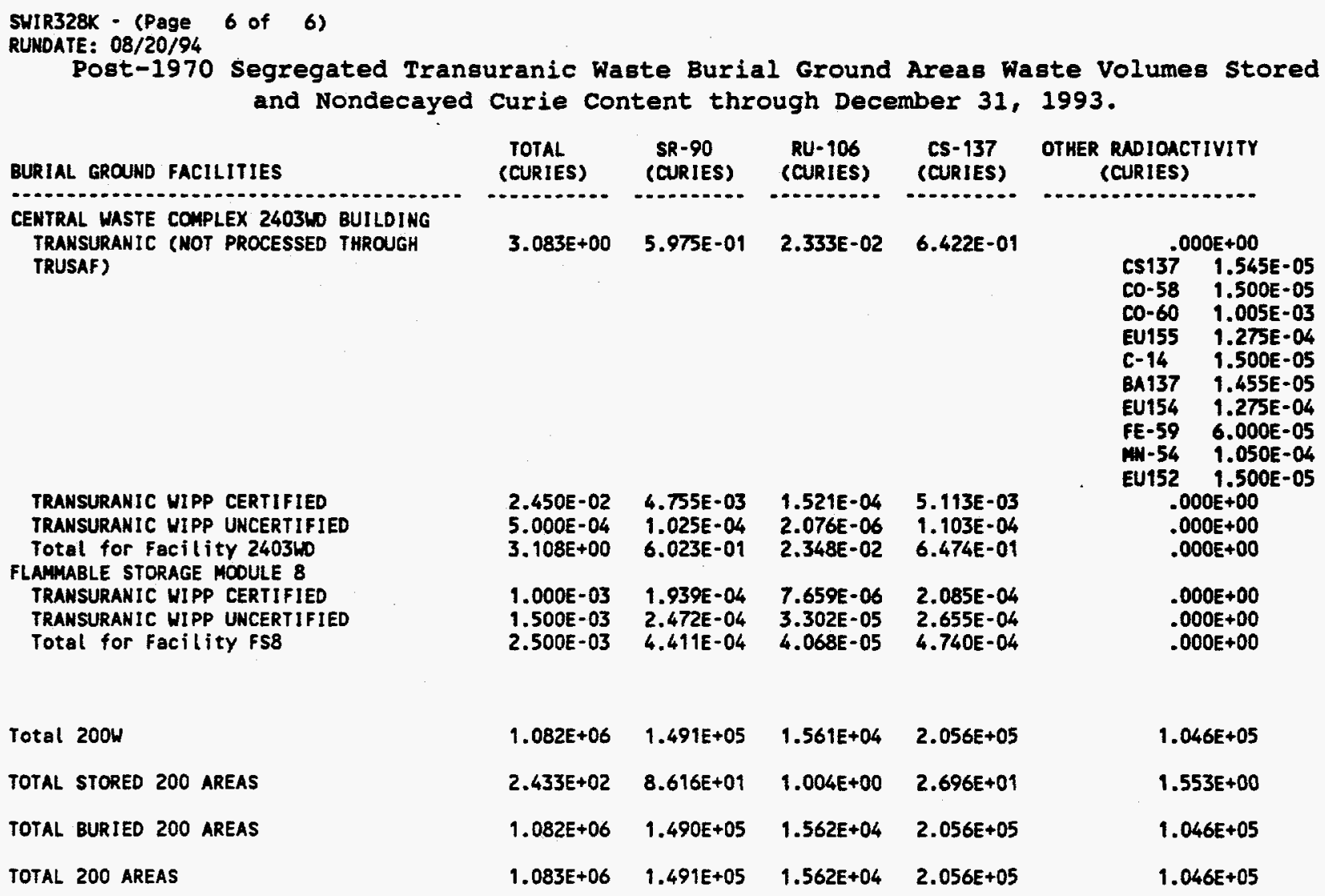


WHC-EP-0125-6

SUIR328L - (Page 1 of 6) RUNDATE: 08/20/94

Post-1970 Segregated Transuranic Waste Burial Ground Areas Decayed Curie Content through December 31, 1993.

BURIAL GROUND FACILITIES

C...............................................

200E AREA

CO.......

BURIAL GROUND 218E12B

TRANSURANIC (NOT PROCESSED THROUGH

TRUSAF)

rotal for Facility 218E12B

Total $200 E$
TOTAL

(CURIES)

SR-90

(CURIES)

(...........

RU-106

(CURIES)

CS-137

(CURIES)

OTHER RADIOACTIVITY (CURIES)

C.....................

$1.488 E+01$

$3.597 E+00$

3.605E-0

$3.933 E+00$

$1.488 E+01$

$3.597 E+00$

$3.605 E-06$

$3.933 E+00$

CE- 141
TA- 182

$.000 E+00$

TA-182 7.229E-25

(1)

$1.488 E+0$

$3.597 E+00$

$3.605 E-06 \quad 3.933 E+00$

$7.229 E-25$ 


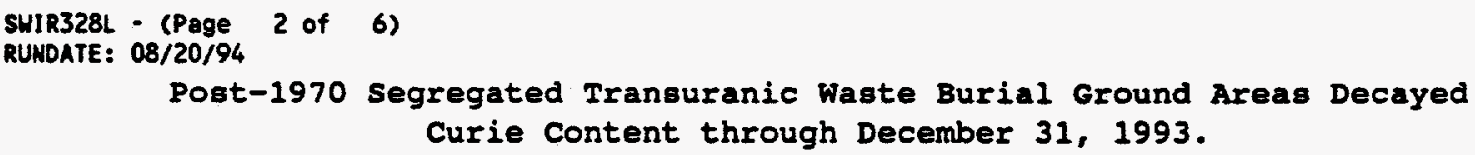

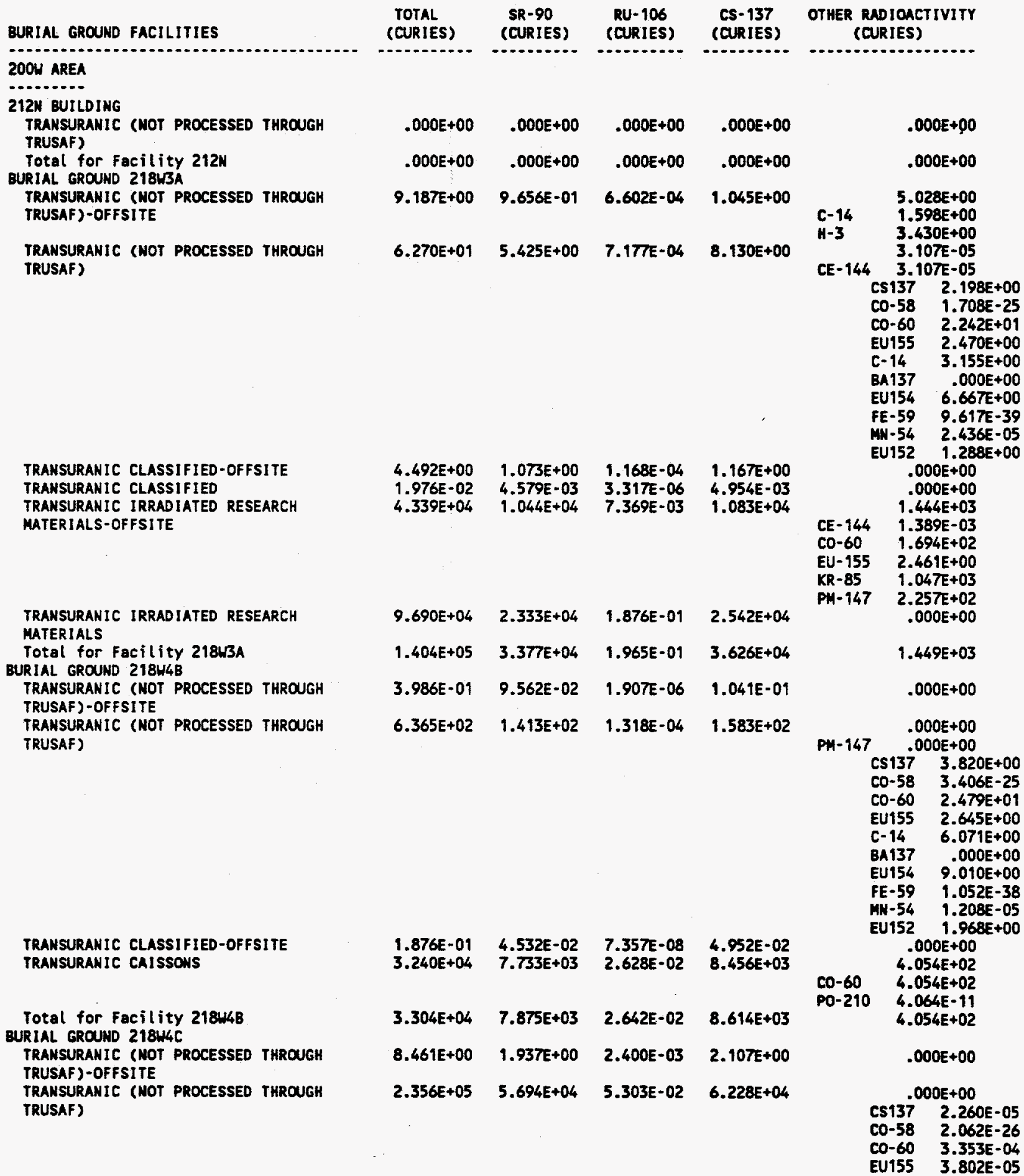


SUIR328L - (Page 3 of 6)

RUNDATE: 08/20/94

Post-1970 segregated Transuranic Waste Burial Ground Areas Decayed

Curie Content through December 31, 1993.

BURIAL GROUND FACILITIES

BURIAL GROUND 218 HC

TRANSURANIC (NOT PROCESSED THROUGH

TRUSAF)

TRANSURANIC WIPP UMCERTIFIED-OFFSITE TRANSURANIC UIPP UNCERTIFIEO
TOTAL

(CURIES)

SR-90

(CURIES)

(CURIES)

CS- 137

(CURIES)

2.677E+0

1.777E+03

$5.624 E+02$

4. 121E+02

8. $082 E+00$

2. $940 \mathrm{E}-01$

$6.056 E+02$

$4.461 E+02$

$\begin{array}{llll}3.296 E-01 & 7.718 E-02 & 2.927 E-05 & 8.357 E-02 \\ 4.360 E+03 & 9.593 E+02 & 4.934 E+00 & 1.034 E+03 \\ 1.154 E+05 & 7.935 E+03 & 1.195 E+02 & 5.052 E+04\end{array}$

TRANSURANIC SPECIAL CASE-OFFSITE TRANSURANIC IRRADIATED RESEARCH MATERIALS-OFFSITE

TRAKSURANIC IRRADIATED RESEARCH MATERIALS

$1.154 E+05$

$7.935 E+03$

$.195 E+02$

$.052 E+04$
Total for facility $218 W 4 C$

$3.599 E+05$

$6.682 E+04$

$1.328 E+02$

$1.149 E+05$

TRANSURAKIC UASTE STORAGE \& ASSAY FACILITY (TRUSAF)

TRANSURANIC (NOT PROCESSED THROUGH

$1.122 E+02$

$5.266 E+01$

$1.025 E-02$

$3.260 E+00$ TRUSAF)

TRANSURANIC MIPP CERTIFIED

$3.153 E+01$

$1.229 E+01$

8.411E-03

$3.308 E+00$
OTHER RADIOACTIVITY (CURIES)

(n.....................

C-14 2.995E-05

BA137 .000E+00

EU154 8.397E-05

FE-59 2.909E-38

MN-54 3.345E-09

EU152 1.467E-05

$.000 E+00$ $.000 E+00$

CS137 4.175E-06

CO-58 $3.170 E-20$

C0-60 1.008E-04

EU155 1.185E-05

C-14 4.995E-06

$8 A 137.000 E+00$

EU154 2.017E-05

FE-59 5.608E-28

MN-54 2.115E-08

EU152 3.094E-06

$.000 E+00$

$.000 E+00$

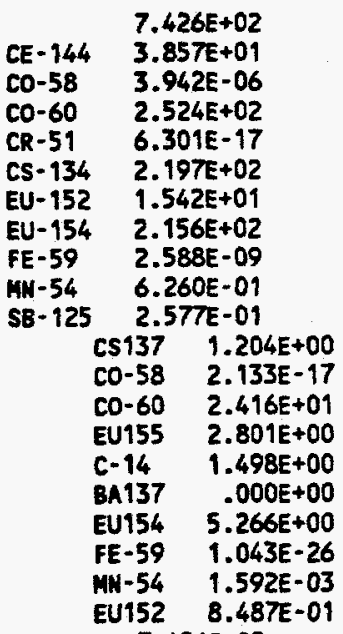

$7.426 E+02$

$$
\begin{array}{ll}
\text { CS137 }^{2} \text {.00E } & 5.132 E-06 \\
\text { CO-58 } & 2.890 E-06 \\
\text { CO-60 } & 3.283 E-04 \\
\text { EU155 } & 4.160 E-05 \\
\text { C-14 } & 5.000 E-06 \\
\text { BA137 } & .000 E+00 \\
\text { EU154 } & 4.197 E-05 \\
\text { FE-59 } & 8.372 E-06 \\
\text { WN-54 } & 3.091 E-05
\end{array}
$$

EU152 4.960E-06

7.839E-04

C0-60 2.649E-05

C5-134 2.940E-04

EU-152 7.757E-05

EU-154 2.749E-04 


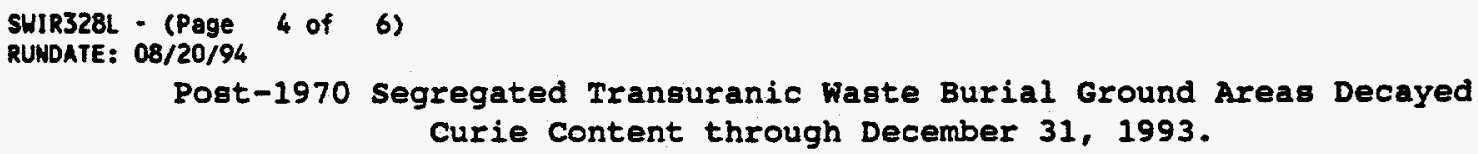

TRANSURANIC WIPP UNCERTIFIED

Total for Facility $224 \mathrm{~T}$

CENTRAL WASTE COMPLEX 24014 BUILDING TRANSURANIC (NOT PROCESSED THROUGH TRUSAF)

TRANSURANIC HIPP UNCERTIFIED

Total for Facility 2401W

CENTRAL HASTE COMPLEX $2402 \mathrm{~W}$ BUILDING TRANSURANIC WIPP UNCERTIFIED-OFFSITE TRANSURANIC MIPP UNCERTIFIED

Total for Facility $2402 \mathrm{~W}$

CENTRAL UASTE COMPLEX 2402UB BUILDING TRANSURANIC (NOT PROCESSED THROUGH TRUSAF)

TRANSURANIC MIPP CERTIFIED

TRANSURANIC UIPP UNCERTIFIED

Total for Facility 2402WB

CENTRAL WASTE COMPLEX 2402WC BUILDING TRANSURANIC (NOT PROCESSED THROUGH

TRUSAF)

TRANSURANIC UIPP CERTIFIED

TRAHSURANIC UIPP UNCERTIFIED

Total for Facility 2402WC

CENTRAL WASTE COMPLEX 2402WG BUILDING TRANSURANIC (NOT PROCESSED THROUGH

TRUSAF)

TRANSURANIC UIPP CERTIFIED

TRANSURANIC WIPP UHCERTIFIED-OFFSITE

TRANSURANIC UIPP UNCERTIFIED

Total for Facility 2402WG

CENTRAL WASTE COMPLEX 2402WH BUILDING TRANSURANIC WIPP CERTIFIED

TRANSURANIC WIPP UNCERTIFIED-OFFSITE TRANSURANIC WIPP UNCERTIFIED

Total for Facility 2402WH

CENTRAL HASTE COMPLEX 2402UL BUILDING TRANSURAMIC (NOT PROCESSED THROUGH TRUSAF)

TRANSURANIC UIPP UNCERTIFIED

Total for Facility 2402WL

CENTRAL WASTE COMPLEX 2403WA BUILDING

\section{$2.703 E+01$ \\ $6.387 E+0$ \\ $1.279 E-02$ \\ $6.947 E+00$}

$1.708 E+02$

$7.134 E+01$

3. 146E-02

$1.351 E+01$

4.814E-02

1.116E-02

8.082E-06

1.207E-02

1.130E-03

4.927E-02

2.358E-04

1. 139E-02

3.657E-06

2.539E-04

8.077E-03

1.622E-03

8.057E-0.

8.883E-03

$1.689 E-04$

4.204E-01

$1.790 E-03$

4.350E-05

$2.415 \mathrm{E}-06$

4.591E-05

$1.744 E-03$

$1.819 E-04$

9.819E-02

5.798E-05

$1.926 E-03$

3.481E-01

$7.746 E-02$

8.743E-03

$1.835 E-03$

7.773E-0 01

1.775E-01

$5.430 \mathrm{E}-04$

1.065E-01

$2.572 E-05$

8.380E-02

1.976E-03

4.399E-04

9.300E-05

(6)

1.922E-01

$1.515 E+00$

2.626E-02

$1.541 E+00$

3.450E-01

5.495E-03

4.973E-04

3.506E-01

$1.145 \mathrm{E}-06$

$1.002 E-04$

1.386E-03

8.113E-05

1.469E-03

3.737E-01

5.916E-03

3.797E-01

$1.965 E-06$

1.101E-04

3.062E-01

1.619E-02

1.023E-04

7.071E-02

2.610E-03

3.255E-01

2.080E-01

3.173E-03

5.422E-04

3.014E-04

$1.125 E-04$

8.988E-06

7.678E-02

3.411E-03

7.452E-02

4.249E-04

.835E-04

4.763E-02

8.495E-04

4.237E-03

2.894E-04

2.125E-01

5.945E-05

4.854E-02

4.643E-03

2.296E-03

2.155E-04

2.312E-05

1.167E-06

2.398E-04

5.166E-02

$9.135 E-04$

6.395E-05

5.264E-02

2.609E-09

2.088E-07

2.536E-03 7.180E-03

5.209E-O4 2.817E-03
1.025E-05 $1.025 E-05$
5.605E-04 5.607E-04
OTHER RADIOACTIVITY (CURIES)

-

SB-125 1.105E-04

TE-125 4.774E-0?

2R-95 1.110E-08

CS137 5.006E-05

CO-58 4.383E-07

CO-60 2.820E-03

EU155 3.539E-04

C-14 5.009E-05

BA137 .000E+00

EU154 3.823E-04

FE-59 1.079E-07

MN-54 1.198E-04

EU152 4.673E-05

4. $149 E-04$

CO-60 3.976E-04

EU-154 1.729E-05

MN-54 1.933E-08

$1.199 E-03$

$.000 E+00$

$.000 E+00$

$.000 E+00$

$.000 E+00$

$.000 E+00$

$.000 E+00$

$.000 E+00$

$.000 E+00$

$.000 E+00$

$.000 E+00$

$.000 E+00$

$.000 E+00$

$.000 E+00$

$.000 E+00$

$.000 E+00$

$.000 E+00$

$.000 E+00$

$.000 E+00$

$.000 E+00$

$.000 E+00$

$.000 E+00$

$.000 E+00$

$.000 E+00$

5. 047E-05

CO-60 1.845E-08

CS- $134 \quad 5.045 E-05$

$\mathrm{NI}-60 \quad .000 \mathrm{E}+00$

$.000 E+00$

5.047E-05 
SHIR328L - (Page 5 of 6)

RUNDATE: 08/20/94

Post-1970 Segregated Transuranic Waste Burial Ground Areas Decayed Curie Content through December 31, 1993.

BURIAL GROUHD FACILITIES
CENTRAL WASTE COMPLEX 2403WA BUILDING
TRANSURANIC (NOT PROCESSED THROUGH
TRUSAF)
TRAMSURANIC WIPP CERTIFIED

TRANSURANIC MIPP UNCERTIFIED Total for facility 2403WA CENTRAL WASTE COMPLEX 2403WC BUILDING TRANSURANIC CNOT PROCESSED THROUGH TRUSAF) -OFFSITE

TRANSURANIC (NOT PROCESSEO THROUGH TRUSAF)

TRANSURANIC HIPP CERTIFIED

TRANSURANIC MIPP UNCERTIFIED Total for Facility 2403 WC

\begin{tabular}{cccc}
$\begin{array}{c}\text { TOTAL } \\
\text { (CURIES) }\end{array}$ & $\begin{array}{c}\text { SR-90 } \\
\text { (CURIES) }\end{array}$ & $\begin{array}{c}\text { RU-106 } \\
\text { (CURIES) }\end{array}$ & $\begin{array}{c}\text { CS-137 } \\
\text { (CURIES) }\end{array}$ \\
\hline $2.591 E-02$ & $5.355 E-03$ & $9.680 E-05$ & $5.763 E-03$ \\
$1.151 E+00$ & $2.588 E-01$ & $8.659 E-04$ & $2.795 E-01$
\end{tabular}

$\begin{array}{llll}1.396 E+01 & 3.351 E+00 & 3.771 E-04 & 3.649 E+00 \\ 1.514 E+01 & 3.615 E+00 & 1.340 E-03 & 3.934 E+00 \\ 1.914 E-01 & 1.410 E-05 & 2.248 E-05 & 1.507 E-05\end{array}$

4.632E-01 2.453E-02 $\quad 7.689 E-04 \quad 4.469 E-02$

$5.490 E+00 \quad 1.288 E+00 \quad 2.409 E-03 \quad 1.416 E+00$

1.389E+01

2.004E+01

$3.331 E+00$

$4.643 \mathrm{E}+00$

5.636E-04

$3.626 E+00$

3.764E-03

$5.086 E+00$
OtHER RADIOACTIVITY (CURIES)

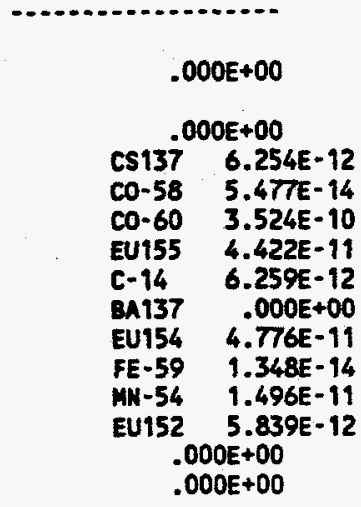

AC-227
$B 1-214$
$C-14$
$C O-58$
$C O-60$
$C R-51$
FE-55
$H-3$
$M N-54$
$M O-93$
$N B-94$
$N I-59$
$M I-63$
PB-214
$P O-210$
$T C-99$
$C E-144$
$C O-60$
$C S-134$
$E U-152$
$E U-154$
$E U-155$
$K-40$
TA- 182

1.911E-01

1.183E-02

$.000 E+00$

8.399E-08

$1.968 E-04$

$1.225 E-03$

$1.273 E-04$

2.047E-03

$2.438 E-07$

$3.467 \mathrm{E}-04$

2.100E-08

$9.900 E-09$

2.600E-06

2.891E-04

$.000 E+00$

1.751E-01

4.500E-09

3.111E-01

2.416E-03

3.028E-04

$1.601 E-03$

$9.736 E-02$

1.823E-01

2.701E-02

4.513E- 05

TA-182 1.239E-04

3.286E-04

CE-144 1.210E-05

CO-60 5.762E-06

CS-134 1.102E-05

EU-152 1.003E-04

EU-154 1.994E-04

C5137 6.954E-08

CO-58 6.089E-10

C0-60 3.918E-06

EU155 4.916E-07

C-14 6.959E-08

BA137 .000E+00

EU154 5.310E-07

FE-59 $1.498 E-10$

MN-54 1.664E-07

EU152 6.492E-08

$.000 E+00$

5.026E-09 


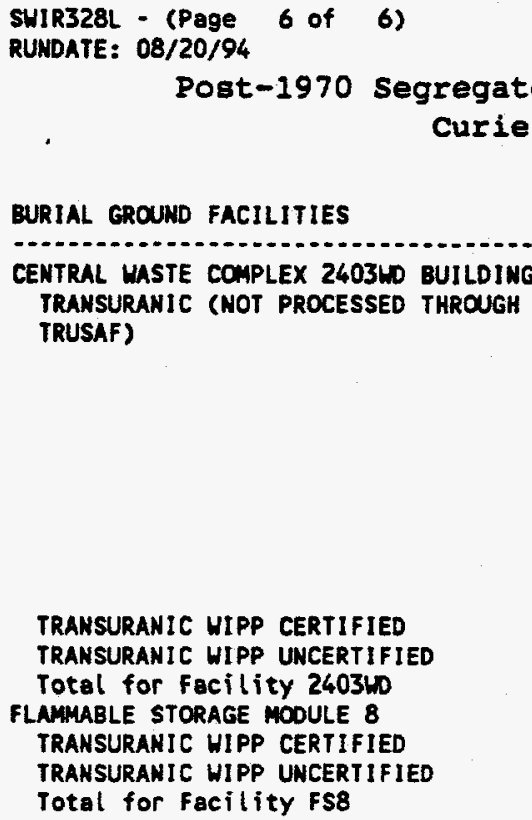
TRUSAF)

TLMMABLE STOR

Total for Facility FSB

$2.821 E+00$

5.814E-O

(CURIES)

CS-137 (CURIES)

OTHER RADIOACTIVITY (CURIES)

\subsection{E-02 4.979E-04 2.844E+00 \\ 8.736E-04 $1.114 E-03$ $1.988 E-03$}

4.673E-03 $1.024 E-04$ 5.862E-O

$8.832 E-05$ $1.991 E-06$

1.084E-02

$5.030 E-03$

$1.102 E-04$

6.310E-01

Total 200W

TOTAL STOREO 200 AREAS

TOTAL BURIED 200 AREAS

TOTAL 200 AREAS
1.854E-04 2.117E-06 1.997E-04

2.330E-04 3.450E-06 2.509E-04

4.184E-04 5.567E-06 4.506E-04

$5.335 E+05$

$1.085 E+05$

$1.331 E+02$

$1.598 E+05$

5.024E-02

2.389E+01

$5.333 E+05$

8.085E+01

$1.331 E+02$

$1.598 E+05$

$5.335 E+05$

$1.085 E+05$

$1.331 E+02$

$1.598 E+05$
$.000 E+00$

$\begin{array}{rr}\text { CS137 } & 1.541 E-05 \\ \text { CO-58 } & 9.981 E-06 \\ \text { CO-60 } & 9.901 E-04 \\ \text { EU155 } & 1.255 E-04 \\ \text { C-14 } & 1.500 E-05 \\ \text { 8A137 } & .000 E+00 \\ \text { EU154 } & 1.263 E-04 \\ \text { FE-59 } & 3.143 E-05 \\ \text { MN-54 } & 9.573 E-05 \\ \text { EU152 } & 1.491 E-05 \\ .000 E+00 \\ .000 E+00 \\ .000 E+00 \\ .000 E+00 \\ .000 E+00 \\ .000 E+00\end{array}$

$2.598 E+03$

5.038E-01

2.597E+03

$2.598 E+03$ 
WHC-EP-0125-6

SUIR328M - (Page 9 of 9)

RUNDATE: $08 / 20 / 94$

Transuranic and Nontransuranic Waste Received for Disposal or Storage for the Calendar Year from January 1 to December 31, 1993.

BURIAL GROUND FACILITIES

VOLINe

BURAL GROUN FACILITIES

(CUBIC $M$ )

VOLUME

URANIU: PLUTONILA OT

200E AREA

BURIAL GROUND 218E10

LON-LEVEL NONINDUSTRIAL

BURIAL GROUND 218E12B

LOW-LEVEL INDUSTRIAL-OFFSITE

9.072E+01

9.072E+01

$.000 E+00$

$.000 E+00$

$.000 E+00$

$6.286 E+03$

$6.286 E+03$

$.000 E+00 \quad .000 E+00$

NH-241

CN-244

PU-239

PU-240

PU-241

1.339E-03

2.261E-05

$9.230 E-07$

9.991E-04

$2.729 E-04$

4.316E-05

Total $200 E$

$6.377 E+03 \quad 6.377 E+03 \quad .000 E+00 \quad .000 E+00$

1.339E-03 


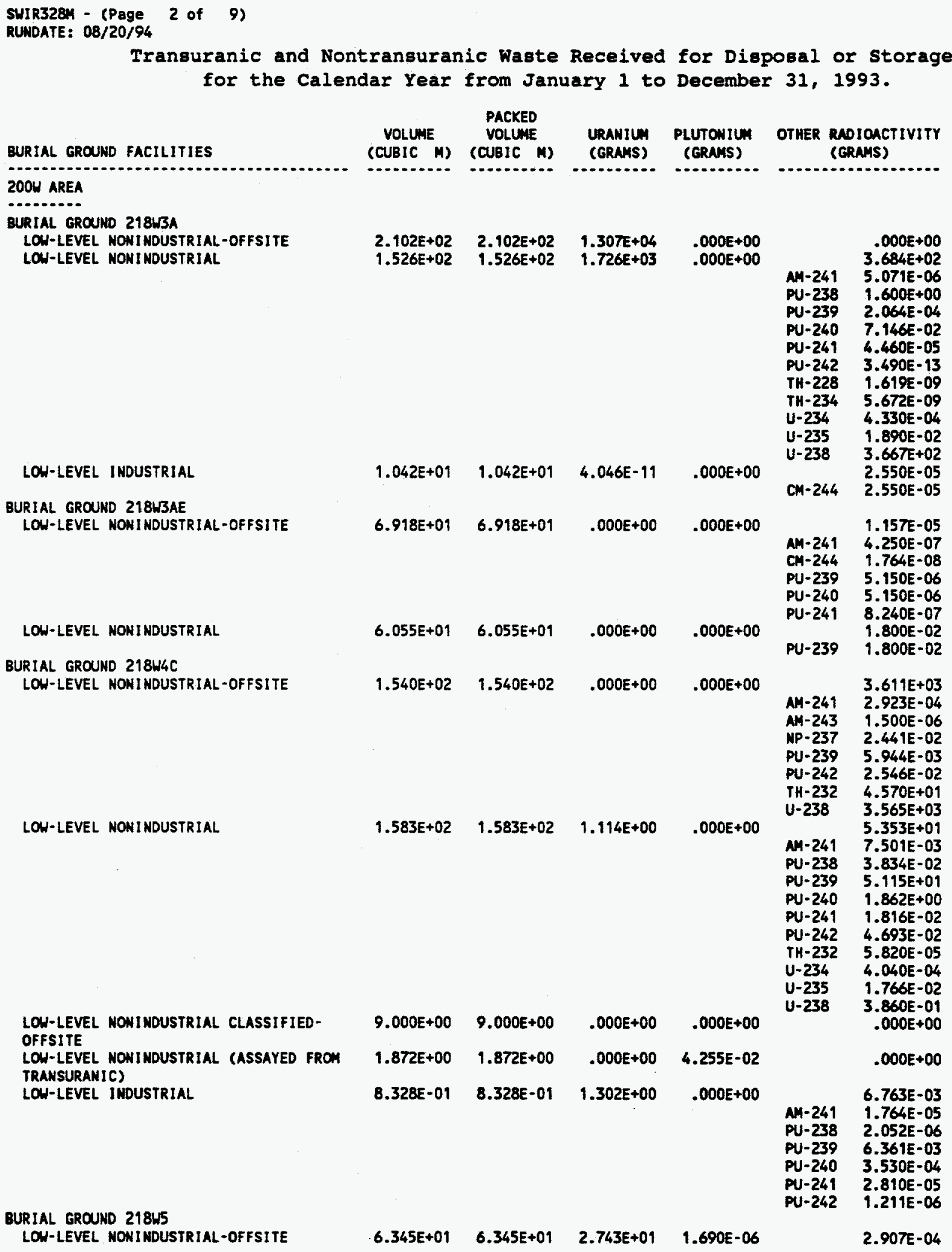




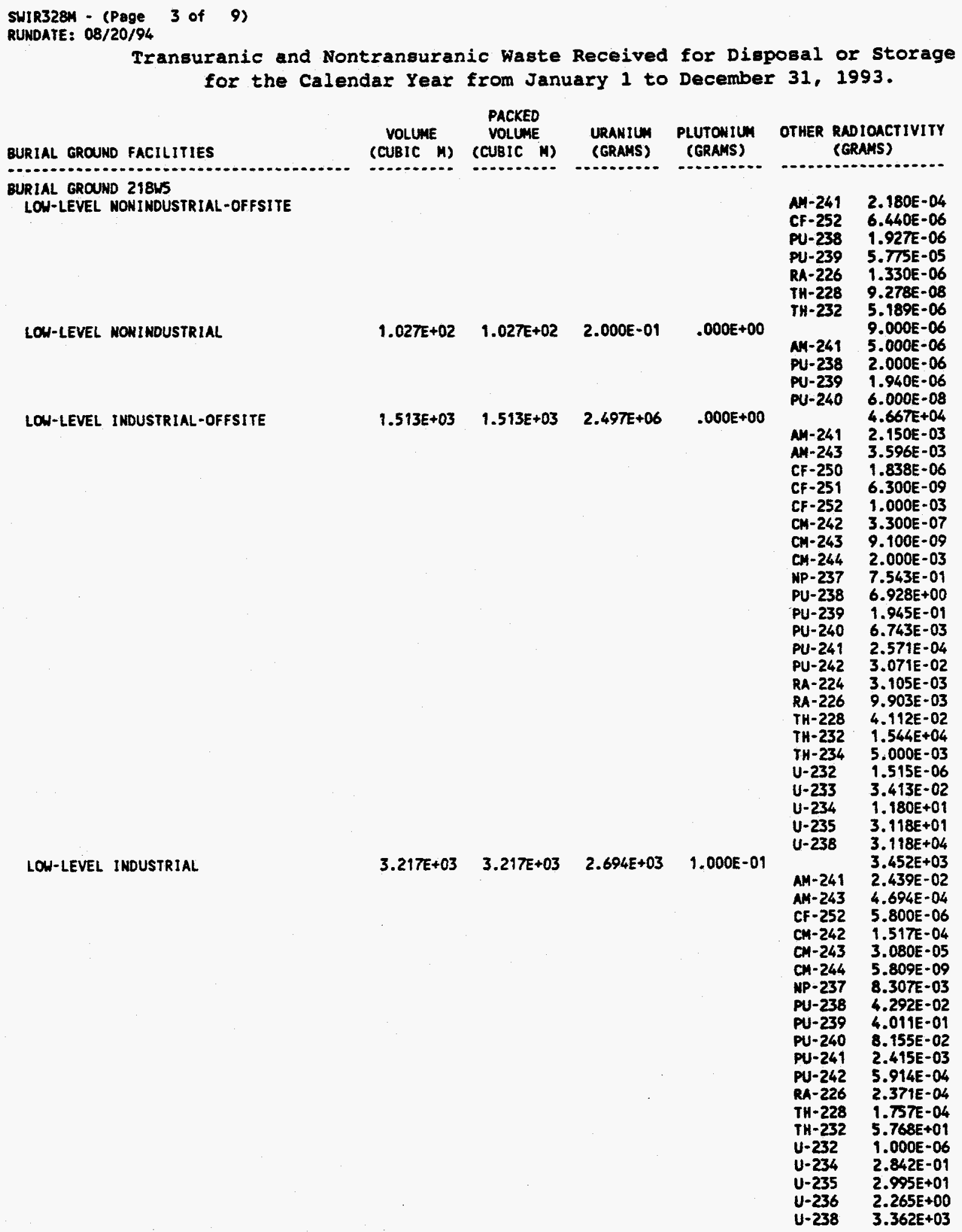

TRANSURANIC MASTE STORAGE ASSAY FACILITY(TRUSAF) 


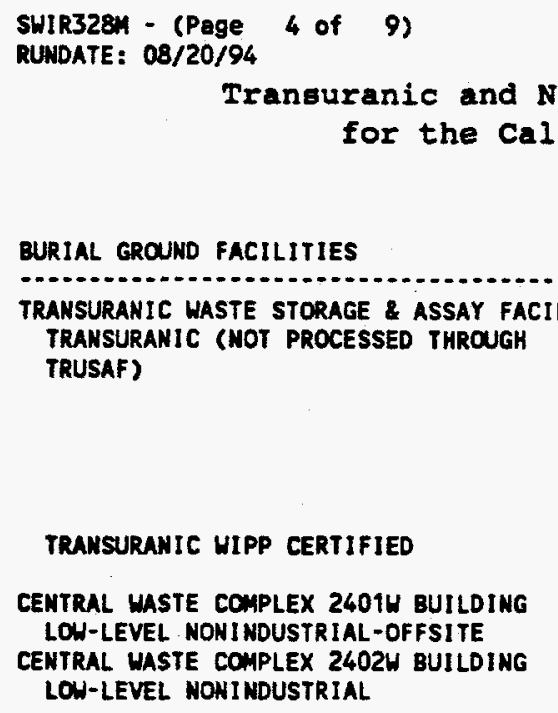

$\begin{array}{cccc}1.019 E+01 & 1.019 E+01 & .000 E+00 & 1.671 E+03 \\ 2.082 E-01 & 2.082 E-01 & .000 E+00 & .000 E+00 \\ 2.082 E-01 & 2.082 E-01 & .000 E+00 & .000 E+00\end{array}$

CENTRAL HASTE COMPLEX 2402WB BUILDING LON-LEVEL NOWINDUSTRIAL

CENTRAL HASTE COMPLEX 240ZWC BUILDING TRANSURANIC (NOT PROCESSED THROUGH TRUSAF)

LOW-LEVEL NONINDUSTRIAL-OFFSITE LOW-LEVEL NONINDUSTRIAL

\begin{abstract}
$4.997 \mathrm{E}+00$
\end{abstract}
OTHER RADI (GRAMS)

$\begin{array}{ll} & 1.439 E+03 \\ M M-241 & 6.256 E+00 \\ P U-238 & 3.970 E-01 \\ P U-239 & 1.348 E+03 \\ P U-240 & 7.685 E+01 \\ P U-241 & 6.933 E+00 \\ P U-242 & 2.740 E-01 \\ & 1.375 E+00 \\ \text { AM-241 } & 1.375 E+00\end{array}$

$.000 E+00$

1.5TOE-OG

AM-241 $1.740 E-07$

PU-238 4.600E-08

PU-239 1.470E-04

PU-240 8.980E-06

PU-241 7.330E-07

PU-242 3.080E-08

1.074E-01

$\begin{array}{ll}\text { AM-241 } & 1.493 E-05 \\ \text { MP-237 } & 7.450 E-02\end{array}$

PU-238 1.935E-06

PU-239 3.722E-03

PU-240 2.235E-04

PU-241 9.973E-05

PU-242 6.476E-07

U-234 2.885E-05

U-235 1.261E-03

U-238 2.756E-02

$2.080 E-01 \quad 2.080 E-01 \quad .000 E+00 \quad .000 E+00$

AM- 241

1.055E-06

1.070E-09

PU-238 3.100E-10

PU-239 9.880E-07

PU-240 6.020E-08

PU-241 5.010E-09

PU-242 2.070E-10

$2.070 E-10$
$.000 E+00$

4. $935 E-01$
Ai-241 2.644E-05

CM-242 5.536E-16

NP-237 4.920E-01

PU-238 7.127E-08

PU-239 1.425E-03

PU-240 1.368E-05

PU-241 1.128E-06

PU-242 5.871E-08

CENTRAL WASTE COMPLEX 2402WG BUILDING TRANSURANIC (NOT PROCESSED THROUGH TRUSAF)

$2.082 E-01 \quad 2.082 E-01 \quad .000 E+00 \quad .000 E+00$

NA-241

PU-238

PU-239

PU-240

PU-241

PU-242

2.002E-03

$1.912 E-06$

5.880E-07

1.875E-03

$1.142 E-04$

$9.624 E-06$

$3.123 E+00 \quad 3.123 E+00 \quad .000 E+00 \quad .000 E+00$

AM-241
$3.920 E-07$

$2.868 E-05$ 


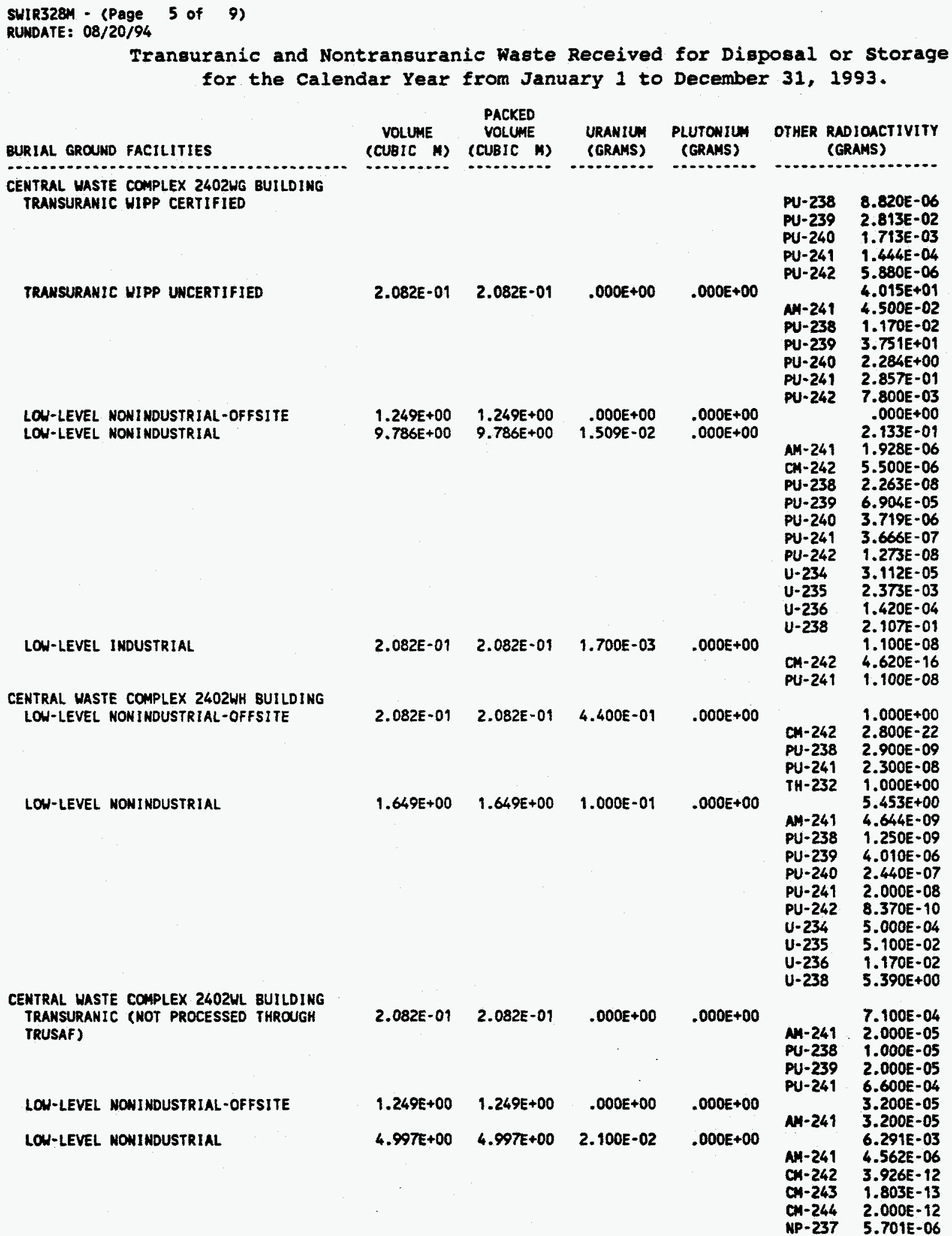




\begin{tabular}{|c|c|c|c|c|c|c|}
\hline \multirow[b]{2}{*}{ BURIAL GROUND FACILITIES } & \multirow[b]{2}{*}{ YOLLME } & \multirow[b]{2}{*}{$\begin{array}{l}\text { PACKED } \\
\text { YOLUME }\end{array}$} & $\begin{array}{l}\text { Received } \\
\text { ary } 1 \text { to }\end{array}$ & \multicolumn{3}{|c|}{$\begin{array}{l}\text { for Disposal or Storage } \\
\text { December } 31,1993 .\end{array}$} \\
\hline & & & $\begin{array}{l}\text { URANIUM } \\
\text { (GRAMS) }\end{array}$ & $\begin{array}{l}\text { PLUTONIUM } \\
\text { (GRAMS) }\end{array}$ & OTHER RI & $\begin{array}{l}\text { IOACTIVITY } \\
\text { ANS) }\end{array}$ \\
\hline $\begin{array}{l}\text { CENTRAL WASTE COMPLEX 2402WL BUILOING } \\
\text { LOU-LEVEL NONINDUSTRIAL }\end{array}$ & & & & & $\begin{array}{l}P U-238 \\
P U-239 \\
P U-240 \\
P U-241 \\
P U-242 \\
R A-226 \\
U-234 \\
U-235 \\
U-238\end{array}$ & $\begin{array}{l}1.256 E-08 \\
3.757 E-05 \\
1.637 E-06 \\
1.307 E-05 \\
3.640 E-09 \\
7.690 E-10 \\
6.220 E-06 \\
2.720 E-04 \\
5.950 E-03\end{array}$ \\
\hline $\begin{array}{l}\text { CENTRAL WASTE COMPLEX 2403HA BUILDING } \\
\text { LOW-LEVEL NOWINDUSTRIAL-OFFSITE }\end{array}$ & $1.064 E+02$ & $1.064 E+02$ & $3.791 E+02$ & $.000 E+00$ & $\begin{array}{l}A H-241 \\
A M-243 \\
C H-242 \\
M P-237 \\
P U-238 \\
P U-239 \\
P U-240 \\
P U-241 \\
R A-226 \\
T H-228 \\
T H-232 \\
T H-234 \\
U-233 \\
U-234 \\
U-235 \\
U-238\end{array}$ & $\begin{array}{l}1.229 E+03 \\
1.869 E-02 \\
2.697 E-03 \\
5.500 E-09 \\
2.430 E+00 \\
1.659 E-05 \\
5.600 E-05 \\
2.533 E-05 \\
5.957 E-05 \\
1.146 E-02 \\
1.400 E-07 \\
4.959 E+02 \\
2.900 E-10 \\
1.711 E-04 \\
5.000 E-02 \\
5.270 E+00 \\
7.256 E+02\end{array}$ \\
\hline LOW-LEVEL NONINDUSTRIAL & $9.095 E+01$ & $9.095 \mathrm{E}+01$ & $3.844 E+04$ & $.000 E+00$ & $\begin{array}{l}M M-241 \\
A M-243 \\
C M-242 \\
C M-243 \\
C M-244 \\
M P-237 \\
P U-238 \\
P U-239 \\
P U-240 \\
P U-241 \\
P U-242 \\
T H-232 \\
T H-234 \\
U-234 \\
U-235 \\
U-236 \\
U-238\end{array}$ & $\begin{array}{l}1.640 E+01 \\
5.907 E-01 \\
1.800 E-05 \\
1.741 E-12 \\
2.506 E-10 \\
2.792 E-09 \\
2.685 E+00 \\
1.830 E-03 \\
1.121 E+01 \\
6.978 E-01 \\
8.086 E-01 \\
1.795 E-03 \\
9.200 E-06 \\
1.030 E-06 \\
1.996 E-04 \\
9.743 E-03 \\
1.420 E-04 \\
3.942 E-01\end{array}$ \\
\hline LOH-LEVEL INDUSTRIAL & $2.082 E-01$ & $2.082 E-01$ & $.000 E+00$ & $.000 E+00$ & $\begin{array}{l}\text { AM-249 } \\
\text { PU-238 } \\
\text { PU-239 } \\
\text { PU-240 }\end{array}$ & $\begin{array}{l}8.138 E-04 \\
7.900 E-06 \\
2.850 E-05 \\
7.700 E-04 \\
7.400 E-06\end{array}$ \\
\hline $\begin{array}{l}\text { CENTRAL WASTE COMPLEX 2403UC BUILDING } \\
\text { TRANSURANIC (NOT PROCESSED THROUGH } \\
\text { TRUSAF)-OFFSITE } \\
\text { TRAWSURAHIC (NOT PROCESSED THROUGH } \\
\text { TRUSAF) }\end{array}$ & $\begin{array}{l}2.082 E-01 \\
2.977 E+01\end{array}$ & $\begin{array}{l}2.082 E-01 \\
2.977 E+01\end{array}$ & $\begin{array}{l}.000 E+00 \\
.000 E+00\end{array}$ & $\begin{array}{r}.000 E+00 \\
4.937 E+02\end{array}$ & $\begin{array}{l}\text { RA-226 } \\
\text { AM-241 } \\
\text { AM-243 } \\
\text { CH-243 } \\
\text { CM-244 } \\
\text { NP-237 }\end{array}$ & $\begin{array}{l}4.000 E-01 \\
4.000 E-01 \\
4.894 E-01 \\
4.499 E-01 \\
2.770 E-04 \\
5.950 E-07 \\
1.930 E-04 \\
3.265 E-02\end{array}$ \\
\hline
\end{tabular}




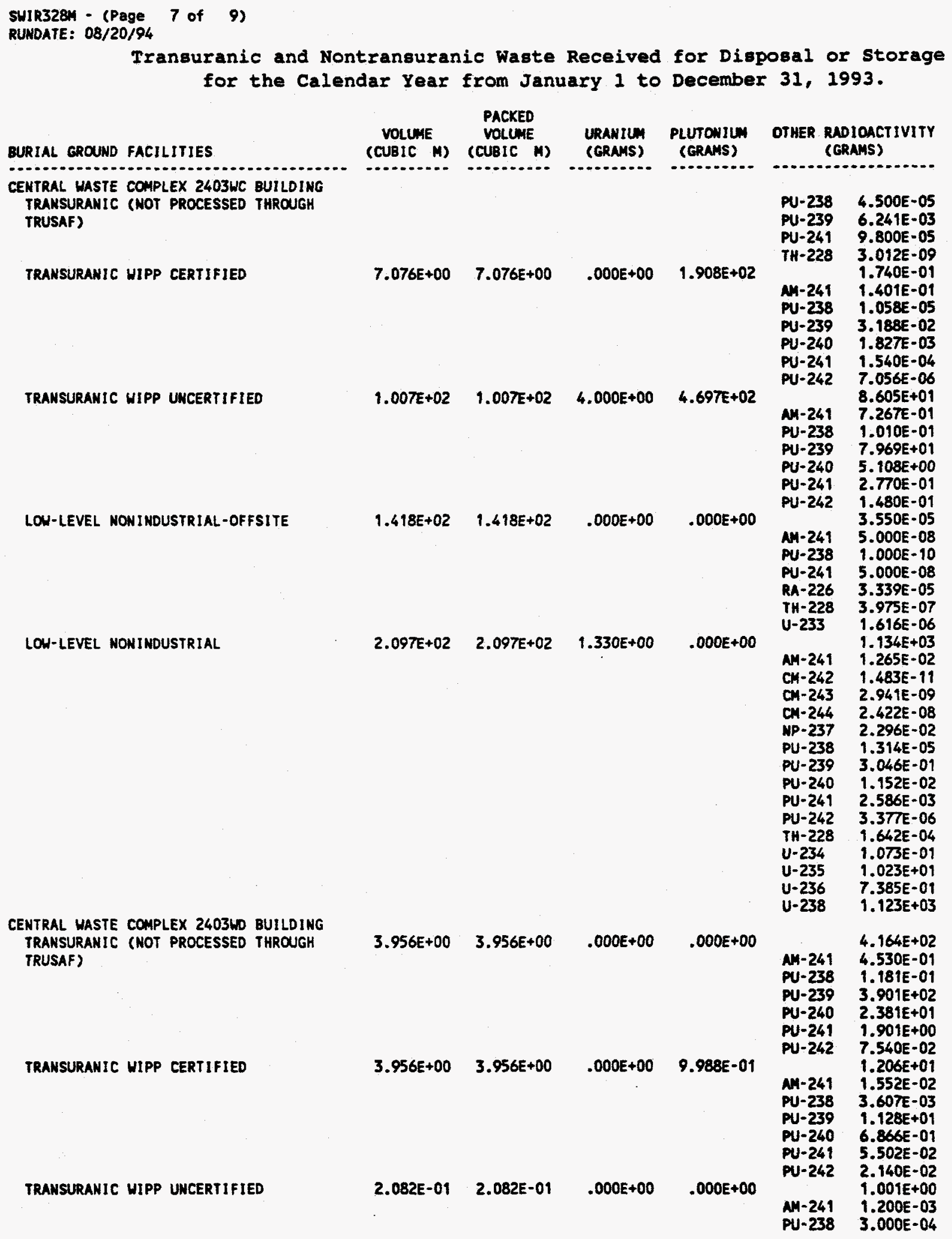




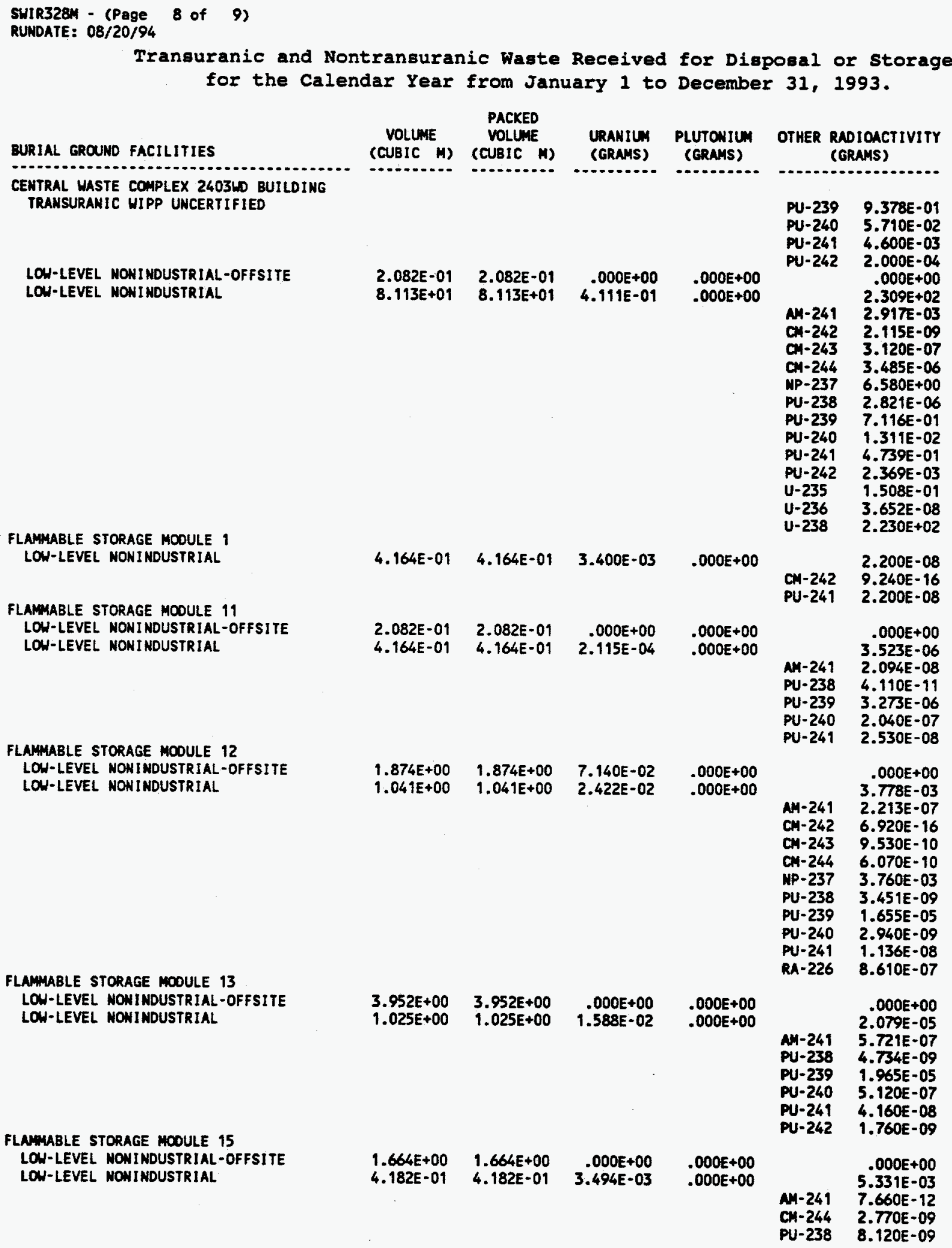




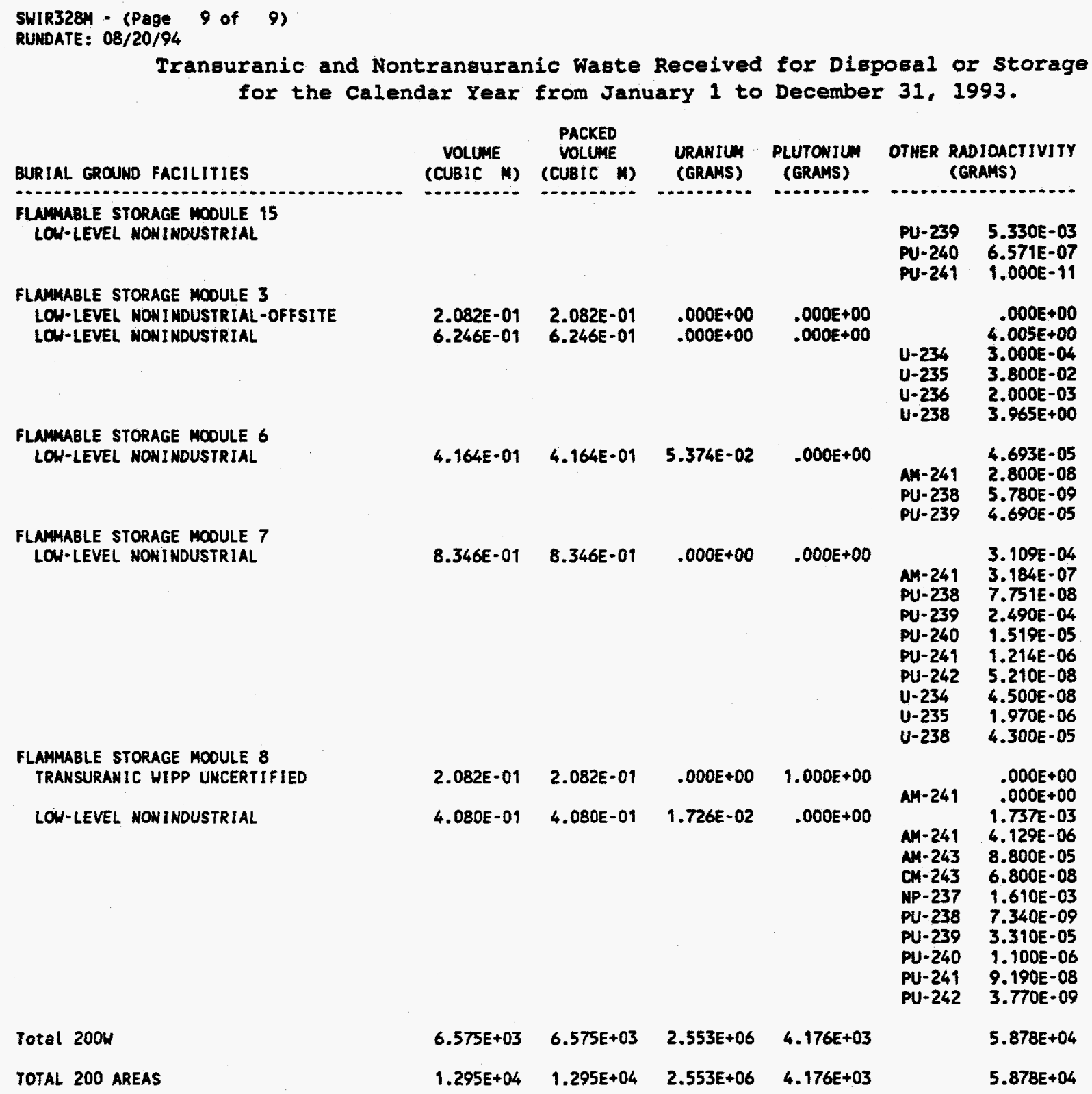




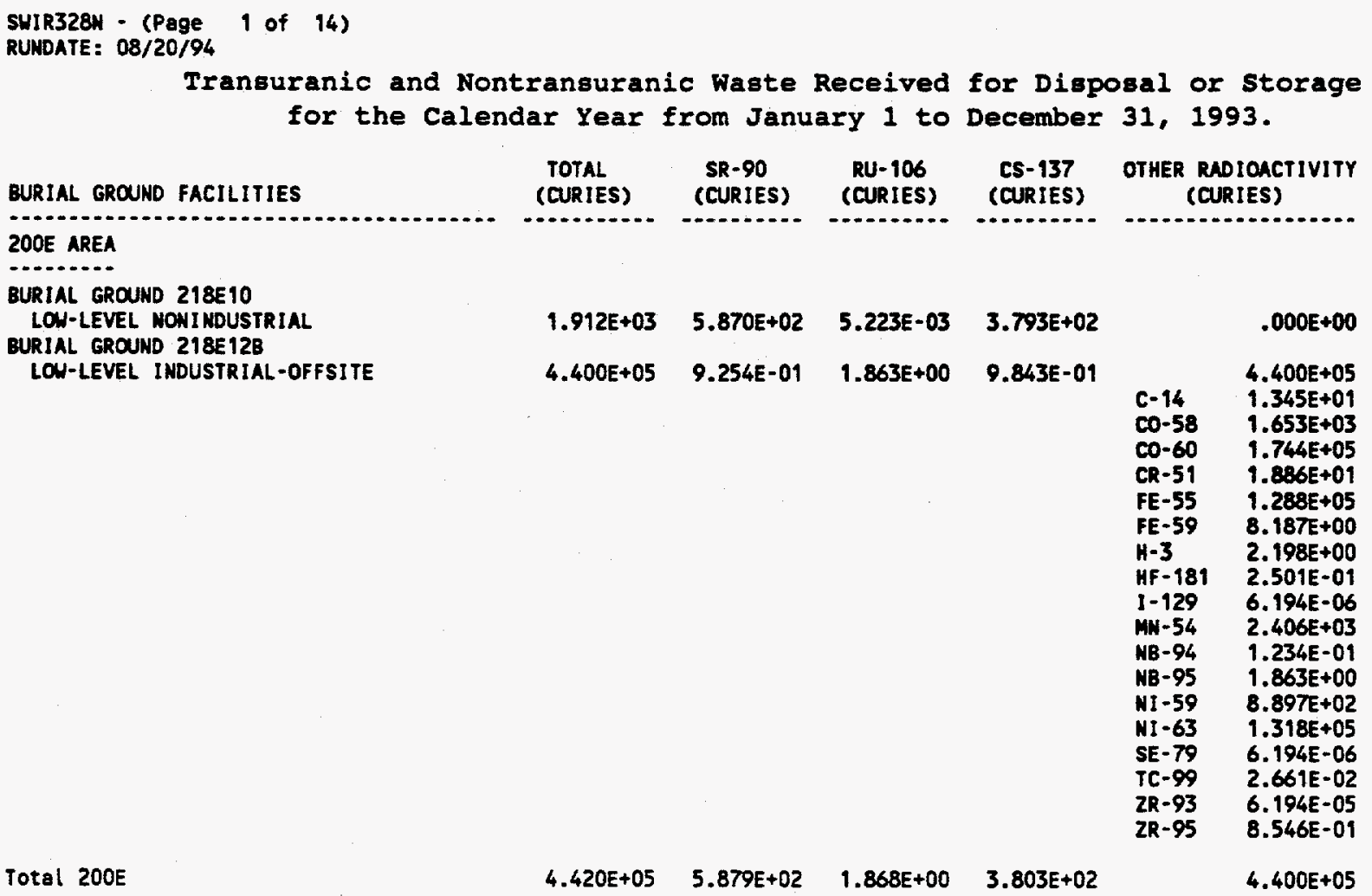


SUIR328N - (Page 2 of 14 )

RUNDATE: 08/20/94

Transuranic and Nontransuranic Waste Received for Disposal or Storage for the Calendar Year from January 1 to December 31, 1993.

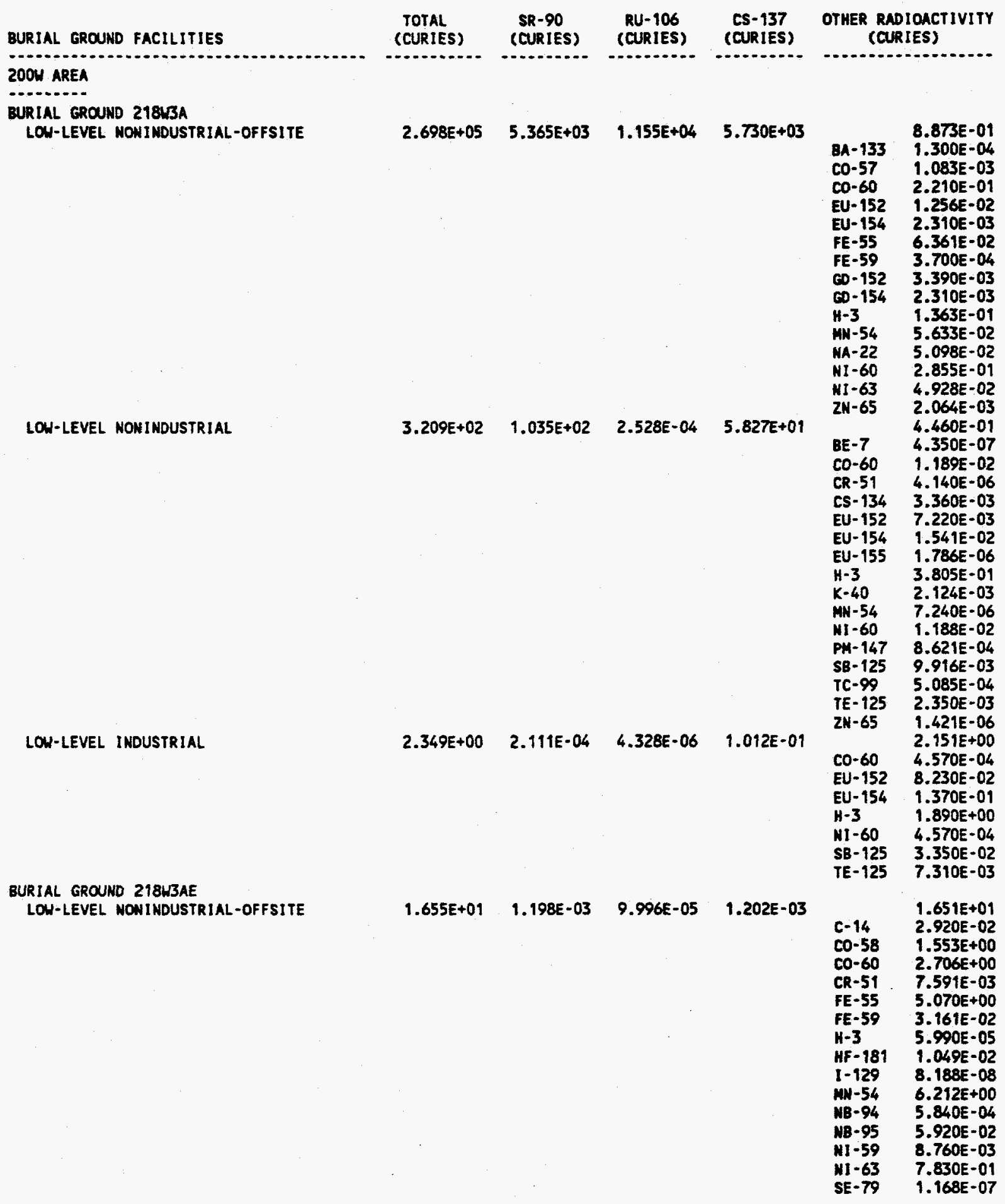




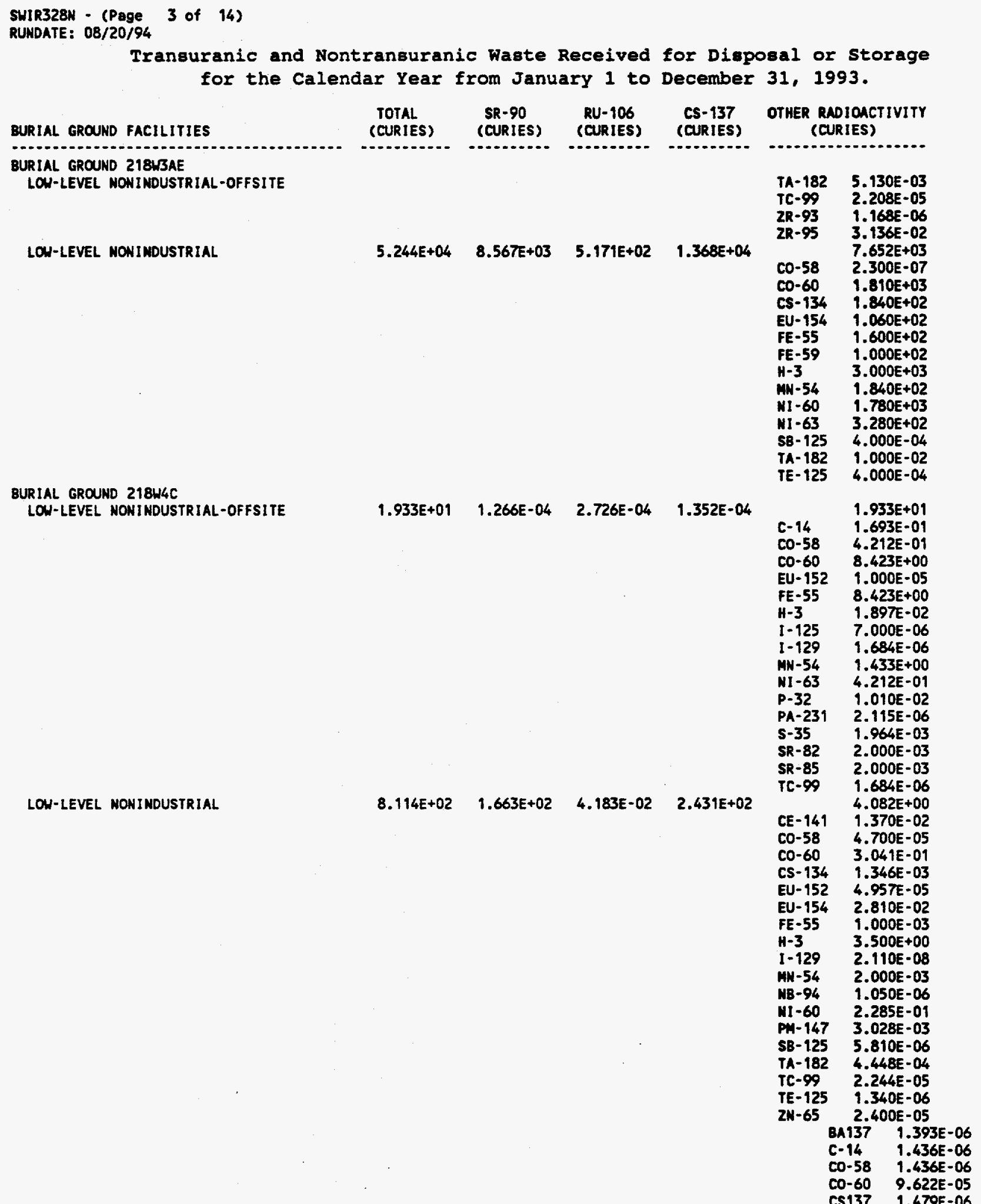




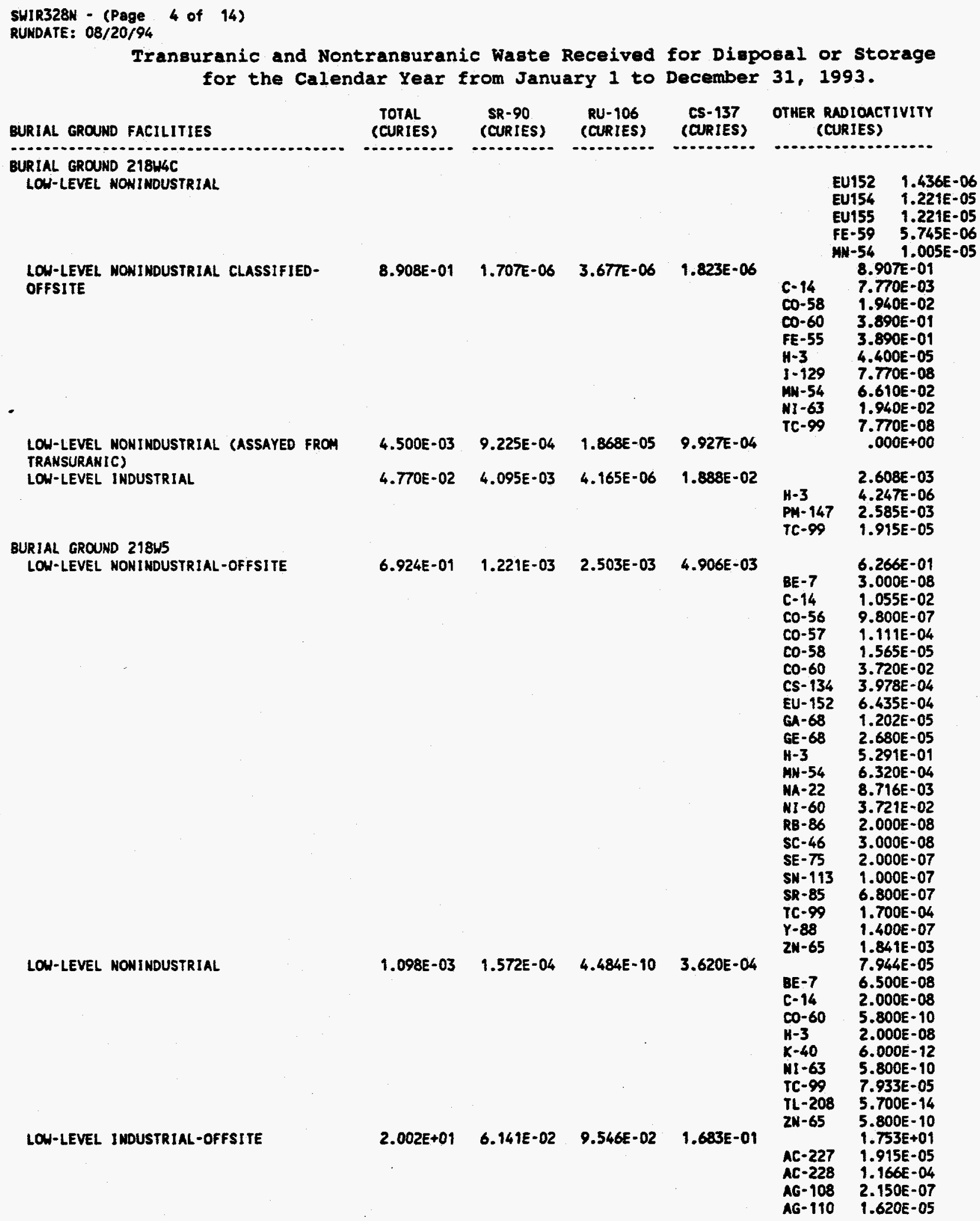


SUIR328N - (Page 5 of 14)

RUNDATE: 08/20/94

Transuranic and Nontransuranic Waste Received for Disposal or storage for the Calendar Year from January 1 to December 31, 1993.

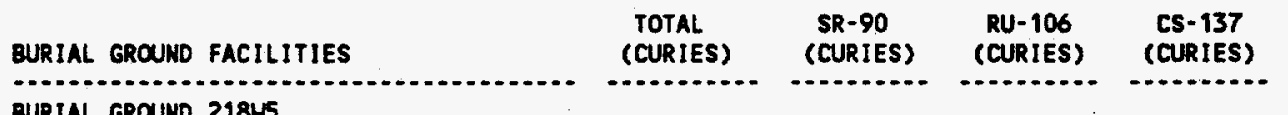

OTHER RADIOACTIVITY (CURIES)

\begin{tabular}{|c|c|}
\hline 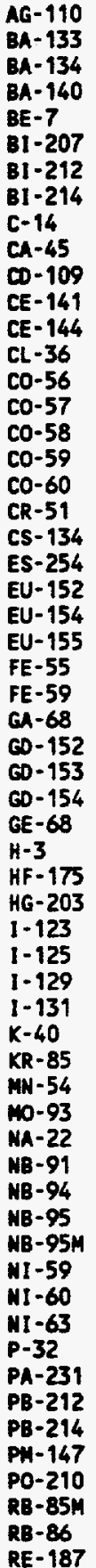 & $\begin{array}{l}2.162 E-04 \\
3.610 E-05 \\
1.270 E-07 \\
1.000 E-06 \\
6.209 E-03 \\
1.509 E-03 \\
6.980 E-03 \\
2.376 E-04 \\
3.010 E-02 \\
1.360 E-02 \\
1.310 E-03 \\
1.701 E-04 \\
1.334 E-05 \\
1.142 E-05 \\
1.203 E-04 \\
1.211 E-02 \\
6.015 E-03 \\
4.000 E-06 \\
1.676 E+00 \\
2.111 E-01 \\
2.100 E-04 \\
1.142 E-04 \\
5.149 E-01 \\
6.206 E-02 \\
7.200 E-04 \\
3.345 E+00 \\
3.679 E-02 \\
5.868 E-03 \\
8.468 E-02 \\
5.000 E-08 \\
4.420 E-02 \\
3.675 E-05 \\
6.041 E+00 \\
1.002 E-06 \\
1.200 E-05 \\
5.600 E-04 \\
2.938 E-02 \\
7.700 E-08 \\
2.100 E-07 \\
2.471 E-04 \\
7.430 E-06 \\
1.600 E-01 \\
1.203 E+00 \\
1.026 E+00 \\
1.000 E-03 \\
1.300 E-07 \\
1.005 E-03 \\
2.000 E-07 \\
2.300 E-04 \\
1.726 E+00 \\
5.3878-02 \\
2.483 E-01 \\
7.688 E-01 \\
3.240 E-03 \\
1.483 E-04 \\
1.221 E-05 \\
2.016 E-06 \\
3.0000 E-06 \\
2.296 E-03 \\
3.000 E-06\end{array}$ \\
\hline
\end{tabular}




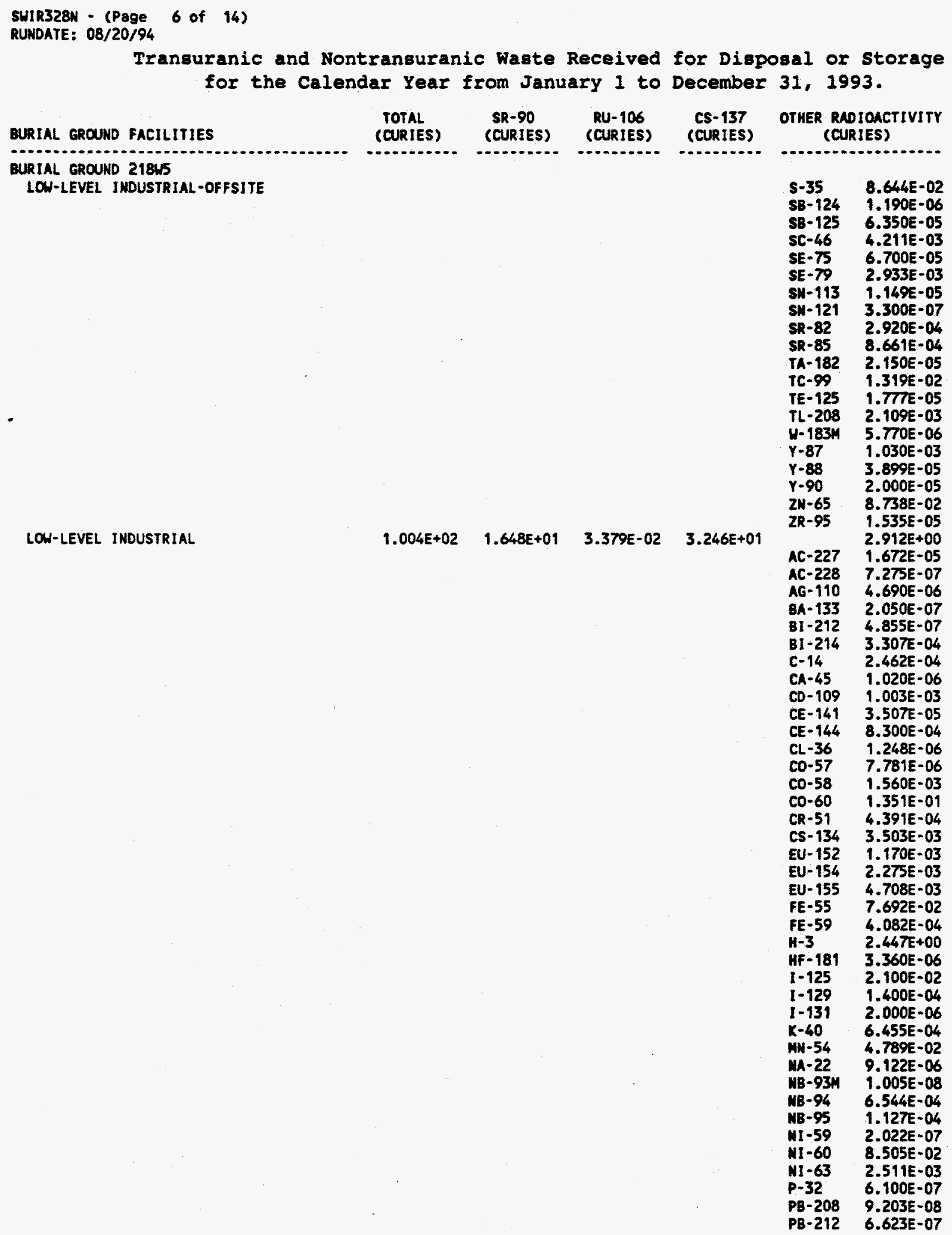




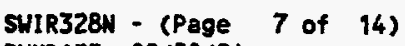

Transuranic and Nontransuranic Waste Received for Disposal or Storage for the Calendar Year from January 1 to December 31, 1993.

BURIAL GROUND FACILITIES

BURIAL GROUND 21845

LOW-LEVEL INDUSTRIAL
TOTAL (CURIES)

SR-90 (CURIES) (2..........
RU- 106 (CURIES) $\operatorname{cs}-137$ (CURIES)
OTHER RNDIOACTIVITY (CURIES)

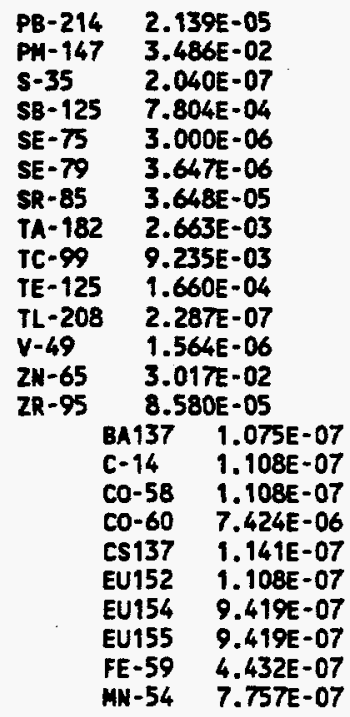

TRANSURANIC WASTE STORAGE \& ASSAY FACILITY(TRUSAF)
TRAKSURANIC (NOT PROCESSED THROUGH

TRUSAF)

4.604E+00

9.429E-01

$1.910 E-02$

$1.015 E+00$

TRANSURANIC HIPP CERTIFIED CENTRAL WASTE COMPLEX $2401 \mathrm{~W}$ BUILDING LON-LEVEL NONIMDUSTRIAL-OFFSITE

CENTRAL WASTE COMPLEX 2402W BUILDING LON-LEVEL NONINDUSTRIAL

CENTRAL WASTE COMPLEX 2402WB BUILDING LOW-LEVEL NONINDUSTRIAL

$\begin{array}{cccc}9.150 E-02 & 1.876 E-02 & 3.799 E-04 & 2.018 E-02 \\ 2.100 E+02 & .000 E+00 & .000 E+00 & .000 E+00 \\ & \cdot & & \\ 5.000 E-04 & 1.025 E-04 & 2.076 E-06 & 1.103 E-04 \\ 8.009 E+00 & 2.237 E-03 & 8.307 E-06 & 1.641 E-03\end{array}$

CENTRAL HASTE COMPLEX 2402WC BUILDING TRANSURANIC (NOT PROCESSED THROUGH TRUSAF)

LON-LEVEL NONINDUSTRIAL-OFFSITE

$\begin{array}{llll}5.000 E-04 & .000 E+00 & .000 E+00 & .000 E+00 \\ 1.000 E-07 & .000 E+00 & .000 E+00 & .000 E+00\end{array}$

\begin{tabular}{lr}
\multicolumn{2}{c}{$.000 E+00$} \\
BA137 & $4.850 E-06$ \\
C- 14 & $5.000 E-06$ \\
C0-58 & $5.000 E-06$ \\
CO-60 & $3.350 E-04$ \\
CS137 & $5.150 E-06$ \\
EU152 & $5.000 E-06$ \\
EU154 & $4.250 E-05$ \\
EU155 & $4.250 E-05$ \\
FE-59 & $2.000 E-05$ \\
MN-54 & $3.500 E-05$ \\
\multicolumn{3}{c}{.000E+00 }
\end{tabular}

$H-3 \quad 2.100 E+02$

$.000 E+00$

2.909E-04

C-14 $1.110 E-08$

CO-60 2.770E-05

EU-152 2.940E-05

EU-154 5.020E-05

EU-155 2.010E-05

H-3 1.830E-07

MA-22 1.780E-05

MB-95M 8.120E-10

MI $-60 \quad 2.770 E-05$

PH-147 1.116E-04

TC-99 1.131E-06

$2 R-95 \quad 5.030 E-06$

$.000 E+00$

1.000E-07 
SUIR328N - (Page 8 of 14)

RUNDATE: $08 / 20 / 94$

Transuranic and Nontransuranic Waste Received for Disposal or Storage for the Calendar Year from January 1 to December 31, 1993.

BURIAL GROUND FACILITIES
CENTRAL WASTE COMPLEX 2402 WC BUILDING
LOW-LEVEL NONINDUSTRIAL-OFFSITE
LOW-LEVEL NONINDUSTRIAL

LOW-LEVEL NONINDUSTRIAL

TOTAL
(CURIES)

$S R-90$

(CURIES)

7.967E-02

$3.548 E-02$
RU-106

(CURIES)

-.
CS-137

(CURIES)

(c..........

$9.308 E-06 \quad 2.711 E-03$
CENTRAL WASTE COMPLEX 2402WG BUILDING TRANSURANIC (NOT PROCESSED THROUGH TRUSAF)

TRANSURANIC HIPP CERTIFIED

TRANSURANIC HIPP UNCERTIFIED LON-LEVEL NONINDUSTRIAL-OFFSITE

LON-LEVEL NONINDUSTRIAL
3.770E-02

$3.962 E-03$

1.191E-05

$1.464 E-02$

\subsection{E-04 \\ 7.500E-03 \\ 5.000E-04 \\ 8.802E-04 \\ . $050 E-04$ \\ $8.154 \mathrm{E}-10$ \\ $4.152 E-06$ \\ $1.756 E-09$ \\ $1.544 \mathrm{E}-03$ \\ 2.206E-04 \\ 8.709E-10}

$3.962 E-03$

$1.464 E-02$

OTHER RADIOACTIVITY (CURIES)

$\begin{array}{ll}\text { C-14 } & 1.000 E-07 \\ & 2.211 E-03 \\ C-14 & 1.632 E-07 \\ \text { CE-141 } & 1.450 E-08 \\ \text { CE-144 } & 2.610 E-06 \\ \text { CO-60 } & 8.131 E-05 \\ \text { EU-152 } & 4.318 E-04 \\ \text { EU-154 } & 7.358 E-04 \\ \text { EU-155 } & 2.950 E-04 \\ H-3 & 7.820 E-08 \\ 1-129 & 6.589 E-10 \\ \text { MA-22 } & 2.620 E-04 \\ \text { MB-94 } & 4.447 E-15 \\ \text { MB-95 } & 1.000 E-12 \\ \text { MB-95M } & 1.150 E-08 \\ \text { NI-60 } & 1.001 E-10 \\ \text { PM-147 } & 7.270 E-06 \\ \text { SE-79 } & 2.936 E-11 \\ \text { TC-99 } & 4.529 E-06 \\ \text { ZR-95 } & 3.908 E-04\end{array}$

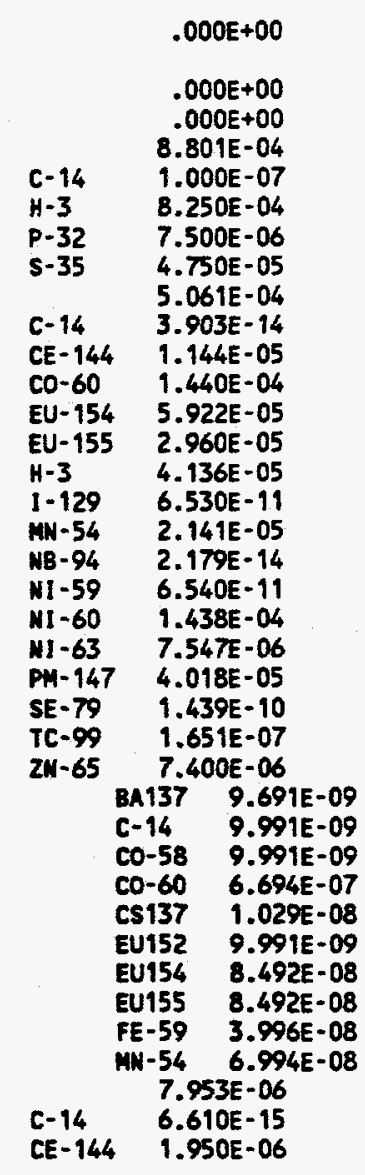




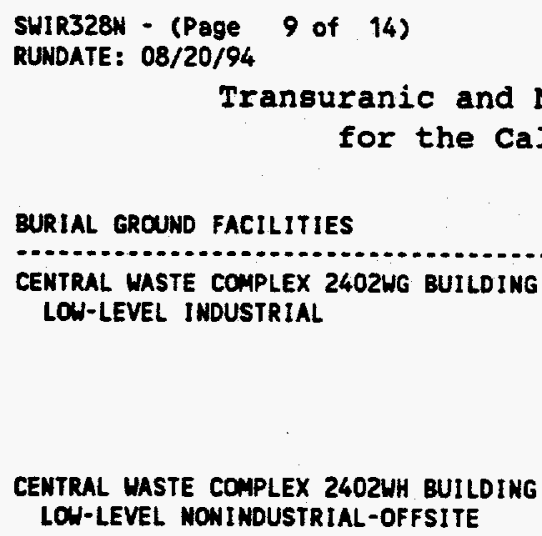
BURIAL GROUND FACILITIES
CENTRAL WASTE COMPLEX 240ZWG BUILDING
LOW-LEVEL INDUSTRIAL
CENTRAL WASTE COMPLEX 2402WH BUILDING
LOW-LEVEL NOWINDUSTRIAL-OFFSITE

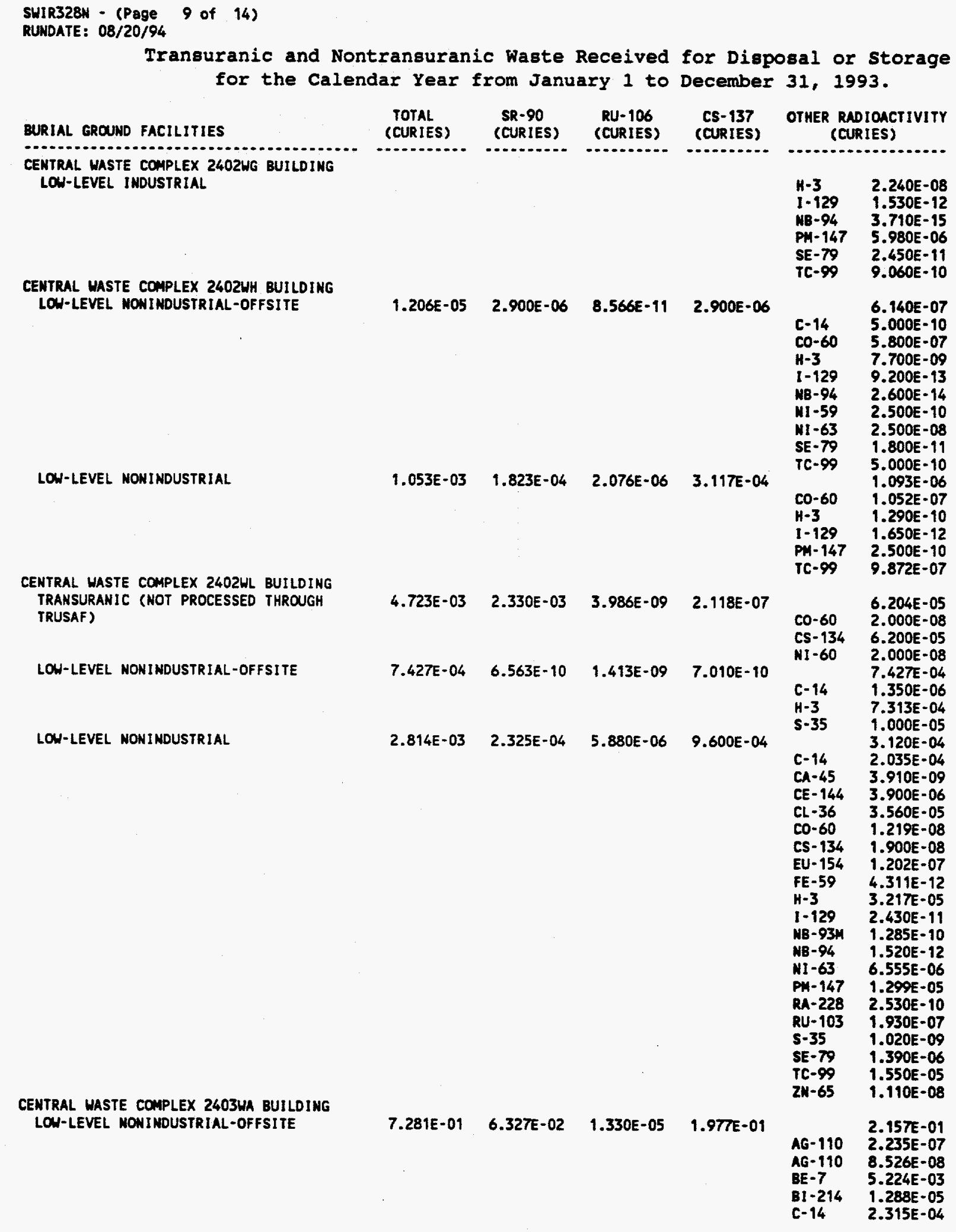

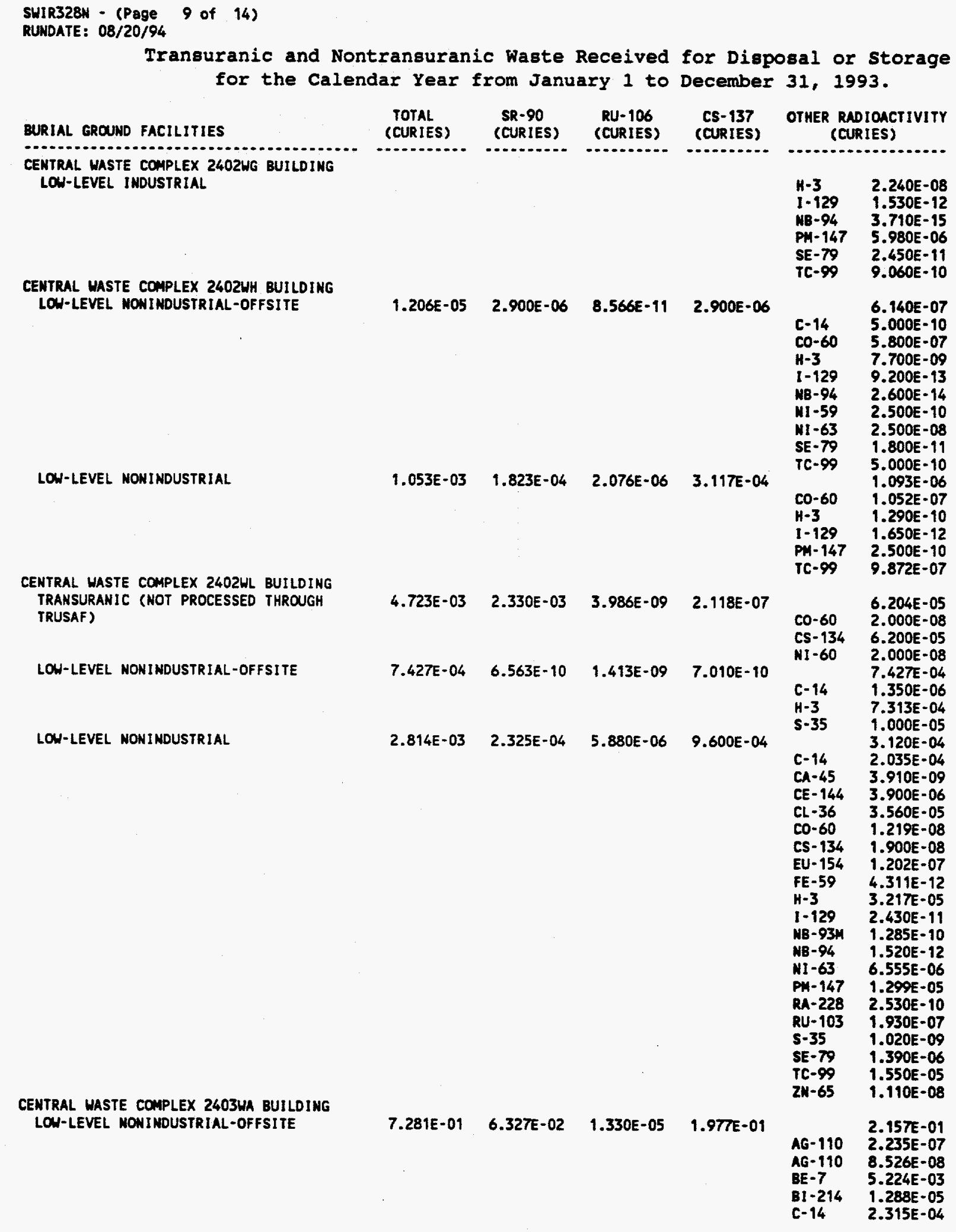

$1.206 E-05 \quad 2.900 E-06 \quad 8.566 E-11 \quad 2.900 E-06$

\begin{tabular}{l} 
SR-90 \\
(CURIES) \\
\hline$-\ldots+\cdots$
\end{tabular}

RU. 106

(CURIES)

CS-137
(CURIES)

OTHER RADIOACTIVITY (CURIES)

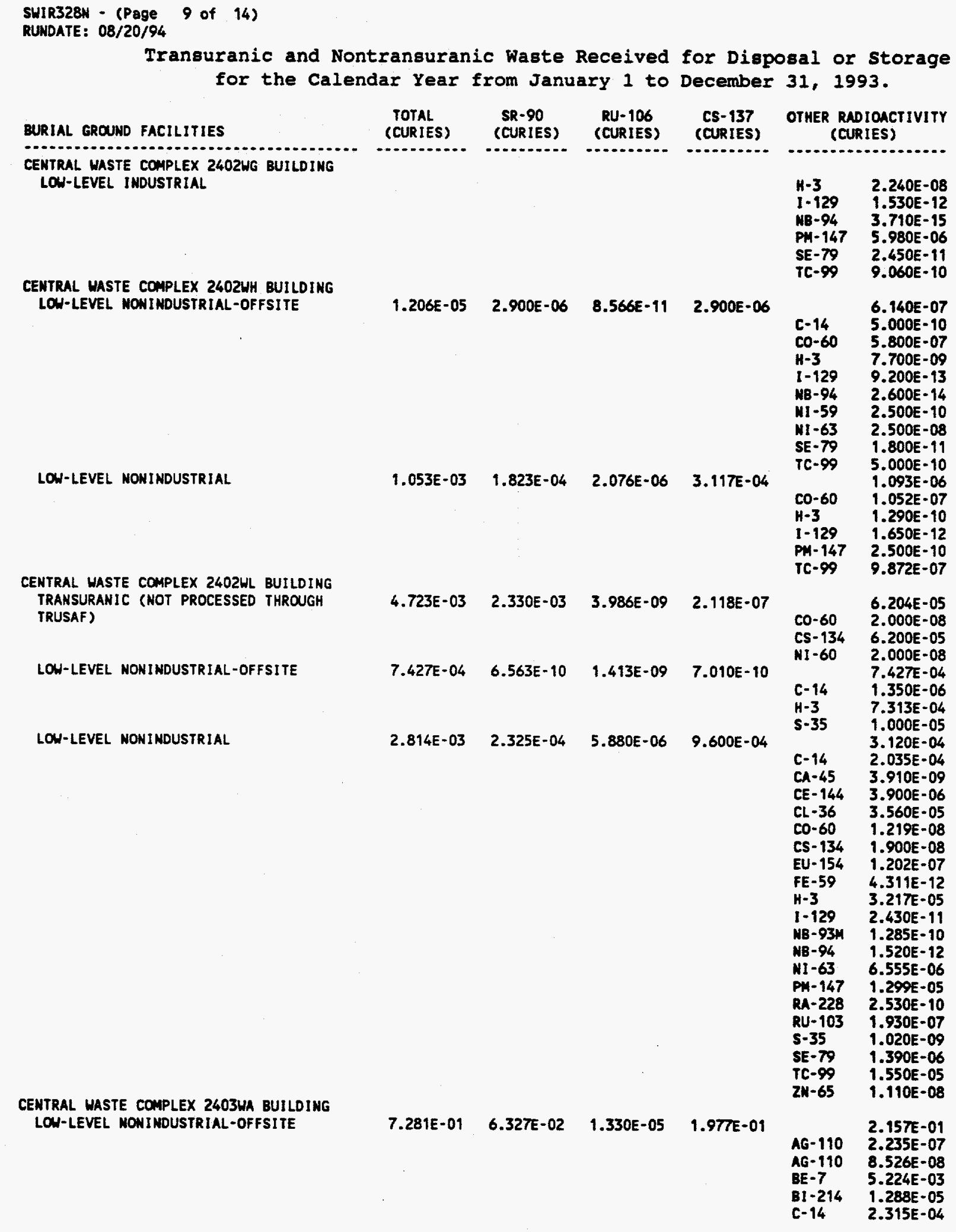

\begin{abstract}
CENTRAL HASTE COMPLEX 2402WL BUILDING TRANSURANIC (NOT PROCESSED THROUGH TRUSAF)

LON-LEVEL NONINDUSTRIAL-OFFSITE

LON-LEVEL NONINDUSTRIAL

CENTRAL MASTE COMPLEX 2403WA BUILOING LON-LEVEL NONINOUSTRIAL-OFFSITE

LOW-LEVEL NONINDUSTRIAL
\end{abstract}


SUIR328N - (Page 10 of 14)

RUNDATE : 08/20/94

Transuranic and Nontransuranic Waste Received for Disposal or storage for the Calendar Year from January 1 to December 31, 1993.

BURIAL GROUNO FACILITIES
TOTAL
(CURIES)

CENTRAL WASTE COMPLEX 2403WA BUILOING

LON-LEVEL NONINDUSTRIAL-OFFSITE

LON-LEVEL NOWINDUSTRIAL

$3.142 E+00 \quad 6.237 E-01 \quad 2.182 E-03 \quad 3.919 E-01$

SR-90 RU-106

CS-137 (CURIES)

OTHER RADIOACTIVITY (CURIES)

(CURIES) (CUR (CURIE)

CE- 144

Co-56

8.500E-04 CO-57

co-58

Co-60

Cs- 134

EU-152

EU-154

EU-155

FE- 55

FE- 59

$\mathrm{H}-3$

HF-175

HG-203

$1-125$

; -129

$k-40$

2.642E-06

7.318E-05

$1.174 E-05$

6.924E-02

1.271E-03

5.182E-04

3.581E-04

4.870E-06

2.522E-04

3.310E-05

$6.814 E-03$

2.000E-04

$1.333 E-04$

1.050E-05

3.544E-06

1.386E-02

2.486E-03

MN-54 1.211E-04

MA-22 $1.338 E-04$

पE-94 1.793E-08

$11-59 \quad 7.644 E-06$

NI-60 6.824E-02

$\mathrm{N1}-63 \quad 9.517 \mathrm{E}-04$

P.32 $\quad 5.430 E-04$

PB-214 1.288E-05

PM-147 2.403E-03

PT-195 2.800E-05

$5-35 \quad 4.611 E-03$

SB-125 1.940E-02

SE-79 3.592E-08

TC- 99 1.156E-06

TE-125 $1.589 E-02$

TE-125 1.792E-03

ZN-65 5.566E-06

5.474E- 0

CA-45 $\quad 9.300 E-06$

CE-144 9.084E-05

CO-60 2.974E-03

CS-134 1.902E-05

EU-152 1.219E-03

EU-154 2.357E-03

EU-155 5.760E-04

FE-55 3.513E-0

CD-153 5.000E-04

H-3 3.035E-03

$1-120 \quad 2.380 E-04$

$K-40 \quad 4.766 E-05$

MN-54 1.130E-01

MO-93 2.400E-07

MA-22 4.711E-04

MB-93M 2.292E-07

NB-94 2.096E-09

MB-95M 2.387E-08

NI-59 $5.984 E-09$

$M 1-60 \quad 5.944 E-05$

NI-63 4.660E-06

PB-214 4.090E-09

PM-147 6.593E-03 
SHIR328N - (Page 11 of 14)

RUNDATE: $08 / 20 / 94$

Transuranic and Nontransuranic Haste Received for Disposal or Storage for the Calendar Year from January 1 to December 31, 1993.

GURIAL GROUND FACILITIES

CENTRAL HASTE COMPLEX 2403WA BUILDING LON-LEVEL NONINDUSTRIAL

LOH-LEVEL INDUSTRIAL

CENTRAL WASTE COMPLEX 2403UC BUILDING TRANSURANIC (NOT PROCESSED THROUGH TRUSAF)-OFFSITE

TRANSURANIC (NOT PROCESSED THROUGH TRUSAF)

TRANSURANIC WIPP CERTIFIED TRANSURANIC HIPP UMCERTIFIED LOW-LEVEL NONINDUSTRIAL-OFFSITE

LOH-LEVEL KONINOUSTRIAL

TOTAL
(CURIES)

SR-90

(CURIES)

RU-106
(CURIES)

cs- 937 (CORiES) (...........
7.162E-07
$1.435 E-19$
2.906E-13
3.680E-07
$1.226 E+00$
$1.426 E-05$
3.071E-05
1.523E-05

4.798E-01

$2.374 E-02$

$1.156 E-03$

4.402E-02

$1.890 \mathrm{E}-02$

3.875E-03

8.918E-03

7.847E-05

$4.400 \mathrm{E}-02$

$4.564 \mathrm{E}-06$

$1.806 E-04$
$1.215 E-06$

4. $169 \mathrm{E}-03$

$9.596 E-03$

$2.908 E-04$

\begin{abstract}
$4.5642-06$
\end{abstract}
2.908E-04
OTHER RADIOACTIVITY (CURIES)

-....................

SB-125 1.048E-06

SC-46 2.600E-08

SE-79 1.554E-08

TC- $99 \quad 6.452 E-02$

TE-125 4.400E-08

TE-125 2.290E-07

$V-49$ 1.301E-04

2K-65 $\quad 1.360 E-10$

2R-95 2.098E-04

$.000 E+00$

$1.225 E+00$

AC-227 1.200E-02

BI-214 4.000E-01

C-14 8.400E-08

CO-58 $9.900 \mathrm{E}-04$

CO-60 1.300E-03

CR-51 7.900E-03

FE-55 2.300E-03

$H-3 \quad 2.500 E-07$

MN-54 5.000E-04

MO-93 2.100E-08

NB-94 9.900E-09

NI -59 2.600E-06

$\mathrm{NI}-63 \quad 2.900 \mathrm{E}-04$

PB-214 4.000E-01

PO-210 4.000E-01

TC- 99 4.500E-09

3.254E-01

CE- 144 3.800E-03

Co-60 3.056E-04

CS-134 $1.900 E-03$

EU-152 1.000E-01

EU-154 1.900E-01

EU-155 2.900E-02

$K-40 \quad 4.513 E-05$

TA-182 3.800E-04

$.000 E+00$

$.000 E+00$

$1.463 E-01$

8I-214 3.657E-05

C- $14 \quad 6.459 E-04$

CO-58 1.354E-03

CO-60 2.703E-02

FE-55 2.698E-02

H-3 8.408E-02

I-129 5.399E-09

MN-54 4.587E-03

N1-60 5.247E-05

NI-63 $1.354 E-03$

P-32 1.670E-04

PB-214 3.65TE-05

TC-99 5.399E-09

2.629E-02

C-14 3.851E-07

CE-144 3.299E-06

CD-60 3.096E-04

CS-134 8.297E-04

EU-152 2.57TE-05

EU-154 1.507E-02 
SUIR328N - (Page 12 of 14)

RUNDATE: 08/20/94

Transuranic and Nontransuranic Waste Received for Dibposal or Storage for the Calendar Year from January 1 to December 31, 1993.

BURIAL GROUND FACILITIES

CENTRAL MASTE COMPLEX 2403UC BUILDING LOW-LEVEL MONINDUSTRIAL

CENTRAL WASTE COMPLEX 2403LD BUILDING TRANSURANIC (NOT PROCESSED THROUGH TRUSAF)

TRANSURANIC MIPP CERTIFIED

TRANSURANIC UIPP UNCERTIFIED

LON-LEVEL NONINDUSTRIAL-OFFSITE

LOW-LEVEL NONINDUSTRIAL
TOTAL

(CURIES)

SR-90

(CURIES)

RU-106

(CURIES)

CS- 137

(CURIES)

(-..........

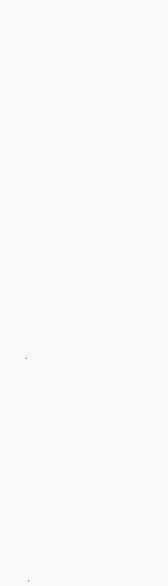

$6.350 E-02$

$1.169 E-02$

$2.367 E-04$

$1.262 E-02$
$9.500 E-03$
$5.000 E-0$.

5.
$2.0000 E-04$
$2.080 E-05$

1.845E-03

$1.025 E-04$

$.000 E+00$

3.737E- 05

$2.076 E-06$

$.000 E+00$

1.985E-03

$3.172 E+03$

3. $107 E+00$

6.355E-03

$1.103 E-04$
$.000 E+00$

2.292E+01

\footnotetext{
fLAMMABLE STORAGE mOOULE 1
} LOW-LEVEL NONINDUSTRIAL 
SUIR328n - (Page 13 of 14)

RUNDATE: $08 / 20 / 94$

Transuranic and Nontransuranic Waste Received for Disposal or storage for the Calendar Year from January 1 to December 31, 1993.

\section{BURIAL GROUNO FACILITIES \\ fLAMUABLE STORAGE MOOULE 1 LON-LEVEL NONINDUSTRIAL \\ FLAMUABLE STORAGE MOOULE 11 \\ LOW-LEVEL NONINDUSTRIAL-OFFSITE \\ LON-LEVEL NONIMDUSTRIAL \\ FLAMABLE STORAGE MOOULE 12 \\ LOW-LEVEL NONINDUSTRIAL-OFFSITE LON-LEVEL NONIMDUSTRIAL}

FLAMMABLE STORAGE MOOULE 13 LOW-LEVEL NONINDUSTRIAL-OFFSITE

LOW-LEVEL NOHINDUSTRIAL

LON-LEVEL NONIMDUSTRIAL

FLAMMABLE STORAGE MOOULE 15

LOW-LEVEL NOWINDUSTRIAL-OFFSITE

LOW-LEVEL NONINDUSTRIAL

FLAMABLE STORAGE MOOULE 3

LOW-LEVEL NOWIMDUSTRIAL-OFFSITE

LOW-LEVEL NOWINDUSTRIAL

FLNMABLE STORAGE MOOULE 6

LON-LEVEL NONINDUSTRIAL

FLAMMABLE STORAGE MOOULE 7 $\begin{array}{lllll}\text { TOTAL } & \text { SR-90 } & \text { RU-106 } & \text { CS-137 OTHER RADIOACTIVITY }\end{array}$

(CURIES) (CURIES) (CURIES) (CURIES) (CURIES)

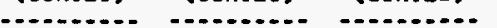

$\begin{array}{llll}1.382 E-04 & 1.492 E-09 & 3.212 E-09 & 1.593 E-09 \\ 3.854 E-04 & 2.950 E-06 & 2.907 E-10 & 1.950 E-04 \\ 9.000 E-04 & 1.790 E-05 & 3.855 E-05 & 1.912 E-05 \\ 5.967 E-05 & 1.063 E-05 & 5.490 E-07 & 1.128 E-05\end{array}$

PH-147 1.196E-05

SE-79 4.900E-11

TC- $99 \quad 1.812 E-09$

1.381E-04

$\begin{array}{ll}C-14 & 2.000 E-06 \\ H-3 & 1.361 E-04\end{array}$

$.000 E+00$

$.000 E+00$

$1.248 E-05$

C-14 9.910E-15

CE-144 2.922E-06

$H-3 \quad 3.360 E-08$

$1-129 \quad 2.339 E-12$

NB-94 5.560E-15

PM-147 8.960E-06

SB-125 1.840E-08

SE-79 3.670E-11

TC-99 5.304E-07

TE-125 1.840E-08

$2.602 E+00 \quad 6.225 E-06 \quad 1.341 E-05 \quad 6.648 E-06$

$c-1$

2.601E+00

C-14 4.828E-02

$5.182 E-04 \quad 1.062 E-04 \quad 2.076 E-06 \quad 1.143 E-04$

Co-58 $\quad 6.100 E-07$

CO-60 8.600E-08

EU- $154 \quad 1.600 E-07$

EU-155 3.530E-08

FE-55 1.200E-06

H-3 $1.347 \mathrm{~F}-08$

$1-129 \quad 1.719 E-10$

MN-54 6.300E -07

NI-60 8.600E-08

NI-63 $1.400 E-07$

PM-147 6.552E-08

TC- 99 1.331E-10

\begin{tabular}{|c|c|c|c|c|c|}
\hline $1.399 E+00$ & $5.032 E-06$ & $1.084 E-05$ & $5.374 E-06$ & $\begin{array}{l}\mathrm{C}-14 \\
\mathrm{H}-3\end{array}$ & $\begin{array}{l}1.399 E+00 \\
7.092 E-03 \\
1.392 E+00\end{array}$ \\
\hline $8.260 E-04$ & $2.770 E-04$ & $2.846 E-10$ & $1.381 E-04$ & $\begin{array}{l}c-14 \\
C 0-60 \\
I-129 \\
M I-60 \\
M I-63\end{array}$ & $\begin{array}{l}3.184 E-06 \\
5.450 E-09 \\
1.580 E-06 \\
1.790 E-08 \\
1.580 E-06 \\
1.680 E-10\end{array}$ \\
\hline
\end{tabular}

$\begin{array}{rrrrrr}3.539 E-03 \quad .000 E+00 \quad .000 E+00 \quad .000 E+00 \quad & & 3.539 E-03 \\ & & & H-3 & 3.537 E-03\end{array}$

7.381E-07 1.025E-10 2.076E-12 1.103E-10 P-32 2.000E-06

7.381E-D7 1.025E-10 2.076E-12 $1.103 E-10 \quad 07.376 E-07$

$\begin{array}{ll}C 0-60 & 5.760 E-08 \\ \text { TC- } 99 & 6.800 E-07\end{array}$

$\begin{array}{lllll}6.630 E-05 & 3.060 E-05 & 2.076 E-11 & 1.420 E-06 & 2.330 E-06\end{array}$

EU-154 2.270E-06

EU-155 6.030E-08 
WHC-EP-0125-6

SUIR328N - (Page 14 of 14)

RUNDATE: 08/20/94

Transuranic and Nontransuranic waste Received for Disposal or storage for the Calendar Year from January 1 to December 31, 1993.

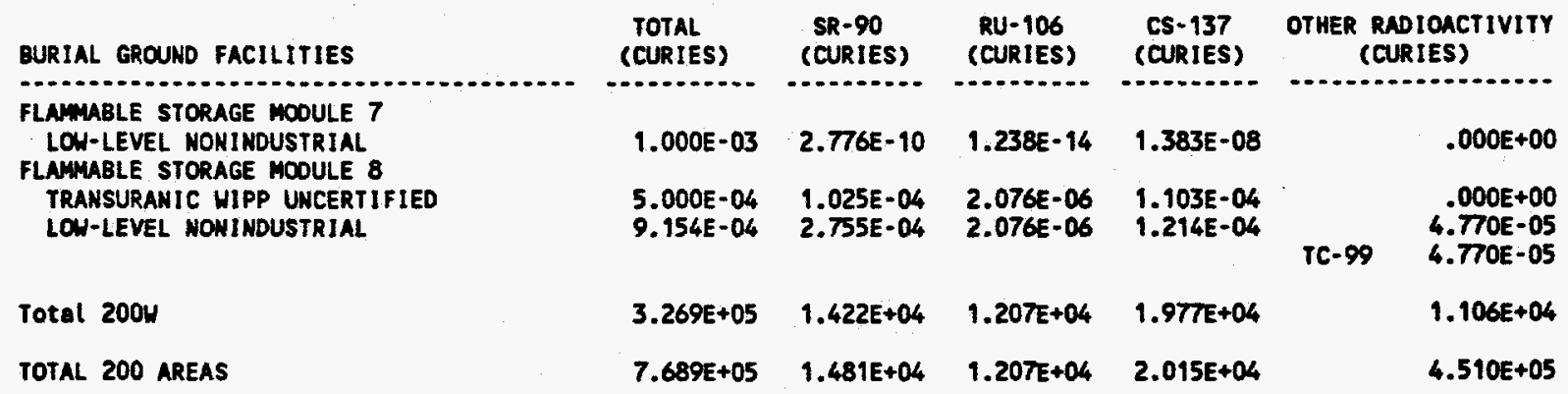


SWIR3280 - (Page 1 of 99 )

RUN DATE: 08/20/94

Land Disposal or storage of Solid Radioactive waste from January 1 to December 31 , 1993

BURIAI GROUND 218E10, 200 EAST AREA, LOW-LEVEL NONINDUSTRIAL.

\begin{tabular}{|c|c|c|c|c|c|c|c|c|c|c|c|}
\hline mpany & $\begin{array}{c}\text { Origin } \\
\text { of } \\
\text { Waste }\end{array}$ & $\begin{array}{c}\text { VOLLUNE } \\
\text { (CUBIC M.) }\end{array}$ & $\begin{array}{l}\text { PACKED } \\
\text { VOLLWNE } \\
\text { (CUBIC M.) }\end{array}$ & $\begin{array}{c}P U \\
\text { (GRAMS) }\end{array}$ & $\begin{array}{l}\text { URANIUM } \\
\text { (GRAMS) }\end{array}$ & $\begin{array}{l}\text { TOTAL } \\
\text { (CURIES) }\end{array}$ & $\begin{array}{l}\text { SR-90 } \\
\text { (CURIES) }\end{array}$ & $\begin{array}{c}\text { RU-106 } \\
\text { (CURIES) }\end{array}$ & $\begin{array}{c}\text { CS-137 } \\
\text { (CURIES) }\end{array}$ & $\begin{array}{l}\text { OTHER } \\
\text { (CURIES) }\end{array}$ & $\begin{array}{c}\text { OTHER } \\
\text { (GRAMS) }\end{array}$ \\
\hline $\begin{array}{l}\text { RENCH O } \\
\text { UHC } \\
\text { OTALS }\end{array}$ & $\begin{array}{l}2258 \\
271 B\end{array}$ & $\begin{array}{l}8.505 E+01 \\
5.670 E+00 \\
9.072 E+01\end{array}$ & $\begin{array}{l}8.505 E+01 \\
5.670 E+00 \\
9.072 E+01\end{array}$ & $\begin{array}{l}.000 E+00 \\
.000 E+00 \\
.000 E+00\end{array}$ & $\begin{array}{l}.000 E+00 \\
.000 E+00 \\
.000 E+00\end{array}$ & $\begin{array}{l}1.855 E+03 \\
5.750 E+01 \\
1.912 E+03\end{array}$ & $\begin{array}{l}5.810 E+02 \\
6.005 E+00 \\
5.870 E+02\end{array}$ & $\begin{array}{l}6.643 E-05 \\
5.157 E-03 \\
5.223 E-03\end{array}$ & $\begin{array}{l}3.560 E+02 \\
2.327 E+01 \\
3.793 E+02\end{array}$ & $\begin{array}{l}.000 E+00 \\
.000 E+00 \\
.000 E+00\end{array}$ & $\begin{array}{l}.000 E+00 \\
.000 E+00 \\
.000 E+00\end{array}$ \\
\hline
\end{tabular}


SWIR3280 - (Page 2 of 99)

RUN DATE: 08/20/94

Land Disposal or storage of Solid Radioactive Waste from January 1 to December 31 , 1993 BURIAL GROUND 218E12B, 200 EAST AREA, LOW-LEVEL INDUSTRIAL-OFFSITE.

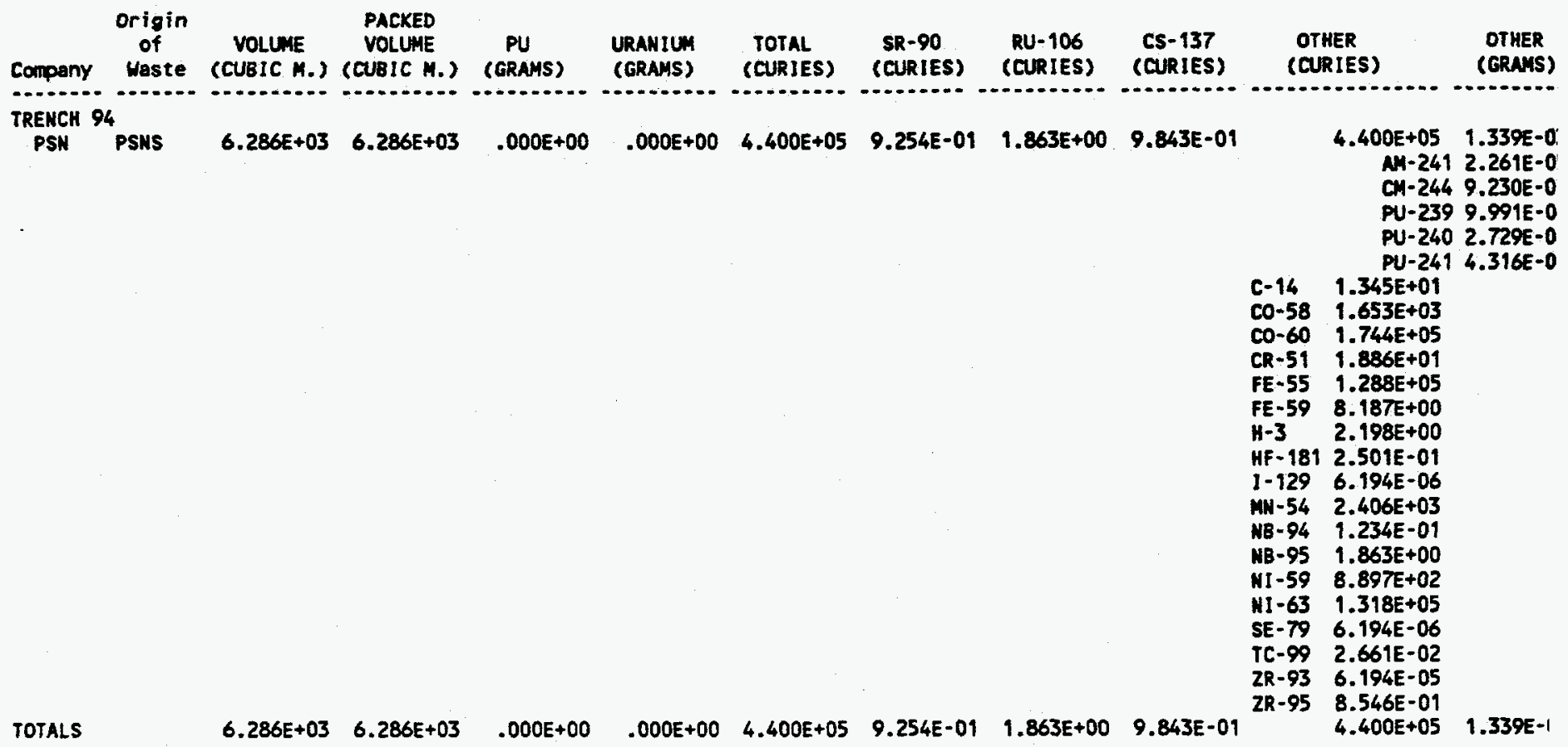




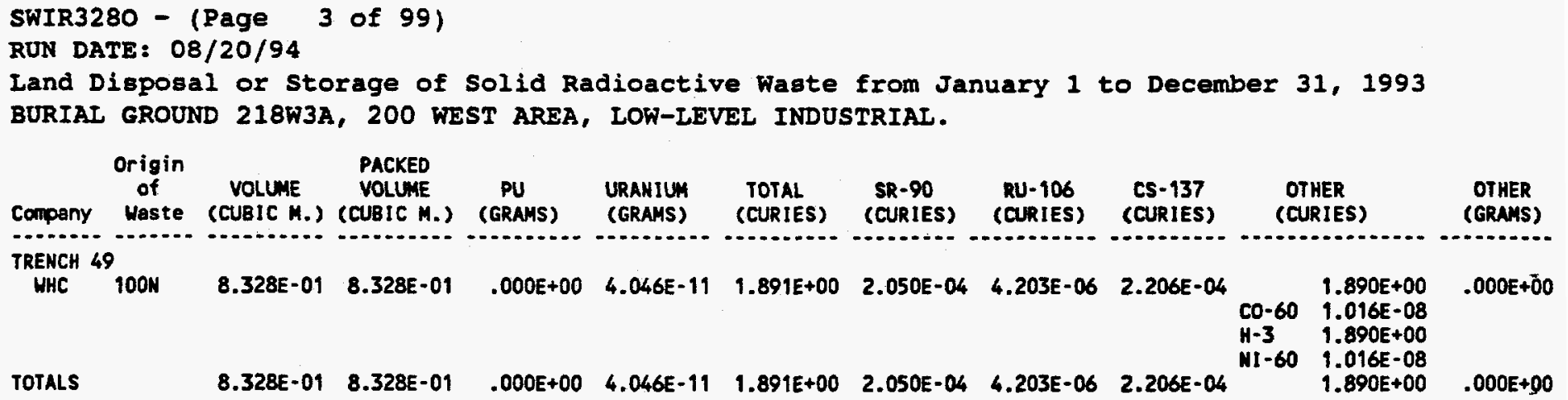




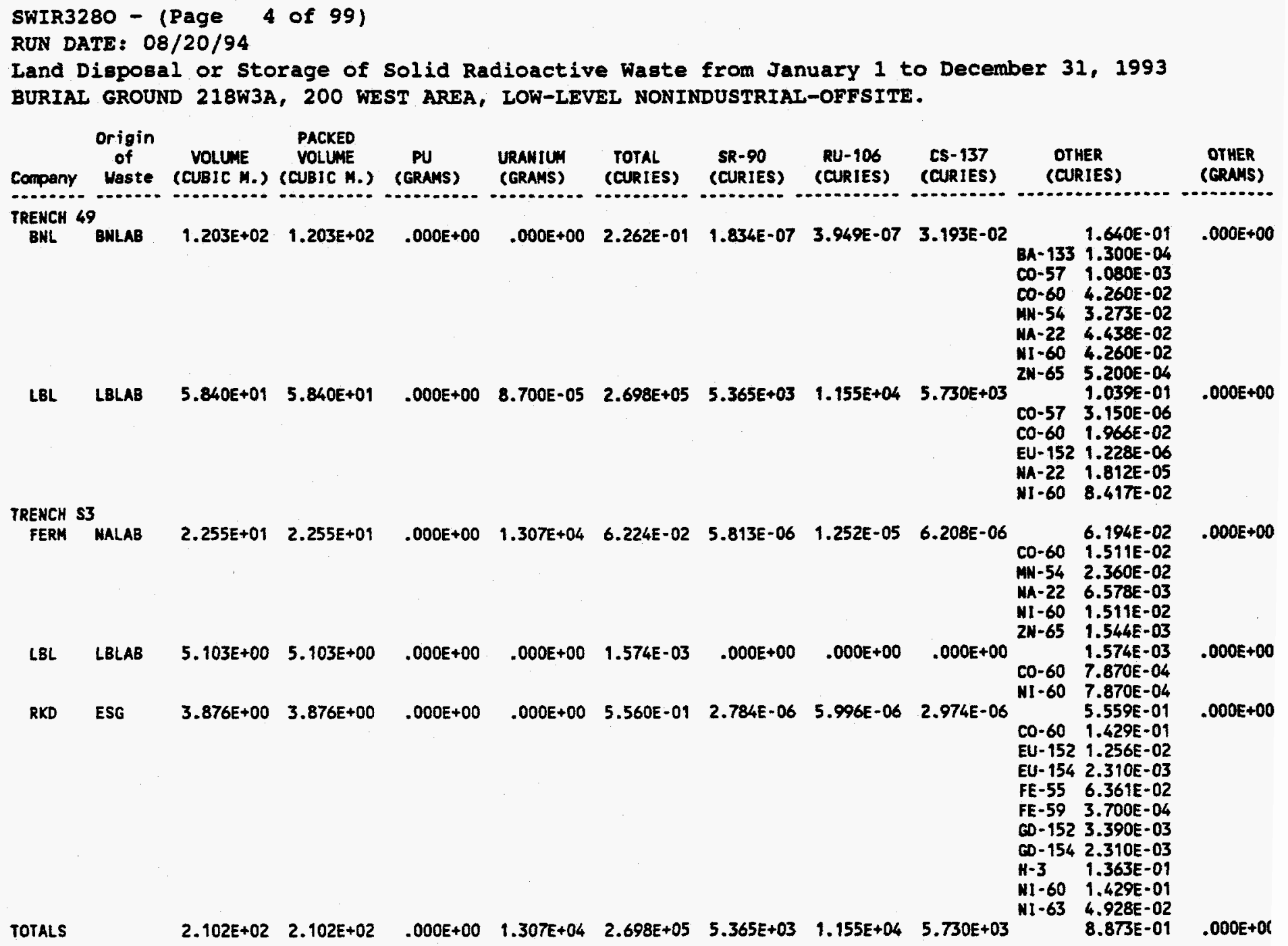




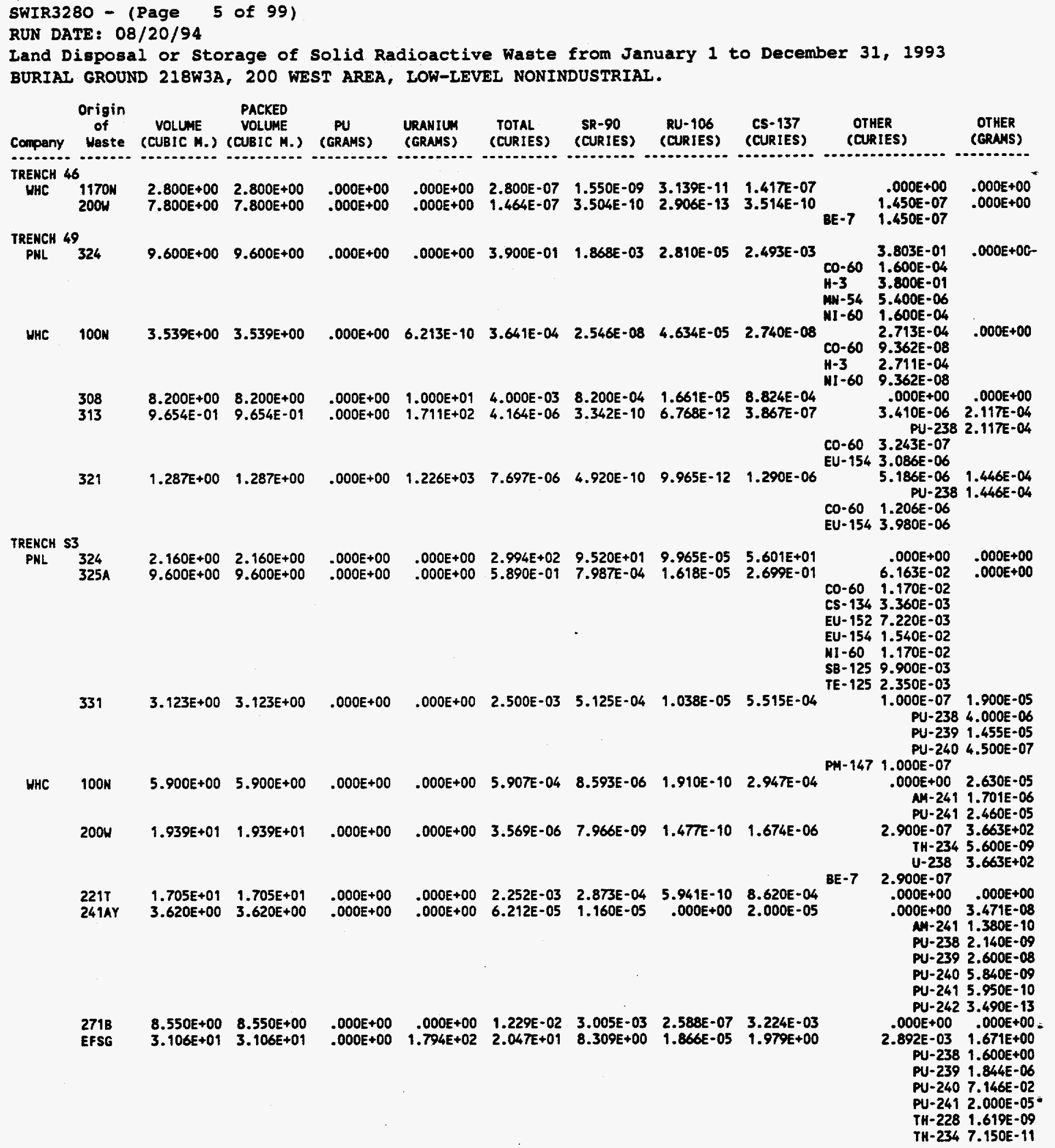


SWIR3280 - (Page 6 of 99)

RUN DATE: 08/20/94

Land Disposal or storage of Solid Radioactive Waste from January 1 to December 31 , 1993

BURIAL GROUND 218W3A, 200 WEST AREA, LOW-LEVEL NONINDUSTRIAL.

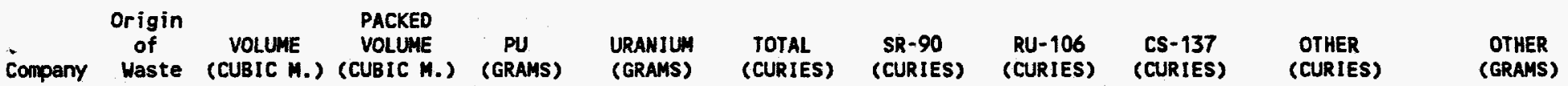
(GRE

TRENCH 53

WHC EFSG

5

$$
\text { EFSG }
$$

TRENCH S6

UHC

$9.577 E+00 \quad 9.577 E+00$

$.000 E+00$

$.000 E+00$

4.702E-05

4. 100E-11

$8.304 E-13 \quad 4.412 E-11$

CO-60 3.524E-06

CR-51 4.140E-06

$\mathrm{H}-3 \quad 2.405 E-04$

$K-40 \quad 2.124 E-03$

$\mathrm{HI}-60 \quad 2.306 \mathrm{E}-06$

SB-125 1.624E-05

TC-99 5.021E-04

(1)

9.5TrE+00 9.5TrE+00

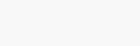

\section{co-}

4.702E-05

EU-154 2.188E-06

EU-155 1.786E-06

MN-54 1.840E-06

NI-60 1.990E-05

ZN-65 1.421E-06

TOTALS

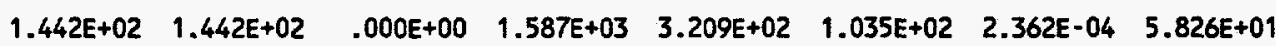

4.452E-01 3.679E+02 
SWIR3280 - (Page 7 of 99)

RUN DATE: $08 / 20 / 94$

Land Disposal or storage of Solid Radioactive Waste from January 1 to December 31 , 1993

BURIAL GROUND 218W3AE, 200 WEST AREA, LOW-LEVEL NONINDUSTRIAL-OFFSITE.

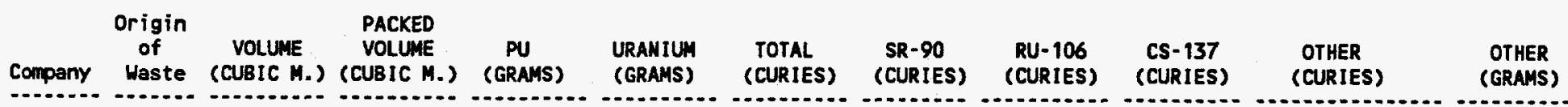

TRENCH 08

MIN NAVY

$2.306 E+01 \quad 2.306 E+01 \quad .000 E+00$

$.000 E+00 \quad 2.342 E+00 \quad 2.994 E-04 \quad 3.538 E-05 \quad 3.005 E-04$

2.336

C- 14

7.200E-03

CO-58 2.080E-01

CO-60 6.550E-01

CR-51 3.910E-04

$\mathrm{FE}-55 \quad 1.200 E+00$

FE-59 2.830E-03

$H-3 \quad 1.980 E-05$

HF- $1818.730 E-04$

I-129 2.880E-08

MN-54 3.950E-02

NB-94 1.440E-04

NI-59 2.160E-03

NI $-63 \quad 2.150 E-01$

SE-79 2.880E-08

TA-182 1.230E-03

TC-99 7.210E-08

ZR-93 2.880E-07

PSN PSNS $\quad 4.612 E+01 \quad 4.612 E+01 \quad .000 E+00 \quad .000 E+00 \quad 1.420 E+01 \quad 8.990 E-04 \quad 6.458 E-05 \quad 9.010 E-04$

2R-95 3.870E-03

$1.417 E+01 \quad 8.715 E-06$ AM-241 3.200E-07 CM-244 1.332E-08 PU-239 3.880E-06 PU-240 3.880E-06 PU-241 6.220E-07

C-14 2.200E-02

Co-58 $1.345 E+00$

CO-60 2.051E+0O

CR-51 7.200E-03

FE-55 3.870E+00

FE-59 2.878E-02

$\mathrm{H}-3$ 4.010E-05

HF-181 9.620E-03

$1-129 \quad 5.308 \mathrm{E}-08$

MN-54 6.173E+00

NB-94 4.400E-04

NB-95 5.920E-02

NI-59 6.600E-03

NI-63 5.680E-01

SE-79 8.800E-08

TA-182 3.900E-03

TC-99 2.201E-05

ZR-93 8.800E-07

TOTALS $\quad 6.918 E+01 \quad 6.918 E+01 \quad .000 E+00 \quad .000 E+00 \quad 1.655 E+01 \quad 1.198 E-03 \quad 9.996 E-05 \quad 1.202 E-03$

ZR-95 $2.749 \mathrm{E}-02$

1.157E-05 
SWIR3280 - (Page 8 of 99)

RUN DATE: 08/20/94

Land Digposal or Storage of Solid Radioactive Waste from January 1 to December 31 , 1993 BURIAI GROUND 218W3AE, 200 WEST AREA, LOW-LEVEL NONINDUSTRIAL.

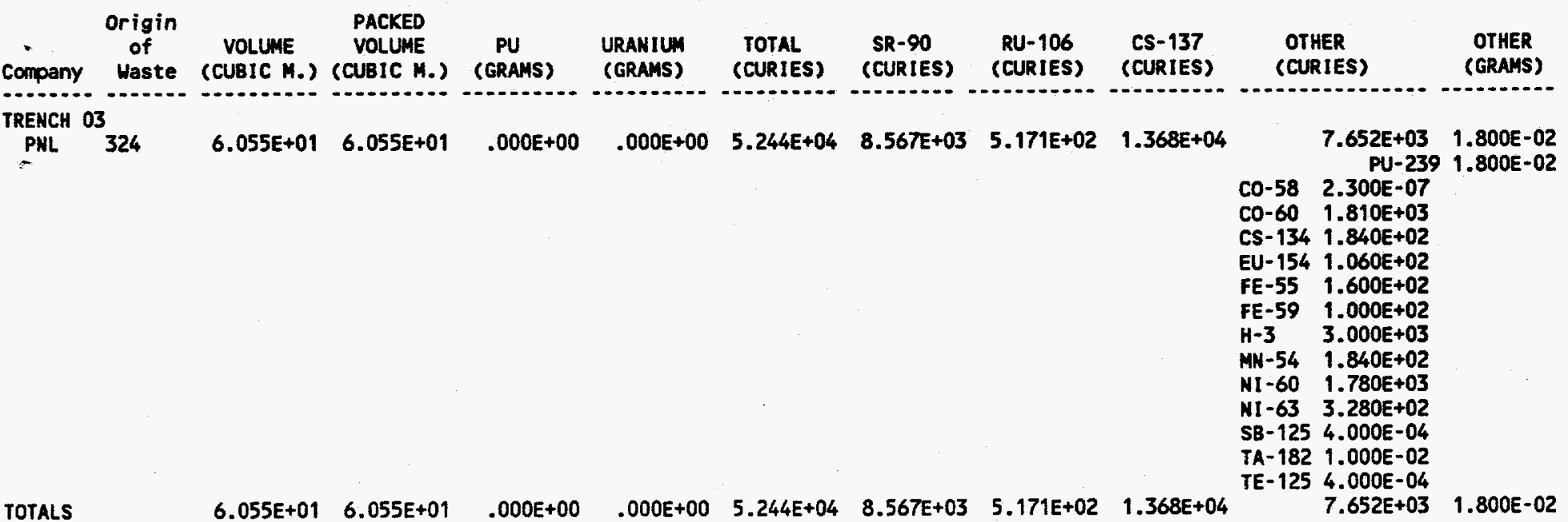




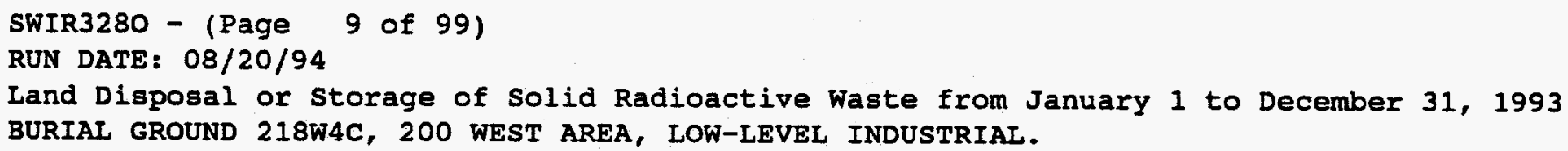


SWIR3280 - (Page 10 of 99 )

RUN DATE: $08 / 20 / 94$

Land Disposal or Storage of Solid Radioactive Waste from January 1 to December 31, 1993

BURIAL GROUND 218W4C, 200 WEST AREA, LOW-LEVEL NONINDUSTRIAL (ASSAYED FROM TRANSURANIC).

\begin{tabular}{|c|c|c|c|c|c|c|c|c|c|c|c|}
\hline Company & $\begin{array}{c}\text { Origin } \\
\text { of } \\
\text { Waste }\end{array}$ & $\begin{array}{l}\text { VOLUME } \\
\text { (CUBIC M.) }\end{array}$ & $\begin{array}{l}\text { PACKED } \\
\text { VOLLUME } \\
\text { (CLBIC M.) }\end{array}$ & $\begin{array}{c}\text { PU } \\
\text { (GRAMS) }\end{array}$ & $\begin{array}{l}\text { URANIUM } \\
\text { (GRAMS) }\end{array}$ & $\begin{array}{l}\text { TOTAL } \\
\text { (CURIES) }\end{array}$ & $\begin{array}{l}\text { SR-90 } \\
\text { (CURIES) }\end{array}$ & $\begin{array}{c}\text { RU-106 } \\
\text { (CURIES) }\end{array}$ & $\begin{array}{c}\text { CS-137 } \\
\text { (CURIES) }\end{array}$ & $\begin{array}{c}\text { OTHE } \\
\text { (CUNGES) }\end{array}$ & $\begin{array}{l}\text { OTHER } \\
\text { (GRAMS) }\end{array}$ \\
\hline $\begin{array}{l}\text { RENCH } \\
\text { WHC } \\
\text { OTALS }\end{array}$ & 23452 & $\begin{array}{l}1.872 E+00 \\
1.872 E+00\end{array}$ & $\begin{array}{l}1.872 E+00 \\
1.872 E+00\end{array}$ & $\begin{array}{l}4.255 E-02 \\
4.255 E-02\end{array}$ & $\begin{array}{l}.000 E+00 \\
.000 E+00\end{array}$ & $\begin{array}{l}4.500 E-03 \\
4.500 E-03\end{array}$ & $\begin{array}{l}9.225 E-04 \\
9.225 E-04\end{array}$ & $\begin{array}{l}1.868 E-05 \\
1.868 E-05\end{array}$ & $\begin{array}{l}9.927 E-34 \\
9.927 \mathrm{E}-04\end{array}$ & $\begin{array}{l}.000 E+00 \\
.000 E+00\end{array}$ & $\begin{array}{l}.000 E+00 \\
.000 E+00\end{array}$ \\
\hline
\end{tabular}


SWIR3280 - (Page 11 of 99)

RUN DATE: 08/20/94

Land Disposal or Storage of Solid Radioactive Waste from January 1 to December 31 , 1993 BURIAL GROUND 218W4C, 200 WEST AREA, LOW-LEVEL NONINDUSTRIAL CLASSIFIED-OFFSITE.

Origin PACKED

of VOLUME VOLUME PU URANIUM TOTAL SR-90 RU-106 CS-137 OTHER

Company Waste (CUBIC M.) (CUBIC M.) (GRAMS) (GRAMS) (CURIES) (CURIES) (CURIES) (CURIES) (CURIES)

TRENCH MAYAL COOE

MIN NAVY

$\begin{array}{llllllll}9.000 E+00 & 9.000 E+00 & .000 E+00 & .000 E+00 & 8.908 E-01 & 1.707 E-06 & 3.677 E-06 & 1.823 E-06\end{array}$

C-14 $\quad \begin{aligned} & 8.907 \mathrm{E}-01 \\ & 7.770 \mathrm{E}-03\end{aligned}$

CO-58 1.940E-02

CO-60 3.890E-01

FE-55 3.890E-01

H-3 4.400E-05

$1-129 \quad 7.770 \mathrm{E}-08$

$M N-54 \quad 6.610 E-02$

NI $-63 \quad 1.940 E-02$

TOTALS

$\begin{array}{lllllll}9.000 E+00 & 9.000 E+00 \quad .000 E+00 \quad .000 E+00 & 8.908 E-01 & 1.707 E-06 & 3.677 E-06 & 1.823 E-06\end{array}$

TC-99 $7.770 \mathrm{E}-08$

$.000 E+00$

8.907E-01 .000E+00 
SWIR3280 - (Page 12 of 99)

RUN DATE: 08/20/94

Land Disposal or Storage of Solid Radioactive Waste from January 1 to December 31 , 1993

BURIAL GROUND 218W4C, 200 WEST AREA, LOW-LEVEL NONINDUSTRIAL-OFFSITE.

\begin{tabular}{|c|c|c|c|c|c|c|c|c|c|c|c|}
\hline ompany & $\begin{array}{l}\text { Origin } \\
\text { of } \\
\text { Waste }\end{array}$ & $\begin{array}{l}\text { VOLUME } \\
\text { (CUBIC M.) }\end{array}$ & $\begin{array}{l}\text { PACKED } \\
\text { VOLUME } \\
\text { (CUBIC M.) }\end{array}$ & $\begin{array}{c}\text { PU } \\
\text { (GRAMS) }\end{array}$ & $\begin{array}{l}\text { URANIUH } \\
\text { (GRAMS) }\end{array}$ & $\begin{array}{l}\text { TOTAL } \\
\text { (CURIES) }\end{array}$ & $\begin{array}{l}\text { SR-90 } \\
\text { (CURIES) }\end{array}$ & $\begin{array}{l}\text { RU-106 } \\
\text { (CURIES) }\end{array}$ & $\begin{array}{l}\text { CS }-137 \\
\text { (CURIES) }\end{array}$ & $\begin{array}{l}\text { OTHER } \\
\text { (CURIES) }\end{array}$ & $\begin{array}{l}\text { OTHER } \\
\text { (GRAMS) }\end{array}$ \\
\hline
\end{tabular}

TRENCH 24

$\begin{array}{llllllllll} & \text { LBL } & 1.041 E+00 & 1.041 E+00 & .000 E+00 & .000 E+00 & 3.514 E-02 & 2.295 E-07 & 4.727 E-07 & 2.344 E-07\end{array}$ $-$

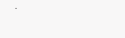

TRENCH NAVAL CORE MIN NAVY $4.500 E+01 \quad 4.500 E+01 \quad .000 E+00 \quad .000 E+00 \quad 5.686 E+00 \quad 3.120 E-05 \quad 6.720 E-05 \quad 3.333 E-05$

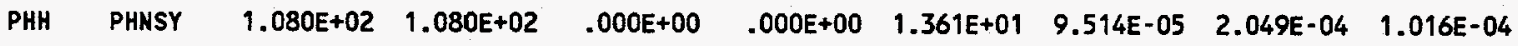

C- $14 \quad 8.311 E-04$

EU-152 1.000E-05

H-3 $1.822 E-02$

I-125 7.000E-06

P-32 1.010E-02

PA-231 2.115E-06

S-35 1.964E-03

SR-82 2.000E-03

$S R-85$ 2.000E-03

$\begin{array}{lll} & 5.685 E+00 & .000 E+00 \\ C-14 & 4.961 E-02 & \\ C O-58 & 1.242 E-01 & \\ C O-60 & 2.482 E+00 & \\ F E-55 & 2.482 E+00 & \\ H-3 & 2.200 E-04 & \\ I-129 & 4.961 E-07 & \\ M N-54 & 4.225 E-01 & \\ \text { NI }-63 & 1.242 E-01 & \\ \text { TC-99 } & 4.961 E-07 & \\ & 1.361 E+01 & .000 E+00 \\ C-14 & 1.188 E-01 & \\ C O-58 & 2.970 E-01 & \\ \text { CO-60 } & 5.941 E+00 & \\ \text { FE-55 } & 5.941 E+00 & \\ H-3 & 5.280 E-04 & \\ I-129 & 1.188 E-06 & \\ M N-54 & 1.010 E+00 & \\ \text { NI }-63 & 2.970 E-01 & \\ \text { TC-99 } & 1.188 E-06 & \\ & 1.933 E+01 & 3.611 E+03\end{array}$


WHC-EP-0125-6

SWIR3280 - (Page 13 of 99)

RUN DATE : 08/20/94

Land Disposal or Storage of Solid Radioactive Waste from January 1 to December 31 , 1993 BURIAL GROUND 218W4C, 200 WEST AREA, LOW-LEVEL NONINDUSTRIAL.

\begin{tabular}{|c|c|c|c|c|c|c|c|c|c|c|c|}
\hline Company & $\begin{array}{c}\text { Origin } \\
\text { of } \\
\text { Waste }\end{array}$ & $\begin{array}{l}\text { VOLUAME } \\
\text { (CUBIC M.) }\end{array}$ & $\begin{array}{c}\text { PACKED } \\
\text { VOLUME } \\
\text { (CUBIC M.) }\end{array}$ & $\begin{array}{c}\text { PU } \\
\text { (GRAMS) }\end{array}$ & $\begin{array}{l}\text { URANIUM } \\
\text { (GRAMS) }\end{array}$ & $\begin{array}{l}\text { TOTAL } \\
\text { (CURIES) }\end{array}$ & $\begin{array}{l}\text { SR-90 } \\
\text { (CURIES) }\end{array}$ & $\begin{array}{l}\text { RU-106 } \\
\text { (CURIES) }\end{array}$ & $\begin{array}{l}\text { CS-137 } \\
\text { (CURIES) }\end{array}$ & $\begin{array}{l}\text { OTHER } \\
\text { (CURIES) }\end{array}$ & $\begin{array}{c}\text { OTHER } \\
\text { (GRAMS) }\end{array}$ \\
\hline
\end{tabular}

TRENCH 24

PNL 324

324

$6.795 E+01 \quad 6.795 E+01 \quad .000 E+00$

$.000 E+00$

$6.745 E+00 \quad 1.148 E+00 \quad 1.039 E-04 \quad 2.266 E+00$

3.530E-02

$.000 E+00$

CO-60 3.650E-03 EU-154 2.800E-02

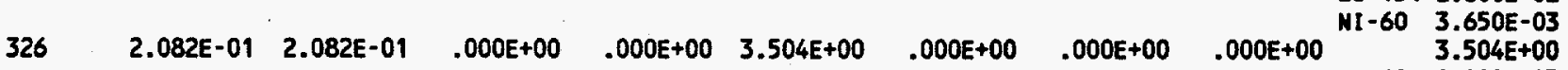

CO-60 $1.000 E-03$

FE-55 1.000E-03

$\mathrm{H}-3$, 3.500E+0O

MN-54 $1.000 E-03$

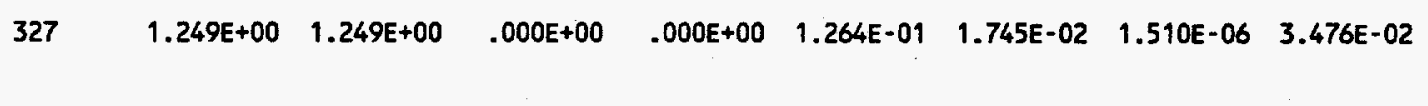

NI -60 1.000E-03

$\begin{array}{ccc}\text { AM-241 } 5.380 E-05 \\ \text { CO-58 } & 4.700 E-05\end{array}$

CO-60 $1.046 \mathrm{E}-02$

CS-134 1.346E-03

EU-152 7.500E-06

EU-154 6.750E-05

MN-54 $9.891 E-04$

NB-94 $1.050 E-06$

NI-60 1.046E-02

SB-125 5.810E-06

TA- 182 4.448E-04

TE-125 1.340E-06

2N-65 2.400E-05

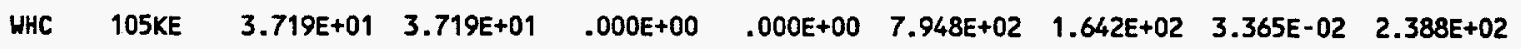

2.895E-01 5.087E+01

AM-241 3.563E-03

PU-238 3.466E-02

PU-239 4.906E+01

PU-240 1.763E+00

PU-241 3.224E-06

CO-60 1.523E-01 PU-242 2.284E-03

NI-60 1.372E-01

CS137 1.479E-06

CO-58 $1.436 E-06$

CO-60 9.622E-05

EU155 1.221E-05

C-14 1.436E-06

BA137 1.393E-06

EU154 1.221E-05

FE-59 5.745E-06

MN-54 1.005E-05

EU152 $1.436 E-06$

$105 \mathrm{KW} \quad 4.164 E+00 \quad 4.164 E+00 \quad .000 E+00 \quad .000 E+00 \quad 2.453 E+00 \quad 4.318 E-01 \quad 7.846 E-03 \quad 5.443 E-01$

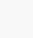

$2.265 E-01 \quad 3.584 E-01$ AM-241 1.895E-03 PU-238 2.754E-07 PU-239 3.489E-01 PU-240 6.374E-03 PU-241 1.733E-06 PU-242 1.227E-03

CE-141 1.370E-02

CO-60 $1.367 \mathrm{E}-01$

NI-60 7.612E-02 
WHC-EP-0125-6

SWIR3280 - (Page 14 of 99)

RUN DATE: $08 / 20 / 94$

Land Disposal or storage of Solid Radioactive Waste from January 1 to Deciaber 31 , 1993

BURIAL GROUND 218W4C, 200 WEST AREA, LOW-LEVEL NONINDUSTRIAL.

\begin{tabular}{|c|c|c|c|c|c|c|c|c|c|c|c|}
\hline Company & $\begin{array}{c}\text { Origin } \\
\text { of } \\
\text { Waste }\end{array}$ & $\begin{array}{l}\text { VOLUME } \\
\text { (CUBIC M.) }\end{array}$ & $\begin{array}{l}\text { PACKED } \\
\text { VOLUHE } \\
\text { (CUBIC M.) }\end{array}$ & $\begin{array}{c}\text { PU } \\
\text { (GRANS) }\end{array}$ & $\begin{array}{l}\text { URANIUN } \\
\text { (GRAMS) }\end{array}$ & $\begin{array}{l}\text { TOTAL } \\
\text { (CURIES) }\end{array}$ & $\begin{array}{c}\text { SR-90 } \\
\text { (CURIES) }\end{array}$ & $\begin{array}{c}\text { RU-106 } \\
\text { (CURIES) }\end{array}$ & $\begin{array}{l}\text { CS-137 } \\
\text { (CURIES) }\end{array}$ & $\begin{array}{l}\text { OTHER } \\
\text { (CURIES) }\end{array}$ & $\begin{array}{l}\text { OTHER } \\
\text { (GRAMS) }\end{array}$ \\
\hline
\end{tabular}

TRENCH 24

UHC 2225

$H-3 \quad 6.613 E-06$
-129

$U-238 \quad 3.860 E-01$

I-129 2.110E-08

PM- 147 3.028E-03

TC-99 2.244E-05

$23452 \quad 2.142 E+01 \quad 2.142 E+01 \quad .000 E+00 \quad .000 E+00 \quad 5.150 E-02 \quad 1.056 E-02 \quad 2.138 E-04 \quad 1.136 E-02$

$.000 E+00 \quad 1.247 E+00$

AM-241 $1.248 E-03$

PU-238 3.563E-03

PU-239 1.154E+00

PU-240 7.066E-02

PU-241 1.744E-02

PU-242 $2.484 E-04$

$2718 \quad 1.690 E+01 \quad 1.690 E+01 \quad .000 E+00 \quad .000 E+00 \quad 3.496 E+00 \quad 3.577 E-01 \quad 1.340 E-05 \quad 1.429 E+00$

$\begin{array}{lllllllll}308 & 7.344 E+00 & 7.344 E+00 & .000 E+00 & .000 E+00 & 1.124 E-01 & 5.611 E-02 & 1.423 E-07 & 3.584 E-05\end{array}$

$.000 E+00 \quad .000 E+00$

1.021E-04 6.496E-01

AM-241 7.286E-04

PU-238 1.258E-04

PU-239 5.830E-01

PU-240 2.191E-02

PU-241 7.129E-04

$\begin{array}{ll}\text { CO-60 } & 1.347 \mathrm{E}-05 \\ \text { EU- } 152 & 4.207 \mathrm{E}-05\end{array}$

PU-242 4.318E-02

EU-154 3.547E-05

$M N-54 \quad 1.107 E-05$

TOTALS

$1.583 E+0 \% \quad+.583 E+02 \quad .000 E+00 \quad 1.114 E+00$

$8.114 E+02 \quad 1.663 E+02 \quad 4.183 E-02 \quad 2.431 E+02$

$4.082 E+00 \quad 5.353 E+01$ 


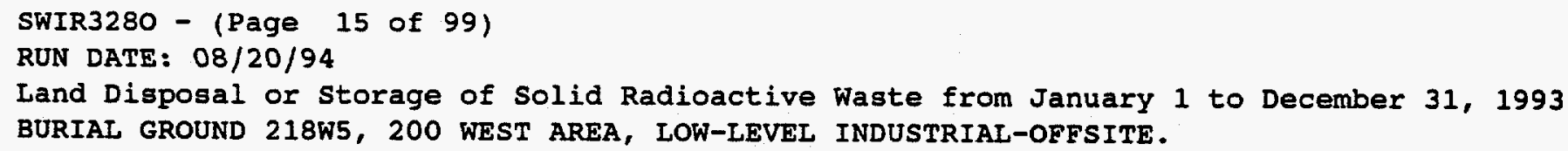

CR-51 1.002E-04

CS-134 8.300E-07

EU-152 2.400E-05

EU-154 2.900E-05

FE-59 1.581E-05

$H-3 \quad 1.162 E+00$

HG-203 1.200E-05

I-125 4.500E-05

K-40 1.073E-04

MN-54 9.476E-05

NA-22 2.208E-04

NB-95M 2.000E-07

N1-60 5.586E-02

P-32 3.938E-03

PA-231 1.800E-08

PB-212 1.130E-04

PB-214 1.190E-04

PM- 147 1.000E-05

RB-85M 3.000E-06 
SWIR3280 - (Page 16 of 99)

RUN DATE: 08/20/94

Land Disposal or Storage of Solid Radioactive Waste from January 1 to December 31 , 1993 BURIAL GROUND 218W5, 200 WEST AREA, LOW-LEVEL INDUSTRIAL-OFFSITE.

\begin{tabular}{|c|c|c|c|c|c|c|c|c|c|}
\hline Company & $\begin{array}{c}\text { Origin } \\
\text { of } \\
\text { Waste }\end{array}$ & $\begin{array}{l}\text { VOLUAE } \\
\text { (CUBIC M.) }\end{array}$ & $\begin{array}{l}\text { PACKED } \\
\text { VOLUME } \\
\text { (CUBIC M.) }\end{array}$ & $\begin{array}{c}\text { PU } \\
\text { (GRAMS) }\end{array}$ & $\begin{array}{l}\text { URANIUM } \\
\text { (GRAMS) }\end{array}$ & $\begin{array}{l}\text { TOTAL } \\
\text { (CURIES) }\end{array}$ & $\begin{array}{l}\text { SR-90 } \\
\text { (CURIES) }\end{array}$ & $\begin{array}{l}\text { RU-106 } \\
\text { (CURIES) }\end{array}$ & $\begin{array}{c}\text { CS-137 } \\
\text { (CURIES) }\end{array}$ \\
\hline $\begin{array}{l}\text { RENCH } \\
\text { ANL }\end{array}$ & ARGON & & & & & & & & \\
\hline
\end{tabular}

ANL ARGON

FERM NALAB $\quad 7.021 E+00 \quad 7.021 E+00 \quad .000 E+00 \quad 6.300 E+01 \quad 1.027 E+00 \quad 2.706 E-06 \quad 5.827 E-06 \quad 2.890 E-06$

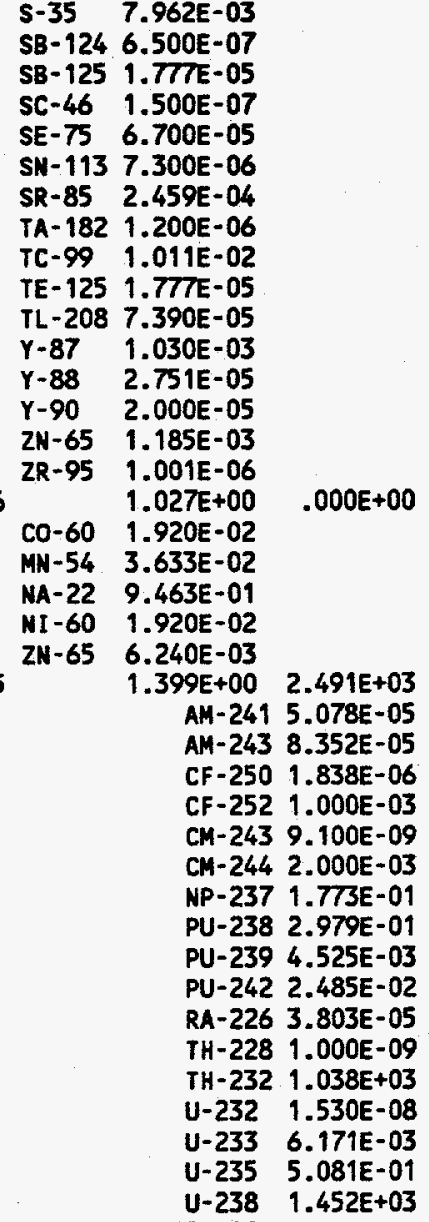

AC-227 1.715E-05

AG-108 3.500E-08

AG-110 1.000E-09

BE-7 1.000E-11

BI-207 2.100E-06

C-14 2.370E-02

CA-45 1.167E-02

CD-109 1.000E-07

CE-141 1.000E-07

CE-144 1.321E-05

CO-56 1.500E-05

CO-57 1.006E-03

CO-58 1.000E-09

CO-60 1.912E-01

CR-51 2.078E-01

CS-134 1.650E-05

ES-254 1.142E-04

EU-152 1.882E-01

EU-154 2.100E-06

FE-55 3.502E-03 
SWIR3280 - (Page 17 of 99)

RUN DATE: 08/20/94

Land Disposal or Storage of Solid Radioactive Waste from January 1 to December 31 , 1993 BURIAL GROUND 218W5, 200 WEST AREA, LOW-LEVEL INDUSTRIAL-OFFSITE.

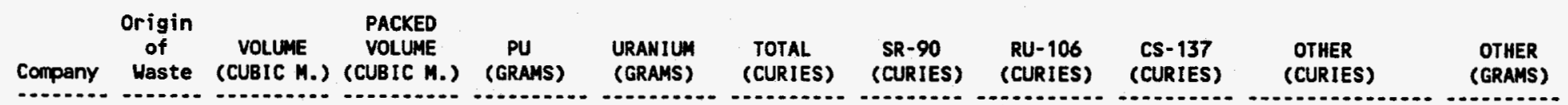

TRENCH 22

LBL LBLAB

TR

PPL PRINC $\quad 1.062 E+01 \quad 1.062 E+01 \quad .000 E+00 \quad .000 E+00 \quad 9.232 E-02 \quad 6.072 E-08 \quad 8.553 E-08 \quad 2.424 E-07$
FE-59 $1.864 E-02$ GA-68 5.850E-03 GD-152 5.000E-08 CD-153 5.000E-08 GE-68 3.040E-05 $\mathrm{H}-3$ 3.608E-01 HF- 175 1.002E-06 I-123 5.600E-04 I-125 2.933E-02 I-131 2.100E-07 MN-54 1.620E-05 NA-22 1.042E-04 NB-91 1.000E-03 NB-95 1.003E-03 N1-59 1.000E-08 P-32 2.442E-01 PA-231 3.178E-02 PO-210 2.011E-06 RB-86 2.296E-03 S-35 6.875E-02 SC-46 4.038E-03 SN-113 1.000E-06 SR-82 2.920E-04 SR-85 6.200E-04 TA-182 1.000E-06 TC- $99 \quad 2.996 E-03$ Y-88 1.000E-05 2N-65 4.000E-07 ZR-95 1.435E-05

9.231E-02 8.300E-06 AM-241 1.300E-06 PU-239 3.000E-07 TH-232 2.200E-06 c- 14 U-238 $4.500 E-06$

C-14 1.300E-06 CD-109 2.500E-06 CL-36 2.000E-08 CO-57 7.900E-08 CO-60 8.500E-07 EU-152 5.400E-06 $\mathrm{H}-3 \quad 9.230 \mathrm{E}-02$ MN-54 9.500E-07 NA-22 2.000E-07 PO-210 3.000E-09 SN-113 3.200E-07 TC-99 2.800E-07 Y-88 4.800E-07 ZN-65 2.900E-07

RFP RF $\quad 1.020 E+01 \quad 1.020 E+01 \quad .000 E+00 \quad .000 E+00 \quad 5.300 E-03 \quad 1.054 E-04 \quad 2.270 E-04 \quad 1.126 E-04$ SLACU $\quad 2.720 E+00 \quad 2.720 E+00 \quad .000 E+00 \quad .000 E+00 \quad 5.368 E-04 \quad 1.571 E-09 \quad 3.384 E-09 \quad 1.678 E-09$ $\begin{array}{ll}\text { CO-60 } & 3.208 E-04 \\ \text { MN-54 } & 2.096 \mathrm{E}-06 \\ \text { NA-22 } & 2.139 \mathrm{E}-04\end{array}$
$.000 \mathrm{E}+00 \quad 3.273 E-04$ AM-241 6.140E-05 PU-238 4.320E-08 PU-239 2.493E-04 PU-240 1.568E-05 PU-249 8.691E-07 PU-242 1.161E-07 .367E-04 .000E+00

RENCH 27 
SWIR3280 - (Page 18 of 99)

RUN DATE: 08/20/94

Land Disposal or Storage of Solid Radioactive Waste from January 1 to December 31 , 1993

BURIAL GROUND 218W5, 200 WEST AREA, LOW-LEVEL INDUSTRIAL-OFFSITE.

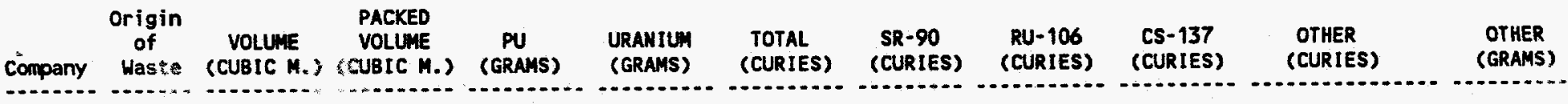

TRENCH 27

ANL ARGON $1.881 E+02 \quad 1.881 E+02 \quad .000 E+00 \quad 2.351 E+06 \quad 4.303 E+00 \quad 4.553 E-04 \quad 9.805 E-04 \quad 5.206 E-03$

$272 E+00 \quad 1.319 E \cdot 6$ AH-241 4.300E-04 AM-243 3.360E-03 PP-237 5.400E-02 PU-238 1.090E-05 PU-239 6.490E-02 PU-240 5.820E-03 PU-241 2.260E-04 PU-242 1.512E-04 RA-224 2.900E-03 RA-226 4.800E-05 TH-228 8.291E-05 AC-228 $9.440 \mathrm{E}-05$ TH-234 3.300E-08 AG-108 1.800E-07 $B E-7 \quad 6.400 E-06$ BI - 212 6.757E-03 $B I-2148.739 E-05$ C-14 1.000E-04 CO-60 7.995E-01 EU-155 7.200E-04 FE-55 7.200E-01 $\mathrm{H}-3$ 3.110E-05 $K-40 \quad 2.060 E-05$ MH-54 1.300E-05 MO- $93 \quad 1.203 \mathrm{E}+00$ NA-22 3.240E-06 NI $-60 \quad 7.990 E-01$ PA-231 7.370E-01 PB-212 3.127E-03 PB-214 2.931E-05 TL-208 2.035E-03

BATC BATCO $\quad 6.775 E+01 \quad 6.775 E+01 \quad .000 E+00 \quad .000 E+00 \quad 2.191 E-02 \quad 2.392 E-03 \quad 3.146 E-07 \quad 3.153 E-03$

$\begin{array}{rr}1.098 E-02 & 2.527 E+04 \\ A M-241 & 4.620 E-06 \\ \text { NP-237 } & 1.400 E-01 \\ P U-239 & 2.520 E-05 \\ \text { RA-226 } & 1.000 E-05 \\ T H-232 & 7.538 E+03 \\ U-234 & 1.177 E+01 \\ U-235 & 2.392 E+01 \\ U-238 & 1.770 E+04\end{array}$

BA-133 7.500E-06

CD-109 1.007E-03

Cl $-36 \quad 8.000 E-07$

CO-57 6.800E-07

CO-60 $9.368 \mathrm{E}-03$

CS-134 1.200E-08

EU-152 7.620E-06

FE-55 1.886E-06

$\mathrm{H}-3 \quad 7.900 \mathrm{E}-06$

I-129 7.700E-08

KR-85 7.430E-06

MN-54 2.400E-08

N8-94 1.300E-07

NI $-60 \quad 4.925 E-04$

NI -63 4.800E-06

PY- 147 2.210E-06

PO-210 2.000E-09

SN-113 2.000E-10

SN-121 3.300E-07 
SWIR3280 - (Page 19 of 99 )

RUN DATE: $08 / 20 / 94$

Land Disposal or Storage of Solid Radioactive Waste from January 1 to December 31 , 1993

BURIAL GROUND 218W5, 200 WEST AREA, LOW-LEVEL INDUSTRIAL-OFFSITE.

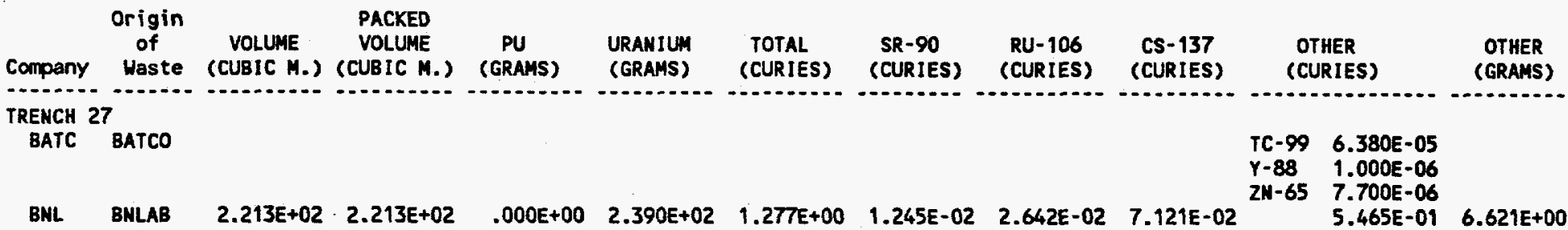

AM-241 1.057E-05

PU-238 6.620E+00

PU-239 8.070E-04

AC-227 2.000E-06

RA-226 1.000E-06

$A C-227$
$A G-110$
$1.595 E-05$

$B E-7 \quad 4.306 E-03$

BI $-2071.492 E-03$

C-14 5.036E-03

CE-141 4.000E-05

CE-144 1.300E-07

CO-56 4.026E-05

CO-57 1.081E-02

CO-58 4.365E-03

CO-60 1.425E-01

CR-51 3.176E-03

CS-134 1.416E-04

EU-154 3.140E-04

FE-59 $1.813 E-02$

GA-68 1.753E-05

GE-68 $6.350 E-06$

$\mathrm{H}-3 \quad 6.890 \mathrm{E}-02$

I-125 6.000E-08

MN-54 2.180E-02

NA-22 3.494E-02

NB-95 2.060E-06

NI-60 1.425E-01

P-32 2.310E-04

RB-86 $2.600 E-07$

RE-187 3.000E-06

S-35 9.732E-03

SB-124 5.400E-07

SB- 125 4.555E-05

SC-46 1.709E-04

SE-79 2.933E-03

SN-113 2.870E-06

SR-82 1.000E-08

SR-85 1.900E-07

TA-182 1.930E-05

TC-99 2.001E-05

FERM NALAB $\quad 4.461 E+01 \quad 4.461 E+01 \quad .000 E+00 \quad 2.026 E+04 \quad 2.957 E-01 \quad 6.874 E-06 \quad 1.480 E-05 \quad 7.341 E-06$

ZN-65 7.475E-02

2.953E-01

$.000 E+00$

$\begin{array}{ll}\text { CO-60 } & 7.211 E-02 \\ M N-54 & 1.016 E-01\end{array}$

NA-22 4.432E-02

NI-60 7.213E-02

ZH-65 5.159E-03

GAS GASD $1.614 E+02 \quad 1.614 E+02 \quad .000 E+00 \quad .000 E+00 \quad 1.100 E-01 \quad 2.148 E-03 \quad 4.626 E-03 \quad 2.294 E-03$

$.000 E+00 \quad 4.473 E+03$

$T H-232 \quad 4.468 E+03$

$\mathrm{U}-234 \quad 3.000 \mathrm{E}-02$

$U-235 \quad 4.200 E+00$

U-238 $3.000 E-01$

$.000 E+00 \quad .000 E+00$

$\begin{array}{lllllllll}\text { PGDF } & 2.050 E+01 & 2.050 E+01 & .000 E+00 & 5.640 E+01 & 5.000 E-04 & 9.944 E-06 & 2.142 E-05 & 1.062 E-05\end{array}$

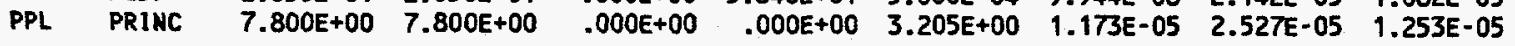

$3.204 E+00$

$.000 E+00$

CO-60 2.300E-03

FE-55 2.300E+00 
SWIR3280 - (Page 20 of 99)

RUN DATE: 08/20/94

Land Disposal or Storage of Solid Radioactive waste from January 1 to December 31 , 1993 BURIAL GROUND 218W5, 200 WEST AREA, LOW-LEVEL INDUSTRIAL-OFFSITE.

\begin{tabular}{|c|c|c|c|c|c|c|c|c|c|c|c|c|}
\hline Eompany & $\begin{array}{c}\text { Origin } \\
\text { of } \\
\text { Waste }\end{array}$ & $\begin{array}{c}\text { VOLLUME } \\
\text { (CUBIC M.) }\end{array}$ & $\begin{array}{l}\text { PACKED } \\
\text { VOLLUME } \\
\text { (CUBIC M.) }\end{array}$ & $\begin{array}{c}\text { PU } \\
\text { (GRAMS) }\end{array}$ & $\begin{array}{l}\text { URANIUM } \\
\text { (GRAMS) }\end{array}$ & $\begin{array}{l}\text { TOTAL } \\
\text { (CURIES) }\end{array}$ & $\begin{array}{l}\text { SR-90 } \\
\text { (CURIES) }\end{array}$ & $\begin{array}{c}\text { RU-106 } \\
\text { (CURIES) }\end{array}$ & $\begin{array}{c}\text { CS-137 } \\
\text { (CURIES) }\end{array}$ & \multicolumn{2}{|c|}{$\begin{array}{l}\text { OTHER } \\
\text { (CURIES) }\end{array}$} & $\begin{array}{c}\text { OTHER } \\
\text { (GRAMS) }\end{array}$ \\
\hline $\begin{array}{l}\text { RENCH } 2 \\
\text { PPL }\end{array}$ & $7_{\text {PRINC }}$ & & & & & & & & & $\begin{array}{l}H-3 \\
M N-54\end{array}$ & $\begin{array}{l}9.020 \mathrm{E}-01 \\
1.100 \mathrm{E}-04\end{array}$ & \\
\hline RKD & ESG & $2.419 E+02$ & $2.419 E+02$ & $.000 E+00$ & $1.899 E+02$ & $5.383 E+00$ & $.159 E-03$ & 7.786E-05 & $3.164 E-03$ & $\begin{array}{l}C O-59 \\
C O-60 \\
\text { EU-152 } \\
\text { EU-154 } \\
\text { FE-55 } \\
\text { CD-152 } \\
\text { CD }-154 \\
H-3 \\
N I-59 \\
N I-60 \\
N I-63\end{array}$ & $\begin{array}{c}5.369 E+00 \\
T H-234 \\
4.000 E-06 \\
3.835 E-01 \\
3.267 E-01 \\
6.169 E-02 \\
3.216 E-01 \\
8.468 E-02 \\
4.420 E-02 \\
3.455 E+00 \\
2.300 E-04 \\
6.372 E-01 \\
5.387 E-02\end{array}$ & $\begin{array}{l}5.000 E-03 \\
5.000 E-03\end{array}$ \\
\hline SLA & SLACU & $1.088 E+01$ & $1.088 E+01$ & $.000 E+00$ & $.000 E+00$ & $9.925 E-04$ & $6.105 E-09$ & 1.315E-08 & $6.521 E-09$ & $\begin{array}{l}\text { CO-57 } \\
\text { CO-60 } \\
\text { CS-134 } \\
\text { EU-152 } \\
\text { EU-154 } \\
K-40 \\
M N-54 \\
N A-22 \\
\text { SC-46 } \\
2 N-65\end{array}$ & $\begin{array}{l}9.922 E-04 \\
2.412 E-05 \\
3.889 E-04 \\
5.108 E-05 \\
1.703 E-05 \\
1.987 E-05 \\
1.192 E-04 \\
2.054 E-05 \\
3.118 E-04 \\
1.391 E-06 \\
3.831 E-05\end{array}$ & $.000 E+00$ \\
\hline UCD & DAVIS & $3.891 E+02$ & $3.891 E+02$ & $.000 E+00$ & $.000 E+00$ & $1.875 E-02$ & $9.326 E-03$ & $2.819 E-06$ & $1.398 E-06$ & & $\begin{array}{r}3.611 E-05 \\
A M-241 \\
P U-240 \\
P U-241 \\
R A-226 \\
T H-228\end{array}$ & $\begin{array}{l}1.468 E-03 \\
7.754 E-08 \\
1.390 E-11 \\
1.286 E-09 \\
1.468 E-03 \\
4.767 E-08\end{array}$ \\
\hline & & & & & & & & & & $\begin{array}{l}\text { BA-134 } \\
C-14 \\
H-3 \\
\text { SB-125 } \\
W-183 M\end{array}$ & $\begin{array}{l}1.270 E-07 \\
1.452 E-05 \\
1.552 E-05 \\
1.789 E-07 \\
5.770 E-06\end{array}$ & \\
\hline & & 13 & $1.513 E+03$ & 0 & 7E+ & 1 & 6.12 & & & & $E+01$ & 4.0 \\
\hline
\end{tabular}


SWIR3280 - (Page 21 of 99)

RUN DATE: $08 / 20 / 94$

Land Disposal or Storage of Solid Radioactive Waste from January 1 to December 31 , 1993 BURIAL GROUND 218W5, 200 WEST AREA, IOW-LEVEL INDUSTRIAL.

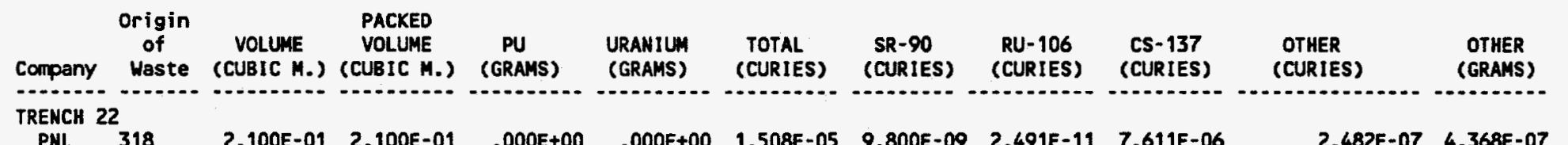
$\mathrm{CH}-242 \quad 4.670 \mathrm{E}-08$ PU-238 1.200E-10 PU-239 2.300E-07 RA-226 1.600E-07 BA-133 1.050E-07 $\begin{array}{ll}\mathrm{BA}-133 \\ \mathrm{C}-14 & 4.530 \mathrm{E}-08\end{array}$ CD-109 4.800E-09 CL-36 2.750E-08 CO-58 1.000E-11

CO-60 4.670E-08

MN-54 4.000E-11

NA-22 1.090E-08

$324 \quad 1.541 E+01 \quad 1.541 E+01 \quad .000 E+00 \quad .000 E+00 \quad 9.408 E-01 \quad 1.103 E-02 \quad 6.884 E-07 \quad 4.360 E-02$ TC-99 7.900E-09

8.338E-01

$.000 E+00$

CO-58 4.751E-05

CO-60 9.107E-03

CS-134 7.660E-05

EU-154 8.598E-06

$\mathrm{H}-3$ 8.131E-01

MN-54 8.907E-04

NI-60 9.097E-03

TA-182 $1.532 E-03$

$325 \quad 1.312 E+01 \quad 1.312 E+01 \quad .000 E+00 \quad 1.000 E-06 \quad 9.383 E-01 \quad 7.531 E-04 \quad 5.609 E-07 \quad 1.586 E-03$

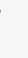

BA-133 1.000E-07

C-14 1.090E-08

CD-109 1.000E-03

CL-36 1.680E-08

CO-60 5.691E-04

CS- 134 2.412E-06

EU-152 9.627E-06

EU-154 2.334E-05

EU-155 3.830E-06

FE-55 1.100E-05

$\mathrm{H}-3$ 9.000E-01

I-129 5.666E-08

$K-40 \quad 1.680 E-06$

MiN-54 2.640E-06

NA-22 1.000E-06

N1-60 5.699E-05

NI-63 2.000E-03

SB-125 1.000E-07

TC-99 1.203E-06

$2 N-65 \quad \begin{array}{r}3.000 E-02 \\ .000 E+00\end{array}$

$\begin{array}{lllllllll}326 & 1.666 E+00 & 1.666 E+00 & .000 E+00 & .000 E+00 & 1.753 E-07 & 4.104 E-12 & 8.312 E-14 & 9.009 E-08 \\ 327 & 1.774 E+01 & 1.774 E+01 & .000 E+00 & 6.606 E+01 & 1.882 E-01 & 7.282 E-03 & 1.477 E-04 & 1.378 E-02\end{array}$ 3.041E+01 AM-241 5.210E-05 AM-243 1.586E-05 CM-244 3.610E-12 NP-237 5.000E-07 
WHC-EP-.0125-6

SWIR3280 - (Page 22 of 99)

RUN DATE: 08/20/94

Land Disposal or Storage of Solid Radioactive Waste from January 1 to December 31 , 1993

BURIAL GROUND 218W5, 200 WEST AREA, LOW-LEVEL INDUSTRIAL.

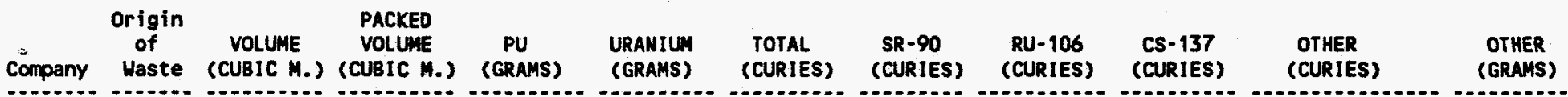

TRENCH 22

PNL $\quad 327$

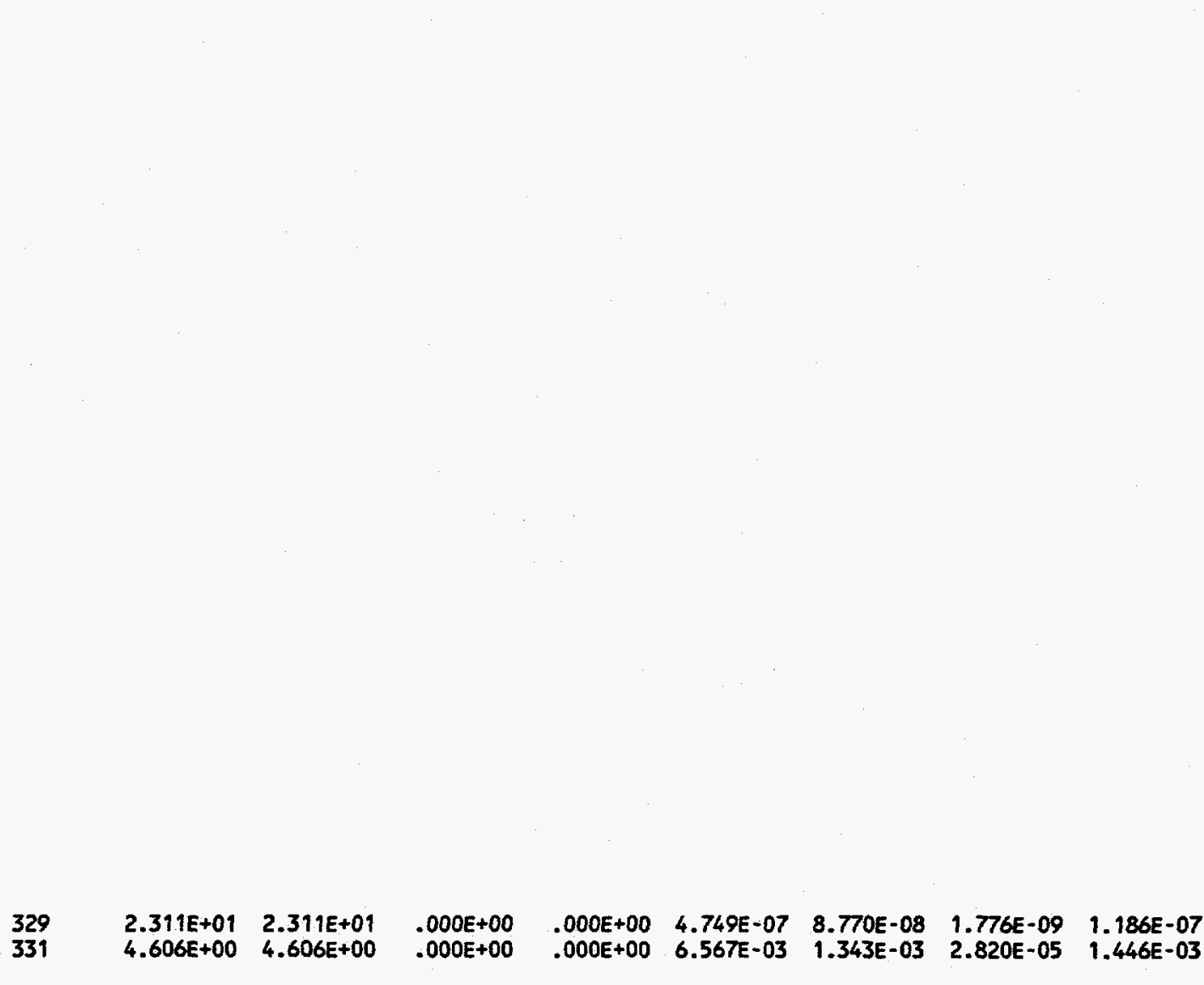

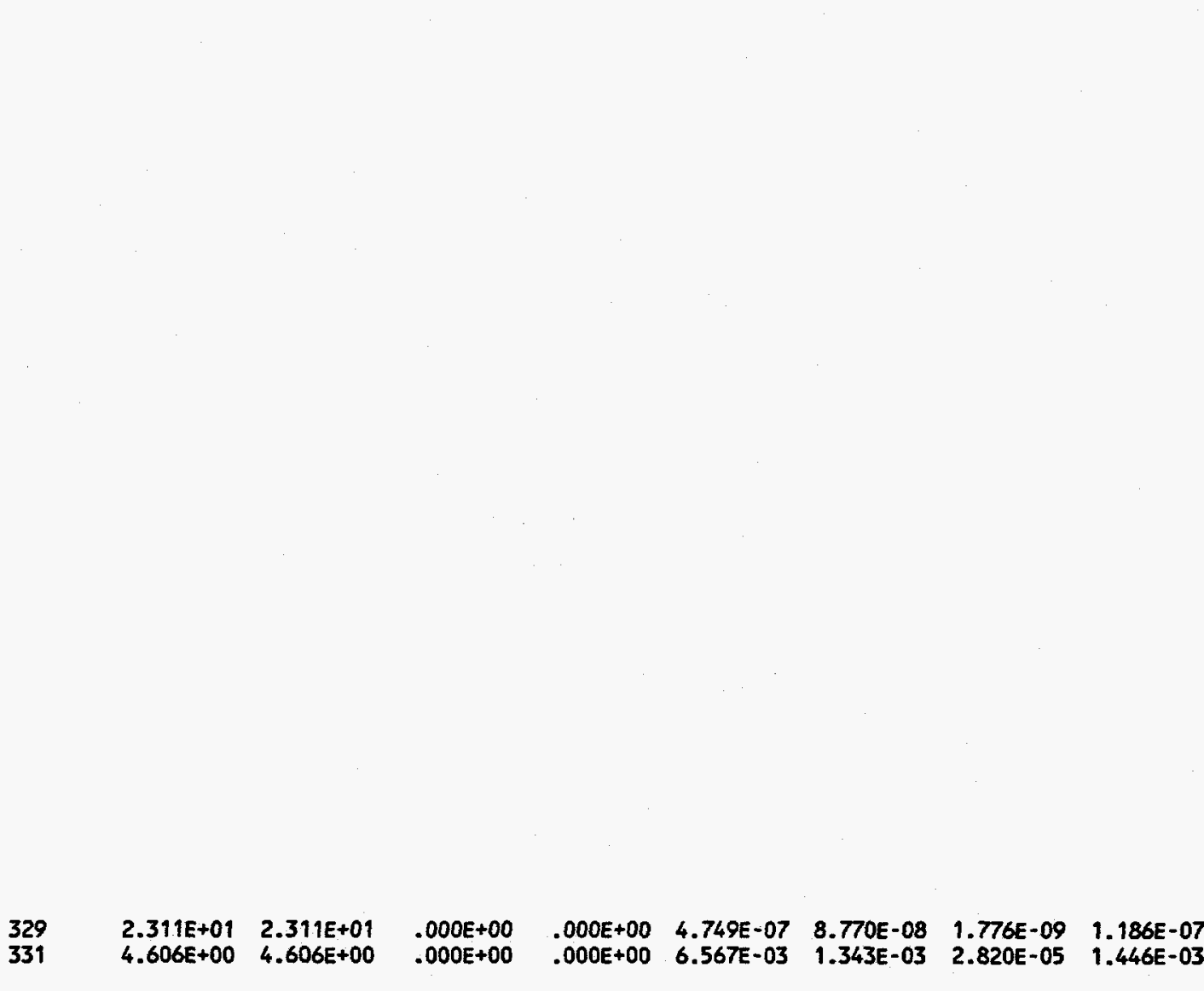

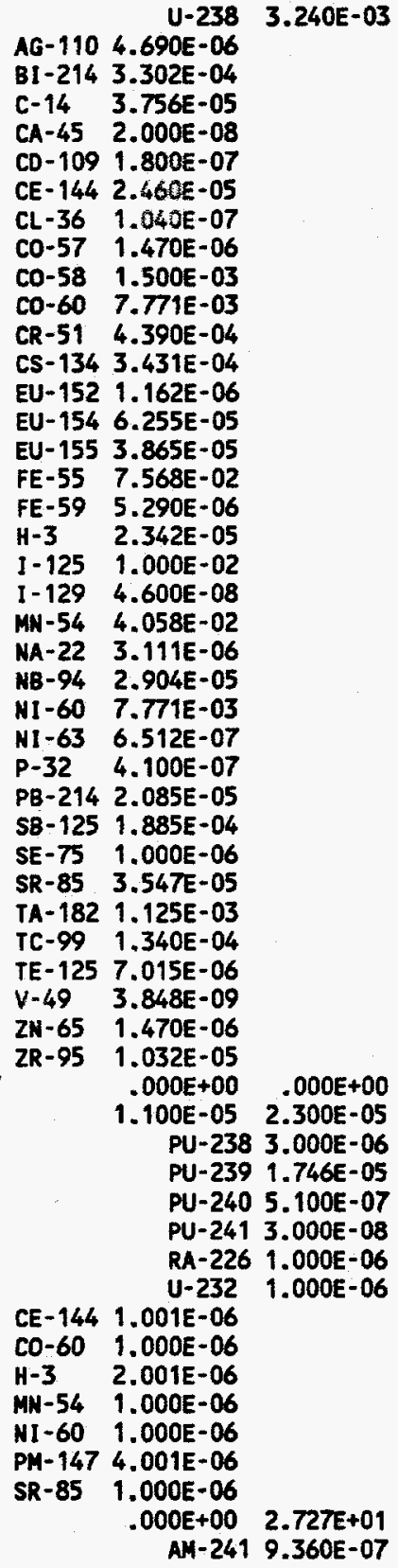

PU-238 4.410E-09 PU-239 1.009E-01 PU-241 1.733E-04 PU-242 $1.874 E-08$ RA-226 $1.403 E-05$ $\begin{array}{lll}\text { RA-226 } & 1.403 E-05 \\ T H-232 & 3.031 E+01\end{array}$ $\begin{array}{ll}\mathrm{TH}-232 & 3.031 \mathrm{E}+01 \\ \mathrm{U}-238 & 3.240 \mathrm{E}-03\end{array}$

$.000 E+00$

$1.150 E-02$

$2.358 E-03$

4.775E-05 2.537E-03 


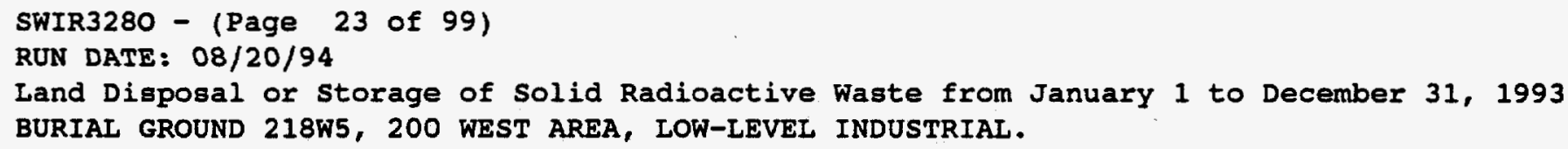
AM- $2413.301 E-04$ PU-238 4.862E-08 PU-239 6.250E-02 PU-240 2.182E-03 
SWIR3280 - (Page 24 of 99)

RUN DATE: 08/20/94

Land Disposal or Storage of Solid Radioactive Waste from January 1 to December 31 , 1993 BURIAL GROUND 218W5, 200 WEST AREA, LOW-LEVEL INDUSTRIAL.

\begin{tabular}{|c|c|c|c|c|c|c|c|c|c|c|c|}
\hline mpany & $\begin{array}{l}\text { of } \\
\text { Waste }\end{array}$ & $\begin{array}{c}\text { VOLUME } \\
\text { (CUBIC H.) }\end{array}$ & $\begin{array}{l}\text { PACKED } \\
\text { YOLUME } \\
\text { (CUBIC M.) }\end{array}$ & $\begin{array}{c}\text { PU } \\
\text { (GRAMS) }\end{array}$ & $\begin{array}{l}\text { URANIUN } \\
\text { (GRAMS) }\end{array}$ & $\begin{array}{l}\text { TOTAL } \\
\text { (CURIES) }\end{array}$ & $\begin{array}{l}\text { SR-90 } \\
\text { (CURIES) }\end{array}$ & $\begin{array}{l}\text { RU-106 } \\
\text { (CURIES) }\end{array}$ & $\begin{array}{c}\text { CS-137 } \\
\text { (CURIES) }\end{array}$ & $\begin{array}{l}\text { OTHER } \\
\text { (CURIES) }\end{array}$ & $\begin{array}{l}\text { OTHER } \\
\text { (GRAMS) }\end{array}$ \\
\hline
\end{tabular}

TRENCH 22

WHC 105KE
PU-241 2.987E-07

PU-242 2.116E-04

CO-60 $1.483 E-02$

EU-154 2.102E-08

EU- 155 1.401E-08

$M N-54 \quad 7.006 E-09$

NI-60 1.423E-02

CS137 1.141E-07

C0-58 1.108E-07

C0-60 $7.424 E-06$

EU155 $9.419 \mathrm{E}-07$

C- $14 \quad 1.108 E-07$

BA137 1.075E-07

EU154 9.419E-07

FE-59 4.432E-07

MN-54 7.757E-07

EU152 1.108E-07

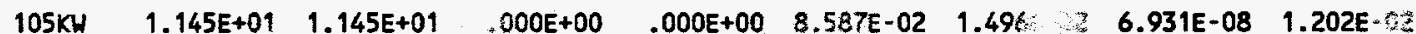

$105 N$

$2.457 E+01 \quad 2.457 E+01 \quad .000 E+00$

$.000 E+00 \quad 2.879 E-03$

$3.279 E-04$

2.074E-09

$1.127 E-03$

EU- 154 1.382E-07

$1-129 \quad 4.678 E-11$

$M N-54 \quad 3.276 E-08$

NI $-60 \quad 1.336 E-05$

NI -63 1.333E-08

TC-99 1.187E-07

ZN-65 1.333E-08

CS137 1.520E-12

CO-58 $1.476 E-12$

Co-60 $9.886 \mathrm{E}-11$

EU155 1.254E-11

C-14 1.476E-12

BA137 1.431E-12

EU154 1.254E-11

FE-59 5.902E-12

MN-54 1.033E-11

EU152 1.476E-12

\begin{tabular}{|c|c|c|c|c|c|c|c|c|c|c|c|}
\hline $1143 N$ & $8.328 E-01$ & $8.328 E-01$ & $.000 E+00$ & $.000 E+00$ & $1.026 E-05$ & $.000 E+00$ & $.000 E+00$ & $.000 E+00$ & EU152 & $\begin{array}{l}1.476 E-12 \\
1.026 E-05\end{array}$ & $.000 E+00$ \\
\hline $200 E$ & $1.666 \mathrm{E}+00$ & $1.666 E+00$ & $.000 E+00$ & $.000 E+00$ & $3.423 E-04$ & $3.130 E-06$ & $6.025 E-08$ & $5.432 E-06$ & & $\begin{array}{r}3.232 E-04 \\
\text { AM-241 } \\
\text { CM-242 } \\
\text { CM-244 } \\
\text { NP-237 } \\
\text { PU-238 } \\
\text { PU-239 } \\
\text { PU-240 } \\
U-234\end{array}$ & $\begin{array}{l}4.991 E-04 \\
9.987 E-09 \\
1.296 E-11 \\
2.073 E-09 \\
4.705 E-09 \\
2.610 E-10 \\
5.700 E-08 \\
5.654 E-08 \\
3.143 E-13\end{array}$ \\
\hline
\end{tabular}

$$
06^{x-4}
$$




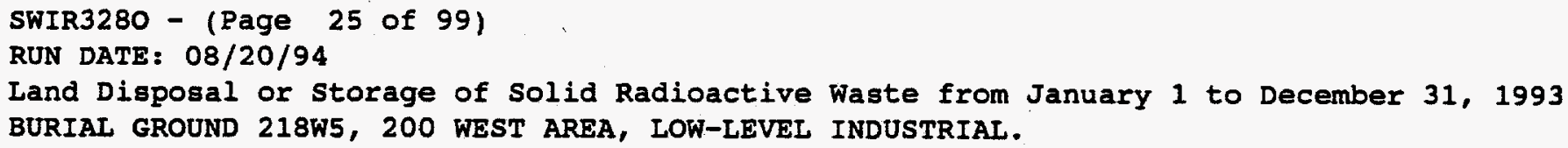


SWIR3280 - (Page 26 of 99)

RUN DATE: 08/20/94

Land Disposal or storage of Solid Radioactive Waste from January 1 to December 31,1993 BURIAL GROUND 218W5, 200 WEST AREA, LOW-LEVEL INDUSTRIAI.

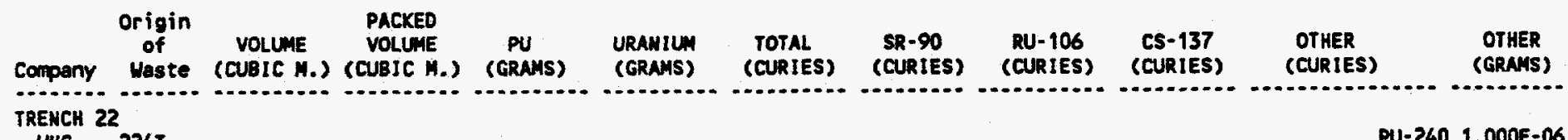

WHC $224 T$

$224 U \quad 1.124 E+01 \quad 1.124 E+01 \quad 1.000 E-01 \quad 4.500 E+00 \quad 8.804 E-06 \quad 1.435 E-10 \quad 2.906 E-12 \quad 2.050 E-06$

2335

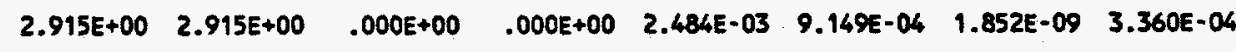

TC-99

PU-241 $1.000 E-06$

PU-242 1.000E-06

4.814E-06 $9.895 E+00$

AM-241 1.120E-06

U-234 1.293E-04

U-235 1.400E-02

U-236 1.055E-03

U-238 $9.880 E+00$

$\begin{array}{rr}4.814 E-06 \\ .000 E+00 & 2.799 E-02\end{array}$

AN-241 1.079E-04 PU-238 2.963E-08 PU-239 2.733E-02 PU-240 4.584E-04 PU-241 $9.838 E-08$ PU-242 9.210E-05

$23452 \quad 1.159 E+02 \quad 1.159 E+02 \quad .000 E+00 \quad .000 E+00 \quad 3.520 E-01 \quad 6.928 E-02 \quad 1.403 E-03 \quad 7.455 E-02$ $.000 E+00$ 3.524E-02 AM-241 5.457E-05 PU-238 1.785E-03 PU-239 3.058E-02 PU-240 1.997E-03 PU-241 8.081E-04 PU-242 7.940E-06

$2436 \quad 1.041 E+00 \quad 1.041 E+00 \quad .000 E+00 \quad .000 E+00 \quad 4.297 E-04 \quad 3.233 E-07 \quad 4.650 E-10 \quad 4.189 E-06$ 4.209E-04 3.593E-07 AM-241 2.145E-08 AM-243 1.211E-12 CM-242 1.203E-14 CM-243 4.382E-09 CM-244 2.325E-09 MP-237 9.459E-08 PU-238 5.710E-10 PU-239 2.480E-07 PU-240 6.796E-08

CO-60 4.181E-04 $\mathrm{H}-3 \quad 1.305 \mathrm{E}-06$ $\begin{array}{ll}1-129 & 2.059 E-10\end{array}$ MI-59 1.275E-09 NI-63 1.051E-06

TC-99 4.156E-07 $\begin{array}{lllllllll}271 B & 3.724 E+01 & 3.724 E+01 & .000 E+00 & .000 E+00 & 6.850 E-02 & 7.018 E-03 & 5.238 E-07 & 2.798 E-02 \\ 2724 \mathrm{H} & 7.287 E+00 & 7.287 E+00 & .000 E+00 & 1.675 E+00 & 3.898 E-03 & 6.064 E-04 & 2.217 E-09 & 2.084 E-04\end{array}$ $.000 E+00$

CE-144 4.136E-05 CO-60 $1.663 \mathrm{E}-03$ EU- 152 6.630E-06 EU-154 2.001E-05 FE-59 8.957E-05 MN-54 2.849E-04 MB-95 2.468E-05 SB-125 1.612E-05 TE-125 1.568E-05 ZN-65 4.42TE-05

$2765 \quad 2.707 E+00 \quad 2.707 E+00 \quad .000 E+00 \quad .000 E+00 \quad 9.245 E-04 \quad 3.477 E-04 \quad 9.134 E-10 \quad 1.177 E-04$ $2 R-95 \quad 1.638 E-05$

$276 U 1.041 E+00 \quad 1.041 E+00 \quad .000 E+00 \quad .000 E+00 \quad 3.47 T E-03 \quad 1.208 E-03 \quad 3.982 E-09 \quad 5.448 E-04$ 
SWIR3280 - (Page 27 of 99)

RUN DATE: 08/20/94

Land Disposal or Storage of Solid Radioactive Waste from January 1 to December 31 , 1993 BURIAL GROUND 218W5, 200 WEST AREA, LOW-LEVEL INDUSTRIAL.

\begin{tabular}{|c|c|c|c|c|c|c|c|c|c|}
\hline \multirow{3}{*}{$\begin{array}{c}\text { CompanY } \\
\text { TRENCH } \\
\text { WHC }\end{array}$} & $\begin{array}{c}\text { Origin } \\
\text { of } \\
\text { Uaste }\end{array}$ & $\begin{array}{l}\text { VOLLUE } \\
\text { (CUBIC M.) }\end{array}$ & $\begin{array}{c}\text { PACKED } \\
\text { VOLUME } \\
\text { (CUBIC M.) }\end{array}$ & $\begin{array}{c}\text { PU } \\
\text { (GRAMS) }\end{array}$ & $\begin{array}{l}\text { URANIUM } \\
\text { (GRAMS) }\end{array}$ & $\begin{array}{l}\text { TOTAL } \\
\text { (CURIES) }\end{array}$ & $\begin{array}{l}\text { SR-90 } \\
\text { (CURIES) }\end{array}$ & $\begin{array}{c}\text { RU-106 } \\
\text { (CURIES) }\end{array}$ & $\begin{array}{c}\text { CS-137 } \\
\text { (CURIES) }\end{array}$ \\
\hline & 22200 & & & & & & & & \\
\hline & $\begin{array}{l}2915 \\
327\end{array}$ & $\begin{array}{l}6.246 E-01 \\
4.164 E-01\end{array}$ & $\begin{array}{l}6.246 E-01 \\
4.164 E-01\end{array}$ & $\begin{array}{l}.000 E+00 \\
.000 E+00\end{array}$ & $\begin{array}{l}.000 E+00 \\
.000 E+00\end{array}$ & $\begin{array}{l}5.116 E-05 \\
6.246 E-03\end{array}$ & $\begin{array}{l}1.876 E-05 \\
1.438 E-05\end{array}$ & $\begin{array}{l}8.179 E-11 \\
5.763 E-09\end{array}$ & $\begin{array}{l}7.004 E-06 \\
2.392 E-03\end{array}$ \\
\hline
\end{tabular}

$340 \quad 3.956 E+00 \quad 3.956 E+00 \quad .000 E+00 \quad .000 E+00 \quad 2.016 E-03 \quad 2.171 E-05 \quad 3.949 E-06 \quad 1.240 E-04$

$\begin{array}{ll}C 0-60 & 7.550 E-04 \\ E U-154 & 5.158 E-05\end{array}$

$\mathrm{NI}-60 \quad 7.550 \mathrm{E}-04$

340 $3.949 E-06 \quad 1.240 E-04$

NI $-60 \quad \begin{array}{r}7.550 E-04 \\ 1.704 E-03\end{array}$

AM-241 1.671E-05

PU-238 2.317E-07

PU-239 3.226E-05

PU-240 3.940E-08

CE-144 1.971E-05

PU-241 4.726E-07

CS- $1349.853 E-06$

EU-152 5.460E-04

EU-154 9.745E-04

EU- $1551.520 E-04$

$437 \quad 4.997 E+00 \quad 4.997 E+00 \quad .000 E+00 \quad .000 E+00 \quad 2.279 E-02 \quad 5.687 E-07 \quad 1.152 E-08 \quad 9.274 E-03$

TA- $182 \quad 1.971 \mathrm{E}-06$

$4.743 E-03$

$.000 E+00$

CS- $1342.318 E-03$

NH $-54 \quad 1.159 E-03$

NI-60 6.854E-04

EFSG $\quad 3.388 E+01 \quad 3.388 E+01 \quad .000 E+00 \quad 1.326 E-15 \quad 8.831 E+01 \quad 1.461 E+01 \quad 4.073 E-03 \quad 2.990 E+01$

$\begin{array}{lrr} & 7.420 E-01 & 2.843 E-02 \\ & \text { CF-252 } & 5.800 E-06 \\ & \text { PU-238 } & 1.892 E-02 \\ & \text { PU-239 } & 7.237 E-03 \\ & \text { PU-240 } & 2.146 E-03 \\ & \text { RA-226 } & 8.698 E-05 \\ & \text { TH-228 } & 3.397 E-05 \\ \text { AC-227 } & 1.672 E-05 \\ \text { CO-60 } & 4.924 E-02 \\ \text { CR-51 } & 5.658 E-08 \\ \text { EU-155 } & 3.400 E-07 \\ H-3 & 6.601 E-01 \\ I-129 & 4.097 E-07 \\ \text { K-40 } & 5.001 E-04 \\ \text { NI-60 } & 3.033 E-02 \\ \text { PB-208 } & 9.203 E-08 \\ \text { SB-125 } & 1.722 E-04 \\ \text { TC-99 } & 1.706 E-03\end{array}$

TRENCH 27

$\begin{array}{rllllllllll}\text { PNL } & 306 \mathrm{H} & 4.078 E+00 & 4.078 E+00 & .000 E+00 & 1.000 E+00 & 5.000 E-04 & 1.025 E-04 & 2.076 E-06 & 1.103 E-04 \\ & 314 & 8.082 E+00 & 8.082 E+00 & .000 E+00 & 2.830 E+00 & 3.860 E-05 & .000 E+00 & .000 E+00 & .000 E+00\end{array}$

324

$\begin{array}{llllllll}1.595 E+02 & 1.595 E+02 & .000 E+00 \quad .000 E+00 & 1.101 E+00 & 1.621 E-01 & 5.868 E-06 & 3.605 E-01\end{array}$

$$
\text { (1) }
$$

$$
\begin{aligned}
& \mathrm{EU}- \\
& \mathrm{H}-3
\end{aligned}
$$$$
\text { NI }
$$

$.000 E+00 \quad .000 E+00$ $3.860 E-05 \quad .000 E+00$

C0-60. $6.005 E-07$

MN-54 3.800E-05

I-60 5.100E-10 $7.490 E-02 \quad .000 E+00$

6. $350 E-06$

.350E-02

$\begin{array}{ll}1-60 & 2.436 E-03\end{array}$

$9.485 E-07 \quad 7.271 E-03$ AM-241 6.852E-05 AM-243 4.420E-04 MP-237 6.760E-03 PU-239 7.580E-07 
SWIR3280 - (Page 28 of 99 )

RUN DATE: 08/20/94

Land Disposal or Storage of Solid Radioactive Waste from January 1 to December 31 , 1993

BURIAL GROUND 218\%5, 200 WEST AREA, LOW-LEVEL INDUSTRIAI.

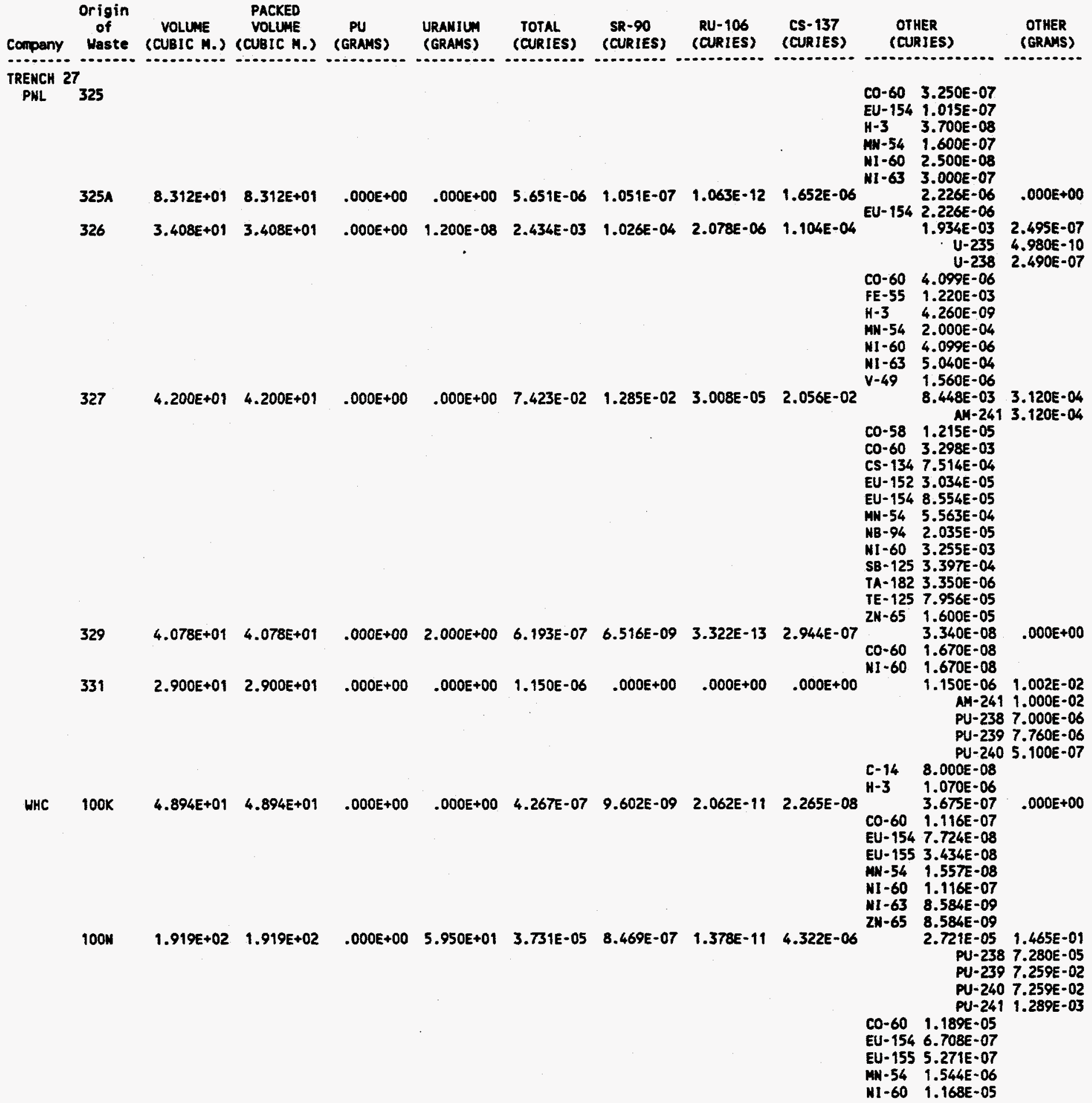


SWIR3280 - (Page 29 of 99)

RUN DATE: $08 / 20 / 94$

Land Disposal or Storage of Solid Radioactive Waste from January 1 to December 31 , 1993 BURIAL GROUND 218W5, 200 WEST AREA, LOW-LEVEL INDUSTRIAL.

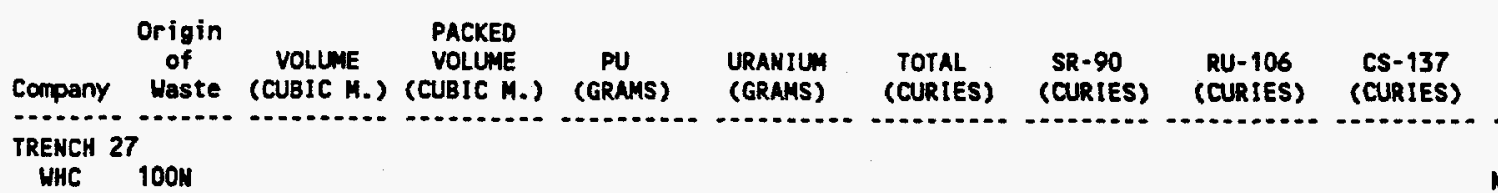

\begin{tabular}{|c|c|}
\hline $\begin{array}{l}N I-63 \\
2 N-65 \\
C S 137 \\
C O-58 \\
C O-60 \\
\text { EU155 } \\
C-14 \\
\text { BA137 } \\
\text { EU154 } \\
\text { FE-59 } \\
\text { NN-54 } \\
\text { EU152 }\end{array}$ & $\begin{array}{l}5.074 E-07 \\
3.881 E-07 \\
3.378 E-13 \\
3.280 E-13 \\
2.198 E-11 \\
2.788 E-12 \\
3.280 E-13 \\
3.182 E-13 \\
2.788 E-12 \\
1.312 E-12 \\
2.296 E-12 \\
3.280 E-13 \\
4.463 E-05\end{array}$ \\
\hline
\end{tabular}

\begin{tabular}{|c|c|c|c|c|c|c|c|c|c|c|c|}
\hline $105 B$ & $4.531 E+00$ & $4.531 E+00$ & $.000 E+00$ & $.000 E+00$ & $7.078 E-05$ & $2.271 E-06$ & $2.906 E-11$ & $1.110 E-05$ & $\begin{array}{l}\text { EU152 } \\
C-14 \\
C O-60 \\
E U-152 \\
E U-154 \\
E U-155 \\
H-3\end{array}$ & $\begin{array}{l}3.280 E-13 \\
4.463 E-05 \\
2.590 E-05 \\
1.160 E-05 \\
1.720 E-06 \\
6.120 E-07 \\
1.470 E-06 \\
3.330 E-06\end{array}$ & $.000 E+00$ \\
\hline 105KE & $3.534 E+01$ & $3.534 E+01$ & $.000 E+00$ & $.000 E+00$ & $2.082 E-04$ & $6.822 E-06$ & $5.199 E-10$ & 3.291E-05 & $\begin{array}{l}C-14 \\
C O-60 \\
E U-152 \\
E U-154 \\
E U-155 \\
H-3 \\
M N-54\end{array}$ & $\begin{array}{l}1.304 E-04 \\
7.600 E-05 \\
3.405 E-05 \\
5.060 E-06 \\
1.176 E-06 \\
4.349 E-06 \\
9.800 E-06 \\
9.450 E-09\end{array}$ & $.000 E+00$ \\
\hline $5 \mathrm{~N}$ & $8.156 E+00$ & $8.156 E+00$ & $.000 E+00$ & $.000 E+00$ & $3.378 E-08$ & $6.910 E-10$ & $3.048 E-13$ & $1.706 E-09$ & $\begin{array}{l}C O-60 \\
\text { EU-154 } \\
\text { EU-155 } \\
M N-54 \\
N I-60 \\
H I-63 \\
Z N-65\end{array}$ & $\begin{array}{l}2.906 E-08 \\
9.058 E-09 \\
5.540 E-09 \\
2.704 E-09 \\
1.352 E-09 \\
9.058 E-09 \\
6.760 E-10 \\
6.760 E-10\end{array}$ & $.000 E+00$ \\
\hline $9 N$ & $5.327 E+01$ & $5.327 E+01$ & $.000 E+00$ & $.000 E+00$ & $1.372 E-04$ & $1.381 E-06$ & $4.152 E-10$ & $1.382 E-06$ & $\begin{array}{l}C 0-60 \\
M N-54 \\
N I-60 \\
N I-63\end{array}$ & $\begin{array}{l}1.318 E-04 \\
6.314 E-05 \\
2.744 E-06 \\
6.314 E-05 \\
2.744 E-06\end{array}$ & $.000 E+00$ \\
\hline $2 U$ & $3.625 E+00$ & $3.625 E+00$ & $.000 E+00$ & $.000 E+00$ & 2.737E-06 & $6.992 E-07$ & $3.322 E-12$ & 2.182E-07 & & $\begin{array}{r}9.140 E-07 \\
A M-241 \\
P U-238 \\
P U-239 \\
P U-240 \\
P U-241 \\
T H-228\end{array}$ & $\begin{array}{l}7.236 E-07 \\
2.210 E-09 \\
4.920 E-11 \\
1.990 E-07 \\
1.280 E-08 \\
1.580 E-09 \\
5.080 E-07\end{array}$ \\
\hline OSKE & $8.156 E+00$ & B.156E+00 & $.000 E+00$ & $.000 E+00$ & $7.368 E-08$ & 1.309E-08 & $3.654 E-14$ & $2.095 E-08$ & $\begin{array}{l}R-40 \\
C O-60 \\
E U-154 \\
E U-155 \\
M N-54 \\
N I-60\end{array}$ & $\begin{array}{l}1.140 E-07 \\
6.732 E-09 \\
1.122 E-09 \\
2.244 E-09 \\
1.496 E-09 \\
7.480 E-10 \\
1.122 E-09\end{array}$ & $.000 E+00$ \\
\hline UE & $3.282 E+01$ & $282 E+01$ & $.000 E+00$ & $960 E+02$ & $1.546 E-03$ & $1.510 E-05$ & $1.291 E-09$ & $E-04$ & & $\begin{array}{r}5.694 E-04 \\
A M-241 \\
P U-238 \\
P U-239 \\
P U-249 \\
R A-226\end{array}$ & $\begin{array}{l}1.745 E-04 \\
2.805 E-06 \\
1.112 E-09 \\
2.766 E-08 \\
4.058 E-05 \\
1.311 E-04\end{array}$ \\
\hline
\end{tabular}


SWIR3280 - (Page 30 of 99 )

RUN DATE: $08 / 20 / 94$

Land Disposal or Storage of Solid Radioactive Waste from January 1 to December 31 , 1993

BURIAL GROUND 218W5, 200 WEST AREA, LOW-LEVEL INDUSTRIAL.

\begin{tabular}{|c|c|c|c|c|c|c|c|c|c|}
\hline Company & $\begin{array}{c}\text { Origin } \\
\text { of } \\
\text { Waste }\end{array}$ & $\begin{array}{c}\text { VOLUNE } \\
\text { (CUBIC M.) }\end{array}$ & $\begin{array}{c}\text { PACKED } \\
\text { VOLUME } \\
\text { (CUBIC } \text { H.) }^{\text {. }}\end{array}$ & $\begin{array}{c}\text { PU } \\
\text { (GRAMS) }\end{array}$ & $\begin{array}{l}\text { URANIUM } \\
\text { (GRAMS) }\end{array}$ & $\begin{array}{l}\text { TOTAL } \\
\text { (CURIES) }\end{array}$ & $\begin{array}{c}\text { SR-90 } \\
\text { (CURIES) }\end{array}$ & $\begin{array}{c}\text { RU-106 } \\
\text { (CURIES) }\end{array}$ & $\begin{array}{l}\text { CS-137 } \\
\text { (CURIES) }\end{array}$ \\
\hline & & & & & & & & & \\
\hline
\end{tabular}

$201 C \quad 7.250 E+00 \quad 7.250 E+00 \quad .000 E+00 \quad .000 E+00 \quad 8.903 E-04 \quad 2.420 E-04 \quad 3.114 E-10 \quad 2.088 E-04$ $202 A \quad 1.921 E+02 \quad 1.921 E+02 \quad .000 E+00 \quad 2.854 E+00 \quad 1.562 E-02 \quad 1.944 E-03 \quad 2.594 E-04 \quad 1.243 E-03$

$.000 E+00$

$000 E+00$

$.696 E-03 \quad 6.225 E-04 \quad 1.699 E-08 \quad 2.314 E-04$

21842A

$3.625 E+00 \quad 3.625 E+00$

$.000 E+00$

3.114E-O

$3.347 E-0$

$1.058 E-06 \quad 3.322 E-11$

$6.459 E-06$

\section{c. \\ CE-}

TH-228 4.584E- 09

$$
\begin{array}{lr}
H-3 & 3.196 E-04 \\
K-40 & 6.900 E-05 \\
T C-99 & 1.808 E-04 \\
& .000 E+00 \\
& 8.302 E-03 \\
& C H-242 \\
& \text { PU-24 } \\
& U-238 \\
& \\
& \\
C-14 & 3.114 E-12 \\
\text { CE-144 } & 4.990 E-04 \\
H-3 & 1.048 E-05 \\
I-129 & 8.519 E-10 \\
M B-94 & 1.695 E-12 \\
\text { PM-147 } & 2.812 E-03 \\
\text { SE-79 } & 1.111 E-08 \\
\text { TC-99 } & 4.980 E-03 \\
\text { ZN-65 } & 4.040 E-10 \\
&
\end{array}
$$

$.000 E+00 \quad .000 E+00$

$8.302 E-03 \quad 5.625 E-02$

CH-242 1.426E-06 PU-241 6.409E-06 $1-238 \quad 5.610 E-02$

$.000 E+00 \quad 8.991 E-04$ AM-241 3.470E-06 PU-238 $9.530 E-10$ PU-239 8.780E-04 PU-240 1.470E-05 PU-249 3.160E-09 PU-242 2.960E-06 1.878E-05 AC-228 7.275E-07 B1-212 4.855E-07 BI-214 5.309E-07 $k=40 \quad 1.561 E-05$

PB-212 6.623E-07

PB-214 5.401E-07

$221 T \quad 2.601 E+02 \quad 2.601 E+02 \quad .000 E+00 \quad .000 E+00 \quad 1.904 E-01 \quad 2.657 E-02 \quad 1.602 E-07 \quad 6.875 E-02$

TL-208 2.287E-07

$\begin{array}{llllllll}2.601 E+02 & 2.601 E+02 \quad .000 E+00 & .000 E+00 & 1.904 E-01 & 2.657 E-02 & 1.002 E-07 & 6.875 E-02\end{array}$

$221 U \quad 7.250 E+00 \quad 7.250 E+00 \quad .000 E+00 \quad .000 E+00 \quad 4.375 E-04 \quad 5.079 E-05 \quad 2.370 E-08 \quad 1.721 E-04$

2225

$4.548 E+02 \quad 4.548 E+02$

$.000 E+00$

$4.692 E+00$

$6.757 E-01$

$9.751 E-02$

2.193E-06 2.287E-01

EU-

$$
\begin{array}{cc}
3.507 E-03 & 7.500 E-06 \\
\text { PU-241 } & 7.500 E-06
\end{array}
$$

8.790E-04

$2.143 E-03$

$.000 E+00 \quad .000 E+00$

3.565E-02 $1.354 E+00$

AM-241 3.404E-04

PU-238 4.315E-06

\begin{tabular}{|c|c|c|c|c|c|c|c|c|c|c|c|}
\hline 2240 & $1.066 E+02$ & $1.066 E+02$ & $.000 E+00$ & $.000 E+00$ & $1.909 E-03$ & $.000 E+00$ & $.000 E+00$ & $.000 E+00$ & & $\begin{array}{r}1.909 E-03 \\
U-234 \\
U-235 \\
U-236 \\
U-238\end{array}$ & $\begin{array}{l}3.119 E+03 \\
2.807 E-01 \\
2.870 E+01 \\
2.215 E+00 \\
3.088 E+03\end{array}$ \\
\hline 23452 & $1.995 E+02$ & $1.995 E+02$ & $.000 E+00$ & $.000 E+00$ & $4.050 E-02$ & 8.303E-03 & $1.682 E-04$ & 8.934E-03 & TC $=99$ & $\begin{array}{r}1.909 E-03 \\
.000 E+00 \\
\text { NH-249 } \\
\text { PU-238 }\end{array}$ & $\begin{array}{l}1.236 E-02 \\
1.388 E-05 \\
3.621 E-06\end{array}$ \\
\hline
\end{tabular}

PU-239 4.355E-03

PU-241 1.500E-08

$U-234 \quad 1.348 E-03$

U-235 5.899E-02

H-3 $\quad 9.528 E-03$

U-238 $1.289 E+00$

I-129 1.216E-O4

PW-147 2.585E-02

$224 U \quad 1.066 E+02 \quad 1.066 E+02 \quad .000 E+00 \quad .000 E+00 \quad 1.909 E-03 \quad .000 E+00 \quad .000 E+00 \quad .000 E+00$

$23452 \quad 1.995 E+02 \quad 1.995 E+02 \quad .000 E+00 \quad .000 E+00 \quad 4.050 E-02 \quad 8.303 E-03 \quad 1.682 E-04 \quad 8.934 E-03$

TC- 


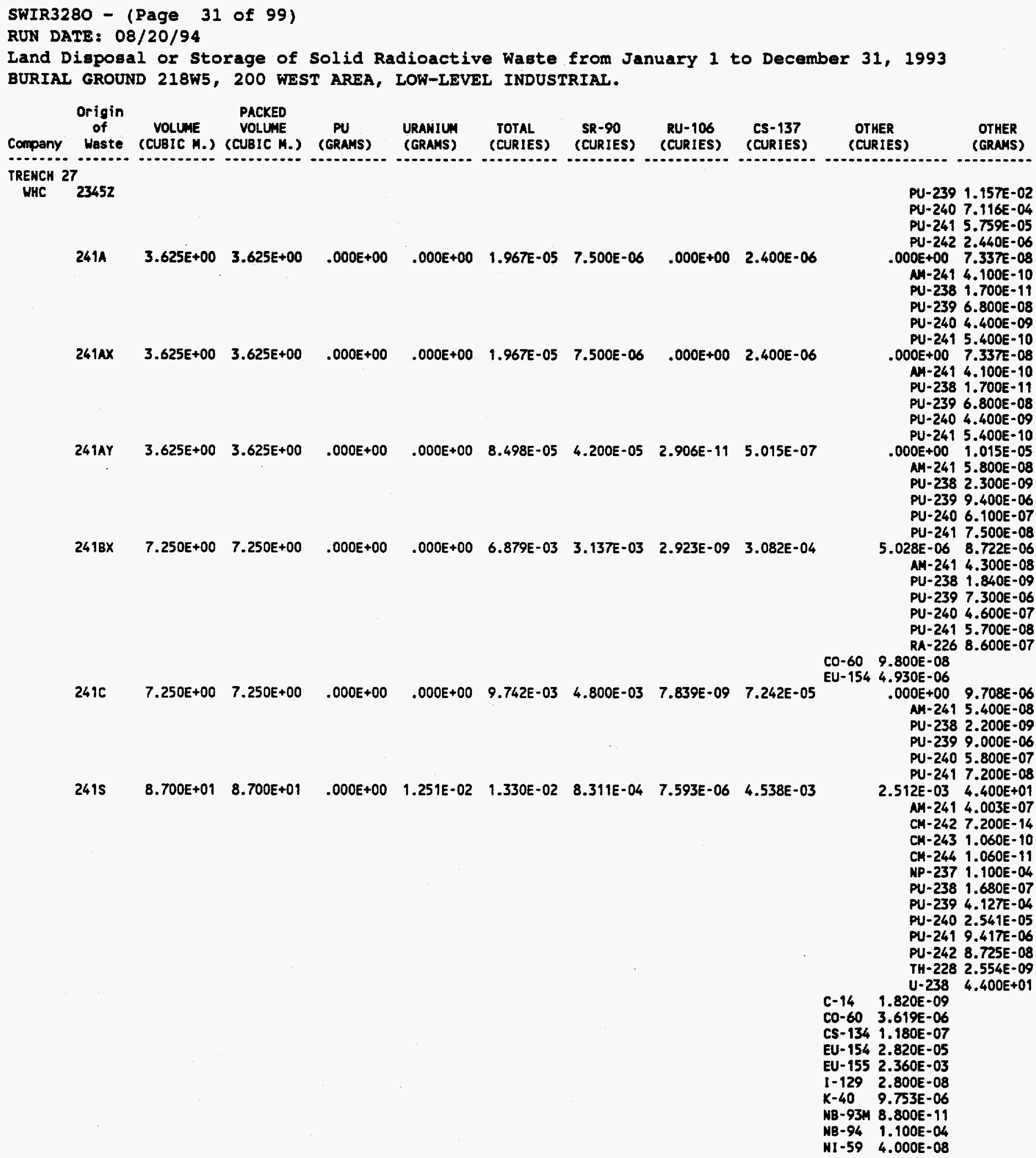


SWIR3280 - (Page 32 of 99 )

RUN DATE: 08/20/94

Land Dieposal or storage of Solid Radioactive Waste from January 1 to December 31 , 1993 BURIAL GROUND 218W5, 200 WEST AREA, LOW-IEVEL INDUSTRIAL.

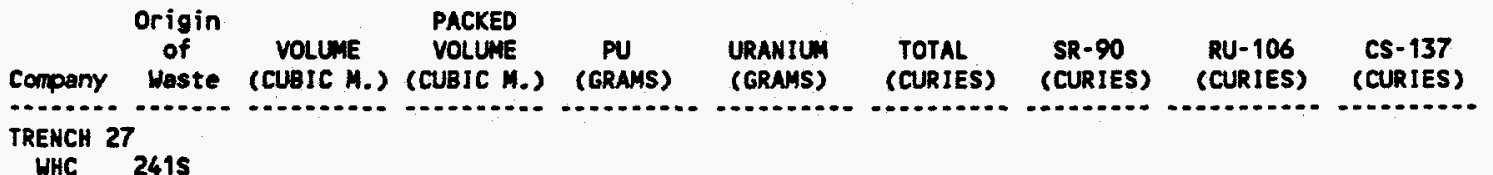

HHC 2415$$
\text { SI }
$$

9 7.000E-07

OTHER OTHER

(CURIES)

(GRAMS)

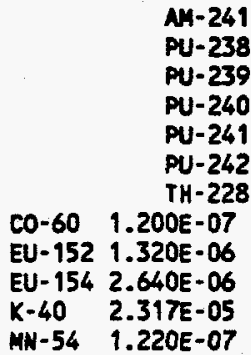

$2415 Y \quad 5.800 E+01 \quad 5.800 E+01 \quad .000 E+00 \quad 6.253 E-03 \quad 9.751 E-03 \quad 5.080 E-04 \quad 3.170 E-09 \quad 4.197 E-03$

MN

5.679E-04 5.108E-02 AM-241 B.075E-07 CM-242 $4.148 E-13$ CM-243 3.080E-05 CM-244 1.967E-10 UP-237 6.361E-04 PU-238 6.638E-10 PU-239 2.432E-06 PU-240 1.609E-07 PU-241 3.241E-06 PU-242 4.280E-10 TH-228 2.041E-09 U-235 2.646E-05 U-236 4.880E-02 U-238 $1.578 E-03$

c-14 1.050E-08

CO-60 7.160E-06

CS-134 3.860E-07

EU-154 4.039E-05

FE-59 2.000E-08

$1-129 \quad 1.415 E-05$

K-40 6.355E-06

NB-934 9.964E-09

NB-94 4.950E-04

NB-95 1.160E-07

NI-59 1.603E-07

NI-63 5.232E-08

SE-79 3.590E-09

TC-99 4.046E-06

$2417 \quad 1.813 E+01 \quad 1.813 E+01 \quad .000 E+00 \quad .000 E+00 \quad 6.632 E+00 \quad 1.361 E+00 \quad 2.751 E-02 \quad 1.462 E+00$

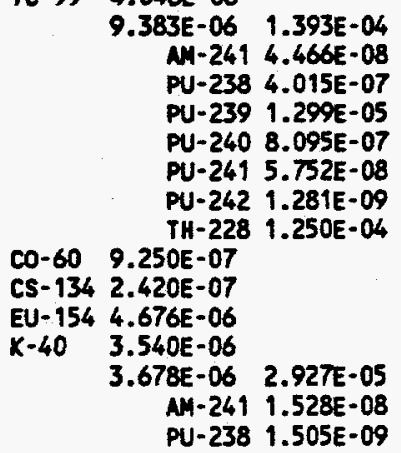


SWIR3280 - (Page 33 of 99)

RUN DATE: 08/20/94

Land Disposal or Storage of Solid Radioactive Waste from January 1 to December 31,1993

BURIAL GROUND 218W5, 200 WEST AREA, LOW-LEVEL INDUSTRIAL.

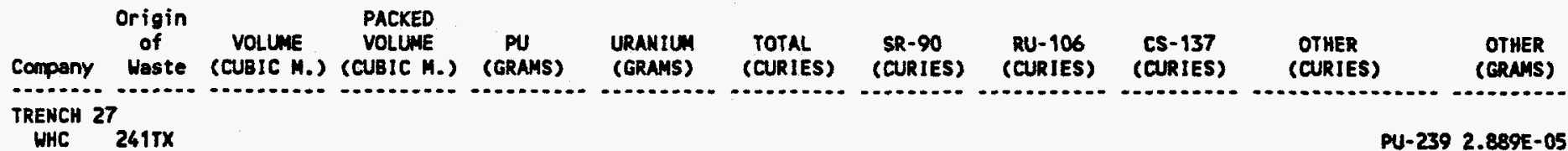

\begin{tabular}{|c|c|c|c|c|c|c|c|c|}
\hline 2410 & $3.625 E+00$ & $3.625 E+00$ & $.000 E+00$ & $.000 E+00$ & $2.737 E-06$ & $6.992 E-07$ & $3.322 E-12$ & $2.182 E-07$ \\
\hline $242 A$ & $2.082 E-01$ & $2.082 E-01$ & $.000 E+00$ & $.000 E+00$ & $3.181 E-06$ & $6.720 E-07$ & $.000 E+00$ & $9.440 E-07$ \\
\hline $\begin{array}{l}2718 \\
2724 \mathrm{~N}\end{array}$ & $\begin{array}{l}1.796 E+02 \\
5.844 E+01\end{array}$ & $\begin{array}{l}1.796 E+02 \\
5.844 E+01\end{array}$ & $\begin{array}{l}.000 E+00 \\
.000 E+00\end{array}$ & $\begin{array}{r}.000 E+00 \\
4.181 E+00\end{array}$ & $\begin{array}{l}3.706 E-01 \\
2.758 E-02\end{array}$ & $\begin{array}{l}4.781 E-02 \\
1.565 E-03\end{array}$ & $\begin{array}{l}1.897 E-06 \\
3.406 E-08\end{array}$ & $\begin{array}{l}1.413 E-01 \\
1.631 E-04\end{array}$ \\
\hline
\end{tabular}

CO-60 8.980E-07

EU-152 4.490E-07

$K-40 \quad 2.331 E-06$

$2.889 E-05$

UU-240 3.380E-07

PU-241 2.004E-08

PU-242 $6.793 E-10$

TH-228 1.627E-09

EU-154 9.140E-07

$2.331 E-06$
$9.140 E-07 \quad 2.163 E-07$

AM-241 2.210E-09

PU-238 4.920E-11

PU-239 1.990E-07

PU-240 $1.280 E-08$

PU-241 $1.580 E-09$

TH-228 $6.190 E-10$

$\begin{array}{llllll}.000 E+00 & 4.181 E+00 & 2.758 E-02 & 1.565 E-03 & 3.406 E-08 & 1.631 E-04\end{array}$

CE-144 1.061E-04

AM-241 8.600E-11 PU-238 $3.490 E-12$

PU-239 1.480E-08 PU-240 9.070E-10 PU-241 $1.130 E-10$ $.000 E+00 \quad .000 E+00$ 2.399E-02 6.994E-06 AM-241 6.994E-06 CO-60 2.256E-02 EU-154 5.209E-05 FE-59 2.353E-O4 HF- $1813.360 E-06$ MN-54 7.645E-04 NB-95 6.329E-05 SB-125 4.111E-05 TE-125 4.111E-05 2N-65 7.255E-05

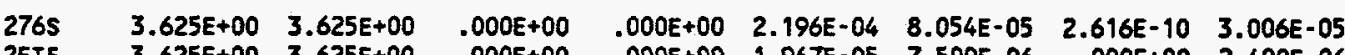

$2 R-95 \quad 4.268 E-05$

2ETF $\quad 3.625 E+00 \quad 3.625 E+00 \quad .000 E+00 \quad .000 E+00 \quad 1.967 E-05 \quad 7.500 E-06 \quad .000 E+00 \quad 2.400 E-06$

$.000 E+00 \quad .000 E+00$ $000 E+00 \quad 7.337 E-08$ AM-241 4.100E-10 PU-238 $1.700 E-11$ PU-239 6.800E-08 PU-240 4.400E-09 PU-241 5.400E-10

300-ARE $\quad 1.088 E+01 \quad 1.088 E+01 \quad .000 E+00 \quad 6.799 E+01 \quad 7.787 E-07 \quad 2.214 E-07 \quad 7.058 E-13 \quad 1.604 E-07$

$328 \quad 7.250 E+00 \quad 7.250 E+00 \quad .000 E+00 \quad 2.468 E-01 \quad 1.517 E-08 \quad 1.025 E-14 \quad 2.076 E-16 \quad 5.225 E-09$ C0-60 $\begin{array}{ll}2.373 E-08 & 2.373 E-08\end{array}$ $.000 E-09 \quad 4.750 E-10$ TH-232 4.750E-10

$33399.379 E+01 \quad 9.379 E+01 \quad .000 E+00 \quad 1.868 E+03 \quad 1.150 E-02 \quad 2.255 E-03 \quad 4.567 E-05 \quad 2.427 E-03$

CO-60 5.000E-09

$.000 E+00 \quad 1.125 E+02$ U-235 $6.800 E-01$ $U-238 \quad 1.118 E+02$

$\begin{array}{lllllllll}351 & 8.000 E+00 & 8.000 E+00 & .000 E+00 & 3.900 E+00 & 2.780 E-06 & .000 E+00 & .000 E+00 & .000 E+00\end{array}$ $2.780 \mathrm{E}-06 \quad 1.570 \mathrm{E}-05$ RA-226 2.950E-06 TH-228 1.275E-05

CuC $\quad 2.922 E+01 \quad 2.922 E+01 \quad .000 E+00 \quad .000 E+00 \quad 2.800 E-05 \quad .000 E+00 \quad .000 E+00 \quad .000 E+00 \quad \begin{array}{lllllll}K-40 & 2.780 E-06 & 2.800 E-05 & .000 E+00\end{array}$

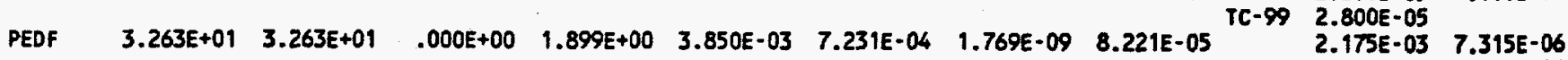
CE-144 5.337E-05 M-241 7.315E-06 
SWIR3280 - (Page 34 of 99)

RUN DATE: $08 / 20 / 94$

Land Disposal or Storage of Solid Radioactive Waste from January 1 to December 31,1993 BURIAL GROUND 218W5, 200 WEST AREA, LOW-IEVEL INDUSTRIAL.

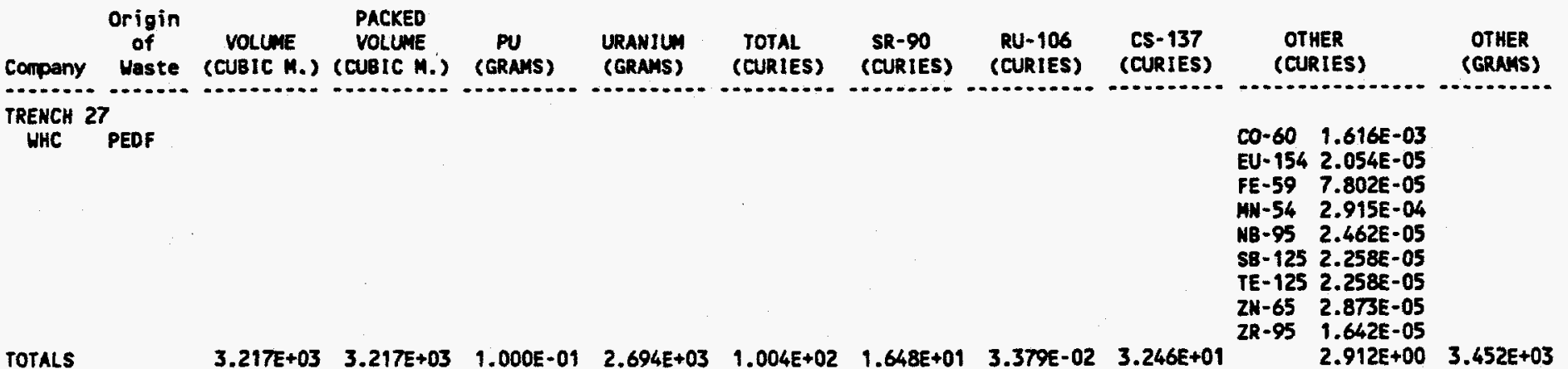


SWIR3280 - (Page 35 of 99)

RUN DATE: 08/20/94

Land Disposal or Storage of Solid Radioactive Waste from January 1 to December 31 , 1993

BURIAL GROUND 218W5, 200 WEST AREA, LOW-LEVEL NONINDUSTRIAL-OFFSITE.

\begin{tabular}{|c|c|c|c|c|c|c|c|c|c|}
\hline Eompany & $\begin{array}{c}\text { Origin } \\
\text { of } \\
\text { Waste }\end{array}$ & $\begin{array}{c}\text { VOLUME } \\
\text { (CUBIC M.) }\end{array}$ & $\begin{array}{c}\text { PACKED } \\
\text { VOLUAE } \\
\text { (CUBIC M.) }\end{array}$ & $\begin{array}{c}P U \\
\text { (GRAMS) }\end{array}$ & $\begin{array}{l}\text { URANIUH } \\
\text { (GRAMS) }\end{array}$ & $\begin{array}{l}\text { TOTAL } \\
\text { (CURIES) }\end{array}$ & $\begin{array}{l}\text { SR-90 } \\
\text { (CURIES) }\end{array}$ & $\begin{array}{c}\text { RU-106 } \\
\text { (CURIES) }\end{array}$ & $\begin{array}{c}\text { CS-137 } \\
\text { (CURIES) }\end{array}$ \\
\hline BNL & $7_{\text {BNLAB }}$ & $6.345 E+01$ & $6.345 E+01$ & $1.690 E-06$ & $2.743 E+01$ & $6.924 E-01$ & $1.221 E-03$ & $2.503 E-03$ & $4.906 \mathrm{E}-03$ \\
\hline
\end{tabular}

\section{BE-}

3.000E-08

-14 1.055E-02

CO-56 $9.800 E-07$

CO-5T 1.111E-06

CO-58 1.565E-05

C0-60 $3.720 E-02$

CS-134 3.978E-04

EU-152 6.435E-04

GA-68 $1.202 E-05$

GE-68 2.680E-05

$\mathrm{H}-3$ 5.291E-01

MN-54 6.320E-04

NA-22 8.716E-03

NI -60 3.721E-02

RB-86 2.000E-08

SC-46 $3.000 E-08$

SE-75 2.000E-07

SN-113 1.000E-07

SR-85 $6.800 E-07$

TC-99 1.700E-04

$Y-88 \quad 1.400 E-07$

ZN-65 1.841E-03

6.266E-01 2.907E-04 
SWIR3280 - (Page 36 of 99)

RUN DATE: 08/20/94

Land Disposal or Storage of Solid Radioactive Waste from January 1 to December 31 , 1993 BURIAI GROUND 218W5, 200 WEST AREA, LOW-LEVEL NONINDUSTRIAL.

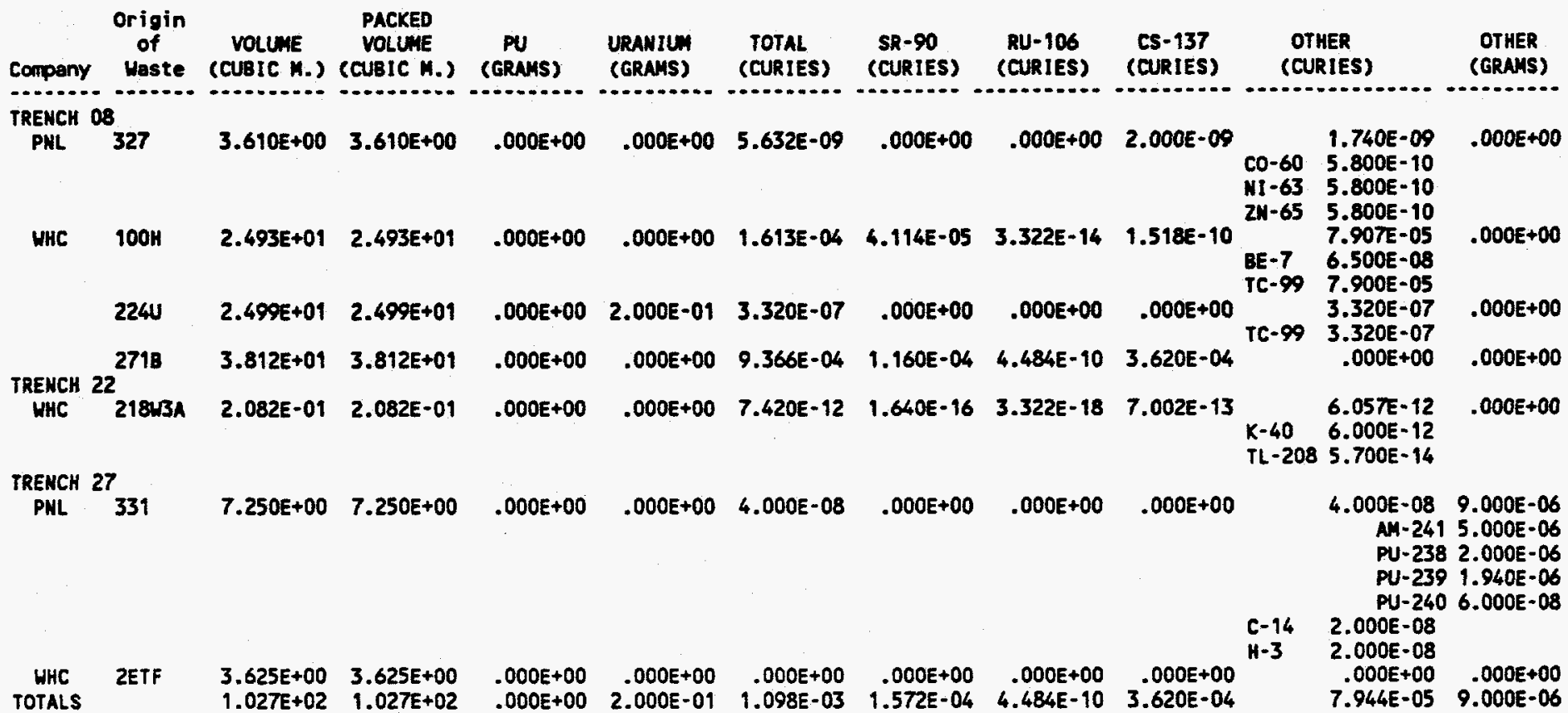




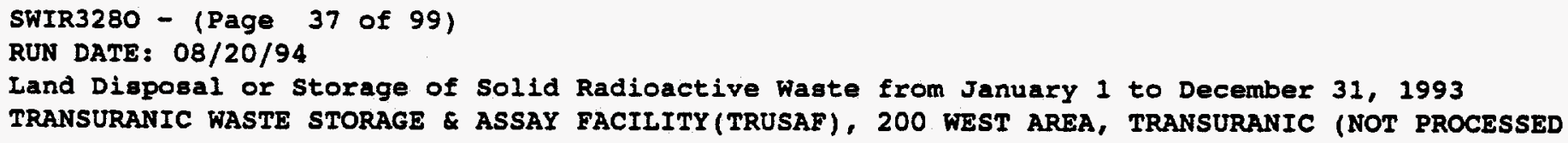

Cs137 5.150E-06

C0-58 5.000E-06

CO-60 3.350E-04

EU155 4.250E-05

C-14 5.000E-06

BA137 4.850E-06

EU154 4.250E-05

FE-59 2.000E-05

MN-54 3.500E-05

EU152 5.000E-06

$.000 E+00 \quad 1.439 E+03$ 


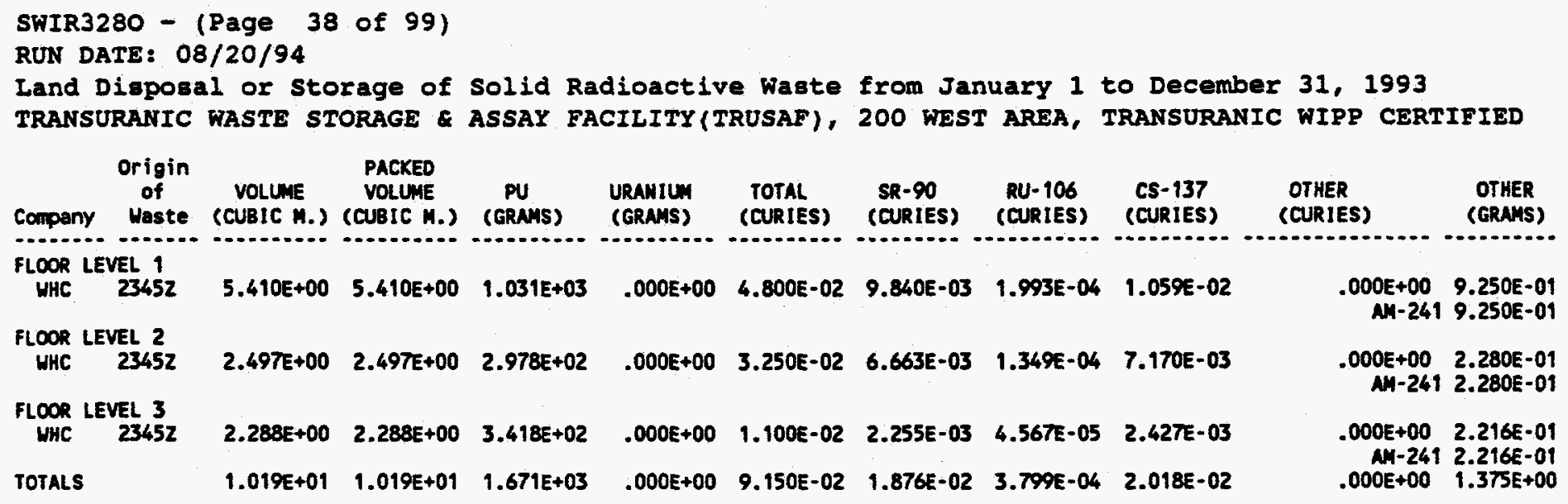


WHC-EP-0125-6

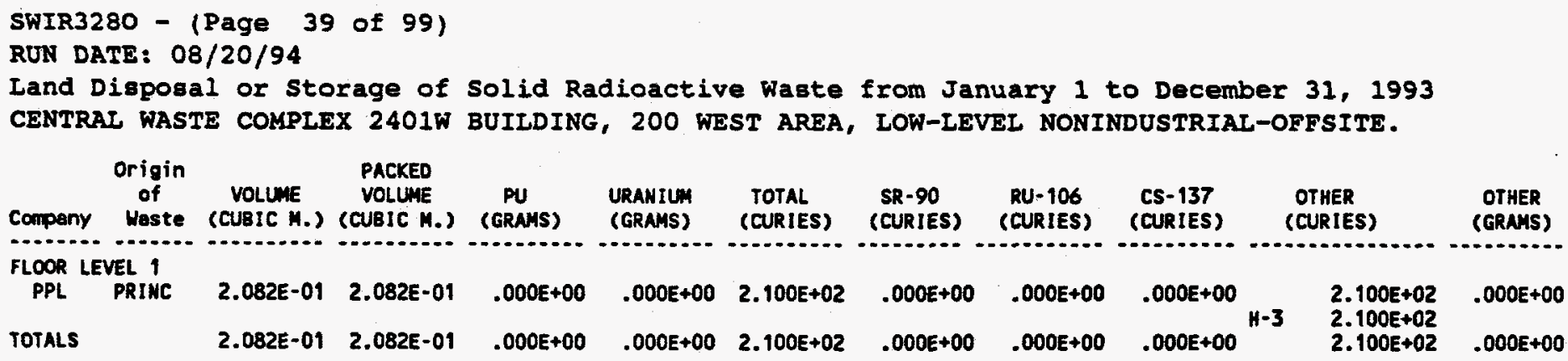


SWIR3280 - (Page 40 of 99)

RUN DATE: 08/20/94

Land Disposal or Storage of Solid Radioactive waste from January 1 to December 31 , 1993

CENTRAL WASTE COMPLEX 2402W BUILDING, 200 WEST AREA, LOW-LEVEL NONINDUSTRIAL.

\begin{tabular}{|c|c|c|c|c|c|c|c|c|c|c|}
\hline Company $\begin{array}{c}\text { Origin } \\
\text { of } \\
\text { Waste }\end{array}$ & $\begin{array}{l}\text { VOLUME } \\
\text { (CUBIC M.) }\end{array}$ & $\begin{array}{l}\text { PACKED } \\
\text { VOLUME } \\
\text { (CUBIC M.) }\end{array}$ & $\begin{array}{c}\text { PU } \\
\text { (GRAMS) }\end{array}$ & $\begin{array}{l}\text { URAMIUN } \\
\text { (GRAMS) }\end{array}$ & $\begin{array}{l}\text { TOTAL } \\
\text { (CURIES) }\end{array}$ & $\begin{array}{l}5 R-90 \\
\text { (CURIES) }\end{array}$ & $\begin{array}{l}\text { RU-106 } \\
\text { (CURIES) }\end{array}$ & $\begin{array}{l}\text { CS-137 } \\
\text { (CURIES) }\end{array}$ & $\begin{array}{l}\text { OTHER } \\
\text { (CURIES) }\end{array}$ & $\begin{array}{l}\text { OTHER } \\
\text { (GRAMS) }\end{array}$ \\
\hline $\begin{array}{c}\text { FLOOR } \\
\text { HHC }\end{array}$ & $2.082 E-01$ & 2.082E-01 & $.000 E+00$ & $.000 E+00$ & $5.000 E-04$ & $1.025 E-04$ & $2.076 E-06$ & $1.103 E-04$ & $\begin{array}{c}.000 E+00 \\
M-241 \\
P U-238 \\
P U-239 \\
P U-240 \\
P U-241 \\
P U-242 \\
.000 E+00\end{array}$ & $\begin{array}{l}1.570 E-04 \\
1.740 E-07 \\
4.600 E-08 \\
1.470 E-04 \\
8.980 E-06 \\
7.330 E-07 \\
3.080 E-08 \\
1.570 E-04\end{array}$ \\
\hline
\end{tabular}


SWIR3280 - (Page 41 of 99)

RUN DATE: 08/20/94

Iand Disposal or Storage of Solid Radioactive Waste from January 1 to December 31,1993

CENTRAL WASTE COMPLEX 2402WB BUIIDING, 200 WEST AREA, LOW-LEVEL NONINDUSTRIAL.

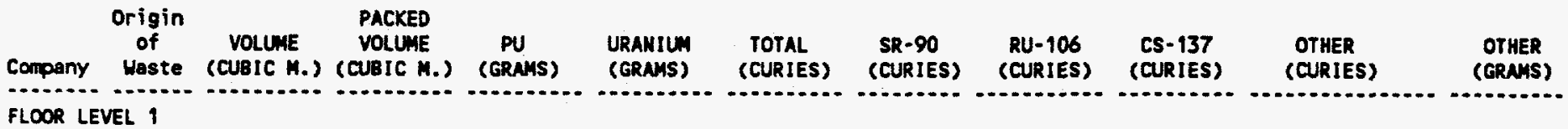

$\begin{array}{lllllllll}\text { WHC } \quad 221 T \quad 2.082 E-01 & 2.082 E-01 & .000 E+00 & .000 E+00 & 2.576 E-03 & 1.190 E-03 & 1.769 E-09 & 6.434 E-06\end{array}$

$222 S \quad 1.249 E+00 \quad 1.249 E+00 \quad .000 E+00 \quad 3.047 E-02 \quad 2.013 E-03 \quad 1.679 E-04 \quad 9.342 E-10 \quad 8.039 E-04$

$241 A N \quad 2.082 E-01 \quad 2.082 E-01 \quad .000 E+00 \quad .000 E+00 \quad 1.240 E-07 \quad 1.036 E-10 \quad 1.661 E-13 \quad 6.361 E-08$

$2418 \quad 4.164 E-01 \quad 4.164 E-01 \quad .000 E+00 \quad .000 E+00 \quad 2.971 E-06 \quad 6.737 E-07 \quad 3.778 E-12 \quad 8.344 E-07$

$241 C \quad 2.082 E-01 \quad 2.082 E-01 \quad .000 E+00 \quad .000 E+00 \quad 1.693 E-03 \quad 4.680 E-04 \quad 2.491 E-11 \quad 3.890 E-04$

TOTALS

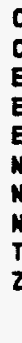

$1.782 E-04 \quad 7.459 E-02$ NM-241 6.810E-06 NP-237 7.450E-02 PU-239 8.180E-05

EU-152 2.940E-05

EU-154 5.020E-05

EU-155 2.010E-05

NA-22 1.780E-05

NB-95M 8.120E-10

NI $-60 \quad 2.770 E-05$

TC-99 3.040E-07

2R-95 5.030E-06

$1.126 E-04 \quad 2.888 E-02$

AN-241 4.633E-07 PU-238 1.155E-08 PU-239 2.608E-05 U-234 2.885E-05 U-235 1.261E-03 U-238 2.756E-02

H-3 1.830E-07

PM-147 1.116E-04

TC- 99 8.271E-07

$.000 E+00 \quad 3.397 E-03$ AM-241 4.566E-06 PU-238 1.798E-06 PU-239 3.106E-03 PU-240 $1.908 E-04$ PU-241 9.384E-05 PU-242 6.476E-07 $.000 E+00 \quad 1.877 E-10$ AM-241 1.060E-12 PU-238 4.320E-14 PU-239 1.740E-10 PU-240 1.120E-11 PU-241 1.390E-12 $.000 E+00 \quad 1.856 E-06$ AM-241 3.260E-11 PU-238 1.330E-12 PU-239 5.380E-09 PU-240 3.460E-10 PU-241 1.850E-06 $.000 E+00 \quad 5.480 E-04$ AN-241 3.090E-06 PU-238 1.250E-07 PU-239 5.080E-04 PU-240 3.270E-05 PU-241 4.040E-06 2.909E-04 1.074E-01 
WHC-EP-0125-6

SWIR3280 - (Page 42 of 99 )

RUN DATE: $08 / 20 / 94$

Land Disposal or storage of Solid Radioactive Waste from January 1 to December 31 , 1993 CENTRAL WASTE COMPLEX 2402WC BUILDING, 200 WEST AREA, LOW-LEVEL NONINDUSTRIAL-OFESITE.

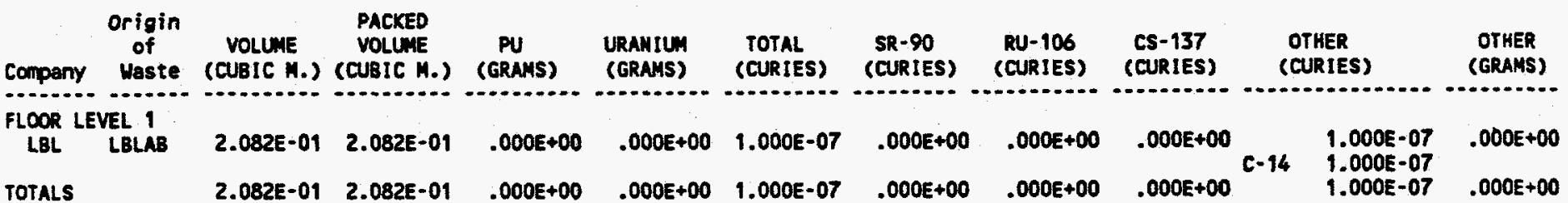




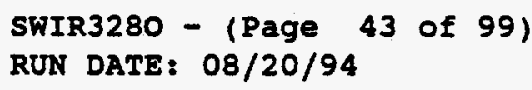




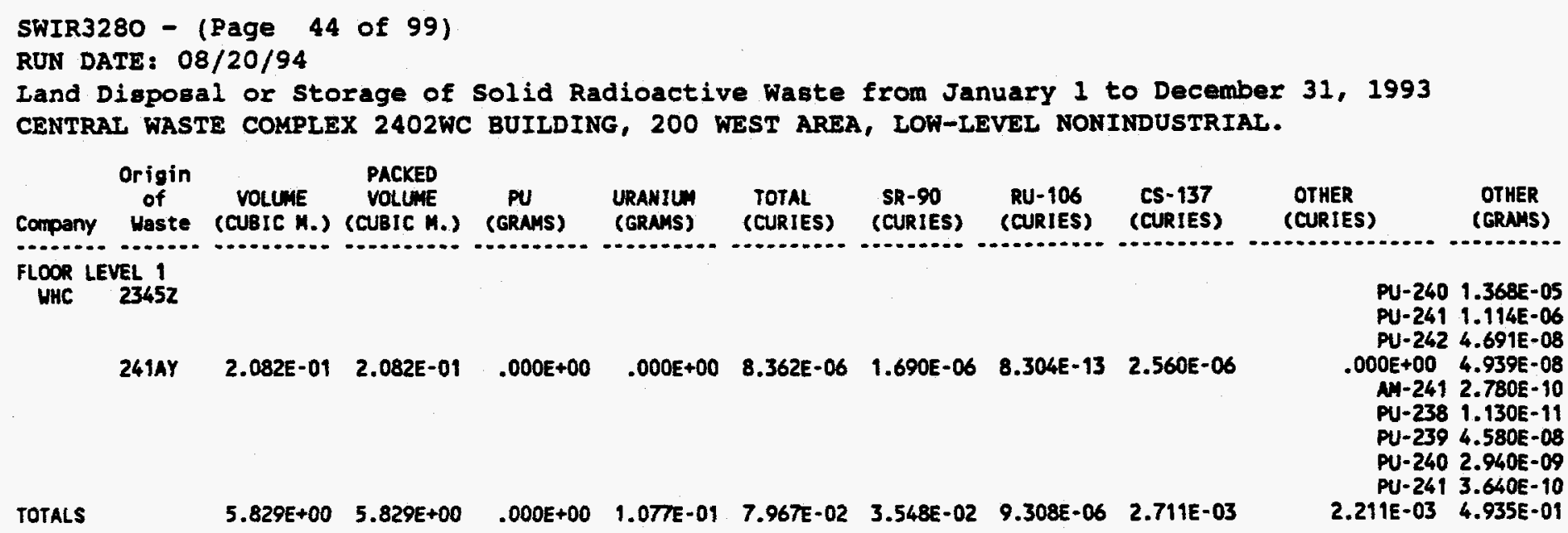


SHIR3280 - (Page 45 of 99 )

RUN DATE: $08 / 20 / 94$

Land Disposal or storage of Solid Radioactive Waste from January 1 to December 31 , 1993

CENTRAL WASTE COMPLEX $2402 W C$ BUILDING, 200 WEST AREA, TRANSURANIC (NOT PROCESSED THROUGH TRUS

Origin
Of voLUME PACKED VOLUME PU URANIUN TOTAL SR-90 RU-106 CS-137 OTHER

Company Haste (CUBIC M.) (CUBIC M.) (GRAMS) (GRAMS) (CURIES) (CURIES) (CURIES) (CURIES) (CURIES) (GRAMS)

FLOOR LEVEL 9

WHC 23452

2.080E-01 2.080E-01 .000E+00

$.000 E+00 \quad 5.000 E-04$

$.000 E+00$

$.000 E+00 \quad .000 E+00$

TOTALS

2.080E-01 2.080E-01 .000E+00

$.000 E+00$

5.000E-0

$.000 E+00$

$.000 E+00$

$.000 E+00$

$.000 E+00 \quad 1.055 E-06$ NH-241 1.070E-09 PU-238 3.100E-10 PU-239 9.830E-07 PU-240 6.020E-08 PU-241 5.010E-09 PU-242 2.070E-10 $.000 E+00 \quad 1.055 E-06$ 
พHC-EP-0125-6

SWIR3280 - (Page 46 of 99 )

RUN DATE: $08 / 20 / 94$

Land Disposal or Storage of Solid Radioactive Waste from January 1 to December 31,1993

CENTRAL WASTE COMPLEX 2402WG BUILDING, 200 WEST AREA, LOW-IEVEL INDUSTRIAL.

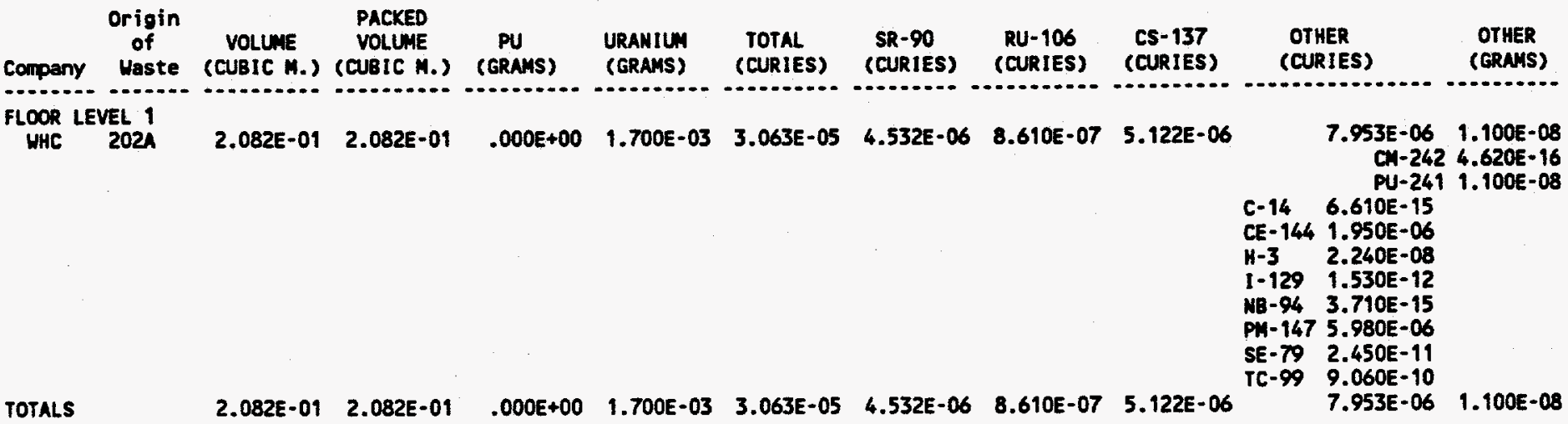


SWIR3280 - (Page 47 of 99 )

RUN DATE: 08/20/94

Iand Disposal or Storage of Solid Radioactive Waste from January 1 to December 31 , 1993 CENTRAL WASTE COMPLEX 2402 WG BUILDING, 200 WEST AREA, LOW-LEVEL NONINDUSTRIAL-OFFSITE.

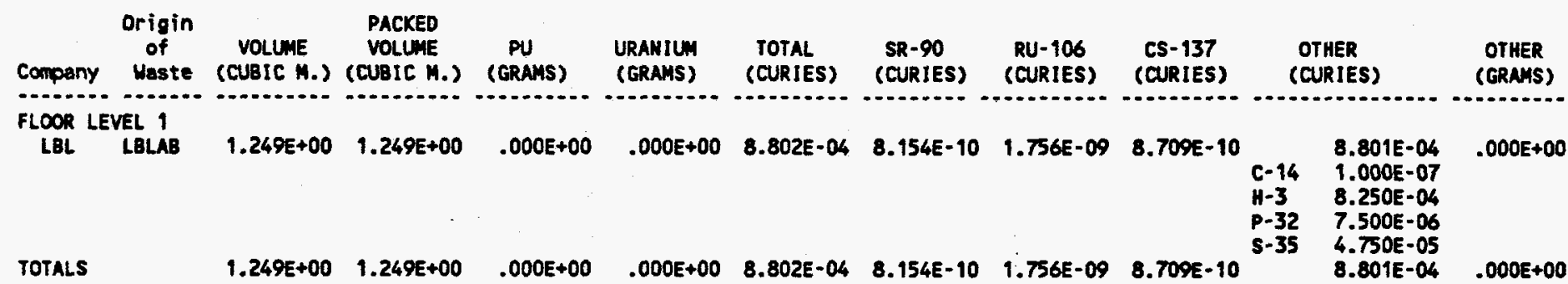


SWIR3280 - (Page 48 of 99 )

RUN DATE: 08/20/94

Land Disposal or storage of Solid Radioactive Waste from January 1 to December 31,1993 CENTRAI WASTE COMPLEX 2402WG BUILDING, 200 WEST AREA, LON-LEVEL NONINDUSTRIAL.

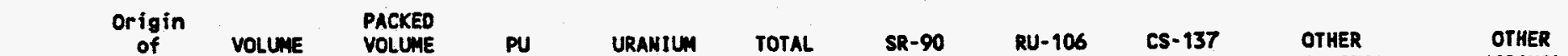
Company Haste (CUBIC M.) (CUBIC M.) (GRAMS) (GRAMS) (CURIES) (CURIES) (CURIES) (CURIES) (CURIES) FLOOR LEVEL 1 .

UHC 100N

\subsection{E-01 2.082E-01}

2.082E-01 2.082E-0

\section{$.000 E+00$}

$.000 E+0$ $\begin{array}{ccc}.000 E+00 & 4.000 E-05 & 4.400 E-12 \\ .000 E+00 & 3.700 E-04 & 7.400 E-06\end{array}$ $\begin{array}{cc}.000 E+00 & .000 E+00 \\ .000 E+00 & 1.853 E-05\end{array}$

\section{$H-3$}

\section{co-} EU-154 5.920E-05 EU-155 2.960E-05 MN-54 1.480E-05 MI $-60 \quad 9.990 E-05$ MI $-63 \quad 7.400 \mathrm{E}-06$ 2N-65 7.400E-06 CS137 1.029E-08 CO-58 9.990E-09 C0-60 6.693E-07

EU155 8.492E-08 C-14 9.990E-09 BA137 9.690E-09

EU154 8.492E-08 FE-59 3.996E-08 MN-54 6.993E-08 EU152 $9.990 \mathrm{E}-09$ 8.160E-10 CO-60 $2.720 E-10$ NI-60 2.720E-10 N1-63 2.720E-10 1.407E-07 CO-60 6.407E-08 EU-154 1.220E-09 EU-155 5.440E-10 MN-54 8.118E-09 NI $-60 \quad 6.407 E-08$ NI-63 2.706E-09

$109 \mathrm{~N} \quad 2.288 E+00 \quad 2.288 E+00 \quad .000 E+00 \quad .000 E+00 \quad 3.024 E-06 \quad 2.994 E-08 \quad 1.046 E-12 \quad 3.036 E-08$ $2.288 E+00 \quad 2.288 E+00 \quad .000 E+00 \quad .000 E+00 \quad 3.024 E-06 \quad 2.994 E-08 \quad 1.046 E-12 \quad 3.036 E-08$ 183H 4.200E-01 $4.200 E-01 \quad .000 E+00 \quad .000 E+00 \quad 9.855 E-07 \quad 4.250 E-07 \quad .000 E+00 \quad 2.273 E-12$ CS137 $9.270 E-13$ FE-59 $3.600 E-12$

\section{NI}

-60 $1.373 E-06$ EU-154 2.176E-09 EU-155 $1.088 E-09$

MN-54 6.006E-08 NI-60 $1.349 E-06$ NI $-63 \quad 8.858 E-08$

$2 N-65 \quad 2.720 E-10$ Co-60 1.231E-07 EU-154 1.231E-08 CO-58 9.000E-13 CO-60 6.030E-11 EU155 $7.650 E-12$ C-14 9.000E-13 BA137 8.730E-13 EU154 7.650E-12 MN-54 $6.300 E-12$ EU152 $9.000 E-13$

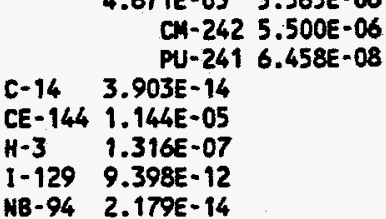

(GRAMS)

$.000 E+00$

$.000 E+00$

$.000 E+00$

$.000 E+00$

$.000 E+00$

$.000 E+00$ 


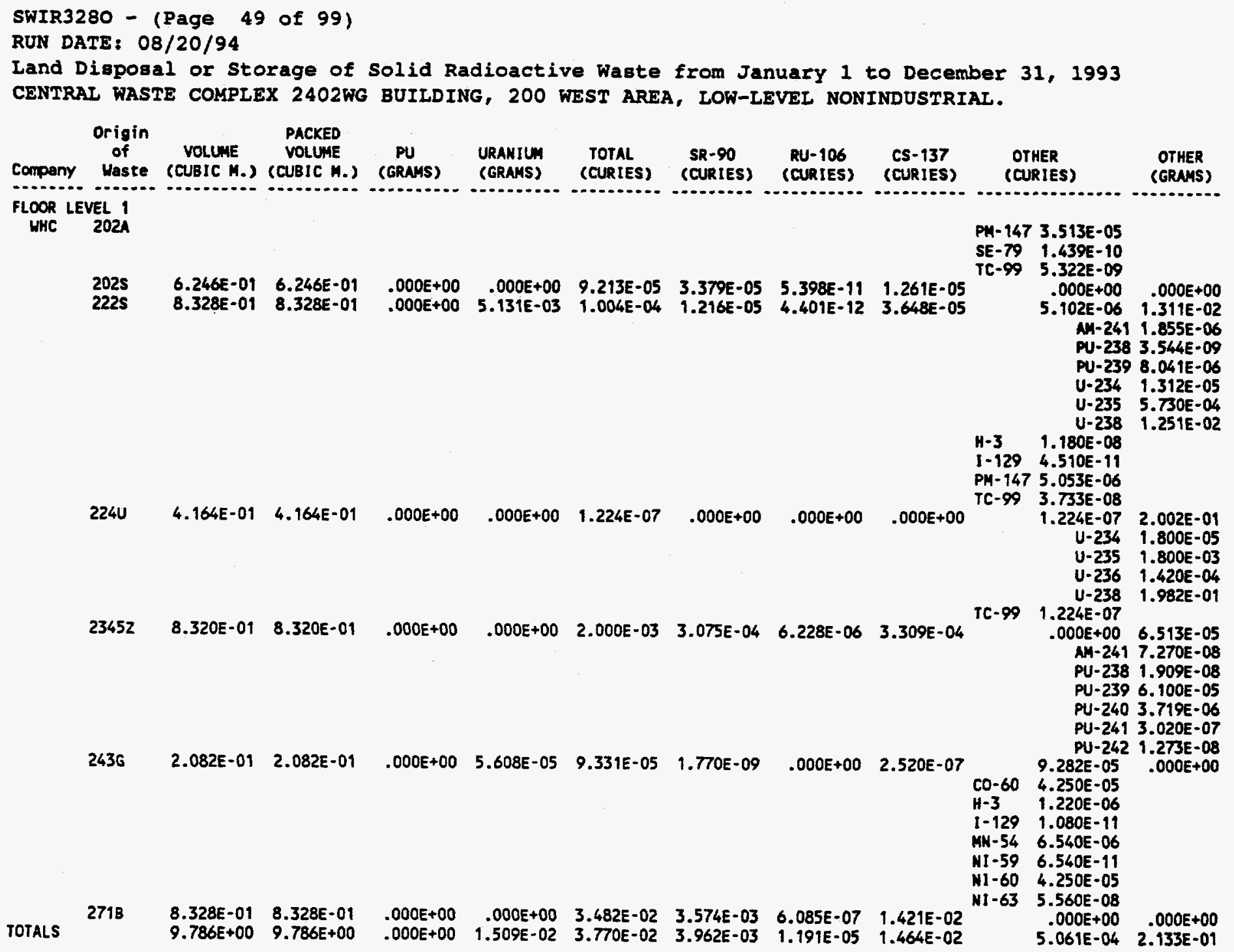


SWIR3280 - (Page 50 of 99 )

RUN DATE: 08/20/94

Land Disposal or Storage of Solid Radioactive Waste from January 1 to December 31, 1993

CENTRAI WASTE COMPLEX 2402WG BUILDING, 200 WEST AREA, TRANSURANIC (NOT PROCESSED THROUGH TRUS

$$
\begin{gathered}
\text { Origin PACKED } \\
\text { of }
\end{gathered} \text { VOLUME VOLUME } \text { PU URANIUM TOTAL } 5 R-90 \quad \text { RU-106 CS-137 } \quad \text { OTHER }
$$

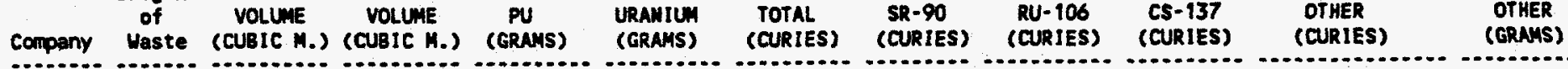

FLOOR LEVEL

WHC 23452

$\begin{array}{lllllll}2.082 E-01 & 2.082 E-01 \quad .000 E+00 & .000 E+00 & 5.000 E-04 & 1.025 E-04 & 2.076 E-06 & 1.103 E-04\end{array}$

TOTALS

$\begin{array}{lllllll}2.082 E-01 & 2.082 E-01 \quad .000 E+00 \quad .000 E+00 & 5.000 E-04 & 1.025 E-04 & 2.076 E-06 & 1.103 E-04\end{array}$

.000E+00 2.002E-03 AM-241 1.912E-06 PU-238 5.880E-07

PU-239 1.875E-03

PU-240 1.142E-04

PU-241 9.624E-06

PU-242 3.920E-07

$.000 E+00 \quad 2.002 E-03$ 
WHC-EP-0125-6

SWIR3280 - (Page 51 of 99)

RUN DATE: $08 / 20 / 94$

Land Disposal or storage of Solid Radioactive Waste from January 1 to December 31 , 1993

CENTRAI WASTE COMPLEX 2402 WG BUILDING, 200 WEST AREA, TRANSURANIC WIPP CERTIFIED.

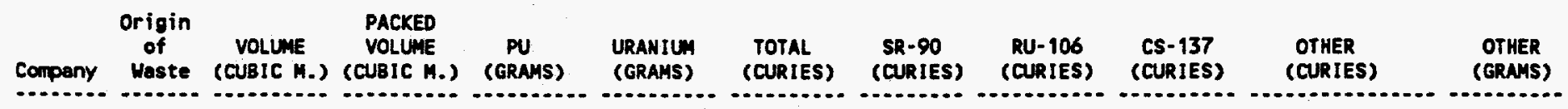

FLOOR LEVEL,

HHC 23452

$\begin{array}{lllllll}3.123 E+00 & 3.123 E+00 \quad .000 E+00 \quad .000 E+00 & 7.500 E-03 & 1.435 E-03 & 2.906 E-05 & 1.544 E-03\end{array}$

TOTALS

$\begin{array}{lllllll}3.123 E+00 & 3.123 E+00 \quad .000 E+00 & .000 E+00 & 7.500 E-03 & 1.435 E-03 & 2.906 E-05 & 1.544 E-03\end{array}$

$.000 E+00 \quad 3.003 E-02$ MH-241 2.868E-05

PU-238 8.820E-06 PU-239 2.813E-02 PU-240 1.713E-03 PU-261 1.444E-04 PU-242 5.880E-06 
SWIR3280 - (Page 52 of 99)

RUN DATE: $08 / 20 / 94$

Land Diaposal or Storage of Solid Radioactive waste from January 1 to December 31,1993

CENTRAL WASTE COMPLEX 2402WG BUILDING, 200 WEST AREA, TRANSURANIC WIPP UNCERTIFIED.

\section{Origin
of PACKEO \\ Waste \\ (CUBIC M.) (CUBIC H.)}

Company

FLOOR LEVEL 1

HHC 23452

2.082E-09 2.082E-01

URANIU: TOTAL

SR-90

Ru- 106

Cs-137

(GRAMS)

(CURIES)

(CUR1ES)

(CURIES)

(CURIES)

OTHER

(CURIES)

OTHER

TOTALS

$2.082 E-01 \quad 2.082 E-01 \quad .000 E+00 \quad .000 E+00 \quad 5.000 E-04 \quad 2.050 E-04 \quad 4.152 E-06 \quad 2.206 E-04$
$.000 E+00 \quad 4.015 E+01$ NM-241 4.500E-02 PU-238 1.170E-02 PU-239 3.751E+01 PU-240 2.284E+00

PU-241 2.85TE-01

PU-242 7.800E-03 $.000 E+00 \quad 4.015 E+01$ 
SWIR3280 - (Page 53 of 99)

RUN DATE: 08/20/94

Land Disposal or storage of Solid Radioactive Waste from January 1 to December 31,1993

CENTRAL WASTE COKPLEX 2402WH BUILDING, 200 WEST AREA, LOW-LEVEL NONINDUSTRIAL-OFFSITE.

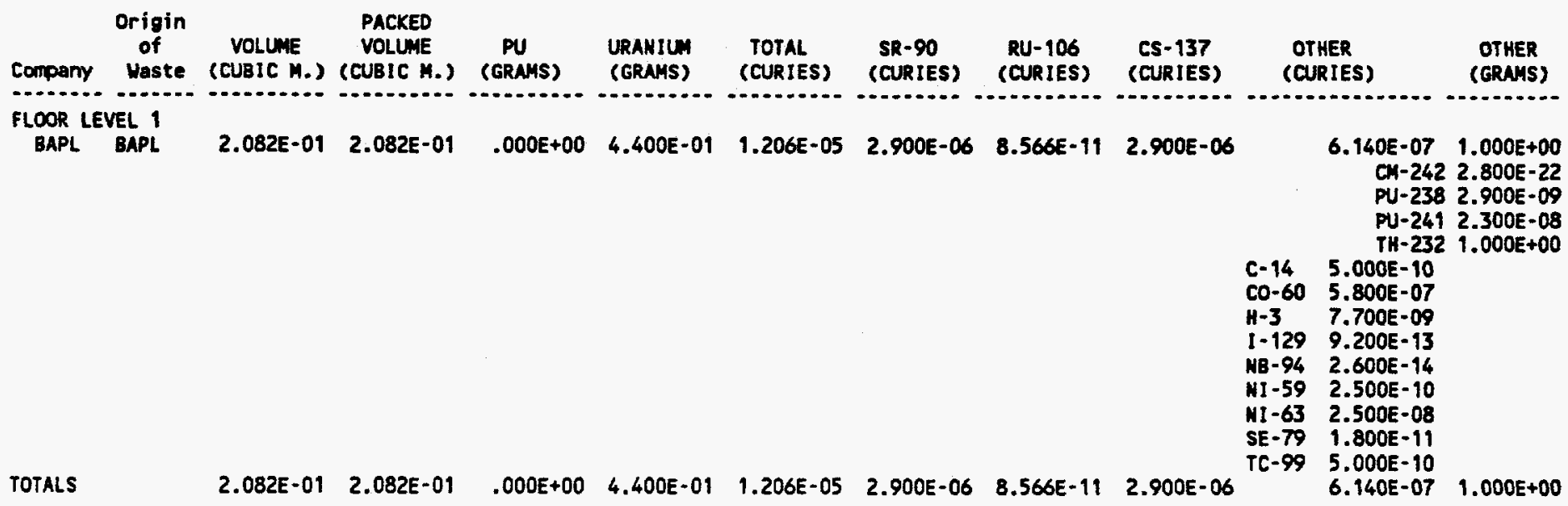


SWIR3280 - (Page 54 of 99 )

RUN DATE: 08/20/94

Land Disposal or Storage of Solid Radioactive Waste from January 1 to December 31 , 1993

CENTRAI WASTE COMPLEX $2402 \mathrm{WH}$ BUILDING, 200 WEST AREA, LOW-IEVEL NONINDUSTRIAI.

\begin{tabular}{|c|c|c|c|c|c|c|c|c|c|}
\hline Company & $\begin{array}{c}\text { Origin } \\
\text { of } \\
\text { Waste }\end{array}$ & $\begin{array}{l}\text { VOLUME } \\
\text { (CUBIC M.) }\end{array}$ & $\begin{array}{l}\text { PACKED } \\
\text { VOLUNE } \\
\text { (CUBIC M.) }\end{array}$ & $\begin{array}{c}\text { PU } \\
\text { (GRAMS) }\end{array}$ & $\begin{array}{l}\text { URANIUN } \\
\text { (GRAMS) }\end{array}$ & $\begin{array}{l}\text { TOTAL } \\
\text { (CURIES) }\end{array}$ & $\begin{array}{c}\text { SR-90 } \\
\text { (CURIES) }\end{array}$ & $\begin{array}{l}\text { RU-106 } \\
\text { (CURIES) }\end{array}$ & $\begin{array}{c}\text { CS-137 } \\
\text { (CURIES) }\end{array}$ \\
\hline $\begin{array}{l}\text { LOOR } \\
\text { WHC }\end{array}$ & $\begin{array}{l}\text { VEL } 1 \\
2025 \\
221 T \\
2225\end{array}$ & $\begin{array}{l}2.082 E-01 \\
2.082 E-01 \\
2.000 E-01\end{array}$ & $\begin{array}{l}2.082 E-01 \\
2.082 E-01 \\
2.000 E-01\end{array}$ & $\begin{array}{l}.000 E+00 \\
.000 E+00 \\
.000 E+00\end{array}$ & $\begin{array}{r}.000 E+00 \\
.000 E+00 \\
3.054 E-08\end{array}$ & $\begin{array}{l}3.943 E-05 \\
5.121 E-04 \\
7.354 E-09\end{array}$ & $\begin{array}{l}1.446 E-05 \\
6.532 E-05 \\
1.170 E-09\end{array}$ & $\begin{array}{l}2.906 E-11 \\
3.488 E-10 \\
2.491 E-15\end{array}$ & $\begin{array}{l}5.399 E-06 \\
1.960 E-04 \\
2.380 E-09\end{array}$ \\
\hline
\end{tabular}

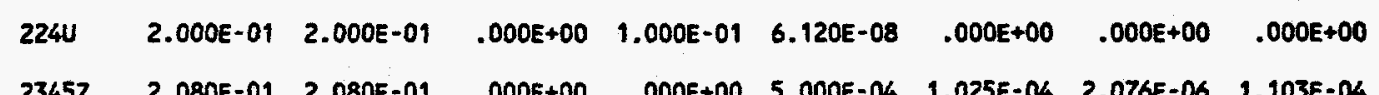

$H-3$

$1.290 \mathrm{E}-10$

$1-129 \quad 1.650 E-12$

PN-147 2.500E-10

TC-99 $1.270 E-12$ TC. $99 \quad 6.120 E-08$

$23452 \quad 2.080 E-01 \quad 2.080 E-01 \quad .000 E+00 \quad .000 E+00 \quad 5.000 E-04 \quad 1.025 E-04 \quad 2.076 E-06 \quad 1.103 E-04$

$303 K \quad 6.246 E-01 \quad 6.246 E-01 \quad .000 E+00 \quad .000 E+00 \quad 1.031 E-06 \quad 4.100 E-11 \quad 8.304 E-13 \quad 4.412 E-11$

$.000 \mathrm{E}+00$ $000 E+00 \quad 6.281 E-06$ AM-24, 4.640E-09 PU-238 1.250E-09 PU-239 4.010E-06 PU-240 2.440E-07 PU-241 2.000E-08

PU-242 8.370E-10

$1.031 E-06 \quad 5.453 E+00$ $\mathrm{U}-234$ 5.000E-04 U-235 5.100E-02 U-236 $1.170 E-02$ U-238 $\quad 5.390 E+00$ 


\section{WHC-EP-0125-6}

SWIR3280 - (Page 55 of 99 )

RUN DATE: 08/20/94

Land Disposal or Storage of Solid Radioactive Waste from January 1 to December 31 , 1993

CENTRAL WASTE COMPLEX 2402WL BUILDING, 200 WEST AREA, LOW-LEVEL NONINDUSTRIAL-OFFSITE.

\begin{tabular}{|c|c|c|c|c|c|c|c|c|c|c|c|c|}
\hline Company & $\begin{array}{c}\text { Origin } \\
\text { of } \\
\text { Waste }\end{array}$ & $\begin{array}{l}\text { VOLUHE } \\
\text { (CUBIC M.) }\end{array}$ & $\begin{array}{c}\text { PACKED } \\
\text { VOLUME } \\
\text { (CUBIC M.) }\end{array}$ & $\begin{array}{c}\text { PU } \\
\text { (GRAMS) }\end{array}$ & $\begin{array}{l}\text { URANIUN } \\
\text { (GRAMS) }\end{array}$ & $\begin{array}{l}\text { TOTAL } \\
\text { (CURIES) }\end{array}$ & $\begin{array}{c}\text { SR-90 } \\
\text { (CURIES) }\end{array}$ & $\begin{array}{l}\text { RU-106 } \\
\text { (CURIES) }\end{array}$ & $\begin{array}{c}\text { CS-137 } \\
\text { (CURIES) }\end{array}$ & \multicolumn{2}{|c|}{$\begin{array}{l}\text { OTHER } \\
\text { (CURIES) }\end{array}$} & $\begin{array}{l}\text { OTHER } \\
\text { (GRAMS) }\end{array}$ \\
\hline $\begin{array}{l}\text { LOOR LE } \\
\text { ANL }\end{array}$ & $\begin{array}{l}\text { EVEL } 1 \\
\text { ARGOW }\end{array}$ & 4.164E-01 & 4.164E-01 & $.000 E+00$ & $.000 E+00$ & $3.400 E-04$ & $.000 E+00$ & $.000 E+00$ & $.000 E+00$ & & $\begin{array}{r}3.400 E-04 \\
\text { AM-241 }\end{array}$ & $\begin{array}{l}3.200 E-05 \\
3.200 E-05\end{array}$ \\
\hline LBL & LBLAB & $1.249 E+00$ & $8.328 E-01$ & $.000 E+00$ & $.000 E+00$ & $4.027 \mathrm{E}-04$ & $6.563 E-10$ & $1.413 E-09$ & $7.010 E-10$ & $\begin{array}{l}H-3 \\
C-14 \\
H-3 \\
s-35\end{array}$ & $\begin{array}{l}3.400 E-04 \\
4.027 E-04 \\
1.350 E-06 \\
3.913 E-04 \\
1.000 E-05 \\
7.427 E-04\end{array}$ & $.000 E+00$ \\
\hline
\end{tabular}


SWIR3280 - (Page 56 of 99)

RUN DATE: 08/20/94

Land Disposal or Storage of Solid Radioactive Waste from January 1 to December 31,1993

CENTRAL WASTE COMPLEX 2402WL BUILDING, 200 WEST AREA, IOW-LEVEL NONINDUSTRIAL.

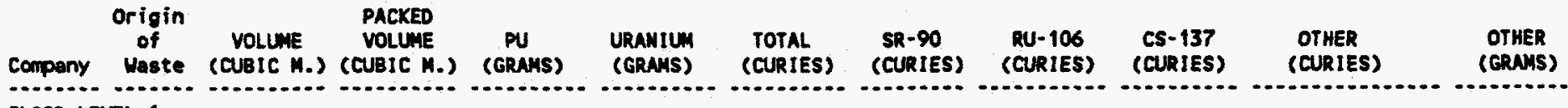

FLOOR LEVEL

PNL LSLII

$6.246 E-01 \quad 6.246 E-01$

$.000 E+00$

$.000 E+00 \quad 7.545 E-04 \quad 1.025 E-04 \quad 2.076 E-06 \quad 1.103 E-04$

$2.544 E-04 \quad 7.008 E-07$ MP- $2377.000 E-07$ RA-226 7.690E-10

C-14 2.011E-04

CA-45 $3.910 E-09$

CL-36 $3.560 E-05$

H-3 8.550E-09

WI $-63 \quad 6.554 E-06$

RA-228 $2.530 E-10$

$5-35$ 1.020E-09

TC-99 1.113E-05

UHC $202 A \quad 4.164 E-01 \quad 4.164 E-01 \quad .000 E+00 \quad 3.400 E-03 \quad 1.049 E-03 \quad 9.263 E-06 \quad 1.726 E-06 \quad 5.173 E-04$

ZN-65 1.110E-08

$1.591 E-05 \quad 2.200 E-08$ CM-242 9.240E-16

$C-14 \quad 1.322 E-14$ PU-241 2.200E-08

$2225 \quad 1.457 E+00 \quad 1.457 E+00 \quad .000 E+00 \quad 1.760 E-02 \quad 6.334 E-05 \quad 5.123 E-06 \quad 1.687 E-09 \quad 5.811 E-06$

CE-144 3.900E-06

H-3 4.480E-08

$1-129 \quad 3.060 E-12$

NB-94 $7.420 E-15$

PH-147 $1.196 E-05$

SE-79 4.900E-11

TC- $99 \quad, .812 E-09$

$1.457 E+00 \quad 1.457 E+00 \quad .000 E+00 \quad 1.760 E-02 \quad 6.334 E-05 \quad 5.123 E-06 \quad 1.687 E-09 \quad 5.811 E-06$

$4.153 E-05 \quad 6.240 E-03$ AM-241 3.964E-07 PU-238 4.471E- 09 PU-239 $1.120 E-05$ $U-234 \quad 6.220 E-06$ U-235 2.720E-0 U-238 $5.950 E-03$

C. $14 \quad 2.419 E-06$

CS-134 1.900E-08

H-3 3.212E-05

PM-147 1.028E-06

RU-103 1.930E-07

SE-79 1.390E-06

$23452 \quad 2.080 E-01 \quad 2.080 E-01 \quad .000 E+00 \quad .000 E+00 \quad 5.000 E-04 \quad 1.025 E-04 \quad 2.076 E-06 \quad 1.103 E-04$

TC-99 4.360E-06 $.000 E+00 \quad 1.858 E-05$ AN-241 1.840E-08 PU-238 5.450E-09 PU-239 1.740E-05 PU-240 1.060E-06 PU-241 8.860E-08 PU-242 3.640E-09

$241 A \quad 2.082 E-01 \quad 2.082 E-01 \quad .000 E+00 \quad .000 E+00 \quad 2.792 E-06 \quad 5.901 E-07 \quad 2.906 E-12 \quad 8.282 E-07$ $.000 E+00 \quad 1.337 E-08$ N. $-2417.540 E-11$ PU-238 $3.050 E-12$ PU-239 1.240E-08 PU-240 7.970E-10 PU-241 9.810E-11

$241 A Y \quad 8.328 E-01 \quad 8.328 E-01 \quad .000 E+00 \quad .000 E+00 \quad 4.044 E-04 \quad 9.865 E-06 \quad 2.948 E-10 \quad 1.977 E-04$ $.000 E+00 \quad 2.657 E-05$ MH-241 4.145E-06 PU-238 2.204E-09 PU-239 8.896E-06 PU-240 5.719E-07 PU-241 1.296E-05

$2418 X \quad 2.082 E-01 \quad 2.082 E-01 \quad .000 E+00 \quad .000 E+00 \quad 2.025 E-07 \quad 5.131 E-08 \quad 2.906 E-13 \quad 5.132 E-08$ .000E+00 6.008E-08 MI-241 3.390E-10 PU-238 1.380E-11 PU-239 5.570E-08 


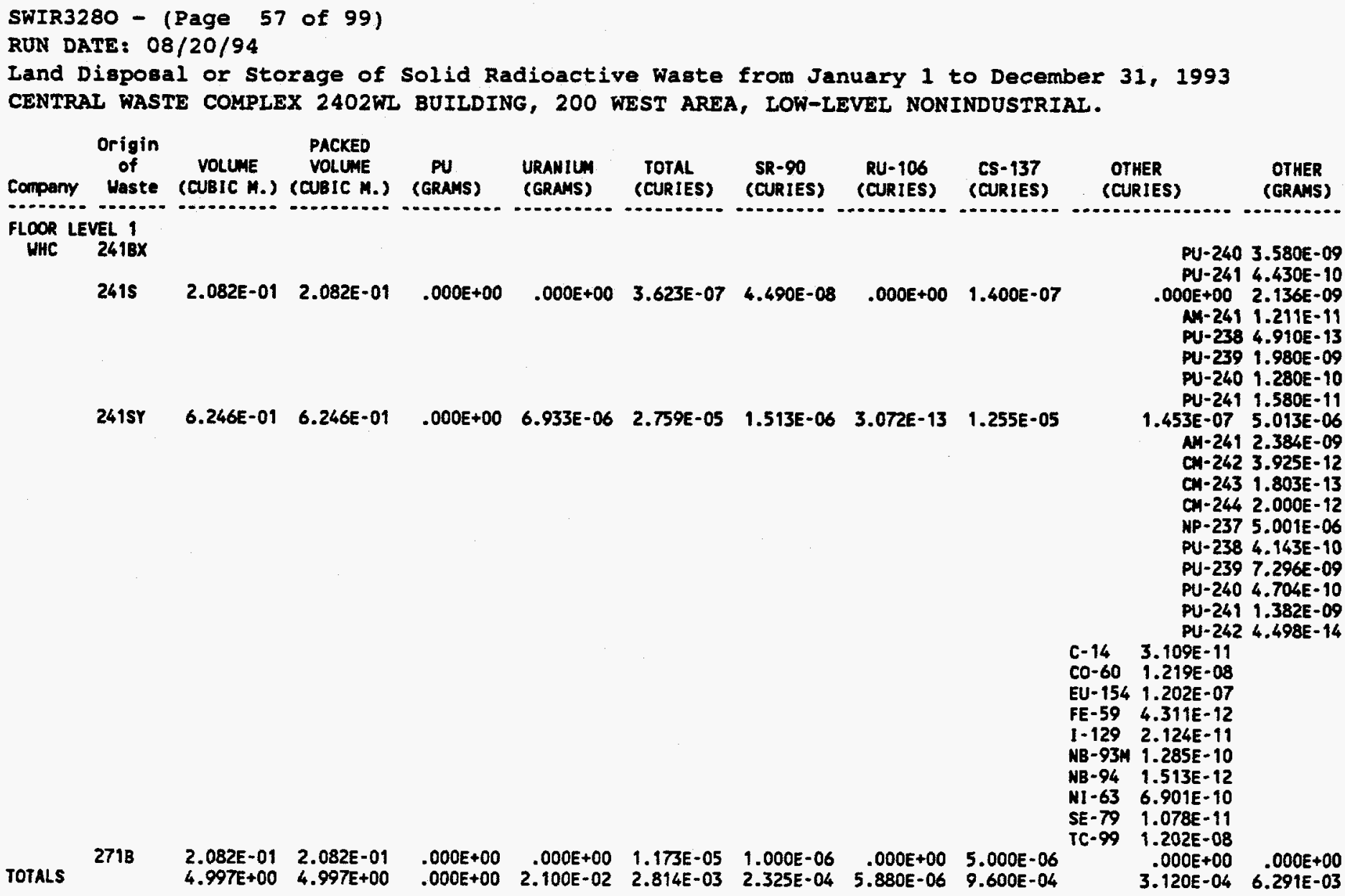

C- 14 3.109E-11

Co-60 1.219E-08

EU-154 1.202E-07

FE-59 4.311E-12

1-129 2.124E- 11

NB-93M $1.285 E-10$

NB-94 1.513E-12

NI-63 6.901E-10

SE-79 $1.078 E-11$

TC-99 1.202E-08

$.000 E+00 \quad .000 E+00$ 3.120E-04 6.291E-03 
SWIR3280 - (Page 58 of 99)

RUN DATE: $08 / 20 / 94$

Land Disposal or storage of Solid Radioactive Waste from January 1 to December 31,1993

CENTRAL WASTE COMPLEX 2402WL BUILDING, 200 WEST AREA, TRANSURANIC (NOT PROCESSED THROUGH TRUS

Origin
of
West

Compary

VOLUNE PACKED

FLOOR LEVEL 1

PNL 325

(cruns)

URANIUA

(GRAMS)

TOTAL

(CURIES)

SR-90

(CURIES)

RU-106

(CURIES)

c5-137

(CURIES)

OTHER

(CURIES)

OTHER

(GRAMs)

$2.082 E-01 \quad 2.082 E-01 \quad .000 E+00$

$.000 E+00$

$4.723 E-03$

$2.330 E-03 \quad 3.986 E-09 \quad 2.118 E-07$

co-60 2.000E-08

CS-134 6.200E-05

TOTALS 
SWIR3280 - (Page 59 of 99)

RUN DATE: 08/20/94

Land Disposal or Storage of Solid Radioactive Waste from January 1 to December 31 , 1993

. CENTRAI WASTE COMPLEX 2403WA BUIIDING, 200 WEST AREA, LOW-LEVEL INDUSTRIAL.

\begin{tabular}{|c|c|c|c|c|c|c|c|c|c|c|}
\hline $\begin{array}{c}\text { Origin } \\
\text { of } \\
\text { Company }\end{array}$ & $\begin{array}{l}\text { VOLUME } \\
\text { (CUBIC N.) }\end{array}$ & $\begin{array}{l}\text { PACKED } \\
\text { VOLUME } \\
\text { (CUBIC M.) }\end{array}$ & $\begin{array}{c}P U \\
\text { (GRAMS) }\end{array}$ & $\begin{array}{l}\text { URANIUH } \\
\text { (GRAMS) }\end{array}$ & $\begin{array}{l}\text { TOTAL } \\
\text { (CURIES) }\end{array}$ & $\begin{array}{l}5 R-90 \\
\text { (CURIES) }\end{array}$ & $\begin{array}{l}\text { RU-106 } \\
\text { (CURIES) }\end{array}$ & $\begin{array}{c}\text { CS-137 } \\
\text { (CURIES) }\end{array}$ & $\begin{array}{l}\text { OTHER } \\
\text { (CURIES) }\end{array}$ & $\begin{array}{c}\text { OTHER } \\
\text { (GRAMS) }\end{array}$ \\
\hline LOOR LEVEL 1 & & & & & & & & & & \\
\hline PML $\quad 325$ & $2.082 E-01$ & 2.082E-01 & $.000 \mathrm{E}+00$ & $.000 E+00$ & $7.162 E-07$ & $1.435 E-11$ & $2.906 E-13$ & $3.680 E-07$ & $\begin{array}{r}.000 E+00 \\
M M-241 \\
P U-238 \\
P U-239 \\
P U-240 \\
.000 E+00\end{array}$ & $\begin{array}{l}8.138 E-04 \\
7.900 E-06 \\
2.850 E-05 \\
7.700 E-04 \\
7.400 E-06 \\
8.138 E-04\end{array}$ \\
\hline
\end{tabular}


SWIR3280 - (Page 60 of 99)

RUN DATE: $08 / 20 / 94$

Land Disposal or Storage of Solid Radioactive waste from January 1 to December 31,1993 CENTRAL WASTE COMPLEX 2403WA BUILDING, 200 WEST AREA, LOW-LEVEL NONINDUSTRIAI-OFFSITE.

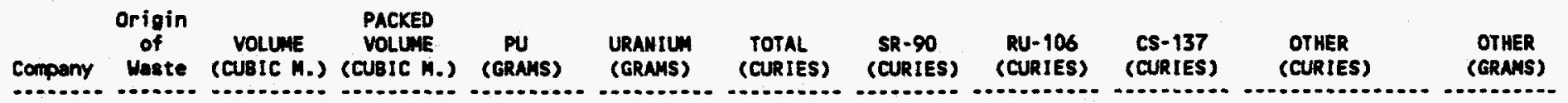

FLOOR LEVEL 1

ANL ARGO!

$7.058 E+01 \quad 7.058 E+01$

$.000 E+00$

1.110E-01

6.708E-01 5.608E-02 3.967E-06 1.905E-01

$1.879 E-01 \quad 1.036 E+01$

AM-241 $1.869 E-02$

AM-243 2.697E-03

NP-237 $2.430 E+00$

PU-239 5.600E-05

RA-226 1.146E-02

TH-228 1.400E-07

TH-232 7.900E+00

U-233 1.711E-04

Bi-214 1.288E-05

CO-60 6.877E-02

EU-155 3.700E-07

FE-59 3.300E-05

$\mathrm{K}-40 \quad 1.386 \mathrm{E}-02$

NA-22 2.980E-05

N1-60 6.815E-02

PB-214 1.288E-05

PT- 195 2.800E-05

SB-125 1.930E-02

BAPL BAPL $\quad 1.832 E+01 \quad 1.832 E+01 \quad 000 E+00 \quad 1.030 E+00 \quad 3.909 E-02 \quad 7.190 E-03 \quad 9.246 E-06 \quad 7.188 E-03$

TE-125 $1.766 E-02$

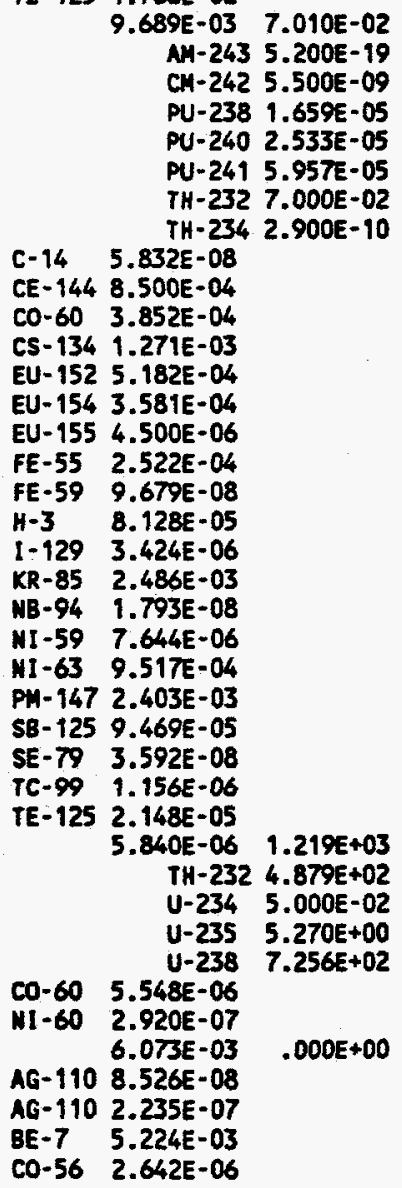


WHC-EP-0125-6

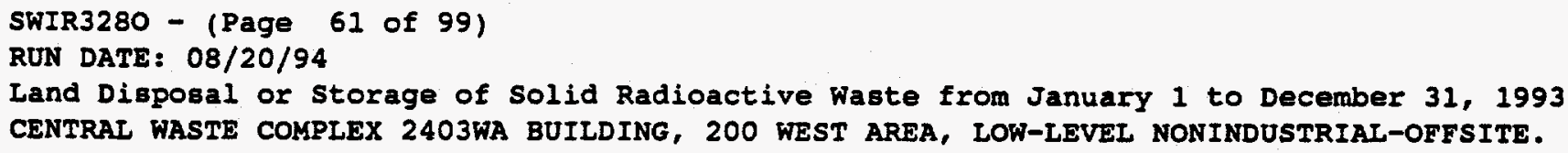

\begin{tabular}{|c|c|c|c|c|c|c|c|c|c|c|c|c|}
\hline Compeny & $\begin{array}{c}\text { Origin } \\
\text { of } \\
\text { Waste }\end{array}$ & $\begin{array}{l}\text { VOLUME } \\
\text { (CUBIC M.) }\end{array}$ & $\begin{array}{c}\text { PACKED } \\
\text { VOLUMEE } \\
\text { (CUBIC M.) }\end{array}$ & $\begin{array}{c}\text { PU } \\
\text { (GRAMS) }\end{array}$ & $\begin{array}{l}\text { URANIUH } \\
\text { (GRAMS) }\end{array}$ & $\begin{array}{l}\text { TOTAL } \\
\text { (CURIES) }\end{array}$ & $\begin{array}{l}\text { SR-90 } \\
\text { (CURIES) }\end{array}$ & $\begin{array}{c}\text { RU-106 } \\
\text { (CURIES) }\end{array}$ & $\begin{array}{l}\text { CS-137 } \\
\text { (CURIES) }\end{array}$ & \multicolumn{2}{|c|}{$\begin{array}{c}\text { OTHER } \\
\text { (CURIES) }\end{array}$} & $\begin{array}{c}\text { OTHER } \\
\text { (GRAMS) }\end{array}$ \\
\hline $\begin{array}{l}.00 R \text { LE } \\
\text { FERM }\end{array}$ & $\begin{array}{l}\text { VEL } 1 \\
\text { NALAB }\end{array}$ & & & & & & & & & $\begin{array}{l}C O-57 \\
\text { CO-58 } \\
C O-60 \\
H-3 \\
H F-175 \\
H G-203 \\
M N-54 \\
M A-22 \\
N I-60 \\
5-35 \\
2 N-65\end{array}$ & $\begin{array}{l}7.318 E-05 \\
1.174 E-05 \\
8.544 E-05 \\
2.264 E-05 \\
2.000 E-04 \\
1.333 E-04 \\
1.211 E-04 \\
1.040 E-04 \\
8.544 E-05 \\
3.965 E-06 \\
5.566 E-06\end{array}$ & \\
\hline LBL & LBLAB & $1.208 E+01$ & $1.208 E+01$ & $.000 E+00$ & $.000 E+00$ & 1.210E-02 & $1.820 E-08$ & 3.919E-08 & $1.944 E-08$ & $\begin{array}{l}C-14 \\
H-3 \\
I-125 \\
I-129 \\
p-32 \\
s-35\end{array}$ & $\begin{array}{l}1.210 E-02 \\
2.314 E-04 \\
6.710 E-03 \\
1.050 E-05 \\
1.200 E-07 \\
5.430 E-04 \\
4.607 E-03 \\
2.157 E-01\end{array}$ & $.000 E+00$ \\
\hline
\end{tabular}


SWIR3280 - (Page 62 of 99 )

RUN DATE: $08 / 20 / 94$

Land Disposal or Storage of Solid Radioactive Waste from January 1 to December 31 , 1993 CENTRAL WASTE COKPLEX 2403WA BUIIDING, 200 WEST AREA, LON-LEVEL NONINDUSTRIAL.

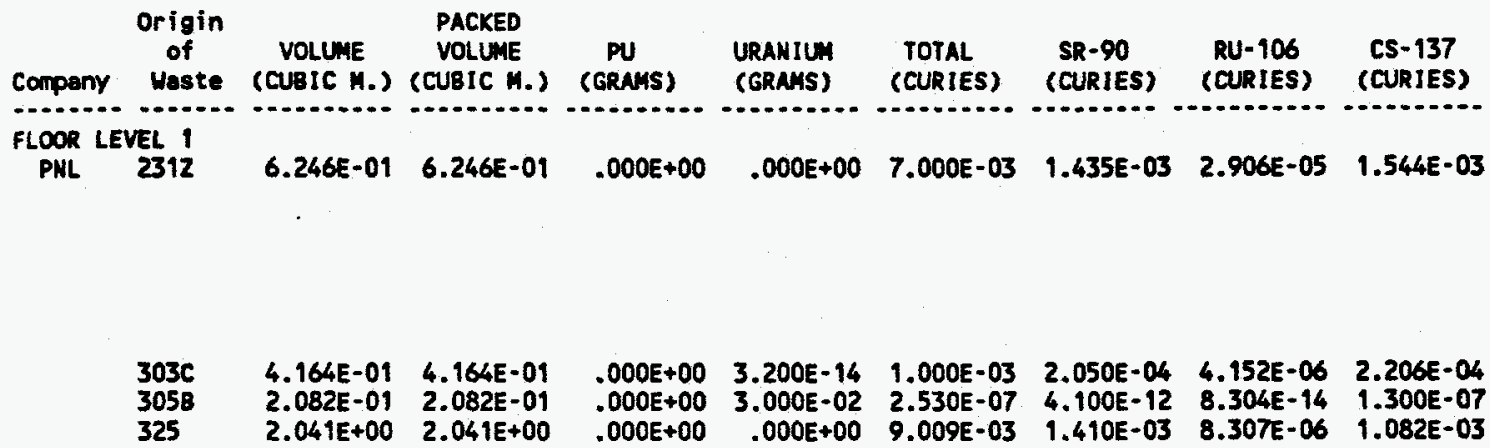

\section{EU}

Co-60 8.700E-O

EU-152 $1.000 E-10$

EU-154 $1.500 E-09$

EU-155 4.000E-10

CD-153 5.000E-04

$\mathrm{H}-3$ 1.890E-03

NA-22 9.405E-07

4.645E-01

CA-45 1.300E-06

CO-60 4.300E-06

FE-55 3.513E-01

$\mathrm{H}-3 \quad 1.904 \mathrm{E}-05$

MN-54 $1.130 E-01$

MO-93 2.400E-07

NB-93M 5.500E-08

NI-60 4.300E-06

$\mathrm{NI}-63 \quad 3.700 \mathrm{E}-06$

SC-46 2.600E-08

TC-99 $1.200 E-08$

$v-49 \quad 1.301 E-04$

4.164E-01 4. I64E-01

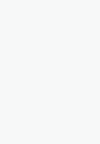

331

$8.328 E-01 \quad 8.328 E-01$

$.000 E+00$

$.000 E+00 \quad 2.030 E-06$

$.000 E+00$

$.000 E+00$

$.000 E+00$

\section{Co-}

$1443.000 E-13$

2.000E-06 9.201E-06 NM-241 6.700E-11 NP-237 6.200E-10 PU- $238 \quad 2.400 E-13$ PU-239 6.500E- 10 PU-240 8.700E-12 TH-232 9.200E-06 TH-234 2.100E-23

-60 1.000E-06

EU-152 4.900E-16

EU-154 5.000E-16

NI-60 1.000E-06

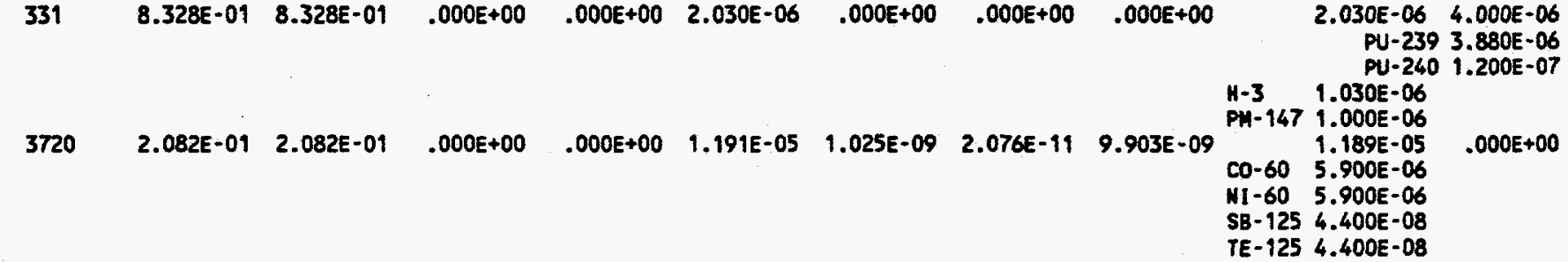




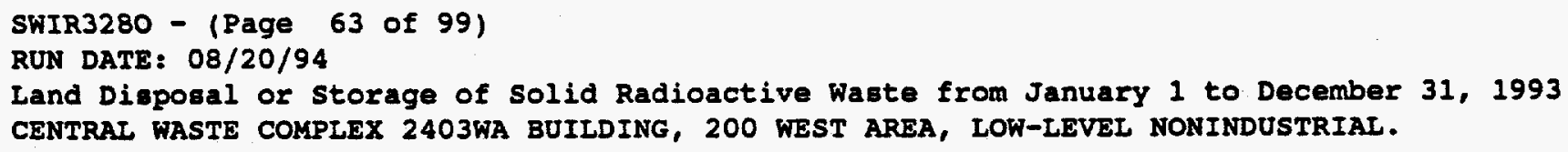

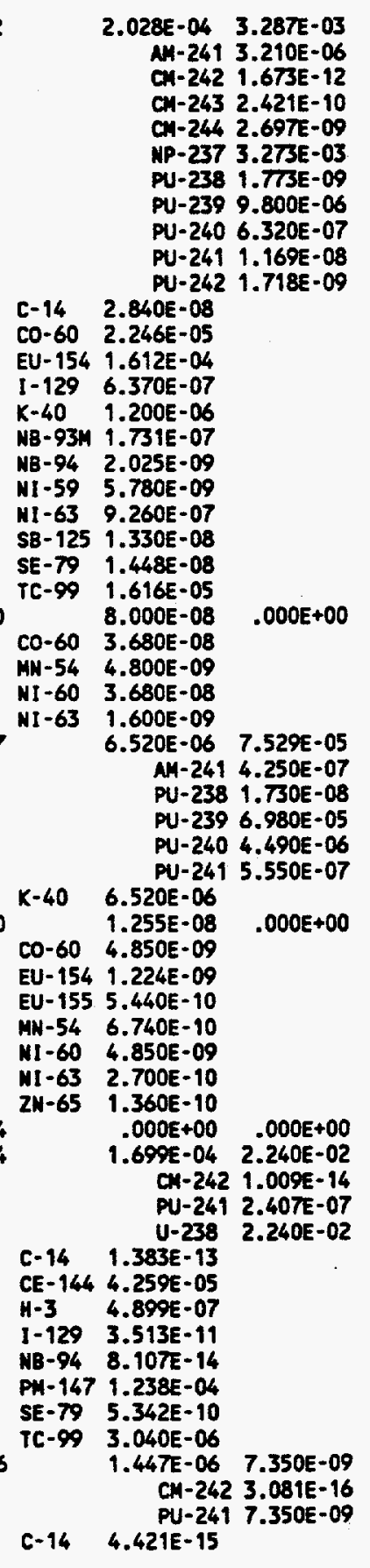


WHC-EP-0125-6

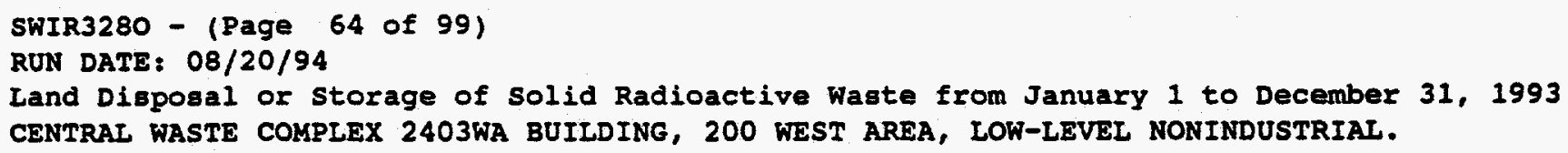
PU-239 9.52BE-04 PU-240 5.813E-05 PU-241 4.640E-06 PU-242 $1.993 \mathrm{E}-07$

$241 A \quad 1.571 E+00 \quad 1.571 E+00 \quad .000 E+00 \quad .000 E+00 \quad 5.834 E-01 \quad 2.002 E-01 \quad 3.108 E-07 \quad 9.403 E-02$

$1.496 E-05 \quad 4.091 E-04$ AM-241 5.143E-08 PU-238 2.105E-09 PU-239 $4.085 E-04$ PU-240 5.456E-07 


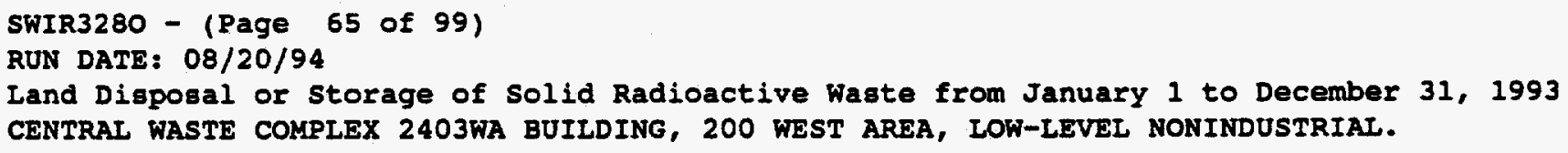

$241 A N \quad 3.956 E+00 \quad 3.956 E+00 \quad .000 E+00 \quad 2.733 E-01 \quad 3.738 E-02 \quad 9.998 E-03 \quad 1.845 E-08 \quad 8.909 E-03$

C0-60 6.470E-07

PU-241 6.748E-08

EU-154 7.390E-06

EU-155 6.920E-06

$241 A P \quad 1.874 E+00 \quad 1.874 E+00 \quad .000 E+00 \quad .000 E+00 \quad 9.717 E-02 \quad 3.742 E-02 \quad 1.804 E-08 \quad 1.147 E-02$

2411 8.328E-01 8.328E-01

$241 A 2$

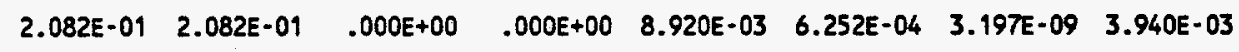

$241 B X \quad 6.246 E-01 \quad 6.246 E-01 \quad .000 E+00 \quad .000 E+00 \quad 5.057 E-04 \quad 3.168 E-05 \quad 2.064 E-10 \quad 2.273 E-04$

$241 B Y \quad 1.457 E+00 \quad 1.457 E+00 \quad .000 E+00 \quad 1.674 E-04 \quad 5.095 E-03 \quad 2.953 E-04 \quad 7.432 E-10 \quad 2.285 E-03$
CS-134 5.210E-06

EU-154 2.304E-05

EU-155 5.820E-06

K-40 7.320E-06

SB-125 9.910E-07

TE-125 2.290E-07

4

S- $134 \quad 1.381 \mathrm{E}-05$

1.381E-05 1.223E-04 NH-241 1.160E-04 PU-238 1.449E-09 PU-239 5.863E-06 PU-240 3.780E-07 PU-241 4.665E-08 $.000 E+00 \quad 6.348 E-06$ AM-241 5.194E-08 PU-238 5.607E-09 PU-239 5.068E-06 PU-240 3.371E-07 PU-241 8.858E-07 $1.990 E-06 \quad 9.039 E-08$ AM-241 5.100E-10 PU-238 2.070E-11 PU-239 8.380E-08 PU-240 5.390E-09 PU -241 $.000 E+00 \quad 1.592 E-06$ NH-241 8.796E-10 PU-238 3.566E-11 PU-239 1.580E-06 PU-240 $9.284 E-09$ PU-241 1.149E-09 4.964E-05 6.325E-05 NH-241 8.530E-08 CH-242 3.966E-14 CM-243 5.837E-12 CM-244 6.504E-11 NP-237 6.149E-05 PU-238 4.326E-08 PU-239 1.521E-06 PU-240 9.896E-08 PU-241 1.057E-08

C-14 1.006E-09

PU-242 3.740E-11 CO-60 3.773E-05

EU-154 1.148E-05 1-129 1.524E-08 NB-93H $1.096 E-09$

NB-94 4.881E- 11 
SWIR3280 - (Page 66 of 99 )

RUN DATE: 08/20/94

Land Disposal or Storage of Solid Radioactive waste from January 1 to December 31,1993

CENTRAL WASTE COMPLEX 2403WA BUILDING, 200 WEST AREA, LON-LEVEL NONINDUSTRIAL.

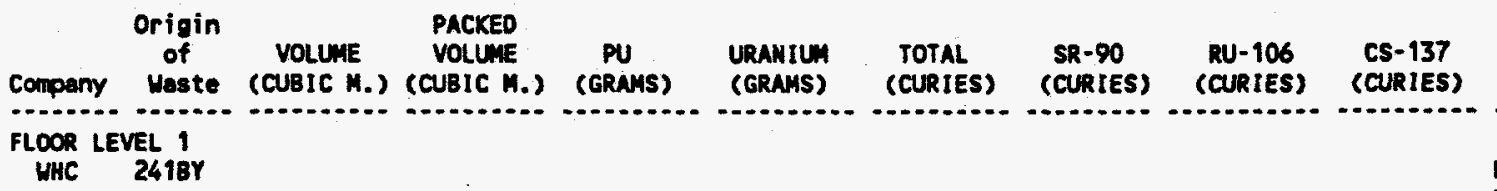

$2416 \quad 3.202 E+00 \quad 3.202 E+00 \quad .000 E+00 \quad .000 E+00 \quad 6.876 E-02 \quad 2.659 E-02 \quad 3.235 E-08 \quad 7.992 E-03$

NI-59 $1.390 E-10$

$N I-63 \quad 2.225 E-08$

SE-79 3.490E-10

TC-99 3.902E-07

$241 E N \quad 2.082 E-01 \quad 2.082 E-01 \quad .000 E+00 \quad .000 E+00 \quad 3.334 E-04 \quad 1.000 E-04 \quad 4.110 E-10 \quad 6.852 E-05$

$\begin{array}{lll}\text { PU-241 } & \text { 7.809E-05 } \\ \text { CO-60 } & \text { 4.190E-06 }\end{array}$

EU-154 2.190E-05

2.609E-05 1.060E-02

M-241 5.987E-05

PU-238 2.685E-06

PU-239 9.829E-03

PU-240 6.321E-04

(1)

1.457.

(1)

$2415 \times \quad 4.164 E-01 \quad 4.164 E-01 \quad 000 E+00 \quad .000 E+00 \quad 3.321 E-04 \quad 9.450 E-06 \quad 4.069 E-10 \quad 1.592 E-04$

$2415 Y \quad 1.457 E+00 \quad 1.457 E+00 \quad .000 E+00 \quad 7.773 E-05 \quad 5.833 E-02 \quad 2.012 E-02 \quad 2.412 E-09 \quad 8.443 E-03$

$.000 E+00 \quad 1.554 E-07$ AM-2418.770E-10 PU-238 $3.560 E-11$ PU-239 $1.440 E-07$ PU-260 9.300E-09 PU-241 1.150E-09

1.040E-04 5.029E-02 $M-241$ 8.126E-03 PU-238 2.338E-05 PU-239 3.668E-02 PU-240 2.348E-03 PU-241 3.103E-03 PU-242 4.390E-06

CE-144 4.760E-05

CO-60 4.325E-06

EU-154 2.314E-05

$E U-155 \quad 3.952 E-06$

K-40 2.497E-05

$1.287 E-05$

Mi-241 $1.842 E-06$

PU-238 4.465E-08

PU-239 1.024E-05

PU-240 6.580E-07

PU-241 8.140E-08

EU- $1545.310 E-07$

K-40 2.790E-06

1.652E-03 8.870E-05

AM-241 3.580E-08

CH-242 1.830E- 14

CM-243 2.690E-12

CH-244 3.000E-11

NP-237 4.020E-05

PU-238 1.970E-11

PU-239 4.847E-05

C-14 4.650E-10

Co-60 1.650E-03

EU-154 1.790E-06

1-129 7.100E-09

NB-94 2.260E-11

UB-95M 1.930E-09

$M 1-59 \quad 6.450 E-11$

N1-63 1.030E-08

SE-79 1.610E-10

TC- 99 1.810E-07

$241 T \quad 5.076 E+00 \quad 5.076 E+00 \quad .000 E+00 \quad .000 E+00 \quad 2.335 E-02 \quad 9.502 E-03 \quad 1.427 E-07 \quad 2.181 E-03$
1.0595-04

AM-241 6.248E-05 PU-238 $1.580 \mathrm{E}-06$ 
SWIR3280 - (Page 67 of 99)

RUN DATE: 08/20/94

Land Diaposal or Storage of Solid Radioactive Wagte from January 1 to December 31 , 1993

CENTRAL WASTE COMPLEX 2403WA BUILDING, 200 WEST AREA, LOW-LEVEL NONINDUSTRIAL.

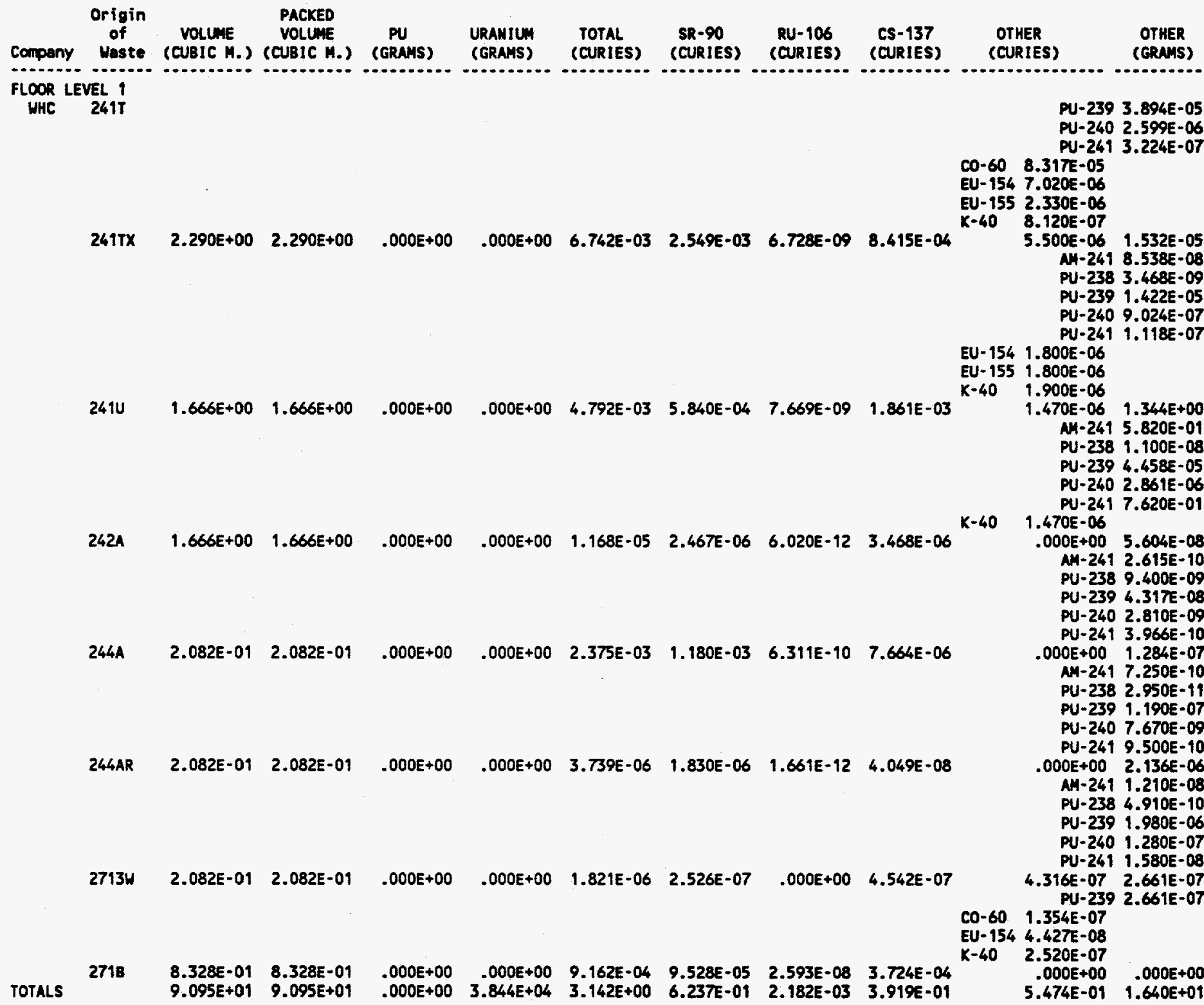


SWIR3280 - (Page 68 of 99)

RUN DATE: 08/20/94

Land Disposal or Storage of Solid Radioactive Waste from January 1 to December 31 , 1993

CENTRAL WASTE COMPLEX 2403WC BUILDING, 200 WEST AREA, LOW-IEVEL NONINDUSTRIAL-OFESITE.

\begin{tabular}{|c|c|c|c|c|c|c|c|c|c|c|c|c|}
\hline Compeny & $\begin{array}{c}\text { Origin } \\
\text { of } \\
\text { Waste }\end{array}$ & $\begin{array}{c}\text { VOLUME } \\
\text { (CUBIC M.) }\end{array}$ & $\begin{array}{l}\text { PACKED } \\
\text { VOLUNE } \\
\text { (CUBIC M.) }\end{array}$ & $\begin{array}{c}\text { PU } \\
\text { (GRAMS) }\end{array}$ & $\begin{array}{l}\text { URANIUM } \\
\text { (GRAMS) }\end{array}$ & $\begin{array}{l}\text { TOTAL } \\
\text { (CURIES) }\end{array}$ & $\begin{array}{c}\text { SR-90 } \\
\text { (CURIES) }\end{array}$ & $\begin{array}{c}\text { RU-106 } \\
\text { (CURIES) }\end{array}$ & $\begin{array}{c}\text { CS-137 } \\
\text { (CURIES) }\end{array}$ & $\begin{array}{l}\text { OTh } \\
\text { cCUR }\end{array}$ & $\begin{array}{l}\text { IER } \\
\text { RIES) }\end{array}$ & $\begin{array}{c}\text { OTHER } \\
\text { (GRAMS) }\end{array}$ \\
\hline $\begin{array}{l}\text { LOOR LE } \\
\text { ANL }\end{array}$ & $\begin{array}{l}\text { LEVEL } 1 \\
\text { ARCOW }\end{array}$ & $5.621 E+01$ & $5.621 E+09$ & $.000 E+00$ & $.000 E+00$ & $7.351 E-04$ & $3.102 E-10$ & $6.682 E-10$ & 2.862E-04 & $\begin{array}{l}81-214 \\
c 0-60 \\
N I-60 \\
P B-214\end{array}$ & $\begin{array}{r}1.781 E-04 \\
R A-226 \\
T H-228 \\
3.657 E-05 \\
5.247 E-05 \\
5.247 E-05 \\
3.657 E-05\end{array}$ & $\begin{array}{l}3.379 E-05 \\
3.339 E-05 \\
3.975 E-07\end{array}$ \\
\hline LBL & LBLAB & $3.538 E+00$ & $3.538 E+00$ & $.000 E+00$ & $.000 E+00$ & $3.709 E-02$ & $8.154 E-10$ & $1.756 E-09$ & $8.709 E-10$ & $\begin{array}{l}c-14 \\
H-3 \\
p-32\end{array}$ & $\begin{array}{c}3.709 E-02 \\
\text { PU-238 } \\
U-233 \\
1.059 E-04 \\
3.682 E-02 \\
1.670 E-04\end{array}$ & $\begin{array}{l}1.616 E-06 \\
1.000 E-10 \\
1.616 E-06\end{array}$ \\
\hline PSN & PSNS & $8.182 E+01$ & $8.182 E+01$ & $.000 E+00$ & $.000 E+00$ & $1.091 E-01$ & $5.589 E-07$ & $1.204 E-06$ & $5.969 E-07$ & $\begin{array}{l}C-14 \\
C O-58 \\
C O-60 \\
F E-55 \\
H-3 \\
I-129 \\
M N-54 \\
M 1-63 \\
T C-99\end{array}$ & $\begin{array}{l}1.090 E-01 \\
5.399 E-04 \\
1.354 E-03 \\
2.698 E-02 \\
2.698 E-02 \\
4.726 E-02 \\
5.399 E-09 \\
4.587 E-03 \\
1.354 E-03 \\
5.399 E-09\end{array}$ & $.000 E+00$ \\
\hline RXD & ESG & $2.082 E-01$ & 2.082E-01 & $.000 E+00$ & $.000 E+00$ & $1.600 E-05$ & 4.004E-06 & $9.251 E-09$ & 4.005E-06 & & $\begin{array}{r}.000 E+00 \\
A M-241 \\
P U-241 \\
1.463 E-01\end{array}$ & $\begin{array}{l}1.000 E-07 \\
5.000 E-08 \\
5.000 E-08 \\
3.550 E-05\end{array}$ \\
\hline
\end{tabular}


SWIR3280 - (Page 69 of 99)
RUN DATE: 08/20/94

Land Disposal or Storage of Solid Radioactive Waste from January 1 to December 31 , 1993 CENTRAL WASTE COMPLEX 2403WC BUILDING, 200 WEST AREA, LON-LEVEL NONINDUSTRIAL.

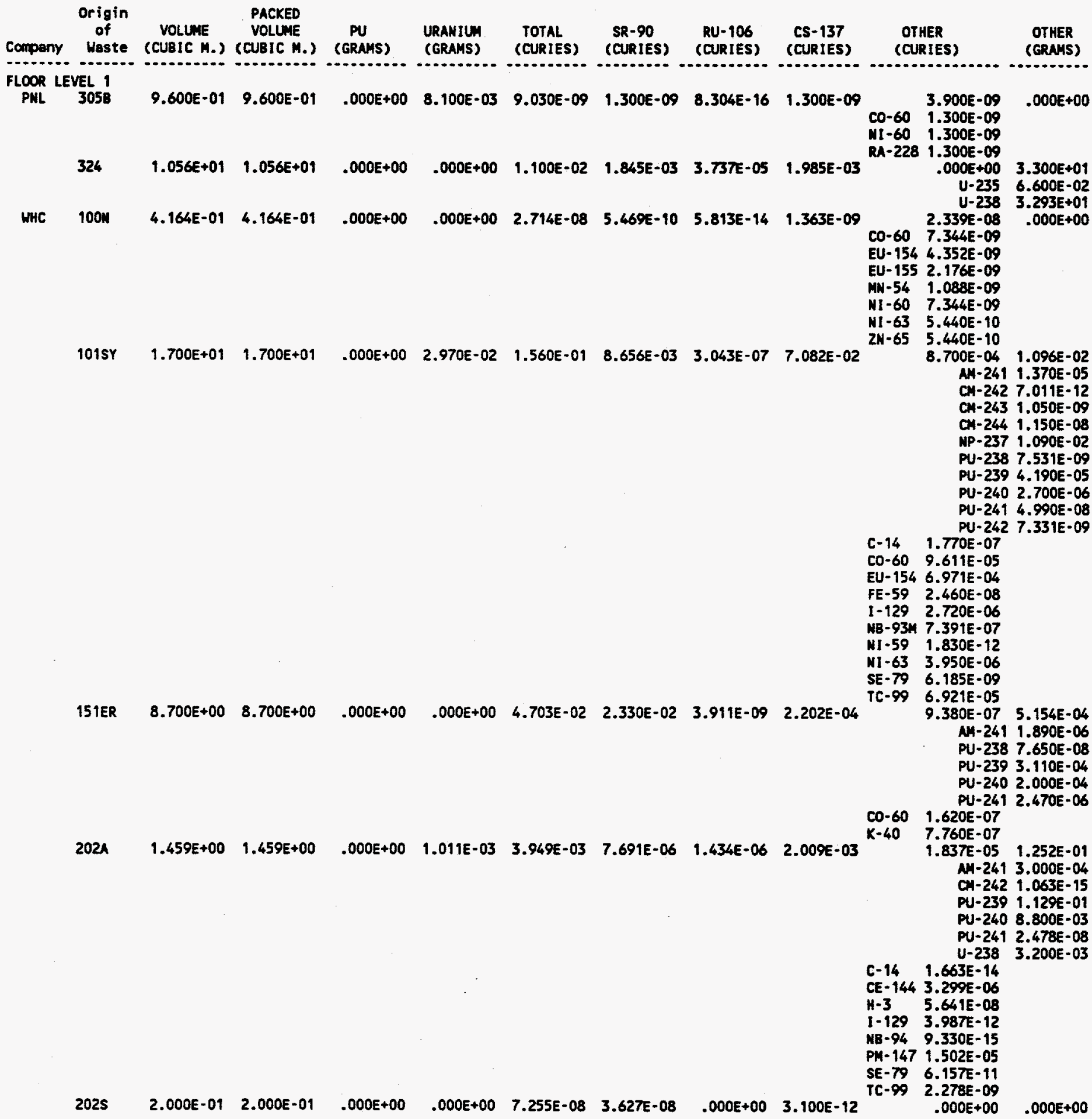


SWIR3280 - (Page 70 of 99 )

RUN DATE: $08 / 20 / 94$

Land Disposal or storage of Solid Radioactive Waste from January 1 to December 31 , 1993

CENTRAL WASTE COMPLEX 2403WC BUILDING, 200 WEST AREA, LOW-LEVEL NONINDUSTRIAL.

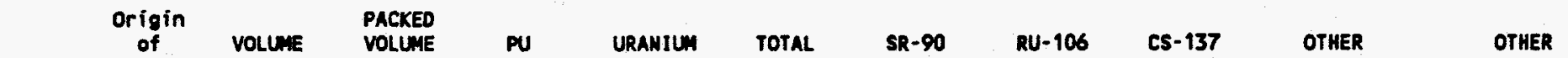

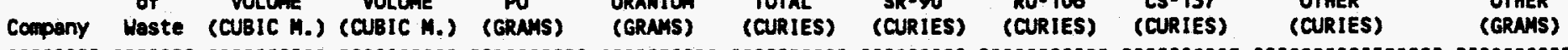

FLOOR IEVEL

WHC 2O4AR

$\begin{array}{llllllll}1.120 E+00 & 1.120 E+00 \quad .000 E+00 \quad .000 E+00 \quad 1.599 E-06 & 7.486 E-08 & 4.144 E-10 & 1.070 E-07\end{array}$ $2217 \quad 4.182 E-01 \quad 4.182 E-01 \quad .000 E+00 \quad .000 E+00 \quad 2.769 E-03 \quad 1.284 E-03 \quad 2.478 E-08 \quad 6.605 E-05$

8.500E-08

$1.225 E-06 \quad 1.922 E-07$ AM-241 $1.230 E-11$ PU-238 4.980E-13 PU-239 2.020E-09 PU-240 1.300E-10 PU-241 1.610E-11 TH-228 $1.900 E-07$ CS-134 $6.500 E-08$ EU-152 6.000E-07 K-40 4.750E-07

6.841E-05 3.2185-05 NA-241 3.220E-06 NP-237 2.630E-05 PU-239 2.658E-06

- $14 \quad 5.920 E-09$ CO-60 2.952E-06 EU-152 1.564E-05 EU-154 2.677E-05 EU-155 1.070E-05 NA-22 9.498E-06 NB-95N 4.320E-10 TC-99 1.619E-07 $2225 \quad 1.524 E+01 \quad 1.524 E+01 \quad .000 E+00 \quad 1.181 E+00 \quad 4.910 E-01 \quad 1.960 E-01 \quad 5.413 E-08 \quad 4.931 E-02$

ZR-95 2.680E-06

$3.063 E-03 \quad 3.513 E+01$ AN-241 2.43TE-05 PU-238 2.675E-07 PU-239 5.293E-03 $U-234 \quad 1.038 E-02$ U-235 3.463E-01 $\mathrm{U}-238 \quad 3.477 \mathrm{E}+01$ C-14 6.120E-09 EU-154 4.840E-06 EU- 155 1.260E-06 H-3 1.054E-05 I-129 7.710E-08 $K-40 \quad 1.876 E-04$ PM- 147 2.751E-03

$224 U \quad 6.246 E-01 \quad 6.246 E-01 \quad .000 E+00 \quad 1.000 E-01 \quad 1.700 E-03 \quad .000 E+00 \quad .000 E+00 \quad .000 E+00$ TC-99 1.077E-04 $1.700 E-03 \quad 1.000 E+03$ U-234 9.001E-02 U-235 $9.201 E+00$ U-236 7.101E-01 U-238 $9.901 E+02$ $2312 \quad 3.140 E+00 \quad 3.140 E+00 \quad .000 E+00 \quad .000 E+00 \quad 5.000 E-04 \quad .000 E+00 \quad .000 E+00 \quad .000 E+00 \quad \begin{array}{llllllll}T C-99 & 1.700 E-03 & .000 E+00 & 1.996 E-04\end{array}$ PU-238 $1.790 E-06$ PU-239 2.080E-05 PU-240 4.630E-06 PU-241 1.710E-04 PU-242 $2.730 E-10$

$23452 \quad 3.772 E+00 \quad 3.772 E+00 \quad .000 E+00 \quad .000 E+00 \quad 2.800 E-03 \quad 5.740 E-04 \quad 1.163 E-05 \quad 6.177 E-04$ $.000 E+00 \quad 1.724 E-02$ Ni-241 2.028E-05 PU-238 5.016E-06 PU-239 1.615E-02 PU-2409.811E-04 PU-2417.873E-05 PU-242 $3.361 E-06$

$241 A \quad 8.508 E+00 \quad 8.508 E+00 \quad .000 E+00 \quad .000 E+00 \quad 5.670 E-01 \quad 1.416 E-01 \quad 3.451 E-04 \quad 1.451 E-01$ 8.210E-04 1.224E-04 


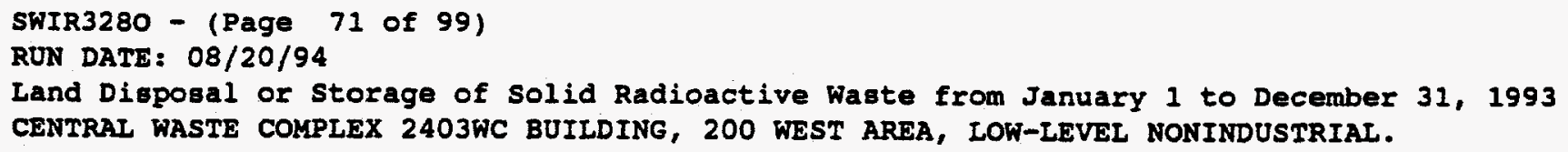

$241 A N \quad 1.120 E+00 \quad 1.120 E+00 \quad .000 E+00 \quad .000 E+00 \quad 2.009 E-03 \quad 8.962 E-04 \quad 4.127 E-09 \quad 1.112 E-04$

$241 \mathrm{AH}$

$8.300 E+00 \quad 8.300 E+00$

$.000 \mathrm{E}+00$

$.000 E+00$

1.

$241 A Z$

$8.300 E+$

$8.300 E+00$

$.000 E+00$

$.000 E+$

5

591E-07 2.532E-08 3.737E-13 5.452E-08

2418

$1.120 E+$

$1.120 E+00$

$.000 E+00$

.

$000 E+00$

$8.538 E-03$

$2.060 E-03$

2.408E-09 2.270E-03

$2418 x$

$7.116 E+$

$7.116 E+00$

$.000 E+0$

$+00$

OOOE+OO

$2.378 E-0$

$1.123 E-01$

9.717E-07 6.774E-03

$\begin{array}{ll}c 0-60 & 1.360 E-06 \\ C S-136 & 8.030 E-04\end{array}$

EU-154 1.200E-05

$K-40 \quad 4.660 E-06$

NM-241 5.483E-07

PU-238 2.934E-08

PU-239 $1.137 E-04$

PU-240 7.230E-06

PU-241 8.490E-07

$.000 E+00$ 1.068E-04

AM-2419.660E-09 PU-238 3.000E-08 PU-239 1.000E-04 PU-240 6.150E-06 PU-241 5.640E-07 4.779E-05 5.579E-05 M. $2492.950 E-07$ PU-238 $1.200 E-08$ PU-239 4.850E-05 PU-240 3.120E-06 PU- $2413.860 E-06$ CD-60 4.400E-07 CS-134 2.660E-05 K-40 6.360E-07 NI-60 4.400E-07 SB- 125 1.620E-05 TE-125 3.470E-06 $.000 E+00 \quad 1.133 E-03$ AM-241 6.420E-06 PU-238 2.600E-07 PU-239 $1.050 E-03$ PU-240 6.780E-05 PU-241 8.390E-06 4.024E-07 1.640E-04 AM-2417.720E-14 PU-238 3.140E-15 PU-239 1.270E- 11 PU-240 8.150E- 13 PU-241 1.010E-13 TH-228 1.640E-04

K-40 4.000E-07

TC-99 2.350E-09 $.000 E+00 \quad 4.649 E-07$ $A M-241 \quad 2.620 E-09$ PU-238 1.070E-10 PU-239 4.310E-07 PU-240 2.770E-08 PU-241 3.430E-09 2.950E-06 $1.222 E-02$ NH-241 6.585E-05 PU-238 2.831E-06 PU-239 $1.134 E-02$ PU-240 7.280E-04 PU-241 8.601E-05

EU-154 1.890E-06

$K-40 \quad 1.060 E-06$

$241 C \quad 8.908 E+00 \quad 8.908 E+00 \quad .000 E+00 \quad .000 E+00 \quad 1.303 E-02 \quad 5.115 E-04 \quad 2.144 E-08 \quad 6.167 E-03$

$.000 E+00 \quad 8.833 E-04$ AM-2417.975E-08 PU-238 2.475E-07 PU-239 8.275E-04 
SWIR3280 - (Page 72 of 99)

RUN DATE: 08/20/94

Land Dieposal or Storage of Solid Radioactive Waste from January 1 to December 31 , 1993 CENTRAL WASTE COMPLEX 2403WC BUILDING, 200 WEST AREA, LOW-LEVEL NONINDUSTRIAL.

\begin{tabular}{|c|c|c|c|c|c|c|c|c|c|}
\hline Company & $\begin{array}{c}\text { Origin } \\
\text { of } \\
\text { Maste }\end{array}$ & $\begin{array}{c}\text { VOLUME } \\
\text { (CUBIC M.) }\end{array}$ & $\begin{array}{l}\text { PACKED } \\
\text { VOLUME } \\
\text { (CUBIC M.) }\end{array}$ & $\begin{array}{c}\text { PU } \\
\text { (GRAMS) }\end{array}$ & $\begin{array}{l}\text { URANIUN } \\
\text { (GRAMS) }\end{array}$ & $\begin{array}{l}\text { TOTAL } \\
\text { (CURIES) }\end{array}$ & $\begin{array}{l}\text { SR-90 } \\
\text { (CURIES) }\end{array}$ & $\begin{array}{c}\text { RU- } 106 \\
\text { (CURIES) }\end{array}$ & $\begin{array}{c}\text { CS-137 } \\
\text { (CURIES) }\end{array}$ \\
\hline $\begin{array}{l}\text { COR } \\
\text { HHC }\end{array}$ & $\begin{array}{l}\text { VEL } 1 \\
241 C\end{array}$ & & & & & & & & \\
\hline & 2415 & $1.562 E+01$ & $1.562 E+01$ & $.000 E+00$ & $2.544 E-03$ & $6.812 E-01$ & 3.070E-01 & $5.229 E-07$ & $3.451 E$ \\
\hline
\end{tabular}

$2415 X \quad 2.085 E+00 \quad 2.085 E+00 \quad .000 E+00 \quad .000 E+00 \quad 6.646 E-02 \quad 2.695 E-02 \quad 2.172 E-08 \quad 6.459 E-03$

C- 1

EU-154 $7.500 E-05$

$\begin{array}{ll}1-129 & 2.330 E-07\end{array}$

$K-40 \quad 1.145 E-05$

NB-93M 6.320E-08

MB-94 7.390E-10

MI -59 2.110E-09

NI -63 3.380E-07

SE-79 5.280E-09

TC-99 5.920E-06

$.000 E+00$ 2.019E-04

AM-241 6.283E-07 PU-238 5.094E-08 PU-239 1.881E-04 $P U-2401.184 E-05$ PU-241 1.295E-06

$2415 Y \quad 3.480 E+01 \quad 3.480 E+01 \quad .000 E+00 \quad 8.157 E-03 \quad 1.732 E-01 \quad 8.830 E-03 \quad 2.194 E-07 \quad 7.290 E-02$

$1362 \mathrm{E}-02 \quad 3.553 \mathrm{E}-02$ AM-241 $1.384 E-05$ $\mathrm{CH}-2427.222 \mathrm{E}-12$ CH-243 $1.803 E-09$ CM-244 $1.173 E-08$ NP- 237 1.110E-02 PU-238 7.982E-09 PU-239 4.254E-05 PU-240 2.699E-06 PU-241 2.200E-03 PU-242 7.439E-09 U-235 3.720E-04 U.236 9.020E-11 U-238 2.180E-02

$c-14 \quad 1.808 E-07$

Co-60 9.803E-05

EU-154 1.345E-02

I-129 2.775E-06

MB-93M 7.534E-07

MB-94 8.819E-09

MI -59 2.516E-08

NI-63 4.035E-06

SE-79 6.303E-08

TC-99 7.056E-05

$241 T X \quad 4.472 E+00 \quad 4.472 E+00 \quad .000 E+00 \quad .000 E+00 \quad 3.686 E-02 \quad 1.606 E-02 \quad 3.307 E-08 \quad 2.341 E-03$
$1.894 E-04 \quad 7.146 E-05$ NH-241 4.029E-07 PU-238 1.636E-08 PU-239 6.626E-05 PU-240 4.256E-06 PU-241 5.269E-07 
WHC-EP-0125-6

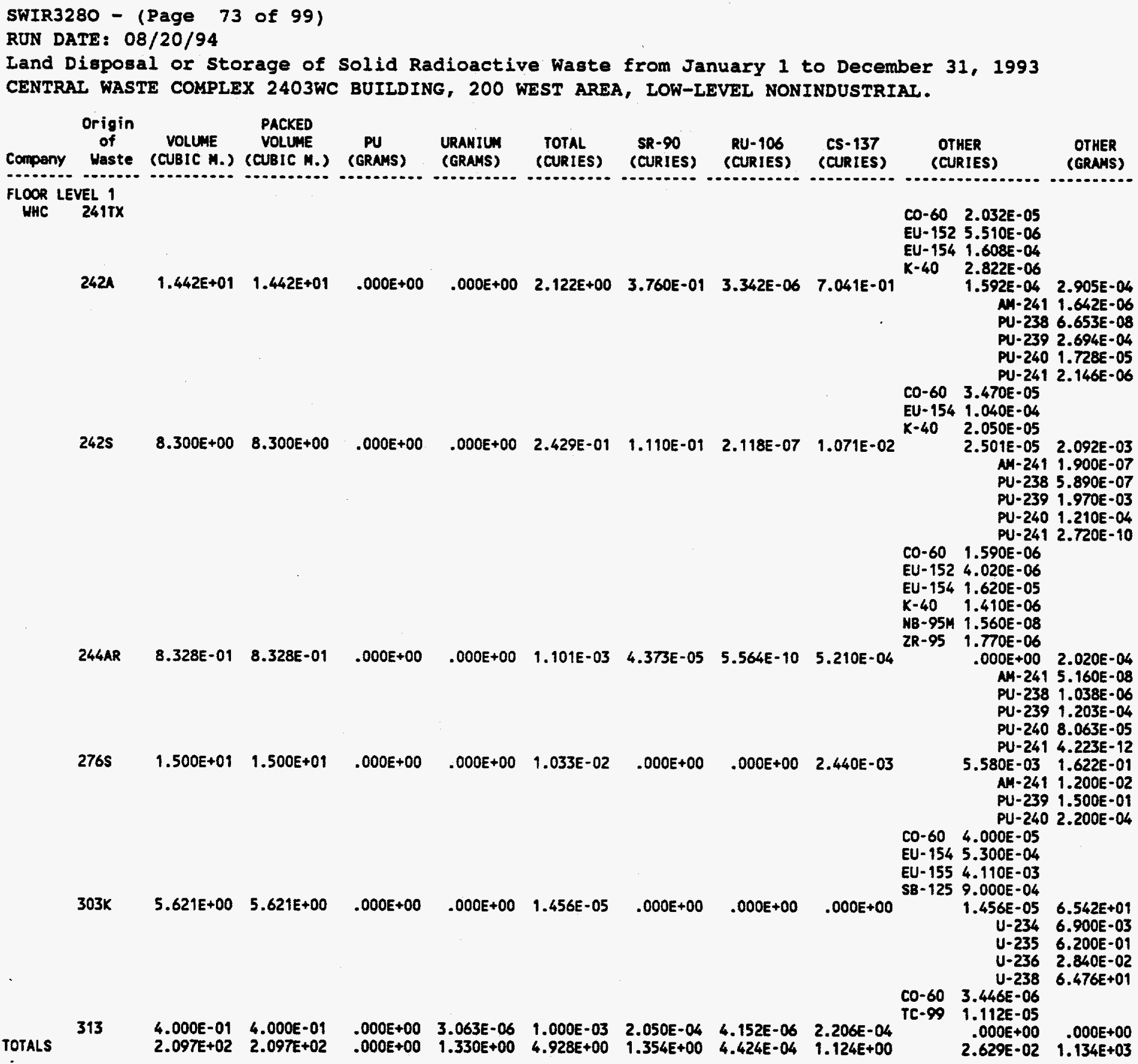


SWIR3280 - (Page 74 of 99 )

RUN DATE: $08 / 20 / 94$

Land Disposal or Storage of Solid Radioactive Waste from January 1 to December 31 , 1993

CENTRAL WASTE COMPLEX 2403WC BUILDING, 200 WEST AREA, TRANSURANIC (NOT PROCESSED THROUGH TRUS

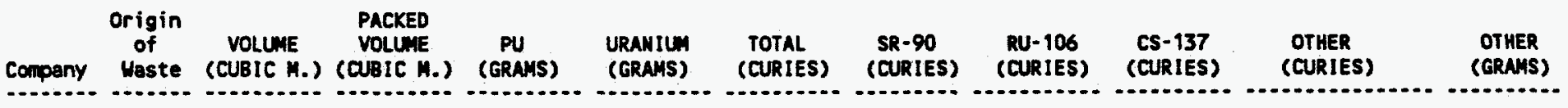

FLOOR LEVEL 1

KAPL KAPL

$2.082 E-01 \quad 2.082 E-01 \quad .000 E+00$

$.000 E+00 \quad 1.226 E+00 \quad 1.426 E-05 \quad 3.071 E-05 \quad 1.523 E-05$

$1.225 E+00 \quad 4.000 E-01$

AC-227 1.200E-02

$R A-226 \quad 4.000 E-01$

B1-214 4.000E-01

$C-14 \quad 8.400 E-08$

CD-58 $9.900 E-04$

CO-60 $1.300 E-03$

CQ-51 7.900E-03

FE-55 2.300E-03

$H-3 \quad 2.500 E-07$

MN-54 5.000E-04

MO-93 2.100E-08

NB-94 9.900E-09

MI -59 2.600E-06

NI-63 2.900E-04

PB-214 4.000E-01

PO-210 4.000E-01

TOTALS

$\begin{array}{llllllll}2.082 E-01 & 2.082 E-01 & .000 E+00 & .000 E+00 & 1.226 E+00 & 1.426 E-05 & 3.071 E-05 & 1.523 E-05\end{array}$

TC- 99 4.500E-09 
SWIR3280 - (Page 75 of 99 )

RUN DATE: 08/20/94

Land Disposal or Storage of Solid Radioactive Waste from January 1 to December 31 , 1993

CENTRAL WASTE COMPLEX 2403WC BUIIDING, 200 WEST AREA, TRANSURANIC (NOT PROCESSED THROUGH TRUS

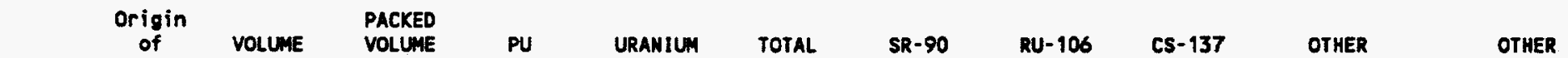

Company Waste (CUBIC M.) (CUBIC M.) (GRAMS) (GRAMS) (CURIES) (CURIES) (CURIES) (CURIES) (CURIES)

FLOR LEVEL 1 (

PNL $325 \quad 2.082 E-01 \quad 2.082 E-01 \quad .000 E+00 \quad .000 E+00 \quad 5.000 E-04 \quad 1.025 E-04 \quad 2.076 E-06 \quad 1.103 E-04$

HHC $2312 \quad 1.900 E+00 \quad 1.900 E+00 \quad .000 E+00 \quad .000 E+00 \quad 3.508 E-04 \quad 1.661 E-08 \quad 3.363 E-10 \quad 1.787 E-08$

$\begin{array}{llllllllll}23452 & 2.746 E+01 & 2.746 E+01 & 4.937 E+02 & .000 E+00 & 9.530 E-02 & 1.943 E-02 & 3.936 E-04 & 2.091 E-02 \\ 340 & 2.082 E-01 & 2.082 E-01 & .000 E+00 & .000 E+00 & 3.836 E-01 & 4.200 E-03 & 7.600 E-04 & 2.300 E-02\end{array}$

$10-60$

$3.056 E-04$

$M-2412.470 E-02$

NM-243 2.770E-04

CN-243 5.950E-07

$\mathrm{CH}-244$ 1.930E-04

$3.507 E-04 \quad 1.138 E-01$

NH-241 8.120E-02 MP-237 3.265E-02 TH-228 3.012E-09 AM-241 3.407E-01

3.251E-01 9.684E-03 AM-241 3.300E-03 PU-238 4.500E-05 PU-239 6.241E-03 PU-249 9.800E-05

CE- $1443.800 E-03$

CS-134 1.900E-03

EU-152 1.000E-01

EU-154 1.900E-01

EU-155 2.900E-02

TA- 182 3.800E-04 
SWIR3280 - (Page 76 of 99)

RUN DATE: 08/20/94

Land Dieposal or Storage of Solid Radioactive Waste from January 1 to December 31 , 1993

CENTRAL WASTE COMPLEX 2403WC BUILDING, 200 WEST AREA, TRANSURANIC WIPP CERTIEIED.

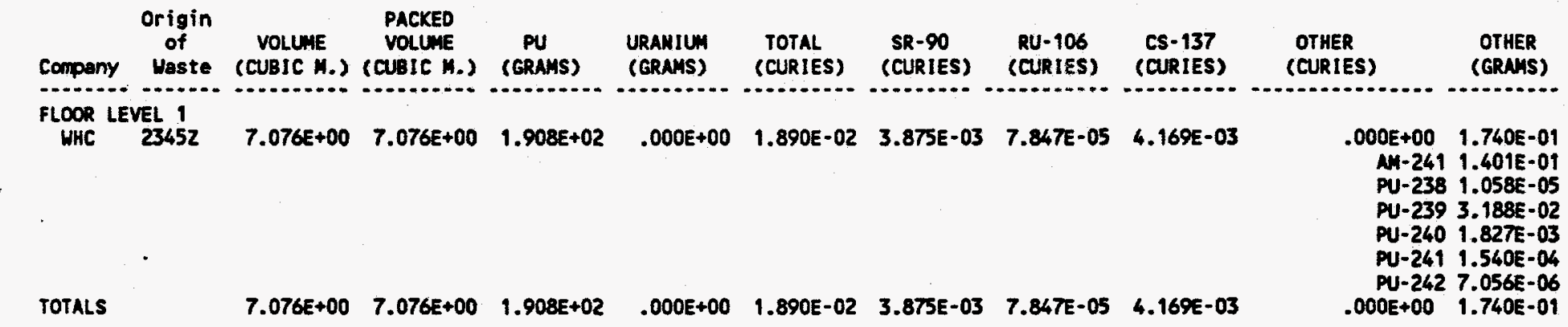




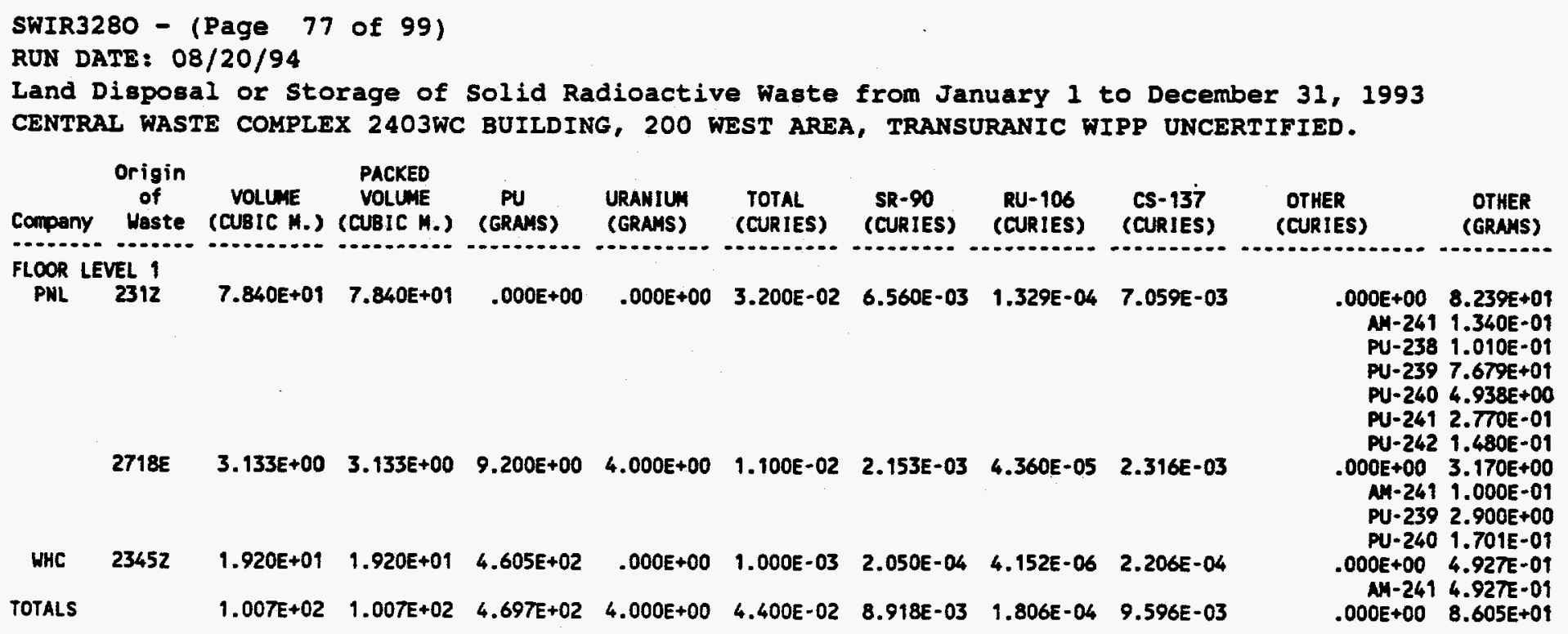


WHC-EP-0125-6

SWIR3280 - (Page 78 of 99)

RUN DATE: $08 / 20 / 94$

Land Disposal or Storage of Solid Radioactive Waste from January 1 to December 31 , 1993

CENTRAL WASTE COMPLEX 2403WD BUILDING, 200 WEST AREA, LON-IEVEL NONINDUSTRIAL-OFESITE.

\section{origin PACKED}

of VOLUAE VOLUME

PU URANTUM

(GRAMS)

TOTAL

SR 90

RU-106

cs-137

OTHER

(CURIES)

OTHER

Company Waste (CUBIC M.) (CUBIC M.) (GRAMS)

(CURIES) (CURIES) (CURIES) (CURIES)

FLOOR LEVEL 1

LBL LBLAB

2.082E-01 $\quad 2.082 E-01 \quad .000 E+00$

$.000 E+00$

2.080E-05

$.000 E+00 \quad .000 E+00$

$.000 E+00$

2.082E-01 2.082E-01

$.000 E+00$

$.000 E+00$

$2.080 E-05$

$.000 E+00 \quad .000 E+00 \quad .000 E+00$

2.080E-05

$2.080 E-05$

2.080E-05

$.000 E+00$

(n)


SWIR3280 - (Page 79 of 99 )

RUN DATE: 08/20/94

Land Disposal or Storage of Solid Radioactive Waste from January 1 to December 31 , 1993

CENTRAL WASTE COMPLEX 2403WD BUIIDING, 200 WEST AREA, LOW-LEVEL NONINDUSTRIAL.

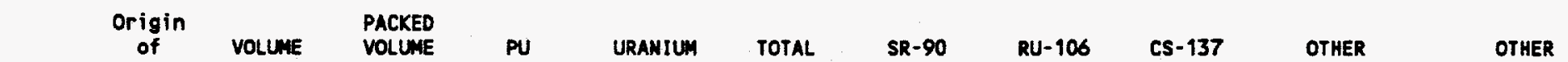
Company Waste (CUBIC M.) (CUBIC M.) (GRAMS) (GRAMS) (CURIES) (CURIES) (CURIES) (CURIES) (CURIES)
(GRAMS)

FLOOR LEVEL 1

$\begin{array}{lllllllll}\text { PNL } 324 & 6.740 E+00 & 6.740 E+00 & .000 E+00 & .000 E+00 & 5.503 E-01 & 1.000 E-01 & 8.304 E-08 & 1.800 E-01\end{array}$

WHC $105 C \quad 1.998 E+01 \quad 1.998 E+01 \quad .000 E+00 \quad .000 E+00 \quad 3.123 E+03 \quad 3.980 E-01 \quad 6.140 E-03 \quad 4.339 E-01$

$202 a$ $6.580 E+00$

$6.580 E+00$

$.000 E+00$

4.11

$17.646 E-03 \quad 1.121 E-03$

$.131 E-04 \quad 1.301 E-03$

2225

$2.000 E-01 \quad 2.000 E-01$

$.000 E+00$

5.780E-06

$1.390 E-06$

$2.210 E-07 \quad 1.951 E-13 \quad 4.500 E-07$

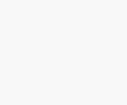

23452

2.080E-01 2.080E-01

$.000 E+00$

$.000 E+00$

5.000E-04 1.025E-04

2.076E-06 1.103E-04

$2415 Y$

4. 200E+0

$.000 E+00$

$.000 E+00$

1

$\begin{array}{rr}.000 E+00 & .000 E+00 \\ 3.121 E+03 & 2.149 E+02 \\ \text { NH-241 } & 4.650 E-05\end{array}$

MM-241
PU-238 $5.430 E-05$
PU-239

PU-239 6.990E-01

PU-240 1.230E-02

PU-241 3.330E-06

PU-242 2.367E-03

$U-238 \quad 2.142 E+02$

CO-60 3.000E+03

EU-152 8.070E-03

EU-154 2.034E-02

EU-155 1.557E-03

NI-59 1.092E+00

NI $-63 \quad 1.200 E+02$

1.971E-03 2.733E-06

CM-242 1.151E-13

C- 14 PU-241 2.733E-06

$\mathrm{H}-3 \quad 5.566 \mathrm{E}-06$

1-129 4.024E-10

NB-94 $9.230 E-13$

PM- 147 1.482E-03

SE-79 6.076E-09

TC-99 2.242E-07

.225E-08 5.722E-09

$A M-2416.810 E-10$

PU-238 5.640E-13

H-3 PU-239 5.040E-09

2.440E-08

$1-129 \quad 3.120 E-10$

PM-147 4.730E-08

TC- $99 \quad 2.410 E-10$

$.000 E+00 \quad 1.174 E-13$

AM-241 1.250E-16 PU-238 3.430E-17 PU-239 1.100E-13 PU-240 6.690E-15 PU-241 5.500E-16 PU-242 2.290E-17 2.605E-01 1.603E+01 AM-241 2.870E-03 CM-242 2.115E-09 CM-243 3.120E-07 $\mathrm{CM}-244$ 3.485E-06 MP $-2376.580 E+00$ PU-238 2.278E-06 PU-239 1.262E-02 PU-240 8.140E-04 PU-241 4.739E-01 PU-242 2.204E-06 U-235 1.508E-01 U-236 3.652E-08 U-238 $8.810 E+00$

C-14 5.380E-05

CO-60 2.904E-02

EU-154 2.074E-01

I-129 1.641E-03

MB-93M 2.230E-04 


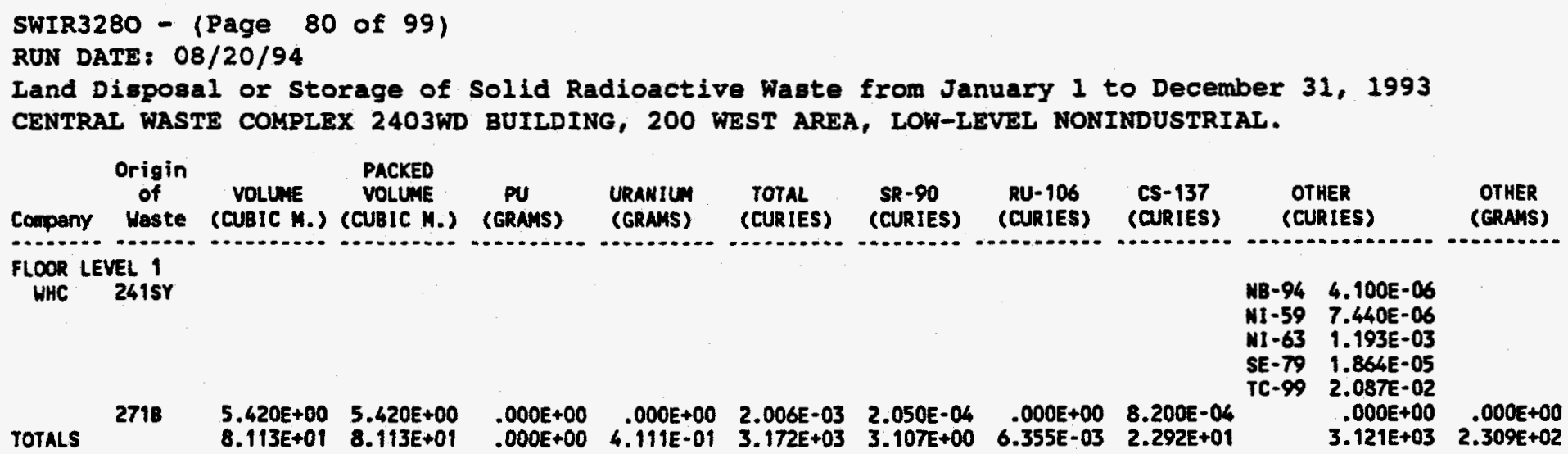


พHC-EP-0125-6

SWIR3280 - (Page 81 of 99 )

RUN DATE: $08 / 20 / 94$

Iand Diaposal or Storage of Solid Radioactive Waste from January 1 to December 31 , 1993

CENTRAL WASTE COMPLEX 2403WD BUILDING, 200 WEST AREA, TRANSURANIC (NOT PROCESSED THROUGH TRUS

\begin{tabular}{|c|c|c|c|c|c|c|c|c|c|c|}
\hline$y$ & $\begin{array}{c}\text { Origin } \\
\text { of } \\
\text { Waste }\end{array}$ & $\begin{array}{l}\text { VOLUME } \\
\text { (CUBIC M.) }\end{array}$ & $\begin{array}{c}\text { PACKED } \\
\text { VOLLME } \\
\text { (CUBIC M.) }\end{array}$ & $\begin{array}{c}\text { PU } \\
\text { (GRAMS) }\end{array}$ & $\begin{array}{l}\text { URANIUH } \\
\text { (GRAMS) }\end{array}$ & $\begin{array}{l}\text { TOTAL } \\
\text { (CURIES) }\end{array}$ & $\begin{array}{l}\text { SR-90 } \\
\text { (CURIES) }\end{array}$ & $\begin{array}{c}\text { RU-106 } \\
\text { (CURIES) }\end{array}$ & $\begin{array}{c}\text { CS-137 } \\
\text { (CURIES) }\end{array}$ & $\begin{array}{c}\text { OTHER } \\
\text { (CURIES) }\end{array}$ \\
\hline
\end{tabular}
FLOOR LEVEL 1

WHC 23452

$3.956 E+00 \quad 3.956 E+00 \quad .000 E+00$

$.000 E+00$

$6.350 E-02$

$1.169 E \cdot 02$

$2.367 E-04 \quad 1.262 E-02$

$.000 E+00 \quad 4.164 E+02$ NH-241 4.530E-01 PU-238 1.1B1E-01 PU-239 3.901E+02

PU-240 2.361E+01 PU-241 1.901E+00 PU-242 7.540E-02

CS137 1.545E-05

CO-5B $1.500 E-05$

CO-60 1.005E-03

EU155 1.275E-04

C-14 1.500E-05

BA137 1.455E-05

EU154 1.275E-04

FE-59 6.000E-05

MN-54 9.050E-04 
SWIR3280 - (Page 82 of 99 )

RUN DATE: 08/20/94

Land Disposal or Storage of Solid Radioactive Waste from January 1 to December 31 , 1993

CENTRAI WASTE COMPLEX 2403WD BUILDING, 200 WEST AREA, TRANSURANIC WIPP CERTIFIED.

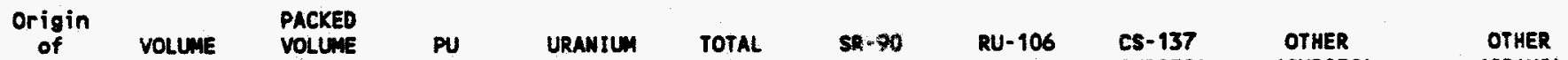
Company Haste (CUBIC M.) (CUBIC M.) (GRAMS) (GRAMS) (CURIES) (CURIES) (CURIES) (CURIES) (CURIES) (GRAMS)

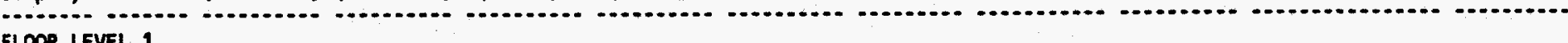

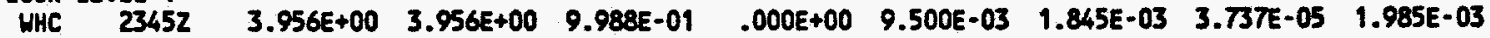

$.000 E+00 \quad 1.206 E+01$

AM-241 $1.552 E-02$

PU-238 3.607E-03

PU-239 1.128E+01

PU-240 6.866E-01

PU-261 5.502E-02

PU-242 2.140E-02

TOTALS

$\begin{array}{llllll}3.956 E+00 \quad 3.956 E+00 \quad 9.988 E-01 \quad .000 E+00 & 9.500 E-03 \quad 1.845 E-03 \quad 3.737 E-05 & 1.985 E-03\end{array}$

$.000 E+00 \quad 1.206 E+01$ 
WHC-EP-0125-6

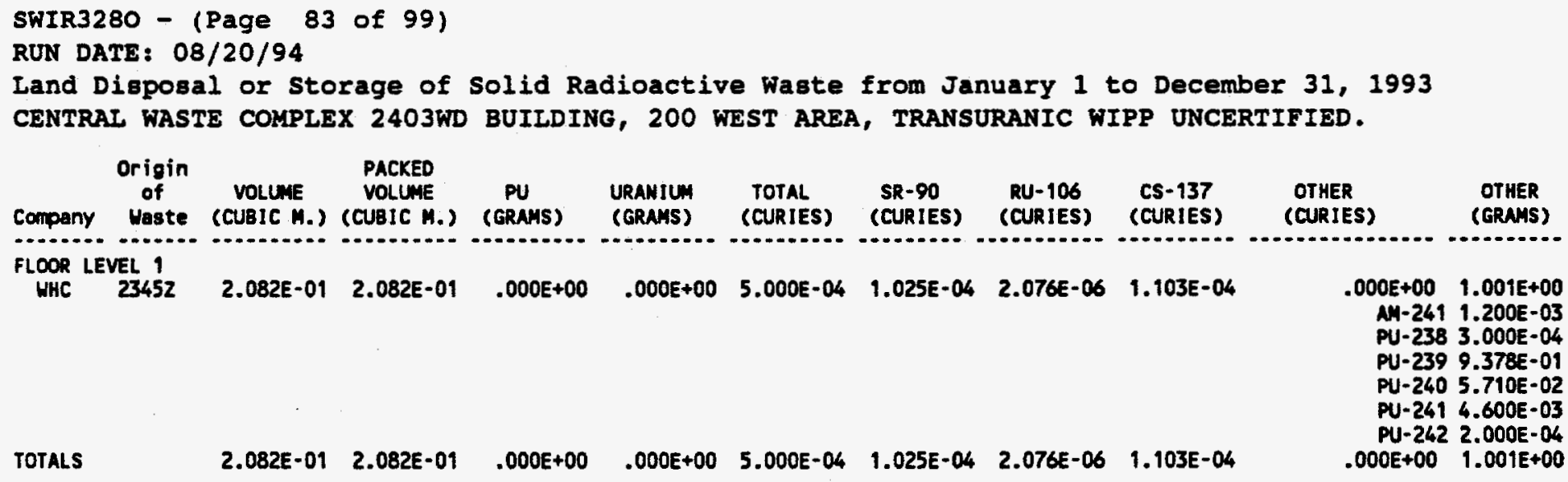


SWIR3280 - (Page 84 of 99)

RUN DATE: $08 / 20 / 94$

Land Disposal or storage of Solid Radioactive Waste from January 1 to December 31 , 1993 FLAMAABLE STORAGE MODULE 1, 200 WEST AREA, LOW-LEVEL NONINDUSTRIAL.

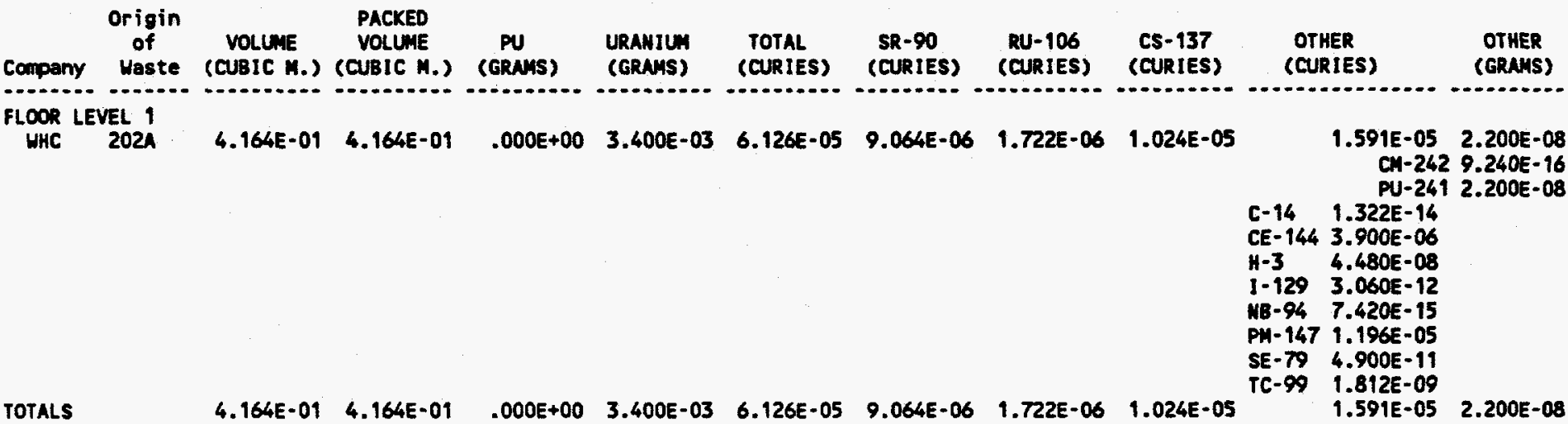


SWIR3280 - (Page 85 of 99)

RUN DATE: 08/20/94

Land Disposal or Storage of Solid Radioactive Waste from January 1 to December 31 , 1993

FLAMMABIE STORAGE MODULE 11, 200 WEST AREA, LOW-LEVEL NONINDUSTRIAL-OFFSITE.

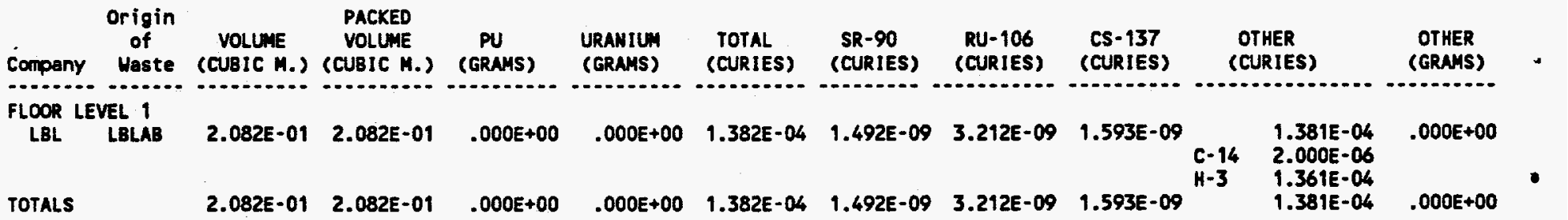


SWIR3280 - (Page 86 of 99)

RUN DATE: 08/20/94

Land Disposal or storage of Solid Radioactive Waate from January 1 to December 31 , 1993

FLAMABALE STORAGE MODULE 11, 200 WEST AREA, LOW-LEVEL NONINDUSTRIAL.

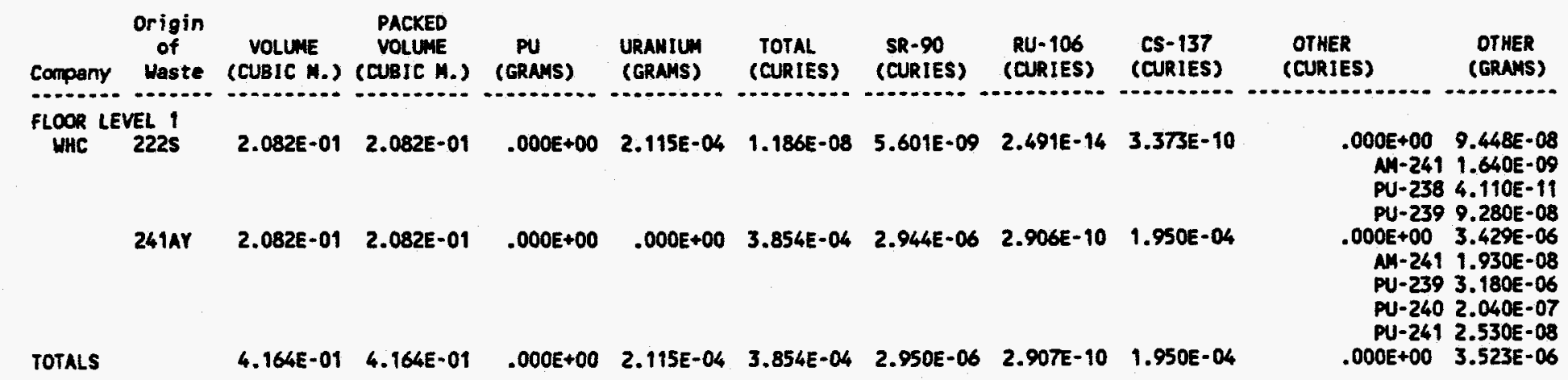


WHC-EP-0125-6

SWIR3280 - (Page 87 of 99)

RUN DATE: $08 / 20 / 94$

Land Disposal or storage of Solid Radioactive Waste from January 1 to December 31 , 1993

FLAMMABLE STORAGE MODULE 12, 200 WEST AREA, LON-LEVEL NONINDUSTRIAI-OFFSITE.

\begin{tabular}{|c|c|c|c|c|c|c|c|c|c|c|c|}
\hline & $\begin{array}{c}\text { Origin } \\
\text { of } \\
\text { Haste }\end{array}$ & $\begin{array}{l}\text { YOLUME } \\
\text { (CUBIC M.) }\end{array}$ & $\begin{array}{c}\text { PACKED } \\
\text { VOLUME } \\
\text { (CUBIC M.) }\end{array}$ & $\begin{array}{c}\text { PU } \\
\text { (GRAMS) }\end{array}$ & $\begin{array}{l}\text { URANIUM } \\
\text { (GRAMS) }\end{array}$ & $\begin{array}{l}\text { TOTAL } \\
\text { (CURIES) }\end{array}$ & $\begin{array}{c}\text { SR-90 } \\
\text { (CURIES) }\end{array}$ & $\begin{array}{c}\text { RU-106 } \\
\text { (CURIES) }\end{array}$ & $\begin{array}{c}\text { CS-137 } \\
\text { (CURIES) }\end{array}$ & $\begin{array}{l}\text { OTHER } \\
\text { (CURIES) }\end{array}$ & $\begin{array}{c}\text { OTHER } \\
\text { (GRAMS) }\end{array}$ \\
\hline B. & $\begin{array}{l}\text { VEL } \\
\text { BAPI }\end{array}$ & $\begin{array}{l}1.874 E+00 \\
1.874 E+00\end{array}$ & $\begin{array}{l}1.874 E+00 \\
1.874 E+00\end{array}$ & $\begin{array}{l}.000 E+00 \\
.000 E+00\end{array}$ & $\begin{array}{l}7.140 E-02 \\
7.140 E-02\end{array}$ & $\begin{array}{l}9.000 E-04 \\
9.000 E-04\end{array}$ & $\begin{array}{l}1.790 E-05 \\
1.790 E-05\end{array}$ & $\begin{array}{l}3.855 E-05 \\
3.855 E-05\end{array}$ & $\begin{array}{l}1.912 E-05 \\
1.912 E-05\end{array}$ & $\begin{array}{l}.000 E+00 \\
.000 E+00\end{array}$ & $\begin{array}{l}.000 E+00 \\
.000 E+00\end{array}$ \\
\hline
\end{tabular}


SWIR3280 - (Page 88 of 99 )

RUN DATE: $08 / 20 / 94$

Land Disposal or Storage of Solid Radioactive Waste from January 1 to December 31,1993

FLAMMABLE STORAGE MODULE 12, 200 WEST AREA, LOW-LEVEL NONINDUSTRIAL.

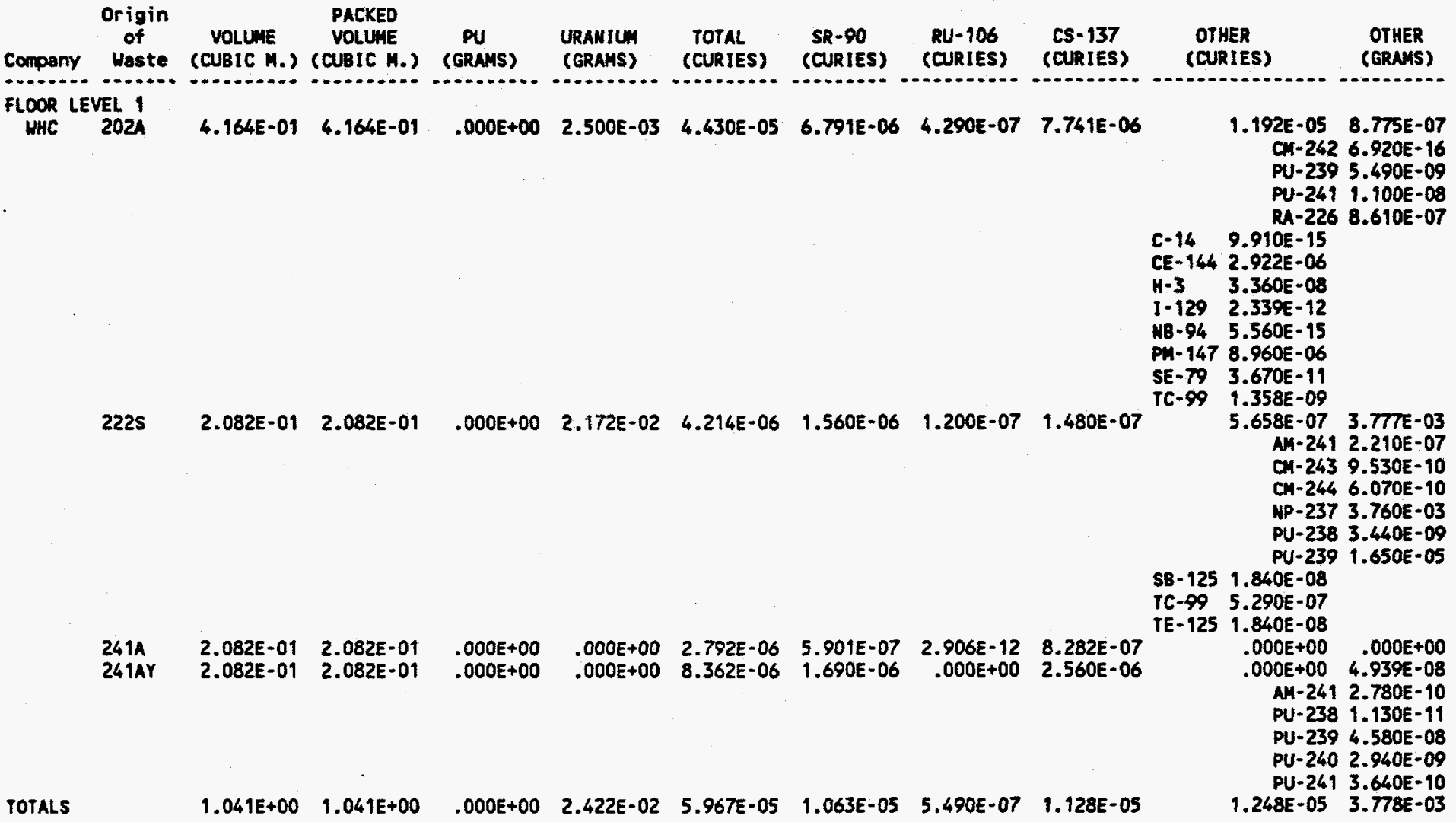


WHC-EP-0125-6

SWIR3280 - (Page 89 of 99)

RUN DATE: $08 / 20 / 94$

Land Disposal or Storage of Solid Radioactive Waste from January 1 to December 31,1993

FLAMGABLE STORAGE MODULE 13, 200 WEST AREA, LOW-LEVEL NONINDUSTRIAL-OFFSITE.

\begin{tabular}{|c|c|c|c|c|c|c|c|c|c|c|c|}
\hline $\begin{array}{c}\text { Origin } \\
\text { of } \\
\text { Company Waste }\end{array}$ & $\begin{array}{l}\text { VOLUME } \\
\text { (CUBIC M.) }\end{array}$ & $\begin{array}{l}\text { PACKED } \\
\text { VOLUME } \\
\text { (CUBIC M.) }\end{array}$ & $\begin{array}{c}\text { PU } \\
\text { (GRAMS) }\end{array}$ & $\begin{array}{l}\text { URANIUM } \\
\text { (GRAMS) }\end{array}$ & $\begin{array}{l}\text { TOTAL } \\
\text { (CURIES) }\end{array}$ & $\begin{array}{l}\text { SR-90 } \\
\text { (CURIES) }\end{array}$ & $\begin{array}{c}\text { RU-106 } \\
\text { (CURIES) }\end{array}$ & $\begin{array}{c}\text { CS- } 137 \\
\text { (CURIES) }\end{array}$ & & $\begin{array}{l}\text { HER } \\
\text { RIES) }\end{array}$ & $\begin{array}{c}\text { OTHER } \\
\text { (GRAMS) }\end{array}$ \\
\hline LOOR LEVEL 1 & & & & & & & & & & & \\
\hline TOTALS & $3.952 E+00$ & $\begin{array}{l}3.952 E+00 \\
3.952 E+00\end{array}$ & $.000 E+00$ & $.000 E+00$ & $2.602 E+00$ & $6.225 E-06$ & $1.341 E-05$ & $6.648 E-0.6$ & $c-14$ & $\begin{array}{l}2.601 E+00 \\
4.828 E-02 \\
2.553 E+00 \\
2.601 E+00\end{array}$ & $.000 E+00$ \\
\hline
\end{tabular}


SWIR3280 - (Page 90 of 99 )

RUN DATE: 08/20/94

Land Disposal or Storage of Solid Radioactive Waste from January 1 to December 31,1993

FLAMMABLE STORAGE MODULE 13, 200 WEST AREA, LOW-LEVEL NONINDUSTRIAL.

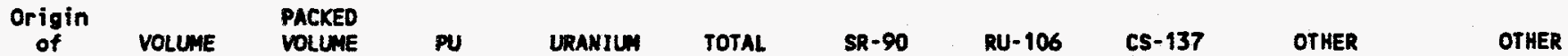

Compeny Haste (CUBIC M.) (CUBIC M.) (GRAMS) (GRAMS) (CURIES) (CURIES) (CURIES) (CURIES) (CURIES)

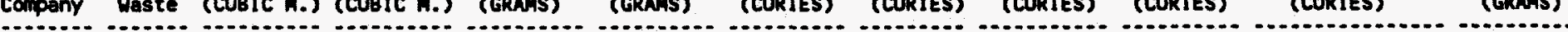

FLOOR LEVEL

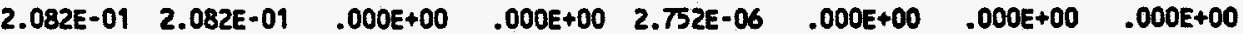

$\begin{array}{lll} & 2.752 E-06 & .000 E+00 \\ \text { CO-58 } & 6.100 E-07 & \\ \text { CO-60 } & 8.600 E-08 \\ \text { FE }-55 & 1.200 E-06 \\ \text { MN-54 } & 6.300 E-07 \\ \text { NI-60 } & 8.600 E-08 \\ \text { NI-63 } & 1.400 E-07\end{array}$

WHC $2225 \quad 6.082 E-01 \quad 6.082 E-01 \quad .000 E+00 \quad 1.588 E-02 \quad 1.543 E-05 \quad 3.653 E-06 \quad 3.350 E-12 \quad 4.036 E-06$

$\begin{array}{lll}.746 E-07 & 1.182 E-05 \\ M-241 & 5.620 E-07\end{array}$

PU-238 2.114E-09

EU-154 1.600E-07

PU-239 $1.125 E-05$

EU-155 3.530E-08

H-3 $\quad 1.347 \mathrm{E}-08$

$1-129 \quad 1.719 E-10$

PW-147 6.552E-08

TC-99 $1.331 E-10$

$23452 \quad 2.082 E-01 \quad 2.082 E-01 \quad .000 E+00 \quad .000 E+00 \quad 5.000 E-04 \quad 1.025 E-04 \quad 2.076 E-06 \quad 1.103 E-04$ $.000 E+00 \quad 8.968 E-06$ AM-241 1.010E-08 PU-238 2.620E-09 PU-239 8.400E-06 PU-240 5.120E-07 PU-241 4.160E-08 
WHC-EP-0125-6

SWIR3280 - (Page 91 of 99)

RUN DATE: $08 / 20 / 94$

Iand Disposal or storage of Solid Radioactive Waste from January 1 to December 31 , 1993

FLAMMABLE STORAGE MODULE 15, 200 WEST AREA, LOW-LEVEL NONINDUSTRIAL-OFFSITE.

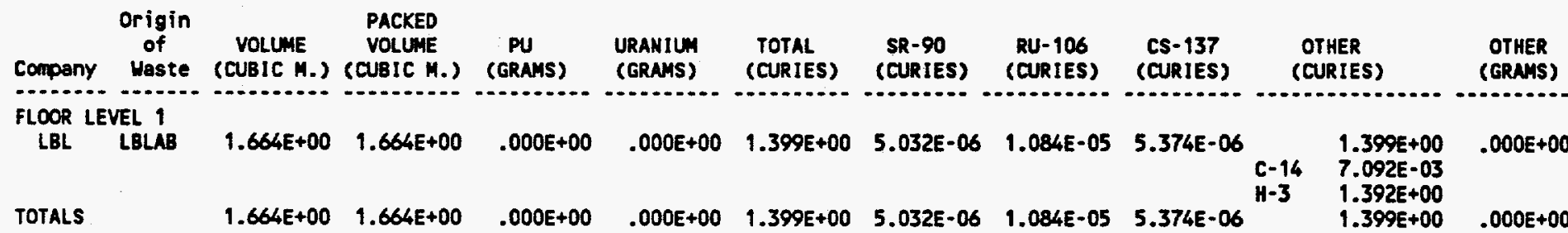


SWIR3280 - (Page 92 of 99 )

RUN DATE: $08 / 20 / 94$

Land Disposal or storage of Solid Radioactive Waste from January 1 to December 31,1993

FLAMMABLE STORAGE MODULE 15, 200 WEST AREA, LON-LEVEL NONINDUSTRIAL.

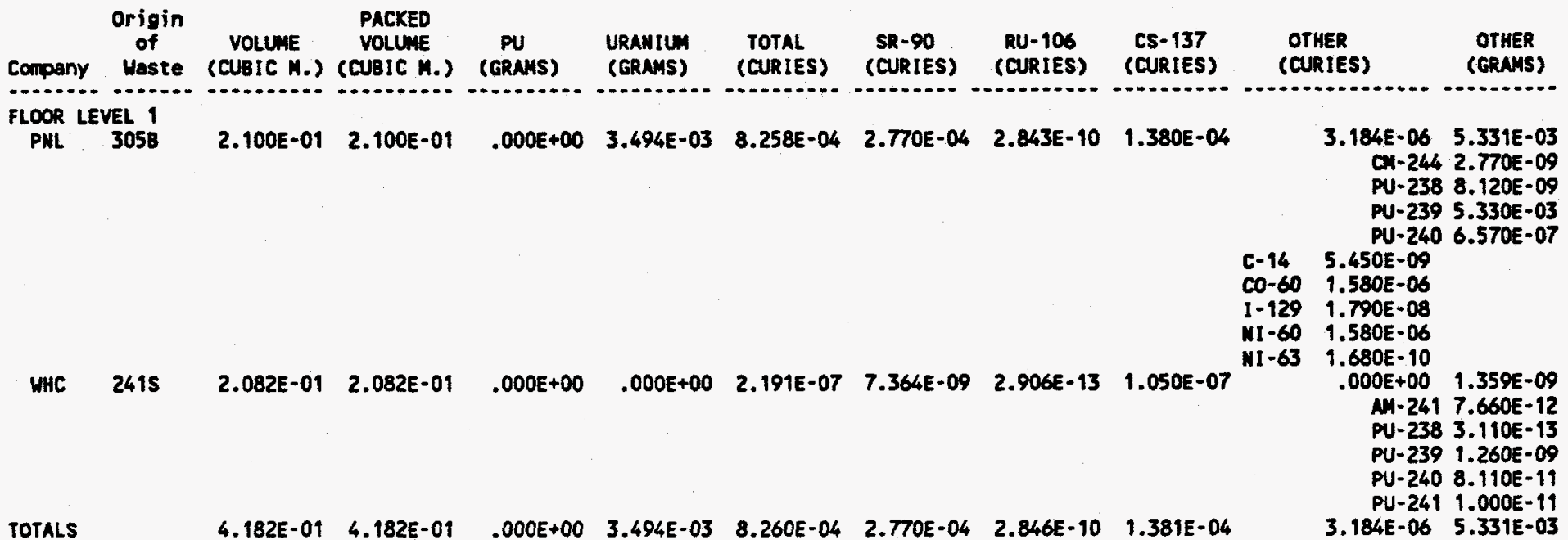


SWIR3280 - (Page 93 of 99)

RUN DATE: 08/20/94

Land Disposal or Storage of Solid Radioactive Waste from January 1 to December 31 , 1993

FLAMMABLE STORAGE MODULE 3, 200 WEST AREA, LOW-LEVEL NONINDUSTRIAL-OFFSITE.

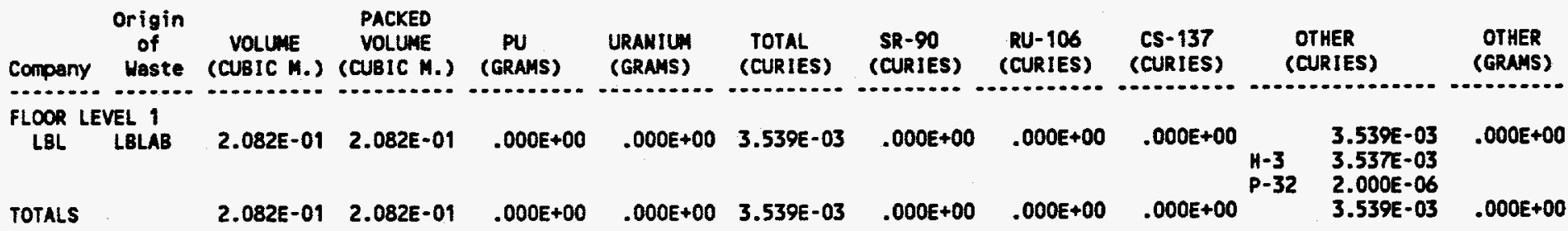


พHC-EP-0125-6

SWIR3280 - (Page 94 of 99 )

RUN DATE: $08 / 20 / 94$

Iand Disposal or Storage of Solid Radioactive waste from January 1 to December 31, 1993 FLAMMABLE STORAGE MODULE 3, 200 WEST AREA, LOW-LEVEL NONINDUSTRIAL.

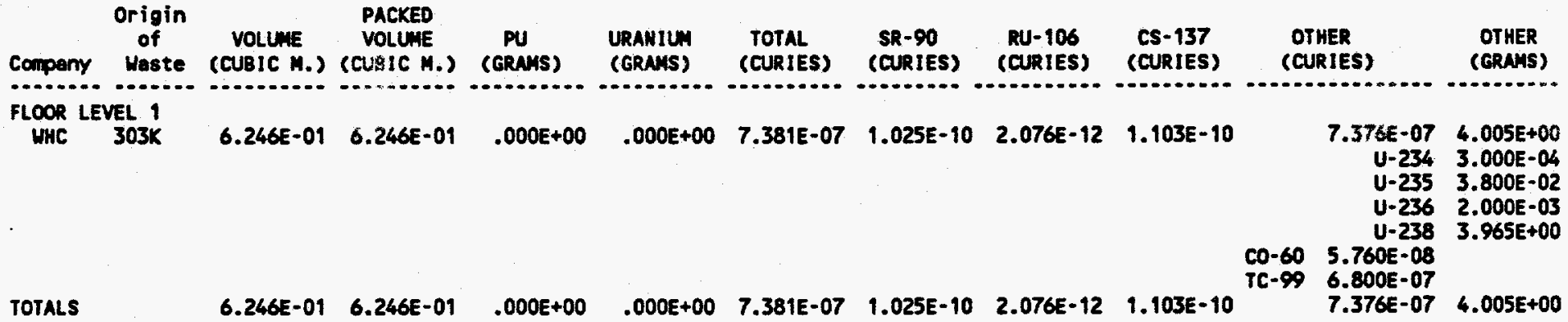


SWIR3280 - (Page 95 of 99 )

RUN DATE: $08 / 20 / 94$

Iand Disposal or Storage of Solid Radioactive Waste from January 1 to December 31 , 1993

FIAMAABLE STORAGE MODULE 6, 200 WEST AREA, IOW-LEVEL NONINDUSTRIAI.

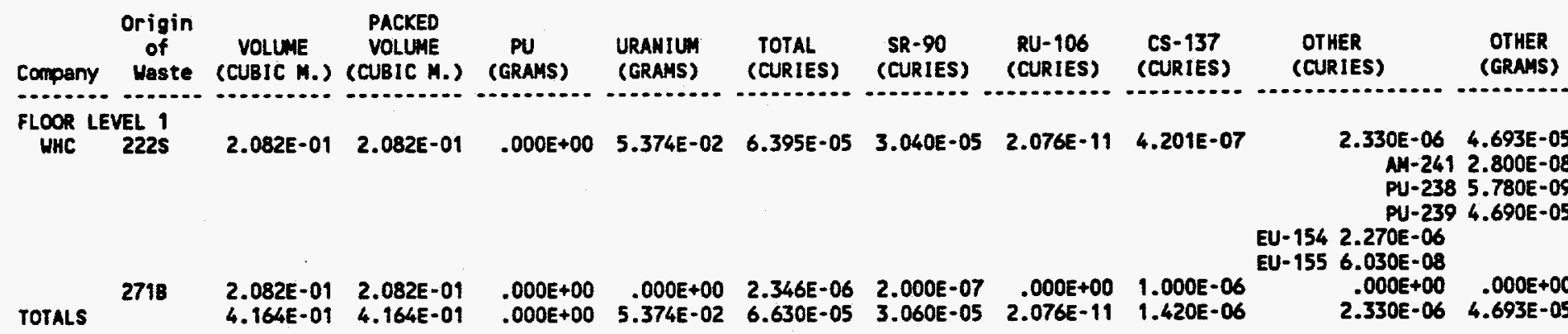


SWIR3280 - (Page 96 of 99 )

RUN DATE: $08 / 20 / 94$

Land Disposal or Storage of Solid Radioactive Waste from January 1 to December 31 , 1993 FLAMMABLE STORAGE MODULE 7, 200 WEST AREA, LOW-IEVEL NONINDUSTRIAL.

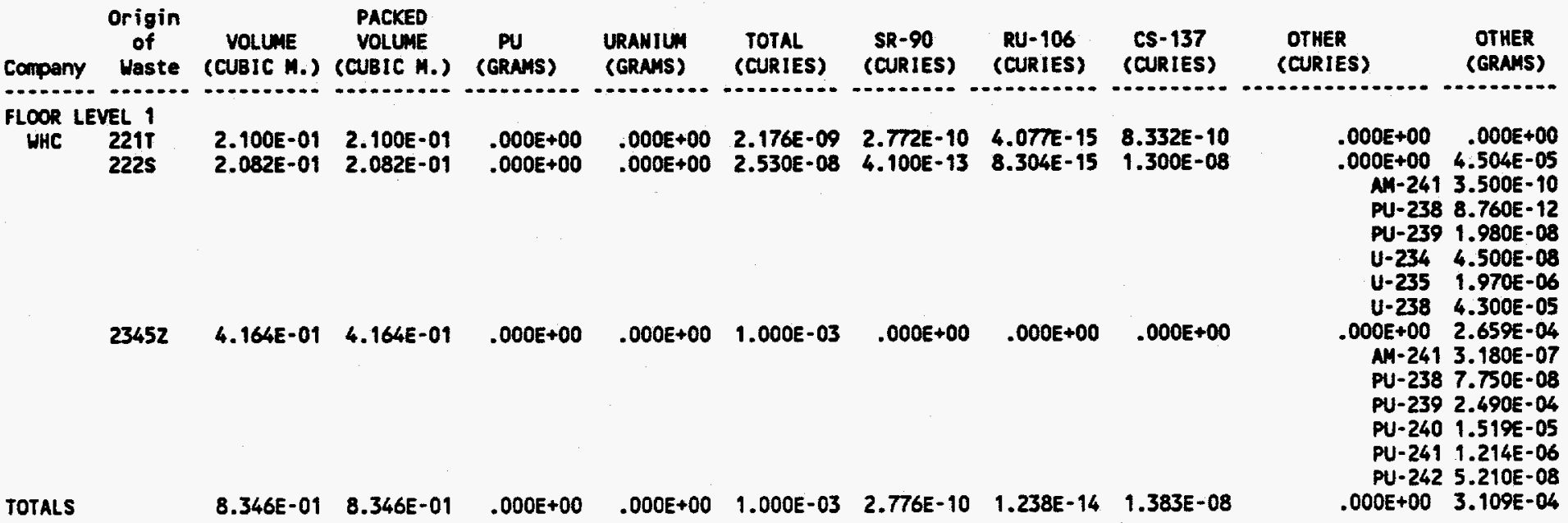


SWIR3280 - (Page 97 of 99 )

RUN DATE: $08 / 20 / 94$

Land Disposal or Storage of Solid Radioactive Waste from January 1 to December 31,1993

FLAMMABLE STORAGE MODULE 8, 200 WEST AREA, LOW-LEVEL NONINDUSTRIAL.

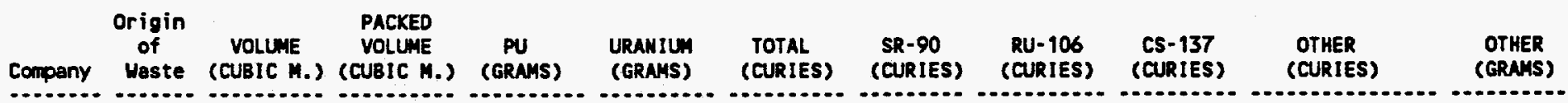

FLOOR LEVEL 1

WHC 2225

$2.000 E-01 \quad 2.000 E-01 . \quad .000 E+00 \quad 1.726 E-02 \quad 4.154 E-04 \quad 1.730 E-04 \quad 4.127 E-10 \quad 1.112 E-05$

4.770E-05 1.717E-03

NM-241 4.110E-06

NH-243 8.800E-05

CH-243 6.800E-08

MP- $2371.610 E-03$

PU-238 1.680E-09

PU-239 1.500E-05

$23452 \quad 2.080 E-01 \quad 2.080 E-01 \quad .000 E+00 \quad .000 E+00 \quad 5.000 E-04 \quad 1.025 E-04 \quad 2.076 E-06 \quad 1.103 E-04$

TC-99 4.770E-05

$.000 E+00 \quad 1.932 E-05$

AM-241 1.910E-08

PU-238 5.660E-09

PU-239 $1.810 E-05$

PU-240 1.100E-06

PU-2419.190E-08

PU-242 3.770E-09 


$$
\text { WHC-EP-0125-6 }
$$

SWIR3280 - (Page 98 of 99 )

RUN DATE: 08/20/94

Land Disposal or Storage of Solid Radioactive Waste from January 1 to December 31 , 1993 FLAMMABLE STORAGE MODULE 8, 200 WEST AREA, TRANSURANIC WIPP UNCERTIFIED.

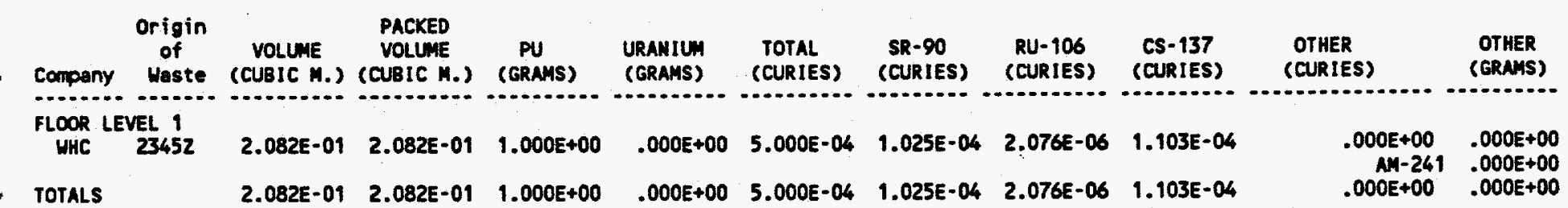


WHC-EP-0125-6

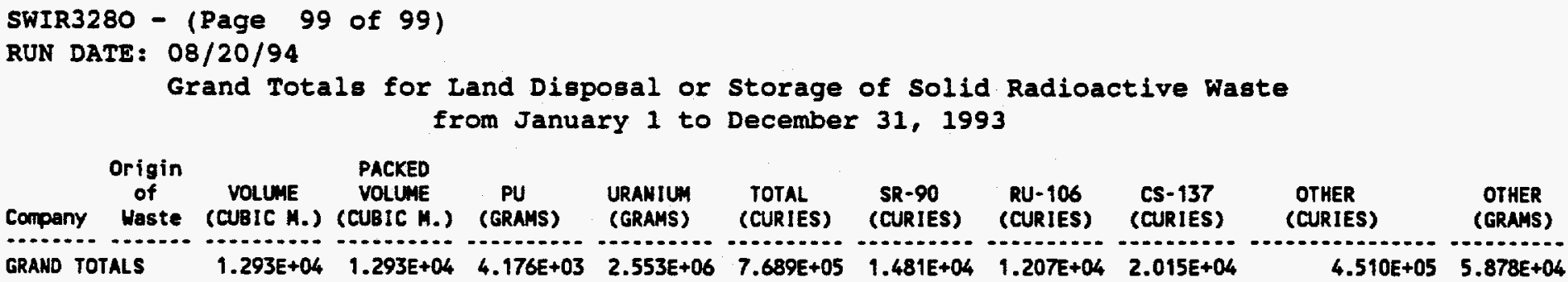




\section{REFHRHCES}

ARH, 1973, Sumary of Radioctive Solid Waste Burials in the 200 Areas During 1972, ARH-2757-part 2, Atlantic Richfield Hanford Company, Richland, Mashington.

ARH, 1975, Summary of Radioactive solid waste Burials in the 200 Areas During 1975, ARH-CD-369-4Q, Atlantlc Richfield Hanford company, Richland, Washington.

RHO, 1978, Summary of Radioactive Solid waste Burials in the 200 Areas During 1977. RHO-CD-27-30, Rockwell Hanford Operations, Richland, Washington.

RHO, 1979, Sumary of Radioactive Solid Waste Burials in the 200 Areas During 1978, RHO-LD-78-24-4Q, Rockwell Hanford Operations, Richland, Washington.

RHO, 1981a, Sumary of Radioactive Solid Waste Burials in the 200 Areas During 1980, RHO-LD-80-24-40, Rockwell Eanford Operations, Richland, Washington.

RHO, 1981b, Summary of Redioactive Solid Wate Burials in the 200 Areas During 1981, RHO-LD-81-24-40, Rockwell Hanford Operations, Richland, Washington.

RHO, 1986, Summary of Radioactive Solid Waste Burials in the 200 Areas During 1986, RHO-SR-86-9-40, Rockwell Hanford Operations, Richland, washington.

WHC, 1988, Hanford Site Solid Waste Acceptance Criteria, WHC-EP-0063, Westinghouse Hanford Company, Richland, Washington.

WHC, 1989, Summary of Radioactive Solid Waste Burials in the 200 Areas During CY 1987, WHC-rP-0125, Westinghouse Hanford Company, Richland, Washington.

WHC, 1989, Summary of Radioactive Solid Waste Received in the 200 Areas During Calendar Year 1988, WHC-EP-0125-1, Westinghouse Hanford Company, Richland, Washington.

WHC, 1990, Summary of Radioactive Solid waste Received in the 200 Areas During Calendar Year 1989, WHC-EP-0125-2, Westinghouse Hanford Company, Richland, Washington.

WHC, 1991, Summary of Radioactive solid Waste Received in the 200 Areas During Calendar Year 1990, WHC-EP-0125-3, Westinghouse Hanford Company, Richland, Washington.

WHC, 1992, Summary of Radioactive Solid Waste Received in the 200 Areas During Calendar Year 1991, WHC-EP-0125-4, Weatinghouse Hanford Company, Richland, Washington.

WHC, 1993, Summary of Radioactive Solid Waste Received in the 200 Areas During Calendar Year 1992, WHC-EP-0125-5, Weatinghouse Hanford Company, Richland, Washington.

wiley and Sons, 1978, Table of Isotopes, Seventh Edition, John wiley and Sons, New York, New York. 
WHC-EP-0125-6

This page intentionally left blank. 
WHC-EP-0125-6

APPETDIX $\boldsymbol{A}$

200 RRMA BURIAI GROURDS

\section{BURIAL GROULDS \\ 200 EAST ABNB}

Burial Ground 218E1

Burial Ground $218 \mathrm{E} 2$

Burial Ground 218E4 (MINOR CONSTR \#4)

Burial Ground 218Es

Burial Ground 218E5A

Burial Ground 218E8 (CONSTRUCTION $* 8$ )

Burial Ground 218EC9

Burial Ground $218 \mathrm{E} 10$

Burial Ground 218E12A
N39821/ $/ 49847$ NORTHWEST N39817/ W49494 NORTHIAST N39532 / W49850 SOUTHWEST N39526 / W49494 SOUTHEAST

N44826 / W53909 NORTHWEST N44827 / W53425 NORTFEAST N44407/W53426 SOUTHEAST N44408 / $\$ 53911$ SOUTHWEST

N44313 / $\$ 54110$ NORTHWEST N43783/ W53503 NORTHEAST N43685 / \$53677 SOUTHEAST

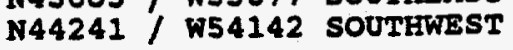

N44826 / W54209 NORTHWEST N44826 / W53909 NORTHEAST N44408/ N53911 SOUTHEAST N44407 / W54165 SOUTHWEST

N44827 / $\$ 54309$ NORTHWEST N44826 / W54209 NORTHEAST N44495, W54164 SOUTHEAST N44494/W54309 SOUTHWEST

N45285/ W48527 NORTHWEST N45281/ W48409 NORTHEAST N44882 / W48418 SOUTHEAST N44885/ W48534 SOUTHWEST

N42717 / W50106 NORTHWEST N42716 / W49855 NORTHEAST N42471/ W49857 SOUTHEAST N42473 / W50108 SOUTHWEST

N45880 / W56725 NORTHWEST N45880 / W54700 NORTHEAST N44398/ $\$ 54660$ SOUTHEAST N44473, W55475 SOUTH N45180 / W56725 SOUTHWEST

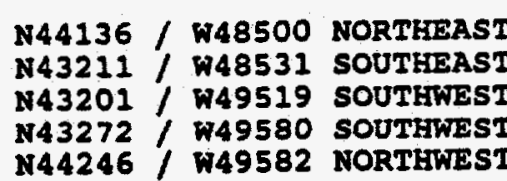

REFERHTCE DRAKINGS

H-2-124

REV. 3

H-2-55534

REV. 16

H-2-55534

REV. 16

H $-2-55534$

REV. 16

$H-2-55534$

REV. 16

$\mathrm{H}-2-33276$

SH 1 REV. 10
$\mathrm{H}-2-92004$

SH 1 REV. 2

SH 2 REV. 1

H-2-32560

REV. 2 
WHC-EP-0125-6

APPEADIX A

200 AREA BURIAT GROUNDS

REFERENCE

BURIAL GROUNDS

COORDIRTAMES

DRAWINGS

200 EAST AREA

Burial Ground 218E12B

N46775 / W51475 NORTHWEST

N46775 / W47445 NORTHEAST

N46675 / W47345 NORTHEAST

N45523 / \$47345 SOUTHEAST

N45485 / W48523 SOUTHEAST

N44485 / W48542 SOUTHEAST

N44504 / W49661 SOUTHAEST

N45220 / $\$ 51475$ SOUTHKEST

218E14

(PUREX TUNNEL $\# 1$ )

N39196 / W48020 NORTH

N39555 / W48020 SOUTH

N37670 / W47960 NORTH

218E15

(PUREX TUNNEL \#2)

N39366 / N47960 SOUTH

222B VAULTS

CENTER POINT

(DRY WASTE E7)

N42269 / W53826 VAULT 1

N42269 / W53806 VAULT 2

N43364 / W53776 VAULT 3

H-2-33276

SH 1 REV. 10

SH 2 REV. 3

SH 3 REV. 3

200 WEST AREA

Burial Ground 218 w

N42099 / W77979 NORTHWEST N42099 / W77458 NORTHEAST N41641 / W77458 SOUTHEAST

N41641 / W77979 SOUTHWEST

Burial Ground 218พ1A

N45464 / W74930 NORTH N45114 \%74431 NORTHEAST N45072/ $/ 74460$ NORTHEAST N45053 / W74668 NORTHEAST N44597 / W74844 SOUTHEAST N44624 / W75113 SOUTHWEST N44781 / W75175 SOUTHWEST N45102 / W75209 NORTHWEST

Burial Ground 218 w2

N41641 / W77979 NORTHWEST N41641/W77458 NORTHEAST N41052 / W77458 SOUTHEAST N41052/ /77979 SOUTHWEST

$H-2-75149$

REV. 0

H-2-2516

REV. 3

$\mathrm{H}-2-2503$

REV. 2 
WHC-EP-0125-6

APPEADIX $A$

200 MRE BURIXI eRound

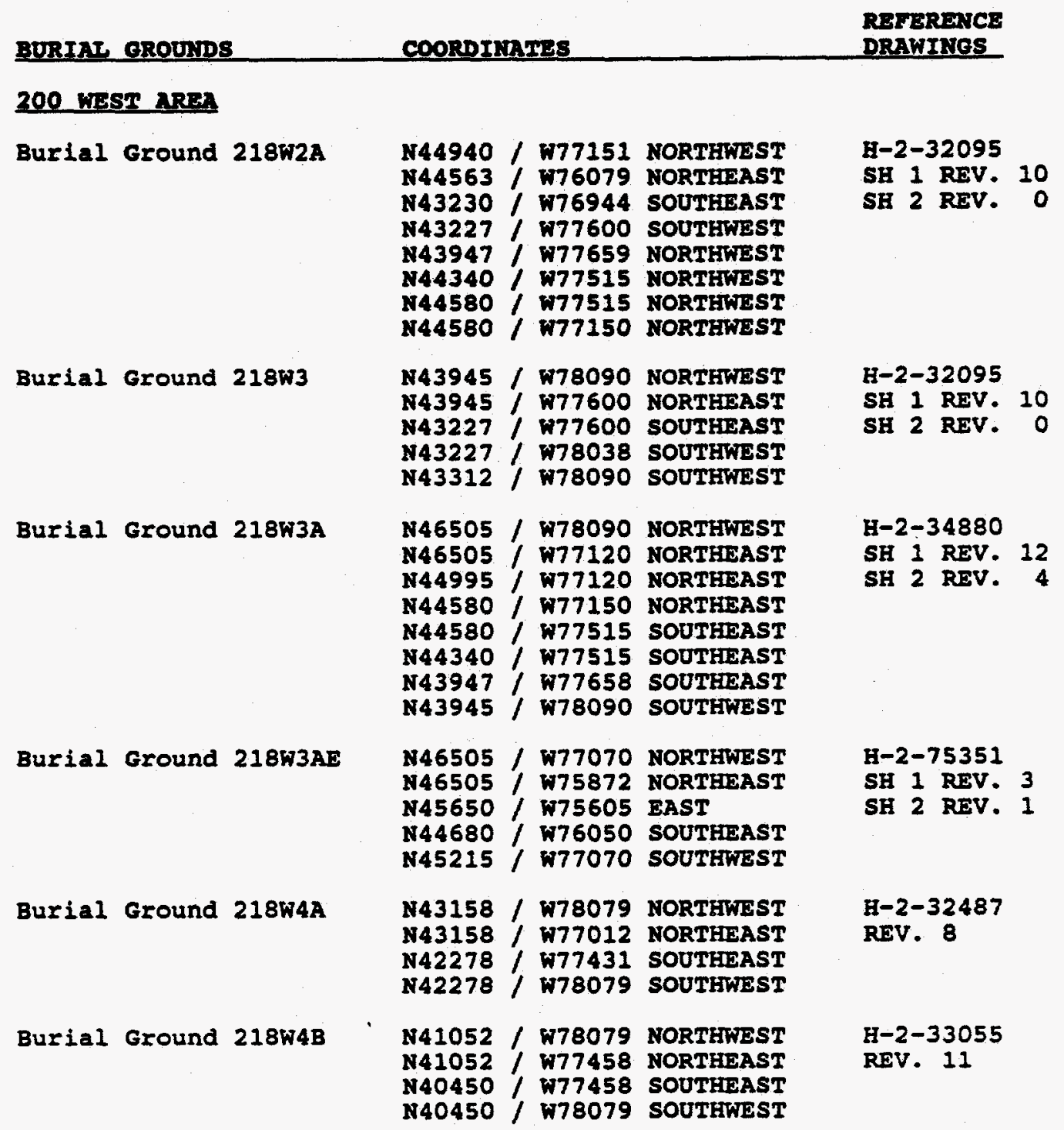


WHC-EP-0125-6

APPEATIX A

200 MREA BURIAI GROUIDS

BURIAL OROUIDS

coORDINATES

REFERENCE DRAWTNGS

\section{WEST ABEA}

Burial Ground 218พ4C

Burial Ground $218 \% 5$

Burial Ground 218w9

$222 T$ VAULT

(DRY WASTE W8)

$222 S$ VAULT

(DRY WASTE W7)

212N Building

212P Building

$224 \mathrm{~T}$ Building

(TRUSAF)

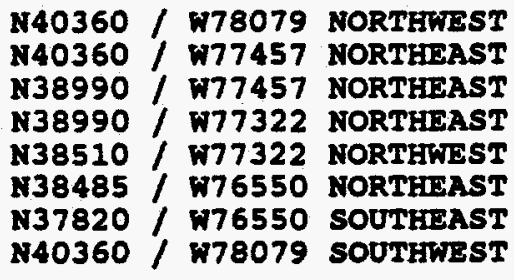

N46535 / W79450 NORTHWEST N46480 / W78250 NORTHIAST 1143270 / K78250 SOUTHEAST N43270 / H79145 SOUTHWEST N44570 / W79450 NORTHWEST

N35647 / W74556 NORTHWEST N35648 / N74458 NORTHEAST N35511/ $/ 74458$ SOUTHEAST N35508 / W74555 SOUTHWEST

N42278 / W77979 NORTHWEST N42278 / W77458 NORTHEAST N42099 / W77458 SOUTHEAST N42099 / W77979 SOUTHWEST

N43721 / $\$ 73007$ NORTHWEST N43696 / W72972 NORTHEAST N43630 / W73019 SOUTHEAST N43655 / $\$ 73054$ SOUTHWEST

N34129/ W73540 CENTER PT.

N55800

NORTH

พ65500

WEST

N55800

พ62800

NORTH

WEST

$N 43550$

พ73350

NORTH

WEST
$\mathrm{E}-2-37437$ SE 1 REV. 7 SE 2 REV. 1 SH 3 REV. 0

$\mathrm{H}-2-94677$ SE 1 REV. 2 SH 2 REV. 1

H-2-94261 REV. 0

$\mathrm{H}-2-94250$

RSV. 0

H-2-2322

REV. 3

H-2 -5170

REV. 4

H-2-32307 H-2-32524

พ-71323

พ-71324

พ-71325

H-2-36215

SK-2-62470 
WEC-EP-0125-6

APPEATI

200 are BURIN GROODDs

\begin{tabular}{|c|c|c|}
\hline RIAI, GROUNDS & coospriatifs & DRAKI \\
\hline 213W COMPACTOR & $N 40608$ / w78722 & $\mathrm{H}-2-93691$ \\
\hline Building 2401W & N40716 / พ78515 & $\mathrm{H}-2-80543$ \\
\hline Building 2402W & N41310 / W78530 & $\mathrm{H}-2-80605$ \\
\hline Building 2402\%B & $N 41310$ / & H-2-80737 \\
\hline Building 2402wC & N41410 / W78530 & $\mathrm{H}-2-80737$ \\
\hline Building $2402 \mathrm{kD}$ & N41410 / W78690 & $\mathrm{H}-2-80737$ \\
\hline Building $2402 \mathrm{WE}$ & N41510 / พ78530 & $\mathrm{E}-2-80737$ \\
\hline Building 2402WF & N41510 / W78690 & $\mathrm{H}-2-80737$ \\
\hline Building 2402WG & N41610 / W78530 & $\mathrm{H}-2-80737$ \\
\hline Building 2402\%H & N41610 / W78690 & $\mathrm{H}-2-80737$ \\
\hline Building 2402WI & $N 41710 / \$ 78530$ & $\mathrm{H}-2-80737$ \\
\hline Building $2402 \mathrm{wJ}$ & N41710 / & $\mathrm{H}-2-80737$ \\
\hline Buflding 2402WK & N41810 / พ78530 & $\mathrm{H}-2-80737$ \\
\hline $\begin{array}{l}\text { FLAROMABLE STORAGE } \\
\text { MODULES }\end{array}$ & $N 40900 /$ / 678700 & H-2-81296 \\
\hline $\begin{array}{l}\text { MIXED WASTE } \\
\text { STORAGE PAD }\end{array}$ & N41560 / w78250 & $\mathrm{H}-2-80580$ \\
\hline STAGING AREA & $\begin{array}{l}\text { N41310 / } \$ 78400 \\
\text { N41510 / }\end{array}$ & H-2-90264 \\
\hline
\end{tabular}


WHC-EP-0125-6

This page intentionally left blank. 


\section{APPETDI}

Solid waste Management Facilities Acreage Report Disposed and stored Acres in the 200 Areas from startup to December 31, 1993 Includes All Waste Received

\begin{tabular}{|c|c|c|c|c|}
\hline Burial ground & $\begin{array}{l}\text { Total } \\
\text { acres }\end{array}$ & $\begin{array}{c}\text { Total } \\
\text { acree ueed }\end{array}$ & $\begin{array}{l}\text { Acres } \\
\text { remaining }\end{array}$ & $\begin{array}{l}\text { Acres used } \\
\text { CY } 1993\end{array}$ \\
\hline $218-E-01$ & 2.38 & 2.38 & 0 & 0 \\
\hline $218-\mathrm{E}-02,-2 \mathrm{~A},-9$ & 5.87 & 5.87 & 0 & 0 \\
\hline $218-E-4$ & 2.75 & 2.75 & 0 & 0 \\
\hline $218-E-5$ & 2.31 & 2.31 & 0 & 0 \\
\hline $218-\mathrm{B}-5 \mathrm{~A}$ & 1.11 & 1.11 & 0 & 0 \\
\hline $218-E-7$ & 0.05 & 0.05 & 0 & 0 \\
\hline $218-E-8$ & 1.15 & 1.15 & 0 & 0 \\
\hline $218-z-010$ & 56.64 & 40.30 & 16.34 & 0.49 \\
\hline $218-E-10 B$ & 32.54 & 0 & 32.54 & 0 \\
\hline $218-E-12 A$ & 24.56 & 24.56 & 0 & 0 \\
\hline $218-\mathrm{E}-12 \mathrm{~B}$ & 169.80 & 39.19 & 130.61 & 1.83 \\
\hline $218-E-14$ & 0.50 & 0.50 & 0 & 0 \\
\hline $218-E-15$ & 2.34 & 2.34 & 0 & 0 \\
\hline $218-E-C 9$ & 4.20 & 4.20 & 0 & 0 \\
\hline subtotal (Bast) & 306.21 & 126.71 & 179.49 & 2,32 \\
\hline 218 W-01 & 5.48 & 5.48 & 0 & 0 \\
\hline $218-W-01 A$ & 8.31 & 8.31 & 0 & 0 \\
\hline $218-W-2$ & 7.04 & 7.04 & 0 & 0 \\
\hline $218-W-3$ & 8.01 & 8.01 & 0 & 0 \\
\hline $218-W-2 A$ & 39.76 & 39.76 & 0 & 0 \\
\hline $218-n-3 A$ & 50.3 & 46.52 & 3.78 & 0.46 \\
\hline $218-W-3 A 2$ & 49.4 & 7.12 & 39.38 & 0.33 \\
\hline $218-W-04 A$ & 17.03 & 17.03 & 0 & 0 \\
\hline $218-W-04 B$ & 8.58 & 8.58 & 0 & 0 \\
\hline $218-n-04 C$ & 51.7 & 10.14 & 41.56 & 0 \\
\hline $218-W-05$ & 84.00 & 13.00 & 69.33 & 1.18 \\
\hline $218-W-06$ & 44.50 & 0 & 44.50 & 0 \\
\hline $218-n-07$ & 0.01 & 0.01 & $\mathbf{0}$ & 0 \\
\hline $218-N-08$ & 0.08 & 0.08 & 0 & 0 \\
\hline 218-N-09 & 0.31 & 0.31 & 0 & 0 \\
\hline
\end{tabular}


WHC-EP-0125-6

IPPETIX $\mathbf{B}$

Solid Waste Management Facilities Acreage Report Disposed and stored Acres in the 200 Areas from startup to December 31, 1993 Includes All waste Received

\begin{tabular}{|c|c|c|c|c|}
\hline Burial ground & $\begin{array}{l}\text { Total } \\
\text { acree }\end{array}$ & $\begin{array}{c}\text { Total } \\
\text { ecres used }\end{array}$ & $\begin{array}{c}\text { Acres } \\
\text { remaining }\end{array}$ & $\begin{array}{l}\text { Acres used } \\
\text { CY } 1993\end{array}$ \\
\hline $218-W-11$ & 2.14 & 2.14 & 0 & 0 \\
\hline 212-N Building & 0.36 & 0.36 & 0 & 0 \\
\hline 224-T Building & 0.14 & 0.14 & 0 & 0 \\
\hline Cwc Facilities & 100.00 & 100.00 & 0 & 0 \\
\hline subtots $($ treet) & 47,15 & 27003 & 198,55 & 1.97 \\
\hline Grand total & 783.36 & 400,74 & 378.04 & 4.29 \\
\hline
\end{tabular}


WEC-EP-0125-6

\section{APPETDIX}

Post-1970 Segregated Nontransuranic and Traneuranic Acres Ueed through

necember 31, 1993 within

the Burial Grounds

\begin{tabular}{|c|c|c|c|}
\hline $\begin{array}{l}\text { Burial } \\
\text { ground }\end{array}$ & Waste type & $\begin{array}{c}\text { square } \\
\text { foet }\end{array}$ & Used acree \\
\hline \multirow[t]{4}{*}{$218-B-12 B$} & TRE & $2.048 \mathrm{E}+04$ & 0.47 \\
\hline & UNSEG TRU & $4.078 \mathrm{E}+05$ & 9.36 \\
\hline & IIN & $1.279 \mathrm{E}+06$ & 29.36 \\
\hline & TOTAL & $1.707 \mathrm{E}+06$ & 39.19 \\
\hline \multirow[t]{4}{*}{$218-W-03 A$} & TRU & $1.671 \mathrm{E}+05$ & 3.84 \\
\hline & UNSEG TRU & $1.952 \mathrm{E}+04$ & 0.45 \\
\hline & LLW & $1.839 \mathrm{E}+06$ & 42.23 \\
\hline & TOTAL & $2.026 E+06$ & 46.52 \\
\hline \multirow[t]{6}{*}{$218-W-04 B$} & TRU & $4.968 \mathrm{z}+04$ & 1.14 \\
\hline & TRU CAIS & $1.728 E+04$ & 0.39 \\
\hline & ILW & $3.348 E+03$ & 0.77 \\
\hline & $\begin{array}{l}\text { UNSEG TRU } \\
\text { CAIS }\end{array}$ & $1.134 \mathrm{E}+04$ & 0.26 \\
\hline & UNSEG TRU & $2.621 \mathrm{E}+04$ & 6.02 \\
\hline & TOTAL & $3.738 \quad E+05$ & 8.58 \\
\hline \multirow[t]{3}{*}{$218-W-04 C$} & TRU & $2.390 \mathrm{E}+05$ & 5.49 \\
\hline & ILW & $2.026 \varepsilon+05$ & 4.65 \\
\hline & Torat & $4.4178+05$ & 10.14 \\
\hline
\end{tabular}


พHC-EP-0125-6

\section{APPEADIX $\mathbf{B}$}

Solid Waste Operatione Storage Facilitiea storage status as of December 31, 1993

\begin{tabular}{|c|c|c|c|c|c|}
\hline $\begin{array}{c}\text { Building } \\
\text { number }\end{array}$ & $\begin{array}{c}\text { Square } \\
\text { feet }\end{array}$ & $\begin{array}{l}\text { Total } \\
\text { acres }\end{array}$ & $\begin{array}{l}\text { storage* } \\
\text { capacity }\end{array}$ & $\begin{array}{l}\text { storage* } \\
\text { capacity } \\
\text { used }\end{array}$ & $\begin{array}{l}\text { storage* } \\
\text { capacity } \\
\text { remaining }\end{array}$ \\
\hline $212 N$ & $1.576 \mathrm{E}+04$ & 0.36 & 1,032 & 1,032 & 0 \\
\hline $224 T$ & $5.9105+03$ & 0.14 & 1,750 & 1,199 & 551 \\
\hline $2401 \%$ & $4.000 \mathrm{E}+03$ & 0.09 & 1,000 & 577 & 423 \\
\hline $2402 W$ & $4.000 \mathrm{E}+03$ & 0.09 & 1,000 & 873 & 127 \\
\hline $2402 \mathrm{WB}$ & $4.000 \mathrm{E}+03$ & 0.09 & 1,000 & 610 & 390 \\
\hline $2402 \% C$ & $4.000 \mathrm{E}+03$ & 0.09 & 1,000 & 281 & 719 \\
\hline $2402 \mathrm{FD}$ & $4.000 \mathrm{E}+03$ & 0.09 & 1,000 & 918 & 82 \\
\hline $2402 \mathrm{WE}$ & $4.0002+03$ & 0.09 & 1,000 & 12 & 988 \\
\hline $2402 \mathrm{HF}$ & $4.000 \mathrm{E}+03$ & 0.09 & 1,000 & 1,051 & 0 \\
\hline 2402WG & $4.000 \mathrm{E}+03$ & 0.09 & 1,000 & 549 & 451 \\
\hline 2402WH & $4.000 \mathrm{E}+03$ & 0.09 & 1,000 & 128 & 872 \\
\hline $2402 \mathrm{WI}$ & $4.000 \mathrm{E}+03$ & 0.09 & 1,000 & 1,015 & 0 \\
\hline 2402 พJ & $4.0002+03$ & 0.09 & 1,000 & 907 & 93 \\
\hline $2402 \mathrm{WK}$ & $4.000 \mathrm{E}+03$ & 0.09 & 1,000 & 0 & 1,000 \\
\hline $2402 \mathrm{WL}$ & $4.000 \mathrm{E}+03$ & 0.09 & 1,000 & 306 & 694 \\
\hline $2403 \% A$ & $3.400 E+04$ & 0.78 & 11,000 & 8,345 & 2,655 \\
\hline $2403 \mathrm{WB}$ & $3.400 \mathrm{E}+04$ & 0.78 & 11,000 & 1,295 & 9,705 \\
\hline 2403 HC & $3.400 \mathrm{E}+04$ & 0.78 & 11,000 & 2,515 & 8,485 \\
\hline $3403 \% D$ & $5.525 \mathrm{E}+04$ & 1.27 & 15,500 & 2,479 & 13,021 \\
\hline ES 1 & $1.509 \mathrm{E}+02$ & 0.003 & 30 & 17 & 13 \\
\hline FS 2 & $1.600 \mathrm{E}+02$ & 0.004 & 30 & 17 & 13 \\
\hline FS 3 & $1.965 \mathrm{E}+02$ & 0.005 & 30 & 19 & 11 \\
\hline FS 4 & $2.289 E+02$ & 0.005 & 30 & 30 & 0 \\
\hline FS 5 & $2.400 \mathrm{E}+02$ & 0.006 & 30 & 36 & 0 \\
\hline FS 6 & $4.800 \mathrm{E}+02$ & 0.011 & 30 & 26 & 4 \\
\hline FS 7 & $1.953 \mathrm{E}+02$ & 0.005 & 30 & 13 & 17 \\
\hline FS 8 & $2.070 \mathrm{E}+02$ & 0.005 & 30 & 20 & 10 \\
\hline Fs 9 & $1.411 \mathrm{E}+02$ & 0.003 & 30 & 0 & 30 \\
\hline FS 10 & $3.200 \mathrm{E}+02$ & 0.007 & 30 & 25 & 5 \\
\hline FS 11 & $1.411 \mathrm{E}+02$ & 0.003 & 30 & 8 & 22 \\
\hline FS 12 & $3.200 B+02$ & 0.007 & 30 & 35 & 0 \\
\hline
\end{tabular}


WHC-EP-0125-6

\section{APPENDIX}

Solid Waste Operation. Storage Facilities storage Status ac of December 31, 1993

\begin{tabular}{|c|c|c|c|c|c|}
\hline $\begin{array}{l}\text { Building } \\
\text { number }\end{array}$ & $\begin{array}{l}\text { square } \\
\text { feet }\end{array}$ & $\begin{array}{l}\text { Total } \\
\text { acres }\end{array}$ & $\begin{array}{l}\text { storage* } \\
\text { capacity }\end{array}$ & $\begin{array}{c}\text { storage* } \\
\text { capacity } \\
\text { uad }\end{array}$ & $\begin{array}{l}\text { storage* } \\
\text { capacity } \\
\text { remaining }\end{array}$ \\
\hline ES 13 & $1.965 \mathrm{E}+02$ & 0.005 & 30 & 0 & 30 \\
\hline Fs 14 & $1.965 \mathrm{E}+02$ & 0.005 & 30 & 0 & 30 \\
\hline FS 15 & $1.965 \mathrm{E}+02$ & 0.005 & 30 & 0 & 30 \\
\hline Is 16 & $1.965 \mathrm{~B}+02$ & 0.005 & 30 & 0 & 30 \\
\hline Is 17 & $1.965 \mathrm{E}+02$ & 0.005 & 30 & 0 & 30 \\
\hline Fs 18 & $1.965 \mathrm{E}+02$ & 0.005 & 30 & 0 & 30 \\
\hline Fs 19 & $1.411 \mathrm{E}+02$ & 0.003 & 30 & 0 & 30 \\
\hline FS 20 & $1.965 \mathrm{E}+02$ & 0.005 & 30 & 0 & 30 \\
\hline AMW 1 & $2.299 E+02$ & 0.005 & 30 & 0 & 30 \\
\hline ANW 2 & $2.299 \mathrm{E}+02$ & 0.005 & 30 & 24 & 6 \\
\hline AMW 3 & $2.299 \mathrm{E}+02$ & 0.005 & 30 & 0 & 30 \\
\hline AMW 4 & $2.299 E+02$ & 0.005 & 30 & 21 & 9 \\
\hline Total & $2,3611+05$ & 5,40 & 65,002 & 24,383 & 40,696 \\
\hline
\end{tabular}

* Number of 55-galion drum equivalents. 
WHC-EP-0125-6

This page intentionally left blank. 
WHC-EP-0125-6

\section{DIgIYRIBuryox}

Number of Copies

OPTSITE

1

4

ONSITE

7
0.S. Department of Fneroy, Beadquarterp

Germantown, Maryland

R. Hartinez, EM-322

U.S. Department of Eneray, Rlchland Operations office
D. W. Clauesen
R. M. Gordon
R. E. Guercia (3)
R. D. Izatt
Public Reading Room
S7 -55
S7-55
S7-55
A7 -50
H2-52

Pacific Northwest Iaboratory

D. A. Dickman

B. H. Killand

M. W. HoCoy

PNL Technical Files

$\mathrm{K} 8-58$

P7-79

P7 -79

R1-11

West inghouse Hanford Company

L. R. Ahrens

M. D. Aichele

S. J. Amir

J. D. Anderson

(2)

H. C. Boynton

F. M. Coony

L. P. Diediker

J. J. Dorian

D. R. Duncan

H. P. Emereon

C. D. Fulwyler

R. J. Giroir

D. L. Hagel

W. H. Hamilton

P. L. Hapke

X. I. HLadek

J. H. Hummer

N. C. Jasen

E. P. Mertene

D. C. Mecann

16-07

T4-04

T3-04

T3-04

T3-04

H5-33

T1-30

H6-20

H5-33

T4-03

T7-37

T4-05

H5-33

N3-10

T4-05

H5-33

G2-02

56-17

N3-13

B1-18

M. M. Mccarthy

J. H. Nielsen 
WHC-EP-0125-6

DIsTriburros (cont.)

Number of Copieg

ONSITE

Westinghouse Hanford company (cont.)

$\begin{array}{ll}\text { B. E. Poremba } & T 4-03 \\ \text { D. B. Powell, Jr. } & \text { T4-03 } \\ \text { J. G. Riddelie } & T 3-28 \\ \text { R. J. Roberte } & \text { N3-13 } \\ \text { N. R. Nilils } & T 3-04 \\ \text { Central Files } & \text { L8-04 } \\ \text { OsTI (2) } & \text { L8-07 }\end{array}$

